Bernd Slaghuis

\title{
Vertragsmanagement für Investitionsprojekte
}




\section{Bernd Slaghuis}

\section{Vertragsmanagement für Investitionsprojekte}

Projekte als meist einmalige, neuartige sowie langfristige und mit hohen Zahlungen verbundene Vorhaben erfordern ein an den Projekt- und Unternehmungszielen ausgerichtetes Projektmanagement. Ein explizites Vertragsmanagement mit methodischer Unterstützung findet selten statt. Diese Arbeit zeigt ein Konzept auf, welches die in Investitionsprojekten vorliegenden Kooperationsmerkmale in die Vertrags- und Projektplanung des Auftraggebers integriert. Die theoretischen und die Thematik systematisch erweiternden Ansätze werden fortlaufend durch ein Beispiel veranschaulicht. Der Auftraggeber kann durch die weiterentwickelten Planungsmodelle unter Berücksichtigung der vermuteten Präferenzen des Auftragnehmers die Konsequenzen verschiedener Zahlungsvereinbarungen bestimmen und ein aus seiner Sicht optimales Vertragsdesign festlegen.

Bernd Slaghuis, geboren 1972 in Mönchengladbach, studierte nach seiner Ausbildung zum Bankkaufmann Wirtschaftswissenschaft an der Ruhr-Universität Bochum. Nach dem Abschluss als Diplom-Ökonom arbeitete er ab 2001 als Wissenschaftlicher Mitarbeiter am Lehrstuhl für Betriebswirtschaftslehre, insbesondere Unternehmensforschung und Rechnungswesen, an der Ruhr-Universität Bochum. Die Promotion erfolgte 2005. 
Vertragsmanagement für Investitionsprojekte 


\section{BOCHUMER BEITRÄGE ZUR UNTERNEHMENSFÜHRUNG}

Herausgegeben von

Prof. Dr. Michael Abramovici, Prof. Dr. Dr. h.c. mult. Walther Busse von Colbe,

Prof. Dr. Dr. h.c. Werner H. Engelhardt, Prof. Dr. Roland Gabriel, Prof. Dr. Gert Laßmann, Prof. Dr. Wolfgang Maßberg, Prof. Dr. Bernhard Pellens, Prof. Dr. Marion Steven, Prof. Dr. Rolf Wartmann, Prof. Dr. Brigitte Werners

Herausgegeben

vom Direktorium des Instituts für Unternehmensführung

der Ruhr-Universität Bochum

Band 71

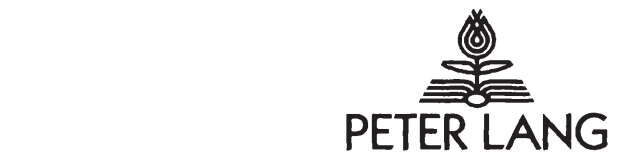

Frankfurt am Main - Berlin - Bern - Bruxelles - New York - Oxford - Wien 
Bernd Slaghuis

\section{Vertragsmanagement für Investitionsprojekte}

Quantitative Projektplanung zur Unterstützung des Contract Managements unter Berücksichtigung

von Informationsasymmetrie

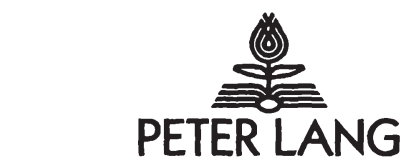

Europäischer Verlag der Wissenschaften 


\title{
Bibliografische Information Der Deutschen Bibliothek
}

Die Deutsche Bibliothek verzeichnet diese Publikation in der Deutschen Nationalbibliografie; detaillierte bibliografische

Daten sind im Internet über <http://dnb.ddb.de> abrufbar.

Open Access: The online version of this publication is published on www. peterlang.com and www.econstor.eu under the international Creative Commons License CC-BY 4.0. Learn more on how you can use and share this work: http://creativecommons.org/licenses/by/4.0.

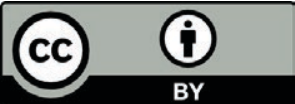

This book is available Open Access thanks to the kind support of ZBW Leibniz-Informationszentrum Wirtschaft.

Zugl.: Bochum, Univ., Diss., 2005

Gedruckt auf alterungsbeständigem, säurefreiem Papier.

\author{
D 294 \\ ISSN 1860-479X \\ ISBN 3-631-54210-0 \\ ISBN 978-3-631-75513-6 (eBook) \\ (c) Peter Lang $\mathrm{GmbH}$ \\ Europäischer Verlag der Wissenschaften \\ Frankfurt am Main 2005 \\ Alle Rechte vorbehalten.
}

Das Werk einschließlich aller seiner Teile ist urheberrechtlich geschützt. Jede Verwertung außerhalb der engen Grenzen des

Urheberrechtsgesetzes ist ohne Zustimmung des Verlages unzulässig und strafbar. Das gilt insbesondere für Vervielfältigungen, Übersetzungen, Mikroverfilmungen und die Einspeicherung und Verarbeitung in elektronischen Systemen.

Printed in Germany $1234 \quad 67$

www.peterlang.de 


\section{Geleitwort}

Zur Unterstützung des Projektmanagements wurde eine Vielfalt quantitativer Modelle und Methoden entwickelt, welche teils sehr aktuelle Problemstellungen lösen, teils seit vielen Jahren erfolgreich und weit verbreitet eingesetzt werden. Über die klassischen Netzplantechniken hinaus, die insbesondere den Projektablauf strukturieren, lassen sich beispielsweise mittels mathematischer Optimierungsmodelle knappe Ressourcen optimal einsetzen, unter Beachtung von Zahlungszeitpunkten Kapitalwerte optimieren oder auch die Projektsteuerung unter Berücksichtigung zeitlicher Risiken unterstützen. Für die Gestaltung von Verträgen für die Durchführung umfangreicher Investitionsprojekte finden derartige Ansätze bisher nur äußerst geringe Verwendung, obwohl sie ein hohes Potenzial für die Entscheidungsunterstützung des Contract Managements bieten. Bestimmungen hinsichtlich der Zahlungshöhen und Zahlungszeitpunkte und Bedingungen für deren Festlegung im Projektablauf sind sowohl für Auftragnehmer als auch Auftraggeber von wesentlichem Einfluss auf den jeweiligen Projekterfolg, wobei deren Interessen nur zum Teil übereinstimmen. Ansätze zur Unterstützung des Vertragsmanagements sollten daher auch diese Aspekte explizit berücksichtigen und Konsequenzen quantitativ ausweisen, um den Interessen der Beteiligten gerecht zu werden.

Herr Dr. Slaghuis untersucht in seiner hervorragenden Arbeit mittels quantitativer Methoden und ihrer Ergebnisse, welche Konsequenzen aus unterschiedlichen typischen Vertragsgestaltungen für Auftraggeber und Auftragnehmer resultieren. Dazu sind die entscheidungstheoretischen Erkenntnisse zur Berücksichtigung konfliktärer Interessen der Vertragspartner, auch vor dem Hintergrund asymmetrischer Informationen, in die Modelle zu integrieren. Ziel ist die Entwicklung eines allgemeinen Vorgehensrahmens zur Unterstützung eines Auftraggebers bei der Entwicklung des in einer spezifischen Situation für ihn günstigsten Vertrags unter Beachtung der Anforderungen des Auftragnehmers und der optimalen Steuerung dessen Verhaltens. Dieses allgemeine theoretische Konzept ist in der konkreten Anwendung unter Berücksichtigung der einzelnen Vertragsinhalte auszugestalten, die umfangreich in der Literatur empirisch basiert vorgeschlagen werden und auf die in dieser Arbeit hingewiesen wird.

Die neuartige, theoretisch sowie praktisch interessante betriebswirtschaftliche Problematik der Unterstützung des Contract Managements mittels quantitativer Methoden wird auf hohem wissenschaftlichem Niveau behandelt. Quantitative Konsequenzen der 
theoretischen Ausführungen werden jeweils begleitend anhand eines Beispiels mit Visualisierung didaktisch sehr gut gelungen veranschaulicht, wozu jeweils umfangreiche Berechnungen mittels Standardsoftware zur linearen Optimierung durchgeführt wurden. Ergebnis dieser Arbeit ist zum einen ein allgemeines Konzept zur systematischen Ermittlung eines optimalen Vertragstyps einschließlich der dazu erforderlichen mathematischen Optimierungsmodelle, welcher die Interessen des Auftragnehmers auf Grundlage eines hierarchischen Prinzipal-Agenten Konzepts berücksichtigt. Zum anderen ist diese Vorgehensweise anhand des umfangreichen Beispiels demonstrativ realisiert worden, sodass die Umsetzung des theoretischen Konzepts in einer Anwendung nach entsprechender Datenbereitstellung möglich ist. In einer konkreten Situation kann aufbauend auf den bereitstehenden Daten ein Projektauftraggeber fundiert bei der Entwicklung des Designs für einen Projektvertrag unterstützt werden, welcher unter Einbeziehung der Anforderungen und des Verhaltens des Auftragnehmers die Ergebnisse des Auftraggebers optimiert. Diese Kombination des Vertragsdesigns mit den quantitativen Planungsmöglichkeiten der mathematischen Optimierung zur Projektplanung ist innovativ und stellt einen besonderen wissenschaftlichen Fortschritt dar, welcher theoretische Erkenntnisse der Betriebswirtschaftslehre für zukünftige Anwendungen erschließt. Damit ist dieser Arbeit eine hohe Beachtung in Theorie und Praxis zu wünschen.

Prof. Dr. Brigitte Werners 


\section{Vorwort}

Die vorliegende Arbeit entstand während meiner Tätigkeit als wissenschaftlicher Mitarbeiter am Lehrstuhl für Betriebswirtschaftslehre, insbesondere Unternehmensforschung und Rechnungswesen, an der Ruhr-Universität Bochum und wurde im Oktober 2004 von der Fakultät für Wirtschaftswissenschaft als Dissertation angenommen. Mein Dank gebührt all denjenigen, die mich in dieser Zeit begleitet und unterstützt haben.

Besonders bedanken möchte ich mich bei Frau Prof. Dr. Brigitte Werners. Sowohl die Diskussionen und wertvollen Anregungen im Rahmen der Betreuung meiner Arbeit als auch die mit den Lehrstuhlaufgaben verbundene gute und vertrauensvolle Zusammenarbeit haben zum Gelingen der Arbeit beigetragen und diesen Abschnitt meiner Ausbildung positiv geprägt. Herrn Prof. Dr. Roland Gabriel danke ich für die Übernahme des Korreferates. Bei Herrn Prof. Dr. Manfred Lösch bedanke ich mich für die Bereitschaft, die Funktion eines Prüfers in der Disputation zu übernehmen. Ich danke dem Institut für Unternehmensführung der Ruhr-Universität Bochum für die Aufnahme meiner Arbeit in die Schriftenreihe.

Allen wissenschaftlichen Mitarbeitern des Lehrstuhls danke ich für das harmonische, kollegiale Verhältnis. Besonders zu nennen ist Stephanie Freiwald, die zeitgleich mit mir diesen Weg am Lehrstuhl begonnen hat. Unseren ständigen gegenseitigen Motivationsanstrengungen ist es zu verdanken, dass wir unsere Arbeit jeweils in relativ kurzer Zeit anfertigen konnten. Für die zahlreichen, langen Diskussionen und für das Korrekturlesen meiner Arbeit bin ich ihr zu Dank verpflichtet. Den studentischen Mitarbeitern und Mitarbeiterinnen danke ich für die tatkräftige Unterstützung auch bei den eher mühseligen Tätigkeiten, die mit der Anfertigung einer Dissertation verbunden sind. Ich möchte hier stellvertretend Ann-Kristin Klas, Jens Kanacher und Thomas Wülfing nennen. Unserer Sekretärin Inge Spieker danke ich für die schöne, auch manchmal sehr erheiternde Zusammenarbeit.

Bei meinen Verwandten und Freunden möchte ich mich bedanken, dass sie während der letzten Monate vor der Abgabe der Arbeit Verständnis aufgebracht haben, als ich mein Privatleben stark eingeschränkt habe. Schließlich gebührt meinen Eltern ein besonders herzlicher Dank dafür, dass sie meine Ausbildung ermöglicht und mich auf allen privaten und beruflichen Wegen jederzeit unterstützt und begleitet haben. 
Bernd Slaghuis - 978-3-631-75513-6 


\section{Inhaltsverzeichnis}

Abbildungsverzeichnis XIII

Tabellenverzeichnis XVII

$\begin{array}{ll}\text { Symbolverzeichnis } & \text { XIX }\end{array}$

1 Einleitung 1

2 Einfuihrung in das Management von Projektkooperationen 5

2.1 Grundlagen des Projektmanagements 6

2.1.1 Merkmale eines Projektmanagements für Investitionsprojekte 6

$\begin{array}{ll}\text { 2.1.2 Zielgrößen des Projektmanagements } & 10\end{array}$

2.1.3 Ziele und Aufgaben der Projektplanung 12

2.2 Die Kooperation zwischen Auftraggeber und Auftragnehmer 17

$\begin{array}{ll}\text { 2.2.1 Projektkooperationen in Organisationen } & 17\end{array}$

$\begin{array}{ll}\text { 2.2.2 Organisationsstrukturen für Projektkooperationen } & 21\end{array}$

2.2.3 Die Kooperationsbeziehung aus entscheidungstheoretischer Sicht 25

2.3 Das Verhalten von Auftraggeber und Auftragnehmer 28

2.3.1 Die Rolle des Auftraggebers in der Kooperationsbeziehung 28

2.3.2 Die Entscheidung des Auftragnehmers über seinen Arbeitseinsatz 29

2.3.3 Möglichkeiten der Beeinflussung des Auftragnehmerverhaltens 32

3 Vertragsmanagement als Bestandteil des Projektmanagements 35

3.1 Einführung in das Vertragsmanagement 36

3.1.1 Der Vertrag als Grundlage einer Kooperation 36

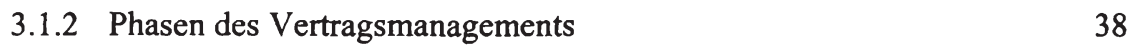

3.1.3 Die Problematik unvollständiger Verträge 43

3.2 Informationsasymmetrie bei Projektkooperationen 48

3.2.1 Ursachen für das Vorliegen von Informationsasymmetrie 48

3.2.2 Prinzipal-Agenten-Probleme bei Projektkooperationen 50

3.2.3 Die Projektkooperation aus der Sicht der hierarchischen Planung 55

3.2.4 Die Kooperations- und Anreizkompatibilitätsbedingungen 57

3.2.5 Informationsmanagement als Teil des Kooperationsmanagements 64 
3.3 Vertragsdesign für Investitionsprojektkooperationen 71

3.3.1 Contracting für das Projektmanagement 71

$\begin{array}{lll}\text { 3.3.2 Kostenerstattungsverträge } & 74\end{array}$

$\begin{array}{lll}3.3 .3 & \text { Festpreisverträge } & 77\end{array}$

3.4 Einbindung von Anreizsystemen in das Vertragsdesign $\quad 80$

3.4.1 Die Bedeutung von Anreizsystemen für die Projektkooperation $\quad 80$

3.4.2 Aufteilung von Projektrisiken durch Verträge mit 83 Anreizsystemen

4 Quantitative Planungsunterstützung für das Management von 91 Projektkooperationen

4.1 Quantitative Methoden der Projektplanung bei Kapitalwertmaximierung 92

4.2 Das Project Scheduling Problem und seine Anwendungen 97

4.2.1 Problemstellung und Stand der Forschung 97

4.2.2 Analyse und Bewertung der Problemcharakteristika 112

$\begin{array}{ll}\text { 4.2.3 Modellierung des Project Scheduling Problems } & 115\end{array}$

4.3 Das Payment Scheduling Problem und seine Anwendungen 119

$\begin{array}{ll}\text { 4.3.1 Problemstellung und Stand der Forschung } & 119\end{array}$

4.3.2 Analyse und Bewertung der Problemcharakteristika 123

4.3.3 Modellierung des Payment Scheduling Problems 126

4.4 Anwendung der Projektplanung anhand eines Beispiels 134

$\begin{array}{ll}\text { 4.4.1 Darstellung des Anwendungsbeispiels } & 134\end{array}$

4.4.2 Ergebnisse des Project Scheduling Problems 136

$\begin{array}{ll}\text { 4.4.3 Ergebnisse des Payment Scheduling Problems } & 138\end{array}$

4.5 Kritische Würdigung 141

$5 \quad$ Einbindung des Vertragsdesigns in die Projektplanung des 143 Auftraggebers

5.1 Projektplanung bei Kostenerstattungsverträgen 144

5.1.1 Erweiterung des Payment Scheduling Modells um relevante $\quad 144$ Vertragscharakteristika

5.1.2 Einfluss der Anzahl der Zahlungen und des Kostenerstattungs- $\quad 147$ faktors auf den Projekterfolg 
5.2 Projektplanung bei Festpreisverträgen 155

5.2.1 Entwicklung von Zahlungsvereinbarungen für Festpreisverträge 155

5.2.2 Projektplanung des Auftraggebers bei Festpreisvereinbarungen 158

5.3 Vergleichende Analyse und Beurteilung der Ergebnisse 163

5.4 Integration der Kooperationsbedingung des Auftragnehmers in die $\quad 166$ Projektplanung

5.4.1 Einbindung der Kooperationsbedingung in das Payment 166 Scheduling

5.4.2 Die Kooperationsbedingung bei Kostenerstattungsverträgen $\quad 168$

5.4.3 Die Kooperationsbedingung bei Festpreisverträgen 173

$\begin{array}{ll}\text { 5.5 Payment Scheduling unter Berücksichtigung des } & 178\end{array}$ Auftragnehmerverhaltens

5.5.1 Erweiterung der Problemstellung um alternative Arbeitsniveaus $\quad 178$

5.5.2 Einbindung der Verhaltensantizipation in das Payment 182 Scheduling

5.5.3 Antizipierendes Payment Scheduling bei Kostenerstattungs- $\quad 185$ verträgen

5.5.4 Antizipierendes Payment Scheduling bei Festpreisverträgen 199

5.6 Ergebnisse der Planungsunterstützung für das Vertragsdesign 212

6 Zusammenfassung und Ausblick 215

$\begin{array}{ll}\text { Literaturverzeichnis } & 221\end{array}$ 
Bernd Slaghuis - 978-3-631-75513-6 


\section{Abbildungsverzeichnis}

Abb. 1.1: Aufbau der Arbeit 2

$\begin{array}{lll}\text { Abb. 2.1: } & \text { Projektmanagement als Führungs- und Organisationskonzept } & 7\end{array}$

Abb. 2.2: Phasen des Projektmanagements 8

Abb. 2.3: Erfolgsfaktoren des Projektmanagements für Investitionsprojekte 11

Abb. 2.4: Verdichtung von Netzplänen unterschiedlicher Planungsebenen 14

Abb. 2.5: Zusammenhang von Kooperation und Koordination sowie deren 19 Einflussfaktoren

Abb. 2.6: Phasen einer Projektkooperation 20

Abb. 2.7: Organisationsform der Generalunternehmerschaft 24

Abb. 2.8: Verknüpfung der Aktionen von Auftraggeber und 26

Auftragnehmer

Abb. 2.9: Entscheidung über den Arbeitseinsatz des Auftragnehmers 30

Abb. 2.10: Einflussfaktoren für das Arbeitsverhalten 31

Abb. 2.11: Motivationsprozess des Auftragnehmers 32

Abb. 3.1: Forschungsrichtungen ökonomischer Vertragstheorie 36

Abb. 3.2: Phasen des Vertragsmanagements 39

Abb. 3.3: Hierarchische Beziehung zwischen den Kooperationspartnern 55

Abb. 3.4: Informationsasymmetrie bei hierarchischen Auftraggeber- 56

Auftragnehmer-Beziehungen

Abb. 3.5: First- und Second-Best-Lösungen des Prinzipal-Agenten- 62 Problems

Abb. 3.6: Aufgaben des Informationsmanagements 65

Abb. 3.7: Einsatz von Informations- und Kommunikationssystemen in 67 Kooperationen

Abb. 3.8: Informationsmanagement aus der Sicht von Transaktionskosten- 68 und Agency-Theorie

Abb. 3.9: Anwendungsbereiche des Informationsmanagements bei 70 Projektkooperationen

Abb. 3.10: Kostenerstattung mit festem Honorar (CPFF) 75

Abb. 3.11: Kostenerstattung mit prozentualem Honorar (CPPC) 75

Abb. 3.12: Zusammenhang von Kosten, Preis und Honorar bei 78

Festpreisverträgen

Abb. 3.13: Das Anreizsystem aus verhaltenswissenschaftlicher Sicht $\quad 80$

Abb. 3.14: Festpreisvereinbarung mit Anreizsystem 85

Abb. 3.15: Einflussfaktoren auf die Aufteilung von Projektrisiken 89 
Abb. 4.1: Beispiel zum Project Scheduling 97

Abb. 4.2: Terminierung der Vorgänge zu frühestmöglichen 98

Startzeitpunkten

Abb. 4.3: Positive und negative Nettozahlungen in Abhängigkeit von der 104 Ereigniszeit

Abb. 4.4: Barwert bei positiven/negativen Zahlungen in Abhängigkeit von 105 der Ereigniszeit

Abb. 4.5: Konstante Zahlungsfunktionen und Barwertfunktionen 106

Abb. 4.6: Linear fallende Zahlungsfunktionen und Barwertfunktionen 106

Abb. 4.7: Activity-Profit-Curve 108

Abb. 4.8: Ende-Start-Beziehung mit Zeitabstand in einem 110

Vorgangsknotennetzplan

Abb. 4.9: Ende-Start-Beziehung mit Zeitabstand als Balkendiagramm 110

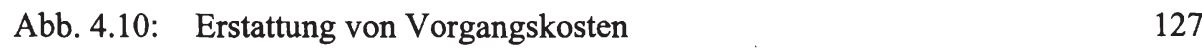

Abb. 4.11: Projektplan des Anwendungsbeispiels 135

Abb. 4.12: Optimale Lösung des Project Scheduling Problems 137

Abb. 4.13: Optimale Lösung des Payment Scheduling Problems 139

Abb. 5.1: Kapitalwerte des Auftraggebers bei Kostenerstattungsfaktoren 148 $\beta \leq 1,0$

Abb. 5.2: Kapitalwerte von Auftraggeber und Auftragnehmer bei 150

Kostenerstattung

Abb. 5.3: Kapitalwerte des Auftraggebers bei Kostenerstattungsfaktoren 153 $\beta>1,0$

Abb. 5.4: Kapitalwerte des Auftraggebers bei prozentualem oder festem $\quad 154$ Honoraraufschlag

Abb. 5.5: Zahlungsvereinbarungen für Festpreisverträge 156

Abb. 5.6: Konstante Zahlungsintervalle in Abhängigkeit von der Anzahl 160 der Zahlungen

Abb. 5.7: Mögliche Zahlungszeitpunkte des Anwendungsbeispiels 161

Abb. 5.8: Kapitalwerte des Auftraggebers bei Festpreisvereinbarungen $\quad 163$

Abb. 5.9: Kapitalwerte des Auftraggebers bei Kostenerstattungs- oder 165

Festpreisvereinbarungen

Abb. 5.10: Höhe des Festpreises bei einer Zahlung in Abhängigkeit von dem Zahlungszeitpunkt 
Abb. 5.11: Kapitalwertdifferenzen zu maximalem Kapitalwert bei einer

Zahlung

Abb. 5.12: Höhe der Festpreise und untere Festpreisgrenze

177

Abb. 5.13: Erlöse in Abhängigkeit von dem Projektende

182

Abb. 5.14: Vierstufiges Vorgehen für die Festlegung des Vertragsdesigns

183

Abb. 5.15: Bestimmung des optimalen Faktors ANTD bei Kostenerstattung auf Stufe 4

Abb. 5.16: Kapitalwerte des Auftragnehmers bei unterschiedlichen Faktoren ANTD bei einer Kostenerstattungsvereinbarung

Abb. 5.17: Bestimmung des optimalen Faktors ANTD bei

Festpreisvereinbarung auf Stufe 4

Abb. 5.18: Kapitalwerte des Auftraggebers in Abhängigkeit von der

Entscheidung des Auftragnehmers bei Festpreisvereinbarungen für Stufe 4 
Bernd Slaghuis - 978-3-631-75513-6 


\section{Tabellenverzeichnis}

Tab. 3.1: Informationsasymmetrie und resultierende Vertragsprobleme $\quad 54$

$\begin{array}{lll}\text { Tab. 3.2: Literaturüberblick Contract Management und Vertragsdesigns } & 73\end{array}$

Tab. 4.1: Literaturüberblick Project Scheduling und 95

Kapitalwertmaximierung

Tab. 4.2: Literaturüberblick Charakteristika Project Scheduling 113

Tab. 4.3: Literaturüberblick Charakteristika Payment Scheduling 124

Tab. 4.4: Kosten aus Sicht von Auftragnehmer und Auftraggeber 128

Tab. 4.5: Daten des Anwendungsbeispiels 135

Tab. 5.1: Optimale Zahlungen und Zahlungszeitpunkte bei Kostenerstattung 149 $(\beta=1,0)$

Tab. 5.2: Kapitalwerte von Auftraggeber und Auftragnehmer bei 150

Kostenerstattung $(\beta=1,0)$

Tab. 5.3: Kostenerstattung mit prozentualem oder festem Honoraraufschlag 153

Tab. 5.4: Ergebnisse der Festpreisvereinbarungen bei vier Zahlungen 162

Tab. 5.5: Charakteristika der betrachteten Zahlungsvereinbarungen 164

Tab. 5.6: Teilmenge optimaler Lösungen bei Kostenerstattung und 170

Kooperationsbedingung

Tab. 5.7: Ergebnisse bei Minimierung des Festpreises und 176

Kooperationsbedingung

Tab. 5.8: Vorgangsdauern und -kosten als Konsequenz des Arbeitsniveaus 181

Tab. 5.9: Frühestmögliches Projektende in Abhängigkeit von dem 181 Arbeitsniveau

Tab. 5.10: Ergebnisse für das geplante Arbeitsniveau bei Kostenerstattung 191

Tab. 5.11: Ergebnisse antizipierendes Payment Scheduling bei 194

Kostenerstattung für Stufe 2

Tab. 5.12: Ergebnisse antizipierendes Payment Scheduling bei

Kostenerstattung für Stufe 3

Tab. 5.13: Ergebnisse für das geplante Arbeitsniveau bei

Festpreisvereinbarung

Tab. 5.14: Ergebnisse antizipierendes Payment Scheduling bei

Festpreisvereinbarung für Stufe 2

Tab. 5.15: Ergebnisse antizipierendes Payment Scheduling bei

Festpreisvereinbarung für Stufe 3

Tab. 5.16: Ergebnisse antizipierendes Payment Scheduling bei

Festpreisvereinbarung für Stufe 3 ohne Bindung von Zahlungsund Ereigniszeitpunkten 
Bernd Slaghuis - 978-3-631-75513-6 


\section{Symbolverzeichnis}

a

$a^{\prime}$

$\mathrm{a}^{\mathrm{AN}}$

$\mathrm{a}_{\text {plan }}$

arg

AG

AN

ANRD

ANTD

ANTFP

ANTK

ANZ

$\beta$

BW

CPFF

CPIF

CPPC

$D_{i j}$

$\mathrm{D}_{\mathrm{ija}}$

DISKF $_{\mathrm{t}}$ Diskontierungsfaktor zur Abzinsung einer Zahlung zum Zeitpunkt $\mathrm{t}$

DISKZ Diskontierungszinssatz

DMax Spätesterlaubtes Projektende

$\mathrm{E}_{\mathrm{i}}$

$\mathrm{E}_{\text {ia }}$

ERL

$\mathrm{ERL}_{\mathrm{t}}$

festp

FESTP

FFP

FP

FPI

g(a)

G

Arbeitsniveau des Auftragnehmers

Antizipiertes Arbeitsniveau

Optimale Handlungsentscheidung des Auftragnehmers

Geplantes Arbeitsniveau

Argument einer Funktion

Auftraggeber

Auftragnehmer

Höhe der Anreizzahlung (Dauer) zum Zeitpunkt t bei Arbeitsniveau a

Bonus/Strafe bei Unter-/Überschreitung des geplanten Projektendes

Prozentualer Anteil des Festpreises

Bonus/Strafe bei Unter-/Überschreitung der geplanten Kosten

Anzahl der Teilzahlungen

Kostenerstattungsfaktor

Barwert

Cost-plus-fixed-fee

Cost-plus-incentive-fee

Cost-plus-percentage-of-cost

Dauer des Vorgangs zwischen Ereignis i und Ereignis $\mathbf{j}$

Dauer des Vorgangs zwischen Ereignis $i$ und Ereignis $j$ bei

Arbeitsniveau a

Frühestmöglicher Zeitpunkt des Ereignisses i

Frühestmöglicher Zeitpunkt des Ereignisses i bei Arbeitsniveau a

Erlöse des Auftraggebers nach Projektabschluss

Erlöse des Auftraggebers nach Projektabschluss zu dem Zeitpunkt $t$

Entscheidung über die Höhe des Festpreises

Höhe des Festpreises

Firm-fixed-price

Festpreisvereinbarung

Fixed-price-incentive

Nutzen aus dem Arbeitseinsatz für den Auftragnehmer

Gewinn 
GE Geldeinheiten

hon ${ }_{t} \quad$ Entscheidung über die Höhe des Auftragnehmerhonorars zum

Zeitpunkt $\mathrm{t}$

$\mathrm{HON}_{\mathrm{t}} \quad$ Festes Auftragnehmerhonorar zum Zeitpunkt $\mathrm{t}$

i Ereignis

I Bezeichnung für das letzte Ereignis

$I_{t}^{A G} \quad$ Informationen des Auftraggebers zum Zeitpunkt $t$

$\mathrm{I}_{\mathrm{t}}^{\mathrm{AN}} \quad$ Informationen des Auftragnehmers zum Zeitpunkt $t$

IN $^{*} \quad$ Optimale Instruktion des Auftraggebers an den Auftragnehmer

INT Zeitintervalle für Zahlungen

$\mathrm{k} \quad$ Index der Anzahl der Teilzahlungen

$\mathrm{K}_{\mathrm{i}}^{\mathrm{AN}} \quad$ Kosten Ereignis i aus der Sicht des Auftragnehmers

$\mathrm{K}_{\mathrm{ia}}^{\mathrm{AN}} \quad$ Kosten Ereignis i aus der Sicht des Auftragnehmers bei

Arbeitsniveau a

$\mathrm{K}_{\mathrm{i}}^{\mathrm{AG}} \quad$ Kosten Ereignis $\mathrm{i}$ aus der Sicht des Auftraggebers

$\mathrm{K}_{\mathrm{Ist}} \quad$ Tatsächlich realisierte Kosten

$\mathrm{K}_{\text {Plan }} \quad$ Geplante Kosten

$\mathrm{KE} \quad$ Kostenerstattungsvereinbarung

KW Kapitalwert

$\mathrm{KW}_{\mathrm{a}} \quad$ Kapitalwert bei Arbeitsniveau a

$\mathrm{L}_{\mathrm{i}} \quad$ Spätesterlaubter Zeitpunkt des Ereignisses i

$\mathrm{L}_{\mathrm{ia}} \quad$ Spätesterlaubter Zeitpunkt des Ereignisses i bei Arbeitsniveau a

m Vorgang

$\max \quad$ Maximum

$\widehat{M} \quad$ Große Zahl

M Bezeichnung für den letzten Vorgang

Prob Wahrscheinlichkeit

$\mathbb{R} \quad$ Menge der reellen Zahlen

$\theta \quad$ Zufallsvariable

t Zeitpunkt

$\mathbf{t}_{\mathrm{i}} \quad$ Zeitpunkt des Ereignisses $\mathrm{i}$

$\mathrm{TB}^{\mathrm{AN}} \quad$ Teilnahmebedingung des Auftragnehmers

$\mathrm{u}^{\mathrm{AG}} \quad$ Risikonutzenfunktion des Auftraggebers

$\mathrm{u}^{\mathrm{AN}} \quad$ Risikonutzenfunktion des Auftragnehmers

$\mathrm{v}(\mathrm{w}) \quad$ Nutzen der Entlohnung für den Auftragnehmer 
V Menge der einem Vorgang unmittelbar vorangehenden und unmittelbar nachfolgenden Ereignisse $(i, j)$

w Monetäre Entlohnung des Auftragnehmers durch den Auftraggeber

$\mathbf{x}_{\text {it }} \quad$ Binärvariable, die angibt, ob Ereignis i zum Zeitpunkt $t$ stattfindet

$\mathrm{x}_{\mathrm{ita}} \quad$ Binärvariable, die angibt, ob Ereignis i zum Zeitpunkt $\mathrm{t}$ bei Arbeitsniveau a stattfindet

$\mathrm{x}(\mathrm{a}, \theta) \quad$ Ergebnis aus den Handlungsentscheidungen des Auftragnehmers

$y_{t} \quad$ Binärvariable, die angibt, ob zum Zeitpunkt t eine Zahlung erfolgt

$\mathrm{y}_{\mathrm{ta}} \quad$ Binärvariable, die angibt, ob zum Zeitpunkt $\mathrm{t}$ bei Arbeitsniveau a eine Zahlung erfolgt

z Zielfunktionswert

$\mathrm{z}_{\mathrm{t}} \quad$ Entscheidung über die Höhe der Zahlung zum Zeitpunkt $\mathrm{t}$

$\mathrm{z}_{\mathrm{ta}} \quad$ Entscheidung über die Höhe der Zahlung zum Zeitpunkt $\mathrm{t}$ bei Arbeitsniveau a

$\mathrm{z}(\mathrm{t}) \quad$ Zahlungsfunktion

$Z_{t} \quad$ Feststehende Zahlung zum Zeitpunkt $t$

$\widehat{Z}_{\mathrm{i}} \quad$ Feststehende Zahlung mit Ereignis i

Zges Gesamtbetrag der Zahlungen des Auftraggebers an den Auftragnehmer 
Bernd Slaghuis - 978-3-631-75513-6 


\section{Einleitung}

Projekte als meist einmalige, neuartige sowie langfristige und mit hohen Zahlungen verbundene Vorhaben erfordern ein an den Projekt- und Unternehmungszielen ausgerichtetes Projektmanagement. Das Management insbesondere von interorganisationalen Projektkooperationen zwischen Auftraggebern und Auftragnehmern gewinnt beispielsweise im Rahmen eines Supply Chain Managements sowohl für die betriebswirtschaftliche Forschung als auch für die Unternehmungspraxis zunehmend an Bedeutung. Ein Vertragsmanagement wird im Rahmen des Projektmanagements jedoch weitgehend vernachlässigt. Hierbei sind rechtswissenschaftliche, ökonomische und verhaltenstheoretische Aspekte integrativ zu berücksichtigen.

Der Schwerpunkt dieser Arbeit liegt in der Unterstützung des Auftraggebers bei der Auswahl der Vertragsart sowie der Gestaltung des Vertrags mit einem das Projekt ausführenden Auftragnehmer. Die Entscheidung für ein Vertragsdesign hat Auswirkungen auf das durch die Informationsasymmetrie geprägte Verhalten der Kooperationspartner und beeinflusst die investitionsspezifischen Performancekriterien durch die Festlegung der Vergütungs- und Zahlungsmodalitäten. Ziel der Arbeit ist daher die Einbindung des bisher separat behandelten betriebswirtschaftlichen Forschungsgebietes des Contract Managements in die Methoden der quantitativen Projektplanung.

Zunächst erfolgt eine detaillierte Analyse der aktuellen Entwicklungen dieser sowie angrenzender Gebiete. Die anschließende Integration der speziell für große Investitionsprojekte entwickelten unterschiedlichen Vertragsdesigns in die um relevante Vertragscharakteristika erweiterten Projektplanungsmodelle zeigt die Konsequenzen für die Vertrags- und Projektplanung des Auftraggebers auf. Zusätzlich werden die Präferenzen des Auftragnehmers durch die in der Prinzipal-Agenten-Theorie diskutierten Teilnahme- und Anreizkompatibilitätsbedingungen abgebildet und bei der Planung des Auftraggebers berücksichtigt. Mittels einer Antizipation alternativer Arbeitsniveaus des Auftragnehmers durch den Auftraggeber werden Vertragsdesigns entwickelt, welche die Kooperationsbereitschaft des Auftragnehmers gewährleisten und diesen durch die Einbindung von Anreizsystemen motivieren, sich entsprechend den Präferenzen des Auftraggebers zu verhalten.

Die dieser Arbeit zugrunde liegende Struktur ist in Abbildung 1.1 dargestellt und wird im Folgenden näher erläutert: 


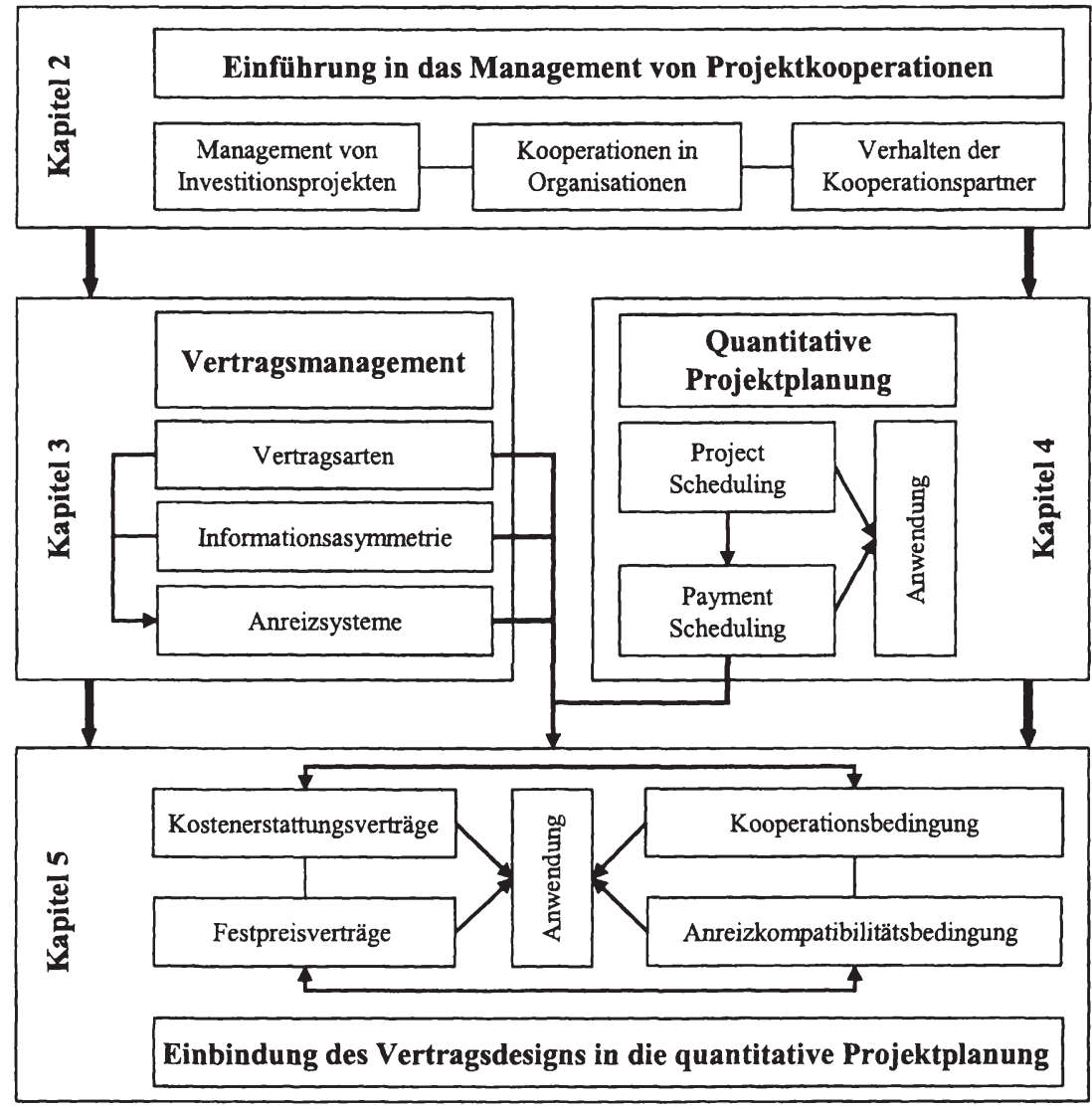

Abbildung 1.1: Aufbau der Arbeit

Im Anschluss an diese Einleitung werden im zweiten Kapitel die Ziele und Phasen des Projektmanagements vorgestellt sowie eine Definition für den Begriff des Investitionsprojektes vorgeschlagen. Die Projektplanung als Teil des Projektmanagements bildet einen Schwerpunkt der Untersuchungen, so dass in dem zweiten Kapitel auch eine Darstellung der Ziele und Aufgaben der Projektplanung erfolgt. Die Projektplanung unterstützt den Auftraggeber des Investitionsprojektes bei der Bestimmung des Vertragsdesigns. Daher werden zunächst die in dieser Arbeit betrachteten Projektkooperationen organisationstheoretisch begründet und verschiedene Arten möglicher Organisationsstrukturen aufgezeigt. Die Entscheidungen eines Kooperationsteilnehmers haben Einfluss auf die Entscheidungen bzw. das Verhalten des anderen Teilnehmers, so dass die Auftraggeber-Auftragnehmer-Beziehung aus entscheidungstheoretischer, insbesondere spieltheoretischer Sicht analysiert wird. Zum Abschluss des zweiten Kapitels werden sowohl die Rolle des Auftraggebers als auch die Entscheidung des Auf- 
tragnehmers hinsichtlich seines Arbeitseinsatzes betrachtet. Es wird aus verhaltenstheoretischer Sicht aufgezeigt, wie die Motivation des Auftragnehmers für dessen Anstrengungsniveau durch die Vorgabe von Anreizen durch den Auftraggeber beeinflusst werden kann.

Das dritte Kapitel behandelt die Thematik eines für ein Management von Investitionsprojekten erforderlichen Vertragsmanagements. Die Vertragsvereinbarungen zwischen dem späteren Projekteigentümer als Auftraggeber und dem mit der Erstellung der Leistungen beauftragten Auftragnehmer legen durch die Spezifikation der Leistungssowie der Zahlungs- und Vergütungsvereinbarungen den Rahmen für die Kooperation fest und sind daher maßgeblich für den Projekterfolg aus der Sicht beider Vertragsparteien verantwortlich. Im Anschluss an die Diskussion der Problematik unvollständiger Verträge werden die Ursachen für das Vorliegen asymmetrisch verteilter Informationen zwischen den Vertragspartnern sowie die aus der Prinzipal-Agenten-Theorie bekannten Lösungsansätze vorgestellt und auf die betrachtete Problemstellung übertragen. Diese Thematik wird durch das Einbeziehen der für die Kooperationsbeziehung typischen hierarchischen Organisationsstruktur ergänzt. Zusätzlich werden Möglichkeiten aufgezeigt, wie ein auf die Kooperationsbeziehung abgestimmtes Informationsmanagement dazu beitragen kann, die aus der Informationsasymmetrie resultierenden Probleme zu reduzieren. Neben diesem informations- und entscheidungstheoretischen Schwerpunkt zeigt das dritte Kapitel unterschiedliche in der Literatur auf dem Gebiet des Contract Managements diskutierte Arten des Vertragsdesigns auf. Im Anschluss an diesen Literaturüberblick werden verschiedene Alternativen einer Ausgestaltung von Kostenerstattungs- und Festpreisvereinbarungen vorgestellt sowie die damit verbundenen Chancen und Probleme für Auftraggeber und Auftragnehmer erläutert. Durch die Einbindung von Anreizsystemen in das Vertragsdesign kann der Auftraggeber die Motivation des Projektpartners so beeinflussen, dass dieser die Leistungen entsprechend seinen Zielvorstellungen erbringt. Die Thematik einer Aufteilung von Projektrisiken durch die Integration von Anreizmechanismen in das Vertragsdesign mit dem Ziel der Beeinflussung des Auftragnehmerverhaltens schließt dieses Kapitel ab.

Das vierte Kapitel beschäftigt sich mit quantitativen Methoden der Projektplanung. Einführend wird die Problematik der Projektplanung bei Kapitalwertmaximierung aufgezeigt. Ein umfassender Literaturüberblick sowie die Analyse und Bewertung der in der Literatur berücksichtigten Problemcharakteristika zeigt neben der chronologischen 
Entwicklung auch den aktuellen Stand der Forschung auf. Es lassen sich zwei unterschiedliche Ansätze der Projektplanung bei Kapitalwertmaximierung identifizieren: Das Project Scheduling Problem sowie als Erweiterung dessen das Payment Scheduling Problem. Für beide Planungsprobleme wird ein lineares Programmierungsmodell vorgeschlagen. Die Anwendung beider Modelle wird im Anschluss an einem Beispiel aufgezeigt. Das vierte Kapitel schließt mit einer kritischen Würdigung der vorgestellten quantitativen Projektplanungsansätze.

Das fünfte Kapitel führt die Untersuchungen des dritten und vierten Kapitels zusammen und zeigt, wie der Auftraggeber bei der Aufgabe der Vertragsgestaltung durch die Methoden der quantitativen Projektplanung unterstützt werden kann. Alle in diesem Kapitel theoretisch aufgezeigten Weiterentwicklungen werden fortlaufend durch die Anwendung des im vierten Kapitel vorgestellten Beispiels ergänzt und hinsichtlich ihrer Einflüsse auf den Projekterfolg von Auftraggeber und Auftragnehmer analysiert. Zunächst wird das Payment Scheduling Modell um relevante Vertragscharakteristika erweitert, wobei eine Unterscheidung in Kostenerstattungs- und Festpreisvereinbarungen erfolgt. Anschließend wird die durch die Kooperationsbeziehung begründete und im Rahmen der hierarchischen Planung formal diskutierte Teilnahmeentscheidung des Auftragnehmers in die quantitative Projektplanung des Auftraggebers integriert sowie die Konsequenzen für das Vertragsdesign von Kostenerstattungs- und Festpreisverträgen untersucht. Die Festlegung des Vertragsdesigns durch den Auftraggeber erfolgt zeitlich vor dem Projektbeginn. Das hieraus resultierende Problem der Informationsasymmetrie hinsichtlich des Auftragnehmerverhaltens als Reaktion auf das Vertragsdesign des Auftraggebers wird durch eine Antizipation möglicher Arbeitsniveaus in Verbindung einer in das Vertragsdesign eingebundenen Anreizfunktion gelöst. Hierfür wird ein vierstufiges Vorgehen vorgeschlagen und anhand der Anwendung umgesetzt. Die Arbeit schließt im sechsten Kapitel mit einer Zusammenfassung der Ergebnisse und einem Ausblick. 


\section{Einführung in das Management von Projektkooperationen}

Dieses Kapitel zeigt die für die weiterführenden Analysen notwendigen Aspekte des Kooperationsmanagements für Investitionsprojekte auf. Zunächst werden die Ziele und Phasen des Projektmanagements vorgestellt sowie eine Definition für den Begriff des Investitionsprojektes vorgeschlagen. Der Schwerpunkt der Arbeit liegt auf der Projektplanung zur Unterstützung des Contract Managements. Daher befasst sich das Kapitel 2.1.3 detailliert mit den Zielen und Aufgaben der Projektplanung, wobei insbesondere die Struktur- und Zeitplanung für Investitionsprojekte im Vordergrund stehen.

Kapitel 2.2 betrachtet die Kooperationsbeziehung zwischen dem Auftraggeber des Investitionsprojektes und dem das Projekt ausführenden Auftragnehmer. Im Anschluss an die Herleitung des für die Arbeit definierten Begriffs einer Projektkooperation wird diese in vier Phasen unterteilt sowie deren Inhalte aufgezeigt. Die Teilnehmer der Kooperation können einer Unternehmung oder verschiedenen Unternehmungen angehören. Daher werden in Kapitel 2.2.2 unterschiedliche Organisationsstrukturen für Kooperationen erläutert und diese von der im Folgenden betrachteten Struktur abgegrenzt. Die arbeitsteilige Zusammenarbeit der Projektpartner führt dazu, dass die Entscheidungen eines Kooperationsteilnehmers Einfluss auf die Entscheidungen bzw. das Verhalten des anderen Teilnehmers haben und somit eine in der Entscheidungstheorie betrachtete Spielsituation vorliegt. Diese Thematik ist daher Bestandteil des Kapitels 2.2.3.

Auftraggeber und Auftragnehmer nehmen innerhalb der Kooperationsbeziehung unterschiedliche Rollen ein. Die Rolle des Auftraggebers als Projekteigentümer wird in Kapitel 2.3.1 näher analysiert. Das darauf folgende Kapitel 2.3.2 untersucht, wie der Auftragnehmer die Entscheidung über seinen Arbeitseinsatz als Reaktion auf das Vertragsdesign triff. Dabei stehen besonders die Grundannahmen ökonomisch-psychologischen Verhaltens sowie die Motivationstheorie im Mittelpunkt der Ausführungen. Wie das aus der Motivation des Auftragnehmers resultierende Verhalten durch den Auftraggeber beeinflusst werden kann, wird in Kapitel 2.3.3 untersucht. 


\subsection{Grundlagen des Projektmanagements}

\subsubsection{Merkmale eines Projektmanagements für Investitionsprojekte}

Der Begriff Projektmanagement lässt sich in seine zwei Bestandteile Projekt und Management teilen und dementsprechend definieren. Der Projektbegriff wird nach dem Deutschen Institut für Normung e. V. definiert als „Vorhaben, das im Wesentlichen durch Einmaligkeit der Bedingungen in ihrer Gesamtheit gekennzeichnet ist, wie z. B. Zielvorgabe, zeitliche, finanzielle, personelle oder andere Begrenzungen, Abgrenzung gegenüber anderen Vorhaben, projektspezifische Organisation." "Diese von Corsten und Corsten als wenig innovativ bezeichnete Sicht wird durch unterschiedliche Definitionen des Projektbegriffs in der betriebswirtschaftlichen Literatur ergänzt. ${ }^{2}$ Eine $\mathrm{Zu}$ sammenfassung dieser Definitionen führt zu den folgenden ein Projekt charakterisierenden Merkmalen: ${ }^{3}$

- Die Projektaufgaben führen zu einem definierten Projektziel.

- Das Projekt ist durch einen definierten Anfang und ein definiertes Ende zeitlich befristet.

- Die für das Projekt verfügbaren Ressourcen sind beschränkt bzw. knapp.

- Die Projektleistungen sind aus der Sicht des Auftraggebers relativ neuartig sowie einmalig.

- Es besteht ein hoher Komplexitätsgrad durch die Anzahl der Projektbeteiligten und der Projektaktivitäten sowie deren Beziehungen untereinander und zu anderen Projekten.

Während die Merkmale der definierten Zielsetzung sowie der zeitlichen Befristung als konstitutiv bezeichnet werden, variieren die Ausprägungen der übrigen Merkmale je nach Eigenart des Projektes. ${ }^{4}$ Eine Definition des in dieser Arbeit zugrunde liegenden Projektbegriffs erfolgt am Ende des Kapitels im Rahmen der Begriffsdefinition eines Investitionsprojektmanagements. Der Managementbegriff als zweiter Bestandteil des Projektmanagementbegriffs lässt sich in einen institutionellen Ansatz sowie einen

\footnotetext{
Deutsches Institut für Normung (1987), S. 1.

Vgl. Corsten/Corsten (2000), S. 1 sowie S. 4. Eine Kritik an der DIN-Definition ubt auch Litke (2004), S. 19.

Für einen ausführlichen Überblick sowie weitere Begriffsdefinitionen vgl. beispielsweise Beck (1996), S. 42ff.; Fiedler (2003), S. 2ff.; George (1999), S. 9ff.; Gido/Clements (2003), S. 4f.; Klein (2000), S. 1f.; Meredith/Mantel (2003), S. 8f.; Project Management Institute (2000), S. 4ff.; Turner/Müller (2003), S. 2.

4 Vgl. George (1999), S. 11 sowie die dort aufgeführte Literatur.
} 
funktionalen Ansatz in der Managementlehre unterteilen. ${ }^{5}$ Hierbei umfasst das Management im funktionalen Sinn die Handlungen, die einer zielgerichteten Steuerung des Leistungsprozesses in einer arbeitsteiligen Organisation dienen. ${ }^{6}$ Für den institutionellen Managementansatz stehen dagegen die Organisationsmitglieder, die eine Vorgesetztenfunktion wahrnehmen, sowie deren Tätigkeiten und Rollen im Mittelpunkt. ${ }^{7}$ Die Funktionen des Managements umfassen die Tätigkeiten der Planung und Organisation, des Personaleinsatzes sowie der Führung und Kontrolle. ${ }^{8}$ Entsprechend diesen Begriffsdefinitionen kann das Projektmanagement als ganzheitliches Führungs- und Organisationskonzept verstanden werden, welches sowohl die für eine erfolgreiche Abwicklung von Projekten notwendigen Tätigkeiten der Planung, Steuerung und Kontrolle sowie die hierfür benötigten Methoden und Verfahren umfasst als auch durch die Schaffung einer geeigneten Organisationsstruktur den leitenden, in die Unternehmung oder in eine Kooperation eingebundenen Institutionen Aufgaben, Kompetenzen und Verantwortung zuweist. ${ }^{9}$ Dieser Zusammenhang ist in Abbildung 2.1 dargestellt:

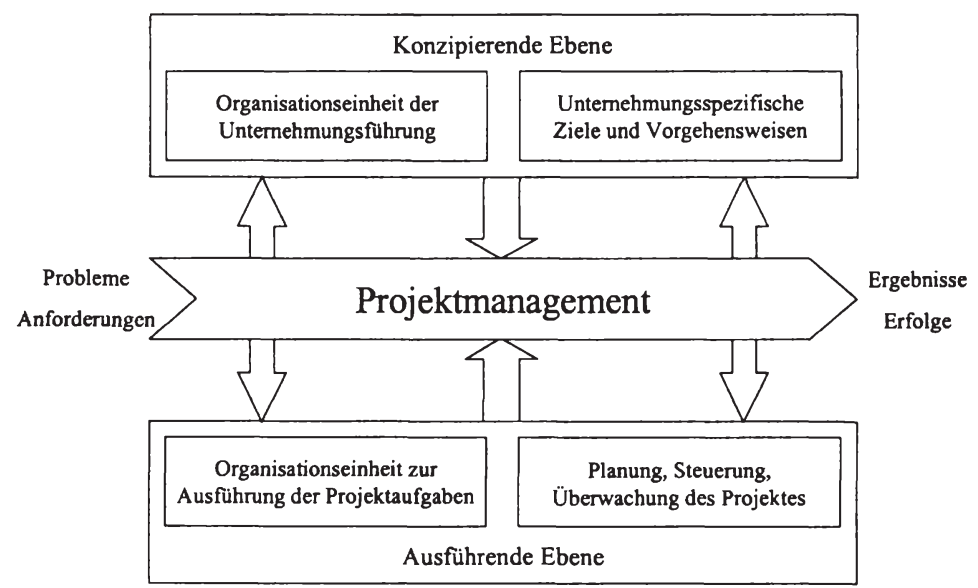

Abbildung 2.1: Projektmanagement als Führungs- und Organisationskonzept (Quelle: In Anlehnung an Keßler/Winkelhofer (2004), S. 11.)

Vgl. etwa Staehle (1999), S. 71.

Vgl. Staehle (1999), S. 89; Steinmann/Schreyögg (2000), S. $5 f$.

Vgl. Wolf (2003), S. 38f.

Vgl. etwa Weihrich/Koontz (1993), S. 17.

Vgl. ähnliche Definitionen in Fiedler (2003), S. 6; George (1999), S. 17; Litke (2004), S. 21; Corsten/Corsten (2000), S. 6ff.; Keßler/Winkelhofer (2004), S. 10f. Für eine umfassende Übersicht mit dem Projektmanagement verbundener Bereiche vgl. Cleland (2001), S. 8f. Für eine Darstellung des Projektmanagements als System vgl. Cleland (2001), S. 15; Litke (2004), S. 22. Cooke-Davies (2002), S. 189 betont, dass die Leistungen durch Personen erbracht werden und somit auch der Projekterfolg von persönlichen Faktoren beeinflusst wird. 
Die Aufgabenbereiche des Projektmanagements können, wie in Abbildung 2.2 dargestellt, in fünf unterschiedliche Phasen unterteilt werden. ${ }^{10}$ Die Vorbereitungsphase, welche zeitlich vor dem Projektstart liegt, umfasst im Anschluss an die Projektinitiierung die Durchführung von Machbarkeitsstudien sowie für den Fall der Entscheidung über die Projektdurchführung die Erstellung des Angebotes. ${ }^{11}$ Die Tätigkeiten der Gestaltung des Vertrags zwischen Auftraggeber und Auftragnehmer sowie des Vertragsabschlusses beenden die Vorbereitungsphase und sind Teil der Projektdefinitionsphase. Das dritte Kapitel wird sich detailliert mit dem Gebiet des Vertragsmanagements für Investitionsprojekte befassen.

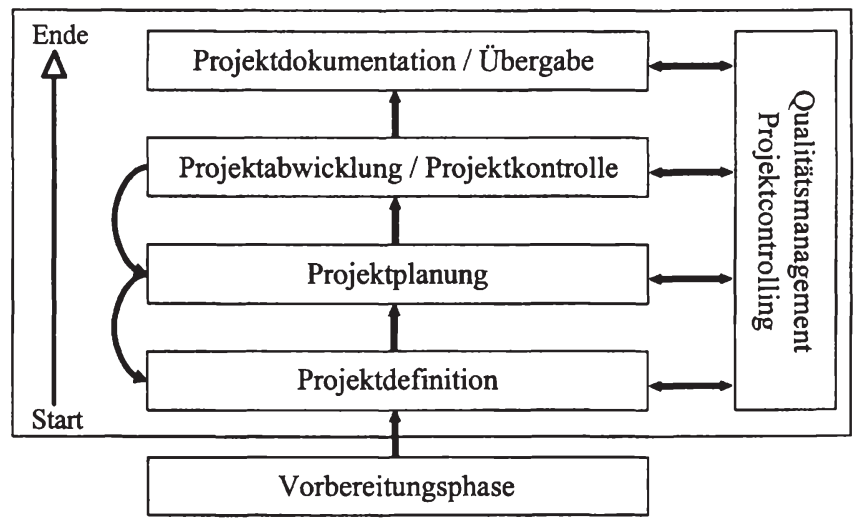

Abbildung 2.2: Phasen des Projektmanagements

Die vier zwischen definiertem Projektstart und Projektende liegenden Phasen werden durch das Qualitätsmanagement sowie das Projektcontrolling ergänzt. Das Qualitätsmanagement umfasst hierbei die präventive und ergebnisspezifische Qualitätssicherung. ${ }^{12}$ Das Projektcontrolling hat die Funktion, die ergebnisorientierte Planung, Steuerung und Kontrolle des Vorhabens durch eine die Entscheidungen unterstützende Koordination zu begleiten. ${ }^{13}$ Die in der Abbildung dargestellten Beziehungen zwischen den einzelnen Phasen des Projektmanagements zeigen neben der zeitlichen Abfolge

$10 \mathrm{Zu}$ den Phasen und Aufgaben des Projektmanagements vgl. ähnliche Darstellungen in Corsten/Corsten (2001), S. 12; Litke (2004), S. 27; Meier (2003), S. 13.

11 Vgl. Corsten/Corsten (2000), S. 12.

12 Vgl. Corsten/Corsten (2000), S. 34f.

$13 \mathrm{Zu}$ den Aufgaben des Controllings im Rahmen des Projektmanagements vgl. beispielsweise Burghardt (2002a), S. 327ff.; Fiedler (2003), S. 9ff.; George (1999), S. 25f.; Horváth (2003), S. 729ff.; Wienhold (2003), S. 46ff. 
auch die möglichen Rückkopplungen insbesondere für die Phasen der Planung und der Abwicklung/Kontrolle auf. Werden durch das Projektcontrolling etwa bei der Projektabwicklung Abweichungen zwischen Ist- und Planzuständen identifiziert, so kann dies zu einer neuen, veränderten Projektplanung führen.

Bevor der Planungsaspekt des Projektmanagements näher betrachtet wird, soll im Folgenden der Begriff des Investitionsprojektes spezifiziert werden. Der Investitionsbegriff wird in der betriebswirtschaftlichen Literatur nicht einheitlich definiert. Lücke unterscheidet vier verschiedene Begriffsgruppen, ${ }^{14}$ wobei die zwei in der Literatur am häufigsten verwendeten Definitionen den vermögensbestimmten Investitionsbegriff, nach dem eine Investition die Umwandlung von Kapital in Vermögen (Realkapital) bedeutet, ${ }^{15}$ und den zahlungsbestimmten Investitionsbegriff bilden, welcher den für eine Anschaffung eines Gutes bzw. Güterverbundes notwendigen Zahlungsstrom aus Aus- und Einzahlungen umfasst. ${ }^{16}$

Im Rahmen dieser Arbeit soll der Auffassung von Schneider und somit dem zahlungsbestimmten Investitionsbegriff gefolgt werden, nach dem bei einer Investition neben der Geldanlage mit der Zielsetzung einer zukünftigen Einnahmenerzielung auch die die Handlungen begleitenden disponierenden und produzierenden Tätigkeiten einbezogen werden. ${ }^{17}$

Der Begriff des Investitionsprojektes wird auf dem Gebiet des Projektmanagements sowie in der Investitionstheorie unterschiedlich verwendet. Der Gebrauch der Einzelbegriffe Investition und Projekt erfolgt zum Teil synonym. ${ }^{18}$ Eine Zusammenführung der hier vorgestellten Begriffsdefinitionen erlaubt an dieser Stelle eine Definition des für diese Arbeit verwendeten Investitionsprojektbegriffs:

14 Vgl. Lücke (1991), S. 151f. Zusätzlich zu den aufgefuhrten Investitionsbegriffen nennt Lücke den kombinationsbestimmten sowie den dispositionsbestimmten Investitionsbegriff. Vgl. Lucke (1991), S. 152.

15 Zum vermögensbestimmten Investitionsbegriff vgl. zusătzlich etwa Bosse (2000), S. 20; Domschke/Scholl (2003), S. 231; Mensch (2002), S. 1; Perridon/Steiner (2003), S. 27; Schmidt/Terberger (1997), S. 11.

16 Zum zahlungsbestimmten Investitionsbegriff vgl. beispielsweise Adam (2000), S. 4; Blohm/Lüder (1995), S. 2; Bosse (2000), S. 20; Busse von Colbe/Laßmann (1990), S. 2; Olfert (2003), S. 25; Perridon/Steiner (2003), S. 28; Schneider (1992), S. 10.

17 Vgl. Schneider (1992), S. 10. Schneider nennt an dieser Stelle auch die Möglichkeit der Vertragsgestaltung mit dem Zweck, die Unsicherheit zukünftiger Einnahmen und Ausgaben zu mindern.

18 Vgl. Johannwille (2000), S. 15, wo ein Investitionsprojekt ,... als zeitliche Abfolge interdependenter Investitionsentscheidungen..." definiert wird. Breuer (2002), S. 7 verwendet den Begriff ohne năhere Spezifikation. Heinrich (2002), S. 169f. schlägt vor, die Entscheidung über die Durchfuhrung von Softwareprojekten mit den Methoden der Investitionsrechnung zu betrachten. Blohm/Lüder (1995), S. 238 definieren das Investitionsprojekt als einen Verbund strategisch ausgerichteter Investitionen. Mensch (2002), S. 1 definiert eine Investition als ein Projekt und verwendet damit die Begriffe synonym. Zell (2003), S. 57 führt als Beispiele für Investitionsprojekte Bau- oder Anlagenbauprojekte auf. 
Ein Investitionsprojekt ist ein zeitlich befristetes, relativ neuartiges und meist einmaliges Vorhaben als zeitliche Abfolge komplexer Entscheidungen und Aktionen, welche zu Aus- und Einzahlungen führen mit dem Ziel, durch die langfristige Bindung von Finanzmitteln neue Nutzenpotenziale zu schaffen.

Ausgehend von dieser Definition erfolgt in dem folgenden Kapitel 2.1.2 eine Vorstellung der für das Projektmanagement relevanten, in der Literatur angewandten Zielgrößen. Im Anschluss daran werden in Kapitel 2.1.3 die im Rahmen der Projektplanung durchzuführenden Aufgaben sowie deren Bedeutung für die in dieser Arbeit untersuchte Problemstellung aufgezeigt.

\subsubsection{Zielgrößen des Projektmanagements}

Die Zielgröße als Teil eines Zielsystems wird in der Entscheidungstheorie als Eigenschaft definiert, durch die der Entscheidungsträger die Konsequenzen seiner Entscheidung hinsichtlich der Auswahl einer Handlungsalternative beschreibt. Unter Berücksichtigung einer Präferenzrelation, welche die Intensität seines Strebens nach einer Zielgröße beschreibt, kann insbesondere bei mehreren Zielgrößen die für ihn beste Handlungsalternative ausgewählt werden. ${ }^{19}$

Für das Projektmanagement werden meist drei unterschiedliche, sowohl finanzielle als auch nicht-finanzielle Zielgrößen aufgeführt: Zeit, Kosten und Qualität. Der zwischen diesen Zielgrößen bestehende Konflikt wird durch das magische Dreieck des Projektmanagements beschrieben, welches in Abbildung 2.3 dargestellt ist und zusätzlich die speziell für Investitionsprojekte relevanten Zielgrößen aufzeigt. ${ }^{20}$ Das Ziel des Managements ist somit, das Projekt innerhalb der vorgegebenen Projektdauer unter Einhaltung des Budgets sowie mit der geforderten Qualität abzuschließen. ${ }^{21}$ Der Zielkonflikt entsteht, sofern das Projekt beispielsweise für eine frühere Verfügbarkeit des Investiti-

19 Vgl. etwa Bamberg/Coenenberg (2002), S. 28; Eisenführ/Weber (2003), S. 31.

20 Das magische Dreieck wird auch als Triade des Projektmanagements bezeichnet. Vgl. beispielsweise Burghardt (2002b), S. 23; Horsch (2003), S. 21; Keßler/Winkelhofer (2004), S. 55; Ling (2004), S. 477; Litke (2004), S. 55f.; Stiasni (1994), S. 9f.; Zell (2003), S. 58. Ling (2004) fuihrt auch solche Faktoren auf, die diese Zielgrößen maßgeblich beeinflussen.

21 Dieser Zielkonflikt kann etwa durch die Formulienung eines Ziels als Extremierungsvorschrift sowie der anderen Ziele als Satisfizierungsvorschrift für ein mathematisches Modell abgebildet werden. So ist beispielsweise ein frühestmögliches Projektende unter Einhaltung des Budgets und Erfüllung des Qualitätsanspruchs möglich. Alternativ ist auch eine Leistungserstellung zu minimalen Kosten mit der geforderten Qualităt innerhalb der festgelegten Projektdauer denkbar. 
onsobjektes und somit einer früheren Markteinführung zu einem früheren Termin abgeschlossen werden soll. Dies führt durch die Beschleunigung der Aktivitäten meist zu einer Erhöhung des Ressourcenbedarfs und somit zu einer Kostensteigerung. Dieses Ziel kann jedoch auch durch das Senken des Qualitätsanspruchs und somit durch Ergebniseinbußen erreicht werden.

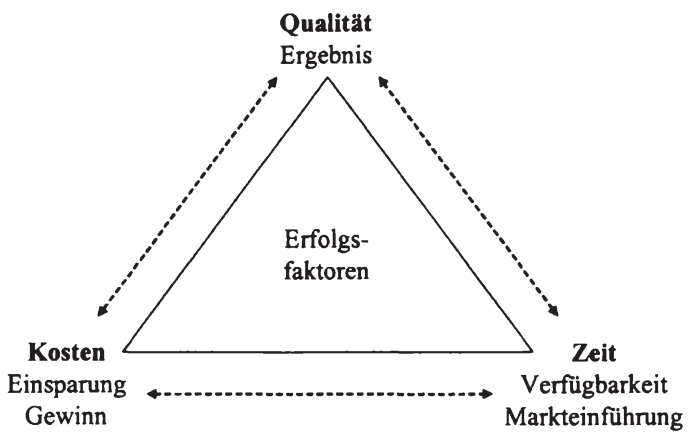

Abbildung 2.3: Erfolgsfaktoren des Projektmanagements für Investitionsprojekte (Quelle: In Anlehnung an Horsch (2003), S. 21.)

Unter Berücksichtigung von begrenzt verfügbaren Ressourcen stehen als mögliche Ziele eine gleichmäßige Ressourcenbeanspruchung oder die für die Einhaltung von Zeitvorgaben notwendige minimale Ressourcenbeanspruchung im Vordergrund. ${ }^{22}$ Aufgrund der mit dem Einsatz von Ressourcen einhergehenden Kosten sowie der Möglichkeit, die Vorgangsdauern durch den Mehr- bzw. Mindereinsatz von Ressourcen in Form unterschiedlicher Ausführungsintensitäten zu beeinflussen, handelt es sich bei diesen Problemstellungen meist um zu lösende Zeit-Kosten-Konflikte. ${ }^{23}$

Besonders bei der Auswahl, Planung und Durchführung von Projekten mit langer Laufzeit, ${ }^{24}$ deren Ausrichtung im Einklang mit strategischen Unternehmungszielen steht, ist die zeitliche Wirkung monetärer Größen sowohl für die Beurteilung der Projektperformance als auch für die Erfolgsrechnung der Unternehmung von großer Be-

22 Vgl. Demeulemeester/Herroelen (2002), S. 88ff.

23 Vgl. Erenguc/Tufekci/Zappe (1993); Werners/Wolf (2004), S. 97f.; Wolf (2004).

24 Derartige Projekte lassen sich etwa durch die charakteristischen Merkmale industrieller Anlagengeschäfte, wie sie bei Backhaus (1980), S. 2ff.; Bröker (1993), S. 5 sowie bei Funk (1986), S. 9ff. untersucht werden, beschreiben. 
deutung. ${ }^{25}$ Aus diesem Grund lassen sich in der wissenschaftlichen Literatur zum Project Scheduling zahlreiche Ansätze finden, welche anstelle des Ziels der Projektdauerminimierung die Zielsetzung der Kapitalwertmaximierung vorschlagen und somit sowohl die Höhenpräferenz als auch die Zeitpräferenz des Entscheidungsträgers erfassen. ${ }^{26}$ Auf den Kapitalwert als Kriterium zur Beurteilung von Projektplänen wird daher zu Beginn des vierten Kapitels im Rahmen der quantitativen Projektplanung für das Management von Projektkooperationen detailliert eingegangen.

\subsubsection{Ziele und Aufgaben der Projektplanung}

Die Projektplanung als dritte Phase des oben beschriebenen Projektmanagementprozesses hat das Ziel, die für die Umsetzung des Projektgegenstandes erforderlichen zukünftigen Handlungsalternativen und Verhaltensweisen gedanklich vorwegzunehmen, hinsichtlich der im Rahmen der Projektdefinition aufgestellten Zielsetzungen zu bewerten und schließlich die zu realisierenden Alternativen auszuwählen. ${ }^{27}$ Das Ergebnis des Planungsprozesses ist ein Projektplan, welcher den Ausgangspunkt der Projektabwicklung bildet und gleichzeitig die Plandaten für den im Rahmen der Projektkontrolle durchzuführenden Soll-Ist-Vergleich bereitstellt. ${ }^{28}$

Als Merkmale der Planung sind ein Zukunftsbezug, eine Prozessbezogenheit, ein systematischer Charakter sowie die Zielorientiertheit zu nennen. ${ }^{29}$ Bezogen auf die Projektplanung lassen sich deren Aufgaben in die Struktur-, Zeit- und Kapazitätsplanung

25 Vgl. Cappels (2003); Gardiner/Stewart (2000), S. 251. Der Begriff der Projektperformance wird hier in Anlehnung an das Konzept des Performance Measurement gewählt und soll insbesondere auf eine zukunftsorientierte Sicht hinweisen. Vgl. zum Performance Measurement etwa Günther/Grünig (2001); Horváth (2002); Klingebiel (1998) sowie fưr eine Anwendung im Supply Chain Management Werners/Thorn/Freiwald (2003).

26 Die Höhenpräferenz drückt aus, dass der Entscheidungsträger zum Beispiel höhere Einzahlungen niedrigeren vorzieht. Die Zeitpräferenz drückt aus, dass der Entscheidungstrăger gegenwărtige Ergebnisse höher als zukunftige Ergebnisse schätzt und somit die Zeitdimension der Ergebnisse berucksichtigt. Vgl. hierzu etwa Bamberg/Coenenberg (2002), S. 29; Johannwille (2000), S. 21. Kapitel 4 gibt einen Überblick über unterschiedliche Problemstellungen bei Kapitalwertmaximierung sowie die aktuelle Entwicklung der wissenschaftlichen Forschung auf diesem Gebiet.

27 Zum Planungsbegriff in der Betriebswirtschaftslehre vgl. etwa Adam (1996), S. 1; Domschke/Scholl (2003), S. 24ff.; Domschke/Scholl/Voß (1997), S. 1; Homburg (2000), S. 3.

28 Vgl. Corsten/Corsten (2000), S. 20. Den Zusammenhang zwischen der Projektplanung und dem Projekterfolg zeigen Dvir/Raz/Shenhar (2003) sowie Shenhar et al. (2002) durch eine empirische Studie auf. Sie kommen zu dem Ergebnis, dass Unsicherheit durch Planung reduziert und die Wahrscheinlichkeit furr einen Projekterfolg erhöht wird.

29 Vgl. Homburg (2000), S. 4. 
unterteilen. ${ }^{30}$ Diese Teilaufgaben sowie die hiermit einhergehenden Planungsprobleme werden im Folgenden näher erläutert sowie deren Relevanz für die in dieser Arbeit untersuchte Problemstellung herausgestellt.

\section{Strukturplanung}

Ziel einer Strukturierung ist die Zerlegung eines Gesamtsystems in seine Elemente sowie die Feststellung der Beziehungen zwischen den Elementen. ${ }^{31}$ Die Projektstrukturplanung nimmt hierfür eine graphische oder tabellarische Aufgliederung des Projektes in Teilprojekte vor. ${ }^{32}$ Wird dieses Vorgehen wiederholt durchgeführt, indem die Teilprojekte auf der nächsten Ebene in Unterprojekte gegliedert werden, entstehen auf der untersten Ebene des Projektstrukturplans einzelne Arbeitspakete, welche meist mehrere Vorgänge umfassen. ${ }^{33}$ Durch die Strukturierung werden die Transparenz des Projektes erhöht und Zusammenhänge aufgedeckt. Die Gliederung in Arbeitspakete soll so erfolgen, dass ein Delegieren an Organisationseinheiten und somit die Festlegung von Verantwortlichkeiten möglich ist. ${ }^{34}$ Nach Abschluss der Strukturierung des Projektes können die Vorgänge sowie deren Reihenfolgebeziehungen mit Hilfe graphentheoretischer Darstellungselemente in einem Netzplan abgebildet werden. ${ }^{35}$ Die Elemente eines Netzplans sind Knoten und Kanten, wobei je nach Darstellungsform eine unterschiedliche Zuordnung von Vorgängen und Ereignissen zu den Knoten und Kanten erfolgt. ${ }^{36}$ Für die in dieser Arbeit betrachteten Problemstellungen eignet sich eine vorgangsorientierte Darstellung, bei der die Vorgänge durch Pfeile (Kanten) und die mit dem Start bzw. Ende von Vorgängen verbundenen Ereignisse als Kreise (Knoten) visualisiert werden. ${ }^{37}$ Eine Untersuchung von Tavares zeigt, dass die Darstellung

30 Vgl. Klein (2000), S. 22ff.; Litke (2004), S. 89f;; Madauss (2000), S. 189. Zusătzlich zu den hier aufgefuhrten drei Aufgaben der Projektplanung lassen sich weitere, jedoch zum Teil diesen Aufgabenbereichen zuzuordnende Aufgaben unterscheiden: die Aufwandsschätzung als Teil der Zeit- bzw. Kapazitătsplanung, die Projektkostenplanung, Finanzierungsplanung und die Personalplanung als Teil der Kapazitatsplanung sowie die Qualitătsplanung, welche allen drei Teilaufgaben untergeordnet werden kann. Vgl. zu weiteren Aufgaben der Planung beispielsweise Heinrich (2002), S. 291ff.; Keßler/Winkelhofer (2004), S. 234ff.; Litke (2004), S. $107 \mathrm{ff}$.

31 Vgl. Litke (2004), S. 90.

32 Vgl. Madauss (2000), S. 190f.

33 Vgl. Schwarze (2001), S. 84f. Hierbei werden funktionsorientierte Projektstrukturpläne und objekt- bzw. erzeugnisorientierte Strukturpläne sowie gemischt-orientierte Strukturplăne unterschieden. Vgl. auch Keßler/ Winkelhofer (2004), S. 237.

${ }_{34}$ Vgl. Federhen/Adlbrecht (2003), S. 806f; Höffken/Schweitzer (1991), S. 69; Litke (2004), S. 91.

35 Vgl. Altrogge (1996), S. 8ff.

36 Während ein Vorgang eine als Zeit beanspruchende und zwischen einem Anfangs- und Endzeitpunkt erfolgende Tätigkeit oder Frist definiert wird, stellen Ereignisse Zeitpunkte dar, die den Beginn oder das Ende von Vorgängen und somit das Erreichen eines bestimmten Projektzustands definieren. Vgl. Schwarze (2001), S. 88f.; Zimmermann (1971), S. $11 \mathrm{f}$.

37 Zu Darstellungsformen von Netzplänen vgl. Zimmermann (1971), S. 13. 
als Vorgangspfeilnetz in der Operations Research Literatur überwiegt. ${ }^{38}$ Kolisch und Padman stellen fest, dass unter Berücksichtigung der Zielsetzung der Kapitalwertmaximierung Vorgangspfeilnetzpläne besser geeignet sind, die Problemstruktur abzubilden und als Grundlage einer Projektplanung zu dienen. ${ }^{39}$ Dies liegt darin begründet, dass sowohl die mit der Ausführung der Vorgänge entstehenden Kosten als auch die hierfür mindestens benötigten Dauern berücksichtigt und zudem durch die Darstellung der Ereignisse als Knoten die für den Projektverlauf bedeutenden Ereignisse als Meilensteine gekennzeichnet werden können. ${ }^{40}$

Für die hier betrachtete Projektplanung des Auftraggebers zur Bestimmung der Zahlungsvereinbarungen als Teil des Vertragsmanagements erfolgt eine Netzplanverdichtung. ${ }^{41}$ Eine solche Verdichtung von Netzplänen unterschiedlicher Planungsebenen ist in Abbildung 2.4 dargestellt. Die dunkel markierten Ereignisknoten kennzeichnen die für den Projektverlauf wichtigen Meilensteinereignisse.

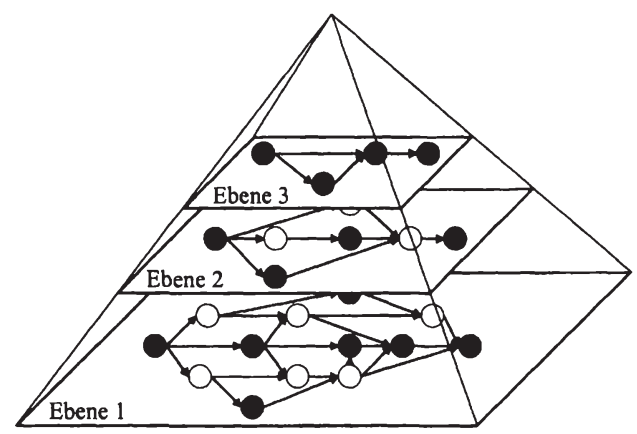

Abbildung 2.4: Verdichtung von Netzplänen unterschiedlicher Planungsebenen (Quelle: In Anlehnung an Burghardt (2002a), S. 257.)

Dieses Vorgehen kann damit begründet werden, dass der Aspekt der Zeitplanung einzelner Vorgänge aus der Sicht des Auftraggebers nicht von Interesse und aufgrund unvollständiger Informationen über die Dauern der in den Arbeitspaketen enthaltenen, durch den Auftragnehmer auszuführenden Vorgänge auch nicht möglich ist.

\footnotetext{
38 Vgl. Tavares (2002), S 3. Während in Deutschland Ereignisknotennetzpläne bevorzugt werden, sind international Vorgangspfeilnetzpläne weiter verbreitet. Vgl. Madauss (2000), S. 209.

39 Vgl. Kolisch/Padman (2001), S. 251.

40 Für eine Begriffsdefinition von Meilensteinen vgl. beispielsweise Keßler/Winkelhofer (2004), S. 241; Schwarze (2001), S. 89.

41 Vgl. Schwarze (2001), S. 139ff.
} 
Für das Entscheidungsproblem des Auftraggebers wird somit die Annahme getroffen, dass Zahlungen während der Projektabwicklung nur zu bestimmten Meilensteinen erfolgen können. Die zwischen diesen Ereignissen stattfindenden Vorgänge werden zu einem Vorgang zusammengefasst, welcher durch seine Dauer sowie die mit der Ausführung verbundenen Kosten charakterisiert wird.

Ein weiterer Aspekt der Strukturplanung liegt in der Festlegung einer geeigneten Organisationsstruktur der an dem Projekt beteiligten Organisationen, Stellen und Personen sowie der Verteilung der Kompetenzen zwischen ihnen. ${ }^{42}$ Verschiedene Formen von Projektkooperationen in Organisationen sowie eine Abgrenzung der in dieser Arbeit untersuchten Kooperationsform sind Gegenstand des Kapitels 2.2.

\section{Zeitplanung}

Das Ziel der Zeitplanung als Bestandteil der Projektplanung ist die zeitliche Festlegung der Arbeitspakete, so dass das Projekt fristgerecht abgeschlossen wird. ${ }^{43}$ Gleichzeitig wird mit der Zeitplanung ermittelt, welche Vorgänge nicht streng termingebunden sind und somit zeitlich verschoben oder ausgedehnt werden können, ohne den Projektendtermin zu verschieben. ${ }^{44}$ Sind die Ausführungsdauern der einzelnen Vorgänge bekannt, ${ }^{45}$ können mit Hilfe der Zeitrechnung beispielsweise die frühestmöglichen und spätesterlaubten Zeitpunkte von Ereignissen bzw. Vorgängen ermittelt werden. Die Ergebnisse dieser Berechnungen zeigen auch, ob ein Ereignis bzw. ein Vorgang Pufferzeiten enthält. ${ }^{46}$ Möglichkeiten einer Visualisierung zeitlicher Vorgangsabfolgen bieten Balkendiagramme beispielsweise in Form von Gantt-Charts. ${ }^{47}$

Während der Auftragnehmer eine detaillierte Zeitplanung der einzelnen für die Leistungserstellung auszuführenden Vorgänge durchführt, sind für die Planung des Auftraggebers insbesondere die Zeitpunkte der Meilensteinereignisse von Interesse, welche jedoch von den Zeitpunkten der zwischen diesen stattfindenden Vorgängen abhän-

42 Vgl. Federhen/Adlbrecht (2003), S. 807.

43 Vgl. Keßler/Winkelhofer (2004), S. 240. Fur eine Klassifikation von Problemen der Zeitplanung vgl. etwa Demeulemeester/Herroelen (2002), S. $71 \mathrm{ff}$.

44 Vgl. Schwarze (2001), S. 159.

45 Bei unbekannten Vorgangsdauern wird beispielsweise im Rahmen einer PERT-Zeitplanung eine Dreizeitenschätzung vorgenommen, bei der die häufigste, die pessimistische und die optimistische Dauer eines Vorgangs geschätzt werden und Bestandteil der Zeitplanung sind. Vgl. etwa Lockyer/Gordon (1991), S. 40ff.; Miller (1970); Schwarze (2001), S. 186ff.

46 Für eine formale Darstellung der Berechnungsvorschriften im Rahmen der Zeitplanung vgl. etwa Corsten/Corsten (2000), S. 153ff.; Homburg (2000), S. 502ff.; Klein (2000), S. 43ff.; Schwarze (2001), S. 157ff.; Werners (2000), S. 423ff.

47 Vgl. Corsten/Corsten (2000), S. 146ff. 
gen. Durch die Annahme, dass der Auftraggeber frühestens mit dem Abschluss des Projektes Erlöse aus der Projektnutzung erhält, ist speziell bei der Anwendung von Methoden der dynamischen Investitionsrechnung auch der frühestmögliche Zeitpunkt des Projektendes für die Projektplanung sowie einer Wirtschaftlichkeitsrechnung des Auftraggebers relevant.

\section{Kapazitätsplanung}

Ziel der Kapazitätsplanung ist die Ermittlung des für die Ausführung der Vorgänge benötigten Kapazitätsbedarfs sowie die Ableitung der Unter- bzw. Überbeschäftigung durch die Gegenüberstellung von Kapazitätsbedarf und verfügbaren Kapazitäten. ${ }^{48}$ Als Kapazitäten bzw. Einsatzmittel werden in der Literatur meist Geldmittel, Personal und Maschinen sowie Material als Betriebsmittel genannt. ${ }^{49}$ Verfahren, wie beispielsweise der Kapazitätsabgleich, können bei der Kapazitätsplanung unterstützen. ${ }^{50}$ Insbesondere für die simultane Kapazitätsplanung mehrerer, identische Ressourcen beanspruchender Projekte kommen Modelle und Methoden des Operations Research zum Einsatz. ${ }^{51}$

Bei der in dieser Arbeit betrachteten Problemstellung entscheidet der Auftragnehmer über den Einsatz von Ressourcen, welche für die Ausführung der durch den Auftraggeber vorgegebenen Leistungen notwendig sind. Für die untersuchte Entscheidungssituation des Auftraggebers wird daher vorausgesetzt, dass der Auftragnehmer zu dem Zeitpunkt der Vertragsplanung eine Zeit- und Kapazitätsplanung auf der Grundlage der Leistungsbeschreibungen durchgeführt hat und das Ergebnis dieser Planung in Form eines Angebotes an den Auftraggeber übermittelt. Die Problematik der Kapazitätsplanung wird daher im Folgenden nicht weiter verfolgt.

Aufgrund des gewählten Schwerpunktes der Vertrags- und Projektplanung wird an dieser Stelle auf die weiteren Phasen des Projektmanagements nicht detailliert eingegangen. Stattdessen widmet sich das folgende Kapitel der zwischen Auftraggeber und Auftragnehmer bestehenden Kooperationsbeziehung. Diese Beziehung wird zunächst von alternativen Kooperationsformen zwischen und innerhalb von Organisationen abgegrenzt und anschließend aus entscheidungstheoretischer Sicht charakterisiert.

48 Vgl. Corsten/Corsten (2000), S. 190.

49 Vgl. Burghardt (2002b), S. 132; Corsten/Corsten (2000), S. 190; Keßler/Winkelhofer (2004), S. 242; Schwarze (2001), S. 259.

so Vgl. Altrogge (1996), S. 194ff.; Homburg (2000), S. 531 ff.; Schwarze (2001), S. $263 \mathrm{ff}$.

51 Vgl. De Maio/Vaganti/Corso (1994); Demeulemeester/Herroelen (2002), S. 203ff.; Kolisch (2001), S. 217f;; Lova/Tormos (2001); Meredith/Mantel (2003), S. 473ff.; Chiu/Tsai (2002). 


\subsection{Die Kooperation zwischen Auftraggeber und Auftragnehmer}

\subsubsection{Projektkooperationen in Organisationen}

Die Kooperation zwischen einem Auftraggeber und einem oder mehreren Auftragnehmern stellt eine spezielle Form der Projektkooperationen in und zwischen Organisationen dar, so dass im Folgenden zunächst eine allgemeine Definition des Kooperationsbegriffs sowie eine Einordnung des in dieser Arbeit verwendeten Begriffs erfolgt.

Der von dem lateinischen Wort cooperare abstammende Begriff Kooperation bedeutet Zusammenarbeit oder die gemeinschaftliche Erfüllung von Aufgaben. ${ }^{52}$ Nach Tröndle können Kooperationen allgemein durch die folgenden konstitutiven Merkmale charakterisiert werden: ${ }^{53}$ Autonomie einerseits und gegenseitige Abhängigkeit oder Interdependenz andererseits. ${ }^{54}$ Während die Kooperationspartner weitgehend autonom handeln, besteht durch die Geschäftsgrundlage der Kooperation eine sachlich-fachliche Verbindung zwischen ihnen. ${ }^{55}$ Hieraus lassen sich die ersten zwei Bestandteile einer Begriffsdefinition ableiten: Bei einer Kooperation behalten die Partner ihre rechtliche und wirtschaftliche Selbstständigkeit und vereinbaren die Koordination und Durchführung gemeinsamer Aktivitäten. ${ }^{56}$

Das Eingehen sowie das Beenden von Kooperationen erfolgt in der Regel auf freiwilliger Basis. ${ }^{57}$ Beck führt als weitere in der Literatur genannte Merkmale die Zielbezogenheit der Zusammenarbeit, die Existenz von Vereinbarungen als Grundlage der Zusammenarbeit sowie die wettbewerbsrechtliche Zulässigkeit der Zusammenarbeit auf. $^{58}$ Der zwischen den Projektpartnern abzuschließende Vertrag enthält die Informationen und Vereinbarungen, welche für die Erfüllung der Kooperationsziele notwendig sind. Das dritte Kapitel wird sich daher detailliert mit dem Problem der Vertragsgestaltung beschäftigen. Die Frage der wettbewerbsrechtlichen Zulässigkeit der vertraglich

52 Vgl. etwa Beck (1994), S. 39; Müller (2003), S. 7; Rey (1999), S. 11; Tröndle (1986), S. 15.

53 Vgl. Tröndle (1986), S. 16f.

54 Vgl. hierzu ausführlich Beck (1994), S. 44f.

ss Vgl. Schertler (1995), S. 24.

s6 Vgl. Gabriel et al. (2002), S. 122; Horsch (2003), S. 87; Muller (2003), S. 8. Die wirtschaftliche Selbststăndigkeit kann durch die notwendigen Abstimmungen zwischen den Partnern sowie unterschiedliche Machtpositionen eingeschränkt werden. Vgl. Schlosser (2001), S. 42.

57 Die freiwillige Zusammenarbeit stellt ein wichtiges Merkmal von Kooperationsbeziehungen dar und steht in engem Zusammenhang mit der rechtlichen und wirtschaftlichen Selbstständigkeit. Vgl. hierzu beispielsweise Beck (1994), S. 44; Schlosser (2001), S. 42; Trumpp (1995), S. 13.

$58 \mathrm{Vgl}$. Beck (1994), S. 43. Das Erreichen eines gemeinsamen Ziels bezieht sich auf das zugrunde liegende Sachziel. Die Kooperationspartner können unterschiedliche Zielvorstellungen und somit verschiedene Prafferenzen für die Auswahl von Aktionen zur Erreichung des Sachziels besitzen. 
geregelten Kooperationsbeziehung zwischen den Projektpartnern muss zu dem Zeitpunkt der Projektdefinition bzw. bei der Auswahl des Projektpartners berücksichtigt werden und wird aufgrund des hier gewählten Schwerpunktes der Projektplanung im Folgenden nicht untersucht.

Primäre Zielsetzung und somit Hauptzweck einer Kooperation ist es, durch die gemeinschaftliche, arbeitsteilige Erfüllung der Aufgaben gegenüber der individuellen Aufgabenerfüllung einen höheren Grad der Zielerfüllung zu erreichen. ${ }^{59}$ Als Folge der Arbeitsteilung resultiert die Notwendigkeit der Koordination von Aktivitäten oder Entscheidungen im Hinblick eines übergeordneten Gesamtziels. ${ }^{60}$

Im Gegensatz zur Kooperation, welche sich bei der organisatorischen Gestaltung der arbeitsteiligen Systeme an Effektivitätsüberlegungen orientiert, ist die Koordination auf eine effiziente Gestaltung dieser Beziehungen ausgerichtet. ${ }^{61}$ Dabei umfasst die Koordinationsfunktion insbesondere die Abstimmung von Aktivitäten während des Handlungsprozesses. ${ }^{62}$ Scholtis unterteilt die Koordination in eine sachliche und eine personelle Komponente und begründet damit sowohl den aus dem Erwerb, der Aufteilung und dem Einsatz von Ressourcen als auch den aus der Informationsasymmetrie und den Interessenkonflikten resultierenden Koordinationsbedarf. ${ }^{63}$ Das Koordinationsproblem innerhalb der Kooperationsbeziehung besteht darin, sowohl die arbeitsteilige Aufgabenaufteilung auf die Projektpartner so durchzuführen, dass die Vorteile durch die Spezialisierung am größten sind, als auch die organisatorische Zusammenarbeit so zu gestalten, dass eine möglichst reibungslose Abstimmung der Aufgaben möglich ist. ${ }^{64}$ Dieser Zusammenhang zwischen Kooperation und Koordination für die zwi-

\footnotetext{
59 Vgl. Backhaus/Piltz (1990), S. 3; Beck (1994), S. 49; Fuchs (1999), S. 21f. Zu den Vorteilen der Spezialisierung durch Arbeitsteilung vgl. etwa Laux/Liermann (1997), S. 3f. Scholtis (1998), S. 6 begründet die Notwendigkeit der kooperativen Arbeitsteilung mit der begrenzten Möglichkeit des Menschen, Informationen zu erfassen, zu verarbeiten und zu speichern. Eine ausführliche Übersicht uber Ziele von Kooperationen gibt Rey (1999), S. 21 ff. Fur eine Anwendung aus der Bauwirtschaft vgl. Girmscheid (2004), S. 240ff.

60 Vgl. etwa Gabriel et al. (2002), S. 119ff.; Laux/Liermann (1997), S. 1; Specht/Kahmann (2000), S. 60; Staehle (1999), S. 555ff. Für unterschiedliche Definitionen des Koordinationsbegriffs vgl. Horváth (2003), S. 117f.; Trumpp (1995), S. 17. Die Koordination innerhalb einer Kooperation lässt sich damit auch von einer Koordination über den Markt bzw. uber hierarchische Ordnungen abgrenzen. Vgl. für eine Einordnung der Kooperation zwischen Markt und Hierarchie etwa Fleischer (1997), S. $10 \mathrm{ff}$.

61 Vgl. Beck (1996), S. 32f.; Trumpp (1995), S. 18. Für den Zusammenhang von Koordination und Planung vgl. etwa Staehle (1999), S. 542.

${ }^{62}$ Vgl. Wolf (2003), S. 85 sowie die dort zitierte Literatur von Fayol. Damit wird die Koordinationsfunktion von der Organisationsfunktion unterschieden, welche die Funktion der apriori Abstimmung von Aktivităten erfullt. Werners/Thorn (2003) zeigen wichtige Merkmale einer unternehmungsübergreifenden Koordination fur das Supply Chain Planning auf.

63 Vgl. Scholtis (1998), S. 7

64 Vgl. Jost (2000), S. 455.
} 
schen einem Auftraggeber und einem Auftragnehmer bestehende Beziehung ist in Abbildung 2.5 dargestellt. In dieser Abbildung sind auch die Einflussfaktoren aufgeführt, welche sowohl für die Wahl der Kooperationsform als auch für die Gestaltung der Koordinationsinstrumente von Bedeutung sind.

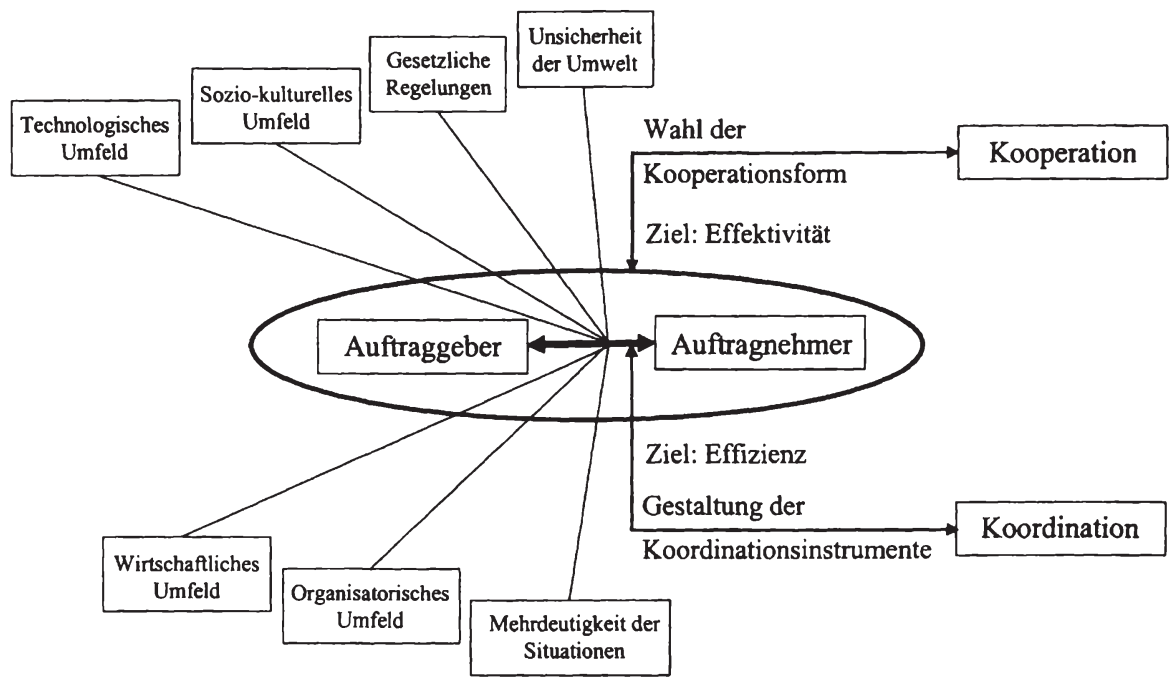

Abbildung 2.5: Zusammenhang von Kooperation und Koordination sowie deren Einflussfaktoren (Quelle: In Anlehnung an Trumpp (1995), S. 20.)

Auf der Grundlage der vorherigen, allgemeinen Ausführungen kann die in dieser Arbeit untersuchte Form der Projektkooperation näher definiert werden:

Die Projektkooperation ist eine zeitlich begrenzte Zusammenarbeit zwischen einem Auftraggeber und mindestens einem Auftragnehmer als Projektpartner zur Erreichung einer vertraglich festgeschriebenen Zielsetzung. Die Projektleistung und/oder die für die Erstellung der Leistung notwendigen Prozesse sind für den Projektauftraggeber relativ einmalig bzw. neuartig. Der Auftraggeber ist im Rahmen der Arbeitsteilung für die Planung, Steuerung und Kontrolle des Gesamtprojektes verantwortlich und delegiert die Aufgabe der Planung, Steuerung und Kontrolle der Ausführung von Teilprojekten bzw. Arbeitspaketen an die Projektpartner, welche aufgrund ihrer Spezialisierung besser in der Lage sind, diese Leistungen zu erbringen.

Diese Definition einer Projektkooperation beschreibt weder die organisatorische Struktur noch das Verhältnis zwischen den Kooperationspartnern. Auf diese Merkmale wird 
im folgenden Kapitel näher eingegangen. Im Anschluss daran erfolgt eine Erweiterung der hier vorgestellten Definition um diese Aspekte.

Die für Projektkooperationen charakteristische zeitliche Befristung der Zusammenarbeit erlaubt eine Gliederung der Kooperationsbeziehung in die in Abbildung 2.6 dargestellten Kooperationsphasen, auf die im Folgenden kurz eingegangen wird. ${ }^{65}$

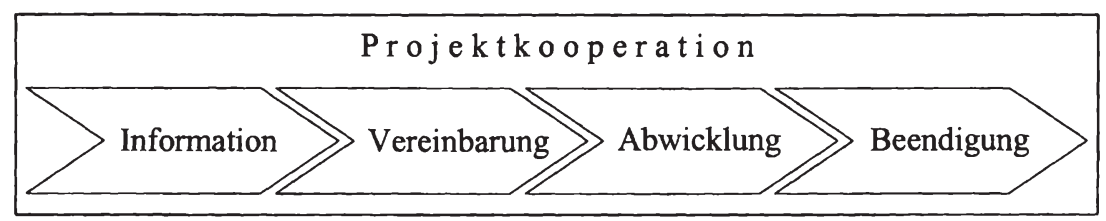

Abbildung 2.6: Phasen einer Projektkooperation

(Quelle: In Anlehnung an Rey (1999), S. 194.)

Die Projektkooperation beginnt mit einer Informationsphase, ${ }^{66}$ in der im Anschluss an die Zielbestimmung die Identifikation und Bewertung der Nutzenpotenziale der Kooperation durch die Bewertung unterschiedlicher Handlungsalternativen erfolgt. ${ }^{67}$ Als Nutzenpotenziale können beispielsweise eine aus der Kooperation resultierende Verkürzung der Projektdauer, die Senkung von Kosten oder das Ausnutzen von Synergieoder Spezialisierungseffekten identifiziert werden. ${ }^{68}$ Sofern als Handlungsalternative das Eingehen einer Kooperationsbeziehung ausgewählt wird, erfolgt eine für das Erreichen der Projektziele notwendige konkrete Aufgabendefinition. ${ }^{69}$ Anschließend werden die für die Ausführung dieser Aufgaben geeigneten Projektpartner ausgewählt. ${ }^{70}$ Dieses definiert den Beginn der Vereinbarungsphase, in der sich die Projektpartner kennen lernen und gemeinsam die Kooperationsinhalte abstimmen. ${ }^{71}$ Der Ab-

${ }^{65}$ Vgl. Link/Marxt (2004), S. 75ff. Für eine Anwendung aus dem Hochbau vgl. die Projektphasen in Girmscheid (2004), S. 130.

66 Diese Phase wird auch als Initiierungs- oder Initialisierungsphase bezeichnet. Vgl. Fuchs (1999), S. 108; Friedli (2000), S. 69; Muller (2003), S. 23.

67 Vgl. Fuchs (1999), S. $110 \mathrm{ff}$. Die Handlungsalternativen schließen hier auch andere Formen der Zusammenarbeit von Unternehmungen, wie beispielsweise Akquisitionen oder Fusionen sowie die Alternative der Eigenfertigung/Eigenentwicklung ein. Vgl. Müller (2003), S. 23.

68 Vgl. Fuchs (1999), S. 113; Müller (2003), S. 23; von Spreckelsen (2004), S. 22.

69 Vgl. Fuchs (1999), S. 136ff.

70 Muller (2003), S. 24f. bezeichnet diese Phase als Anbahnungsphase und misst ihr einen bedeutenden Stellenwert fur eine Projektkooperation bei. Während Fuchs (1999), S. 123 die Partnerauswahl als ersten Schritt der Konfigurationsphase betrachtet, erscheint die hier vorgestellte Reihenfolge aus der Sicht des Auftraggebers sinnvoller, da die Auswahl der Auftragnehmer von den auszuführenden Leistungen abhängt.

71 Vgl. Rey (1999), S. 188. Friedli (2000), S. 69 unterteilt diese Phase in die Konfigurations- und die Designphase. 
schluss eines Kooperationsvertrags, der den Vertragsgegenstand, die Termin- und Preisvereinbarungen, die Nutzungs- und Verwertungsrechte sowie die Gewährleistung regelt, beendet die Vereinbarungsphase. ${ }^{72}$ Die anschließende Abwicklungsphase wird durch die Planung, Durchführung und Steuerung der Leistungserstellung geprägt. Gleichzeitig führen die Kooperationspartner Kontrolltätigkeiten durch. ${ }^{73}$ Die letzte Phase beendet die Projektkooperation. Hierbei stehen Verteilungsfragen zwischen den Kooperationspartnern im Vordergrund. ${ }^{74}$ Diese Phase tritt ein, wenn die gemeinsamen sowie die partnerindividuellen Projektziele erreicht worden sind oder bei Nichterreichen dieser Ziele bzw. durch das Verlassen von Projektpartnern die Kooperation beendet wird. ${ }^{75}$ Insbesondere die Vereinbarungsphase ist im Folgenden Gegenstand der Untersuchungen dieser Arbeit. Dabei liegt der Schwerpunkt auf der Planung des Vertragsdesigns durch den Auftraggeber auf der Grundlage seiner und der während der Informationsphase gewonnenen Informationen über das Projekt sowie über potenzielle Auftragnehmer. Für die Bewertung eines Vertragsdesigns sowie die Auswahl der Projektpartner müssen verschiedene Kriterien berücksichtigt werden, welche im Mittelpunkt der Thematik des dritten Kapitels stehen.

\subsubsection{Organisationsstrukturen für Projektkooperationen}

Projektkooperationen können zunächst nach ihrem Verflechtungsgrad der an der Kooperation beteiligten Unternehmungen in intraorganisationale und interorganisationale Kooperationen unterschieden werden. ${ }^{76}$ Eine intraorganisationale Kooperation liegt vor, sofern die Leistungserstellung ausschließlich durch unternehmungseigene Ressourcen erfolgt und somit keine Verbindung zu anderen Organisationen existiert. ${ }^{77}$

72 Vgl. Burghardt (2002a), S. 50.

73 Vgl. Fuchs (1999), S. 175. Diese Phase bezeichnet Fuchs als Betriebsphase. Müller (2003), S. 23 unterteilt die Phase in die Phasen der Planung und Durchfuhrung. Diese Form der Gliederung erscheint hier nicht sinnvoll, da die Kooperation durch einen begleitenden Planungs-, Steuerungs- und Kontrollprozess unterstutzt werden soll.

74 Vgl. Rey (1999), S. 193.

75 Diese Phase bezeichnet Fuchs (1999), S. 183 auch als Rekonfigurationsphase und bezieht auch die Morglichkeit einer erneuten Kooperationsbeziehung in veränderter Form ein. Dieser Aspekt wird hier jedoch aufgrund der Einmaligkeit und Neuartigkeit der Projekte nicht berulcksichtigt.

76 Vgl. Beck (1994), S. 24. Burghardt (2002a), S. 94 bezeichnet die Kooperation bei inter-industriellen Projekten als unternehmensüberschreitende Projektorganisation. Trumpp (1995), S. 24 spricht von externer bzw. organisationsinterner Kooperation. Fuchs (1999), S. 81 fuhrt zusătzlich eine integrative Ebene auf, welche alle Kooperationspartner innerhalb und zwischen den Unternehmungen auf einer höheren Abstraktionsebene miteinander verbindet.

77 Beck schließt hiervon die Verbindung im Rahmen normaler Abnehmer- und Lieferbeziehungen aus, die eine Unternehmung unterhält. Vgl. Beck (1994), S. 24, Fußnote 36. 
Charakteristisches Kriterium für intraorganisatorische Projekte ist die Möglichkeit des selbstständigen Erreichens des Projektziels durch die eigene Unternehmung. ${ }^{78}$ Im Gegensatz hierzu handelt es sich um eine interorganisationale Projektkooperation, ${ }^{79}$ sofern mindestens zwei Unternehmungen an der projektorientierten Leistungserstellung durch eine gemeinschaftliche arbeitsteilige Aufgabenerfüllung zielgerichtet und zeitlich befristet zusammenarbeiten. ${ }^{80}$ Das von Tröndle geprägte und von Beck übernommene für eine interorganisationale Kooperation charakteristische Merkmal der fehlenden Weisungsbefugnis zum Zweck der Koordination muss für die in dieser Arbeit betrachtete Kooperationsbeziehung teilweise aufgehoben werden, wenngleich davon ausgegangen wird, dass bestimmte Entscheidungsbefugnisse auf die einzelnen Kooperationsteilnehmer verteilt sind. ${ }^{81}$ Der Auftraggeber übernimmt die Gesamtplanung, -steuerung und -kontrolle des Gesamtprojektes und überlässt der als Auftragnehmer fungierenden Unternehmung die Entscheidungsbefugnisse bezüglich der Planung, Steuerung und Kontrolle der auszuführenden Teilprojekte bzw. Arbeitspakete. ${ }^{82}$

Hinsichtlich der Kooperationsrichtung in der Wertschöpfungskette lassen sich horizontale, vertikale und diagonale Projektkooperationen unterscheiden. ${ }^{83}$ Eine horizontale Kooperation zeichnet sich durch die Zusammenarbeit von Unternehmungen der gleichen Wirtschaftsstufe aus, eine vertikale Kooperation liegt beispielsweise vor, wenn die kooperierenden Unternehmungen aufeinander folgenden Handelsstufen angehö-

78 Vgl. Beck (1994), S. 24. Es ist fraglich, ob die Teilnehmer einer intraorganisationalen Kooperation das Kriterium der rechtlichen und wirtschaftlichen Selbstständigkeit erfullen und somit der Kooperationsbegriff an dieser Stelle verwendbar ist.

79 Schlosser (2001), S. 58 bezeichnet diese Form der Projektkooperation als Projekt-Allianz und ordnet sie somit als spezielle Form einer strategischen Allianz ein. Aufgrund der Einmaligkeit des Projektes sowie der niedrigen Intensität der Zusammenarbeit der Kooperationspartner wird die in dieser Arbeit betrachtete Projektkooperation nicht als strategische Allianz definiert.

80 Vgl. Beck (1994), S. 25. Für einen Überblick uber Grundformen interorganisatorischer Beziehungen sowie für eine Abgrenzung der Projektkooperation von alternativen Formen vgl. Fuest (1998), S. 51. Wildforster/Wingen (2001), S. 82 kommen zu dem Ergebnis, dass das interorganisationale Projektmanagement bisher in der Literatur kaum behandelt worden ist. Turner/Müller (2003), S. 3 bezeichnen ein Projekt als eine zeitlich befristete Organisation.

81 Vgl. Tröndle (1986), S. 2; Beck (1994), S. 25. Tröndle unterscheidet die Unternehmungsverbindungen in Kooperationen und Konzentrationen und grenzt diese insbesondere durch die Merkmale der Gleich- bzw. Unter-/Überordnung voneinander ab.

${ }^{82}$ Durch die hierarchische Organisationsstruktur sowie das Merkmal der nicht gemeinsam genutzten Ressourcen lässt sich die untersuchte Projektkooperation etwa von einer virtuellen Unternehmenskooperation abgrenzen. Vgl. hierzu beispielsweise Bauer (2003), S. 114ff.; Specht/Kahmann (2003); Steven/Otterpohl (2000), S. 179ff. Backhaus (2003), S. 268f. unterscheidet strategische Allianzen und strategische Netzwerke nach der Kooperationsrichtung.

83 Vgl. Bauer (2003), S. 112 ff.; Fleischer (1997), S. 15; Fuest (1998), S. 48; Horsch (2003), S. 88; Rey (1999), S. 29; Schlosser (2001), S. 51. Für diagonale Kooperationen wird synonym auch der Begriff der lateralen Kooperationen verwendet. Vgl. Rey (1999), S. 29. 
ren. ${ }^{84}$ Die hier untersuchte interorganisationale Kooperationsbeziehung kann einer vertikalen Kooperationsrichtung zugeordnet werden. Der Auftragnehmer erstellt in Abstimmung mit dem Auftraggeber durch den Einsatz seiner eigenen Ressourcen die vertraglich festgelegte Projektleistung und verkauft diese an den Auftraggeber, der entweder eine Weiterentwicklung oder die sofortige Nutzung des Projektgegenstandes vornimmt. Die Analyse, Planung und Gestaltung des Organisationsaufbaus sind Problemstellungen innerhalb des als Aufbauorganisation bezeichneten Teilbereichs der Organisationstheorie. $^{85}$

Für die effektive Koordination der Projektkooperation ist eine Organisationsstruktur festzulegen, welche unter Beachtung der bestehenden Strukturen innerhalb der einzelnen Unternehmungen die interorganisatorische Beziehung zwischen Auftraggeber und Auftragnehmer so gestaltet, dass eine effiziente gemeinsame Leistungserstellung möglich ist. ${ }^{86}$ In Anlehnung an die für zwischenbetriebliche Kooperationen in der Organisationstheorie vorgeschlagenen Formen der Zusammenarbeit lassen sich diese auf Projektkooperationen übertragen. ${ }^{87}$ Hierbei werden häufig die Einzelauftrags-, die Konsortial- sowie die Generalunternehmerorganisation aufgeführt. ${ }^{88}$ Die Organisationsformen unterscheiden sich insbesondere durch die hierarchische Beziehung der ausführenden Unternehmungen untereinander und $\mathrm{zu}$ dem Auftraggeber sowie durch die Aufteilung der Gesamtprojektaufgabe auf unterschiedliche Auftragnehmer. ${ }^{89}$

Für die weiteren Ausführungen wird ausschließlich die vertragliche Beziehung zwischen einem Auftraggeber und einem Auftragnehmer betrachtet, wobei die Möglichkeit weiterer, dem Auftragnehmer hierarchisch untergeordneter Sub-Unternehmungen nicht ausgeschlossen wird, welche wiederum Teilaufgaben der Gesamtprojektaufgabe

84 Vgl. etwa Bauer (2003), S. 112ff.; Rey (1999), S. 29. Eine diagonale Kooperation umfasst sowohl horizontale als auch vertikale Beziehungen.

85 Vgl. etwa Laux/Liermann (1997), S. 183ff.; Olfert/Steinbuch (2003), S. 50.

86 Vgl. Beck (1994), S. 99ff. Es wird hier zwischen der äußeren und inneren Projektorganisation unterschieden. Auf der Ebene der ăußeren Projektorganisation ist es möglich, dass der Auftraggeber eines Projektes auch die Funktion des Auftragnehmers bei einem anderen Projekt einnimmt. Horváth (2003), S. 125 bezeichnet diese Aufgabe als System bildende Koordination. Nordqvist/Hovmark/Zika-Viktorsson (2004) untersuchen die Zufriedenheit der Kooperationspartner oder den empfundenen Zeitdruck als soziale Aspekte innerhalb von Projektkooperationen.

87 Fur eine Abgrenzung des interorganisationalen Projektmanagements und der zwischenbetrieblichen Kooperation vgl. Beck (1994), S. 66.

88 Vgl. Beck (1994), S. $101 \mathrm{ff}$; Burghardt (2002a), S. 94. Von der Problematik der Bestimmung einer Struktur für die Projektorganisation ist die Problematik der Einordnung des Projektmanagements in die Unternehmungsorganisation abzugrenzen. Vgl. hierzu etwa Litke (2004), S. 69ff.; Wicher (2003), S. $73 \mathrm{ff}$.

89 Für Vor- und Nachteile der jeweiligen Organisationsformen vgl. beispielsweise Beck (1994), S. 101ff.; Burghardt (2002a), S. 96; Corsten/Corsten (2000), S. $81 \mathrm{ff}$. 
erfüllen. Eine solche Organisationsstruktur ist in Abbildung 2.7 dargestellt. Diese Form der Zusammenarbeit ist mit der Generalunternehmerorganisation bzw. der Leitfirmenorganisation vergleichbar. ${ }^{90}$

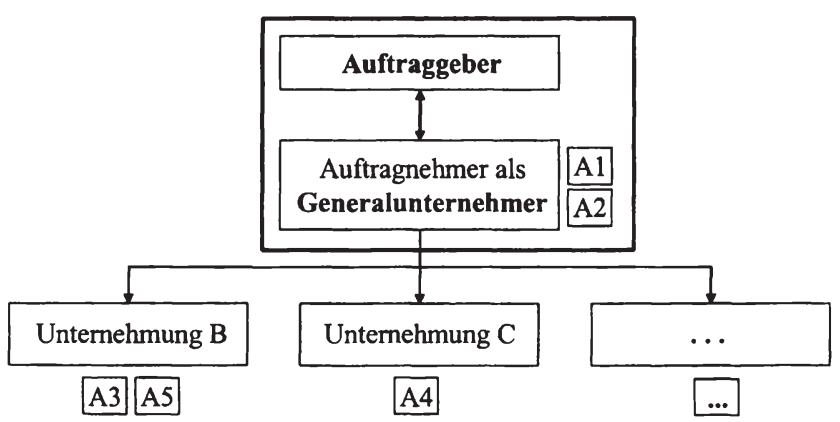

Abbildung 2.7: Organisationsform der Generalunternehmerschaft (Quelle: In Anlehnung an Beck (1994), S. 103.)

Der Auftraggeber schließt hierbei mit einem Auftragnehmer einen Projektvertrag ab, welcher die Leistungen und Gegenleistungen für beide Vertragspartner festlegt. Der Auftraggeber besitzt keine Entscheidungs- und Weisungskompetenz gegenüber den dem Auftragnehmer untergeordneten Unternehmungen. ${ }^{91}$ Der Generalunternehmer entscheidet über die Auswahl dieser und schließt mit ihnen in eigenem Namen Unterverträge über die zu erbringenden Teilaufgaben $a b .{ }^{92}$

Aufgrund des in dieser Arbeit gewählten Schwerpunktes der Vertrags- und Projektplanung des Auftraggebers für die Projektkooperation mit einem Auftragnehmer wird das Verhältnis des Auftragnehmers zu weiteren Unternehmungen im Folgenden ausgeklammert und ausschließlich die in dem stark umrandeten Bereich der Abbildung dargestellte Organisationsform behandelt. Durch diese Kooperationsbeziehung wird somit eine Organisationsstruktur mit zwei Entscheidungsträgern geschaffen. Die Entscheidung des Auftraggebers über das Vertragsdesign sowie der Erfolg des Investitionsprojektes werden durch die Entscheidungen des Auftragnehmers, etwa über dessen Arbeitseinsatz, beeinflusst. Daher wird die Kooperationsbeziehung im Folgenden aus entscheidungstheoretischer, insbesondere spieltheoretischer Sicht analysiert.

90 Der Begriff der Leitfirmenorganisation wird von Beck (1994), S. 102 benutzt. Eine ähnliche Darstellung ist bei Madauss (2000), S. 363 zu finden.

91 Dies entspricht dem Weisungssystem des Einliniensystems, bei dem jeder nachgeordnete Entscheidungstrăger nur von der ubergeordneten Instanz Weisungen erhält. Vgl. etwa Laux/Liermann (1997), S. 187.

92 Vgl. Beck (1996), S. 117f.; Corsten/Corsten (2000), S. 86f. 


\subsubsection{Die Kooperationsbeziehung aus entscheidungstheoretischer Sicht}

Der in dieser Arbeit betrachtete präskriptive Zweig der Entscheidungstheorie beschäftigt sich mit der Fragestellung, wie Entscheidungen bei gegebenen Entscheidungsprämissen durch den Entscheidungsträger möglichst rational zu treffen sind. ${ }^{93}$ Hierfür wird dessen Entscheidungsfeld in die Bestandteile Aktionenraum, Zustandsraum und Ergebnisfunktion unterteilt. Der Aktionenraum umfasst die zu einem bestimmten Zeitpunkt möglichen Handlungsalternativen des Entscheidungsträgers, welche unter Berücksichtigung der durch ihn nicht beeinflussbaren Umweltzustände mit einer Handlungskonsequenz verbunden sind. Die Ergebnisfunktion ordnet jeder Aktion die Konsequenzen dieser Aktion für jeden Zustand zu, so dass die beste Aktion mit Hilfe bestimmter den Zielgrößen und Präferenzrelationen des Entscheiders entsprechenden Entscheidungsregeln ausgewählt werden kann. ${ }^{94}$

Bei der in dieser Arbeit untersuchten Problemstellung wird der Auftraggeber durch die Anwendung quantitativer, auf entscheidungstheoretischen Modellen basierender Methoden der Projektplanung unterstützt, für die Kooperationsbeziehung mit dem Auftragnehmer aus der Menge möglicher Vertragsdesigns ein Vertragsdesign auszuwählen. Hiermit werden die mit der Projektdurchführung verbundenen und aus der Sicht des Auftraggebers zu dem besten Ergebnis führenden Leistungen des Auftragnehmers sowie die Gegenleistungen des Auftraggebers festgelegt.

Die Phasen der Projektkooperation sind in Kapitel 2.2.1 vorgestellt worden. Während dieser Phasen besteht eine Verknüpfung der Aktionen von Auftraggeber und Auftragnehmer, so dass der Auftraggeber für die Vertrags- und Projektplanung auch das vermutete zukünftige Verhalten des Auftragnehmers berücksichtigen muss. Die Rollen der Kooperationspartner sowie deren Verknüpfung miteinander zeigt die Abbildung 2.8. Es lässt sich erkennen, dass besonders die Vertragsverhandlungen, welche der Auftraggeber mit dem Auftragnehmer führt, eine Verbindung beider Kooperationspartner herbeiführen und maßgeblich die anschließende Projektdurchführung beeinflussen.

93 Vgl. Bamberg/Coenenberg (2002), S. 11; Laux (2003), S. 2; Eisenführ/Weber (2003), S. 4f.

94 Vgl. Bamberg/Coenenberg (2002), S. 15ff.; Eisenfuhr/Weber (2003), S. 16ff;; Laux (2003), S. $3 \mathrm{ff}$. 


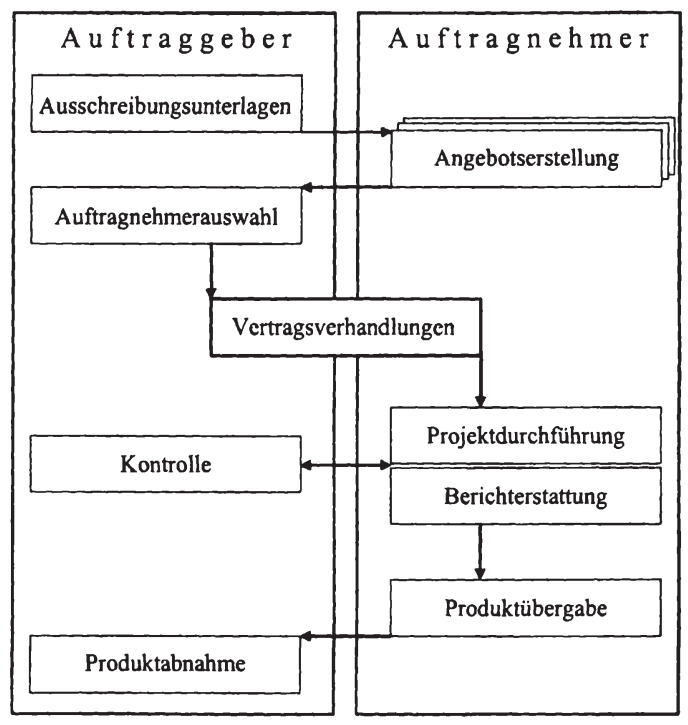

Abbildung 2.8: Verknüpfung der Aktionen von Auftraggeber und Auftragnehmer (Quelle: In Anlehnung an Madauss (2000), S. 364.)

Beeinflusst ein Entscheidungsträger mit der Wahl seiner Aktionen die Konsequenzen bzw. das Verhalten des anderen Entscheidungsträgers und sind sich diese der Beeinflussung bewusst, liegt eine Entscheidungssituation vor, die Gegenstand der Spieltheorie ist. ${ }^{95}$ Die Auftraggeber-Auftragnehmer-Beziehung kann als ein Zweipersonenspiel interpretiert werden, ${ }^{96}$ da insbesondere die von dem Auftragnehmer durchgeführten Aktivitäten von den mit dem Auftraggeber vertraglich vereinbarten Gegenleistungen abhängen und damit auch das Ergebnis des Auftraggebers aus der Nutzung des Projektgegenstandes beeinflusst wird. Obwohl die Kooperationspartner die Erreichung eines gemeinsamen Projektziels anstreben, treffen sie ihre Entscheidungen auf der Grundlage der individuellen Nutzenmaximierung. ${ }^{97}$ Die Spieltheorie bezeichnet ein Spiel als kooperativ, sofern die Spieler verbindliche Abmachungen treffen können und Mechanismen existieren, die die Einhaltung von Verträgen bindend durchsetzen kön-

95 Vgl. etwa Dixit/Skeath (2004), S. 14; Sieg (2000), S. 2.

96 Vgl. Bamberg/Conenberg (2002), S. $209 \mathrm{ff}$. Von Zweipersonenspielen sind Koalitionsspiele abzugrenzen, bei denen eine Verabredung von Spielern untereinander gegenüber anderen Spielern möglich ist. Vgl. zu Koalitionsspielen beispielsweise Sieg (2000), S. $77 \mathrm{ff}$.

97 Damit lässt sich die Spielsituation von einer im Rahmen der Verhandlungstheorie betrachteten Situation abgrenzen, bei der die Kooperationspartner eine Gruppe mit gemeinsamen Zielen bilden. Vgl. Eisenfuhr/Weber (2003), S. 316. Zur Verhandlungstheorie vgl. Holler/Illing (2003), S. 240ff.; Raifa/Richardson/Metcalfe (2003). 
nen. ${ }^{98}$ Die Spieler sind zu einer Kooperation bereit, wenn jeder einzelne Spieler durch die Kooperation einen Nutzen erreicht, welcher mindestens dem Nutzen ohne Kooperation entspricht. ${ }^{99}$ Somit ist das Vorliegen einer Win-Win-Situation, bei der allen Beteiligten aus der Kooperation Vorteile entstehen, charakteristisch für kooperative Spiele. ${ }^{100}$ Obwohl besonders während der Projektdurchführung die in der Spieltheorie als Konstantsummenspiel bezeichnete Situation besteht, bei der sich der Nutzen des Auftraggebers etwa durch die Minderung der Zahlungen an den Auftragnehmer zu dessen Lasten erhöhen lässt und damit nach Meinung der Spieltheorie keine Kooperation zustande kommt, ${ }^{101}$ gehen die Projektpartner aufgrund der aus der Arbeitsteilung resultierenden Kooperationsziele die Zusammenarbeit ein. ${ }^{102}$

Die dargestellten spieltheoretischen Aspekte der Auftraggeber-AuftragnehmerBeziehung tragen zu einer Strukturierung sowie entscheidungstheoretischen Einordnung dieser Kooperationsbeziehung bei. Methoden zur Lösung des zwischen den Vertragspartnern bestehenden Konfliktes lassen sich aus der Spieltheorie nur indirekt über die Formulierung des Entscheidungsproblems als mathematisches Modell ableiten. ${ }^{103}$ Das im fünften Kapitel entwickelte und durch mathematische Modelle unterstützte vierstufige Vorgehen für die Unterstützung des Auftraggebers bei der Projekt- und Vertragsplanung berücksichtigt diese hier aus der Sicht der Spieltheorie betrachteten Reaktionen des Auftragnehmers auf die Aktionen des Auftraggebers.

Aus den Ausführungen des Kapitels 2.2 ist erkennbar, dass der Erfolg der Kooperationsbeziehung und somit das Erreichen der Kooperations- und Projektziele sowohl durch die Organisationsstruktur der Kooperation als auch durch das Verhalten der Kooperationspartner, insbesondere des Auftragnehmers, beeinflusst wird. Daher wird sich das folgende Kapitel näher mit der Problematik der Verhaltensbeeinflussung von Individuen beschäftigen.

98 Vgl. Bamberg/Coenenberg (2002), S. 223f;; Holler/llling (2003), S. 23 und S. 191. Das kooperative Spiel kann zusătzlich um ein Drohspiel ergänzt werden. Vgl. hierzu Borgwardt (2001), S. 587ff.; Holler/Illing (2003), S. 235ff. Steven/Otterpohl (2000), S. 179 stellen fest, dass die kooperative Spieltheorie auf virtuelle Unternehmen nicht anwendbar ist, da hier auf die die Vertragseinhaltung überwachenden Institutionen verzichtet wird.

99 Vgl. Holler/llling (2003), S. 25.

$100 \mathrm{Vgl}$. Fuest (1998), S. 104.

101 Zu Konstantsummenspielen vgl. etwa Bamberg/Coenenberg (2002), S. 194f.; Dixit/Skeath (2004), S. $80 \mathrm{f}$.

102 Vgl. Kapitel 2.2.1.

${ }^{103}$ Eine kritische Würdigung der Spieltheorie ist in Bamberg/Coenenberg (2002), S. 240ff. sowie in Ellinger/ Beuermann/Leisten (2003), S. 13 zu finden. Durch die im füften Kapitel bei der Planung des Auftraggebers erfolgende Berücksichtigung des Verhaltens des Auftragnehmers kommt die Spielsituation zum Ausdruck. 


\subsection{Das Verhalten von Auftraggeber und Auftragnehmer}

\subsubsection{Die Rolle des Auftraggebers in der Kooperationsbeziehung}

Der Auftraggeber als oberste Instanz innerhalb der Kooperationsbeziehung gibt die Rahmenziele und inhaltlichen Weisungen hinsichtlich der Ausführung des Projektes an den Auftragnehmer weiter. ${ }^{104}$ Innerhalb der Organisation des Auftraggebers genehmigt dieser die mit der Leistungserstellung verbundenen Kosten und ist für die Ergebnisse des Projektes verantwortlich. ${ }^{105}$ Der Begriff und die Aufgaben des Projektleiters werden in der Literatur zum Projektmanagement unterschiedlich dargestellt. ${ }^{106}$ Während Litke den Projektleiter als Führungskraft bezeichnet und durch die Aufzählung der Anforderungen und Aufgaben tendenziell dem Management zuordnet, ${ }^{107}$ weist Horsch dem Projektleiter die Rolle eines mit Prozesskompetenz ausgestatteten und auf der operativen Ebene für die Projektdurchführung verantwortlichen und dem Top-Management unterstellten Entscheidungsträgers zu. ${ }^{108}$ Das Hauptunterscheidungsmerkmal beider möglichen aufgezeigten und mit Leitungsfunktionen versehenen Rollen des Projektleiters entweder als Leitungsorgan des ausführenden Projektteams oder als Teil des Managements der das Projekt beauftragenden Unternehmung lässt sich darin erkennen, ob der Projektleiter die Aufgabe der für die Ausführung erforderlichen Projektplanung übernimmt und daher auch über Prozesskompetenz verfügt. ${ }^{109}$

Im Rahmen dieser Arbeit nimmt der Auftraggeber im Folgenden die Rolle des Kooperationsträgers ein. Dieser besitzt die Kompetenz, sämtliche für die Planung und Steuerung der Kooperationsbeziehung notwendigen Entscheidungen zu treffen. ${ }^{110}$ Tröndle unterscheidet den Kooperationsträger nach dessen Funktionen in Zielsetzungs- und Zielerreichungsträger. ${ }^{111}$ Während der Zielsetzungsträger vor allem für das Zustandekommen der Kooperation verantwortlich ist, befasst sich der Zielerreichungsträger

\footnotetext{
104 Vgl. Beck (1996), S. 98.

${ }^{105}$ Vgl. Keßler/Winkelhofer (2004), S. 96.

106 Die englischsprachige Literatur verwendet hierfur den Ausdruck Project Manager sowie Project Leader. Vgl. etwa Archibald (2001), S. 441; Kerzner (2003), S. 9; Walker (2002), S. 202.

107 Vgl. Litke (2004), S. $168 \mathrm{f}$.

108 Vgl. Horsch (2003), S. $186 \mathrm{f}$.

109 Die Ausführungen in den folgenden aufgefuhrten Literaturstellen deuten jeweils auf die Rolle des Projektleiters hin. Zu der Rolle als Leitungsorgan des das Projekt ausfuhrenden Projektteams vgl. Beck (1996), S. 103ff.; Hansel/Lomnitz (2003), S. 153; Höffken/Schweitzer (1991), S. 85; Horsch (2003), S. 188; Kerzner (2003), S. 10; Keßler/Winkelhofer (2004), S. 100; Madauss (2000), S. 87; Olfert/Steinbuch (2003), S. 130. Zu der Rolle als Teil des Managements der Unternehmung des Auftraggebers vgl. Burghardt (2002a), S. 103; Litke (2004), S. 169.

110 Vgl. Tröndle (1986), S. 71.

111 Vgl. Tröndle (1986), S. 71f.
} 
auch mit den das Kooperationsziel herbeiführenden Maßnahmen. ${ }^{112}$ Beide Aufgaben werden durch den Auftraggeber für die untersuchte Projektkooperation wahrgenommen: Er entscheidet sowohl über die Definition und Formulierung der Projektziele als auch über die für die Zielerreichung erforderlichen Maßnahmen der Planung, Steuerung und Überwachung des Gesamtprojektes. ${ }^{13}$

Der Auftraggeber besitzt durch die hierarchische Organisationsstruktur der Kooperation in Form einer Über-/Unterordnungsbeziehung die Entscheidungs- und Weisungskompetenz gegenüber dem Auftragnehmer. ${ }^{114}$ Durch diese Position ist es für den Auftraggeber möglich, Einfluss auf das Verhalten des Auftragnehmers auszuüben. ${ }^{115}$ Möglichkeiten einer Verhaltensbeeinflussung durch in den Projektvertrag integrierte Anreizsysteme werden in Kapitel 2.3.3 allgemein sowie in Kapitel 3.4.1 detailliert analysiert und im Rahmen der Ausführungen der Kapitel 5.4 und 5.5 in die Projektplanung des Auftraggebers eingebunden sowie anhand des Anwendungsbeispiels umgesetzt. Hierfür wird in dem folgenden Kapitel das mögliche Verhalten des Auftragnehmers innerhalb der Kooperationsbeziehung betrachtet. Dieses Verhalten kann als Konsequenz der Entscheidung des Auftragnehmers über die zur Erbringung der vertraglich festgelegten Projektleistungen gewählte Arbeitsanstrengung definiert werden.

\subsubsection{Die Entscheidung des Auftragnehmers über seinen Arbeitseinsatz}

Das Erreichen der Kooperations- und somit der Projektziele hängt maßgeblich von dem durch den Auftragnehmer für die Leistungserstellung gewählten Arbeitseinsatz ab. Das dem Handeln des Auftragnehmers zugrunde liegende Niveau des Arbeitseinsatzes ist das Ergebnis einer Entscheidung über die ihm zur Verfügung stehenden Handlungsalternativen. ${ }^{116}$ Das Vorgehen der für die Entscheidung über den Arbeitseinsatz erforderlichen individuellen Bewertung der Konsequenzen der Handlungsalternativen kann in Anlehnung an das Erwartungs-Valenz-Modell von Vroom verdeut-

\footnotetext{
112 Diese Unterteilung ähnelt den zwei zuvor unterschiedenen Rollen eines Projektleiters.

113 Vgl. Litke (2004), S. 168f.; Madauss (2000), S. 87.

114 Vgl. Beck (1996), S. 100.

115 Der Auftraggeber besitzt aufgnund der Selbstständigkeit der Organisationen der Projektpartner nicht die Macht, auch gegen den Willen des Auftragnehmers eine Verhaltensänderung herbeizuführen. Für die Abgrenzung der Begriffe Macht und Einfluss vgl. etwa Staehle (1999), S. 398; Steinmann/Schreyögg (2000), S. 581f. Das Vertrauen in den Kooperationspartner beeinflusst den Projekterfolg. Vgl. hierzu Vaaland (2004); Wong/Cheung (2004).

116 Vgl. Jost (2000), S. 121. Das Handeln eines Menschen kann als eine Teilklasse seines Verhaltens definiert werden und umfasst ausschließlich dessen zielgerichtetes und sinnhaftes Verhalten. Vgl. Wolf (2003), S. 183.
} 
licht werden. ${ }^{117}$ Dieses als Teil der Prozesstheorie den kognitiven Wahltheorien zugehörige Modell erklärt die Entstehung, Ausrichtung und Stärke von Aktivitäten als Ergebnis einer zukunftsorientierten Wahlentscheidung über Anstrengungs-ErgebnisVerknüpfungen. ${ }^{118}$ Dieser Weg-Ziel-Ansatz kann auf die Kooperationsbeziehung zwischen Auftraggeber und Auftragnehmer übertragen werden und ist in Abbildung 2.9 dargestellt:

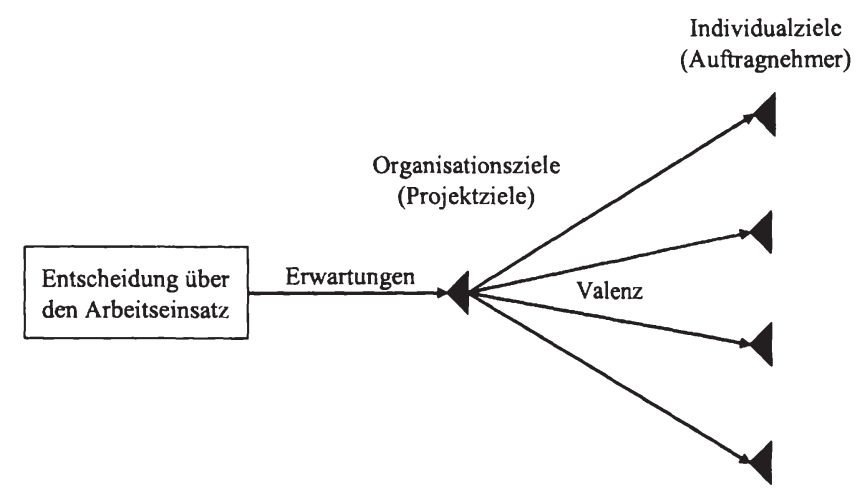

Abbildung 2.9: Entscheidung über den Arbeitseinsatz des Auftragnehmers (Quelle: In Anlehnung an Steinmann/Schreyögg (2000), S. 485.)

Dem Auftragnehmer stehen verschiedene Arbeitsniveaus als Handlungsalternativen zur Verfügung. Mit der Entscheidung des Auftragnehmers für ein Arbeitsniveau sind unterschiedliche Ausprägungen der Handlungen und somit Erwartungen darüber verbunden, zu welchem Ergebnis diese Handlungen führen können. ${ }^{119}$ Die Individualziele des Auftragnehmers ergeben sich sowohl aus den für ihn mit der Wahl des Arbeitsniveaus verbundenen Konsequenzen als auch aus den von der Erreichung der Organisationsziele abhängigen Konsequenzen. Der Wert der Valenz gibt dabei an, welche Bedeutung die verschiedenen Individualziele, wie beispielsweise eine hohe Entlohnung oder die Erfüllung der Anforderungen des Auftraggebers, für den Auftragnehmer haben. ${ }^{120}$ Die Motivation des Auftragnehmers, eine bestimmte Alternative zu wählen, ist somit abhängig von dessen subjektiven Erwartungen, mit den Handlungen ein bestimmtes Organisationsziel zu realisieren mit der Folge, in Abhängigkeit von dem die-

\footnotetext{
${ }^{117}$ Vgl. Vroom (1964), S. 8ff.; Kirchler/Rodler (2001), S. 40ff.; Steinmann/Schreyögg (2000), S. 484ff.

118 Vgl. Staehle (1999), S. 231.

${ }_{119}$ Das Ergebnis ist von den zukünftig eintretenden Umweltzuständen abhängig.

${ }^{120}$ Vgl. Vroom (1964), S. 15f.
} 
sen Zielzuständen beigemessen Wert die durch ihn hoch geschätzten Individualziele erreichen zu können. ${ }^{121}$

Das Ergebnis der Analyse dieser Entscheidungssituation ist in Abbildung 2.10 dargestellt und zeigt, dass das Arbeitsverhalten des Auftragnehmers aus dem Zusammenspiel von Person und Situation hervorgeht: ${ }^{122}$

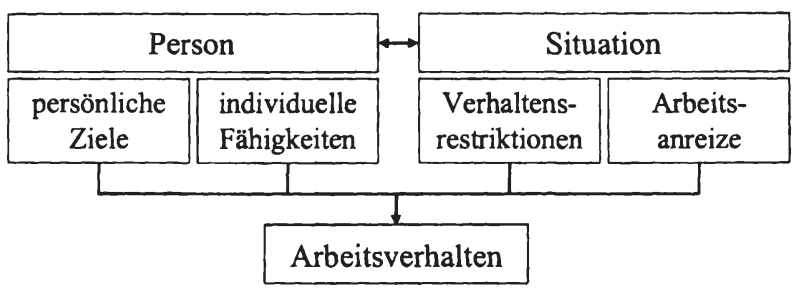

Abbildung 2.10: Einflussfaktoren für das Arbeitsverhalten (Quelle: In Anlehnung an Jost (2000), S. 120.)

Sowohl die persönlichen individuellen Fähigkeiten sowie die individuellen Motive und somit Ziele des Auftragnehmers als auch die durch die Arbeitssituation des Auftragnehmers vorherrschenden Verhaltensrestriktionen ${ }^{123}$ sowie die mit der Leistungserstellung verbundenen Anreize bestimmen das Arbeitsverhalten des Auftragnehmers und damit auch den individuellen Erfolg der Kooperation. ${ }^{124}$ Die als Einflussfaktor für das Arbeitsverhalten aufgefuihrten Anreize stellen intrinsische Arbeitsanreize dar, welche direkt aus der Arbeitsaufgabe resultieren und den Auftragnehmer motivieren, durch einen höheren Arbeitseinsatz auch ein höheres persönliches Ziel zu erreichen. ${ }^{125}$ Die Anreizwirkung entsteht somit nicht durch die Folgen seiner Arbeitsanstrengung, sondern durch die Arbeitsanstrengung selbst. Im Gegensatz hierzu besteht die Möglichkeit, die Motive des Auftragnehmers durch extrinsische Anreize etwa in Form von Belohnungen oder Bestrafungen eines bestimmten Arbeitsverhaltens $\mathrm{zu}$ beeinflussen. Dieser durch den Auftraggeber initiierte Motivationsprozess ist daher Gegenstand der Ausführungen des folgenden Kapitels.

\footnotetext{
121 Vgl. Steinmann/Schreyögg (2000), S. 487; Staehle (1999), S. $231 \mathrm{ff}$; Vroom (1964), S. 14ff.

122 Vgl. Jost (2000), S. 119; Staehle (1999), S. 196ff.

${ }^{123}$ Die Verhaltensrestriktionen schränken den Verhaltensspielraum des Auftragnehmers ein. Ursachen hierfür können ressourcenbedingte, rechtliche oder soziale Restriktionen sein. Vgl. hierzu Jost (2000), S. 82f.

124 Tröndle (1986), S. 77 bezeichnet die für den individuellen Erfolg der Kooperation verantwortlichen Faktoren mit Kooperationsbereitschaft und Kooperationsfähigkeit. Damit wird auch hier auf den Zusammenhang von Person und Situation hingewiesen.

125 Vgl. Jost (2000), S. 82.
} 


\subsubsection{Möglichkeiten der Beeinflussung des Auftragnehmerverhaltens}

Der Motivationsbegriff wurde bereits mehrfach in den vorherigen Kapiteln erwähnt, ist bisher jedoch nicht grundlegend definiert worden. Nach Staehle , ... soll (Motivation) Aufschluss geben über die Beweggründe des Handelns und Verhaltens eines Menschen. “126 Er bezeichnet Motivation als „... Voraussetzung für zielorientiertes Verhalten und deshalb aus Managementperspektive (als) Hauptansatzpunkt für leistungssteigernde Beeinflussungsstrategien. “127 Durch diese Definition des Motivationsbegriffs kommt zum Ausdruck, dass Motivation zwar durch Dritte beeinflussbar ist, jedoch vorwiegend als das Zusammenwirken eines Wahrnehmungsprozesses mit den individuellen Zielen und Erwartungen eines Menschen aufgefasst werden muss. ${ }^{128}$

Für die Kooperationsbeziehung bedeutet dies, dass der Auftragnehmer für die Erreichung seiner individuellen Ziele zunächst für unterschiedliche Anstrengungsniveaus unter Beachtung der von ihm wahrgenommenen Verhaltensrestriktionen die erwarteten möglichen Arbeitsergebnisse bestimmt. Unter Berücksichtigung der Arbeitsanreize lassen sich die Konsequenzen aus den Arbeitsergebnissen ableiten und der aus der Sicht des Auftragnehmers beste und zu realisierende Arbeitseinsatz identifizieren. Dieser Zusammenhang ist in Abbildung 2.11 dargestellt:

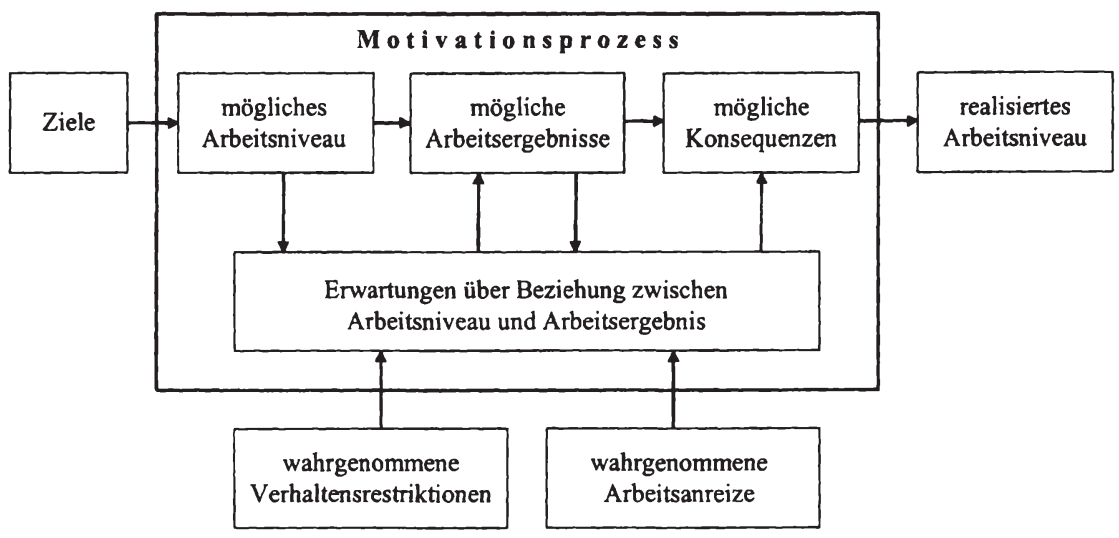

Abbildung 2.11: Motivationsprozess des Auftragnehmers

(Quelle: In Anlehnung an Jost (2000), S. 108.)

\footnotetext{
126 Staehle (1999), S. 219.

127 Staehle (1999), S. 218.

${ }^{128}$ Vgl. Jost (2000), S. 104f.
} 
Der in Abbildung 2.11 dargestellte Motivationsprozess beruht insbesondere auf den Erwartungen des Auftragnehmers hinsichtlich der Beziehung zwischen den möglichen Arbeitsniveaus und den hieraus resultierenden Konsequenzen. ${ }^{129}$

Besteht ein Konflikt zwischen den individuellen Zielen des Auftragnehmers und den Projektzielen des Auftraggebers, liegt ein Motivationsproblem vor. Das Ziel der Lösung dieses Motivationsproblems ist nicht nur das Schaffen eines Interessenausgleichs zwischen den Kooperationspartnern, sondern auch das Sicherstellen der Kooperation mit dem Auftragnehmer. ${ }^{130}$ Staehle bezeichnet diese zwei Arten der Motivation als Leistungsentscheidung und Teilnahmeentscheidung. ${ }^{131}$

Die Teilnahmeentscheidung, welche den Auftragnehmer motiviert, die Kooperationsbeziehung mit dem Auftraggeber einzugehen, wird formal in Kapitel 3.2.4 vorgestellt und in Kapitel 5.4 in die quantitative Projektplanung eingebunden. Auch die Leistungsentscheidung wird zunächst theoretisch in Form der Anreizkompatibilitätsbedingung in Kapitel 3.2.4 analysiert und anschließend in Kapitel 5.5 in Verbindung beispielhaft vorgeschlagener Anreizfunktionen in die quantitative Planung integriert.

Es ist die Aufgabe des Auftraggebers, durch den Einsatz von Motivationsinstrumenten zu versuchen, die intrinsische Motivation des Auftragnehmers durch extrinsische Arbeitsanreize so zu beeinflussen, dass ein zielkonformes Verhalten erreicht wird. ${ }^{132}$ Die intrinsische Motivation als Zusammenspiel von Strebensziel und Handlung des Auftragnehmers umfasst nicht nur positive Charakteristika, sondern kann auch zu unerwünschtem, beispielsweise opportunistischem Verhalten führen. ${ }^{133}$

Durch die Integration von Anreizsystemen als Motivationsinstrumente in die Vertragsgestaltung kann die Motivation des Auftragnehmers beeinflusst werden. Bei der Auswahl der Anreizsysteme durch den Auftraggeber ist hinsichtlich der gewünschten Motivationswirkungen $\mathrm{zu}$ beachten, dass durch die resultierende Aufgabenerfüllung gleichzeitig die von dem Auftragnehmer hoch geschätzten individuellen Ziele erreicht werden können. ${ }^{134}$ Gleichzeitig besteht bei dem Gebrauch von Anreizsystemen die Ge-

\footnotetext{
${ }^{129}$ Für eine ausfuhrlichere Darstellung vgl. auch Heinrich (2002), S. 426.

${ }^{130}$ Vgl. Jost (2000), S. 458.

131 Vgl. Staehle (1999), S. 817.

132 Vgl. Jost (2000), S. 456. Für eine Abgrenzung extrinsischer und intrinsischer Motivation vgl. etwa Frey/ Osterloh (2002), S. 8f.; Kirchler/Rodler (2001), S. $11 \mathrm{ff}$.

${ }^{133}$ Vgl. Staehle (1999), S. 243.

${ }^{134}$ Vgl. Steinmann/Schreyögg (2000), S. 492. Heinrich (2002), S. 436f. zăhlt allgemeine Faktoren zur Motivation von Mitarbeitern auf, welche die Motivationswirkungen unterstützen können.
} 
fahr, dass die intrinsische Motivation des Auftragnehmers insbesondere durch den wiederholten Einsatz der Motivationsinstrumente so verändert wird, dass dieser ausschließlich Aufgaben durchführt, wenn hiermit Anreize für ihn verbunden sind. ${ }^{135}$

In Kapitel 3.4 werden unterschiedliche Arten von Anreizen speziell für die Projektkooperation diskutiert, so dass auf diesen Aspekt an dieser Stelle nicht detailliert eingegangen wird. Dort wird auch aufgezeigt, in welcher Form diese Anreize in Anreizsysteme eingebunden werden können und welche Konsequenzen sich hieraus für die Kooperationspartner ergeben.

In diesem in das Management von Projektkooperationen einführenden Kapitel ist bereits mehrfach der Aspekt der Vertragsgestaltung erwähnt worden. Die im Rahmen dieser Arbeit betrachtete und in Kapitel 2.2 erläuterte Organisationsstruktur der Projektkooperationsbeziehung zwischen einem Auftraggeber und einem Auftragnehmer erfordert ein Vertragsmanagement als Bestandteil des Projektmanagements des Auftraggebers. Die Bestimmung des Vertragsdesigns durch den Auftraggeber findet während der Vereinbarungsphase sowie der Phase der Projektdefinition statt und dient der Festlegung der durch den Auftragnehmer zu erbringenden Projektleistungen sowie der durch den Auftraggeber zu leistenden Vergütungen.

Das dritte Kapitel analysiert die aus betriebswirtschaftlicher Sicht relevanten Aspekte des Vertragsmanagements sowie des Vertragsdesigns und berücksichtigt hierbei insbesondere die aus der Kooperationsbeziehung resultierenden Probleme asymmetrisch verteilter Informationen.

${ }^{135}$ Vgl. Frey/Osterloh (2002), S. 9f., die dieses Phänomen als Crowding-out-effect bezeichnen; Kirchler/Rodler (2001), S. 14f. 


\section{Vertragsmanagement als Bestandteil des Projektmanagements}

Die Ausführungen des zweiten Kapitels zeigen, dass eine erfolgreiche Planung und Durchführung von Projektkooperationen nicht nur ein zielgerichtetes Projektmanagement voraussetzt, sondern zusätzlich um ein Vertragsmanagement ergänzt werden muss. Der Begriff der Projektkooperation sowie seine Auslegung und Bedeutung für die in dieser Arbeit untersuchte Kooperationsform zwischen dem Auftraggeber und einem Auftragnehmer ist in Kapitel 2.2 behandelt worden. Ziel dieses dritten Kapitels ist eine umfassende Analyse der Vertragsbeziehung zwischen den Projektpartnern.

Kapitel 3.1 zeigt hierfür die Ursprünge der ökonomischen Vertragstheorie sowie Anforderungen an ein Vertragsmanagement auf. Eine Vertragsbeziehung stellt immer eine Verbindung zwischen mindestens zwei Vertragsparteien her. Insbesondere im Rahmen der in dieser Arbeit unterstellten Vertragsbeziehung zwischen Auftraggeber und Auftragnehmer sind Interessenkonflikte sowie Probleme der ungleich verteilten Information von großer Bedeutung.

Das Kapitel 3.2 wird sich daher zunächst der Problematik der Informationsasymmetrie widmen und anschließend einen Bezug zur Prinzipal-Agenten-Theorie sowie dem Konzept der hierarchischen Planung herstellen. Dieses Kapitel schließt mit Überlegungen, welche Anforderungen an ein Informationsmanagement zu stellen sind, um die durch die Informationsasymmetrie begründeten Probleme zu mindern.

Kapitel 3.3 gibt einen Überblick über die vielfältigen Möglichkeiten des Vertragsdesigns im Allgemeinen sowie über die Besonderheiten von Investitionsprojektkooperationen. Es werden sowohl die für Kostenerstattungs- als auch für Festpreisverträge typischen Charakteristika sowie die mit diesen Vertragsdesigns einhergehenden Chancen und Probleme für Auftraggeber und Auftragnehmer dargestellt und analysiert.

Im Anschluss daran werden in Kapitel 3.4 Möglichkeiten aufgezeigt, wie Anreizsysteme für die in Kapitel 3.3 vorgestellten Vertragsdesigns dazu beitragen können, die aus der Informationsasymmetrie resultierenden Probleme zu reduzieren. 


\subsection{Einführung in das Vertragsmanagement}

\subsubsection{Der Vertrag als Grundlage einer Kooperation}

Ein Vertrag kann allgemein als eine Vereinbarung zwischen mindestens zwei Parteien definiert werden, die aus der Sicht der Parteien bindende Verpflichtungen für eine oder mehrere Seiten auslöst und somit durch die Festlegung von Verfügungs- und Kontrollrechten die Aufgaben und das Verhalten der Parteien regelt. ${ }^{1}$ Inhalt einer vertraglichen Vereinbarung und damit gleichzeitig Funktion eines Vertrags ist die Regelung der Sachverhalte, die nicht in allgemeiner Form durch Gesetze vorgegeben sind. ${ }^{2}$ Ebenso beinhaltet ein Vertrag Sachverhalte, die zwar durch gesetzliche Vorschriften geregelt, jedoch von den Parteien nicht akzeptiert werden, sofern diese Änderungen gesetzlich zulässig sind. ${ }^{3}$ Das Werkvertragsrecht, welches bei Werkvertragsleistungen wie beispielsweise im Bereich des Industrieanlagenbaus oder komplexer Bauvorhaben Anwendung findet, ist ein Beispiel dafür, dass durch den Gesetzgeber nicht jeder Sachverhalt geregelt worden ist, sondern Freiräume für die individuellen Bedürfnisse der Beteiligten geschaffen wurden, die somit einer vertraglichen Regelung bedürfen. ${ }^{4}$

Die Theorie der Neuen Institutionenökonomik unterscheidet drei Forschungsrichtungen, die sich mit vertragstheoretischen Fragestellungen beschäftigen und in folgender Abbildung aufgeführt sind: ${ }^{5}$

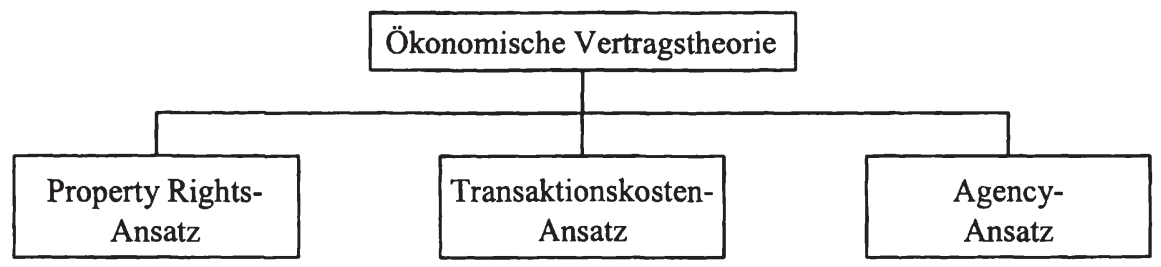

Abbildung 3.1: Forschungsrichtungen ökonomischer Vertragstheorie

\footnotetext{
Vgl. Pfaffmann (1996), S. 646. Macho-Stadler/Pérez-Castrillo (2001), S. 5f. betonen, dass die vereinbarten Leistungen und Gegenleistungen verifizierbar sein müssen, um sie ggf. vor Gerichten durchsetzen zu können.

2 Zu juristischen Aspekten einer Vertragsbeziehung zwischen Auftraggeber und Auftragnehmer vgl. beispielsweise Schmidt (2004).

3 Vgl. Heussen (2002), S. If.

4 Vgl. Heussen (2002), S. 3.

5 Vgl. beispielsweise Werin/Wijkander (1992), S. 2ff.; Wolff (2000), S. 31 ff. Für einen Überblick uber Property Rights-Ansatz und Transaktionskosten-Ansatz vgl. etwa Richter (1991). Für eine Gesamtbeurteilung der Neuen Institutionenökonomie vgl. Wolf (2003), S. 283ff.
} 
Grundbedingung jedes wirtschaftlichen Handelns und damit gleichzeitig Voraussetzung jeder praktischen und theoretischen Erforschung marktwirtschaftlicher Ordnungen ist die Garantiertheit und Handelbarkeit privaten Eigentums. ${ }^{6}$ Auf Coase geht der Gedanke zurück, dass anstelle von Gütern Rechte oder Bündel von Rechten (PropertyRights) an ihnen gehandelt werden, wobei es für die Zielsetzung ökonomischer Effizienz irrelevant ist, wem diese Rechte zugeordnet sind. ${ }^{7}$ Der Property-Rights-Ansatz erklärt sowohl die Koordination von Leistungsprozessen als auch die Motivation für einen ökonomischen Ressourceneinsatz. ${ }^{8}$

Die von Coase begründete und von Williamson fortgefürte Transaktionskostentheorie stellt die Organisation in den Vordergrund. ${ }^{9}$ Sie geht der Frage nach, bei welcher Organisationsform innerhalb des Spektrums zwischen Markt und organisatorischer Hierarchie die zur Abwicklung ökonomischer Transaktionen entstehenden Transaktionskosten am geringsten sind. ${ }^{10}$ Transaktionskosten fallen bei der Vertragsabfassung und dem Vertragsabschluss in Form von Suchkosten, Anbahnungskosten, Verhandlungskosten, Abschlusskosten sowie nach Vertragsabschluss durch Kontroll- und Anpassungskosten an. ${ }^{11}$

Im Zentrum des Agency-Ansatzes steht die Annahme, dass eine Leistungsbeziehung zwischen Akteuren einer Transaktion durch Informationsasymmetrien geprägt ist. ${ }^{12}$ Die aus der Informationsasymmetrie resultierenden Prinzipien der Arbeitsteilung und der Kooperation zwischen den Akteuren wirken sich ökonomisch zwar wohlstandssteigernd aus, bergen jedoch auch Gefahren in sich. Opportunistisches Verhalten sowie Grenzen der Informiertheit der Akteure können zu einem Vorteil des besser informierten bzw. zu einer Ausbeutung des schlechter informierten Akteurs führen. ${ }^{13}$ Die Prinzipal-Agenten-Theorie untersucht die Beziehung zwischen dem Prinzipal, der eine bestimmte Aufgabe an den Agenten delegiert, welcher die Aufgabe im Sinne des Prinzipals erfüllen soll, sowie die aus dieser Beziehung resultierenden Probleme. ${ }^{14}$

\footnotetext{
Vgl. Wolff (2000), S. 34.

7 Dieser unter dem Begriff des Coase-Theorems geprägten Aussage liegt die Annahme zugnunde, dass keine Transaktionskosten berucksichtigt werden. Vgl. Coase (1937).

8 Vgl. Wolff (1995), S. 25f; Wolff (2000), S. 35.

9 Vgl. Coase (1937); Williamson (1975). Einen Überblick über die empirische Forschung der Transaktionskostentheorie geben Shelanski/Klein (1995).

10 Vgl. Erlei (1998), S. 30f.; Wolff (2000), S. 36f.

"Vgl. Adler et al. (1998); Hammann et al. (2001), S. 257ff.; Wolf (2003), S. 269. Für unterschiedliche Beispiele von Transaktionkosten vgl. Richter/Furubotn (2003), S. 57ff.

12 Vgl. Picot (1991), S. 150.

13 Vgl. Wolff (2000), S. 41.

14 Vgl. Jost (2001), S. 12ff. Die Prinzipal-Agenten-Theorie wird in Kapitel 3.2.2 ausfuhrlich betrachtet.
} 
Alle drei Ansätze der Neuen Institutionenökonomik lassen sich zu einer ökonomischen Vertragstheorie integrieren. Der Vertrag als Grundlage einer die Transaktionskosten der Leistungserstellung senkenden eingegangenen Kooperation zwischen Akteuren regelt die Übertragung von Property Rights beispielsweise in Form von Entscheidungs-, Eigentums- oder Gewinnrechten. Er kann durch die arbeitsteilige Organisationsform und die mit ihr verbundenen Informationsasymmetrien jedoch zu Wohlfahrtsverlusten führen.

Ziel des folgenden Kapitels ist es, zunächst die mit dem Abschluss von Verträgen verbundenen Managementaufgaben zu analysieren sowie deren Bedeutung für den Erfolg von Investitionsprojektkooperationen herauszustellen.

\subsubsection{Phasen des Vertragsmanagements}

Es ist die Aufgabe des Managements, komplexe Vorhaben zu planen und mit Hilfe geeigneter Führungs- und Kontrollmaßnahmen zu realisieren. ${ }^{15}$ Der Begriff des Vertragsmanagements (Contract Management) beschreibt somit alle planerischen und organisatorischen Tätigkeiten, die mit der Gestaltung und Realisation des Vertrags verbunden sind. ${ }^{16}$ Hierbei sind die Planungsaufgaben des Managements mit den rechtlichen Bewertungsaufgaben der Rechtsabteilungen zu koordinieren. ${ }^{17}$ In Anlehnung an die Funktionen des Managements Planung, Organisation, Durchsetzung und Kontrolle kann das Vertragsmanagement in die in Abbildung 3.2 aufgeführten Phasen untergliedert werden. ${ }^{18}$ Durch die Definition des Ablaufs zeitlich-logischer, zusammenhängender Aktivitäten werden die funktionalen Ansätze in einen Zeitbezug gestellt und können somit als Phasen eines Managementprozesses angesehen werden. ${ }^{19}$

15 Es wird hier der funktionale Ansatz der Managementlehre betrachtet. Institutionale Aspekte des Vertragsmanagements werden in Kapitel 3.3.3 im Rahmen der Betrachtung hierarchischer Entscheidungen bei Projektkooperationen diskutiert. Zu den Aufgaben des Managements vgl. beispielsweise Staehle (1999); Steinmann/Schreyögg (2000).

16 Vgl. etwa Weber (2003), S. 984.

17 Vgl. Heussen (2002), S. 15f.

18 Diese vier Managementfunktionen, die auf die funktionale Gliederung der Unternehmung durch Fayol zurückgehen, finden nach Staehle (1999), S. 81 in den meisten Managementlehrbüchern Beachtung. Zu den Elementen des Vertragsmanagements vgl. Gilbreath (1992), S. 6; Heussen (2002), S. 15. Hier wird eine Unterteilung in die Phasen Vertragsplanung, Vertragsgestaltung, Vertragsverwaltung und Vertragsüberwachung gewählt.

19 Für eine Abgrenzung der Begriffe Ablauf und Prozess vgl. Völkner (1998), S. 29. Zum Prozessbegriff vgl. etwa Staehle (1999), S. 81. Crocker/Masten (1991), S. 96 stellen fest, dass das Vertragsmanagement als Prozess betrachtet werden kann, und erklären die Verhandlung und Festsetzung von Preisen in Langzeitverträgen. 


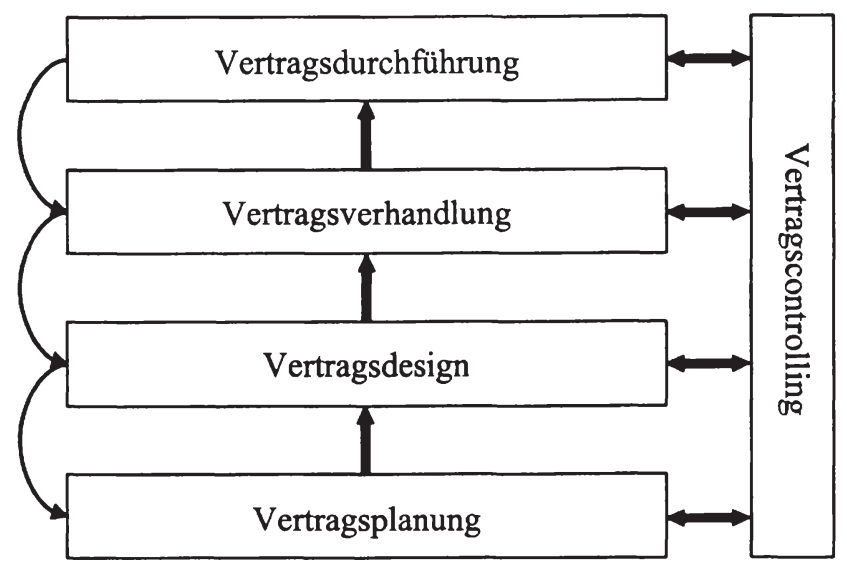

Abbildung 3.2: Phasen des Vertragsmanagements

Als Erweiterung der in der Literatur erfolgten Aufzählung der Elemente des Vertragsmanagements zeigt Abbildung 3.2 durch die mit Pfeilen angedeuteten Beziehungen zwischen den einzelnen Elementen, dass zwar eine zeitliche Reihenfolge zwischen Vertragsplanung, -design, -verhandlung und -durchführung besteht, jedoch auch Rückkopplungen zwischen diesen möglich und notwendig sind, um die Option zu eröffnen, auch nach Vertragsabschluss steuernd in das Vertragsmanagement eingreifen zu können. ${ }^{20}$ Der Ablauf der Vertragsmanagementprozesse wird im Folgenden näher erläutert sowie deren Relevanz für die weiteren Untersuchungen dieser Arbeit herausgestellt. $^{21}$

Der Prozess der Vertragsplanung beginnt mit der Festlegung der Vertragsstrategie und der Vertragstaktik und endet mit der Vertragsvorbereitung. ${ }^{22}$ Während die Vertragsstrategie die Bestimmung der Zielrichtung umfasst, die mit dem Vertrag erreicht werden soll, ist es die Funktion der Vertragstaktik, die für das Erreichen dieser Ziele möglichen Handlungsalternativen zu identifizieren. ${ }^{23}$ Die Vertragsziele können sowohl monetäre als auch nicht-monetäre Ziele sein und müssen im Einklang mit den Projektzielen stehen. Die charakteristischen Merkmale der hier untersuchten Kooperationsbe-

20 Auf den Begriff und die Funktionen des Vertragscontrollings wird später eingegangen.

21 Als Prozessablauf werden Aktivităten definiert, die zu Zustandsänderungen im Zeitverlauf fuhren und als mögliches Ziel das Erreichen eines Endzustandes verfolgen. Vgl. Völkner (1998), S. 29; Völkner/Werners (2000), S. 634.

22 Vgl. Heussen (2002), S. 28.

23 Vgl. allgemein zur Aufgabe der Planung Steinmann/Schreyögg (2000), S. 9 sowie speziell zur Vertragsplanung Heussen (2002), S. $28 \mathrm{ff}$. 
ziehung führen dazu, dass der Prozess der Vertragsplanung insbesondere durch folgende Faktoren beeinflusst wird: ${ }^{24}$

- Güte des Informations- und Kommunikationsprozesses zwischen den Partnern

- Machtverhältnisse/Beziehungen der Partner zu- und untereinander

- Ressourcen (Zeit- und Finanzplanung) der Projektkooperation

Die Vertragsplanung legt somit die strategische Ausrichtung der mit dem Kooperationsvertrag verbundenen Ziele und Handlungsmöglichkeiten fest. Gleichzeitig muss bereits in dieser frühen Phase des Vertragsmanagements das mögliche Verhalten des bzw. der Kooperationspartner einbezogen werden.

Der Begriff des Vertragsdesigns kann nach Heussen charakterisiert werden: „Die Auslegung und das Design des Vertrags sollten so sein, dass der Vertrag realisierbar, verifizierbar und seine Durchführung lenkbar ist. ${ }^{\text {‘25 }}$ Branconi und Loch führen Vereinbarungen zwischen den Kooperationspartnern auf, die in einen Vertrag aufgenommen werden sollten. ${ }^{26}$ Sie unterteilen diese in projektspezifische Vereinbarungen und solche, die aufgrund der Kooperation Beachtung finden müssen. $\mathrm{Zu}$ der ersten Gruppe zählen etwa Vereinbarungen über den Projektgegenstand, Schätzungen für Preise und Kosten, Vereinbarungen über den zeitlichen Projektablauf sowie über die Abwicklung von Zahlungen. Die zweite Gruppe enthält Vereinbarungen über Garantieregelungen nach Projektende, Strafen für die Nichterfüllung von Leistungen sowie Haftungsbegrenzungen für den Auftragnehmer. Für die insbesondere im fünften Kapitel erfolgenden weiteren Untersuchungen spezieller Zahlungsvereinbarungen sowie deren Konsequenzen auf die zeitliche Projektplanung ist die erste Gruppe von Vereinbarungen von besonderem Interesse.

Aus betriebswirtschaftlicher Sicht ist die Frage zu stellen, welchen Einfluss das Vertragsdesign auf den Erfolg des dem Vertrag zugrunde liegenden Projektes hat. Hierbei interessieren insbesondere die Fragestellungen, in welcher Form das Vertragsdesign dazu beitragen kann, das Verhalten der Vertragspartner zu steuern und die mit der Kooperation verbundenen Risiken und Probleme zu bewältigen. ${ }^{27}$

\footnotetext{
24 Vgl. Heussen (2002), S. 34.

25 Heussen (2002), S. 102. Zum Vertragsdesign vgl. auch Gilbreath (1992), S. 67ff.

26 Vgl. Branconi/Loch (2004), S. 5.

27 Vgl. Werners/Slaghuis (2004), S. 353.
} 
Obwohl zum Zeitpunkt der Vertragsplanung und des Vertragsdesigns erste Kontakte zwischen den Vertragspartnern bestehen, stellt die Phase der Vertragsverhandlung einen wichtigen Prozess im Rahmen des Vertragsmanagements dar, bei dem beide Seiten versuchen, ihre gegenseitigen Interessen durchzusetzen. ${ }^{28}$ Dieser Informationsaustausch mit dem Ziel des Vertragsabschlusses ist nicht an eine bestimmte Form gebunden, sondern kann schriftlich durch den Austausch von Dokumenten oder durch eine Konferenz der Vertragspartner erfolgen. Heussen bezeichnet die Vertragsverhandlung als ein Spiel mit offenen, versteckten und unbewussten Regeln zwischen Personen. ${ }^{29}$ Um beide Parteien vor opportunistischem Verhalten oder gar Täuschung durch den anderen Geschäftspartner zu schützen, hat der Gesetzgeber Regelungen getroffen, die bei Verletzung einer Offenbarungs-, Hinweis- oder Anzeigepflicht zu Schadenersatz verpflichten. ${ }^{30}$ Dennoch ist die Phase der Angebotsabgabe durch den Auftragnehmer sowie die nachfolgende Verhandlung über die Vertragsinhalte von herausragender Bedeutung für die Entscheidung des Auftraggebers und den Erfolg der Kooperation. Während die deutschsprachigen Lehrbücher des Projektmanagements diesen Prozess kaum oder gar nicht berücksichtigen, nehmen sich einige wissenschaftliche Fachbeiträge der Fragestellung an, wie sich die Parteien insbesondere während der Phasen des Bieter- und Auswahlprozesses verhalten sollen. ${ }^{31}$ Diese Thematik wird im Zuge der Einbindung von Anreizsystemen in das Vertragsdesign in Kapitel 3.4 aufgegriffen.

Die Phase der Vertragdurchführung startet im Anschluss an die Verhandlung und Unterzeichnung des Vertrags mit dem Beginn der Handlungen zur Realisation der dem Vertrag zugrunde liegenden Leistungen und endet mit der vollständigen Erfüllung des Vertragsgegenstandes oder mit Abbruch der Vertragsbeziehung bei Nichterfüllung. ${ }^{32}$ Dieser Managementprozess beinhaltet neben der eigentlichen Leistungserstellung

28 Während der Phasen vor der Vertragsverhandlung müssen Informationen uber mőgliche Vertragspartner eingeholt werden sowie Angebote abgegeben werden. Es kann angenommen werden, dass der Auftraggeber insbesondere bei großen Investitionsprojekten mit einem oder mehreren potenziellen Auftragnehmern in Kontakt steht, um projektspezifische Informationen auszutauschen. Das Pflichtenheft legt beispielsweise fest, wie und womit die Leistung zu erbringen ist. Vgl. Weber (2003), S. 986.

29 Vgl. Heussen (2002), S. 165.

30 Vgl. Schmid (2001), S. 395 sowie die hier genannten Paragraphen des Bürgerlichen Gesetzbuches. Gierl (2000), S. 110f. führt drei Maßnahmen zur Vermeidung von Opportunismus in einer Geschaftsbeziehung auf. Hierbei handelt es sich um vertragliche Regelungen, gute Beziehungsqualităt sowie ausgleichende Investitionen.

31 Für Lehrbücher des Projektmanagements vgl. etwa Corsten/Corsten (2000); KeBler/Winkelhofer (2004); Meier (2003). Zum Bieter- und Auswahlprozess vgl. etwa Ip/Yung/Wang (2004); Marzouk/Moselhi (2003); Turner (2003), S. $117 \mathrm{ff}$. Backhaus (2003), S. 554 nennt als zentrale Verhandlungspunkte technische Probleme, Finanzierungsprobleme, Preisnachlässe und Lieferzeitfragen.

32 Vgl. Gilbreath (1992), S. 145ff.; Heussen (2002), S. $281 \mathrm{ff}$. 
durch den Auftragnehmer Informationspflichten in Form von Hinweis- und Warnpflichten vor allem durch den Auftragnehmer, da dieser den Projektfortschritt besser beobachten und bewerten kann. ${ }^{33}$ Einseitige oder beiderseitige Leistungsänderungen während der Vertragsdurchführung können aus organisatorischen oder personellen Umstellungen, technischen Änderungen oder Veränderungen der finanziellen Bedingungen notwendig werden. Auch das während der Phase der Verhandlung nicht planbare Verhalten Dritter sowie zufällige Umwelteinflüsse können zu Abänderungen des Vertrags oder zu einem Wegfall der Geschäftsgrundlage führen. ${ }^{34}$ Heussen beschränkt die Tätigkeiten des Vertragscontrollings auf die Vertragsdokumentation, die Nachkalkulation sowie das gemeinsame Controlling von Ergebnissen und betrachtet diesen Prozess als zeitlichen Abschluss des Vertragsmanagements. ${ }^{35}$ Der betriebswirtschaftliche Begriff des Controllings beschränkt sich jedoch nicht nur auf eine Erfolgskontrolle. Vielmehr hat sich in den letzten Jahren eine koordinationsorientierte ControllingKonzeption herausgebildet, ${ }^{36}$ deren Ziel die „Koordination des Führungsgesamtsystems zur Sicherstellung einer zielgerichteten Lenkung “ ist. ${ }^{37}$ Durch die in Abbildung 3.2 gewählte Anordnung des Vertragscontrollingprozesses wird deutlich, dass das Controlling nicht nur als eine am Ende des Prozesses durchgeführte Erfolgskontrolle definiert werden darf, sondern als eine die Vertragsmanagementprozesse begleitend überwachende, koordinierende und steuernde Tätigkeit verstanden werden muss.

Zusammenfassend kann festgestellt werden, dass für das Erreichen der Ziele der Projektkooperation eine Integration des Vertragsmanagements in das Projektmanagement notwendig ist. Insbesondere bei langfristigen Kooperationsbeziehungen ist es vorteilhaft, die für das Projektmanagement verantwortlichen Organisationsmitglieder in die Phasen der Vertragsplanung und des Vertragsdesigns einzubinden. ${ }^{38}$ Gelingt es, durch ein entsprechendes Vertragsdesign neben den speziellen Projektanforderungen auch die organisations- und informationsrelevanten Gegebenheiten zu berücksichtigen, kann sich dies positiv auf die Projektdurchführung und das Kooperationsergebnis auswirken.

33 Wahrend dieser Phase spielen die in der Prinzipal-Agenten-Theorie bekannten Gefahren Hidden Action und Hidden Information eine große Rolle. Vgl. hierzu die Ausfuhrungen in Kapitel 3.2.2.

34 Der Vergleich von Ergebnis und Planung steht hierbei im Vordergrund. Vgl. Heussen (2002), S. 289.

35 Vgl. Heussen (2002), S. 305ff.

36 Vgl. Horváth (2003), S. 152ff.; Küpper (2001), S. 5 ff.

37 Küpper(2001), S. 12. Horváth und Schneider kritisieren diese Definition als zu weit gefasst und grenzen damit die Controllingfunktion von der allgemeinen Managementfunktion der Unternehmensleitung ab. Vgl. Horváth (2003), S. 154; Schneider (1994), S. 330.

38 Zur Bedeutung des Vertragsmanagements für das Projektmanagement vgl. Mulcahy (2001), S. 151ff. 
Bevor in Kapitel 3.2 näher auf die Thematik der Informationsasymmetrie zwischen den Kooperationspartnern eingegangen wird, beschäftigt sich der letzte Abschnitt dieses in das Vertragsmanagement einführenden Kapitels mit der Problematik unvollständiger Verträge.

\subsubsection{Die Problematik unvollständiger Verträge}

Ein Vertag wird dann als vollständig bezeichnet, sofern der Vertragsinhalt für alle möglichen Umweltzustände die jeweils zu realisierende Handlungsalternative vorsieht und die aus ihr resultierenden Konsequenzen bekannt sind, so dass alle Vertragsparteien auf der Grundlage vollständiger Informationen bei einer Situation unter Sicherheit handeln. ${ }^{39}$ Leistung und Gegenleistung sind damit in einem vollständigen Vertrag klar vereinbart sowie jederzeit beobachtbar und verifizierbar. Hart bezeichnet die oben beschriebenen vollständigen Verträge als umfassende Verträge und grenzt sie damit von Verträgen $a b$, die unabhängig von dem zukünftigen Umweltzustand zu festgelegten Handlungen und Konsequenzen führen. ${ }^{40}$ Artz und Norman definieren die Vollständigkeit von Verträgen als „,.. degree to which the obligations of the exchange (e.g., price, quality, delivery, other terms and conditions) are outlined upfront." ${ }^{\text {"41 }}$ Aus dieser Definition lässt sich erkennen, dass ein Spektrum zwischen der Abfassung vollständiger und unvollständiger Verträge existiert. Während bei eher vollständigen Verträgen aufgrund der notwendigen Informationsbeschaffung ex ante hohe Kosten entstehen, jedoch nach Vertragsabschluss opportunistisches Verhalten oder bewusste Verletzung festgelegter Regeln durch die Vertragspartner kaum möglich sind, können mit eher unvollständigen Verträgen ex post hohe Kosten verbunden sein, die aus der Notwendigkeit von Nachverhandlungen resultieren. Gleichzeitig ermöglichen es unvollständige Verträge, flexibel auf veränderte Umweltsituationen zu reagieren. ${ }^{42}$ So können etwa nicht realisierbare Projektanforderungen nach Absprache mit dem Auftraggeber während der Vertragsdurchführung durch den Auftragnehmer geändert werden, ohne Vertragsänderungen vornehmen zu müssen.

39 Vgl. Hart (1987), S 752ff.; Macho-Stadler/Pérez-Castrillo (2001), S. 5; Scholtis (1998), S. 27.

40 Vgl. Hart (1995), S. $21 \mathrm{f}$.

41 Artz/Norman (2002), S. 400.

42 Vgl. Artz/Norman (2002), S. 401; Jost (2000), S. 475ff.; Schnedler (2004), S. 11; Wolf (1995), S. 42ff. Crocker/Reynolds (1993) untersuchen als bedeutende Entscheidung im Rahmen des Vertragsdesigns empirisch den optimalen Grad der Vertragsunvollständigkeit unter der Zielsetzung der Minimierung der mit dem Vertragsgeschäft für die Parteien verbundenen Transaktionskosten. 
Verträge der Realität sind weder vollkommen vollständig noch vollkommen unvollständig, da die Beschaffung aller für die Formulierung eines vollständigen Vertrags notwendigen Informationen über zukünftig mögliche Umweltzustände und Handlungskonsequenzen nicht nur kaum möglich, sondern mit sehr hohen Kosten verbunden ist und vollkommen unvollständige Verträge aufgrund der fehlenden Regelungen den Handel zwischen den Vertragsparteien so sehr erschweren, dass der Vertrag selbst entbehrlich ist. ${ }^{43}$ Unabhängig von der Entscheidung für einen bestimmten Grad an Vertragsunvollständigkeit lassen sich fünf exogene Gründe für das Vorliegen von Unvollständigkeit von Verträgen identifizieren: ${ }^{44}$

- Die Sprache, in der der Vertrag abgefasst ist, beinhaltet Spielräume bei der Auslegung von Begriffen und Sachverhalten.

- Bestimmte Vertragsinhalte werden durch Unachtsamkeit der Vertragspartner nicht berücksichtigt.

- Die Kosten der Aufnahme eines Vertragsinhalts übersteigen dessen Nutzen.

- Asymmetrische Information zwischen den Vertragspartnern sowie Dritten führt zu mangelnder Fähigkeit der Beobachtbarkeit und/oder Verifizierbarkeit von Vertragsinhalten. $^{45}$

- Screening-Mechanismen zur Identifikation tatsächlich vorliegender Eigenschaften der Vertragspartner sind nicht möglich und es kommt zu einer mit Effizienzverlusten verbundenen Pooling-Lösung. ${ }^{46}$

Diese Tatbestände führen dazu, dass Verträge der Realität als unvollständige Verträge formuliert werden und die hieraus resultierenden Probleme durch geeignete, in das Vertragsdesign integrierte Anreizmechanismen, wie sie auch in der Prinzipal-AgentenTheorie diskutiert werden, zu lösen sind. ${ }^{47}$ Als Folge dieser Feststellung der Existenz unvollständiger Verträge ist die Frage zu beantworten, welche Faktoren die Wahl des

43 Vgl. Crocker/Reynolds (1993), S. 127f.

44 Vgl. Schwartz (1992), S. 80ff.

45 Hierbei können zwei Fälle unterschieden werden: (1) Die Parteien sind ex post symmetrisch informiert und können die Leistungen beobachten. Diese lassen sich jedoch nicht durch eine dritte Partei, z. B. ein Gericht, verifizieren. (2) Die Parteien sind ex post asymmetrisch informiert, die Leistungen sind weder beobachtbar noch verifizierbar. Vgl. Schwartz (1992), S. 81.

46 Auf den Begriff des Screenings wird in Kapitel 3.2.2 năher eingegangen. Zur Definition von PoolingGleichgewichten vgl. Dixit/Skeath (2004), S. 416ff.; Hartmann-Wendels (1989), S. 724ff.

47 Die Theorie unvollständiger Vertrăge als Zweig der Theorie der Vertragsgestaltung beschäftigt sich mit der (meist empirischen) Untersuchung realer Verträge, um hieraus Aussagen abzuleiten, in welchen Kooperationsformen und -bereichen durch die Unvollstăndigkeit Kooperationsprobleme entstehen kőnnen. Vgl. Scholtis (1998), S. 30. 
Vertrags sowie den Grad der Unvollständigkeit bestimmen. Artz und Norman nennen in Anlehnung an die Transaktionskostentheorie drei Faktoren, welche Einfluss auf die vertragliche Kooperationsbeziehung haben: ${ }^{48}$

\section{Unsicherheit}

Lässt sich beispielsweise die zukünftige Preisentwicklung der zur Erbringung der vertraglich vereinbarten Leistung notwendigen Produktionsfaktoren zu dem Zeitpunkt des Vertragsabschlusses nicht hinreichend genau bestimmen, müssen sich die Vertragsparteien darüber einigen, ob sie bereit sind, die höheren Kosten eines besseren Informationsstandes zu zahlen oder bei zukünftigem Eintritt eines Umweltzustandes die Kosten der Nachverhandlung und damit möglicher Effizienzverluste zu tragen. ${ }^{49}$ Neben dieser Form der parametrischen Unsicherheit kann auch Unsicherheit in Bezug auf das Verhalten des jeweils anderen Vertragspartners bestehen. ${ }^{50}$ Empirische Untersuchungen haben gezeigt, dass der Grad der Unsicherheit mit der Unvollständigkeit von Verträgen positiv korreliert. Hieraus folgt, dass je schlechter die Vertragsparteien informiert sind, desto eher sehen sie die Notwendigkeit, durch die Abfassung unvollständiger Verträge flexibel auf veränderte Umweltzustände oder Verhaltensweisen reagieren zu können. ${ }^{51}$

\section{Investitionsspezifität}

Die Investitionsspezifität gibt an, ob die für die Erbringung der vereinbarten Sachleistung notwendigen Investitionen des Auftragnehmers in Ressourcen ausschließlich für diese Transaktion genutzt werden oder diese auch für andere Transaktionen nutzbar sind. ${ }^{52}$ Bei einer hohen Spezifität von Investitionen sind die Geschäftspartner stark aneinander gebunden, ein Wechsel des Geschäftspartners ist daher insbesondere für den Auftraggeber kaum möglich. ${ }^{53}$ Gleichzeitig besteht bei hoher Spezifität die Tendenz

48 Vgl. Artz/Norman (2002), S. 402.

49 Für eine sehr anschauliche Analyse dieses Konfliktes vgl. Crocker/Reynolds (1993), S. 128f.

so Vgl. Wolf (2003), S. 271.

5I Vgl. Artz/Norman (2002), S. 402; Chiappori/Salanié (2002), S. 26; Crocker/Masten (1991), S. 96; Crocker/Reynolds (1993), S. 127.

52 Vgl. Adler et al. (1998), S. 190. Die Autoren nennen neben der hier aufgeführten Investitionsspezifităt noch zwei weitere Arten von Spezifität: physische Investitionsspezifität bei neuartigen und einmaligen Leistungen und die Spezifität durch Humankapital. Hier werden auch Moglichkeiten zur Berechnung und Messung der Spezifität vorgestellt.

53 Vgl. Jost (2000), S. 470ff.; Wolf (2003), S. 270f. Aufgrund der durch den Auftragnehmer erfolgten speziell für die Leistungserstellung notwendigen Investitionen muss der Auftraggeber bei einem Wechsel des Projektpartners auch Zahlungen für diese Investitionen des neuen Auftragnehmers leisten, was zu einer Erhöhung des Gesamtpreises führt. 
zu langfristigen Kooperationsbeziehungen. ${ }^{54}$ Werden Verträge mit einer zeitlich langen Vertragdauer abgeschlossen, so erhöht sich mit der Vertragsdauer die Wahrscheinlichkeit für sich ändernde Umweltzustände. Hierdurch werden beide Vertragspartner eher einen unvollständigen Vertrag schließen, um sich gegen ungünstige Entwicklungen abzusichern und die Option für Nachverhandlungen zu sichern. ${ }^{55}$ Eine dieser These widersprechende Meinung vertreten Goldberg und Erickson. ${ }^{56}$ Sie sehen gerade in der Spezifität der Leistung und der hierdurch erzielbaren Produzentenrente, die auf beide Vertragspartner aufzuteilen ist, den Grund für besonders große Anstrengungen in der Phase der Vertragsverhandlungen, den Vertrag möglichst vollständig abzufassen, da es für den Auftragnehmer nach Annahme des Vertrags nicht mehr attraktiv ist, Nachverhandlungen zuzulassen. Die empirischen Untersuchungen von Artz und Norman zeigen, dass Investitionen in spezifische Anlagen positiv mit dem Gebrauch unvollständiger Verträge korrelieren. ${ }^{57}$

\section{Potenzial für opportunistisches Verhalten}

Opportunistisches Verhalten beschreibt das von Individuen konsequente Ausnutzen von Gelegenheiten und ist mit einer Minderung des Zielerreichungsgrades anderer verbunden. ${ }^{58}$ Durch Opportunismus wird das Konzept der individuellen Nutzenmaximierung erweitert. ${ }^{59}$ Zur Verfolgung eigener Vorteilsinteressen setzen sich Individuen auch über Vertragsabsprachen und allgemeine Normen hinweg. ${ }^{60}$ Bei hoher Spezifität der Investition ist das Potenzial für opportunistisches Verhalten durch die enge Bindung der Vertragspartner aneinander gegeben. Erkennt der Auftragnehmer, dass sein Geschäftspartner keinen alternativen Anbieter mit der Erbringung der Leistung beauftragen kann, so kann er die aufgrund der begrenzten Rationalität zwischen den Vertragspartnern vorhandene Informationsasymmetrie zu seinen Gunsten ausnutzen. ${ }^{61}$ Die Gefahr opportunistischen Verhaltens kann durch einen höheren Grad an Vertragsvollständigkeit, was bei entsprechendem Vertragsdesign einer Minderung der Ausbeutungsmöglichkeiten entspricht, reduziert werden. ${ }^{62}$ Insbesondere bei interorganisatio-

\footnotetext{
54 Vgl. Jost (2000), S. 471. Zu Langzeitverträgen sowie dem Verhalten der Vertragspartner bei Langzeitverträgen vgl. Lambert (1983).

55 Vgl. Artz/Norman (2002), S. 403.

56 Vgl. Goldberg/Erickson (1987), S. 398.

$57 \mathrm{Vgl}$. Artz/Norman (2002), S. 412.

58 Vgl. Jost (2000), S. 482ff.

59 Vgl. Wolf (2003), S. 270.

60 Vgl. Gierl (2000), S. 107; Hax (1991), S. 56.

61 Vgl. Artz/Norman (2002), S. 402f.; Crocker/Reynolds (1993).

62 Vgl. Crocker/Masten (1991), S. 96; Schäffer (2002), S. 86f.
} 
nalen Kooperationen müssen eine sorgfältige Ausarbeitung gemeinsamer Ziele sowie eine präzise Festlegung von Verantwortlichkeiten erfolgen, um die aus opportunistischem Verhalten begründeten Transaktionskosten $\mathrm{zu}$ mindern. ${ }^{63}$

Aus der Sicht empirischer transaktionskostentheoretischer Untersuchungen erscheint daher insbesondere bei langfristigen Kooperationen das Abfassen eher unvollständiger Verträge als vorteilhaft. Dabei ist ein unvollständiger Vertrag nicht nur eine Folge exogener, in Kooperationsbeziehungen bestehender Probleme, sondern auch eine Variable, die den Trade-Off zwischen Kosten der ex ante Informationsbeschaffung sowie Kosten der ex post Vertragsverhandlungen bestimmt. Die ex post entstehenden Kosten der Nachverhandlungen können reduziert werden, sofern die Kooperationspartner bestimmte Normen der Zusammenarbeit akzeptieren und ihr Verhalten hieran wie folgt ausrichten: ${ }^{64}$ Durch die Anpassung der Zusammenarbeit bei veränderten Umweltzuständen zeigen die Partner die Bereitschaft, gemeinsam effiziente Lösungen zu finden. Binden sich die Partner durch Langzeitverträge, so ändert sich die dominierende Strategie des nicht-kooperativen Verhaltens bei Einzelspielen in die Strategie der Kooperation bei wiederkehrenden Spielen. ${ }^{65}$ Auch die Möglichkeit der nicht an zwanghafte Regeln gebundenen Kommunikation zwischen den Kooperationspartnern reduziert die Höhe der nachträglichen Verhandlungskosten.

Die Ausführungen in diesem Kapitel verdeutlichen, dass in Abhängigkeit von der in einer Kooperationsbeziehung vorhandenen Unsicherheit hinsichtlich zukünftiger Entwicklungen sowie dem Verhalten der Kooperationspartner unterschiedliche Anforderungen an das Vertragsdesign bestehen. Während die Vertragsparteien keinen Einfluss auf zukünftige Entwicklungen haben, lässt sich das Verhalten der Kooperationspartner steuern. Hierbei interessiert insbesondere die Fragestellung, wie in AuftraggeberAuftragnehmer-Beziehungen der Auftragnehmer so durch den Auftraggeber beeinflusst werden kann, dass dieser in seinem Sinne handelt. Hierfür werden in einem ersten Schritt in Kapitel 3.2.1 die Ursachen für das Vorliegen von Informationsasymmetrie bei Auftraggeber-Auftragnehmer-Kooperationen analysiert sowie in Kapitel 3.2.2 die aus der Prinzipal-Agenten-Theorie bekannten Lösungsansätze diskutiert.

63 Vgl. Dahlstrom/Nygaard (1999), S. 167.

64 Für die hier aufgefuhrten Normen der Zusammenarbeit vgl. Artz/Norman (2002), S. 404ff. Backhaus/Köhl (1999), S. 17ff. betrachten die Ursachen und Folgen von Nachverhandlungen furVertrăge des internationalen Anlagengeschäftes.

65 Zum Informationstransfer-Dilemma in Anlehnung an das in der Spieltheorie diskutierte Gefangenendilemma vgl. die Ausfuhrungen in Picot/Reichwald/Wigand (2003), S. 129f;; Schrader (1990), S. $21 \mathrm{ff}$. 


\subsection{Informationsasymmetrie bei Projektkooperationen}

\subsubsection{Ursachen für das Vorliegen von Informationsasymmetrie}

Der Begriff der Informationsasymmetrie setzt sich aus zwei Begriffsteilen zusammen, die zunächst einer näheren Definition bzw. Erläuterung bedürfen. Der Informationsbegriff kann je nach Anwendungsbezug auf vielfältige Weise definiert werden. ${ }^{66}$ Viele Autoren betriebs- und volkswirtschaftlicher Veröffentlichungen verweisen auf die Definition von Wittmann, nach der Information zweckorientiertes Wissen ist. ${ }^{67}$ Hierbei sind nicht die mit der Information verbunden Daten von Interesse, sondern der Beitrag, wie die Informationsbotschaft zur Erreichung von Zielen genutzt werden kann. ${ }^{68}$ Wissen als komplexer und aus vernetzten Daten gewonnener Sachverhalt wird somit dann zu einer Information, sobald es von einer Person oder Gruppe von Personen als Entscheidungsträger zur Zielerreichung verwendet wird. $^{69}$

Wittmann unterscheidet die Formen der Information in die Extremfälle der vollkommenen Voraussicht und der vollkommenen Ignoranz. ${ }^{70}$ Beide Fälle sind in der Realität nicht existent. Es lassen sich weder sichere Aussagen über zukünftige Entwicklungen machen, noch können - von isolierten kleinen Bereichen abgesehen - gegenwärtige und vergangene Ereignisse vollständig erklärt werden, um den Grad der vollkommenen Information zu erreichen. Obwohl die Vermutung, dass der Grad der vollkommenen Ignoranz realitätsfremd ist, schwerer zugänglich erscheint, verweist Wittmann auf die Ausführungen von Meinong aus dem Jahr 1890, der feststellte, dass die völlige Unbekanntschaft keine leicht erfüllbare Bedingung ist. ${ }^{71}$ Der Grad der Information

66 Für einen Überblick vgl. Bode (1997).

67 Vgl. Wittmann (1959), S. 14. Diese Definition findet auch Akzeptanz bei Gabriel/Beier (2003), S. 30; Horváth (2003), S. 348; Kiener (1990), S. 7; Rey (1999), S. 45; Scholtis (1998), S. 10f.; Staehle (1999), S. 300; Vincenti (2002), S. 56; Wolf (2003), S. 235. Diese Kennzeichnung von Information bezeichnet Schneider (1997), S. 80 als unbrauchbar, Meinhövel (1999) schließt sich Schneider an und stellt die Zweckorientierung von Information gegenuber Wittmann deutlicher in den Vordergrund. Mag (1990), S. 6 benutzt anstelle des Begriffs der Zweckorientierung den Ausdruck der Entscheidungsorientierung. Eine kritische Diskussion über Vorzüge und Schwächen unterschiedlicher Definitionen des Informationsbegriffs findet sich in Bode (1997).

68 Vgl. Wolf (2003), S. 235.

${ }^{69}$ Vgl. Wolf (2003), S. 236. Für eine ausführliche Abgrenzung der Begriffe Daten - Informationen - Wissen vgl. Gabriel/Beier (2003), S. 29ff. Streubel (2000), S. 64 grenzt die Begriffe zusätzlich nach der maschinellen Verarbeitbarkeit des Wissens ab. Hiernach sind Informationen nicht-maschinell verarbeitbares Wissen mit Zweckeignung.

70 Vgl. Wittmann (1959), S. 18ff. Vollkommene Ignoranz wird hier als Unwissenheit und nicht als willentliches Übersehen bezeichnet. Vgl. Wittmann (1959), S. 23, Fußnote 20.

71 Vgl. Wittmann (1959), S. 23. 
bzw. der Informationsstand eines Individuums kann daher als Verhältnis der tatsächlich vorhandenen Information $\mathrm{zu}$ der notwendigen Information definiert werden. ${ }^{72}$

Ist das unvollständige Wissen bei allen Wirtschaftssubjekten gleich verteilt, das heißt alle Menschen einer Gesellschaft besitzen dieselben Vorstellungen über zukünftige Entwicklungen und verwenden unter Berücksichtigung ihrer persönlichen Präferenzen dieselben Entscheidungsregeln, liegt der Zustand symmetrischer Information vor. ${ }^{73}$ Demnach kann der Zustand asymmetrischer Information definiert werden, wenn sich der Informationsgrad mindestens zweier Individuen voneinander unterscheidet. ${ }^{74}$ Durch den Zeitpunktbezug wird deutlich, dass sich der Zustand asymmetrischer Information durch bestimmte Maßnahmen verändern lässt und somit das Informationsdefizit eines Marktteilnehmers gegenüber einem anderen verringert oder auch vergröBert werden kann. ${ }^{75}$

Es wird dabei angenommen, dass der Auftragnehmer private Informationen besitzt und somit gegenüber dem Auftraggeber einen Wissensvorsprung besitzt, der ihn zu Handlungen bei der Auftragsdurchführung zu Lasten des Auftraggebers befähigt. Diese Situation bezeichnet Schneider als das Problem des Auftragshandelns und grenzt sie zu den seiner Meinung nach zu offenen und irreführenden Definitionen der PrinzipalAgenten-Theorie $a b .^{76}$

Sowohl die Theorie des Auftragshandelns als auch die Prinzipal-Agenten-Theorie unterstellen in der Regel, dass der Auftraggeber gegenüber dem Beauftragten ein Informationsdefizit besitzt. ${ }^{77}$ Dies resultiert aus der Annahme, dass der Beauftragte, der mit der Ausführung der Aufgabe vertraut ist und bei regelmäßigen Aufträgen ähnlicher Art von Lerneffekten profitieren kann, möglicherweise eine größere Anzahl von Entscheidungsalternativen kennt und im Gegensatz zum Auftraggeber einen Informationsvorsprung bezüglich der Erwartung zukünftig eintretender Umweltzustände besitzt. ${ }^{78}$

\footnotetext{
Vgl. Wittmann (1959), S. 25.

Vgl. Schneider (1995), S. 43.

Vgl. Vincenti (2002), S. 56.

Vgl. Kiener (1990), S. 22.

$76 \mathrm{Vgl}$. Schneider (1995), S. 47. Schneider kritisiert, dass die bestehende Möglichkeit einer Beeinflussung des Nutzens eines Individuums durch die Handlungen eines anderen für das Vorliegen einer Prinzipal-AgentenBeziehung nicht ausreicht. Auch Team-Entscheidungen unter Gleichberechtigten stellen keine PrinzipalAgenten-Situationen dar. Notwendige Bedingung ist vielmehr eine hierarchische Beziehung, zum Beispiel in Form eines Vorgesetzten-Untergebenen-Verhältnisses.

77 Vgl. Jost (2001), S. 21; Kiener (1990), S. 22; Schiller (1994), S. 32; Schneider (1995), S. 48f.

78 Vgl. Breid (1995), S. 824.
} 
Bei einer Auftraggeber-Auftragnehmer-Beziehung lassen sich nach dem Zeitpunkt innerhalb der Kooperationsbeziehung insbesondere zwei Arten asymmetrisch verteilter Information unterscheiden: ${ }^{79}$

- Vor Vertragsabschluss besitzt der Auftraggeber keine ausreichenden Informationen über die Qualität der durch die möglichen Kooperationspartner angebotenen Leistungen (Qualitätsunsicherheit durch verborgene Information).

- Nach Vertragsabschluss bietet sich für den Auftragnehmer die Möglichkeit, aufgrund seines Wissensvorsprungs gegenüber dem Auftraggeber, von dem ursprünglich vereinbarten Verhalten abzuweichen. (Verhaltensunsicherheit durch verborgene Handlungen).

Das folgende Kapitel wird sich näher mit den aus einer Auftraggeber-AuftragnehmerBeziehung resultierenden möglichen Problemen beschäftigen und einen Überblick über die in der Prinzipal-Agenten-Theorie diskutierten Vertragsprobleme und Möglichkeiten zur Überwindung dieser Probleme geben. Hierbei erfolgt insbesondere eine Unterteilung in Probleme, die vor bzw. zu dem Zeitpunkt des Vertragsabschlusses auftreten sowie in Probleme, die während der Vertragsdurchführung zu einer Störung der Kooperationsbeziehung führen können.

\subsubsection{Prinzipal-Agenten-Probleme bei Projektkooperationen}

Die Prinzipal-Agenten-Theorie beschäftigt sich mit arbeitsteiligen AuftraggeberAuftragnehmer-Beziehungen, die durch Situationen geprägt werden, welche sich insbesondere durch Informationsasymmetrie, Zieldivergenz, unterschiedliche Risikoeinstellungen sowie individuelle Nutzenmaximierung der beteiligten Parteien auszeichnen. ${ }^{80}$

Die Kooperationsbeziehung zwischen mindestens zwei Vertragspartnern muss für die Durchführung einer Analyse resultierender Beziehungsprobleme an dieser Stelle näher detailliert werden. ${ }^{81}$ Dabei werden die Ausdrücke Auftraggeber und Prinzipal sowie Auftragnehmer, Beauftragter und Agent synonym verwendet. Wie die Bezeichnungen

\footnotetext{
79 Vgl. Meinhövel (1999), S. 13f.; Vincenti (2002), S. 57.

80 Vgl. Erlei/Leschke/Sauerland (1999), S. 106; Jost (2001), S. 22; Kaluza/Dullnig/Malle (2003), S. 18; Mikus (1998), S. 451 ff.; Picot (1991), S. 150f. Pfaff/Zweifel (1998), S. $187 \mathrm{ff}$. zeigen Anwendungsbereiche der Prinzipal-Agenten Theorie auf.

$81 \mathrm{Zu}$ den folgenden Ausführungen vgl. Jost (2001), S. $11 \mathrm{ff}$.
} 
bereits andeuten, besteht durch die arbeitsteilige Beziehung zwischen den Kooperationspartnern eine vertikale hierarchische Struktur. Der Prinzipal delegiert eine Aufgabe an den Agenten, der diese durch die Durchführung bestimmter Tätigkeiten und das Treffen von Entscheidungen ausführen soll. Der Agent erhält für die Aufgabendurchführung eine Entlohnung durch den Prinzipal, die durch die Kosten der Leistungserstellung gemindert wird. Der Prinzipal erwartet einen festgelegten Nutzen durch den Erfolg der Aufgabendurchführung. Unter der Annahme, dass beide Parteien Nutzen maximierende Individuen sind, ist der Agent bestrebt, mit einem möglichst geringen Arbeitseinsatz die Leistung zu erstellen und als Entlohnung einen möglichst großen Anteil des Kooperationsgewinns zu erhalten. Der Prinzipal hat ein Interesse an einer bestmöglichen Leistungserstellung und somit einem höchstmöglichen Arbeitseinsatz des Agenten. Gleichzeitig versucht er, die Entlohnung des Agenten gering zu halten, da diese seinen Kooperationsgewinn schmälert. Die aus diesem zwischen den Parteien bestehenden Zielkonflikt resultierenden Fragestellungen betreffen einerseits die Entscheidung des Agenten, wie er die übertragene Aufgabe ausführt und andererseits die Entscheidung des Prinzipals, wie er den Agenten beeinflussen kann, damit dieser die Aufgabe in seinem Sinne ausführt. ${ }^{82}$

Das Problem der Informationsasymmetrie ist im vorhergehenden Kapitel grundlegend behandelt worden. Im Folgenden wird daher ein Überblick über die Typen asymmetrischer Informationsverteilung vorgestellt. Auch hier kann eine Unterteilung nach dem Entstehungszeitpunkt des Informationsdefizites sowie nach dessen Ursprung erfolgen.

Die vor Vertragsabschluss bestehende Qualitätsunsicherheit in Bezug auf die Leistungen des Agenten werden in der Prinzipal-Agenten-Literatur mit dem Begriff Hidden Characteristics bezeichnet. ${ }^{83}$ Spremann nennt folgende Erklärungen für das Vorliegen dieser zeitlich vor der Entscheidung des Prinzipals entstehenden Informationsasymmetrie: ${ }^{84}$ Der Prinzipal muss entweder so frühzeitig eine Entscheidung über die Wahl des Beauftragten treffen, dass keine Möglichkeit besteht, den Vertragspartner kennen zu lernen, oder er kann aufgrund der Heterogenität des Umfeldes nicht von der Qualität eines Kandidaten auf die eines anderen Kandidaten schließen. Des Weiteren besteht die Möglichkeit, dass die zusätzliche Informationsbeschaffung des Prinzipals nicht

\footnotetext{
82 Zum Grundmodell der ökonomischen Agency-Theorie vgl. etwa Hartmann-Wendels (1992), Spalte 72ff.; Jost (2001), S. 20ff.; Laux/Schenk-Mathes (1992), S. 2ff.; Macho-Stadler/Pérez-Castrillo (2001), S. 4ff.; Pfaff/Zweifel (1998), S. 184f.

83 Vgl. Picot (1991), S. 152.

84 Vgl. Spremann (1990), S. 567f.
} 
erfolgreich ist und durch exogene Einflüsse gestört wird. Diese Form der Qualitätsunsicherheit kann auf der Seite des Prinzipals zu einer Verhaltensunsicherheit und der Gefahr der adversen Selektion führen. ${ }^{85}$ Um den Vertragsabschluss herbeizuführen, täuscht der Agent gegenüber dem Prinzipal Eigenschaften vor (Qualifikation, Begabung, Risikoeinstellung), die zur Zielerfüllung notwendig sind. ${ }^{86}$ Der Prinzipal wird versuchen, durch geeignete Vertragsgestaltung den für das Erreichen seiner Ziele geeigneten Beauftragten zu finden. Bietet er einen Vertrag an, der auf einen Beauftragten mit durchschnittlichen Eigenschaften zugeschnitten ist, so besteht die Gefahr, dass der Vertrag für Agenten mit guten Eigenschaften nicht attraktiv genug ist und er einen Partner auswählt, der diese durchschnittlichen Eigenschaften nicht erfüllt, diese jedoch vortäuscht. Damit kommt es zu einer systematischen Auswahl unvorteilhafter Agenten. ${ }^{87}$ Als Ansätze zur Lösung des Hidden Characteristics Problems werden in der Literatur die Möglichkeiten des Screening und Signalling diskutiert. ${ }^{88}$ Durch das Anbieten sich unterscheidender Verträge und das Beobachten der Reaktionen der Kooperationskandidaten hofft der Auftraggeber, von diesen private Informationen zu erhalten und hierdurch eine bessere Auswahl des Vertragspartners treffen zu können (Screening). Während die Initiative hier von der Person des Auftraggebers ausgeht, kann auch der an einem Vertragsabschluss interessierte Auftragnehmer Teile seiner privaten Information im Rahmen der vorvertraglichen Verhandlungen preisgeben und damit die Informationsasymmetrie mindern (Signalling). ${ }^{89}$

Eine weitere Form vorvertraglicher Informationsasymmetrie wird unter dem Begriff Hidden Intention zusammengefasst. ${ }^{90}$ Hierbei besteht die Kooperationsunsicherheit darin, dass der Auftraggeber die Motive des Auftragnehmers für eine Zusammenarbeit sowie das Verhalten während der Kooperation schlecht oder gar nicht einschätzen kann. ${ }^{91}$ Entsteht nach Vertragsabschluss beispielsweise durch einen hohen Grad von Investitionsspezifität ein Abhängigkeitsverhältnis zwischen Auftraggeber und Auf-

85 Zum Begriff der adversen Selektion vgl. ausführlich Theilen (1996), S. $23 \mathrm{ff}$.

86 Vgl. Kaluza/Dullnig/Malle (2003), S. 21.

87 Vgl. Jost (2001), S. 28.

${ }^{88}$ Vgl. Göbel (2002), S. 101; Jost (2001), S. 29.

89 Zum Screening und Signalling vgl. Göbel (2002), S. 113ff.; Schneider (1995), S. 44f.; Vincenti (2002), S. 58. Auch das Vorgehen Self Selection dient dazu, gepoolte Märkte zu separieren, indem sich die Agenten für einen für sie effizienten Vertrag aus mehreren vom Prinzipal angebotenen Verträgen entscheiden. Vgl. hierzu Picot/Dietl/Franck (2002), S. 93 und S. 102.

90 Vgl. Breid (1995), S. 825; Kaluza/Dullnig/Malle (2003), S. 22; Scholtis (1998), S. 17.

91 Im Gegensatz zu Hidden Characteristics sind dies Eigenschaften des Agenten, die dessen Willen unterliegen. Als Beispiele werden Verhaltensmerkmale, wie zum Beispiel Entgegenkommen, Fairness, Kulanz oder die Bereitschaft fur Folgeinvestitionen genannt. Vgl. Scholtis (1998), S. 17; Spremann (1990), S. 566. 
tragnehmer, so besteht die Gefahr, dass der Auftraggeber nicht mehr in der Lage ist, den Beauftragten so zu beeinflussen, dass sich dieser konform zu den Interessen des Auftraggebers verhält. Hierfür wird der Begriff Hold-up in der Literatur geprägt, der teilweise synonym mit dem Begriff Hidden Intention verwendet wird. ${ }^{92}$

Nach Vertragsabschluss bestehende Informationsasymmetrien werden in die Fälle Hidden Action und Hidden Information unterteilt. ${ }^{93}$ Der Hidden Action-Fall zeichnet sich dadurch aus, dass der Prinzipal zwar die Leistungen des Agenten beobachten und verifizieren kann, jedoch nicht in der Lage ist, die für die Erbringung der Leistungen notwendigen Arbeitsanstrengungen zu bewerten. ${ }^{94}$ Es ist für den Auftraggeber in dieser Situation nicht möglich, von dem realisierten Ergebnis ohne zusätzliche Informationskosten Rückschlüsse auf die eingetretenen Umweltzustände und die hierbei gewählten Aktionen des Beauftragten zu ziehen. ${ }^{95}$ Die in Kombination mit Hidden Action bestehende Gefahr des Moral Hazard liegt darin begründet, dass der Auftragnehmer die Erbringung einer für den Auftraggeber nicht zufrieden stellenden Leistung mit dem Eintreten bestimmter, durch den Auftraggeber nicht beobachtbarer Umweltzustände entschuldigen kann, auch wenn er ein besseres Ergebnis durch die Wahl eines höheren Aktivitätsniveaus hätte erreichen können. ${ }^{96}$ Der Agent nutzt hiermit das Informationsdefizit des Prinzipals aus, um sein Ziel der Nutzenmaximierung zu verfolgen. ${ }^{97}$

Eine Hidden Action-Situation birgt für den Prinzipal auch die Gefahr von Drückebergerei (Shirking) oder privatem Konsum (Consumption on the job) durch den Agenten. ${ }^{98}$ Im Gegensatz zu einer Nichtbeobachtbarkeit der gewählten Aktionen zur Erreichung des Ergebnisses im Fall des Hidden Action kann der Prinzipal bei Vorliegen von Informationsasymmetrie in Form von Hidden Information die Aktivitäten beo-

92 Vgl. Spremann (1990), S. 566. Zu Hold-up vgl. Spremann (1990), S. 568ff.

93 Vgl. Gobel (2002), S. 100; Jost (2001), S. 25ff.; Picot (1991), S. 151 f.

94 Vgl. Scholtis (1998), S. 14f.

95 Vgl. Kleine (1995), S. 34f.

96 Vgl. Trebilcock (1993), S. 102ff. Hierbei werden auch Handlungen, wie etwa Tăuschung, fahrlăssige bzw. schuldlose Falschdarstellung sowie die Nichtangabe von Tatsachen als ein møgliches Verhalten des Agenten aufgefuhrt.

97 Vgl. Jost (2001), S. 26. Göbel (2002), S. 103 fasst den Begriff Moral Hazard weiter und bezieht ihn auf alle Probleme innerhalb einer Prinzipal-Agenten-Beziehung mit Ausnahme bestimmter Fälle der adversen Selektion, bei denen die Moral der Handelnden nicht relevant ist.

98 Vgl. Wolf (2003), S. 280. 
bachten, jedoch wegen seines fehlenden Sachverstandes nicht bewerten. ${ }^{99}$ Der Auftragnehmer kann durch die während der Auftragsdurchführung erlangten Informationen den Erfolg seiner Anstrengungen besser beurteilen als der Auftraggeber. ${ }^{100}$ Auch hierbei besteht die Gefahr, dass sich der Beauftragte nicht moralisch verhält (Moral Hazard) und ein niedrigeres Anstrengungsniveau wählt, welches seinen Nutzen erhöht, jedoch dem Auftraggeber ein zielkonformes Ergebnis vortäuscht.

Alle hier vorgestellten Formen von ungleich verteilter Information innerhalb einer Auftraggeber-Beauftragten-Beziehung sind in Tabelle 3.1 zusammengefasst:

\begin{tabular}{|c|c|c|c|c|}
\hline & $\begin{array}{c}\text { Hidden } \\
\text { Characteristics }\end{array}$ & $\begin{array}{l}\text { Hidden } \\
\text { Intention }\end{array}$ & $\begin{array}{l}\text { Hidden } \\
\text { Action }\end{array}$ & $\begin{array}{c}\text { Hidden } \\
\text { Information }\end{array}$ \\
\hline $\begin{array}{l}\text { Entstehungs- } \\
\text { zeitpunkt der } \\
\text { Informations- } \\
\text { asymmetrie }\end{array}$ & $\begin{array}{c}\text { vor } \\
\text { Vertragsabschluss }\end{array}$ & $\begin{array}{l}\text { vor und nach } \\
\text { Vertragsabschluss }\end{array}$ & $\begin{array}{c}\text { nach } \\
\text { Vertragsabschluss }\end{array}$ & $\begin{array}{c}\text { nach } \\
\text { Vertragsabschluss }\end{array}$ \\
\hline $\begin{array}{l}\text { Ursache der } \\
\text { Informations- } \\
\text { asymmetrie }\end{array}$ & $\begin{array}{l}\text { verborgene } \\
\text { Eigenschaften des } \\
\text { Beauftragten }\end{array}$ & $\begin{array}{l}\text { verborgene } \\
\text { Absichten des } \\
\text { Beauftragten }\end{array}$ & $\begin{array}{l}\text { nicht beobachtbare } \\
\text { Aktivitäten des } \\
\text { Beauftragten }\end{array}$ & $\begin{array}{l}\text { nicht beobachtbarer } \\
\text { Informationsstand } \\
\text { des Beauftragten }\end{array}$ \\
\hline $\begin{array}{l}\text { Anforderungen } \\
\text { an das } \\
\text { Vertragsdesign }\end{array}$ & $\begin{array}{l}\text { Auswahl des } \\
\text { "richtigen" } \\
\text { Beauftragten }\end{array}$ & $\begin{array}{l}\text { Auswahl und } \\
\text { Verhaltens- } \\
\text { beeinflussung }\end{array}$ & $\begin{array}{c}\text { Möglichkeiten zur } \\
\text { Verhaltens- } \\
\text { beeinflussung }\end{array}$ & $\begin{array}{c}\text { Möglichkeiten zur } \\
\text { Ergebnis- } \\
\text { beeinflussung }\end{array}$ \\
\hline $\begin{array}{c}\text { Gefahren } \\
\text { für den } \\
\text { Auftraggeber }\end{array}$ & Adverse Selection & $\begin{array}{c}\text { Hold-up } \\
\text { Adverse Selection }\end{array}$ & $\begin{array}{l}\text { Moral Hazard } \\
\text { Shirking } \\
\text { Consumption }\end{array}$ & $\begin{array}{c}\text { Moral Hazard } \\
\text { Adverse Selection }\end{array}$ \\
\hline Lösungsansätze & $\begin{array}{l}\text { Signalling } \\
\text { Screening }\end{array}$ & Signalling & $\begin{array}{l}\text { Anreizsysteme } \\
\text { Kontrollsysteme }\end{array}$ & $\begin{array}{l}\text { Anreizsysteme } \\
\text { Kontrollsysteme }\end{array}$ \\
\hline
\end{tabular}

Tabelle 3.1: Informationsasymmetrie und resultierende Vertragsprobleme ${ }^{101}$

99 Vgl. Göbel (2002), S. 102; Kleine (1995), S. 39ff.; Scholtis (1998), S. 15. Arrow (1986), S. 1183f. verwendet die Begriffe Hidden Information und Hidden Knowledge synonym. Durch die Bezeichnung verborgenes Wissen konkretisiert er den allgemein verständlichen und jede Prinzipal-Agenten-Situation definierenden Begriff der verborgenen Information.

$100 \mathrm{Vgl}$. Jost (2001), S. 30.

101 Vgl. ahnliche Übersichtsdarstellungen in Breid (1995), S. 824; Göbel (2002), S. 100; Jost (2001), S. 25; Mikus (1998), S. 454; Picot/Diet1/Franck (2002), S. 92; Schiller (1994), S. 33; Spremann (1990), S. 572; Wolff (1995), S. 73. 


\subsubsection{Die Projektkooperation aus der Sicht der hierarchischen Planung}

Das durch die Projektkooperation entstehende Verhältnis zwischen Auftraggeber und Auftragnehmer lässt sich in Form einer hierarchischen Organisationsstruktur beschreiben. ${ }^{102}$ Der Auftraggeber trifft auf einer übergeordneten Stufe, der Top-Ebene, sowohl Entscheidungen über den Projektgegenstand sowie die Projektziele als auch Entscheidungen über das Vertragsdesign. Der Auftragnehmer trifft auf der Grundlage dieser Vorgaben der Top-Ebene auf der Basis-Ebene Entscheidungen, etwa über seinen Arbeitseinsatz. Diese hier vorliegende hierarchische Struktur wird insbesondere durch einen unterschiedlichen Informationsstatus der Entscheidungsträger geprägt. ${ }^{103}$

Verhalten sich die Entscheidungsträger zudem als Gegenspieler und somit antagonistisch, entsteht aus der Über-Unterordnungs-Beziehung eine Prinzipal-AgentenSituation. Die Abbildung 3.3 veranschaulicht diese verschiedenen hierarchischen Beziehungen zwischen den Kooperationspartnern.
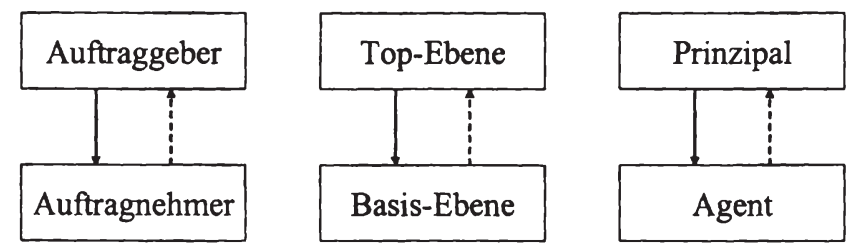

Abbildung 3.3: Hierarchische Beziehung zwischen den Kooperationspartnem (Quelle: In Anlehnung an Schneeweiß (2003a), S. 8.)

Durch die gestrichelten Pfeile wird angedeutet, dass nicht nur der Auftragnehmer durch die Entscheidung des Auftraggebers beeinflusst wird, sondern auch eine Beziehung in umgekehrter Richtung existiert. Durch das Konstrukt der Kooperation muss der Auftraggeber durch seine Entscheidungen Strukturen schaffen, die den Auftragnehmer veranlassen, die aus der Sicht des Auftraggebers beste Handlungsalternative seines Arbeitseinsatzes zu wählen (Anreizkompatibilitätsbedingung) und gleichzeitig sein eigenes Anspruchsniveau zu erreichen (Kooperationsbedingung).

102 Vgl. Picot/Reichwald/Wigand (2003), S. 236. Zu hierarchischen Organisationsstrukturen vgl. beispielsweise Krüger (1994), S. 62ff.

$103 \mathrm{Vgl}$. Schneeweiß (2003a), S. 9. Schneeweiß unterteilt die organisatorisch bedingten hierarchischen Entscheidungen in den Fall eines Entscheidungstrăgers, welcher zu verschiedenen Zeitpunkten einen unterschiedlichen Informationsstand besitzt, wie zum Beispiel bei der taktisch-operativen Planung, sowie den hier vorliegenden Fall mehrerer Entscheidungsträger in einer Fürer-Geführten-Beziehung. 
Problematisch ist jedoch, dass der Auftraggeber durch die Annahme der Informationsasymmetrie sowie des für eine Prinzipal-Agenten-Situation typischen opportunistischen Verhaltens keine ausreichenden Informationen sowohl über die Präferenzen als auch über die Handlungsalternativen des Auftragnehmers besitzt. Abbildung 3.4 verdeutlicht die in dem hierarchischen Prinzipal-Agenten-System möglichen Formen der Informationsasymmetrie sowohl zum Zeitpunkt vor Vertragsabschluss als auch in der nach Vertragsabschluss folgenden Projektphase. ${ }^{104}$

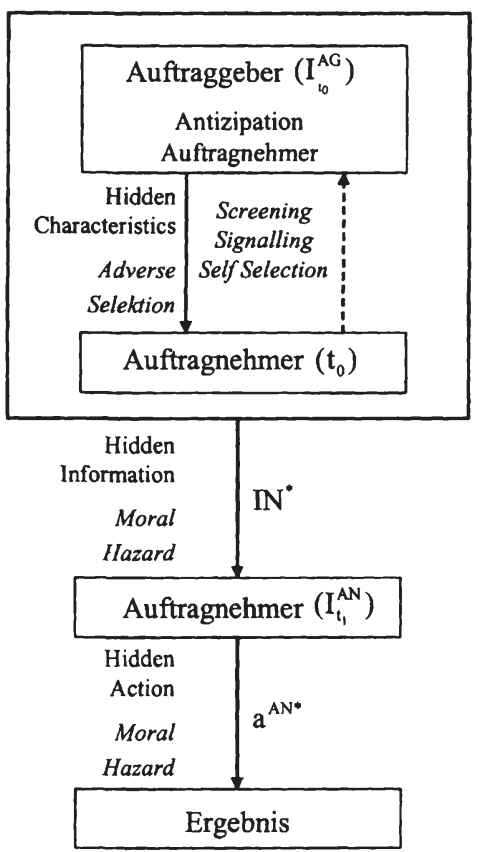

Abbildung 3.4: Informationsasymmetrie bei hierarchischen Auftraggeber-AuftragnehmerBeziehungen (Quelle: In Anlehnung an Schneeweiß (2003a), S. 128.)

Dabei stellt der eingefasste Bereich die Phase des Vertragsdesigns bzw. der Vertragsverhandlungen zu dem Zeitpunkt $t_{0}$ dar. Der Auftraggeber berücksichtigt bei seiner Entscheidung seine Einschätzungen hinsichtlich des Informationsstandes des Auftrag-

${ }^{104} \mathrm{Vgl}$. zu den unterschiedlichen Formen der Informationsasymmetrie die Ausführungen in Kapitel 3.3.2. Für das Konzept des hierarchischen Prinzipal-Agenten-Systems vgl. Schneeweiß (1995), S. 23ff.; Schneeweiß (2003a), S. 126f.; Schneeweiß (2003b), S. 246f. Das Konzept der hierarchischen Überlagerung der Entscheidungsmodelle von Top- und Basisebene ist als Grundlage einer hierarchischen Controllingkonzeption in Homburg (2001), S. 26ff. zu finden. Eine Anwendung fur eine hierarchische Produzenten-LieferantenBeziehung stellen Schneeweiß/Zimmer (2004) vor. 
nehmers sowie seiner Präferenzvorstellung und seiner möglichen Handlungsalternativen bei der Festlegung seines Arbeitseinsatzes. ${ }^{105}$ Lassen sich diese Informationen nicht perfekt durch den Auftraggeber antizipieren, so kann die Informationsasymmetrie in Form von Hidden Characteristics durch zusätzliche Informationen (Screening, Signalling, Self-Selection) gemindert werden. ${ }^{106}$

Auftraggeber und Auftragnehmer gehen nach Festlegung des Vertragsdesigns die Kooperationsbeziehung ein. Während der Projektphase $\left(t_{1}\right)$ erhält ausschließlich der Auftragnehmer zusätzliche Informationen und trifft auf der Grundlage dieser seine Handlungsentscheidungen $\mathrm{a}^{\mathrm{AN}}$, die $z u$ dem aus seiner Sicht besten Ergebnis führen. Sind die Handlungen des Auftragnehmers für den Auftraggeber nicht beobachtbar und besteht somit eine Hidden Action-Situation mit der Gefahr des moralischen Risikos, ist der Auftraggeber am Ende der Projektlaufzeit in der Lage, das Ergebnis der Kooperation festzustellen und zu bewerten.

Die Entscheidung des Auftraggebers für die Wahl eines Vertragsdesigns wird maßgeblich durch die Fähigkeit der Antizipation des Auftragnehmerverhaltens beeinflusst. Aus diesem Grund soll in den folgenden zwei Kapiteln eine detaillierte theoretische Diskussion sowohl der Kooperationsbedingung als auch der Anreizkompatibilitätsbedingung erfolgen.

\subsubsection{Die Kooperations- und Anreizkompatibilitätsbedingungen}

Nach Hax kommt ein effizienter Vertrag dann zustande, sofern eine Partei das für sie optimale Vertragsdesign auswählt, jedoch berücksichtigt, dass der Vertrag durch die andere Partei akzeptiert wird. ${ }^{107}$ Diese in das Entscheidungsmodell des Auftraggebers zu integrierende Nebenbedingung wird in der Prinzipal-Agenten-Theorie als Teilnahme-, Partizipations- oder Kooperationsbedingung bezeichnet. ${ }^{108}$ Die Bedingung soll sicherstellen, dass der Beauftragte durch das Eingehen der Auftragsbeziehung einen Mindestnutzen erzielt, welcher mindestens dem einer alternativen Beziehung bzw. an-

\footnotetext{
$105 \mathrm{Zu}$ hierarchischen Aushandlungen vgl. ausfuhrlich Homburg (1996).

${ }_{106}$ Zu den Typen der Antizipation vgl. Schneeweiß (2003a), S. 42f.

$107 \mathrm{Vgl}$. Hax (1991), S. 58. Es ist für die Definition eines effizienten Vertrags unbedeutend, welche Partei das Vertragsdesign auswählt, sofern das Maximierungsproblem die Akzeptanzbedingung beinhaltet. Vgl. hierzu Macho-Stadler/Pérez-Castrillo (2001), S. 57.

108 Vgl. Erlei/Leschke/Sauerland (1999), S. 117; Göbel (2002), S. 105; Jost (2001), S. 19; Laux (1990), S. 13; Macho-Stadler/Pérez-Castrillo (2001), S. 21. Jost (2000), S. 463 diskutiert die Teilnahmeentscheidung im Rahmen des Motivationsproblems innerhalb einer Arbeitsbeziehung.
} 
derer am Markt erzielbarer Renditen entspricht. ${ }^{109}$ Dieses auch als Reservationsnutzen bezeichnete Mindestgehalt des Agenten muss in dem durch den Auftraggeber vorgegebenen Vertragsdesign Berücksichtigung finden, um die Teilnahme des Agenten an der Kooperation sicherzustellen. ${ }^{110}$

Im Folgenden wird anhand eines Grundmodells unter der Annahme von Informationssymmetrie aufgezeigt, wie sich die aus der normativen Prinzipal-Agenten-Theorie ${ }^{111}$ bekannte Bedingung formal in das Vertragsproblem zwischen Auftraggeber und Auftragnehmer einbinden lässt. ${ }^{112}$ Es wird hierbei angenommen, dass der Auftraggeber (Prinzipal) in Abhängigkeit von dem Arbeitseinsatz des Auftragnehmers (Agent) ein Ergebnis in Form eines monetären Äquivalentes erzielt. Das Ergebnis wird sowohl durch den Arbeitseinsatz des Auftragnehmers als auch zusätzlich durch eine Zufallsgröße $\theta$ beeinflusst. Beide Vertragsparteien besitzen identische Erwartungen bezüglich der Wahrscheinlichkeit des Eintretens eines bestimmten Umweltzustandes $\theta .{ }^{113}$ Bei Annahme einer endlichen Menge möglicher, von einem Arbeitseinsatz $a \in\{1, \ldots, A\}$ sowie des zum Zeitpunkt des Vertragsabschlusses nicht beobachtbaren Umweltzustandes abhängiger Ergebnisse $x_{n}, n \in\{1, \ldots, N\}$, gibt $p_{n}(a)$ an, mit welcher Wahrscheinlichkeit das Ergebnis bei der Wahl des Arbeitseinsatzes a eintritt: ${ }^{114}$

$\operatorname{Prob}\left(\mathrm{x}=\mathrm{x}_{\mathrm{n}} \mid \mathrm{a}\right)=\mathrm{p}_{\mathrm{n}}(\mathrm{a}) \quad \forall \mathrm{n}, \mathrm{a}$

Durch die Definition der Menge möglicher Ergebnisse $X=\left\{x_{1}, \ldots, x_{N}\right\}$ gelten zusätzlich die folgenden Bedingungen:

${ }^{109}$ Vgl. Erlei/Leschke/Sauerland (1999), S. 117; Jost (2001), S. 19; Theilen (1996), S. 11.

${ }^{110}$ Vgl. Laux/Lierman (1997), S. 514; Petersen (1989), S. 45. Backhaus (1980) untersucht in seiner Arbeit das Entscheidungsproblem der Auftragsplanung aus der Sicht des Auftragnehmers. Es wird ein Lðsungsverfahren speziell für die Auftragsplanung auf dem Gebiet des industriellen Anlagengeschäftes vorgestellt.

111 Die normative Prinzipal-Agenten-Theorie untersucht die Auftraggeber-Beauftragten-Beziehung mit Hilfe mathematischer Entscheidungsmodelle und gibt auf der Grundlage von Erkenntnissen und Methoden der traditionellen Mikroökonomik Empfehlungen für die Gestaltung von Vertragsbeziehungen. Vgl. Meyer (2002); Wall (2003), S. 522. Die positive Prinzipal-Agenten-Theorie beschäftigt sich hingegen mit der institutionellen Abwicklung solcher Beziehungen. Sie weist einen stärkeren empirischen Bezug auf und gibt Gestaltungsempfehlungen fur in der Realităt beobachtbare Organisations- und Vertragsformen. Zur Abgrenzung beider Theorierichtungen vgl. Beier (2002), S. 245; Eisenhard (1989); Wenger/Terberger (1988), S. 506ff.

112 Es handelt sich hierbei um ein Standard-Modell der Prinzipal-Agenten-Theorie. Bei der betrachteten Entscheidungssituation bleiben zunächst spezielle Formen der Informationsasymmetrie zwischen Auftraggeber und Auftragnehmer unberücksichtigt. Vgl. für weiterfuhrende Untersuchungen Jost (2001); Kleine (1995); Meyer (1992); Petersen (1989).

113 Es wird fur dieses Standard-Modell von einer symmetrischen Informationsverteilung zwischen Prinzipal und Agent ausgegangen, das heißt, beide besitzen die gleichen Vorstellungen über die Wahrscheinlichkeitsverteilung zuklinftig möglicher Umweltzustănde. Vgl. Macho-Stadler/Pérez-Castrillo (2001), S. 21.

114 Vgl. Macho-Stadler/Pérez-Castrillo (2001), S. 18f. 


$$
\begin{array}{ll}
\sum_{\mathrm{n}=1}^{N} \mathrm{p}_{\mathrm{n}}(\mathrm{a})=1 & \forall \mathrm{a} \\
\mathrm{p}_{\mathrm{n}}(\mathrm{a})>0 & \forall \mathrm{n}, \mathrm{a}
\end{array}
$$

Während die Bedingung (3.2) sicherstellt, dass die Summe der Einzelwahrscheinlichkeiten für jedes Arbeitsniveau eins betragen muss, wird durch die Bedingung (3.3) angenommen, dass die Realisation eines bestimmten Ergebnisses mit jedem Arbeitsniveau mit einer bestimmten Wahrscheinlichkeit möglich ist. Anders ausgedrückt heißt dies, dass keine Ergebnisausprägung existiert, die bei einem Arbeitsniveau möglich und bei einem alternativen Arbeitsniveau nicht möglich ist. ${ }^{115}$

Die Bewertung des mit Unsicherheit behafteten und von dem Arbeitseinsatz des Agenten abhängigen Ergebnisses erfolgt mittels Risikonutzenfunktionen des Agenten und des Prinzipals, ${ }^{116}$ wobei in diesem Grundmodell der Prinzipal-Agenten-Theorie für beide Parteien eine Bewertung nach dem Bernoulli-Prinzip und somit eine Orientierung an dem Nutzenerwartungswert unterstellt wird. ${ }^{117}$

Die Nutzenfunktion des Auftraggebers $\mathrm{u}^{\mathrm{AG}}$ ist abhängig von dem erzielten Ergebnis $\mathbf{x}$ sowie der Entlohnung $w$, die dieser an den Auftragnehmer für die Arbeitsanstrengung und das hiermit verbundene Ergebnis zahlt. Der Auftraggeber orientiert sich für die Auswahl einer Handlungsalternative somit an dem Nutzen des Gewinns:

$$
\mathrm{u}^{\mathrm{AG}}(\mathrm{G})=\mathrm{u}^{\mathrm{AG}}(\mathrm{x}-\mathrm{w})
$$

Mit der Annahme, dass der Auftraggeber risikoneutral oder risikoavers eingestellt ist, verläuft diese Nutzenfunktion monoton steigend linear bzw. konkav, das heißt, es gilt: $\left(\mathrm{u}^{\mathrm{AG}}\right)^{\prime}>0,\left(\mathrm{u}^{\mathrm{AG}}\right)^{n} \leq 0 .{ }^{118}$

Die Nutzenfunktion des Auftragnehmers $u^{\text {AN }}$ berücksichtigt neben dem Nutzen aus der Entlohnung durch den Auftraggeber v(w) auch den mit dem Arbeitseinsatz verbundenen Nutzen g(a). ${ }^{119}$ Für den Auftragnehmer gilt, dass dieser Arbeit mit negativem Einkommen gleichsetzt, das heißt, dass der Nutzen mit steigendem Arbeitseinsatz

\footnotetext{
115 Vgl. Macho-Stadler/Pérez-Castrillo (2001), S. 18.

${ }^{116}$ Vgl. zu Nutzenfunktionen etwa Bamberg/Coenenberg (2002), S. 35f.; Eisenführ/Weber (2003), S. $211 \mathrm{ff}$.; Laux (2003), S. $180 \mathrm{ff}$.

117 Vgl. Ross (1973), S. 134. Zum Bernoulli-Prinzip vgl. beispielsweise Bamberg/Coenenberg (2002), S. 85ff.

${ }^{118} \mathrm{Zu}$ unterschiedlichen Verlăufen von Risikonutzenfunktionen sowie der Risikoeinstellung von Entscheidungsträgern vgl. beispielsweise Bamberg/Coenenberg (2002), S. 92ff.; Eisenfuhr/Weber (2003), S. 222ff.; Laux (2003), S. 180ff.; Saliger (2003), S. 52ff.

119 Vgl. Jost (2001), S. 47; Kleine (1995), S. 30; Petersen (1989), S. 42.
} 
sinkt. ${ }^{120}$ Die Nutzenfunktion des Auftragnehmers kann somit wie folgt formuliert werden: ${ }^{121}$

$u^{\mathrm{AN}}(\mathrm{w}, \mathrm{a})=\mathrm{v}(\mathrm{w})-\mathrm{g}(\mathrm{a})$

Bei Betrachtung dieser Nutzenfunktionen lassen sich die folgenden zwei Interessenskonflikte zwischen Prinzipal und Agent identifizieren: ${ }^{122}$

- Das Ergebnis $\mathbf{x}$ ist Bestandteil der Nutzenfunktion des Prinzipals, jedoch nicht Teil der Nutzenfunktion des Agenten. Das heißt, dass der Agent seine Handlungsentscheidungen nicht direkt nach dem Ergebnis bestimmt, der Prinzipal jedoch eine Präferenz für ein möglichst hohes monetäres Ergebnis besitzt.

- Der Arbeitseinsatz des Agenten geht als negativer Bestandteil in dessen Nutzenfunktion ein, ist jedoch nicht Teil der Nutzenfunktion des Prinzipals. Das heißt, dass der Agent bemüht ist, die mit dem Arbeitseinsatz verbundene Nutzenreduzierung zu minimieren, dies jedoch die Präferenzen des Prinzipals nicht direkt beeinflusst.

Zusätzlich hat der Prinzipal die Vermutung, dass die Wahrscheinlichkeit, ein besseres Ergebnis zu erzielen, mit der Wahl eines höheren Arbeitsniveaus steigt. Das Entscheidungsproblem des Prinzipals bei Informationssymmetrie liegt somit darin, sowohl die für ihn beste Arbeitsleistung a des Agenten zu bestimmen als auch die Entschädigung für diese Arbeitsleistung $w\left(x_{n}\right)$ in Abhängigkeit von dem möglichen Ergebnis so festzulegen, dass er seinen erwarteten Nutzen maximiert und der erwartete Nutzen des Auftragnehmers aus der Arbeitsleistung der Teilnahmebedingung $\mathrm{TB}^{\mathrm{AN}}$ entspricht. ${ }^{123}$ Das Maximierungsproblem aus der Sicht des Auftraggebers lautet somit:

$\max _{\left[a,\left\{w\left(x_{n}\right)\right\}_{n=1 \ldots, N}\right]} z=\sum_{n=1}^{N} p_{n}(a) \cdot u^{A G}\left(x_{n}-w\left(x_{n}\right)\right)$

so dass

\footnotetext{
${ }^{120} \mathrm{Vgl}$. Hartmann-Wendels (1989), S. 717. Es wird angenommen, dass die Funktion v(w) steigend konkav verläuft, das heißt, es gilt: $v^{\prime}(w)>0, v^{\prime \prime}(w) \leq 0$. Für die steigend konvexe Funktion des mit dem Arbeitseinsatz verbundenen Nutzens gilt: $g^{\prime}(a)>0, g^{\prime \prime}(a) \geq 0$. Dies bedeutet, dass der marginale Nutzen aus dem Arbeitseinsatz nicht fallend ist. Vgl. Jost (2001), S. 47.

121 Durch die additive Verknüpfung des Nutzens aus der Arbeitsanstrengung und der Zahlungen des Auftraggebers wird ausgedrückt, dass sich die Risikoaversion des Auftragnehmers nicht mit dem Arbeitseinsatz ändert. Vgl. Macho-Stadler/Pérez-Castrillo (2001), S. 19.

122 Vgl. Macho-Stadler/Pérez-Castrillo (2001), S. 19.

${ }^{123}$ Vgl. Jost (2001), S. 16f.; Trumpp (1995), S. 174 ff.
} 
$\sum_{n=1}^{N} p_{n}(a) \cdot v\left(w\left(x_{n}\right)\right)-g(a) \geq T B^{A N}$

Es kann durch folgende Überlegungen gezeigt werden, dass die Kooperationsbedingung (3.7) für die optimale Lösung bindend ist und damit Gleichheit vorausgesetzt werden kann: Erzielt der Agent einen höheren als den mindestens geforderten Nutzen aus der Kooperation, so kann dies mit einer Prämienzahlung durch den Prinzipal gleichgesetzt werden. Der Agent würde den Vertrag jedoch auch ohne diese zusätzliche Prämie akzeptieren und der Prinzipal somit eine niedrigere Entschädigung zahlen und damit seinen Nutzen erhöhen. ${ }^{124}$ Durch die Kooperationsbedingung wird das multikriterielle Entscheidungsproblem mit den Zielsetzungen der Nutzenmaximierung von Prinzipal und Agent in ein Problem transformiert, welches unter Beibehaltung der Extremierungsvorschrift des Prinzipals die Zielsetzung des Agenten als Satisfizierungsvorschrift in Form des mindestens zu erreichenden Anspruchsniveaus erfasst. ${ }^{125}$

Die Lösung des Vertragsproblems bei symmetrischer Information soll im Folgenden skizziert werden. Hierbei wird unterstellt, dass der Prinzipal risikoneutral und der Agent risikoavers eingestellt ist. Aufgrund der Annahme der Informationssymmetrie kann der Prinzipal dem Agenten das gewünschte Arbeitsniveau vorschreiben, welches seinen Nutzen maximiert. Er wählt als Entlohnung eine von dem Arbeitseinsatz unabhängige Vergütung in der Höhe, dass die Bedingung (3.7) erfüllt ist. ${ }^{126}$

Dieses Ergebnis ist aus entscheidungstheoretischer Sicht intuitiv verständlich: Es ist aus der Sicht des Prinzipals vorteilhaft, das Risiko vollständig zu übernehmen, da der Agent für eine Übernahme des Risikos aufgrund seiner Risikoaversion hierfür eine Risikoprämie verlangen würde, was einer höheren Zahlung des Prinzipals an den Agenten entspricht und zu einer Nutzeneinbuße des Prinzipals führt. ${ }^{127}$ Zudem ist es

\footnotetext{
124 Vgl. Macho-Stadler/Pérez-Castrillo (2001), S. 23. Zu dieser Aussage gelangen auch Mohnen (2002), S. 244 sowie Schneeweiß (2003a), S. 138.

$125 \mathrm{Zu}$ Entscheidungsproblemen bei mehrfacher Zielsetzung vgl. beispielsweise Bamberg/Coenenberg (2002), S. 47ff.; Saliger (2003), S. 24. Eine allgemeine Kritik an der Berücksichtigung von Anspruchsniveaus in Form von Restriktionen ist bei Eisenfuhr/Weber (2003), S. 86f. zu finden. Kim/Wang (2004), S. 114 integrieren die Nutzenfunktion des (risikoneutralen) Agenten in die Maximierungszielfunktion des Prinzipals und ersetzen damit die Teilnamebedingung.

$126 \mathrm{Vgl}$. hierzu die Ausführungen von Grossman/Hart (1983a) sowie die formale Lösung in Jost (2001), S. 51.

127 Zum Begriff der Risikoprämie vgl. beispielsweise Bamberg/Coenenberg (2002), S. 95. Die Risikoprämie wird hier als Differenz aus dem Sicherheitsäquivalent eines Ergebnisses und dessen Erwartungswert definiert. Dabei gibt das Sicherheitsäquivalent an, bei welchem sicheren Ergebnis der Entscheidungsträger zwischen diesem und dem zufallsabhăngigen Ergebnis indifferent ist. Ist der Entscheidungsträger risikoavers eingestellt, so ist das Sicherheitsäquivalent kleiner als der Erwartungswert und die Risikoprämie positiv. Vgl. Bamberg/Coenenberg (2002), S. 97.
} 
durch den Zustand der Informationssymmetrie nicht notwendig, Anreize zu setzen, die den Agenten dazu bewegen, ein hohes Arbeitsniveau zu wählen. ${ }^{128}$

Diese Lösung wird in der Literatur als First-Best-Lösung bezeichnet und ist in Abbildung 3.5 dargestellt. ${ }^{129}$ Dies ist das maximale Nutzenniveau, das der Auftraggeber bei Zahlung eines Festpreises zur Erfüllung der Kooperationsbedingung erreicht.

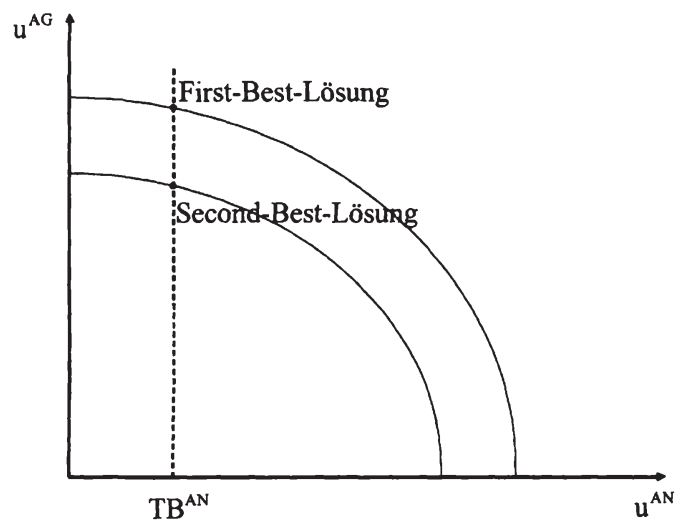

Abbildung 3.5: First- und Second-Best-Lösungen des Prinzipal-Agenten-Problems (Quelle: In Anlehnung an Schneeweiß (2003a), S. 139.)

Wird nun angenommen, dass der Auftraggeber nach Vertragsabschluss in der Lage ist, ausschließlich das Ergebnis der Leistungen des Auftragnehmers und nicht dessen Anstrengungsniveau zu beobachten, muss der Auftraggeber das moralische Risiko berücksichtigen, dass der Auftragnehmer nach Vertragsabschluss nicht das vorgegebene Arbeitsniveau wählt, sondern hiervon abweicht, um durch das Ausnutzen der Informationsasymmetrie bei identischer Vergütung und einer geringeren Arbeitsanstrengung ein höheres Nutzenniveau zu erreichen. Daher wird die folgende Anreizkompatibilitätsbedingung als zusätzliche Restriktion in das Maximierungsproblem des Auftraggebers integriert: ${ }^{130}$

$a \in \underset{a^{\prime}}{\arg \max }\left\{\sum_{n=1}^{N} p_{n}\left(a^{\prime}\right) \cdot v\left(w\left(x_{n}\right)\right)-g\left(a^{\prime}\right)\right\}$

${ }^{128}$ Vgl. Jost (2001), S. 22.

${ }^{129} \mathrm{Vgl}$. Holmström (1979), S. 76. Für eine ausfuhrliche formale Darstellung vgl. Kiener (1990), S. $49 \mathrm{ff}$.

130 Vgl. Jost (2001), S. 48. 
Diese Restriktion gewährleistet, dass aus der Menge aller durch den Auftraggeber antizipierten, die Kooperationsbedingung erfüllenden möglichen Arbeitsniveaus a' dasjenige von dem Auftragnehmer realisiert wird, welches seinen Nutzen als Differenz aus dem erwarteten Nutzen der von dem Ergebnis abhängigen Entschädigung sowie dem Nutzen der Arbeitsanstrengung maximiert. ${ }^{131}$ Anders formuliert besteht das Entscheidungsproblem des Prinzipals darin, dem Auftragnehmer beispielsweise durch eine leistungsbezogene Entlohnung Anreize zu setzen, so dass dieser das vom Auftraggeber gewünschte Anstrengungsniveau realisiert. ${ }^{132}$ Durch die an das beobachtbare und neben der Arbeitsanstrengung von der Realisation eines zum Zeitpunkt des Vertragsabschlusses unsicheren Umweltlage abhängige Ergebnis geknüpfte Vergütung übernimmt jedoch der Auftragnehmer einen Teil des Risikos, so dass die für den Fall der Informationssymmetrie mögliche First-Best-Lösung nicht mehr erreicht werden kann. Es ergibt sich daher fur den Zustand der Informationsasymmetrie die in Abbildung 3.5 dargestellte Second-Best-Lösung. ${ }^{133}$

In der Literatur zur Prinzipal-Agenten-Theorie sind eine Vielzahl über dieses Grundmodell hinausgehende Entwicklungen festzustellen. ${ }^{134}$ Hierbei stehen insbesondere auf allgemeinen Modellen sowie grafischen Analysen basierende Untersuchungen zur Bestimmung optimaler Verträge bei Vorliegen unterschiedlicher Arten der Informationsasymmetrie zwischen Prinzipal und Agent sowie unterschiedlicher Formen und Kombinationen der Risikoeinstellung im Vordergrund. Beispielsweise lassen sich Hinweise auf ein Vorgehen finden, sofern der Prinzipal zwischen unterschiedlichen Agententypen mit individuellen Anspruchsniveaus auswählen kann. ${ }^{135}$ Eine Diskussion bei Vorliegen asymmetrischer Information über den Agentennutzen, das heißt der Prinzipal kennt den Reservationsnutzen und/oder die Risikoeinstellung des Agenten nicht, findet sich bei Göx, Budde und Schöndube. ${ }^{136}$

Die in diesem Kapitel vorgestellte Prinzipal-Agenten-Problematik aus der Sicht einer hierarchischen Planung wird im Zuge der Integration verschiedener Vertragsdesigns in

\footnotetext{
${ }^{131}$ Vgl. Theilen (1996), S. 10f. Für eine alternative Darstellung der Restriktion (3.8) vgl. Grossman/Hart (1983a), S. 13.

132 Ohne eine leistungsbezogene Vergütung wird der Agent unbeobachtet das niedrigste Arbeitsniveau realisieren und durch die feste Vergütung einen höheren als den geforderten Mindestnutzen realisieren. Vgl. Theilen (1996), S. 16.

133 Vgl. Schneeweiß (2003a), S. 139f.

134 Vgl. etwa Göbel (2002); Holmström (1979); Kiener (1990), S. 60ff.; Laffont/Martimort (2002); MachoStadler/Pérez-Castrillo (2001), S. 35ff.; Petersen (1989); Picot/Dietl/Franck (2002), S. 96ff.

135 Vgl. Hartmann-Wendels (1989), S. 717; Laffont/Martimort (2002), S. 84.

${ }^{136} \mathrm{Vgl}$. Göx/Budde/Schöndube (2002).
} 
die quantitative Projektplanung des Auftraggebers im fünften Kapitel erneut aufgegriffen. Zum Abschluss dieses Kapitels werden Möglichkeiten aufgezeigt, wie das Informationsmanagement zu einer Verringerung der Informationsasymmetrie zwischen Auftraggeber und Auftragnehmer beitragen kann.

\subsubsection{Informationsmanagement als Teil des Kooperationsmanagements}

Eine einheitliche Abgrenzung und Definition des Informationsmanagementbegriffs lässt sich in der Literatur nicht identifizieren. ${ }^{137}$ Vielmehr erfolgt häufig eine aus den Begriffen Management und Information abgeleitete, sowohl funktionale als auch institutionale Aspekte des Managements integrierende Sichtweise. ${ }^{138}$ Das Informationsmanagement umfasst somit sämtliche auf ein computergestütztes Informations- und Kommunikationssystem einer Unternehmung bezogenen Führungs- und Durchführungsaufgaben. ${ }^{139}$ Dies beinhaltet sowohl die Ermittlung und Modifikation von Informationsbedarf und -bereitstellung ${ }^{140}$ als auch die Gestaltung und Steuerung eines Informationssystems einer Unternehmung für die zielgerichtete Beeinflussung von Aktivitäten der Informationsverarbeitung. ${ }^{141}$

Das von Wollnik entwickelte und von Krcmar um die Aufgaben des Managements erweiterte Modell des Informationsmanagements ist in Abbildung 3.6 dargestellt: ${ }^{142}$

${ }^{137}$ Eine Übersicht unterschiedlicher Lehrmeinungen zum Informationsmanagement ist in Voß/Gutenschwager (2001), S. 58ff. zu finden. Der Begriff des Wissensmanagements kann von dem Begriff des Informationsmanagements abgegrenzt werden. Vgl. zur Abgrenzung etwa Gabriel/Beier (2003), S. 41; Stelzer (2003), S. 28ff. Zum Wissensmanagement vgl. etwa Burghardt (2002a), S. 276ff.; Gabriel/Beier (2003), S. 215ff.; Krcmar (2003), S. 417 ff.; Rose et al. (2002); Voß/Gutenschwager (2001), S. 317ff. Mit der Problematik der Wissensübertragung zwischen und innerhalb von Unternehmungen beschaftigt sich die Arbeit von Kriwet (1997). Den Einsatz des Wissensmanagements bei Unternehmensberatungen zeigt Disterer (2001).

${ }^{138}$ Vgl. Beier (2002), S. 25ff.; Krcmar (2003), S. 15ff.; Streubel (2000), S. 60ff. Vgl. auch die Ausfuhrungen in Kapitel 3.1.2 sowie Kapitel 3.2.1.

139 Vgl. Domschke/Scholl (2003), S. 376f.; Gabriel/Beier (2003), S. 41.

${ }^{140}$ Vgl. Streubel (2000), S. 83.

${ }^{141}$ Vgl. Beier (2002), S. 44 sowie zum strategischen Informationsmanagement Werners (1993), S. $21 \mathrm{ff}$.

142 Vgl. Wollnik (1988), S. 38; Picot/Reichwald/Wigand (2003), S. 144f. Das von Wollnik entwickelte DreiEbenen-Modell des Informationsmanagements wird von Krcmar (2003), S. 46 um eine vierte Ebene erweitert, die Ebenen übergreifend die generellen Führungsaufgaben des Informationsmanagements beinhaltet. Voß/Gutenschwager (2001), S. 74 wählen eine ähnliche Darstellung für die Definition von Aufgaben des Informationsmanagements. Streubel (2000), S. 81f. stellt einen Zwei-Ebenen-Ansatz vor und zeigt den bidirektionalen Zusammenhang zwischen immateriellen, die informationale Beziehung zwischen Akteuren umfassenden, und materiellen, insbesondere die Informationstechnik sowie die Beziehung zwischen Mensch und Technik berücksichtigenden Ebenen auf. Einen weiteren alternativen Ansatz für eine Systematisierung der Aufgaben des Informationsmanagements stellen Gabriel/Beier (2003), S. 66 vor. Hierbei erfolgt eine Unterscheidung in eine strategische/operative Dimension sowie nach den Führungsaufgaben Planung/Entscheidung/Steuerung/Kontrolle und nach den Elementen eines Informations- und Kommunikationssystems Menschen/Aufgaben/Technik. Eine Anwendung des Informationsmanagements am Beispiel des deutschen Pharmagroßhandels zeigen Engelhardt/Gersch (1995), S. $201 \mathrm{ff}$. auf. 


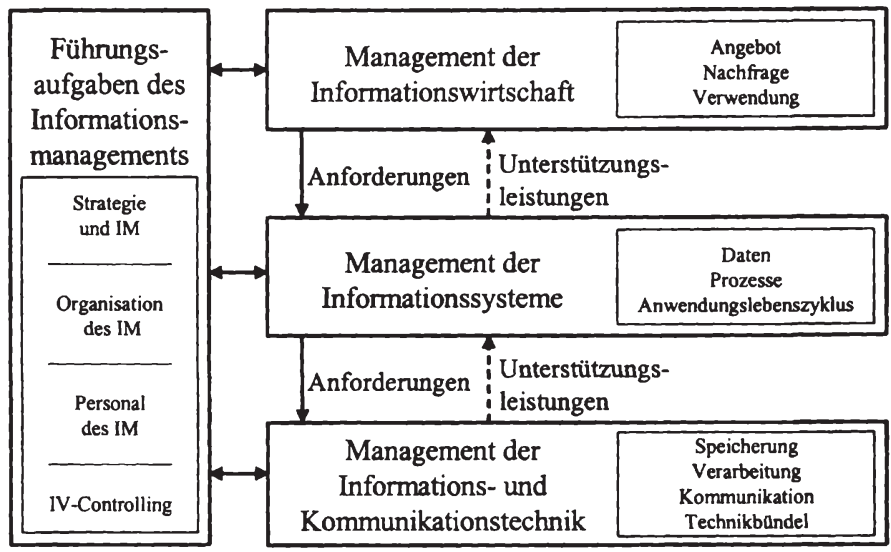

Abbildung 3.6: Aufgaben des Informationsmanagements (Quelle: In Anlehnung an Krcmar (2003), S. 46.)

Auf der obersten Ebene des Managements der Informationswirtschaft sind Entscheidungen über den Informationsbedarf, das Informationsangebot sowie die Informationsverwendung zu treffen. Die Planung, Organisation und Kontrolle von Informationsangebot und -nachfrage sollte alle Bereiche einer Unternehmung umfassen. ${ }^{143}$ Die Informationsverwendung ist insbesondere abhängig von dem Einsatz betriebswirtschaftlicher Entscheidungsmodelle. ${ }^{144}$

Das Management der Informationssysteme verfolgt die Zielsetzung der Deckung des Informationsbedarfs der obersten Ebene. Hierzu zählen beispielsweise Systeme der Produktionsplanung und -steuerung oder Standardsysteme des Rechnungswesens. Diese Informations- und Kommunikationssysteme setzen sich aus aufeinander abgestimmten personellen, organisatorischen und technischen Elementen zusammen. ${ }^{145}$

Auf der untersten Ebene stehen die mit der Bereitstellung und Verwaltung der Technikinfrastruktur verbundenen Managementaufgaben im Mittelpunkt. Insbesondere hierbei wird die Verbindung der Ebenen untereinander erkennbar. Die unteren Ebenen

${ }^{143} \mathrm{Vgl}$. Krcmar (2003), S. 46f.

$144 \mathrm{Vgl}$. Voß/Gutenschwager (2001), S. 74ff. In welcher Form das Wissensmanagement als organisationsweiter Prozess für eine Entscheidungsunterstützung eingesetzt werden kann, zeigen Hasan/Gould (2001), S. 79f. Anforderungen an die Gestaltung von Informationssystemen zur Unterstützung multikriterieller Entscheidungen zeigt Scheubrein (2003), S. 227ff. auf.

145 Vgl. Picot/Reichwald/Wigand (2003), S. 145; Bloodgood/Salisbury (2001), S. $61 \mathrm{ff}$. Nach DIN 69901 wird ein Projektinformationssystem definiert als „Gesamtheit der Einrichtungen und Hilfsmittel und deren $\mathrm{Zu}$ sammenwirken bei der Erfassung, Weiterleitung, Be- und Verarbeitung, Auswertung und Speicherung der Projektinformationen.“ S. Deutsches Institut für Normung (1987), S. 3. 
des Informationsmanagements müssen die Anforderungen der oberen Ebenen berücksichtigen und hierfür die Erbringung von Unterstützungsleistungen in Form von Anwendungssystemen sowie der hierauf abgestimmten physischen Rechnernetzwerke gewährleisten. ${ }^{146}$ Das Controlling der Informationsverarbeitung als Bestandteil der Ebenen übergreifenden Führungsaufgaben ist von besonderer Bedeutung und wird daher in einigen Lehrbüchern intensiv diskutiert. ${ }^{147}$

Die von Voß und Gutenschwager stammende folgende Definition des Informationsmanagements umfasst die hier dargestellten unterschiedlichen Ebenen und kann im Hinblick auf ein Informationsmanagement für Kooperationsbeziehungen erweitert werden: „Informationsmanagement ist die wirtschaftliche (effiziente) Planung, Beschaffung, Verarbeitung, Distribution und Allokation von Informationen als Ressource zur Vorbereitung und Unterstützung von Entscheidungen (Entscheidungsprozessen) sowie die Gestaltung der dazu erforderlichen Rahmenbedingungen. “148

Diese Definition beschränkt sich nicht auf das Informationsmanagement innerhalb einer Unternehmung, sondern behält auch für die unternehmungsübergreifenden, in dieser Arbeit betrachteten Entscheidungsprozesse ihre Gültigkeit. Die folgenden Ausführungen zeigen auf, welche Bedeutung Informations- und Kommunikationssystemen als Teil des Informationsmanagements in Kooperationen zukommt und welche Möglichkeiten durch deren Einsatz existieren, die im Rahmen der Agency-Theorie sowie der Transaktionskostentheorie aufgezeigten Probleme asymmetrisch verteilter Informationen zu mindern.

Rey untersucht ausführlich, welche besonderen Anforderungen an die Planung und Nutzung von Informations- und Kommunikationssystemen in Projektkooperationen zu stellen sind. ${ }^{149}$ Die folgende Abbildung 3.7 gibt einen zusammenfassenden Überblick über den Einsatz von Informations- und Kommunikationssystemen für die einzelnen Phasen des Kooperationsmanagements:

\footnotetext{
146 Vgl. Krcmar (2003), S. 47.

147 Vgl. Gabriel/Beier (2003), S. 136ff.; Krcmar (2003), S. 346ff.; Martiny/Klotz (1989), S. 118; Voß/Gutenschwager (2001), S. $79 \mathrm{ff}$.

148 Voß/Gutenschwager (2001), S. 70.

149 Vgl. Rey (1999). Für eine Anwendung aus dem Bereich des Gesundheitswesens vgl. Pedersen/Larsen (2001). Für die Bedeutung von Kommunikation in Kooperationen vgl. Gabriel et al. (2002), S. 125f.; Mohr (1995), S. $370 \mathrm{ff}$.
} 


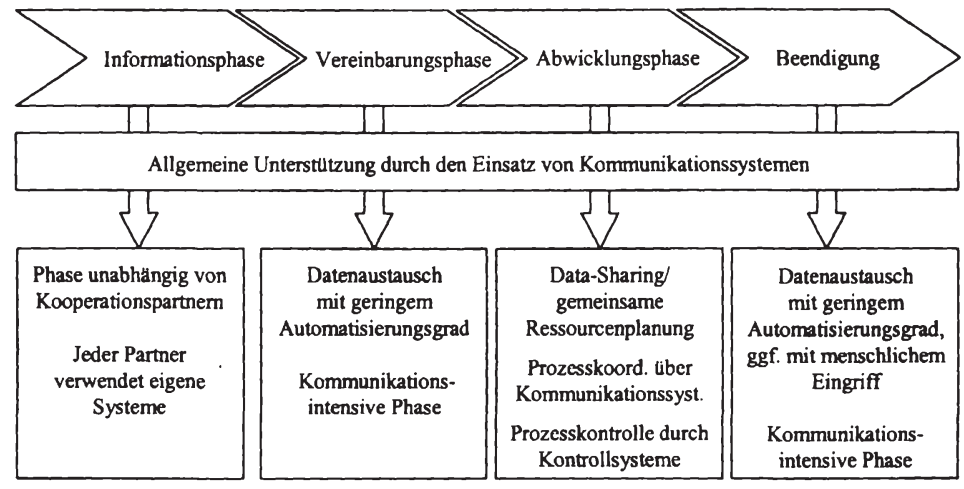

Abbildung 3.7: Einsatz von Informations- und Kommunikationssystemen in Kooperationen (Quelle: In Anlehnung an Rey (1999), S. 194.)

Während der Informationsphase findet zwar eine Kommunikation zwischen möglichen Kooperationspartnern statt, jedoch nutzen diese ihre eigenen Systeme für die Entscheidungsvorbereitung beispielsweise zur Formulierung der individuellen Kooperationsziele sowie der Suche und Auswahl der Kooperationspartner. ${ }^{150}$ Hierbei steht die Beschaffung von Marktinformationen im Vordergrund, was eigenständig durch die potenziellen Kooperationsteilnehmer durchgeführt wird. ${ }^{151}$

Innerhalb der Vereinbarungsphase erfolgt die Abstimmung der Kooperationspartner untereinander hinsichtlich der Kooperationsziele sowie der vertraglichen Vereinbarungen. Ein Datenaustausch findet während dieser kommunikationsintensiven Phase nur in begrenztem Umfang statt und geschieht meist durch die Anforderung durch einen und anschließende Bereitstellung von Daten durch einen anderen Kooperationspartner. ${ }^{152}$ Im Gegensatz hierzu ist für die Planung des Prozessablaufs, die Ressourcenplanung sowie die Koordination und Kontrolle der Prozessabwicklung während der Abwicklungsphase ein umfangreicher Datenaustausch zwischen den Kooperationsteilnehmern notwendig. ${ }^{153}$ Insbesondere ist hier der Einsatz von Systemen zur Geschäftsprozessoptimierung ${ }^{154}$ sowie von Kennzahlensystemen für das Controlling von Bedeu-

\footnotetext{
${ }^{150} \mathrm{Zu}$ den Anforderungen an das Informationsmanagement insbesondere für interorganisationale Projektkooperationen vgl. Beck (1994), S. 134ff.

151 Vgl. Rey (1999), S. 187.

152 Vgl. Rey (1999), S. 188. Unterschiedliche Formen des Informations- und Kommunikationsverhaltens von Entscheidungstrăgern werden bei Picot/Reichwald/Wigand (2003), S. 80ff. behandelt. Für eine empirische Untersuchung der Verschlüsselungs- und Entschlusselungskompetenz von Projektmanagern in einem Projektteam vgl. Henderson (2004).

153 Vgl. Rey (1999), S. 189ff.

154 Zu Systemen der Geschăftsprozessoptimierung vgl. Völkner (1998).
} 
tung. ${ }^{155}$ Die eine Kooperationsbeziehung beendende Phase kann wie die Vereinbarungsphase als kommunikationsintensiv charakterisiert werden. Insbesondere die aus der Kooperation resultierenden, vertraglich formulierten Verteilungsfragen zwischen den Partnern stehen während dieser Phase im Vordergrund. ${ }^{156}$

Beier beschäftigt sich in seiner Arbeit unter anderem mit der Fragestellung, welche Erkenntnisse aus der Theorie der Neuen Institutionenökonomik für das Informationsmanagement gewonnen werden können. ${ }^{157}$ Es werden die Zielsetzungen eines Informationsmanagements aus der Sicht der Transaktionskostentheorie sowie der AgencyTheorie detailliert untersucht. Während die Transaktionskostentheorie die mit der Koordination des Güter- und Leistungsaustauschs verbundenen Kosten betrachtet, welche durch den Einsatz eines Informationssystems gesenkt werden sollen, steht aus der Sicht der Agency-Theorie die durch ein geeignetes Informationssystem unterstützte Gestaltung und Steuerung der Auftraggeber-Auftragnehmer-Beziehung zur Senkung der Agency-Kosten im Vordergrund. ${ }^{158}$ Die Ansatzpunkte des Informationsmanagements aus der Sicht beider Theorierichtungen werden in der folgenden Abbildung 3.8 aufgezeigt:
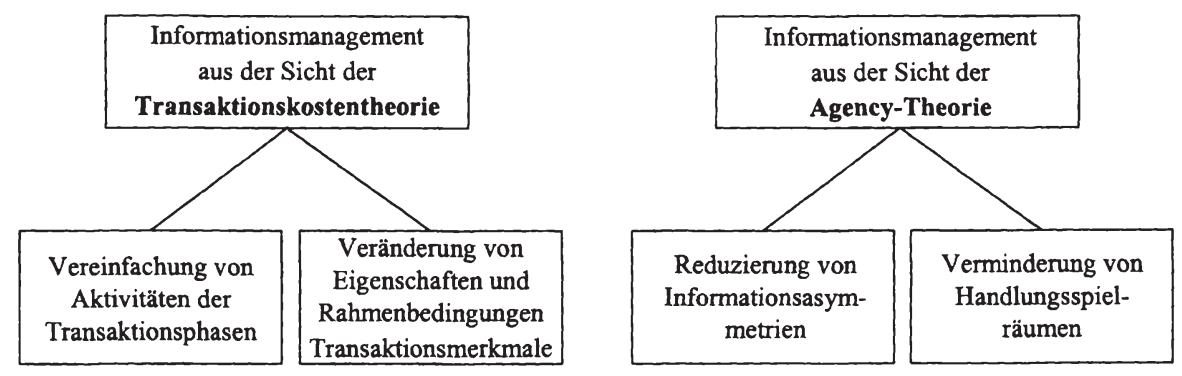

Abbildung 3.8: Informationsmanagement aus der Sicht von Transaktionskosten- und Agency-Theorie (Quelle: In Anlehnung an Beier (2002), S. 252 sowie S. 254.)

Insbesondere während der Informations- und Verhandlungsphase können die hier anfallenden Transaktionskosten in Form von Anbahnungs- und Verhandlungskosten durch eine informationstechnische Unterstützung der Kommunikation zwischen den

\footnotetext{
155 Zum Informationsversorgungsystem aus der Sicht des Controllings vgl. etwa Horváth (2003), S. 357ff. sowie zu der Beziehung zwischen Controlling und Informationsmanagement S. $690 \mathrm{ff}$.

156 Vgl. Rey (1999), S. 193.

157 Vgl. Beier (2002), Kapitel 5.

158 Vgl. Beier (2002), S. 254 und S. 266. Zu den Anforderungen an Systeme fur informationstechnisch vernetzte Geschäftsbeziehungen vgl. Gersch (1998).
} 
Kooperationspartnern sowie die Bereitstellung von Anwendungssystemen zur Beobachtung des Marktgeschehens gesenkt werden. ${ }^{159}$ Die nach Vertragsabschluss entstehenden Kontroll- und Abwicklungskosten können durch den Einsatz von Berichts- und Überwachungssystemen sowie Qualitätssicherungssystemen reduziert werden. ${ }^{160}$ Die vor allem bei unvollständigen Verträgen und bei Realisation von zum Zeitpunkt des Vertragsabschlusses nicht berücksichtigten Umweltzuständen notwendigen Änderungen des Vertrags verursachten Anpassungskosten können durch die Nutzung von Kommunikationssystemen verringert werden. Zusätzlich zu den hier skizzierten Vereinfachungen der Transaktionsaktivitäten kann der Einsatz technikunterstützter Informationsverarbeitung auch eine Veränderung der Vorteilhaftigkeit einer Koordinationsform herbeiführen. ${ }^{161}$

Aus der Sicht der Agency-Theorie lassen sich die in Kapitel 3.2.2 analysierten Probleme als Ursachen asymmetrisch verteilter Information innerhalb der AuftraggeberAuftragnehmer-Beziehung durch den Einsatz von Informationssystemen mindern. Grundsätzlich können die Agency-Kosten durch die Einbindung von Anreizsystemen, die direkte Verhaltenssteuerung sowie die Verbesserung des Informationsstandes des Auftraggebers reduziert werden. ${ }^{162}$ Ansatzpunkte für den Einsatz des Informationsmanagements zur Reduzierung der Agency-Kosten stellen beispielsweise Anwendungssysteme dar, die den Auftraggeber bei der Auswahl der Vertragspartner unterstützen, indem Marktinformationen oder Planungssysteme bereitgestellt werden, die unter Einbeziehung unternehmungsinterner sowie -externer Einflussgrößen eine bessere Prognose von Umweltzuständen bzw. konkret des vermuteten Verhaltens des Auftragnehmers ermöglichen. ${ }^{163}$

Bei der Koordination der Aktivitäten während der Projektdurchführung können Systeme unterstützen, welche die von beiden Projektpartnern genutzten Informationen sammeln, kommentieren und verteilen. Diese Anwendungssysteme werden als Computer Supported Cooperative Work (CSCW)-Systeme bezeichnet. ${ }^{164}$

\footnotetext{
159 Vgl. Beier (2002), S. 254ff.

160 Vgl. Beier (2002), S. 256.

161 Vgl. Beier (2002), S. 329ff. Hierbei steht die Spezifität von Informationssystemen im Vordergrund der Untersuchungen. Beier benutzt den in dieser Arbeit verwendeten Begriff des Vertragsdesigns in einem anderen Zusammenhang, indem er verschiedene Formen zwischen marktlicher und hierarchischer Koordination charakterisiert und voneinander abgrenzt.

162 Vgl. Beier (2002), S. 266.

163 Für eine ausfuhrliche Diskussion der Maßnahmen des Informationsmanagements zur Reduzierung der Agency-Problematik vgl. Beier (2002), S. $265 \mathrm{ff}$.

${ }^{164}$ Vgl. Beier (2002), S. 317; Carbon (1999), S. 47ff.; Ramesh/Tiwana (1999), S. $217 \mathrm{ff}$.
} 
Anwendungen, welche die Zusammenarbeit von Partnern entlang einer Wertschöpfungskette unterstützen, werden unter dem Begriff Collaborative Commerce (cCommerce) zusammengefasst und sind Bestandteil des Supply Chain Managements. ${ }^{165}$

Zusätzlich können die Technologien, welche sich mit dem Begriff des Mobile Business subsumieren lassen, zu einer effektiven und effizienten Gestaltung der Informations- und Kommunikationssysteme beitragen. ${ }^{166}$

Zum Schluss dieses Kapitel können dem Informationsmanagement zusammenfassend insbesondere im Hinblick auf die in dieser Arbeit betrachtete AuftraggeberAuftragnehmer-Beziehung die in Abbildung 3.9 angeordneten unterschiedlichen Anwendungsbereiche hinsichtlich einer möglichen Unterstützung des Auftraggebers für das Vertrags- und Projektmanagement beigemessen werden:

\section{Kooperations-Informationsmanagement}

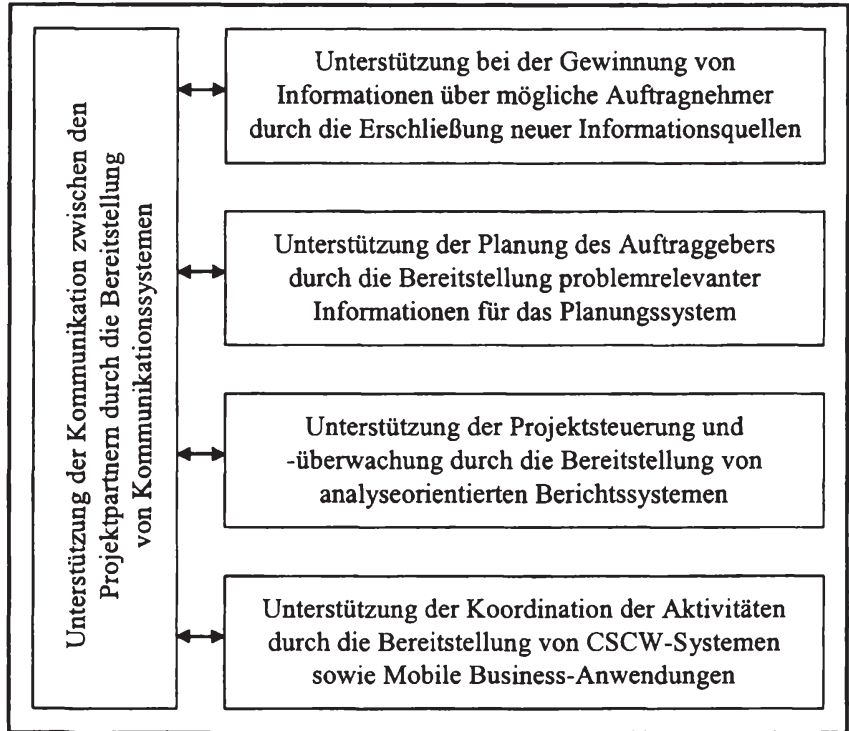

Abbildung 3.9: Anwendungsbereiche des Informationsmanagements bei Projektkooperationen

${ }^{165}$ Vgl. Dudek (2004), S. 35ff.; Krcmar (2003), S. 400. Voß/Schneidereit (2002) untersuchen den Zusammenhang zwischen Transaktionskosten und Informationsmanagement für Supply Contracts. Vgl. zu Supply Contracts auch Cachon (2003); Chopra/Meindl (2004); Elmaghraby (2000).

${ }^{166}$ Fur eine Definition des Begriffs Mobile Business vgl. beispielsweise Beier (2002), S. 385. Anwendungen und Potenziale des Mobile Business insbesondere für das Informationsmanagement zeigen Beier (2002), S. 377ff. sowie Gabriel/Beier (2002), S. 200ff. auf. 


\subsection{Vertragsdesign für Investitionsprojektkooperationen}

\subsubsection{Contracting für das Projektmanagement}

Sowohl die Prinzipal-Agenten-Theorie als auch das Contract Management befassen sich mit der Gestaltung von Verträgen und der Möglichkeit der Integration von Anreizsystemen in Vertragsbeziehungen. Die Arbeiten von Scholtis und Theilen betrachten das Problem der Vertragsgestaltung aus der Sicht der Prinzipal-Agenten-Theorie und schlagen je nach Art der vorliegenden Informationsasymmetrie quantitative Modelle bzw. Instrumente zur Erklärung und Lösung der Problematik vor. ${ }^{167}$

Ziel des in dieser Arbeit im Mittelpunkt stehenden Contract Managements ist es, durch die Festlegung von Zahlungsbedingungen im Rahmen des Vertragsdesigns Organisationsstrukturen in Kooperationsbeziehungen zu schaffen, die Verhaltensanreize zur Erreichung der individuellen Präferenzen setzen. ${ }^{168}$ Obwohl die Prinzipal-AgentenTheorie durch die Formulierung von Anreizkompatibilitätsbedingungen sowie Teilnahmebedingungen ${ }^{169}$ die Art der Vergütungen des Auftragnehmers durch den Auftraggeber implizit berücksichtigt, stehen die Zahlungsbedingungen und -strukturen nicht im Vordergrund der Betrachtungen. Bevor die Thematik des Contract Managements näher behandelt wird, müssen zunächst drei Begriffe sowie deren Bedeutung im Rahmen dieser Arbeit näher definiert werden:

\section{Kosten}

Durch die vorwiegend englischsprachigen wissenschaftlichen Beiträge auf dem Gebiet des Contract Managements wird häufig der Terminus ,cost' als Bestandteil der Kapitalwertberechnung verwendet. ${ }^{170}$ Diese die Begriffe Kosten und Auszahlung gleichsetzende Definition entspricht nicht der Begriffsabgrenzung deutschsprachiger Lehrbücher des betrieblichen Rechnungswesens. Zu den Auszahlungen zählen hiernach ausschließlich Geldbeträge, denen ein tatsächlicher Zahlungsstrom in der Abrechnungsperiode zugrunde liegt. Kosten umfassen hingegen den für eine Periode bewerteten Verbrauch von Produktionsfaktoren, welche sowohl für die Leistungserstellung als auch für die Aufrechterhaltung der Kapazitäten notwendig sind. ${ }^{171}$ Um dem Verfahren

\footnotetext{
167 Vgl. Scholtis (1998); Theilen (1996).

168 Vgl. Artz/Norman (2002); Turner (2004); Turner/Simister (2001).

169 Vgl. Jost (2001), S. 19 sowie Kapitel 3.2.4.

${ }^{170}$ Es werden beispielsweise die Begriffe costs bzw. expenses mit cash-outflows oder payments synonym verwendet. Vgl. etwa Al-Subhi Al-Harbi (1998), S. 73; Berends (2000), S. 167; Bubshait (2003), S. 63; Dayanand/Padman (1997), S. 908.

171 Zur Begriffsabgrenzung vgl. Fandel et al. (2004), S. 9ff.
} 
der Kapitalwertberechnung auf der Grundlage von Ein- und Auszahlungen zu entsprechen, jedoch gleichzeitig die für das Contract Management geläufigen Bezeichnungen zu verwenden, wird der Begriff Kosten im Rahmen dieser Arbeit als die für den Auftragnehmer sowie weiterer Subunternehmer anfallenden Zahlungen definiert, die für die Leistungserstellung des Vertragsgegenstandes getätigt werden. Hierzu zählen die Herstellkosten als Summe aus Materialeinzel- und Materialgemeinkosten, Fertigungseinzel- und Fertigungsgemeinkosten sowie die Sondereinzelkosten der Fertigung. ${ }^{172}$ Darüber hinaus kann auch ein Zuschlag auf die Herstellkosten durch die anteilige Anrechnung der Verwaltungs- und Vertriebskosten bzw. der Forschungs- und Entwicklungsgemeinkosten erfolgen. ${ }^{173}$

\section{Honorar}

Für die Leistungserstellung kann der Auftraggeber an den Auftragnehmer zusätzlich zu den entstandenen Kosten ein Honorar zahlen. Es deckt alle über die Herstellkosten hinausgehenden und in den Selbstkosten enthaltenen Kosten, wie zum Beispiel Entwicklungsgemeinkosten, Verwaltungs- und Vertriebsgemeinkosten oder Wagniszuschläge ab. Zusätzlich ist der Gewinn des Auftragnehmers Teil des Honorars. ${ }^{174}$

\section{Preis}

Als Preis wird in dieser Arbeit die Summe aus Kosten und Honorar bezeichnet und damit derjenige Gesamtbetrag, den der Auftraggeber an den Auftragnehmer für die Leistungserstellung zahlt. Der Preis ist Gegenstand der Vertragsverhandlungen zwischen den Vertragsparteien. Für den Auftraggeber mindert der zu zahlende Preis die aus der Investition resultierenden Einzahlungen bzw. Erlöse und damit seinen Gewinn. Er ist also bemüht, einen niedrigen Preis festzulegen. Der Auftragnehmer erhöht unter der Annahme der Kenntnis der Kosten sowie eines positiven Honorars mit einem höheren Preis seinen Gewinn und ist damit bemüht, einen möglichst hohen Preis zu vereinbaren. Gleichzeitig senkt der Auftragnehmer bei Abgabe eines mit einem hohen Preis kalkulierten Angebotes insbesondere bei Auftragsvergaben durch Ausschreibun-

\footnotetext{
${ }^{172}$ Ein beispielhaftes Kalkulationsschema zur Ermittlung des Selbstkostenpreises im Rahmen der Angebotskalkulation findet sich in Corsten/Corsten (2000), S. 30.

173 Vgl. Jórasz (2003), S. 161. Für eine Abgrenzung der im Rahmen einer Kostenträgerrechnung ermittelten Herstellkosten von den durch die Normen des Handels- und Steuerrechts bestimmten Herstellungskosten vgl. beispielsweise Jórasz (2003), S. 65; Macha (2003), S. $220 \mathrm{ff}$.

174 Vgl. Gilbreath (1992), S. 38.
} 
gen die Wahrscheinlichkeit des Zuschlags. ${ }^{175}$ Eine diese Zielkonflikte sowie die hieraus resultierenden betriebswirtschaftlichen und spieltheoretischen Fragestellungen betrachtende Forschungsrichtung wird in der Literatur auch als Bidding-Problem bezeichnet. ${ }^{176}$ Um die Entwicklung und den Stand wissenschaftlicher Forschung auf dem Gebiet des Contract Managements aufzuzeigen, erfolgt zunächst ein Überblick über wissenschaftliche Beiträge zum Contract Management, die sich als Schwerpunkt mit den verschiedenen Möglichkeiten des Vertragsdesigns auseinandersetzen. Die Tabelle 3.2 zeigt eine Übersicht der in den wissenschaftlichen Veröffentlichungen zum Contract Management behandelten Vertragsarten. ${ }^{177}$

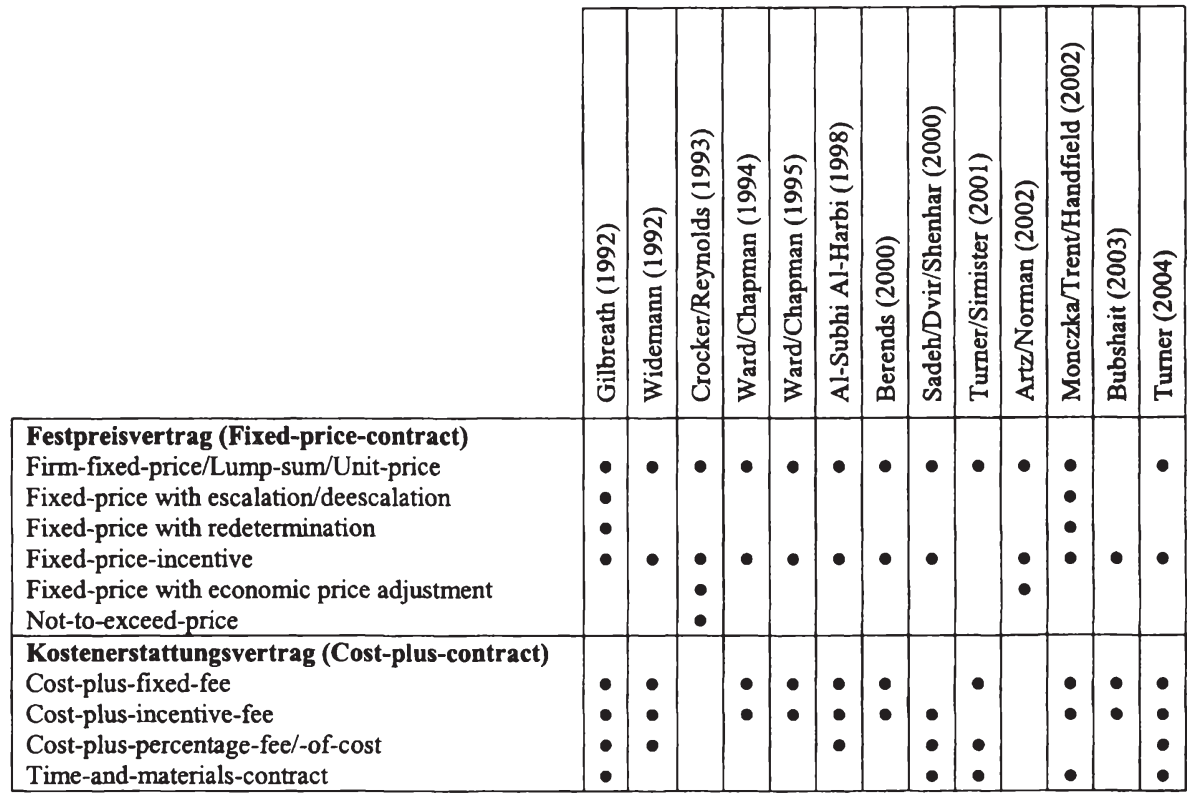

Tabelle 3.2: Literaturüberblick Contract Management und Vertragsdesigns

$175 \mathrm{Vgl}$. Shr/Chen (2003). Die Autoren beziehen in das Bidding-Problem des Auftragnehmers auch die Wirkungen von Bonus-/Strafzahlungen auf den Preis mit ein. Der Anbieter einer Leistung kann beispielsweise ein Angebot mit niedrigem Preis abgeben und somit den Zuschlag im Rahmen der Ausschreibung erhalten, sofern er die private Information besitzt, das Projektende vor dem festgelegten Termin zu erreichen und den Preis durch eine vereinbarte Bonuszahlung zu erhöhen. Eine Anwendung fur den Bereich des Straßenbaus ist in Herbsman/Chen/Epstein (1995) zu finden.

176 Vgl. Simister (2003). Marzouk/Moselhi (2003) entwickeln ein Entscheidungsunterstützungssystem für die Erstellung und Abgabe von Angeboten. Samuelson (1986) analysiert das Bidding-Problem aus der Perspektive des Auftraggebers. Hier stehen die Zielsetzung der Ausgestaltung von Ausschreibungen sowie die Auswahl der Kooperationspartner im Vordergrund. Elmaghraby (1990) erweitert das Bidding-Problem um stochastische Vorgangsdauern.

177 Bei den hier aufgefuhrten Quellen handelt es sich um eine Auswahl von wissenschaftlichen Veroffentlichungen, die das Gebiet des Contract Managements behandeln. Aus Grunden der Aktualităt sind Veroffentlichungen vor 1992 hier nicht aufgefuhrt. Als erster Beitrag, der unterschiedliche Vertragsarten aus der Sicht von Auftraggeber und Auftragnehmer auffürt, kann Scherer (1964) genannt werden. 
Es lässt sich erkennen, dass vier Vertragsarten häufig aufgeführt sind. Dies sind der starre Festpreisvertrag (Firm-fixed-price, FFP), der Festpreisvertrag mit Anreizen (Fixed-price-incentive, FPI), der Kostenerstattungsvertrag mit festem Honorar (Costplus-fixed-fee, CPFF) sowie der Kostenerstattungsvertrag mit Anreizen (Cost-plusincentive-fee, CPIF). Die folgenden zwei Kapitel werden sich daher näher mit Kostenerstattungs- bzw. Festpreisverträgen sowie den mit ihnen verbundenen Auswirkungen auf die Kooperationsbeziehung befassen.

\subsubsection{Kostenerstattungsverträge}

Vereinbaren Auftraggeber und Auftragnehmer die Erstattung aller für die Erbringung der vertraglich festgelegten Leistung entstandenen Kosten des Auftragnehmers in Form von Zahlungen durch den Auftraggeber, so liegt der Kooperationsvereinbarung ein Kostenerstattungsvertrag zugrunde. ${ }^{178}$ Über die Erstattung der Kosten hinausgehende Zahlungen, etwa in Form eines prozentualen Aufschlags (Cost-plus-percentageof-cost, CPPC) oder eines festen Aufschlags (Cost-plus-fixed-fee, CPFF) auf die Kosten sind das Honorar des Auftragnehmers. ${ }^{179}$ Die Abbildung 3.10 bzw. die Abbildung 3.11 zeigt das Verhältnis zwischen Kosten und Honorar des Auftragnehmers sowie zu entrichtendem Preis des Auftraggebers bei einem CPPC- bzw. CPFF-Vertrag. In diesem Beispiel kalkuliert der Auftragnehmer mit vermuteten Kosten in Höhe von 10.000 GE und berechnet als Honorar einen Gewinnzuschlag von $15 \%$, so dass der Festpreis $11.500 \mathrm{GE}$ beträgt. ${ }^{180}$

Es lässt sich erkennen, dass der prozentuale Aufschlag des Honorars auf die Kosten des Auftragnehmers mit sinkenden tatsächlich entstandenen Kosten bei einem CPFFVertrag steigt und bei einem CPPC-Vertrag annahmegemäß konstant ist.

Für den Auftragnehmer besteht bei einem Kostenerstattungsvertrag kein Kostenrisiko, da dieser unabhängig von den zum Zeitpunkt des Vertragsabschlusses erwarteten Kos-

\footnotetext{
${ }^{178}$ Vgl. Turner/Simister (2001), S. 459. Durch die Verordnung über die Preise bei öffentlichen Aufträgen dürfen Kostenerstattungsverträge nur bei mangelnder Feststellbarkeit des Preises oder eingeschränktem Wettbewerb geschlossen warden und unterliegen der Preisprüfung. Vgl. Madauss (2000), S. 336f.

179 Vgl. Ward/Chapman (1994), S. 218. Kostenerstattungsverträge ohne Honorar sind bei Auftragsvergaben öffentlicher oder kirchlicher Institutionen (Universitäten, wohltătige Einrichtungen) teilweise zu beobachten. Dies ist auch bei Forschungs- und Entwicklungsprojekten, bei denen der Auftragnehmer durch die Partizipation an der Kooperation etwa von einem Imagegewinn profitiert, möglich. Vgl. Gilbreath (1992), S. 50.

180 Vgl. Martin/Webster (1986), S. 169.
} 
ten sämtliche Kosten erstattet bekommt, sofern keine Preisobergrenze vereinbart wurde. Eine Risikoprämie als Teil des Honorars des Auftragnehmers entfällt daher.

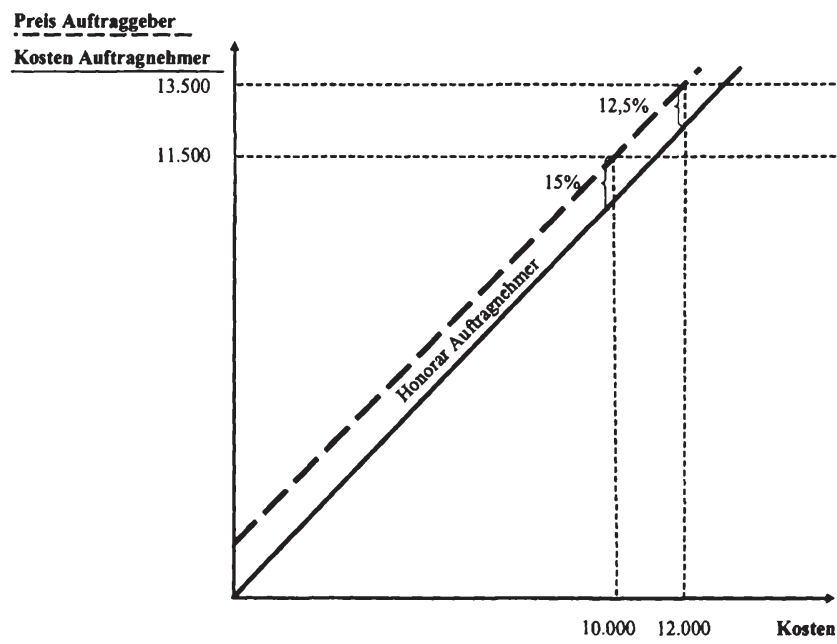

Abbildung 3.10: Kostenerstattung mit festem Honorar (CPFF)

(Quelle: In Anlehnung an Werners/Slaghuis (2004), S. 355.)

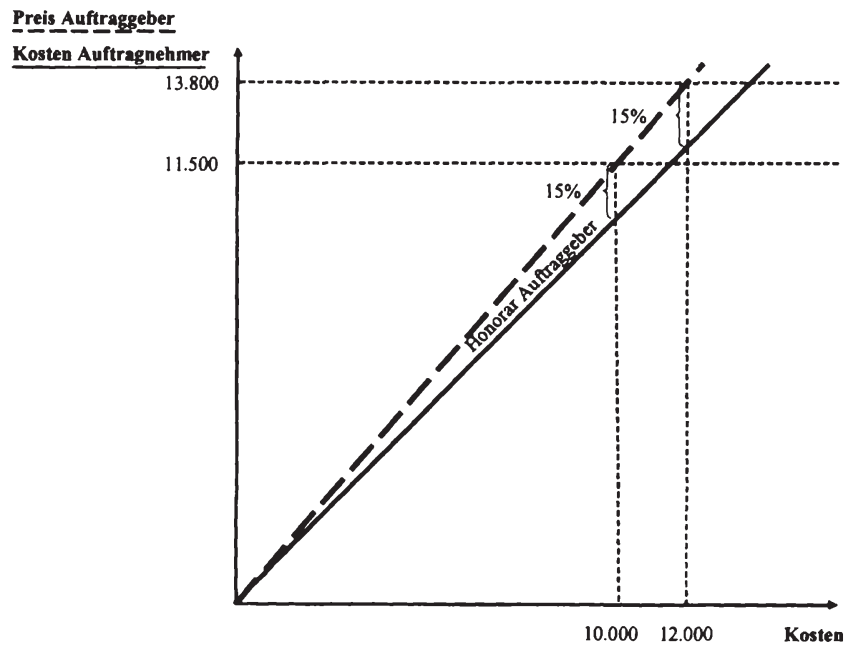

Abbildung 3.11: Kostenerstattung mit prozentualem Honorar (CPPC)

(Quelle: In Anlehnung an Werners/Slaghuis (2004), S. 355.)

Es kann sich jedoch als problematisch erweisen, die mit der Leistungserstellung direkt verbundenen Herstellkosten zu bestimmen. Besonders bei der gleichzeitigen Ausfüh- 
rung von mehreren Projekten unterschiedlicher Auftraggeber lassen sich beispielsweise die Kosten für das Management, die Verwaltung oder die Bereitstellung von Anlagen und Maschinen nur schwer einzelnen Projekten zurechnen. ${ }^{181}$ Es ist im Rahmen der Vertragsvereinbarungen genau zu definieren, welche Kosten der Auftraggeber erstattet und welche Kosten durch das zusätzlich zu zahlende Honorar abgedeckt werden müssen. ${ }^{182}$

Für den Auftraggeber besteht bei einem Kostenerstattungsvertrag die Gefahr, dass der Auftragnehmer zu erstattende Kosten vorweist, die für die Erbringung der Leistung nicht entstanden sind. ${ }^{183}$ Auch besteht die Möglichkeit der Abrechnung projektfremder Kosten zu Lasten des Auftraggebers. Es existiert zudem kein Anreiz für den Auftragnehmer, die vereinbarte Leistung effizient, das heißt mit möglichst geringem Mitteleinsatz zu erbringen. ${ }^{184}$ Dies hat zur Folge, dass dem Auftraggeber hohe Transaktionskosten für die Überwachung der Leistungen des Auftragnehmers entstehen, um beurteilen zu können, ob die in Rechnung gestellten Beträge tatsächlich angefallen sind sowie für die Erstellung der vertraglichen Leistung notwendig waren. ${ }^{185}$

Bei Kostenerstattungsverträgen mit prozentualem Aufschlag erhöht sich die Honorarzahlung und damit der Gewinn des Auftragnehmers mit jeder zusätzlich abgerechneten Einheit entstandener Kosten. ${ }^{186}$ Dieser Anreiz, die Kosten über die notwendigen Kosten hinaus zu steigern, besteht bei einem Kostenerstattungsvertrag mit festem Aufschlag hingegen nicht, da hierbei mit jeder Einheit zusätzlicher Kosten die konstante Honorarzahlung den Anteil des Honorars in Bezug auf die tatsächlichen Kosten prozentual verringert. ${ }^{187}$ Obwohl die Gefahr der Überschreitung geplanter Kosten durch den Auftragnehmer bei beiden hier vorgestellten Kostenerstattungsverträgen besteht, zeigen die Untersuchungen von Sadeh, Dvir und Shenhar, dass diese Form der Zahlungsvereinbarung für die Vertragsparteien auch vorteilhaft sein kann. ${ }^{188}$

\footnotetext{
${ }^{181}$ Vgl. Chapman/Ward (2003), S. 325; Ward/Chapman (1994), S. 218.

$182 \mathrm{Vgl}$. Monczka/Trent/Handfield (2002), S. 490.

${ }^{183}$ Farell (2003), S. 533 bezeichnet den Kostenerstattungsvertrag als verhaltensorientierten Vertrag, da die Kostenerstattungsvereinbarung eine Kontrolle des Verhaltens des Auftragnehmers voraussetzt.

184 Vgl. Bamberg/Coenenberg (2002), S. 173; Turner/Simister (2001), S. 459.

185 Vgl. Gilbreath (1992), S. 53.

186 Gilbreath (1992), S. 54 bezeichnet es als die beste Möglichkeit, diese Vertragsform zu kontrollieren, sie zu vermeiden bzw. nur bei Projekten mit kurzer Laufzeit und niedrigen Kosten einzusetzen.

${ }^{187} \mathrm{Vgl}$. Turner (2003), S. 41; Turner (2004), S. 77f.

$188 \mathrm{Vgl}$. Sadeh/Dvir/Shenhar (2000), S. 20. Die Autoren untersuchen in ihrer empirischen Studie 93 während der letzten 20 Jahre durchgeführte Verteidigungsprojekte hinsichtlich der technologischen Unsicherheit, der Art des Vertragsdesigns sowie unterschiedlicher Erfolgsmerkmale. Ob die Ergebnisse auch auf Projekte des nicht-militărischen Bereichs übertragbar sind, ist aufgrund des untersuchten Anwendungsgebietes fraglich.
} 
Insbesondere bei Investitionsprojekten, die sich durch einen hohen Grad an Prozessunsicherheit auszeichnen, ist die Höhe der für die Leistungserstellung notwendigen Kosten zum Zeitpunkt des Vertragsabschlusses meist nicht bekannt. Die Hauptaufgabe des Auftragnehmers ist hierbei darin zu sehen, die gewünschte Leistung durch die Auswahl und Entwicklung eines geeigneten Produktionsprozesses zu erbringen. Der Auftraggeber ist damit an einer Innovationstätigkeit seines Vertragspartners interessiert und gibt ihm hierfür durch die Erstattung aller entstehenden Kosten die notwendige Flexibilität. $^{189}$

\subsubsection{Festpreisverträge}

Vereinbaren Auftraggeber und Auftragnehmer, dass für die Erstellung einer genau spezifizierten Leistung durch den Auftragnehmer diesem hierfür eine festgesetzte und von den entstandenen Kosten unabhängige Vergütung in bestimmter Höhe durch den Auftraggeber zusteht, handelt es sich um einen Festpreisvertrag. ${ }^{190}$ In der Literatur werden Festpreisverträge als Firm-fixed-price-, Lump-sum- oder Unit-price-contracts bezeichnet. Während die Mehrzahl der Autoren den Begriff Fixed-price-contract gebraucht, ${ }^{191}$ unterscheiden Gilbreath und Widemann den Fixed-price-contract in den Lump-sum- und den Unit-price-contract. ${ }^{192}$ Der Lump-sum-contract entspricht dem Festpreisvertrag mit den oben genannten Eigenschaften, bei dem beispielsweise nach Abschluss des Projektes eine Pauschalsumme an den Auftragnehmer gezahlt wird. Werden Einheitspreise (unit-prices) vereinbart, erhält der Auftragnehmer eine feste Vergütung in Abhängigkeit von der Anzahl fertig gestellter Einheiten, so dass sich die Gesamtzahlung aus dem Produkt von Einheitspreis und Einheiten berechnet. Hierbei besteht jedoch die Problematik, dass der Auftraggeber in der Lage sein muss, während der Projektdauer die Anzahl der Einheiten zu erfassen, die fertig gestellt worden sind. Die Kosten für die Beobachtung und Überwachung der Leistungserstellung sind in diesem Fall höher als bei einem Lump-sum-contract. ${ }^{193}$ Besteht etwa eine Teilleistung

\footnotetext{
${ }^{189}$ Der Begriff der Prozessunsicherheit spiegelt die Neuartigkeit des Projektes wider. Hierbei kann der Auftragnehmer für die Erstellung der Leistung nicht auf bekannte Prozesse zurückgreifen, sondern muss zunăchst ein geeignetes Prozessdesign entwickeln. Hierfur wird der Begriff design-and-build-contract in der Literatur verwendet. Vgl. Turner (2003), S. 20; Turner (2004), S. 80; Turner/Simister (2001), S. 452.

190 Vgl. Turner/Simister (2001), S. 459; Veld/Peeters (1989), S. 156; Ward/Chapman (1995), S. 49.

191 Vgl. Artz/Norman (2002); Chapman/Ward (1994); Crocker/Reynolds (1993); Martin/Webster (1986); Monczka/Trent/Handfield (2002); Sadeh/Dvir/Shenhar (2000); Turner (2004); Turner/Simister (2001); Veld/ Peeters (1989); Ward/Chapman (1994).

192 Vgl. Gilbreath (1992); Widemann (1992).

193 Vgl. Gilbreath (1992), S. 44.
} 
des Gesamtprojektes darin, in einer Produktionshalle eine bestimmte Anzahl neuer Maschinen zu installieren, so kann ein Einheitspreis-Vertrag die Zahlung eines festen Preises im Anschluss an die Installation einer Maschine vorsehen. Dies hat für den Auftragnehmer den Vorteil, auch während der Projektdauer Zahlungen in Form von Fortschrittszahlungen zu erhalten und der Auftraggeber setzt damit gleichzeitig einen Anreiz, fertig gestellte Maschinen frühzeitig aufzustellen und damit die Projektdauer zu verkürzen.

Die Beziehung zwischen den Kosten und dem Honorar des Auftragnehmers, dem zu zahlenden Preis für den Auftraggeber sowie dem Festpreis wird für das in Kapitel 3.3.2 eingeführte Beispiel in Abbildung 3.12 dargestellt:

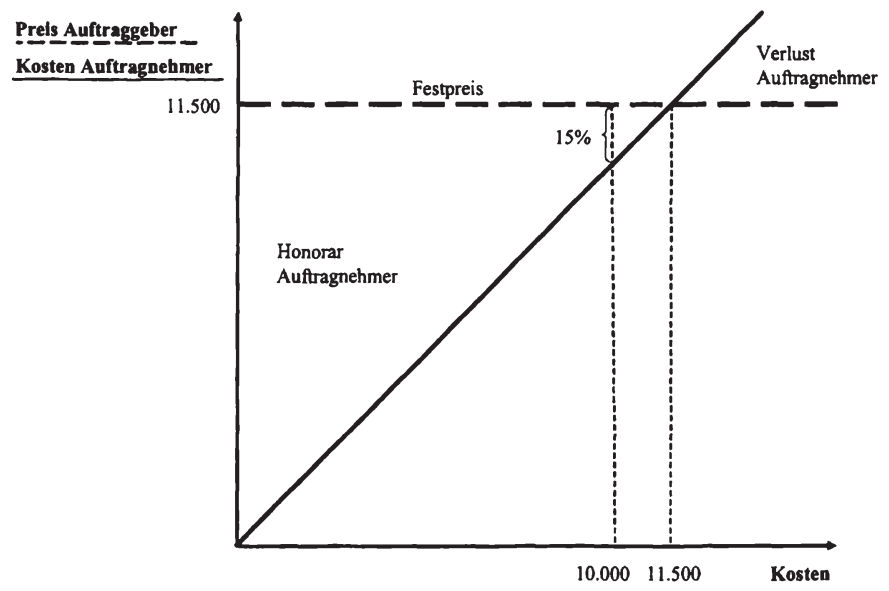

Abbildung 3.12: Zusammenhang von Kosten, Preis und Honorar bei Festpreisverträgen (Quelle: In Anlehnung an Werners/Slaghuis (2004), S. 354.)

Generell sind mit einem Festpreisvertrag bestimmte Risiken und Chancen für den Auftragnehmer und den Auftraggeber verbunden: Übersteigen die für die vertraglich festgeschriebene Leistungserstellung tatsächlich notwendigen Kosten die zum Zeitpunkt der Kalkulation durch den Auftragnehmer vermuteten Kosten, so stellt diese Differenz einen Verlust für den Auftragnehmer dar. Ausschließlich der Auftragnehmer ist in dieser Vertragsbeziehung mit der Unsicherheit der Kostenüberschreitung konfrontiert. ${ }^{194}$ Für die Übernahme dieser Unsicherheit verlangt er daher einen Wagniszuschlag als

\footnotetext{
194 Vgl. Ward/Chapman (1994), S. 217. Hoffken/Schweitzer (1991), S. 22f. betrachten das Festpreisrisiko bei Verträgen über Anlagenbauprojekte.
} 
Teil seines Honorars. Kann der Auftragnehmer die vereinbarte Leistung jedoch zu niedrigeren als den kalkulierten Kosten erstellen, so erhöht diese Differenz das Honorar und damit seinen Gewinn. ${ }^{195}$

Der Auftraggeber trägt bei einem Festpreisvertrag kein Kostenrisiko, er verpflichtet sich lediglich, den vereinbarten, von den entstandenen Kosten unabhängigen Preis an den Auftragnehmer zu zahlen. Existieren für den Auftraggeber keine Möglichkeiten, die Leistungen des Auftragnehmers während der Projektdauer zu kontrollieren (Hidden Action) oder am Ende des Projektes dessen Arbeitseinsatz zu beurteilen (Hidden Information), besteht die Gefahr, dass der Auftragnehmer zur Erhöhung der Differenz zwischen Festpreis und Kosten und damit einer Steigerung seines Gewinns die Kosten durch einen verminderten Arbeitseinsatz in so hohem Ausmaß senkt, dass die Leistungen nicht mehr den vertraglichen Anforderungen entsprechen. Daher wird der Festpreisvertrag in dieser Form als Vertrag mit den stärksten Anreizwirkungen bezeichnet, ohne einen konkreten Anreizmechanismus vorgesehen zu haben. ${ }^{196}$ Insbesondere bei Projektkooperationen, bei denen der Auftraggeber die entstehenden Kosten zum Zeitpunkt des Vertragsabschlusses nicht exakt kalkulieren kann, weil entweder das Produkt durch den Auftraggeber nicht genau spezifiziert werden kann oder die Unsicherheit in dem Prozess der Leistungserstellung begründet ist, wird der Auftragnehmer nur einem Festpreisvertrag zustimmen, wenn er für dieses Risiko entsprechend kompensiert wird und sich damit die Möglichkeit eines Verlustes für ihn reduziert.

Der Abschluss von Festpreisverträgen erscheint daher für solche Projektkooperationen sinnvoll, bei denen der Prozess der Leistungserstellung für den Auftragnehmer bekannt ist und beide Vertragspartner den Vertragsgegenstand eindeutig beschreiben können. Durch die Fähigkeit des Auftragnehmers, die Kosten des Leistungserstellungsprozesses gut schätzen zu können, reduziert sich das Kostenrisiko. Bedingung hierfür ist jedoch, dass während der Projektdurchführung keine durch Änderungswünsche des Auftraggebers resultierenden Prozessmodifikationen unter Beibehaltung des Festpreises notwendig werden.

\footnotetext{
195 Vgl. Ward/Chapman (1995), S. 49.

196 Vgl. Martin/Webster (1986), S. 169; Turner (2004), S. 80. Der Anreiz für die Kostensenkung ist hierbei jedoch meist mit Qualitătseinbußen verbunden, da der Auftragnehmer die Leistungen nicht entsprechend den Zielgrößen des Auftraggebers erbringt. Diese Anreizwirkung ist somit nicht mit den im füften Kapitel durch den Auftraggeber initiierten Anreizwirkungen gleichzusetzen.
} 
Ward und Chapman schlagen für Projektkooperationen, die besonders zu Beginn des Projektes mit Unsicherheit behaftet sind vor, das Gesamtprojekt in mehrere Projektteile zu trennen, wobei zu Beginn eine Vergütung in Form der Kostenerstattung vereinbart wird und im Projektverlauf, sofern eine genauere Spezifikation von Prozess bzw. Produkt möglich ist, zu einem Festpreisvertrag gewechselt wird. ${ }^{197}$

Der von Artz und Norman neben dem starren Festpreisvertrag aufgeführte Festpreisvertrag mit ökonomischer Preisanpassung beruht auf einer Vergütungsvereinbarung, die sich nach festen Verrechnungssätzen für die geleisteten Arbeits- oder Materialeinheiten richtet. ${ }^{198}$ Diese Vertragsform gleicht dem oben aufgefuihrten Einheitspreisvertrag mit den genannten Vor- und Nachteilen.

\subsection{Einbindung von Anreizsystemen in das Vertragsdesign}

\subsubsection{Die Bedeutung von Anreizsystemen für die Projektkooperation}

Ein Anreizsystem kann als Summe unterschiedlicher in einer Unternehmung gewährter Anreize definiert werden. ${ }^{199}$ Ziel dieser Anreizsysteme ist es, das persönliche Verhalten von Individuen so $\mathrm{zu}$ beeinflussen, dass bestimmte Ziele erreicht werden. ${ }^{200}$ Abbildung 3.13 zeigt die Einbindung eines Anreizsystems in einen Leistungserstellungsprozess aus verhaltenswissenschaftlicher Sicht:

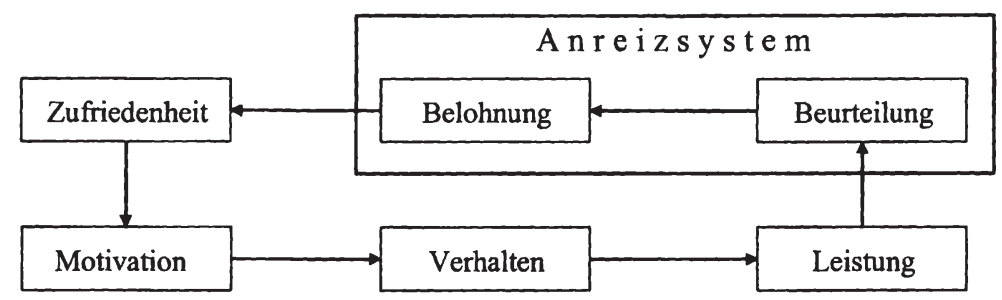

Abbildung 3.13: Das Anreizsystem aus verhaltenswissenschaftlicher Sicht

(Quelle: In Anlehnung an Riegler (2000), S. 30.)

\footnotetext{
197 Vgl. Chapman/Ward (2003), S. 327; Ward/Chapman (1994), S. 218. Dieser Vorschlag findet sich auch bei Madauss (2000), S. 338f.

$198 \mathrm{Vgl}$. Artz/Norman (2002), S. 408.

199 Vgl. Laux (1992), Sp. 112; Welge/Al-Laham (2003), S. 541.

${ }^{200}$ Vgl. Riegler (2000), S. 30; Schanz (1991), S. 8. Die Anreiz-Beitrags-Theorie betrachtet die Wirkung von Anreizen bei Beitritts- und Beitragsentscheidungen von Organisationsteilnehmern und wird den prozessorientierten Motivationstheorien zugeordnet. Vgl. hierzu Staehle (1999), S. 431; Wolf (2003), S. 192; Wolff (1995), S. $11 \mathrm{ff}$.
} 
Durch das Setzen von Anreizen werden die mit persönlichen Zielen verbundenen Motive einer Person aktiviert und somit deren Verhalten beeinflusst. ${ }^{201}$ Verhaltensorientierte Anreizsysteme verwenden als Bezugsgröße das Verhalten bzw. den Arbeitseinsatz und sind daher insbesondere bei komplexen Problemstellungen mit hohen Transaktionskosten der Überwachung bzw. Beurteilung des Verhaltens für die Bestimmung der Belohnung bzw. Bestrafung verbunden. ${ }^{202}$

Ist die Kooperationsbeziehung durch eine asymmetrische Informationsverteilung zwischen den Kooperationspartnern beispielsweise durch das Vorliegen einer Hidden Action-Situation geprägt, bieten ergebnisorientierte Anreizsysteme den Vorteil, dass ein Ergebnis einer Handlung durch die Beteiligten besser beobachtbar und überprüfbar ist. ${ }^{203}$ Anreizarten lassen sich in materielle und immaterielle Anreize unterteilen. $\mathrm{Zu}$ den materiellen Anreizen zählen finanzielle Anreize durch Prämien oder Beteiligungen sowie nicht-finanzielle Anreize, wie beispielsweise Dienstreisen, Dienstwagen, Konsumvorteile oder eine Dienstwohnung. Zu den immateriellen Anreizen zählen etwa die Übernahme zusätzlicher Verantwortung oder ein möglicher Imagegewinn der Unternehmung. ${ }^{204}$

Aus ökonomischer Sicht stehen als Basiselemente eines Anreizsystems die Belohnungs- bzw. die Bestrafungsart, die Bemessungsgrundlage sowie die Anreizfunktion, welche die Beziehung zwischen Anreizart und Bemessungsgrundlage definiert, im Mittelpunkt der Betrachtung. ${ }^{205}$ Als Bemessungsgrundlage sind solche Erfolgskriterien geeignet, anhand derer die Leistung des Kooperationspartners beurteilt werden kann. $^{206}$

Für Anreizsysteme bei Projektkooperationen kommen hier insbesondere quantifizierbare und durch beide Vertragsparteien beobachtbare Größen in Betracht, wie zum Beispiel die Einhaltung von Termin- und/oder Kostenvorgaben. Voraussetzungen für die Wirksamkeit eines Anreizsystems sind die Beeinflussbarkeit der Erfolgsfaktoren durch den Entscheidungsträger, die Verifizierbarkeit der Beurteilungsgröße durch einen un-

\footnotetext{
201 Vgl. Jost (2000), S. 79f.; Weinert (1992), Sp. 123. Meyer (2002), S. 182f. stellt als Ergebnis seiner Arbeit fest, dass es aus ökonomischer Sicht nicht Ziel eines Anreizsystems ist, das Verhalten direkt zu beeinflussen, sondern durch wechselseitige Verhaltenserwartungen das Interaktionsproblem zu lösen.

202 Vgl. Harris/Raviv (1979), S. 232.

203 Vgl. Bosse (2000), S. 233.

204 Vgl. Bosse (2000), S. 233f.; Petersen (1989), S. 4f.; Welge/Al-Laham (2003), S. 542.

205 Vgl. Laux (1992), Sp. 113f.; Laux (1999), S. 25ff.; Riegler (2000), S. 31.

206 Vgl. Riegler (2000), S. 32. Für Erfolgskriterien in Anreizverträgen vgl. etwa Baker (2000); Baker/Gibbons/Murphy (1994). Farell (2003), S. 553 bezeichnet Anreizvertrăge als ergebnisorientierte Vertrăge.
} 
beteiligten Dritten sowie der in zeitlicher Nähe zu der Aktivität liegende Zeitpunkt der Ermittlung der Belohnung bzw. Bestrafung. ${ }^{207}$

Die Bestimmung der optimalen Prämienfunktion eines Anreizsystems stellt ein Entscheidungsproblem dar, welches ausführlich in der wissenschaftlichen Literatur diskutiert wird. Diese vorwiegend theoretischen und auf allgemeingültigen Modellen basierenden Untersuchungen für Entscheidungsträger mit unterschiedlichen Risikoeinstellungen und asymmetrisch verteilter Information zeigen den Zusammenhang zwischen Erfolg, Anreiz, Kontrolle, Belohnung/Bestrafung, Motivation und Risiko auf. ${ }^{208}$ Allgemein kann festgestellt werden, dass ein Entscheidungsträger eine umso höhere Prämie für die Übernahme des Risikos verlangt, je höher das Erfolgs- und somit das Verlustrisiko ist. ${ }^{209}$ Die Motivation, die Erfolgssituation zu verbessern ist umso höher, je stärker die Belohnung bzw. Bestrafung mit dem Erfolg variiert. Daraus folgt, dass der Preis des Anreizsystems in Form der zu zahlenden Risikoprämie umso höher ist, je höher das Risiko in Form der Varianz des Ergebnisses ist. ${ }^{210}$ Die Wahl der Anreizfunktion kann damit als ein Trade-Off zwischen Anreizwirkung und der damit verbundenen Kosten sowie der Aufteilung des mit der Leistungserstellung verbundenen Risikos auf die Kooperationspartner dargestellt werden.

Im Anschluss an diese allgemeinen Ausfuhrungen mit dem Ziel der Einordnung von Anreizsystemen in die ökonomische Theorie sollen im Folgenden für die in Kapitel 3.3.2 und 3.3.3 vorgestellten Kostenerstattungs- und Festpreisverträge beispielhaft Möglichkeiten aufgezeigt werden, wie sich diese durch die Einbindung von Anreizsystemen modifizieren lassen.

Der Festpreisvertrag und der Kostenerstattungsvertrag mit den in den vorigen Kapiteln dargestellten Charakteristika bilden zwei Extremfälle eines möglichen Vertragsdesigns. Bei beiden Vertragsarten trägt entweder der Auftragnehmer (Festpreisvertrag)

\footnotetext{
207 Vgl. Laux (1999), S. 29f.; Riegler (2000), S. 36f. Die Motivationswirkung eines Anreizsystems sinkt, je groBer das Zeitintervall zwischen einer Aktivität und der Gewährung einer Belohnung ist. Gleichzeitig sinkt die Transparenz der Belohnung, da die Akteure dazu neigen, die Belohnung mit gegenwärtig geleisteten Aktivităten zu verbinden. Vgl. hierzu Riegler (2000), S. 37. Zu Anforderungen an Anreizsysteme vgl. auch Bosse (2000), S. 238ff. Anforderungen an Anreizsysteme bei strategischen Entscheidungen sind in Guthof (1995), S. 36ff. aufgefuhrt.

208 Vgl. Bamberg (1991); Jost (2001); Laffont/Martimort (2002); Laux (1992); Laux (1999); Laux/SchenkMathes (1992); Macho-Stadler/Pérez-Castrillo (2001); Petersen (1989); Theilen (1996).

209 Es wird hicrbei unterstellt, dass eine Bestrafung in Form einer negativen Belohnung möglich ist, so dass der Entscheidungstrăger auch an schlechten Ergebnissen partizipiert.

210 Vgl. Laux (1992), Sp. 121.
} 
oder der Auftraggeber (Kostenerstattungsvertrag) vollständig das Kostenrisiko. Hierdurch bedingt werden mit diesen Vertragsdesigns keine Anreize gesetzt, die vereinbarte Leistung effizient sowie unter Beachtung bestimmter Anforderungskriterien, wie zum Beispiel der Einhaltung von Qualitäts- und Sicherheitsstandards, zu erbringen. Wird ein Festpreisvertrag vereinbart, hat ausschließlich der Auftragnehmer einen Anreiz zu einer Kostensenkung. Er wird diese jedoch bei fehlender Kontrollmöglichkeit durch den Auftraggeber und unter der Annahme opportunistischen Verhaltens nicht durch Innovationstätigkeit, sondern zu Lasten der Leistungsqualität erzielen. Hier sind demnach Anreize zu setzen, die den Auftragnehmer veranlassen, von Kosteneinsparungen durch Innovationstätigkeit zu profitieren und gleichzeitig die Leistung mit den vereinbarten Eigenschaften zu erstellen.

\subsubsection{Aufteilung von Projektrisiken durch Verträge mit Anreizsystemen}

Für die Wahl eines Anreizsystems ist zunächst die Festlegung der Bemessungsgrundlage für die Belohnung bzw. Bestrafung durchzuführen. ${ }^{211}$ Für Vertragsdesigns des Projektmanagements werden als Bemessungsgrundlagen Kosten-, Zeit-, Qualitäts-, Sicherheits- sowie Ressourceneinsatz-Kriterien vorgeschlagen. ${ }^{212}$ Die Mehrzahl der untersuchten Literatur im Bereich des Contracting verwendet die Projektkosten als Grundlage der Anreizfunktion und erklärt dies damit, dass die Kosten im Gegensatz zu Qualität oder Sicherheit ein für beide Projektpartner sowie unbeteiligte Dritte kontrollierbares Kriterium darstellen. ${ }^{213}$ Diese Annahme ist besonders für die in der Arbeit untersuchten Investitionsprojekte sowie unter Beachtung der zwischen den Projektpartnern vorhandenen Informationsasymmetrie problematisch. Daher wird im fünften Kapitel die Projektdauer, welche für die Kooperationsteilnehmer beobachtbar ist, als Bemessungsgrundlage für die Bestimmung der Anreizzahlungen verwendet.

Ein zweiter Gesichtspunkt ist der Zusammenhang zwischen der Wahl des Vertragsdesigns und der Auswahl der Projektpartner durch den Auftraggeber. Obwohl es sich hierbei um ein Entscheidungsproblem bei Mehrfachzielsetzung handelt, bei dem der Auftraggeber neben der Auswahl des Projektpartners mit den geringsten erwarteten

\footnotetext{
211 Im Folgenden wird der Begriff der Anreizfunktion verwendet, welcher neben einer Belohnung auch eine Bestrafung bei der Realisation eines schlechten Ergebnisses etwa als Minderung des Gewinns beinhaltet.

${ }^{212}$ Vgl. Berends (2000), S. 167; Bubshait (2003), S. 63.

${ }^{213}$ Vgl. beispielsweise Al-Subhi Al-Harbi (1998); Berends (2000); Chapman/Ward (1994); Raghu/Sen/Rao (2003); Ward/Chapman (1994); Ward/Chapman (1995).
} 
Kosten zusätzlich weitere Zielkriterien, wie bisherige Erfahrungen, Qualität früherer Leistungen oder die Beachtung von Sicherheitsvorschriften berücksichtigen sollte, stehen die in dem Angebot des Auftragnehmers an den Auftraggeber gegebenen kalkulierten Kosten im Vordergrund. Shr und Chen betrachten die Ermittlung eines Angebotes aus der Sicht des Auftragnehmers. ${ }^{214}$ Dieses Angebot setzt sich aus den kalkulierten Kosten und damit dem Preis für die Leistungserstellung sowie der kalkulierten Projektdauer zusammen. Bei Einbeziehung von Anreizsystemen während der Angebotsermittlung kann der Auftragnehmer ein Angebot mit einem niedrigen Preis abgeben und damit die Wahrscheinlichkeit erhöhen, als Projektpartner ausgewählt zu werden. Enthält das Angebot zusätzlich eine Projektdauer, die länger als die kalkulierte Projektdauer ist, kann der Auftragnehmer bei frühzeitigem Projektende durch die vorgesehene Gewährung einer Bonuszahlung durch den Auftraggeber seine Einzahlungen erhöhen und somit den Nachteil des niedrigen Preises kompensieren. ${ }^{215}$ Voraussetzung hierfür ist jedoch eine die erwartete Projektdauer betreffende Informationsasymmetrie zwischen den Kooperationspartnern.

Die Einbindung von Anreizsystemen in die betriebswirtschaftliche Vertragstheorie geht auf Scherer zurück, ${ }^{216}$ der als Anwendung den Kauf militärischer Ausrüstung durch den Staat betrachtet. ${ }^{217}$ Um Vertragsdesigns zu schaffen, die eine Aufteilung von Risiken zwischen den Projektpartnern ermöglichen, schlägt Scherer eine lineare Anreizfunktion vor, nach der die Differenz zwischen den tatsächlichen Kosten (Istkosten $\mathrm{K}_{\text {Ist }}$ ) und den kalkulierten Kosten (Plankosten $\mathrm{K}_{\text {Plan }}$ ) Auswirkungen auf die Zahlungen und damit den Kapitalwert des Auftraggebers sowie auf die Erlöse des Auftragnehmers hat. ${ }^{218}$ Aus der Sicht des Auftragnehmers kann dies in Anlehnung an Scherer formal durch die folgende Gleichung beschrieben werden: ${ }^{219}$

$\mathrm{G}_{\mathrm{ges}}^{\mathrm{AN}}=\mathrm{G}^{\mathrm{AN}}+\mathrm{ANTK} \cdot\left(\mathrm{K}_{\mathrm{Plan}}-\mathrm{K}_{\mathrm{Ist}}\right)$

\footnotetext{
${ }^{214} \mathrm{Vgl}$. Shr/Chen (2003). Für eine Angebotskalkulation im Anlagenbau vgl. Hoffken/Schweitzer (1991), S. $50 \mathrm{f}$.

${ }^{215}$ Die Untersuchungen von Shr/Chen (2003) beziehen sich auf eine Anwendung des Straßenbaus. Der Zusammenhang zwischen Baukosten und Bauzeit wird durch Daten des Florida Department of Transportation (FDOT) ermittelt. Als Anreizfunktionen werden sowohl lineare als auch nicht-lineare Funktionen in Abhängigkeit von dem fruhzeitigen Projektende verwendet.

216 Vgl. Scherer (1964).

217 Anreizverträge zwischen Regierung und Auftragnehmern werden auch von Canes (1975); Cummins (1977); Mc Call (1970) betrachtet.

${ }^{218}$ Es wird beispielhaft eine Anreizfunktion vorgestellt, bei der die Kosten als Bemessungsgrundlage verwendet werden. Diese Form der Anreizfunktion kann auch auf alternative Bemessungsgrundlagen ubertragen werden.

${ }^{219}$ Vgl. Scherer (1964), S. 259.
} 
Der tatsächliche Gewinn des Auftragnehmers $G_{\text {ges }}^{\text {AN }}$ bestimmt sich durch die bei Vertragsabschluss festgelegte Gewinnprämie $G^{A N}$ zuzüglich der mit dem Faktor ANTK zwischen Auftraggeber und Auftragnehmer aufgeteilten Differenz aus Ist- und Plankosten, wobei ANTK den Teilungsfaktor aus der Sicht des Auftragnehmers angibt $(0 \leq \mathrm{ANTK} \leq 1)$.

Der Faktor ANTK $=0$ entspricht einem Kostenerstattungsvertrag mit festem Honorar, der Faktor ANTK = 1 einem Festpreisvertrag. Damit können diese zwei Vertragsformen als Sonderfälle des Anreizvertrags dargestellt werden. ${ }^{220}$ Wird beispielsweise ein Teilungsfaktor in Höhe von 0,4 vereinbart und übersteigen/unterschreiten die Istkosten die Plankosten um $100 \mathrm{GE}$, vermindert/erhöht sich der vereinbarte Gewinn des Auftragnehmers um 40 GE. Abbildung 3.14 zeigt am Beispiel eines Anreiz-Festpreisvertrags den Zusammenhang zwischen den Projektkosten und der Zahlung des Auftraggebers auf. ${ }^{221}$ Wie in Kapitel 3.3 wird angenommen, dass die Plankosten mit 10.000 GE kalkuliert werden und ein fester Gewinnzuschlag von $15 \%$ vereinbart wird, so dass der Festpreis 11.500 GE beträgt. Als Teilungsfaktor wird ANTK =0,4 sowie ein maximaler Festpreis von 12.500 GE festgelegt. Ferner wird angenommen, dass eine bestimmte Festpreisuntergrenze nicht unterschritten wird.

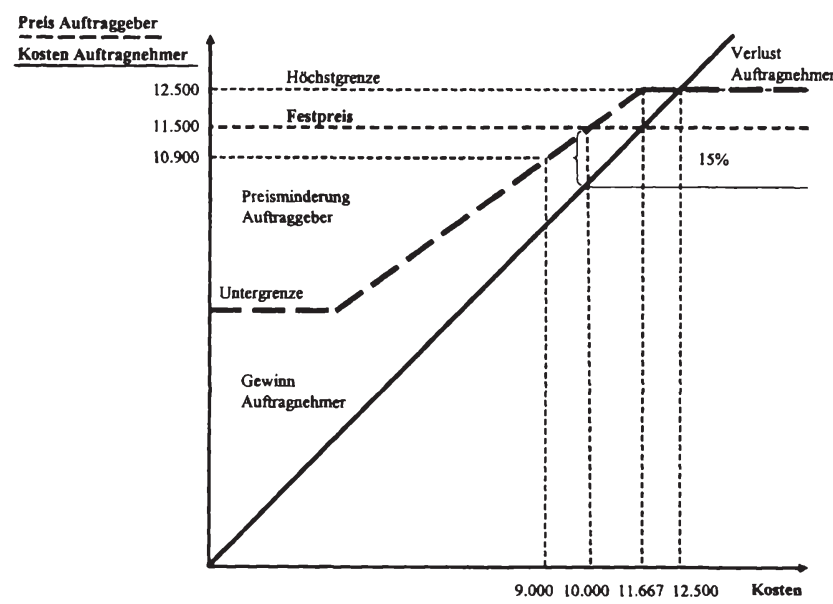

Abbildung 3.14: Festpreisvereinbarung mit Anreizsystem (Quelle: In Anlehnung an Werners/Slaghuis (2004), S. 355.)

${ }^{220}$ Vgl. Al-Subhi Al-Harbi (1998), S. 73.

${ }^{221}$ Für eine ăhnliche Darstellung vgl. Berends (2000), S. 167; Ward/Chapman (1995), S. 52f. 
Übersteigen die Kosten den Wert von $12.500 \mathrm{GE}$, führt diese Differenz zu einem Verlust für den Auftragnehmer. Gelingt es dem Auftragnehmer, die Kosten zu senken, so dass diese niedriger als 10.000 GE sind, erhöht sich mit jeder eingesparten GE der Gewinn bis zum Erreichen der Untergrenze um 0,4 GE. Der durch den Auftraggeber zu zahlende Festpreis reduziert sich entsprechend um 0,6 GE. Dieses Vorgehen kann auf einen Kostenerstattungsvertrag übertragen werden, wobei hierbei jedoch keine Höchstgrenze existiert und damit kein Verlustrisiko für den Auftragnehmer besteht. ${ }^{222}$

Die Bestimmung der Teilungsrate zwischen den Projektpartnern ist von unterschiedlichen Faktoren abhängig. Wird unterstellt, wie dies bereits in den Ausführungen zur Prinzipal-Agenten-Theorie angenommen wurde, dass das Ergebnis der Arbeitsleistung nicht nur von dem gewählten Arbeitseinsatz des Auftragnehmers, sondern auch von zufällig eintretenden Umweltzuständen beeinflusst wird, kann dies auf die Kosten übertragen werden. Zum Zeitpunkt des Vertragsabschlusses, zu dem die Bestimmung der Teilungsrate erfolgt, können die kalkulierten Kosten und damit der Preis nur als Zufallsvariablen abgebildet und, sofern Wahrscheinlichkeiten bezüglich des Eintritts möglicher Umweltlagen bekannt sind, Erwartungswerte berechnet werden. ${ }^{223}$

Bei der Festlegung des Teilungsfaktors ist zusätzlich die Einstellung der Entscheidungsträger gegenüber unsicheren Ergebnissen zu berücksichtigen. Es wird vielfach unterstellt, dass der Auftraggeber risikoneutral und der Auftragnehmer risikoavers eingestellt ist. ${ }^{224}$ Dies kann damit begründet werden, dass der Projekteigentümer ein Projektportfolio besitzt und damit der Effekt einer Risikodiversifikation möglich ist, während der Auftragnehmer insbesondere bei den hier betrachteten Investitionsprojekten aufgrund einer geringeren Beschäftigung einen großen Verlust überproportional und große Gewinne unterproportional hoch bewertet. ${ }^{225}$ Die Risikoeinstellung eines Entscheiders lässt sich unter Anwendung des Bernoulli-Prinzips mit Hilfe seiner Nutzenfunktion abbilden. Demnach existiert eine Nutzenfunktion, die jedem möglichen Ergebnis einen Nutzen zuordnet, so dass der Entscheider die Handlungsalternativen nach

\footnotetext{
222 Vgl. Scherer (1964), S. 258f., Fußnote 4.

${ }^{223}$ Vgl. Carr (1977), S. 154; Gandhi (1979), S. 374f. Für die Abbildung unsicherer Vorgangskosten geben Chapman/Ward (1994), S. 538f. eine Verteilungsfunktion der Projektkosten an. Für eine Anwendung aus dem Bereich der Landwirtschaft vgl. Allen/Lueck (1999).

${ }^{224}$ Vgl. Raghu/Sen/Rao (2003), S. 161; Jost (2001), S. 22.

${ }^{225}$ Dies entspricht auch den im Zusammenhang mit der Prinzipal-Agenten-Theorie in Kapitel 3.3.2 getroffenen Annahmen. Zu Risikoeinstellungen von Entscheidungsträgern vgl. beispielsweise Bamberg/Coenenberg (2002), S. 92ff.; Laux (2003), S. 180ff.
} 
dem Kriterium des Nutzenerwartungswertes beurteilen kann. ${ }^{226}$ Ein Vorgehen zur Ermittlung der Nutzenfunktion eines sich in der Phase der Vertragsverhandlungen befindenden Auftragnehmers wird von Willenbrock beschrieben. ${ }^{227}$ Das Ergebnis seiner Untersuchungen, welches sich mit den Ausführungen von Carr deckt, ist, dass die Nutzenfunktion eines Auftragnehmers von unterschiedlicher Gestalt sein kann, wobei der Verlauf der Nutzenfunktion von der Höhe des aktuellen verfügbaren Kapitals sowie der Schwankung seiner finanziellen Situation abhängt. ${ }^{228}$

Aus der Sicht des Auftragnehmers ergibt sich somit das folgende Entscheidungsproblem: Gibt der Auftragnehmer im Anschluss an eine Projektausschreibung ein Angebot $\mathrm{ab}$, so wird er für ein vertragliches Anreizsystem zusätzlich zu den kalkulierten Gesamtkosten sowie der erwarteten Projektdauer die für ihn optimale Teilungsrate bestimmen. Ein hoher Teilungsfaktor gibt ihm einerseits die Möglichkeit, in hohem Ausmaß an Kosteneinsparungen zu partizipieren und damit den Gewinn zu erhöhen, bedeutet jedoch andererseits auch die Übernahme eines höheren Risikos bei Überschreitung der kalkulierten Kosten. Legt der Auftragnehmer das mit der Projektdurchführung verbundene Risiko für seine Entscheidung zugrunde, wird er bei einem hohen Risiko einen Kostenerstattungsvertrag ( ANTK $=0$ ) bevorzugen, da hierbei eine Überschreitung der geplanten Kosten keine Auswirkung auf seinen Gewinn hat. ${ }^{229}$ Die Untersuchungen von Al-Subhi Al-Harbi zeigen unter der Annahme dieses Verlaufs der Nutzenfunktion, dass der Auftragnehmer seinen erwarteten Nutzen für den Fall einer erwarteten Unterschreitung der geplanten Kosten mit einem Festpreisvertrag $($ ANTK $=1)$ maximiert. ${ }^{230}$

Schlägt der Auftraggeber dem Auftragnehmer als Projektpartner einen Vertrag vor, wie es in dieser Arbeit betrachtet wird, so muss er insbesondere bei mit hoher Unsi-

\footnotetext{
${ }^{226}$ Zum Bernoulli-Prinzip sowie Annahmen und Kritik vgl. etwa Bamberg/Coenenberg (2002), S. 81ff.; Eisenführ/Weber (2003), S. 208ff.; Laux (2003), S. 171ff.; Saliger (2003), S. $45 \mathrm{ff}$.

227 Vgl. Willenbrock (1973).

${ }^{228}$ Vgl. Carr (1977), S. 158. Ein Auftragnehmer in einer stabilen Finanzsituation bzw. mit hohen Finanzreserven bewertet einen geringen Verlust mit einem hoheren Nutzen als ein Auftragnehmer, fur den mit diesem Verlust etwa die Aufgabe der Geschäftstătigkeit verbunden ist. Al-Subhi Al-Harbi (1998), S. 79 legt für seine Untersuchungen eine in drei Bereiche unterteilte Nutzenfunktion zugrunde, nach der der Auftragnehmer gegenuber geringen erwarteten Verlusten risikoneutral, erwarteten Gewinnen risikofreudig und hohen erwarteten Verlusten risikoavers eingestellt ist.

229 Levitt/Ashley/Logcher (1980), S. 299 unterscheiden kontrollierbare und unkontrollierbare Risiken und bemerken, dass ein Anreizsystem nur Einfluss auf kontrollierbare Risiken haben kann. Entscheidungstheoretisch bedeutet dies, dass durch das Setzen von Anreizen bspw. Anstrengungen unternommen werden, den Informationsstand zu verbessern und somit neue Informationen uber das Eintreten möglicher Umweltlagen zu erlangen.

${ }^{230}$ Vgl. Al-Subhi Al-Harbi (1998), S. 78.
} 
cherheit behafteten Projekten abwägen, welches Vertragsdesign seinen erwarteten Nutzen maximiert. Zunächst erscheint die Vorgabe eines Festpreisvertrags sinnvoll, um die zu erstattenden Kosten zu begrenzen und das Kostenrisiko somit auf den Auftragnehmer zu übertragen. Hierbei wird der Auftragnehmer jedoch für das übernommene Risiko eine Prämie und damit einen höheren Preis verlangen. ${ }^{231}$ Zusätzlich besteht die Gefahr, dass er bei einer erwarteten Kostenüberschreitung seine Arbeitsanstrengungen senkt und damit die Qualität der Leistungen sinkt bzw. die Projektdauer steigt, woraus eine Minderung des erwarteten Erlöses aus der Nutzung des Projektes für den Auftraggeber folgt. Durch die Vereinbarung eines Kostenerstattungsvertrags gewährt er jedoch dem Auftragnehmer auch bei Überschreitung der geplanten Kosten die Flexibilität, das Projekt mit Erfolg abzuschließen. Hierbei ist die erwartete Minderung des Nutzens durch die Zahlung des höheren Preises mit dem erwarteten Nutzen aus den Projekterlösen gegenüber zu stellen.

Sieht er zusätzlich eine Aufteilung des Risikos durch ein Anreizsystem vor, so hat die Festlegung eines hohen Teilungsfaktors zwei Effekte: Zum einen wird der Auftragnehmer entsprechend seiner Risikoeinstellung eine Anpassung seiner Risikoprämie fordern, was einer Preiserhöhung entspricht, zum anderen besteht ein Anreiz für den Auftragnehmer, die Differenz aus kalkulierten und tatsächlichen Kosten zu minimieren, da diese mit der Teilungsrate multiplizierte Differenz eine Minderung seines Gewinns bewirkt. Für den Auftraggeber wird es jedoch kaum möglich sein, eine funktionale Beziehung zwischen dem bei einem bestimmten (Anreiz-)Vertragsdesign gewählten Anstrengungsniveau des Projektpartners und den von der Qualität der Projektleistung abhängigen Erlösen herzustellen.

Diese Überlegungen zeigen, dass eine allgemeine Aussage zu der Entscheidungsproblematik, welcher der Projektpartner welches Risiko bei Vereinbarung bestimmter Vertragsdesigns in welchem Ausmaß tragen sollte, nicht möglich ist. ${ }^{232}$ Auch der Hinweis, dass derjenige Projektpartner das Kostenrisiko übernehmen sollte, der am besten in der Lage ist, das Risikomanagement zu übernehmen, ${ }^{233}$ beantwortet die in diesem Kapitel

\footnotetext{
${ }^{231}$ Vgl. Ward/Chapman (1994), S. 219. Ward/Chapman (1994), S. 217 weisen darauf hin, dass die Festpreisvereinbarung in diesem Fall nicht generell zu einem maximalen Nutzen für den Auftraggeber führt. Die Begrundung erfolgt hier durch die Annahme unterschiedlicher Grade der Risikoaversion und somit durch die Hobhe der Kosten der Übernahme des Risikos durch die Vertragsparteien.

232 Vgl. Al-Subhi Al-Harbi (1998), S. 74; Martin/Webster (1986), S. 167; Widemann (1992), S. IX-2. Hier wird furr die unterschiedlichen Vertragsdesigns das Risiko für Auftraggeber und Auftragnehmer dargestellt.

233 Vgl. Berends (2000), S. 165.
} 
aufgezeigte Problematik nur unzureichend. Die Abbildung 3.15 zeigt zusammenfassend solche Faktoren auf, welche eine Aufteilung von Projektrisiken auf die Kooperationspartner beeinflussen:

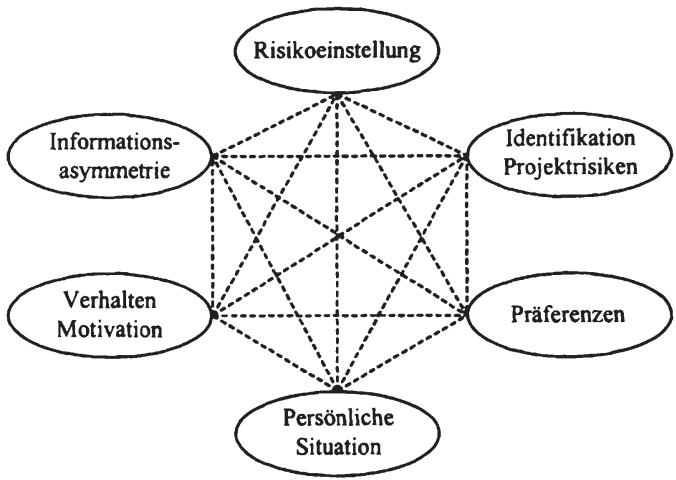

Abbildung 3.15: Einflussfaktoren auf die Aufteilung von Projektrisiken

Beispielsweise wird das durch die Motivation geprägte Verhalten eines Auftragnehmers durch seine aktuelle persönliche Situation sowie durch seine Präferenzen und Risikoeinstellung beeinflusst. Der Begriff der Informationsasymmetrie kann hierbei aus zwei Blickwinkeln interpretiert werden. Zum einen führt der Zustand der Informationsasymmetrie dazu, dass sich der Auftragnehmer aufgrund seiner Präferenzen sowie seiner persönlichen Situation opportunistisch verhält und somit die mangelnde Informiertheit des Auftraggebers ausnutzt. Zum anderen ist die Informationsasymmetrie Ursache für einen Teil der Kooperationsrisiken, deren Auswirkungen auf die Projektkooperation durch die individuelle Risikoeinstellung der Projektpartner beeinflusst wird. Die durch die Verbindungen visualisierten gegenseitigen Abhängigkeiten aller Faktoren deuten die hier vorliegende komplexe Problemstellung an, bei der sowohl verhaltenstheoretische, organisationstheoretische als auch entscheidungstheoretische Aspekte Beachtung finden müssen. Die in diesem Kapitel aufgezeigten Zusammenhänge und Probleme bei der Aufteilung von Projektrisiken durch das Design von Anreizverträgen runden das dritte Kapitel $a b$ und werden im fünften Kapitel im Rahmen der Integration von Vertragsdesigns in die quantitative Projektplanung des Auftraggebers erneut aufgegriffen.

Eine zeitgerechte Ermittlung der tatsächlich entstandenen Kosten sowie eine Feststellung der Abweichung dieser von den kalkulierten Kosten sind insbesondere dann für 
den Auftraggeber kaum möglich, wenn eine Hidden Action Situation vorliegt. Auch die Beurteilung der Qualität der erbrachten Leistungen zum Zeitpunkt des Projektabschlusses kann sich aus der Sicht des Auftraggebers als problematisch erweisen, wenn dieser die Leistungen des Auftragnehmers nicht beobachten oder aufgrund fehlender Informationen nicht kontrollieren kann. Daher wird im fünften Kapitel vorgeschlagen, den Zeitpunkt des Projektabschlusses, welcher durch beide Kooperationspartner leicht identifizierbar ist, als Bemessungsgrundlage eines Anreizsystems $\mathrm{zu}$ verwenden. Durch diese Bemessungsgrundlage kann der Auftragnehmer jedoch nicht motiviert werden, die Leistungserstellung zu niedrigen Kosten zu erbringen. Vielmehr wird er versuchen, durch einen mit höheren Kosten verbundenen Arbeitseinsatz ein früheres Projektende zu realisieren, um seinen Gewinn durch den hierfür gezahlten Bonus zu erhöhen.

Zusammenfassend kann festgestellt werden, dass der im Rahmen des Projektmanagements vielfach vernachlässigte Prozess des Vertragsmanagements großen Einfluss auf den Erfolg eines Projektes haben kann. Durch die Auswahl eines Vertragsdesigns, welches sowohl die spezifischen Projekt- als auch die individuellen Kooperationscharakteristika berücksichtigt, erhält der Auftraggeber die Chance, den Erfolg seines Projektes aktiv zu beeinflussen. Die Entscheidung für ein Vertragsdesign hat Auswirkungen auf das Verhalten der Kooperationspartner und wirkt durch die Festlegung der Zahlungsvereinbarungen auf die Zielsetzung der Kapitalwertmaximierung ein. Die vorwiegend durch die hierarchische Organisationsstruktur der AuftraggeberAuftragnehmer-Beziehung begründete Informationsasymmetrie zwischen den Kooperationspartnern führt zu der Notwendigkeit, Anreizsysteme in das Vertragsdesign zu integrieren. In Abhängigkeit von den Zielkriterien des Auftraggebers sowie der bestehenden Form asymmetrischer Information kann der Auftraggeber durch die Wahl einer geeigneten Bemessungsgrundlage sowie die Festlegung des Teilungsfaktors eine Anreizfunktion bestimmen, welche den Auftragnehmer motiviert, sich entsprechend den Präferenzen des Auftraggebers zu verhalten.

Die in diesem Kapitel aufgezeigten und aus der Sicht des Auftraggebers für die Planung und Durchführung der Kooperation mit dem Auftragnehmer notwendigen Teilnahme- und Anreizkompatibilitätsbedingung werden im fünften Kapitel sowohl für Kostenerstattungs- als auch für Festpreisverträge als Bestandteil der quantitativen Projektplanung, welche im Mittelpunkt des folgenden Kapitels steht, angewandt. 


\section{Quantitative Planungsunterstützung für das Management von Projektkooperationen}

Für eine Unterstützung des Entscheidungsträgers bei der Projektplanung mit der Zielsetzung der Kapitalwertmaximierung existieren verschiedene Ansätze mathematischer Modellformulierungen zur Abbildung unterschiedlicher Problemstellungen. Ziel dieses Kapitels ist daher eine vergleichende Analyse und Diskussion dieser Ansätze im Hinblick auf die in dieser Arbeit untersuchte Kooperationsbeziehung zwischen einem Auftraggeber und einem Auftragnehmer.

Ausgehend von der im zweiten Kapitel festgestellten Eignung des Kapitalwertes als Zielkriterium für das Projektmanagement erfolgt in Kapitel 4.1 eine weiterführende Diskussion der mit diesem Kriterium verbundenen Merkmale. Die Analyse grundlegender sowie aktueller wissenschaftlicher Beiträge auf dem Gebiet der Projektplanung bei Kapitalwertmaximierung zeigt, dass sich zwei Problemstellungen differenzieren lassen: das Project Scheduling Problem und das Payment Scheduling Problem.

Das Kapitel 4.2 betrachtet daher zunächst die Project Scheduling Problematik und zeigt neben der chronologischen Entwicklung den aktuellen Stand der Forschung auf. Hierbei stehen die verschiedenen Modellierungsansätze zur Berücksichtigung relevanter Problemcharakteristika im Vordergrund. Abschließend wird ein mathematisches Modell zur Abbildung des Project Scheduling Problems vorgestellt.

Das Payment Scheduling Problem kann als Erweiterung des Project Scheduling Problems betrachtet werden. Die Untersuchung der bisher geringen Anzahl wissenschaftlicher Beiträge des Payment Schedulings zeigt, dass durch die Modellierung der durch den Auftraggeber zu leistenden Projektzahlungen als Entscheidungsvariablen eine realitätsnahe Abbildung der Beziehung zwischen Auftraggeber und Auftragnehmer möglich wird, indem das Verhandlungsproblem in den Vordergrund rückt. Daher stehen auch in Kapitel 4.3 die unterschiedlichen in den Modellen berücksichtigten Problemcharakteristika im Fokus der Untersuchungen. Schließlich wird ein Payment Scheduling Modell vorgestellt, auf dessen Grundlage die weiterführenden Untersuchungen im Rahmen der Verbindung von Contract Management und quantitativer Projektplanung durchgeführt werden.

Die Anwendung beider Modelle anhand eines konkreten Beispiels erfolgt in Kapitel 4.4. Eine kritische Würdigung schließt dieses Kapitel ab. 


\subsection{Quantitative Methoden der Projektplanung bei Kapitalwert- maximierung}

Die Ziele des Projektmanagements lassen sich in Form des in Kapitel 2.1.2 aufgeführten magischen Dreiecks verdeutlichen, welches aus den Teilzielen Kosten, Zeit und Leistung bzw. Qualität besteht. ${ }^{1}$ Gardiner und Stewart stellen fest, dass eine Einhaltung von Budget-, Zeit- und Kostenvorgaben bei Problemstellungen der Realität aufgrund nicht geplanter Störungen des Projektfortschrittes nur selten möglich ist und somit diese Kriterien für eine Beurteilung des Projekterfolgs unzureichend geeignet sind. $^{2}$ Es wird daher vorgeschlagen, den Kapitalwert als Planungs-, Steuerungs- und Erfolgsgröße für das Projektmanagement heranzuziehen. ${ }^{3}$ Auch Drews gelangt zu der Schlussfolgerung, dass der Kapitalwert als in der Praxis weit verbreitetes und gegenüber alternativen, insbesondere statischen Verfahren der Investitionsrechnung überlegenes Verfahren anerkannt ist. ${ }^{4}$ Gleichzeitig erfüllt das Kapitalwertverfahren die von Drews aufgestellten Anforderungen an eine kooperationsbezogene Investitionsrechnung und erscheint damit auch für die dieser Arbeit zugrunde liegende Problemstruktur anwendbar. 5

Als Schwächen des Kapitalwertverfahrens werden die realitätsfremde Annahme eines vollkommenen Kapitalmarktes ${ }^{6}$ sowie die Probleme der Bestimmung geeigneter Diskontierungsfaktoren aufgeführt. ${ }^{7}$ Schmidt und Terberger diskutieren diese Annahmen ausführlich und kommen zu dem Ergebnis, dass das Kapitalwertverfahren gegenüber

Vgl. Kapitel 2.1.2 sowie beispielsweise Fiedler (2003), S. 6.

Vgl. Gardiner/Stewart (2000), S. 251.

Vgl. Gardiner/Stewart (2000), S. 251. Auch George (1999), S. 111 stellt fest, dass der Kapitalwert als monetäre Kennzahl für die Planung, Steuerung und Kontrolle von Projekten geeignet ist.

4 Vgl. Drews (2001), S. 129. Für eine Abgrenzung der statischen und dynamischen Verfahren der Investitionsrechnung vgl. beispielsweise Adam (2000), S. 105ff.; Blohm/Lüder (1995), S. 54ff.; Breuer (2002), S. 83ff.; Mensch (2002), S. 44ff.; Olfert (2003), S. 147ff.; Perridon/Steiner (2003), S. 37ff.

5 Die Eignung und den Einsatz monetärer Zielgrößen fur die Beurteilung von Projektkooperationen durch die Anwendung des Kapitalwertverfahrens stellen auch Mohnen (2002), S. 41; von Spreckelsen (2004), S. 57 fest. $\mathrm{Zu}$ den Anforderungen an die kooperationsbezogene Investitionsrechnung zăhlt Drews beispielsweise die Einbeziehung von Entscheidungsvariablen des Kooperationsmanagements zur Bildung von Handlungsalternativen, die Berucksichtigung der Zielgröße des Unternehmenswertes durch die Investitionsrechnung, die Integration der unmittelbaren Kooperationsziele als Zielgrößen sowie die Einbeziehung der Zielfunktion des Partners als Nebenbedingung. Vgl. Drews (2001), S. $125 \mathrm{ff}$.

6 Die Annahme eines vollkommenen Kapitalmarktes bedeutet, dass die für Investitionszwecke verfuggbaren finanziellen Mittel nicht beschränkt sind und jederzeit zu einem einheitlichen Kapitalmarktzins aufgenommen werden können. Finanzinvestitionen können zu diesem durch den Investor nicht beeinflussbaren Zins jederzeit getătigt werden. Vgl. etwa Blohm/Luder (1995), S. 74f.; Bosse (2000), S. 37. Zu den Vorzügen dieser Annahmen vgl. beispielsweise Mensch (2002), S. $113 \mathrm{ff}$.

7 Fur Ansätze, nach welchen Kriterien eine Bestimmung des Diskontierungsfaktors für die Bewertung von Investitionsprojekten erfolgen kann vgl. Drews (2001), S. 141f.; Rolfes (2003), S. 22ff.; von Spreckelsen (2004), S. 62. 
alternativen Verfahren der Investitionsrechnung, insbesondere gegenüber der Methode des Internen Zinsfußes überlegen ist. ${ }^{8}$ Besonders für die Bewertung und Auswahl langfristiger Investitionsprojekte erfolgt in den letzten Jahren eine zunehmende Erweiterung der Kapitalwertverfahren um die Ansätze der Realoptionstheorie. ${ }^{9}$ Diese bezieht den Wert zukünftiger Handlungsflexibilitäten in die Bewertung der Investitionsprojekte ein und verwendet hierzu die aus der Optionspreistheorie bekannten Berechnungsansätze. ${ }^{10}$ Boute et al. schlagen die Anwendung dieser Methoden auch für Entscheidungen im Rahmen des Projektmanagements vor. ${ }^{11}$ Aufgrund der Einmaligkeit und Spezifität der hier untersuchten Investitionsprojekte sowie deren Nichtvergleichbarkeit mit Finanzoptionen erweist sich die konkrete Anwendung der Realoptionstheorie als problematisch, da die Ermittlung der für die Berechnung der Optionswerte notwendigen Informationen etwa hinsichtlich eines Duplikationsportfolios, von Kapitalkostensätzen oder von Pseudowahrscheinlichkeiten kaum möglich ist. ${ }^{12}$

Trotz der hier nur kurz dargestellten Möglichkeiten und Probleme der mit der Realoptionstheorie verbundenen Bewertung von Handlungsflexibilität bei Investitionsentscheidungen zeigen diese Ansätze die Notwendigkeit auf, die mit Investitionsprojekten verbundene Unsicherheit in Form eines mehrstufigen Entscheidungsprozesses in Abhängigkeit von der Realisation von Umweltzuständen zu berücksichtigen. Die Entscheidung über die Durchführung einer Investition und die hierfür erforderliche Bewertung verschiedener Investitionsalternativen wird in dieser Arbeit nicht betrachtet, so dass eine möglichst exakte Bewertung des Investitionsprojektes durch die zusätzliche Bewertung der Handlungsflexibilität für die im Folgenden durchgeführten Untersuchungen kein Zielkriterium darstellt. Vielmehr wird durch die Anwendung des Kapitalwertverfahrens die Zeitpräferenz der Kooperationspartner, insbesondere des Auftraggebers, für die Vertrags- und Projektplanung abgebildet.

8 Vgl. Schmidt/Terberger (1997), S. 164ff. Die Autoren kommen zu dem Ergebnis, dass die Annahme des vollkommenen Kapitalmarktes zu der Kapitalwertmethode passt und insbesondere bei sicheren Erwartungen realistisch sein kann. Auch die für die Kapitalwertberechnung getroffene Annahme, Reinvestitionen und Finanzierungen zum Kalkulationszinsfuß durchzuführen, wird als konsequent und sinnvoll bewertet.

9 Vgl. Drews (2001), S. 140; Johannwille (2000), S. 157ff.; Lucke (2001).

10 Zur Realoptionstheorie und deren Anwendung bei Investitionsentscheidungen vgl. beispielsweise Bowman/Moskowitz (2001); Breuer/Gürtler/Schumacher (1999); Crasselt (2003); Dixit/Pindyck (1994); Lucke (2001). Ward/Chapman (2003), S. $101 \mathrm{ff}$. stellen fest, dass das Projektmanagement unter Unsicherheit auch die Chancen einbeziehen sollte.

11 Vgl. Boute/Demeulemeester/Herroelen (2004); Schmitt (2002).

12 Zur Problematik der Anwendung der Realoptionstheorie fur Investitionsprojekte vgl. etwa Brockhoff (2000); Drews (2001), S. 140; Erner/Olinger/Werthschulte (2003), S. 249; Johannwille (2000), S. 166ff.; Magni (2002). 
Der Kapitalwert als Barwert aller erwarteten zukünftig erzielbaren positiven Zahlungen abzüglich des Barwertes aller für die Leistungserstellung notwendigen Auszahlungen kann sowohl im Rahmen einer Wertsteigerungsanalyse (Shareholder-ValueKonzept) als Berichtsgröße als auch für das Management als Kontrollgröße eingesetzt werden. ${ }^{13}$ Bei der Anbahnung langfristiger Projektkooperationen stehen die geplanten bzw. erwarteten finanziellen Mittelzu- und -abflüsse im Mittelpunkt der Verhandlungen zwischen den Projektvertragsparteien, so dass der Kapitalwert sowohl bei der Angebotspreisermittlung des Auftragnehmers als auch bei der Vorteilhaftigkeitsbeurteilung des erhaltenen Angebotes durch den Auftraggeber als geeignete Rechengröße erscheint. ${ }^{14}$ Für die Berechnung des Kapitalwertes kann eine diskrete oder eine kontinuierliche Diskontierung angenommen werden. Hierzu sowie für die mathematische Darstellung wird auf das Kapitel 4.2.3 verwiesen.

Bezogen auf das magische Dreieck des Projektmanagements stellt der Kapitalwert eine Verbindung zwischen dem Ziel der Projektdauerminimierung und dem Ziel der Einhaltung des Projektbudgets her. ${ }^{15}$ Durch die Berücksichtigung des Zeitwertes von Zahlungen und der Annahme, dass die aus dem Projekt resultierenden Einzahlungen nach Abschluss des Projektes erfolgen, erhöht sich der Kapitalwert, je früher das Projektende erreicht werden kann. Es lässt sich jedoch zeigen, dass ein unter der Zielsetzung der Dauerminimierung erstellter Projektplan nicht gleichzeitig den maximal erzielbaren Kapitalwert aufweisen muss. ${ }^{16}$

Übersteigen die tatsächlichen Kosten während der Projektdurchführung die geplanten Kosten, so kann mit Hilfe des Kapitalwertes die Änderung der Vorteilhaftigkeit des Projektes schnell erkannt und steuernd in den Projektablauf eingegriffen werden. Die Untersuchungen von Gardiner und Stewart in Form von Befragungen von Projektverantwortlichen machen deutlich, dass zwar häufig investitionstheoretische Ansätze im Rahmen der Projektauswahl, -planung und -steuerung Anwendung finden, jedoch einige der Projektmanager die Frage nach den Auswirkungen von Änderungen der Zah-

13 Vgl. Drews (2001), S. 130; Gardiner/Stewart (2000), S. 251f. Zur Wertsteigerungsanalyse vgl. etwa Hardtmann (1996). Für die Kapitalwertberechnung ist es zweckmäßig, die pagatorischen Grőßen der Finanzbuchhaltung in Form von Aus- und Einzahlungen zu verwenden. Vgl. Busse von Colbe/Laßmann (1990), S. 1. Eine Berechnung des Periodenerfolgs ist für die in dieser Arbeit betrachtete Problemstellung nicht relevant. Für eine Abgrenzung der Begriffe in der Betriebswirtschaftslehre vgl. etwa Domschke/Scholl (2003), S. 300ff.

14 Vgl. Dayanand/Padman (1998), S. 479ff. Einen finanzmathematischen Ansatz für die Projekterfolgsrechnung auf der Grundlage von Ein- und Auszahlungen wählt auch Bröker (1993), S. 101. Zu allgemeinen Aspekten eines finanzwirtschaftliche Aspekte berucksichtigenden Projektmanagements vgl. Cappels (2003).

15 Vgl. Abbasi/Arabiat (2001), S. 17.

16 Vgl. Doersch/Patterson (1977), S. 885. 
lungszeitpunkte während der Projektdauer auf den Kapitalwert falsch einschätzen. ${ }^{17}$ Sowohl diese Erkenntnisse als auch die steigende Bedeutung monetärer Größen im Rahmen der Unternehmungsführung sind die Ursachen dafür, dass sich eine zunehmende Anzahl wissenschaftlicher Beiträge dem Problem der Projektplanung unter der Zielsetzung der Kapitalwertmaximierung annimmt. ${ }^{18}$

Die folgende Tabelle 4.1 gibt einen Überblick über einige der zu diesem Gebiet in der wissenschaftlichen Literatur erschienenen Beiträge, wobei eine Einordnung in Problemstellungen mit und ohne Berücksichtigung von Ressourcen sowie in exakte und heuristische Lösungsverfahren vorgenommen wird. ${ }^{19}$

\begin{tabular}{|c|c|c|}
\hline & ohne Berucksichtigung von Ressourcen & mit Berücksichtigung von Ressourcen \\
\hline $\begin{array}{l}\text { exaktes } \\
\text { Lösungs- } \\
\text { verfahren }\end{array}$ & $\begin{array}{l}\text { Russell (1970) } \\
\text { Grinold (1972) } \\
\text { Elmaghraby/Herroelen (1990) } \\
\text { Erenguc/Tufekci/Zappe (1993) } \\
\text { Kazaz/Sepil (1996) } \\
\text { Dayanand/Padman (1997) } \\
\text { Shtub/Etgar (1997) } \\
\text { Etgar/Shtub (1999) } \\
\text { Dayanand/Padman (2001b) } \\
\text { Vanhoucke/Demeulemeester/Herroelen (2001b) } \\
\text { Vanhoucke/Demeulemeester/Herroelen (2003) }\end{array}$ & $\begin{array}{l}\text { Doersch/Patterson (1977) } \\
\text { Smith-Daniels/Smith-Daniels (1987) } \\
\text { Yang/Talbot/Patterson (1992) } \\
\text { Baroum/Patterson (1996) } \\
\text { Icmeli/Erenguc (1996) } \\
\text { De Reyck/Herroelen (1998) } \\
\text { Vanhoucke/Demeulemeester/Herroelen (2001a) }\end{array}$ \\
\hline $\begin{array}{l}\text { heuristisches } \\
\text { Lösungs- } \\
\text { verfahren }\end{array}$ & $\begin{array}{l}\text { Etgar/Shtub/LeBlanc (1996) } \\
\text { Dayanand/Padman (1998) } \\
\text { Dayanand/Padman (2001a) }\end{array}$ & $\begin{array}{l}\text { Smith-Daniels/Aquilano (1987) } \\
\text { Padman/Smith-Daniels (1993) } \\
\text { Ulusoy/Özdamar (1995) } \\
\text { Pinder/Marucheck (1996) } \\
\text { Smith-Daniels/Padman/Smith-Daniels (1996) } \\
\text { Padman/Roehrig (1997) } \\
\text { Sepil/Ortac (1997) } \\
\text { Ulusoy/Cebelli (2000) } \\
\text { Abbasi/Arabiat (2001) } \\
\text { Ulusoy/Sivrikaya-Serifoglu/Sahin (2001) } \\
\text { Chiu/Tsai (2002) }\end{array}$ \\
\hline
\end{tabular}

Tabelle 4.1: Literaturüberblick Project Scheduling und Kapitalwertmaximierung

17 Vgl. Gardiner/Stewart (2000), S. 253.

18 Für Beitrăge mit einem Überblick über die Forschung auf dem Gebiet des Project Schedulings bei Kapitalwertmaximierung vgl. beispielsweise Baroum/Patterson (1996); Brucker (2001); Brucker et al. (1999), S. 23ff.; Demeulemeester/Herroelen (2002); Herroelen/De Reyck/Demeulemeester (1998), S. 293ff.; Herroelen/Van Dommelen/Demeulemeester (1997); Icmeli/Erenguc/Zappe (1993); Kolisch/Padman (2001). Steuer/ $\mathrm{Na}$ (2003), S. 496ff. geben einen Literaturüberblick über Entscheidungsprobleme der Investition und Finanzierung bei Mehrfachzielsetzung.

19 Für die Art der Klassifizierung vgl. auch Baroum/Patterson (1998), S. 108. Beiträge aus Buchern beziehungsweise Sammelbänden wurden nicht aufgenommen. Es sind auch keine Beiträge berlicksichtigt, die eine Klassifikation beziehungsweise einen Überblick über Project Scheduling Probleme enthalten. 
Es lässt sich erkennen, dass im Fall der Nichtberücksichtigung von Ressourcen wenig Forschungsbedarf nach heuristischen Lösungsverfahren besteht, da eine Lösung dieser Problemstellungen oft mit exakten Verfahren möglich ist. Bei den innerhalb der heuristischen Lösungsverfahren in Tabelle 4.1 aufgeführten Beiträgen ohne Berücksichtigung von Ressourcen liegen Problemstellungen vor, welche sich durch besondere Annahmen von einem klassischen Project Scheduling Problem unterscheiden und aufgrund ihrer Problemkomplexität heuristische Lösungsverfahren erfordern. ${ }^{20}$

Wegen des in dieser Arbeit vorliegenden Schwerpunktes der Untersuchungen auf dem Gebiet des Vertragsdesigns für das Projektmanagement sollen mit Hilfe des Project Schedulings Handlungsempfehlungen entwickelt werden, die den Projektauftraggeber bei der Auswahl eines Vertragsdesigns und der hiermit verbundenen Zahlungsvereinbarungen unterstützen. Hiervon kann das nach Vertragsabschluss vorliegende Problem der Zeit- und Ressourcenplanung im Zusammenhang mit einer Projektsteuerung abgegrenzt werden. ${ }^{21}$

Im Folgenden werden die in Tabelle 4.1 aufgeführten Beiträge vorgestellt und hinsichtlich ihrer jeweils zugrunde liegenden Problemstellung analysiert. Dabei liegt der Fokus der Untersuchungen auf den in den Beiträgen aufgegriffenen Problemcharakteristika. Aufgrund der Zielsetzung der Kapitalwertmaximierung sind die unterschiedlichen Ansätze einer Berücksichtigung von Aus- und Einzahlungen sowie die zeitliche Abfolge von Vorgängen oder Ereignissen von Interesse. Insbesondere die Einbeziehung von Vertragscharakteristika in die in den Beiträgen vorgestellten Ansätze findet im Hinblick auf die im fünften Kapitel erfolgende Verbindung des Vertragsmanagements mit der quantitativen Projektplanung Beachtung.

Das folgende Kapitel beginnt zunächst mit einer kurzen Darstellung der Project Scheduling Problematik in seiner allgemeinen Form. Im Anschluss daran erfolgt die Auseinandersetzung mit den bisherigen Forschungsbeiträgen zum Project Scheduling bei Kapitalwertmaximierung.

$20 \mathrm{Vgl}$. beispielsweise Etgar/Shtub/LeBlanc (1996). Bei dem in dieser Veroffentlichung vorgestellten Problem ist die Höhe der Zahlungen von dem Zahlungszeitpunkt abhängig und unterscheidet sich damit von dem klassischen Project Scheduling Problem bei Kapitalwertmaximierung, bei dem die Höhe der Zahlungen als von dem Zahlungszeitpunkt unabhängig angenommen wird.

21 Einen Überblick uber dieses Planungsproblem sowie quantitative Methoden für dessen Lösung insbesondere bei stochastischen Vorgangsdauern sowie unterschiedlichen Risikoeinstellungen des Entscheiders zeigt Wolf (2004). 


\subsection{Das Project Scheduling Problem und seine Anwendungen}

\subsubsection{Problemstellung und Stand der Forschung}

Die grundlegende Problemstellung des deterministischen Project Schedulings mit der Zielsetzung der Kapitalwertmaximierung lässt sich anschaulich durch den in Abbildung 4.1 dargestellten Netzplan verdeutlichen. Die in einem Vorgangspfeilnetzplan zwischen den 13 Ereignissen angeordneten 20 Vorgänge sind jeweils mit ihrer Vorgangsbezeichnung (A bis T) sowie der Vorgangsdauer gekennzeichnet. Die Ausführung von Vorgängen ist für den Auftragnehmer mit Kosten verbunden, deren Höhe an dieser Stelle nicht näher spezifiziert wird. Nach Abschluss der in die Ereignisse 7, 10 und 12 einmündenden Vorgänge erhält der Auftragnehmer eine Zahlung des Auftraggebers, so dass mit diesen Ereignissen nach Abzug der Vorgangskosten eine positive Nettozahlung verbunden ist.

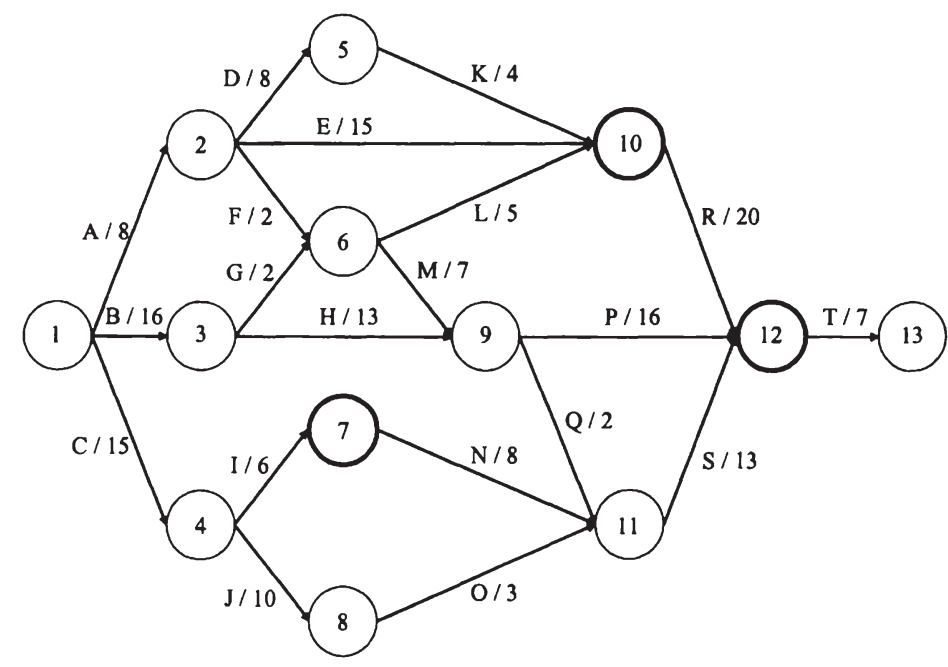

Abbildung 4.1: Beispiel zum Project Scheduling

Wird für einen zulässigen Projektplan als Startzeitpunkt $t=0$ vorausgesetzt und jeder Vorgang zu seinem frühestmöglichen Startzeitpunkt geplant, ergeben sich die in Abbildung 4.2 dargestellten frühesten Start- und Endzeitpunkte der Vorgänge und somit ein Projektplan mit einer Gesamtprojektdauer von 52 Zeiteinheiten. 


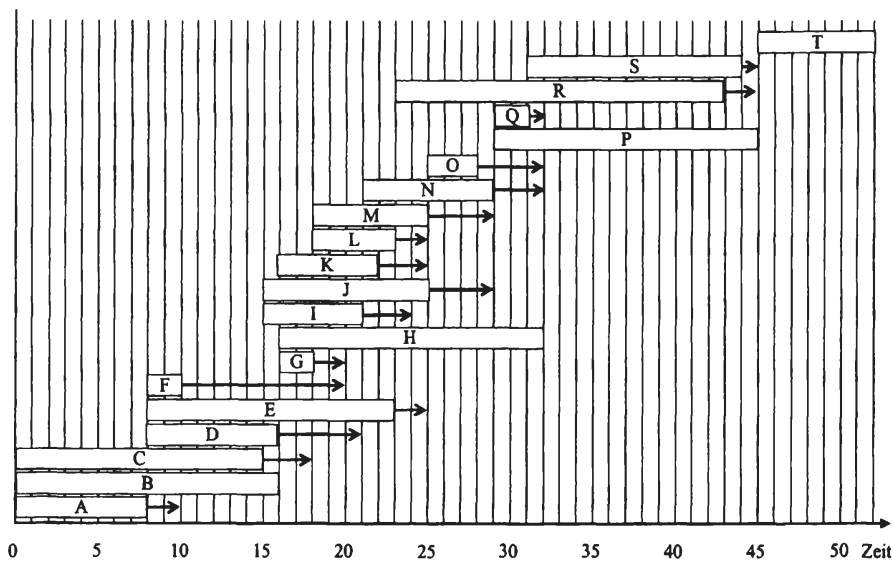

Abbildung 4.2: Terminierung der Vorgänge zu frühestmöglichen Startzeitpunkten

Die Pfeile hinter den Vorgängen in Abbildung 4.2 visualisieren den Gesamtpuffer eines Vorgangs. Dieser lässt sich als Differenz aus dem spätesten Endzeitpunkt und dem frühesten Endzeitpunkt des Vorgangs ermitteln. ${ }^{22}$ Hierbei ist jedoch zu berücksichtigen, dass durch die Abhängigkeitsbeziehungen zwischen den Vorgängen, welche in der Abbildung nicht explizit gekennzeichnet sind, die Inanspruchnahme des Gesamtpuffers eines Vorgangs dazu führen kann, dass sich die frühesten Anfangszeitpunkte folgender Vorgänge verschieben. ${ }^{23}$ Durch die mit der Zielsetzung der Kapitalwertmaximierung verbundene Zeitpräferenz für Zahlungen kann der Kapitalwert erhöht werden, wenn Vorgänge, die mit Auszahlungen verbunden sind, später terminiert werden. ${ }^{24}$ Dabei ist jedoch zu beachten, dass eine spätere Terminierung der mit Auszahlungen verbundenen Vorgänge nicht zu einer späteren Terminierung der mit Einzahlungen verbundenen Ereignisse führt. Beispielsweise bedeutet eine Terminierung des Vorgangs $\mathrm{C}$ zum Zeitpunkt $\mathrm{t}=3$, dass dieser zwar möglichst spät abgeschlossen wird und das Ereignis 4 somit zum Zeitpunkt $t=18$ stattfindet, jedoch das mit einer Einzahlung verbundene Ereignis 7 durch den zwischen Ereignis 4 und Ereignis 7 er-

22 Für die Berechnung des Gesamtpuffers in einem Vorgangspfeilnetzplan vgl. etwa Schwarze (2001), S. 168; Zimmermann (1971), S. 24. Der freie Puffer eines Vorgangs als Differenz zwischen dem kleinsten frühesten Anfangszeitpunkt aller Nachfolger und dem frühesten Endzeitpunkt dieses Vorgangs gibt an, um wie viele Zeiteinheiten eine Verzögerung des Vorgangs stattfinden kann, ohne dass dies Auswirkungen auf die nachfolgenden Vorgänge hat. Vgl. beispielsweise Schwarze (2001), S. 168.

23 Vgl. Werners (2000), S. 424. Es wird vorgeschlagen, den Vorgängen entsprechend bestimmten Aspekten Pufferzeiten zuzuweisen. Im Rahmen der hier betrachteten Problemstellung erscheint es sinnvoll, Vorgängen, die mit hohen Auszahlungen verbunden sind, mehr Pufferzeit zuzuweisen, so dass diese Vorgänge möglichst spät terminiert werden können. Vgl. Werners (2000), S. 427.

24 Dieses Grundproblem ist auch anschaulich anhand eines Beispiels dargestellt in Kimms (2001), S. $19 \mathrm{ff}$. 
folgenden Vorgang I mit einer Dauer von 6 Zeiteinheiten nicht mehr zu seinem frühestmöglichen Zeitpunkt $\mathrm{t}=21$ stattfinden kann.

Für dieses unter der Bezeichnung des unkapazitierten, deterministischen, Kapitalwert maximierenden Project Scheduling bekannte Problem sind zahlreiche Modelle und Lösungsverfahren entwickelt worden, welche im Folgenden in chronologischer Reihenfolge vorgestellt werden. Es lässt sich neben der zeitlichen Entwicklung dieses Forschungsgegenstandes insbesondere erkennen, welche Problemcharakteristika von besonderer praktischer Relevanz sind und daher häufig Teil der theoretischen Untersuchungen sind. ${ }^{25}$

Die erste in der wissenschaftlichen Literatur erfolgte Betrachtung von Zahlungsströmen in Projektnetzplänen stammt aus dem Jahr $1970 .{ }^{26}$ Russell begründet seine Forschungsidee damit, dass neben einer Ressourcenallokation und der Bestimmung zeitkritischer Pfade innerhalb eines Netzplans auch die Untersuchung von Kostenstrukturen, insbesondere zum Zweck des Projektcontrollings, sowie ihrer Auswirkungen auf die Vorteilhaftigkeit und Performance von Projekten von großer Bedeutung ist. ${ }^{27}$ Die Problematik der Bestimmung eines problemadäquaten Diskontierungsfaktors findet auch bei Russell Erwähnung. Dennoch wird der Kapitalwert - als eine Möglichkeit der Investitionsrechnung - als am besten geeignetes Kriterium erachtet, über die Vorteilhaftigkeit von Projekten zu entscheiden. ${ }^{28}$ Dem von ihm vorgestellten mathematischen Modell liegt ein Vorgangspfeilnetzplan zugrunde. Die Dauer der Vorgänge sowie die den Ereignissen zugewiesenen Zahlungen werden als bekannt vorausgesetzt. Ferner wird angenommen, dass das erste Ereignis zu dem Zeitpunkt $t=0$ beginnt. Für die Lösung des von Russell vorgestellten Modells wird die nichtlineare Zielfunktion durch eine lineare Ersatzzielfunktion approximiert, ${ }^{29}$ so dass das Problem als lineares Pro-

25 Es existiert nur eine geringe Anzahl von Beitrăgen, die neben der Zielsetzung der Kapitalwertmaximierung auch stochastische Einflüsse berücksichtigen. Für die Annahme stochastischer Vorgangsdauern vgl. Buss/Rosenblatt (1997); Elmaghraby/Ferreira/Tavares (2000). Fü stochastische Zahlungen vgl. Özdamar/Dundar (1997). Fur stochastische Dauern und stochastische Zahlungen vgl. Tavares (1994); Tavares/Ferreira/Coelho (1998); Yang/Tay/Sum (1995). Aufgrund der bei diesen Beitrăgen im Vordergrund stehenden Problematik der Projektsteuerung wird hierauf im Folgenden nicht năher eingegangen.

26 Vgl. Russell (1970). Die Aussage, dass diese Veroffentlichung die erste ist, die die Verbindung von Kapitalwerttheorie und Projektmanagement herstellt, findet sich u. a. bei Elmaghraby/Herroelen (1990), S. 36; Klein (2000), S. 106; Kolisch/Padman (2001), S. 254; Pinder/Marucheck (1996), S. 230; Ulusoy/Cebelli (2000), S. 262; Vanhoucke/Demeulemeester/Herroelen (2003), S. 604.

27 Vgl. Russell (1970), S. 357.

28 Vgl. Russell (1970), S. 359.

29 Die Zielfunktion beinhaltet die Zeit $t$ als Entscheidungsvariable. Die im Exponent der Funktion stehende Variable ist Ursache dafür, dass die Zielfunktion nichtlinear ist und daher dieses Lösungsverfahren vorgeschlagen wird. 
gramm dargestellt werden kann. Die Dualisierung dieses linearen Modells führt zu einem Problem, welches als Umlade- bzw. Flussproblem in einem Netzwerk interpretiert werden kann. ${ }^{30}$ Durch einen hierauf aufbauenden, die Ereigniszeitpunkte schrittweise ändernden Algorithmus wird ein optimaler Fluss innerhalb des Netzplans bestimmt, der für die approximierte Zielfunktion zu einem maximalen Kapitalwert und damit einer optimalen Lösung für die Vorgangsstart- und -endzeitpunkte führt. ${ }^{31}$ Russell zeigt weiterhin, dass sich der zeitkritische Pfad von einem kostenkritischen Pfad unterscheiden kann. ${ }^{32}$

Bedingt durch die mit der Anwendung der Kapitalwertmaximierung verbundene Zeitpräferenz für Zahlungen ergibt sich das Problem, dass das Projektende bei einem Projekt mit einer negativen Abschlusszahlung unendlich spät terminiert wird, da das Verzögern einer negativen Zahlung um eine Zeiteinheit positiven Einfluss auf den Kapitalwert hat. Grinold fügt daher einen Vorgang mit der Dauer $D_{11}=D M a x$ ein, der das erste Ereignis und das letzte Ereignis I in einem Vorgangspfeilnetzplan miteinander verbindet und als obere Grenze für die maximal erlaubte Projektdauer interpretiert werden kann. ${ }^{33}$ Wird mit $\mathrm{E}_{\mathrm{I}}$ die längste minimale Dauer von Ereignis 1 bis Ereignis I bezeichnet, welche dem frühestmöglichen Zeitpunkt des letzten Ereignisses und somit dem frühestmöglichen Projektende entspricht, muss für die Erzeugung einer zulässigen Lösung DMax $\geq E_{1}$ gelten. ${ }^{34}$ Die Zielfunktion des von Grinold formulierten mathematischen Modells entspricht der von Russell. Die Restriktionen zur Einhaltung der Reihenfolgebeziehungen der Vorgänge sind auch bei Grinold nur für unmittelbar aufeinander folgende Vorgänge bindend. ${ }^{35}$

Doersch und Patterson erweitern die Problemstellung durch das Hinzufügen einer Restriktion, die das für die Ausführung der Vorgänge benötigte verfügbare Kapital berücksichtigt, und formulieren das Project Scheduling Problem als gemischt-ganzzahliges lineares Modell. ${ }^{36}$ Die Zielfunktion beinhaltet zusätzlich eine Größe, die bei

\footnotetext{
$30 \mathrm{Zu}$ Flussproblemen in Netzwerken vgl. Ahuja/Magnanti/Orlin (1993).

31 Vgl. Russell (1970), S. 362ff. Es ist möglich, dass die ermittelte Lösung für das Ursprungsproblem nicht optimal ist.

32 Vgl. Russell (1970), S. 364. Eine Verlängerung der Dauer von Vorgängen auf dem zeitkritischen Pfad hat definitionsgemäß eine Wirkung auf die Gesamtprojektdauer. Liegen Vorgänge auf dem kostenkritischen Pfad, so hat eine Verlängerung der Dauer Einfluss auf den Kapitalwert des Projektes.

33 Vgl. Grinold (1972), S. 124.

34 Vgl. Grinold (1972), S. 125. Als Alternative zu dem frühestmöglichen Zeitpunkt des Projektendes kann auch ein späterer, auf der Grundlage vertraglicher Regelungen festgelegter Zeitpunkt als Projektende gewählt werden. Vgl. Werners (2000), S. 423.

35 Vgl. Grinold (1972), S. 124.

36 Vgl. Doersch/Patterson (1977), S. 883f.
} 
Überschreitung einer vorgegebenen Projektdauer Strafkosten ansetzt und damit den Projektkapitalwert vermindert. Durch das Festlegen eines spätesterlaubten Projektendes besteht die Möglichkeit, das Projektende zeitlich nach dem frühestmöglichen Projektende festzusetzen und damit auch dem letzten Ereignis einen Puffer zuzuweisen. Dies hat zur Folge, dass Ereignisse, die zuvor auf dem zeitkritischen Pfad lagen, Pufferzeiten erhalten und somit die Anzahl der Möglichkeiten der Terminierung von Vorgängen und Ereignissen erhöht wird. Beispielsweise führt eine Verlängerung der Projektdauer um eine Einheit zu Strafkosten in bestimmter Höhe sowie zu einer Minderung des Barwertes der zu dem Zeitpunkt des Projektendes erfolgenden Einzahlung. Dieser negative Einfluss auf den Kapitalwert kann jedoch möglicherweise durch das zeitliche Verzögern eines mit einer hohen Auszahlung zu einem frühen Zeitpunkt stattfindenden Vorgangs kompensiert werden.

Der Konflikt zwischen der Einhaltung einer vorgegebenen Projektdauer und den mit einem Projektplan festgelegten Projektkosten unter der Annahme unsicherer Vorgangsdauern steht im Mittelpunkt der Betrachtungen von Shtub. ${ }^{37}$ Das von ihm vorgestellte heuristische Vorgehen vergleicht einen Zeitplan mit möglichst früh startenden Vorgängen und somit auch zeitlich frühen Auszahlungen mit einem Zeitplan, dessen Vorgänge mit dem spätesterlaubten Startzeitpunkt terminiert sind und damit unter Berücksichtigung der Zeitpräferenz von Zahlungen zu einem höheren Projektkapitalwert führen. Es ist die Aufgabe des Entscheidungsträgers, die nicht kritischen Vorgänge so (zeitlich spät) zu terminieren, dass unter Berücksichtigung der hieraus resultierenden Gefahr eines aufgrund der mit Unsicherheit behafteten Vorgangsdauern verspäteten Projektendes der Kapitalwert maximiert wird. Auch Smith-Daniels und Aquilano untersuchen die Auswirkungen, die eine Verschiebung der Startzeiten auf die spätesterlaubten Zeiten auf den Projektkapitalwert haben. ${ }^{38}$ Unter Beachtung von Ressourcenbeschränkungen kommen die Autoren zu dem Ergebnis, dass dieser so genannte rightshifted-start-schedule sowohl zu einem 2,5\% höheren Kapitalwert als der early-startschedule als auch zu einer Verkürzung der Projektdauer führt. Dieses Vorgehen kann jedoch nur dann zu dem geschilderten Ergebnis führen, wenn, wie dies auch angenommen wird, mit den Ereignissen oder Vorgängen ausschließlich Auszahlungen verbunden sind und die Einzahlung am Ende des Projektes erfolgt. ${ }^{39}$

37 Vgl. Shtub (1986).

38 Vgl. Smith-Daniels/Aquilano (1987).

39 Vgl. Smith-Daniels/Aquilano (1987), S. 619. 
Bei dieser Annahme handelt es sich für reale Problemstellungen des Projektmanagements um eine äußerst restriktive Annahme, da davon auszugehen ist, dass auch während der Projektlaufzeit, beispielsweise zu bestimmten Meilensteinen, Zahlungen durch den Auftraggeber in Form von Fortschrittszahlungen an den Auftragnehmer geleistet werden. Diese Kritik üben auch Elmaghraby und Herroelen an den frühen Modellen und zeigen zwei weitere Annahmen auf, die bei bisherigen Problemstellungen vorausgesetzt wurden, jedoch kaum mit realen Fragestellungen des Project Schedulings kongruent sind. ${ }^{40}$ Dies ist zum einen die Annahme, dass die Höhe der während der Projektlaufzeit stattfindenden und Ereignissen bzw. Vorgängen zugehörigen Einund Auszahlungen unabhängig von den Zeitpunkten der Ereignisse bzw. Vorgänge ist, und zum anderen die Annahme, dass die Höhe der Zahlungen als bekannt vorausgesetzt wird und damit das Verhandlungs-Problem zwischen Auftraggeber und Auftragnehmer ausgeklammert wird.

Obwohl diese Kritik für eine realitätsnähere Abbildung der Problemstellung berechtigt ist, werden die zusätzlichen Annahmen bei der Formulierung des von Elmaghraby und Herroelen vorgestellten Modells nicht berücksichtigt. ${ }^{41}$ Beiträge, die insbesondere den Zeit-Kosten-Konflikt der Projektplanung in den Vordergrund stellen, unterstützen den Projektmanager vorwiegend bei der ausführenden Planung und Steuerung eines Projektes und können daher nur in begrenztem Umfang zu der in dieser Arbeit untersuchten Problemstellung einen Beitrag leisten. Durch die Zielsetzung der Kapitalwertmaximierung erfolgt dennoch im Folgenden eine Berücksichtigung dieser Ansätze.

Yang, Talbot und Patterson formulieren ein mathematisches Modell, welches unter Berücksichtigung erneuerbarer und nicht erneuerbarer Ressourcen sowie mit Vorgängen verbundenen Ein- und Auszahlungen die Zielsetzung der Kapitalwertmaximierung verfolgt. ${ }^{42}$ Hierbei werden alle während der Dauer eines Vorgangs anfallenden Zahlungen zum Zeitpunkt des Vorgangsendes zusammengefasst und auf den Startzeitpunkt des Projektes diskontiert. Ein Bezug der Vorgangszahlungen auf den Startzeitpunkt der Vorgänge erscheint jedoch durch die Annahme der Finanzierung der Kosten durch den Auftragnehmer als sinnvoller.

\footnotetext{
40 Vgl. Elmaghraby/Herroelen (1990), S. 35.

41 Vielmehr folgt als Ergänzung zu diesem Beitrag eine Umsetzung des vorgeschlagenen Modells durch Softwareunterstultzung. Vgl. Herroelen/Gallens (1993).

42 Vgl. Yang/Talbot/Patterson (1992), S. 188ff.
} 
Im Gegensatz zu Modellen mit der Zielsetzung der Projektdauerminimierung, bei denen im Rahmen eines optimierenden Verfahrens mit jeder Verkürzung der Projektdauer eine Einschränkung des Lösungsraums möglich ist, ist dies bei der hier untersuchten Problemstellung nicht möglich, da das Kapitalwertmaximum je nach Zahlungs- und Projektstruktur zwischen dem frühestmöglichen Zeitpunkt und dem spätesterlaubten Zeitpunkt eines Ereignisses bzw. Vorgangs erreicht werden kann. ${ }^{43}$

Eine Verbindung des Zeit-Kosten-Konfliktes mit dem Scheduling Problem bei Kapitalwertmaximierung wird von Erenguc, Tufekci und Zappe vorgeschlagen. ${ }^{44}$ Dabei hängen die Vorgangsdauern sowie die Vorgangskosten von einem zu wählenden Ausführungsmodus ab, die Einzahlungen erfolgen hierbei zu den Ereigniszeitpunkten. Ziel des vorgestellten Problems ist die Maximierung des Kapitalwertes unter Abwägung höherer Vorgangsauszahlungen zugunsten verkürzter Vorgangsdauern und damit früherer Ereigniszeiten, die zu Einzahlungen führen. Eine ähnliche Problemstellung betrachten Ulusoy und Özdamar und schlagen ein heuristisches Verfahren vor, das durch sukzessive Vorwärts- und Rückwärtsterminierung von Vorgängen bei unterschiedlichen Ausführungsmodi und Ressourcenbeschränkungen einen Projektplan mit möglichst hohem Kapitalwert erzeugt. ${ }^{45}$ Eine Erweiterung dieses heuristischen Vorgehens ist bei Baroum und Patterson zu erkennen. ${ }^{46}$ Sie erstellen eine Prioritätenliste mit allen Vorgängen und bestimmen damit, welche Vorgänge vorrangig zeitlich eingeplant und mit Ressourcen versorgt werden. Die Berechnung der Prioritäten geschieht über die Summierung der jeweiligen zu einem Vorgang erfolgenden Zahlung sowie aller Zahlungen (Ein- und Auszahlungen) der auf diesen Vorgang folgenden Vorgänge. Damit wird sichergestellt, dass beispielsweise ein Vorgang mit einer negativen Zahlung nicht so spät wie erlaubt terminiert wird, wenn dadurch ein nachfolgender Vorgang mit einer hohen positiven Zahlung auch spät eingeplant wird und somit negativen Einfluss auf das Ziel der Kapitalwertmaximierung hat. Obwohl die letztgenannten Ansätze das Ziel der Kapitalwertmaximierung verfolgen, besteht auch hierbei das Optimierungsproblem in der Entscheidung über die optimale zeitliche Projektsteuerung. Diese die operative Planung während der Projektdurchführung unterstützenden Problemformulierungen und Lösungswege stehen im Rahmen dieser Arbeit nicht im Mittelpunkt der

\footnotetext{
43 Vgl. Yang/Talbot/Patterson (1992), S. 191.

44 Vgl. Erenguc/Tufekci/Zappe (1993).

45 Vgl. Ulusoy/Özdamar (1995), S. 89ff. Ein Vergleich unterschiedlicher heuristischer Ansătze für die Losung dieser Problemstellung ist bei Padman/Smith-Daniels (1993) zu finden.

46 Vgl. Baroum/Patterson (1996).
} 
Betrachtungen. Dennoch zeigen sie durch die Berücksichtigung unterschiedlicher Ausführungsmodi einen Bezug zu den im fünften Kapitel diskutierten Anreizmechanismen auf.

Eine neue Richtung des Project Schedulings unter Berücksichtigung von Zahlungsströmen geben Etgar, Shtub und LeBlanc vor. ${ }^{47}$ Sie berücksichtigen den Fall von zeitabhängigen Zahlungen, die zum Beispiel in Form von Bonus- oder Strafzahlungen bei Zeitunter- bzw. -überschreitung der vereinbarten Projektdauer vertraglich vereinbart sein können. Bei positiven Zahlungen sinkt die positive Nettozahlung durch die Strafzahlung, je später ein Ereignis bzw. ein Vorgang beendet wird. Bei negativen Zahlungen verringert sich gleichermaßen die negative Nettozahlung. Es wird ein stückweise definierter, linearer konstanter und in Abbildung 4.3 skizzierter Verlauf der Nettozahlungen $\mathrm{z}(\mathrm{t})$ in Abhängigkeit von dem Zeitpunkt des Ereignisendes zwischen frühestmöglichem und spätesterlaubtem Ende angenommen und als bekannt vorausgesetzt. ${ }^{48}$

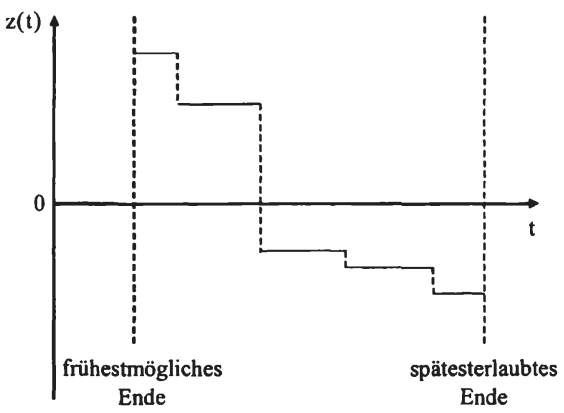

Abbildung 4.3: Positive und negative Nettozahlungen in Abhängigkeit von der Ereigniszeit (Quelle: In Anlehnung an Etgar/Shtub/LeBlanc (1996), S. 91.)

Hierdurch können insbesondere bei Großprojekten, bei denen Zahlungen als Fortschrittszahlungen zu Meilensteinen stattfinden, für definierte Zeitintervalle innerhalb der Zeitspanne zwischen dem frühestmöglichen und spätesterlaubten Ereignisende Boni sowie Strafen in Form von absoluten oder relativen Zahlungen vertraglich ver-

47 Vgl. Etgar/Shtub/LeBlanc (1996).

48 Vgl. Etgar/Shtub/LeBlanc (1996), S. 91. Das von den Autoren vorgeschlagene Lösungsverfahren basiert auf der Heuristik Simulated Annealing. Zu Simulated Annealing vgl. Kirkpatrick/Gelatt/Vecchi (1983). Nach Berechnung der Kapitalwerte fur den fruhesten und spätesten Projektplan werden die Zeiten der nichtkritischen Ereignisse um bestimmte Zeitintervalle verändert und eine Neuberechnung des Kapitalwertes durchgefuhrt. Aufgrund der angenommenen stückweise linearen konstanten Abhängigkeit der Zahlung von dem Ereignisendzeitpunkt erweist sich ein Vorgehen als besonders geeignet, welches die Zeitintervalle so wählt, dass mit jeder Veränderung der Ereigniszeiten eine Änderung des Kapitalwertes erfolgt. 
einbart werden. ${ }^{49}$ Für die Berücksichtigung des Zeitwertes der Zahlungen werden diese auf den Zeitpunkt des Projektbeginns diskontiert, so dass sich für die an einem Ereignis anfallende Zahlung je nach Ereigniszeitpunkt ein unterschiedlicher Barwert $\mathrm{BW}(\mathrm{t})$ ergibt, wie es in Abbildung 4.4 angedeutet wird.

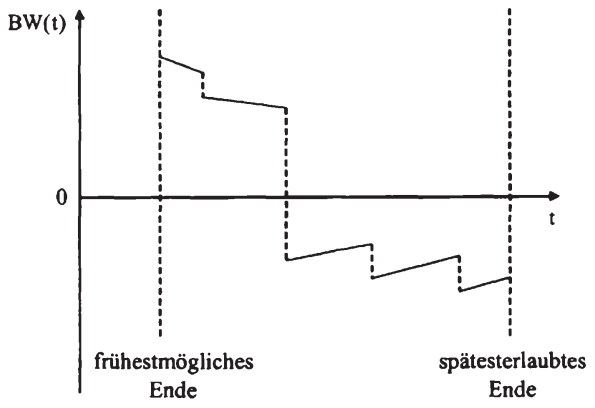

Abbildung 4.4: Barwert bei positiven/negativen Zahlungen in Abhängigkeit von der Ereigniszeit (Quelle: In Anlehnung an Shtub/Etgar (1997), S. 3370.)

Die Abbildung 4.4 zeigt, dass aufgrund der Diskontierung kein monotoner Verlauf des Barwertes vorliegt und somit im Fall positiver Zahlungen der höchste Barwert zum frühestmöglichen Ereigniszeitpunkt erreicht wird und im Fall negativer Zahlungen zu dem Ereigniszeitpunkt, bevor eine höhere Strafe angesetzt wird. ${ }^{50}$ Dies ist damit zu erklären, dass durch die Diskontierung der Zahlungen der Barwert einer Einzahlung bzw. Auszahlung sinkt bzw. steigt, je später diese Zahlung erfolgt.

Die Annahme einer stückweise linearen und konstanten Funktion der Höhe von Zahlungen in Abhängigkeit von dem Ereigniszeitpunkt wird in einer 1999 erschienen Arbeit von Etgar und Shtub aufgehoben und ein linearer stetiger Verlauf von Zahlungen in Abhängigkeit von den Ereigniszeiten unterstellt. ${ }^{51}$ Dabei unterscheiden Etgar und

49 Vgl. Bubshait (2003), S. 66f

so Vgl. Shtub/Etgar (1997), S. 3370. Shtub und Etgar stellen in einer später veroffentlichten Quelle einen Branch-and-Bound-Algorithmus fur diese Problemstellung vor und berichten von durchgefuhrten Tests, die einen Vergleich der in ihrer früheren Quelle veröffentlichten Heuristiken auf der Grundlage von Simulated Annealing mit dem Branch-and-Bound-Vorgehen durchfuhren. Der Branch-and-Bound-Algorithmus führte im Rahmen der untersuchten Testprobleme bei einer kurzen Rechenzeit zu einer jeweils optimalen Lösung. Der Branch-and-Bound-Algorithmus zählt zu den optimierenden Verfahren, d. h. er fulhrt immer zu einer optimalen Lösung. Die Autoren schlagen jedoch vor, die Berechnung bei zu langen Laufzeiten nach einer definierten Anzahl Iterationen abzubrechen. Hierbei kann nicht mit Sicherheit festgestellt werden, ob es sich bei der ermittelten Lơsung und die optimale Lossung handelt. In diesem Fall kann das Vorgehen als heuristisch angesehen werden. Vgl. Shtub/Etgar (1997), S. 3376.

51 Vgl. Etgar/Shtub (1999). 
Shtub die vier folgenden Funktionstypen der allgemeinen Form $z(t)=A+B \cdot t$, $\mathrm{A}, \mathrm{B} \in \mathbb{R}, \mathrm{B} \leq 0:^{52}$

(1) Konstante positive Zahlungsfunktion: $\mathrm{A} \geq 0, \mathrm{~B}=0$

(2) Konstante negative Zahlungsfunktion: $\mathrm{A}<0, \mathrm{~B}=0$

(3) Linear fallende Zahlungsfunktion mit positivem Startwert: $A \geq 0, B<0$

(4) Linear fallende Zahlungsfunktion mit negativem Startwert: $\mathrm{A}<0, \mathrm{~B}<0$

Die Abbildung 4.5 undAbbildung 4.6 zeigen die vier Zahlungsfunktionen $z(t)$ sowie die jeweiligen mit dem Faktor DISKF $F_{t}=(1+D I S K Z)^{-t}$ auf den Zeitpunkt $t=0$ abgezinsten Zahlungen in Form einer Barwertfunktion $B W(z(t))=z(t) \cdot D I S K F_{t}$.

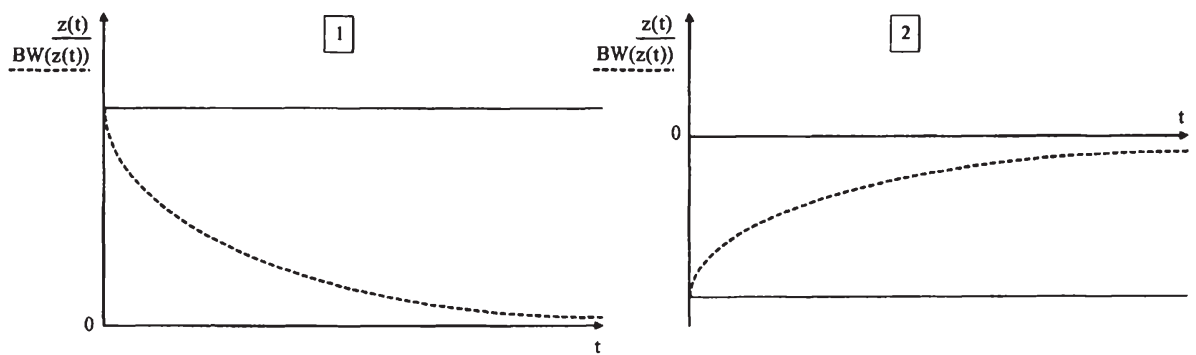

Abbildung 4.5: Konstante Zahlungsfunktionen und Barwertfunktionen

(Quelle: In Anlehnung an Shtub/Etgar (1999), S. 332.)
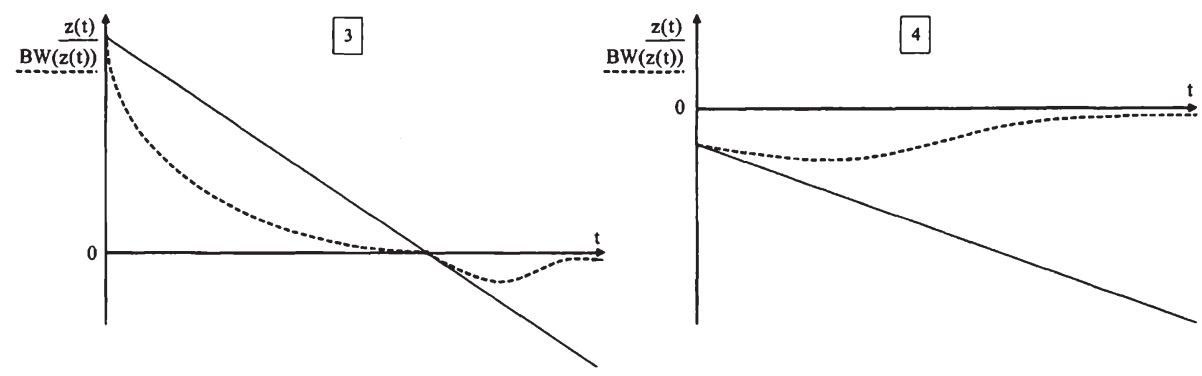

Abbildung 4.6: Linear fallende Zahlungsfunktionen und Barwertfunktionen (Quelle: In Anlehnung an Shtub/Etgar (1999), S. 332f.)

52 Vgl. Etgar/Shtub (1999), S. 331. Sowohl die positive Zahlungsfunktion als auch die Zahlungsfunktion mit positivem Startwert beinhalten auch die Möglichkeit $\mathrm{A}=0$. 
Für den dargestellten Fall einer konstanten positiven bzw. negativen Zahlungsfunktion gilt, dass der Barwert dieser Zahlung umso größer ist, je früher bzw. später das Ereignis terminiert wird. Aus dem Fall einer Zahlungsfunktion mit negativer Steigung resultiert eine nichtlineare Barwertfunktion, so dass eine wie oben getroffene allgemeingültige Aussage nicht möglich ist und eine differenzierte Betrachtung bei der Festlegung der Ereigniszeit notwendig wird. ${ }^{53}$ Im Rahmen der im vierten und fünften Kapitel erfolgenden Untersuchungen sind diese hier vorgestellten Zahlungsfunktionen relevant. Der regelmäßig unterstellte Fall konstanter und vom Zeitpunkt unabhängiger Zahlungen lässt sich etwa von Zahlungen abgrenzen, die Bestandteil eines Anreizsystems sind und deren Höhe von dem Zeitpunkt der Zahlung abhängt.

Kazaz und Sepil untersuchen eine Problemstellung aus der Sicht des Auftragnehmers, bei der Auszahlungen am Ende jedes Vorgangs anfallen und Einzahlungen in Form von Fortschrittszahlungen am Monatsende erfolgen. ${ }^{54}$ Unter der Annahme der Kenntnis der Vorgangskosten und -dauern sowie des Gewinnzuschlagsatzes, des Diskontierungszinssatzes und des spätesterlaubten Endes der Vorgänge lässt sich für jeden Vorgang eine so genannte Activity-Profit-Curve ermitteln, die den Kapitalwert in Abhängigkeit von dem geplanten Vorgangsende visualisiert. ${ }^{55}$

In Abbildung 4.7 wird beispielhaft ein Vorgang mit einer Dauer von 20 Tagen betrachtet, dessen Durchführung aufgrund der Vorgänger-/Nachfolgerbeziehungen in einem Zeitintervall zwischen $t=20$ und $t=90$ stattfindet. Die aus dem Vorgang resultierenden Einzahlungen erfolgen in diesem Beispiel jeweils zum Monatsende, d. h. nach 30, 60 und 90 Tagen, wobei teilweise abgeschlossene Vorgänge nach Anteilen vergütet werden. Auszahlungen sind bei Abschluss des Vorgangs fällig. ${ }^{56}$ Es zeigt sich, dass ein Vorgang den Kapitalwert des Projektes in größtem Ausmaß erhöht, wenn das Vorgangsende mit dem Monatsende identisch ist. Dann können die zu diesem Zeitpunkt erforderlichen Auszahlungen sofort durch die um den Gewinnzuschlag höheren Einzahlungen kompensiert werden. ${ }^{57}$

53 Insbesondere furr den Fall $\mathrm{A}>0$ und $\mathrm{B}<0 \mathrm{vgl}$. die Ausführungen in Vanhoucke/Demeulemeester/Herroelen (2001b). Hier wird auch das Minimum der Kapitalwertfunktion in Abhängigkeit von den Faktoren A und $B$ berechnet.

54 Vgl. Kazaz/Sepil (1996).

55 Diese Abhängigkeit kann fur Diskontraten kleiner als 0,02\% pro Tag als linear angenommen werden. Vgl. Kazaz/Sepil (1996), S. 1265ff.

56 Vgl. Vanhoucke/Demeulemeester/Herroelen (2003), S. 605.

57 Vgl. Kazaz/Sepil (1996), S. 1265. 


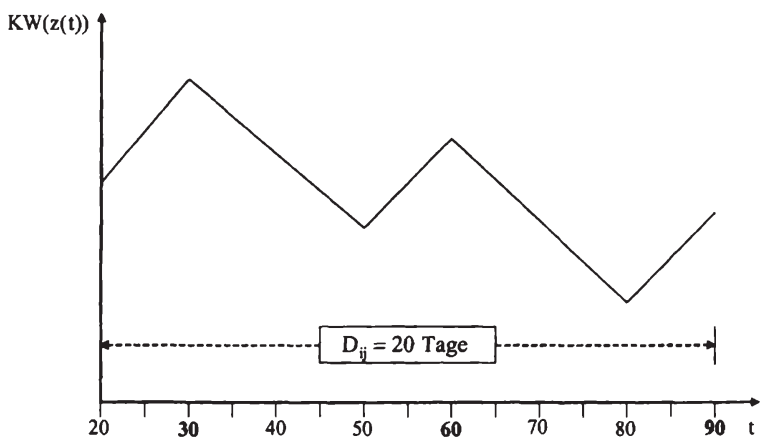

Abbildung 4.7:Activity-Profit-Curve

(Quelle: In Anlehnung an Vanhoucke/Demeulemeester/Herroelen (2003), S. 607.)

Vanhoucke, Demeulemeester und Herroelen knüpfen an der von Kazaz und Sepil diskutierten Problematik an und leiten für den Fall eines Vorgangsknotennetzplans anhand der Activity-Profit-Curve für jeden mit einem Puffer versehenen Vorgang dessen Start- und Endzeitpunkt ab. ${ }^{58}$ Für die Lösung derartiger Problemstellungen unter Berücksichtigung knapper Ressourcen werden von Sepil und Ortac drei Heuristiken getestet, deren Einsatz zu annähernd optimalen Lösungen führt. ${ }^{59}$ Diese Bindung der Fortschrittszahlungen an durch den Kalender definierte Termine kann beispielsweise im Rahmen einer Festpreisvereinbarung von Bedeutung sein.

Eine Erweiterung der in der Literatur diskutierten Resource Contrained Project Scheduling Probleme stellen Smith-Daniels, Padman und Smith-Daniels vor, bei der das für das Projekt verfügbare Kapital die knappe Ressource darstellt. ${ }^{60}$ Im Unterschied zu früheren Veröffentlichungen ${ }^{61}$ ist es im Rahmen dieser Problemstellung aus der Sicht des Auftragnehmers möglich, erhaltene Fortschrittszahlungen als neues Kapital in das Projekt einzubringen und damit eine größere Anzahl von Vorgängen gleichzeitig durchzuführen, was zu einer verkürzten Projektdauer und einem höheren Projektkapitalwert führen kann. ${ }^{62}$

\footnotetext{
58 Vgl. Vanhoucke/Demeulemeester/Herroelen (2003), S. 608. Dabei wird die Activity-Profit-Curve in Teilabschnitte untergliedert, die jeweils ein steigendes und ein fallendes Teilstück enthalten. Über die Bestimmung des weighted-earliness-tardiness-trade-off wird dasjenige Teilstück ausgewăhlt, welches den Trade-Off minimiert.

59 Vgl. Sepil/Ortac (1997).

60 Vgl. Smith-Daniels/Padman/Smith-Daniels (1996). Es handelt sich um eine Erweiterung einer früher veröffentlichten Arbeit, vgl. Smith-Daniels/Smith-Daniels (1987).

61 Vgl. als ersten Ansatz zum Capital Constrained Project Scheduling Doersch/Patterson (1977). Hier wird ein maximal fur die Dauer des Projektes verfugbares Kapital angenommen, dessen Hobhe durch die wăhrend der Projektdauer erfolgenden Aus- und Einzahlungen unberuhrt bleibt.

62 Vgl. Smith-Daniels/Padman/Smith-Daniels (1996), S. 242.
} 
Özdamar und Dündar schlagen als Erweiterung die Lösung einer Problemstellung vor, bei der der Auftragnehmer in Abhängigkeit von dem Projektfortschritt sowie bereits erhaltener bzw. erwarteter Einzahlungen die Ausführungsmodi und damit die Kosten und die Zeitpunkte von zukünftigen Vorgängen bestimmen kann. ${ }^{63}$ Die Zielfunktion des mathematischen Optimierungsmodells berücksichtigt sowohl die Zielsetzung der Kapitalwertmaximierung als auch das Ziel der Minimierung der Projektdauer, indem bei Terminüberschreitungen eine Strafzahlung festgelegt wird. ${ }^{64}$

De Reyck und Herroelen legen den Schwerpunkt ihres 1998 veröffentlichten Beitrags auf die Annahme unterschiedlicher möglicher Abhängigkeitsbeziehungen zwischen Vorgängen. ${ }^{65}$ Ist bisher davon ausgegangen worden, dass ein Vorgang zeitlich unmittelbar nach dem Ende des vorangegangenen und abgeschlossenen Vorgangs folgt, erweitern die Autoren die Scheduling Problematik um die Möglichkeit der Festlegung von minimalen und maximalen Zeitabständen zwischen aufeinander folgenden Vorgängen. Eine Start-Start-Beziehung legt beispielsweise fest, dass ein Vorgang frühestens bzw. spätestens eine bestimmte Dauer nach dem Beginn des Vorgängervorgangs beginnen kann bzw. beginnen muss.

Derart lassen sich auch Start-Ende-, Ende-Start- und Ende-Ende-Beziehungen zwischen Vorgängen abbilden, so dass zum Beispiel ein zeitgleicher Start von Vorgängen erzwungen oder die Möglichkeit eines verzögerten Starts von Vorgängen verboten wird. ${ }^{66}$ Dies ist unter anderem bei Vorgängen notwendig, bei denen Wartezeiten zwischen dem Ende des einen und dem Beginn des nachfolgenden Vorgangs eingehalten werden müssen. Als Anwendung kann beispielsweise das Abbinden von Beton oder die Trocknung eines Anstrichs vor dem Lackieren betrachtet werden. Hierbei werden keine Arbeitskräfte und Maschinen benötigt, so dass diese reine Wartezeit nicht als gesonderter Vorgang abgebildet werden muss, sondern wie in Abbildung 4.8 und Abbildung 4.9 veranschaulicht, als Ende-Start-Beziehung mit Zeitabstand definiert wird.

63 Vgl. Özdamar/Dündar (1997). Die Autoren betrachten eine Anwendung aus dem Wohnungsbau, wobei die Kenntnisse über Höhe und Zeitpunkt der Einzahlungen für fertig gestellte Wohnungen wăhrend der Projektlaufzeit unsicher sind.

64 Zur mathematischen Modellierung vgl. Özdamar/Dündar (1997), S. 1190.

$65 \mathrm{Vgl}$. De Reyck/Herroelen (1998) sowie Herroelen/De Reyck/Demeulemeester (1998), die einen Überblick über das Resource Contrained Project Scheduling geben.

66 Vgl. De Reyck/Herroelen (1998), S. 2f. Kuhlmann (2003) bezieht die Berücksichtigung verschiedener Reihenfolgebziehungen von Vorgängen sowie insbesondere die Möglichkeit, Vorgånge zu unterbrechen, in den entwickelten heuristischen Lösungsansatz zum Project Scheduling ein. 


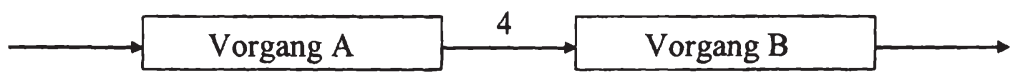

Abbildung 4.8: Ende-Start-Beziehung mit Zeitabstand in einem Vorgangsknotennetzplan

(Quelle: In Anlehnung an Schwarze (2001), S. 120.)

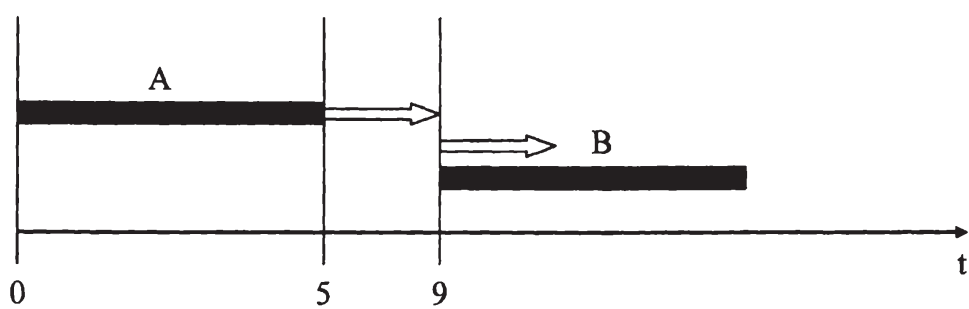

Abbildung 4.9: Ende-Start-Beziehung mit Zeitabstand als Balkendiagramm (Quelle: In Anlehnung an Schwarze (2001), S. 120.)

Hier wird ein Mindestabstand von vier Zeiteinheiten zwischen Vorgang A und Vorgang B erzwungen. Der in Abbildung 4.9 dargestellte Pfeil über Vorgang B deutet an, dass dieser Vorgang nicht zum Zeitpunkt 9 beginnen muss, sondern auch auf einen späteren Zeitpunkt verschoben werden kann, sofern dies die nachfolgenden Vorgänge zulassen. ${ }^{67}$

Eine Erweiterung des Kapitalwert maximierenden, ressourcenbeschränkten Project Scheduling Problems führen Chiu und Tsai ein. ${ }^{68}$ Sie übertragen die bisherigen Forschungsansätze auf den Fall der Multi-Projektplanung. Hierbei sind die Vorgänge innerhalb mehrerer zeitlich parallel durchzuführender Projekte so zu terminieren, dass unter Beachtung der Ressourcenbeanspruchung und -verfügbarkeit die Summe der Projektkapitalwerte aus der Differenz der mit Vorgängen oder Ereignissen verbundenen diskontierten Aus- und Einzahlungen maximal ist. Das mathematische gemischtganzzahlige, nichtlineare, deterministische Modell enthält eine zu maximierende Zielfunktion, die neben Barwerten der Aus- und Einzahlungen sowie einer möglichen Abschlusseinzahlung auch Bonus- bzw. Strafzahlungen für den frühzeitigen bzw. verspäteten Abschluss von Projekten beinhaltet. ${ }^{69}$ Obwohl durch die Modellierung der Bonus- und Strafzahlungen Aspekte des Vertragsdesigns berücksichtigt werden, liegt der

67 Vgl. Schwarze (2001), S. 120.

$68 \mathrm{Vgl}$. Chiu/Tsai (2002).

69 Vgl. Chiu/Tsai (2002), S. 56 f. 
Schwerpunkt der Untersuchungen auf der Entwicklung eines Lösungsalgorithmus sowie der Lösung verschiedener Testprobleme.

Eine Anwendung des Project Schedulings bei einer belgischen Wasserversorgungsunternehmung zeigen Vanhoucke und Demeulemeester. ${ }^{70}$ Für ein Investitionsprojekt zur Erweiterung der Produktionsanlagen, welches über einen Zeitraum von sechs Jahren hohe Investitionen verursacht, soll untersucht werden, welche zeitliche Abfolge der Projektvorgänge am besten geeignet ist, den Barwert der mit der Investition verbundenen Auszahlungen zu minimieren und gleichzeitig den geplanten Projektendtermin einzuhalten. ${ }^{71}$ Ausgehend von den jeweils frühestmöglichen Startzeitpunkten für jeden Vorgang wird vorgeschlagen, Vorgänge mit einem Puffer sukzessive später zu terminieren und die Veränderung des Kapitalwertes zu beobachten. Werden alle Vorgänge zu ihren spätesterlaubten Terminen geplant und der Puffer zu 100\% ausgenutzt, besteht die Gefahr, dass der Projektendtermin nicht eingehalten werden kann. Daher sollte jeder Vorgang so spät geplant werden, dass ein vorgegebener Mindestpuffer erhalten bleibt. ${ }^{72}$ Das dargestellte Vorgehen erhöht mit jeder Verschiebung eines Vorgangs den Barwert der Zahlungen, da lediglich Auszahlungen in die Betrachtung eingehen. Eine differenzierte Betrachtung von Vorgängen, die evtl. zu Terminüberschreitungen führen können und daher bei der Planung mit einem Mindestpuffer berücksichtigt werden sollten, erfolgt an dieser Stelle nicht. Es ist ebenfalls keine Aussage zu finden, wie viel Pufferzeit ein Vorgang beinhalten sollte, damit das Projektende termingerecht erreicht wird. ${ }^{73}$

Für einen Vergleich der in diesem Kapitel vorgestellten Ansätze für die Modellierung und Lösung von Projektplanungsproblemen mit der Zielsetzung der Kapitalwertmaximierung werden die einzelnen Beiträge im Folgenden hinsichtlich ausgewählter und für die weiteren Untersuchungen im Rahmen dieser Arbeit relevanten Problemcharakteristika analysiert.

\footnotetext{
70 Vgl. Vanhoucke/Demeulemeester (2003).

"Es wird davon ausgegangen, dass aus der Sicht des Auftragnehmers während der Projektdauer ausschließlich Auszahlungen stattfinden und nach Projektabschluss Einzahlungen erfolgen, die die Rentabilität des Projektes sicherstellen. Vgl. hierzu Vanhoucke/Demeulemeester (2003), S. 37f. Insbesondere aus der Sicht des Auftragnehmers wird im Rahmen dieser Arbeit jedoch unterstellt, dass dieser während der Projektlaufzeit mehrere Teilzahlungen von dem Auftraggeber erhălt.

72 Vgl. Vanhoucke/Demeulemeester (2003), S. 34ff.

73 Ein Vorgehen fur die Zuweisung von Pufferzeiten nach bestimmten Kriterien zeigt Werners (2000).
} 


\subsubsection{Analyse und Bewertung der Problemcharakteristika}

Die im vorigen Abschnitt vorgestellten Project Scheduling Problemstellungen lassen sich durch die Berücksichtigung unterschiedlicher Problemcharakteristika beschreiben. Tabelle 4.2 gibt einen Überblick über die Problemcharakteristika und deren Berücksichtigung in den einzelnen untersuchten Literaturstellen.

Alle Beiträge betrachten die Problematik aus der Sicht des Auftragnehmers. Das heißt, dass Auszahlungen die für die Leistungserstellung notwendigen Zahlungen, beispielsweise für Löhne und Gehälter oder Material, darstellen. Einzahlungen sind die für die Leistungserstellung durch den Auftraggeber erbrachten Zahlungen. Die Sichtweise des Auftraggebers, für den die zu leistenden Zahlungen Auszahlungen und die nach Projektabschluss erwarteten Erlöse aus dem Projekt Einzahlungen darstellen, wird bei den hier untersuchten Beiträgen nicht berücksichtigt. Zwar lassen sich die Modelle zur Planungsunterstützung des Auftragnehmers auf die Situation des Auftraggebers übertragen, dennoch werden die Auswirkungen eines aus der Sicht des Auftragnehmers erstellten Projektplanes auf den Projektkapitalwert des Auftraggebers nicht berücksichtigt. Die Fokussierung der Untersuchungen auf die Sichtweise des Auftragnehmers zeigt, dass das Project Scheduling diesen insbesondere bei der Planung und Steuerung von Vorgängen sowie Ereignissen unterstützt.

Die Frage nach der richtigen Wahl der für die Modelle und Lösungsverfahren notwendigen Problem-Repräsentation in Form einer Darstellung als Vorgangspfeil- oder Vorgangsknotennetzplan kann nicht eindeutig beantwortet werden. Die Untersuchungen zeigen, dass die Anzahl der Darstellungen als Vorgangspfeilnetzplan überwiegt und damit häufig sowohl Aus- als auch Einzahlungen den Ereignissen zugeordnet sind. Wird hingegen ein Vorgangsknotennetzplan zugrunde gelegt, bei dem eine Berücksichtigung von Ereignissen nicht vorgesehen ist, so müssen die Zahlungen an die Projektvorgänge bzw. die Einzahlungen an das Projektende gekoppelt sein. Mit Ausnahme des Beitrags von De Reyck/Herroelen (1998) sind die Vorgänge durch eine feste Ende-Start-Beziehung gekennzeichnet, so dass die Modellierung einer Einhaltung von Maximal- oder Minimalzeitabständen zwischen Vorgängen keine Beachtung findet. Die Notwendigkeit der Festlegung eines Zeitpunktes für das Projektende wurde von Grinold (1972) erkannt, jedoch nicht bei allen zeitlich folgenden Beiträgen berücksichtigt. 


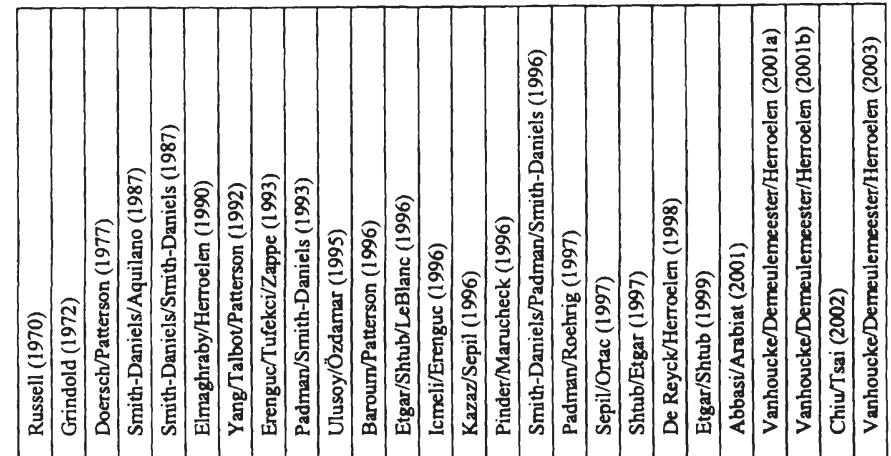

\begin{tabular}{|c|c|c|c|c|c|c|c|c|c|c|c|c|c|c|c|c|c|c|c|c|c|c|c|c|c|c|}
\hline $\begin{array}{l}\text { Projektplanung } \\
\text { Auftragnehmer } \\
\text { Auftraggeber }\end{array}$ & $\bullet$ & $\bullet$ & $\bullet$ & $\bullet$ & $\bullet$ & $\bullet$ & $\bullet$ & $\bullet$ & $\bullet$ & $\bullet$ & $\bullet$ & $\bullet$ & $\bullet$ & $\bullet$ & $\bullet$ & $\bullet$ & $\bullet$ & $\bullet$ & $\bullet$ & - & $\bullet$ & $\bullet$ & $\bullet$ & $\bullet$ & $\bullet$ & $\bullet$ \\
\hline $\begin{array}{l}\text { Netzplandarstellung } \\
\text { Vorgangspfeilnetz } \\
\text { Vorgangsknotennetz }\end{array}$ & $\bullet$ & - & $\bullet$ & $\bullet$ & $\bullet$ & $\bullet$ & $\bullet$ & $\bullet$ & $\bullet$ & $\bullet$ & $\bullet$ & - & $\bullet$ & $\bullet$ & $\bullet$ & $\bullet$ & - & $\bullet$ & $\bullet$ & $\bullet$ & - & $\bullet$ & $\bullet$ & $\bullet$ & $\bullet$ & $\bullet$ \\
\hline $\begin{array}{l}\text { Anordnungsbexiehung } \\
\text { Ende-Start } \\
\text { Min-/Max-Abstände } \\
\text { Projektende fest }\end{array}$ & $\bullet$ & - & $\bullet$ & - & - & $\bullet$ & $\bullet$ & - & - & - & • & - & - & - & $\bullet$ & $\bullet$ & $\bullet$ & - & - & $\bullet$ & $\bullet$ & $\bullet$ & - & $\bullet$ & $\bullet$ & $\bullet$ \\
\hline $\begin{array}{l}\text { Auszahlungen } \\
\text { zu Ereignissen } \\
\text { zu Vorgăngen } \\
\text { Zeitpunkt variabel } \\
\text { Betrag fest } \\
\text { Betrag variabel } \\
\text { Höhe zeitabhängig } \\
\text { Hőhe leistungsabhăngig }\end{array}$ & $\bullet$ & $\bullet$ & $\begin{array}{l}\bullet \\
\bullet \\
\bullet\end{array}$ & $\bullet$ & $\bullet$ & $\bullet$ & $\bullet$ & $\bullet$ & $\bullet$ & $\bullet$ & • & - & $\bullet$ & • & $\bullet$ & $\bullet$ & - & - & - & - & $\begin{array}{l}\bullet \\
\bullet \\
\bullet \\
\bullet\end{array}$ & $\bullet$ & - & - & $\bullet$ & - \\
\hline $\begin{array}{l}\text { Einzahlungen } \\
\text { zu Ereignissen } \\
\text { zu Vorgängen } \\
\text { zum Projektende } \\
\text { zum Monatsende } \\
\text { Zeitpunkt variabel } \\
\text { Betrag fest } \\
\text { Betrag variabel } \\
\text { Hőhe zeitabhångig } \\
\text { Hőhc leistungsabhängig } \\
\text { Hőhe kostenabhăngig }\end{array}$ & $\bullet$ & $\bullet$ & $\bullet$ & $\bullet$ & $\bullet$ & • & • & $\bullet$ & $\bullet$ & $\bullet$ & $\bullet$ & $\bullet$ & $\bullet$ & - & $\bullet$ & $\bullet$ & $\bullet$ & • & - & • & - & $\bullet$ & • & - & $\bullet$ & $\bullet$ \\
\hline $\begin{array}{l}\text { Ressourcen } \\
\text { keine } \\
\text { Ressource Kapital } \\
\text { Ressourcen (sonstige) }\end{array}$ & $\bullet$ & $\bullet$ & $\bullet$ & $\bullet$ & $\bullet$ & $\bullet$ & $\bullet$ & - & $\bullet$ & $\bullet$ & $\bullet$ & $\bullet$ & $\bullet$ & $\bullet$ & $\bullet$ & $\bullet$ & $\bullet$ & - & $\bullet$ & $\bullet$ & $\bullet$ & $\bullet$ & $\bullet$ & $\bullet$ & $\bullet$ & - \\
\hline $\begin{array}{l}\text { Vertragscharakteristika } \\
\text { Anzahl Einzahlungen fest } \\
\text { Intervalle Einzahlungen fest } \\
\text { Häufigkeit Einzahlungen fest } \\
\text { Anreizsystem }\end{array}$ & & & $\bullet$ & & $\bullet$ & & $\bullet$ & & & & & $\bullet$ & & $\bullet$ & & & & - & $\bullet$ & & $\bullet$ & & & $\bullet$ & $\bullet$ & - \\
\hline mathematisches Modell & $\bullet$ & $\bullet$ & $\bullet$ & & $\bullet$ & & - & $\bullet$ & & & $\bullet$ & - & $\bullet$ & - & - & $\bullet$ & & & - & $\bullet$ & $\bullet$ & & & - & - & $\bullet$ \\
\hline $\begin{array}{l}\text { Lösungsverfahren } \\
\text { exakt } \\
\text { heuristisch }\end{array}$ & $\bullet$ & $\bullet$ & - & & $\bullet$ & $\bullet$ & $\bullet$ & $\bullet$ & $\bullet$ & & $\bullet$ & & - & - & $\bullet$ & $\bullet$ & - & $\bullet$ & $\bullet$ & $\bullet$ & - & - & $\bullet$ & $\bullet$ & $\bullet$ & $\bullet$ \\
\hline
\end{tabular}

Tabelle 4.2: Literaturüberblick Charakteristika Project Scheduling 
Alle untersuchten Probleme des Project Schedulings besitzen die Gemeinsamkeit, dass die Höhe der Auszahlungen des Auftragnehmers bekannt ist und die Festlegung der Zeitpunkte der Zahlungen Teil des Planungsproblems ist. Ist die Höhe der Auszahlungen von dem Zahlungszeitpunkt oder von bestimmten Ausführungsmodi der Vorgänge abhängig, so ist diese im Rahmen der Zeitplanung ebenfalls für jeden Zeitpunkt determiniert. Auch die Höhe der Einzahlungen ist für jeden Planungszeitpunkt bekannt. In fünf Beiträgen hängt die Höhe der Einzahlungen von dem Zeitpunkt der Zahlung $a b,{ }^{74}$ bei zwei Arbeiten ist die Höhe der Zahlungen an die bis zu dem Zeitpunkt der Zahlung angefallenen Auszahlungen geknüpft. ${ }^{75}$ Mit der Festlegung des Zahlungszeitpunktes sind die von bestimmten Faktoren abhängigen Zahlungen ebenfalls im Rahmen einer Projektplanung genau bestimmbar. In drei Beiträgen ist die Planung des Zahlungszeitpunktes nicht Teil der Planungsaufgabe, da in diesen Fällen die Zahlungen regelmäßig, beispielsweise zum Monatsende, geleistet werden. ${ }^{76}$

Die Einbindung von Ressourcen in die Problemstellung ist vorwiegend davon abhängig, welche weiteren Problemcharakteristika Berücksichtigung finden. Werden etwa Anreizmechanismen in Form von zeitabhängigen Zahlungen oder Vertragscharakteristika, wie etwa konstante Zahlungsintervalle, in die Problemformulierung aufgenommen, wird das Problem häufig als Modell ohne Berücksichtigung von Ressourcen formuliert. Es kann zudem festgestellt werden, dass die Einbeziehung betriebswirtschaftlicher Aspekte etwa durch die Einbindung von Anreizwirkungen oder zeit- bzw. kostenabhängigen Zahlungen mit zunehmender Aktualität der Beiträge zunimmt, jedoch durch die einseitige Betrachtung des Auftragnehmers als Entscheidungsträger wichtige Fragen der Projektkooperation zwischen dem Auftragnehmer und dem Auftraggeber als Projekteigentümer unberücksichtigt bleiben. Diese Aspekte werden daher im Rahmen des fünften Kapitels näher analysiert. Der Schwerpunkt der Betrachtungen lag bisher auf den unterschiedlichen Problemcharakteristika, so dass die mathematischen Modellformulierungen bewusst ausgeklammert worden sind. Als Grundlage einer quantitativen Planungsunterstützung für die Projektplanung wird das Project Scheduling Problem daher im folgenden Kapitel als mathematisches Modell formuliert. Hierfür werden die verschiedenen zuvor diskutierten Modellierungsaspekte in die für diese Arbeit verwendete und einheitliche Notation überführt.

\footnotetext{
74 Vgl. Etgar/Shtub/LeBlanc (1996); Shtub/Etgar (1997); Etgar/Shtub (1999); Vanhoucke/Demeulemeester/ Herroelen (2001a); Vanhoucke/Demeulemeester/Herroelen (2001b).

75 Vgl. Kazaz/Sepil (1996); Vanhoucke/Demeulemeester/Herroelen (2003).

76 Vgl. Kazaz/Sepil (1996); Sepil/Ortac (1997); Vanhoucke/Demeulemeester/Herroelen (2003).
} 


\subsubsection{Modellierung des Project Scheduling Problems}

In allgemeiner Form lässt sich das Project Scheduling Problem wie folgt beschreiben: Es existiert ein Projekt mit M Vorgängen und I Ereignissen. Jeder Vorgang verbindet in einem Vorgangspfeilnetzplan ein Ereignis i mit einem Ereignis j. Die Dauern der Vorgänge $\mathrm{D}_{\mathrm{ij}}$ sind bekannt. Die aus der Sicht des Auftragnehmers erfolgenden Zahlungen $\widehat{Z}_{i}$, die aufgrund der mit der Leistungserstellung verbundenen Kosten negativ (Auszahlung) oder durch die Zahlung einer Vergütung durch den Auftraggeber positiv (Einzahlung) sein können, sind an die Zeitpunkte der Ereignisse geknüpft und werden ebenfalls als bekannt vorausgesetzt. ${ }^{77}$ Unter Berücksichtigung des Barwertes dieser Zahlungen werden die Ereigniszeitpunkte im Rahmen des Project Scheduling Problems unter Beachtung einer vorgegebenen Vorgangsreihenfolge so geplant, dass der Projektkapitalwert des Auftragnehmers maximal ist. ${ }^{78}$

Für die Bestimmung des Projektkapitalwertes lassen sich in den untersuchten Veröffentlichungen zum Project Scheduling zwei unterschiedliche Diskontierungsansätze identifizieren. Der Barwert BW einer in t Perioden zu leistenden oder zu erhaltenden Zahlung $\mathrm{Z}_{\mathrm{t}}$ lässt sich unter Annahme des Diskontierungszinssatzes DISKZ, zu diskreten Zeitpunkten erfolgender Zahlungen sowie jährlicher Verzinsung wie folgt ermitteln:

$$
\mathrm{BW}=\mathrm{Z}_{\mathrm{t}} \cdot(1+\mathrm{DISKZ})^{-\mathrm{t}}
$$

Dabei wird der hintere Ausdruck des Produktes als Abzinsungsfaktor bezeichnet. ${ }^{79}$ Obwohl diese Form der Diskontierung von Zahlungen zur Bestimmung des Kapitalwertes in den Lehrbüchern der Investitionstheorie vorherrschend ist, ${ }^{80}$ findet sie nur wenig Anwendung in den im Rahmen dieser Arbeit untersuchten Literaturstellen. ${ }^{81}$ Die Mehrzahl der Autoren unterstellt für die Modellierung der Kapitalwert maximierenden Zielfunktion eine kontinuierliche Verzinsung mit einem über die Zeit konstan-

\footnotetext{
Vgl. Etgar/Shtub (1999), S. 329.

Vgl. Demeulemeester/Herroelen (2002), S. 139.

79 Vgl. Busse von Colbe/Laßmann (1990), S. 30.

${ }^{80}$ Zur Berechnung des Barwertes bzw. Kapitalwertes vgl. etwa Blohm/Lüder (1995), S. 58ff.; Breuer (2002), S. 59ff.; Drukarczyk (2003), S. 12; Schmidt/Terberger (1997), S. 128f.

81 Für Modelle mit diskreter Verzinsung vgl. Etgar/Shtub (1999); Etgar/Shtub/LeBlanc (1996); Shtub/Etgar (1997); Smith-Daniels/Smith-Daniels (1987); Vanhoucke/Demeulemeester/Herroelen (2001b).
} 
ten Zinssatz DISKZ und verwendet den folgenden Ausdruck für die Bestimmung des Barwertes BW einer zum Zeitpunkt $t$ anfallenden Zahlung $Z_{\mathrm{t}}:^{82}$

$\mathrm{BW}=\mathrm{Z}_{\mathrm{t}} \cdot \mathrm{e}^{-\mathrm{DISKZ} \cdot \mathrm{t}}$

Dieser Ausdruck lässt sich aus der Grenzwertbetrachtung der Formel (4.1) für den Fall unendlich vieler Zinszahlungen pro Periode herleiten. ${ }^{83}$ Hax bezeichnet den Unterschied zwischen den zwei Vorgehensweisen als rein formaler Art und empfiehlt die Anwendung der kontinuierlichen Diskontierung bei Problemstellungen, bei denen bestimmte Größen als kontinuierliche Funktionen der Zeit abgebildet werden sollen. ${ }^{84}$

Für die in dieser Arbeit untersuchte Problemstellung erscheint die Verwendung der diskreten Verzinsung als sinnvoll, da sowohl die Zahlungen der mit der Leistungserstellung verbundenen Kosten durch den Auftragnehmer als auch die Vergütungszahlungen des Auftraggebers ausschließlich zu diskreten Zeitpunkten stattfinden.

Für die Abbildung der Problemstellung in Form eines mathematischen Modells kommen zwei unterschiedliche Ansätze in Betracht. Die Untersuchung der relevanten Project Scheduling Literatur zeigt, dass oft eine Formulierung als nichtlineares Optimierungsproblem ausgewählt wird. ${ }^{85}$

Hierbei werden die Zeitpunkte $t_{i}$, zu denen ein Ereignis i geplant wird, als Entscheidungsvariablen modelliert, so dass die Kapitalwert maximierende Zielfunktion bei kontinuierlicher Diskontierung dem Ausdruck (4.3) und bei diskreter Diskontierung dem Ausdruck (4.4) entspricht:

$$
\begin{array}{ll}
\max & \sum_{i=1}^{I} \widehat{Z}_{i} \cdot e^{-D I S K Z \cdot t_{i}} \\
\max & \sum_{i=1}^{I} \widehat{Z}_{i} \cdot(1+D I S K Z)^{-t_{i}}
\end{array}
$$

Es werden die zu einem Ereignis $i$ anfallenden und zu einer Nettozahlung $\widehat{Z}_{i}$ zusammengefassten Ein- und Auszahlungen von dem Ereigniszeitpunkt $t_{i}$ auf den Zeitpunkt

82 Zur Diskontierung mit stetiger Verzinsung vgl. Busse von Colbe/Laßmann (1990), S. 32ff. Alle in Tabelle 4.2 aufgeführten und nicht in der vorherigen Fußnote genannten Beiträge zum Project Scheduling verwenden die kontinuierliche Zinszahlung bei der Modellierung der Kapitalwert maximierenden Zielfunktion.

83 Vgl. Busse von Colbe/Laßmann (1990), S. 33.

84 Vgl. Hax (1993), S. 12.

85 Vgl. etwa Chiu/Tsai (2002), S. 57; De Reyck/Herroelen (1998), S. 4; Erenguc/Tufekci/Zappe (1993), S. 28; Etgar/Shtub (1999), S. 331; Grinold (1972), S. 125; Russell (1970), S. 359. 
null mit dem Zinssatz DISKZ diskontiert und für alle Ereignisse summiert. Die reellwertige Variable $t_{i}$, die bei der Zielfunktion (4.3) als Argument in die Exponentialfunktion $\mathrm{e}^{\mathrm{x}}$ und bei der Zielfunktion (4.4) als Exponent in den Ausdruck 1/x eingeht, ist Ursache für die Nichtlinearität der Zielfunktion. Da kein universelles Verfahren zur Lösung nichtlinearer Optimierungsprobleme existiert, sind spezielle auf die Problemformulierung zugeschnittene Lösungsverfahren notwendig. ${ }^{86}$

Die Formulierung der Problemstellung als ganzzahliges lineares Optimierungsproblem stellt eine Variante zu der vorgestellten Formulierung als nichtlineares Problem dar und wird im Folgenden Teil des Grundmodells zum Project Scheduling sein. Für die Modellierung des Project Scheduling Problems werden die folgenden Bezeichnungen der Indizes, der Daten sowie der Variablen vereinbart:

\section{Indizes und Indexmengen:}

$i, j=1, \ldots, I \quad:$ Ereignisse

$\mathrm{t}=0, \ldots, \mathrm{T} \quad$ : Ereigniszeitpunkte

\section{Daten:}

DISKZ : Diskontierungszinssatz

DISKF $_{t} \quad$ : Diskontierungsfaktor, DISKF $_{t}=\left(1+\right.$ DISKZ $^{-t}$

$D_{i j} \quad:$ Dauer des Vorgangs zwischen Ereignis i und Ereignis $j$

DMax : Spätesterlaubter Zeitpunkt des letzten Ereignisses

$\mathrm{V} \quad$ : Menge der einem Vorgang unmittelbar vorangehenden und unmittelbar nachfolgenden Ereignisse (i,j)

$\widehat{Z}_{\mathrm{i}} \quad:$ Höhe der Nettozahlung zu Ereignis $\mathrm{i}$

\section{Variablen:}

$\mathrm{x}_{\mathrm{it}}=\left\{\begin{array}{l}\mathrm{l} \text {, wenn Ereignis i zum Zeitpunkt } \mathrm{t} \text { stattfindet } \\ 0, \text { sonst }\end{array}\right.$

Das Project Scheduling Problem kann als ganzzahliges lineares Programmierungsmodell formuliert werden: ${ }^{87}$

${ }_{87}^{86}$ Vgl. Domschke/Drexl (2002), S. 160.

87 Die Modellformulierung orientiert sich an den in der zuvor aufgefuihrten Literatur vorgeschlagenen Modellen und wird im Hinblick auf die folgenden Kapitel mit der in dieser Arbeit verwendeten Notation versehen. Die Grundlage für die Formulierung der Restriktion (4.6) ist bei Talbot (1982), S. $1197 \mathrm{zu}$ finden. Die Bedingung (4.7) geht auf Russell (1970), S. 359 zurück. Die Anforderung der Modellierung eines feststehenden Projektendes und damit der Formulierung der Restriktion (4.8) basiert auf Grinold (1972), S. 124. 
$\max \mathrm{z}=\sum_{\mathrm{i}=1}^{\mathrm{I}} \sum_{\mathrm{t}=0}^{\mathrm{T}} \mathrm{DISKF}_{\mathrm{t}} \cdot \widehat{\mathrm{Z}_{\mathrm{i}}} \cdot \mathrm{x}_{\mathrm{it}}$

so dass

$\sum_{\mathrm{t}=0}^{\mathrm{T}} \mathbf{x}_{\mathrm{it}}=1$

$\forall \mathrm{i}$

$\sum_{t=0}^{T} t \cdot x_{j t}-\sum_{t=0}^{T} t \cdot x_{i t} \geq D_{i j}$

$\forall(\mathrm{i}, \mathrm{j}) \in \mathrm{V}$

$\sum_{\mathrm{t}=0}^{\mathrm{T}} \mathrm{t} \cdot \mathrm{x}_{\mathrm{It}} \leq \mathrm{DMax}$

$\mathbf{x}_{\text {it }} \in\{0,1\}$

$\forall \mathrm{i}, \mathrm{t}$

Die Zielfunktion (4.5) dieses Grundmodells zum Project Scheduling maximiert den Kapitalwert als Summe der Barwerte aller während des Projektes zu den Ereigniszeitpunkten stattfindenden Zahlungen des Auftragnehmers. Diese Zahlungen können sowohl mit der Leistungserstellung verbundene Kosten und somit Auszahlungen als auch Einzahlungen in Form von Vergütungen des Auftraggebers sein. Die Nebenbedingung (4.6) stellt sicher, dass für jedes Ereignis genau ein Zeitpunkt festgelegt wird, zu dem es stattfindet. Mit Hilfe der Restriktion (4.7) werden die durch den Projektnetzplan vorgegebenen Reihenfolgebeziehungen sowie die durch die Vorgangsdauern bestimmten zeitlichen Mindestabstände zwischen zwei unmittelbar aufeinander folgenden Ereignissen $\mathrm{i}$ und $\mathrm{j}$ eingehalten. Durch die Restriktion (4.8) wird der Zeitpunkt für das Stattfinden des letzten Ereignisses I durch das späteste Projektende DMax begrenzt. Die Definition der Entscheidungsvariablen als Binärvariablen schließt das Optimierungsproblem mit der Bedingung (4.9) ab.

Die Anwendung dieses Modells erfolgt in Kapitel 4.4.2 anhand eines Beispiels. Zunächst wird die Problemstellung des Project Schedulings erweitert, so dass eine Berücksichtigung unterschiedlicher Zahlungsmodalitäten im Rahmen der Projektplanung von Auftraggeber und Auftragnehmer möglich ist. 


\subsection{Das Payment Scheduling Problem und seine Anwendungen}

\subsubsection{Problemstellung und Stand der Forschung}

Das Payment Scheduling Problem mit der Zielsetzung der Kapitalwertmaximierung lässt sich wie folgt von dem in Kapitel 4.2 dargestellten Project Scheduling Problem abgrenzen: Im Rahmen des Project Schedulings sind die zu bestimmten Vorgängen bzw. Ereignissen zu leistenden Aus- und Einzahlungen als bekannt vorausgesetzt und aus der Sicht des Auftragnehmers die Zeitpunkte der Ereignisse unter Berücksichtigung der durch den Projektnetzplan vorgegebenen Reihenfolgebeziehungen so bestimmt worden, dass der Projektkapitalwert des Auftragnehmers maximiert wird.

Das Payment Scheduling Problem besteht hingegen darin, aus der Sicht des Auftraggebers neben den Zeitpunkten von Zahlungen und damit der Festlegung der Ereigniszeiten auch die Höhe der Zahlungen, insbesondere der Auszahlungen an den Auftragnehmer zu bestimmen, um das Ziel eines maximalen Projektkapitalwertes zu erreichen. ${ }^{88}$ Damit rückt der im Rahmen der Projektplanung bedeutsame Verhandlungsprozess zwischen Auftraggeber und Auftragnehmer in den Vordergrund der Betrachtungen.

Aus der Perspektive des Auftragnehmers ist ein Projektplan dann kapitalwertoptimal, wenn er eigene mit der Projektdurchführung entstehende Kosten so spät wie möglich und somit möglichst zu dem Zeitpunkt des Projektendes zahlt und die durch den Auftraggeber für seine Leistungen zu zahlenden Vergütungen so früh wie möglich, das heißt zum Projektbeginn erhält. ${ }^{89}$

Aus der Sicht des Auftraggebers stellen die Zahlungen an den Auftragnehmer Auszahlungen dar, so dass für diese Zahlungen von umgekehrten Zeitpräferenzen ausgegangen werden muss. Die durch den Projektvertrag festgelegten Zahlungsbestimmungen sind daher als Kompromisslösung und Folge der Verhandlungen zwischen Auftraggeber und Auftragnehmer anzusehen. ${ }^{90}$ Beispielsweise können beide Vertragsparteien vereinbaren, dass dem Auftragnehmer zu bestimmten Projektereignissen ein Betrag von konstanter Höhe gezahlt wird (Festpreisvertrag mit konstanten Zahlungen). Alter-

\footnotetext{
88 Vgl. Dayanand/Padman (1997), S. 907. Dies ist die zeitlich erste Literaturquelle, die das Project Scheduling Problem erweitert und damit die von Elmaghraby/Herroelen (1990), S. 35 geubte Kritik bezllglich der Annahme bei der Zeitplanung bekannter Zahlungsgrőßn aufgreift.

89 Vgl. Dayanand/Padman (1997), S. 907.

90 Vgl. Ulusoy/Cebelli (2000), S. 263.
} 
nativ kann eine Orientierung der Höhe der Zahlungen am Projektfortschritt durch die Vergütung der bis zu einem Ereignis entstandenen Kosten erfolgen (Kostenerstattungsvertrag). ${ }^{91}$ Die Fragestellung der Wahl einer Vergütungsart insbesondere aus der Sicht des Auftraggebers wird Gegenstand des fünften Kapitels sein.

Im Folgenden wird ein Überblick über die wissenschaftlichen Beiträge gegeben, die sich mit der Problemstellung des Payment Schedulings befasst haben. Dayanand und Padman erweitern die von Russell, Grinold und Talbot für das Project Scheduling Problem und die Planung des Auftragnehmers entwickelten mathematischen Modelle um Aspekte des Payment Schedulings. ${ }^{92}$ Sie vergleichen die auf Russell und Grinold aufbauenden Payment Scheduling Modelle, denen eine ereignisorientierte Problemstellung in einem Vorgangspfeilnetzplan zugrunde liegt, mit einem Modell, bei welchem die Zahlungen an Vorgänge geknüpft sind. Es zeigt sich, dass der Kapitalwert der Zahlungen bei einer vorgangsorientierten Problemstellung niedriger ist. Dies ist damit zu erklären, dass die Auszahlungen in den vorgangsorientierten Modellen zu dem Startzeitpunkt eines Vorgangs und die Einzahlungen als Fortschrittszahlungen nach Abschluss einer bestimmten Menge von Vorgängen stattfinden. Bei ereignisorientierten Modellen hingegen wird davon ausgegangen, dass die Auszahlungen aller in ein bestimmtes Ereignis einmündenden Vorgänge sowie die Fortschrittszahlungen zum Zeitpunkt des Ereignisses gezahlt werden. Damit werden die Auszahlungen um die Dauern der Vorgänge verzögert, was aus der Sicht des Auftragnehmers einen positiven Einfluss auf den Kapitalwert hat. ${ }^{93}$

Dayanand und Padman schlagen daher eine modifizierte Netzplanstruktur vor, der eine ereignisorientierte Darstellung zugrunde liegt und jedem Vorgang ein Scheinvorgang vorangeht, der in ein Scheinereignis mündet. Somit können die mit den Vorgängen verbundenen Auszahlungen zum Zeitpunkt der Scheinereignisse und damit zu dem Zeitpunkt des tatsächlichen Endes eines Vorgangs und die Einzahlungen zum Zeitpunkt der Meilensteinereignisse, in die mehrere abgeschlossene Vorgänge münden, terminiert werden. ${ }^{94}$

\footnotetext{
91 Vgl. Dayanand/Padman (1997), S. 908 sowie die Ausführungen in Kapitel 3.3.

92 Vgl. Dayanand/Padman (1997), S. 909ff. Zu den Modellen des Project Schedulings vgl. Grinold (1972); Russell (1970); Talbot (1982).

93 Vgl. Dayanand/Padman (1997), S. 914.

94 Vgl. Dayanand/Padman (1997), S. $915 \mathrm{ff}$.
} 
Für die dieser Arbeit zugrunde liegende Problemstellung werden die durch die Anwendung eines Vorgangspfeilnetzplans verursachten Ungenauigkeiten bei der Berechnung des Kapitalwertes im Vergleich zu dem vorgeschlagenen modifizierten Netzplan, welcher durch die zusätzlichen Vorgänge und Ereignisse eine höhere Komplexität aufweist, als unbedeutend erachtet, da dies für das Projektmanagement bei der vorliegenden Fragestellung weder Einfluss auf die Auswahl eines bestimmten Vertragstyps noch Auswirkung auf das Verhalten der Vertragsparteien hat.

Dayanand und Padman erweitern die Untersuchungen um Vertragsarten mit zeit-, kosten- und performanceorientierten Zahlungen. ${ }^{95}$ Sie stellen fest, dass kostenorientierte Zahlungen meist dann vertraglich vereinbart werden, sofern der Auftragnehmer als Entscheidungsträger das Vertragsdesign bestimmen kann. Aus der Sicht des Auftraggebers zieht dieser als Entscheidungsträger performanceorientierte Zahlungsvereinbarungen vor, um auch Qualitätskriterien in die Vergütung des Auftragnehmers einbeziehen zu können. ${ }^{96}$ Die Autoren führen ein Modell auf, welches aus der jeweiligen Sicht der zwei Vertragsparteien zu einem kapitalwertoptimalen Projektplan führt. Ulusoy und Cebelli greifen die von Dayanand und Padman untersuchte Problemstellung auf und schlagen ein Vorgehen vor, welches ausgehend von den für beide Vertragsparteien individuellen optimalen Projektplänen eine Kompromisslösung ermittelt, bei der beide Projektpartner in Höhe eines möglichst gleichen Prozentsatzes von den individuellen optimalen Lösungen abweichen.$^{97}$ Dies wird durch eine Zielfunktion erreicht, die eine möglichst gleich große prozentuale Abweichung von Kompromisslösung und individueller optimaler Lösung für Auftragnehmer und Auftraggeber erzeugt. ${ }^{98}$ Für die im Rahmen dieser Arbeit unterstellten Kooperationsbeziehungen wird jedoch davon ausgegangen, dass die Vertragsparteien nicht in gleichem Ausmaß über die Möglichkeiten des Vertragsdesigns entscheiden können, sondern das Management des Auftraggebers als Kooperationsträger über das Vertragsdesign der Kooperation mit dem Auftragnehmer bestimmt und dieser wiederum über den Vertrag mit möglichen Subunternehmern entscheidet. In einer weiteren im Jahr 2001 erschienenen Veröffentlichung konzentrieren sich Dayanand und Padman auf die Sicht des Auftraggebers und

95 Vgl. Dayanand/Padman (1998).

96 Vgl. Dayanand/Padman (1998), S. 484.

97 Vgl. Ulusoy/Cebelli (2000).

$98 \mathrm{Vgl}$. Ulusoy/Cebelli (2000), S. 264. Die Zielfunktion gewährleistet nicht, dass diese Abweichungen mog lichst gering sind. Das von den Autoren vorgeschlagene auf einem genetischen Algorithmus basierende Verfahren geht jedoch von den individuellen kapitalwertoptimalen Projektplänen aus und gelangt uber einen iterativen Verhandlungsprozess zwischen den Projektpartnern zu der angestrebten Kompromisslösung. 
vergleichen drei unterschiedliche Vertragsarten bzw. Zahlungsvereinbarungen miteinander, bei denen die Anzahl der Zahlungen und/oder Zahlungszeitpunkte bzw. -intervalle vertraglich festgelegt werden. ${ }^{99}$ Ergebnis der Untersuchungen ist, dass sich für den Auftraggeber Zahlungsvereinbarungen als vorteilhaft erweisen, bei denen zwar die Anzahl der Zahlungen, die dieser an den Auftragnehmer während der Projektdauer zu zahlen hat, festgelegt wird, jedoch der Zeitpunkt der Zahlungen frei wählbar ist. Als weiteres Ergebnis zeigt sich, dass der Auftraggeber bei Vereinbarung einer geringen Anzahl an Zahlungen diese mit Ereignissen verbindet, die möglichst spät innerhalb des Projektverlaufs stattfinden. ${ }^{100}$ Auch das Durchsetzen eines niedrigen Kostenerstattungsfaktors, welcher angibt, wie hoch der Anteil der in Form von Teilzahlungen während der Projektlaufzeit durch den Auftraggeber zu erstattenden Kosten ist, führt dazu, dass die mit dem letzten Ereignis verbundene Zahlung größer wird und daraus ein positiver Einfluss auf den Kapitalwert des Auftraggebers resultiert. Trotz des Zeitwertes der Zahlungen erweist es sich für den Auftraggeber meist nicht als vorteilhaft, das Projektende und damit die Zahlungen zu verzögern, da die verspäteten oder entgangenen Erlöse aus dem Projekt die eingesparten Kapitalkosten in der Regel übersteigen. ${ }^{101}$

Ulusoy, Sivrikaya-Serifoglu und Sahin betrachten ähnlich wie Dayanand und Padman vier verschiedene Zahlungsmodalitäten, erweitern diese jedoch dadurch, dass sowohl Ressourcen berücksichtigt werden als auch die Möglichkeit der Ausführung von Vorgängen in unterschiedlichen Modi möglich ist. ${ }^{102}$ Es besteht einerseits ein RessourcenZeit-Trade-Off, da eine zusätzliche Zuweisung von Ressourcen die Dauer der Vorgänge verkürzt, andererseits besteht ein Ressourcen-Ressourcen-Trade-Off, da eine Nutzung mehrerer substituierbarer Ressourcen für die Ausführung der Vorgänge möglich ist. ${ }^{103}$ Für die Lösung dieser als Multi Mode Resource Constrained Payment Scheduling bezeichneten Problemstellung schlagen die Autoren die Anwendung eines genetischen Algorithmus vor und gelangen neben einem wenig aussagekräftigen Vergleich der betrachteten Zahlungsmodalitäten im Rahmen eines Beispiels zu den folgenden allgemeinen Ergebnissen: ${ }^{104}$ Wird nur die Zahlung einer Abschlussvergütung verein-

\footnotetext{
99 Vgl. Dayanand/Padman (2001b).

${ }^{100}$ Diese Aussage wird in Kapitel 4.4.2 anhand der Anwendung als nicht allgemeingültig belegt.

101 Vgl. Dayanand/Padman (2001b), S. 1666.

102 Vgl. Ulusoy/Sivrikaya-Serifoglu/Sahin (2001).

$103 \mathrm{Vgl}$. Ulusoy/Sivrikaya-Serifoglu/Sahin (2001), S. 238.

${ }^{104}$ Heuristiken in Form genetischer Algorithmen zăhlen zu den naturanalogen, evolutionären Verfahren. Grundlegend zu der Idee genetischer Algorithmen vgl. beispielsweise Nissen (1997). Eine genetische Programmierung fur das kapitalwertmaximierende Project Scheduling Problem stellen Padman/Roehrig (1997) vor.
} 
bart, so kann dies mit dem Ziel der Minimierung der Projektdauer gleichgesetzt werden. Werden die Ereignisse festgelegt, bei denen eine Zahlung zu erfolgen hat, so werden die Ereignisse, die insbesondere mit hohen Zahlungen verbunden sind, möglichst früh geplant. In den Fällen, bei denen die Anzahl der zeitlich regelmäßigen Zahlungen oder das Zahlungszeitintervall festgelegt sind, werden die mit zu vergütenden Kosten verbundenen Vorgänge zeitlich so geplant, dass sie vor dem jeweiligen Zahlungszeitpunkt abgeschlossen sind und damit zu möglichst frühen Einzahlungen führen. ${ }^{105}$ Ein weiterer von Dayanand und Padman veröffentlichter Beitrag stellt ein auf der Heuristik Simulated Annealing basierendes Vorgehen für große, nicht durch Ressourcen beschränkte Problemstellungen vor. ${ }^{106}$ Das zweistufige Verfahren beginnt mit einem Projektplan, dessen Ereignisse zu den frühestmöglichen Zeitpunkten geplant werden. Hierauf aufbauend wird die Anzahl und Höhe der Zahlungen ermittelt, die zu einem maximalen Kapitalwert führen. In einem nächsten Schritt werden die Ereigniszeiten verändert und erneut eine den Kapitalwert maximierende Zahlungsreihe ermittelt. Dieses Vorgehen wird unter Berücksichtigung der Parameter der Heuristik Simulated Annealing wiederholt durchgeführt.

Es zeigt sich, dass die in diesem Kapitel untersuchten Beiträge bereits Ansätze einer Verbindung von Contract Management und quantitativer Planung enthalten. Insbesondere die von Dayanand und Padman durchgeführten Untersuchungen motivieren, diese Verbindung unter Berücksichtigung der im dritten Kapitel aufgezeigten verschiedenen Vertragsdesigns sowie der durch die Informationsasymmetrie der Kooperationsbeziehung begründeten Problemstellungen eingehend systematisch zu analysieren und quantitativ in Form eines erweiterten Payment Scheduling Modells abzubilden.

\subsubsection{Analyse und Bewertung der Problemcharakteristika}

Zusammenfassend werden an dieser Stelle als Ergänzungen zu den in Kapitel 4.2.2 vorgestellten Beiträgen zum Project Scheduling in gleicher Weise die Veröffentlichungen auf dem Gebiet des Payment Schedulings vergleichend analysiert. Tabelle 4.3 gibt einen Überblick über die Problemcharakteristika der aufgeführten Literaturstellen:

\footnotetext{
${ }^{105} \mathrm{Vgl}$. Ulusoy/Sivrikaya-Serifoglu/Sahin (2001), S. 252.

106 Vgl. Dayanand/Padman (2001a).
} 


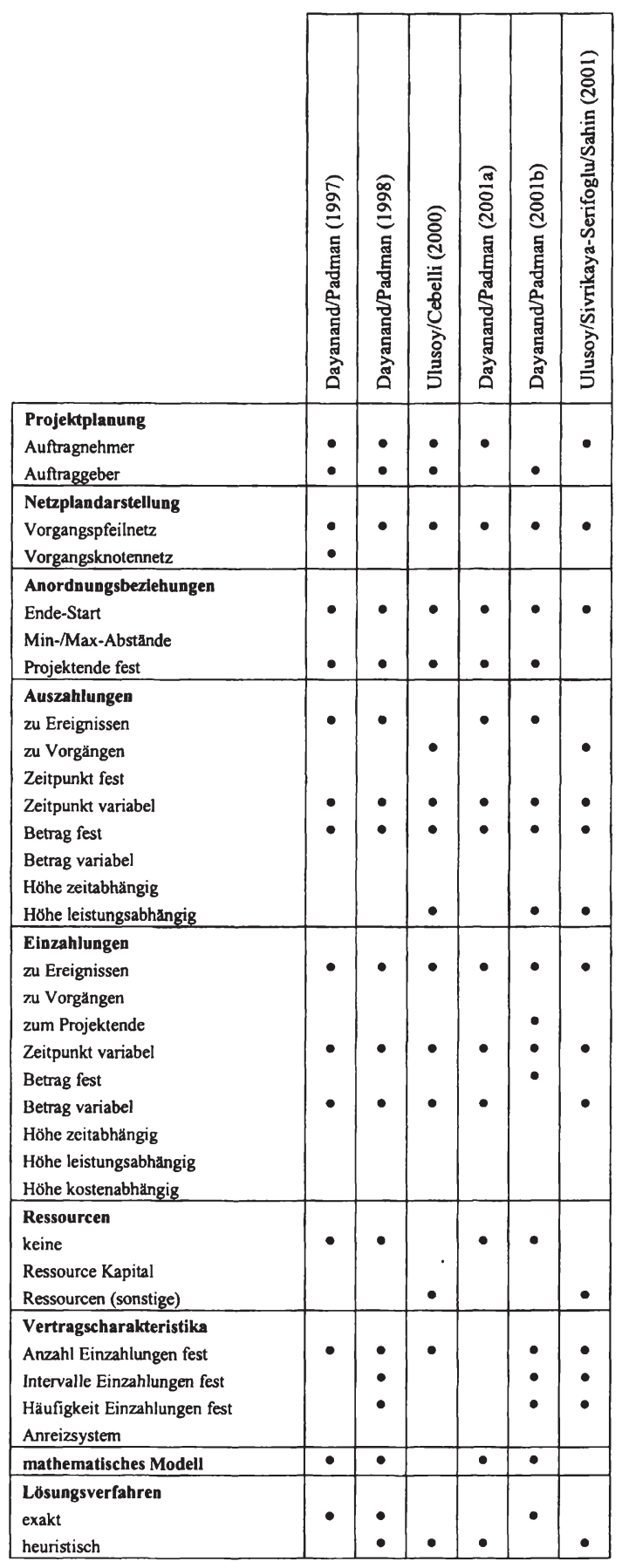

Tabelle 4.3: Literaturüberblick Charakteristika Payment Scheduling 
Es ist zu erkennen, dass die Perspektive des Auftraggebers im Rahmen der Payment Scheduling Problematik in die Ansätze zur Planungsunterstützung einbezogen sowie der Zielkonflikt, der bei der Kapitalwertmaximierung zwischen Auftragnehmer und Auftraggeber vorliegt, vertiefend behandelt wird.

Besonders die Arbeiten von Dayanand und Padman konzentrieren sich auf die Entwicklung mathematischer Modelle aus der Sicht beider Kooperationspartner und modifizieren diese durch die Einbeziehung von Vertragscharakteristika. Dies erfolgt beispielsweise durch die Festlegung einer Mindestanzahl von Zahlungen oder die Modellierung von Zahlungsintervallen. Es lässt sich weiterhin erkennen, dass ein Vorgangspfeilnetzplan als Problemrepräsentation als am besten geeignet erscheint, da die Einzahlungen als Entscheidungsvariablen häufig den Ereignissen zugeordnet werden. Während die Höhe der Auszahlungen in allen betrachteten Ansätzen als bekannt angenommen wird, liegt die Planungsaufgabe darin, die Zeitpunkte von Aus- und Einzahlungen sowie die Höhe der Zahlungen des Auftraggebers an den Auftragnehmer so zu bestimmen, dass hieraus ein kapitalwertmaximaler Projektplan resultiert. Bei allen untersuchten Literaturstellen wird die zeitliche Abfolge der Vorgänge ausschließlich durch die Vorgangsdauern bestimmt. Minimal- oder Maximalabstände werden nicht berücksichtigt. Die Einbeziehung von Ressourcen bei der quantitativen Planung steht ebenfalls nicht im Vordergrund der betrachteten Quellen.

Zusammenfassend kann festgestellt werden, dass sich die Beschäftigung mit dem Payment Scheduling Problem bisher auf wenige wissenschaftliche Beiträge beschränkt und weniger ökonomische Fragestellungen im Vordergrund der Betrachtungen stehen, sondern zunehmend die Entwicklung von heuristischen Verfahren für die Lösung groBer Problemstellungen an Bedeutung gewinnt.

Für die in dieser Arbeit betrachtete Unterstützung des Auftraggebers bei Entscheidungen der Vertragsgestaltung steht das Erstellen eines Projektplans sowie der Zeitplanung für alle Projektereignisse und -vorgänge im Hintergrund. Vielmehr soll das im Folgenden dargestellte und im fünften Kapitel erweiterte Payment Scheduling Modell als Grundlage dienen, die unterschiedlichen Vertragsarten quantitativ zu bewerten und hieraus Aussagen abzuleiten, welche Vertragsart besonders geeignet ist, den Erfolg eines Kooperationsprojektes zu fördern. 


\subsubsection{Modellierung des Payment Scheduling Problems}

Das in diesem Abschnitt dargestellte Modell für das Payment Scheduling Problem basiert auf den Ausführungen von Dayanand und Padman und wird in ausgewählten Teilbereichen modifiziert. ${ }^{107}$ Dieses Modell betrachtet im Gegensatz zu dem Project Scheduling Modell das Planungsproblem aus der Sicht des Auftraggebers als Kooperationsträger und Projekteigentümer. Das Projekt besteht aus M Vorgängen und I Ereignissen und wird als Vorgangspfeilnetzplan dargestellt. Die Dauern $\mathrm{D}_{\mathrm{ij}}$ der Vorgänge sowie deren Reihenfolgebeziehungen sind bekannt. Die Ausführung eines Vorgangs ist für den Auftragnehmer mit Kosten verbunden, deren Höhe für beide Projektpartner als bekannt vorausgesetzt wird. Obwohl der Auftraggeber durch die zu dem Zeitpunkt des Projektendes erfolgende Einzahlung eine Präferenz für einen frühen Projektabschluss hat, wird auch für das Payment Scheduling Modell der spätesterlaubte Zeitpunkt des letzten Ereignisses I mit DMax festgelegt.

Der Auftraggeber des Projektes zahlt an den Auftragnehmer als Vergütung der Leistungserstellung insgesamt einen Betrag in Höhe von Zges . Diese Zahlungen erhält der Auftragnehmer in Form von ANZ Fortschrittszahlungen. Die Zahlungen des Auftraggebers an den Auftragnehmer erfolgen ausschließlich zu Ereigniszeitpunkten, so dass eine Vergütung in Abhängigkeit von dem Projektfortschritt möglich ist. Der Auftraggeber vereinbart während der Vertragsverhandlungen mit dem Auftragnehmer die Anzahl der Zahlungen ANZ sowie die Rate $\beta$ für die Kostenerstattungen. Dabei bedeutet eine Rate $\beta<1$, dass dem Auftragnehmer mit jeder Fortschrittszahlung nur ein Teil seiner durch die Leistungserstellung entstandenen Kosten erstattet wird und zu dem Zeitpunkt des Projektendes eine Abschlusszahlung in Höhe der Differenz zuzüglich einer ebenfalls zu vereinbarenden Gewinnprämie erfolgt. ${ }^{108}$

Die aus der Leistungserstellung resultierenden und durch den Auftraggeber zu erstattenden Kosten des Auftragnehmers werden durch die Ausführung der zeitlich zwischen den Ereignissen stattfindenden Vorgänge verursacht. Abbildung 4.10 zeigt einen Ausschnitt des Anwendungsbeispiels und wird im Folgenden die Problematik der Zuordnung von Kosten zu Ereignissen sowie der Erstattung der Kosten durch den Auftraggeber verdeutlichen:

\footnotetext{
107 Vgl. Dayanand/Padman (1997); Dayanand/Padman (1998); Dayanand/Padman (2001a); Dayanand/Padman (2001b).

108 Vgl. Dayanand/Padman (1998), S. 486.
} 


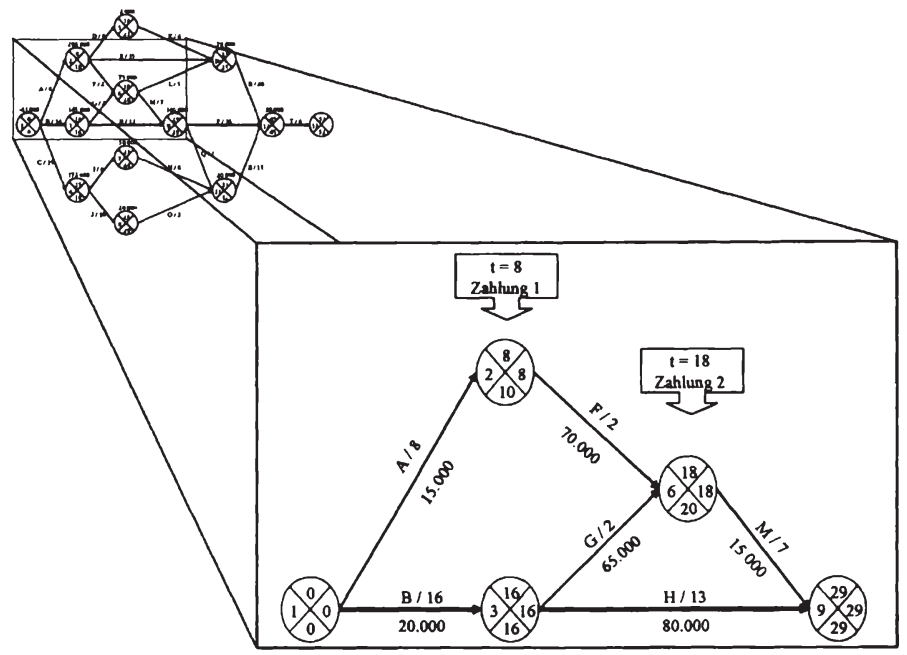

Abbildung 4.10: Erstattung von Vorgangskosten

Unter der Annahme, dass für die Projektausführung Ressourcen benötigt werden, deren Beschaffung und Finanzierung durch den Auftragnehmer vor dem Beginn des Vorgangs erfolgen müssen, wird für die anschließenden Untersuchungen angenommen, dass die Kosten eines Vorgangs aus der Sicht des Auftragnehmers dem Ereignis zugeordnet werden, welches dem jeweiligen Vorgang vorangeht. Die Höhe der einem Ereignis zugeordneten Kosten entspricht somit der Summe der Kosten der Vorgänge, die direkt im Anschluss an das Ereignis beginnen. ${ }^{109}$ Die für den Auftragnehmer entstehenden und einem Ereignis i zugeordneten Kosten werden im Folgenden mit $\mathrm{K}_{\mathrm{i}}^{\mathrm{AN}}$ bezeichnet.

Aus dem Blickwinkel des Auftraggebers erfolgt die Erstattung der Kosten eines Vorgangs erst nach Abschluss des Vorgangs, also frühestens zu dem Zeitpunkt des Ereignisses, welches unmittelbar auf den Vorgang folgt. Die für den Auftraggeber zu erstattenden und einem Ereignis i zugeordneten Kosten werden daher im Folgenden mit $\mathrm{K}_{\mathrm{i}}^{\mathrm{AG}}$ bezeichnet.

${ }^{109}$ Hierbei handelt es sich um eine das Problem vereinfachende Annahme. Für eine exakte Zeitberucksichtigung der aus der Ausfuhrung der Vorgänge resultierenden Zahlungen muss als Problemrepräsentation ein Vorgangsknotennetzplan vorausgesetzt oder Scheinvorgänge ohne Zahlungen in einen Vorgangspfeilnetzplan integriert werden, die jeweils das Ende der in ein Ereignis mündenden Vorgănge anzeigen. Bei einem Vorgangsknotennetzplan ist jedoch eine direkte Abbildung von Ereignissen nicht möglich. Eine Einfuhrung von Scheinvorgăngen in einen Vorgangspfeilnetzplan erhöht die Komplexităt der Problemstellung. Für eine vorgangsorientierte Darstellung vgl. Dayanand/Padman (1997), S. 913f. Für eine Darstellung mit Scheinvorgängen vgl. Dayanand/Padman (1997), S. $915 f$. 
Für den in Abbildung 4.10 dargestellten Ausschnitt zeigt die Tabelle 4.4 die Zurechnung der für den Auftragnehmer mit der Ausführung der Vorgänge entstehenden und durch den Auftraggeber bei einem Kostenerstattungsvertrag zu einem Ereignis zu erstattenden Kosten: ${ }^{110}$

\begin{tabular}{|c|c|c|}
\hline Ereignis & $\mathrm{K}_{\mathrm{i}}^{\mathrm{AN}}$ & \multicolumn{1}{c|}{$\mathrm{K}_{\mathrm{i}}^{\mathrm{AG}}$} \\
\hline 1 & 35.000 & 0 \\
\hline 2 & 70.000 & 15.000 \\
\hline 3 & 145.000 & 20.000 \\
\hline 6 & 15.000 & 135.000 \\
\hline 9 & $\ldots$ & 95.000 \\
\hline
\end{tabular}

Tabelle 4.4: Kosten aus Sicht von Auftragnehmer und Auftraggeber

Diese für die Vertragsparteien individuelle Zuordnung der Kosten zu Ereignissen innerhalb des Vorgangspfeilnetzplans ermöglicht eine realitätsnahe Berechnung der Fortschrittszahlungen und führt zu einer genaueren Ermittlung der Kapitalwerte von Auftragnehmer und Auftraggeber.

Darüber hinaus wird für die Planung des Auftraggebers unterstellt, dass die aus dem Projekt während der Nutzung der Investition resultierenden und zum Zeitpunkt des Vertragsdesigns geschätzten Erlöse bereits zu dem Zeitpunkt des Projektabschlusses zu Einzahlungen führen. Die zeitlich früheren Einzahlungen erhöhen zwar den durch die Optimierung ausgewiesenen Kapitalwert, bei der betrachteten Problemstellung wird jedoch davon ausgegangen, dass die Ermittlung der Vorteilhaftigkeit der Investition durch den Auftraggeber zu dem Zeitpunkt der Vertrags- und Projektplanung abgeschlossen ist und zu einem positiven Ergebnis geführt hat, so dass die Annahme der dem Zeitpunkt des Projektendes zugeordneten Erlöse keine Auswirkungen auf die Projektplanung des Auftraggebers und die Auswahl eines geeigneten Vertragsdesigns hat.

Das Optimierungsproblem besteht bei dieser Problemstellung darin, sowohl die Höhe und Zeitpunkte der Zahlungen des Auftraggebers an den Auftragnehmer als auch die Ereigniszeitpunkte, zu denen keine Zahlungen erfolgen, derart zu bestimmen, dass der Kapitalwert als Summe der Barwerte aller während des Projektverlaufs entstehenden

\footnotetext{
${ }^{110}$ Die Tabelle enthält für Ereignis 9 keinen Eintrag der Kosten aus Sicht des Auftragnehmers, da die Höhe dieser Kosten von den auf Ereignis 9 folgenden und fur diesen Ausschnitt nicht betrachteten Vorgăngen abhăngt.
} 
Auszahlungen des Auftraggebers und des Barwertes der zu dem Zeitpunkt des Projektendes erfolgenden Einzahlung maximal ist.

In Analogie zu dem in Kapitel 4.2.3 aufgeführten Project Scheduling Modell wird die diskrete Diskontierung der Zahlungen auch für die Modellierung des Payment Scheduling Modells vorgeschlagen. Im Folgenden werden die in dieser Modellformulierung verwendeten Indizes, Daten und Variablen vorgestellt:

\section{Indizes und Indexmengen:}

$\mathrm{i}, \mathrm{j}=1, \ldots, \mathrm{I} \quad$ : Ereignisse

$\mathrm{t}, \mathrm{u}=0, \ldots, \mathrm{T}:$ Ereigniszeitpunkte

\section{Daten:}
ANZ : Anzahl der durch den Auftraggeber zu leistenden Zahlungen
: Kostenerstattungsfaktor
$D_{i j} \quad$ : Dauer des Vorgangs zwischen Ereignis i und Ereignis $j$

DISKZ : Diskontierungszinssatz

DISKF $_{t}$ : Diskontierungsfaktor, DISKF $_{t}=\left(1+\right.$ DISKZ $^{-t}$

DMax : Spätesterlaubter Zeitpunkt des letzten Ereignisses I

ERL : Erlöse für den Auftraggeber nach Projektabschluss

$\mathrm{K}_{\mathrm{i}}^{\mathrm{AG}} \quad$ : Kosten der direkt vor dem Ereignis i abgeschlossenen Vorgänge

$\widehat{M} \quad$ : Hinreichend große Zahl

$\mathrm{V} \quad$ : Menge der einem Vorgang unmittelbar vorangehenden und unmittelbar nachfolgenden Ereignisse (i,j)

Zges : Summe der Zahlungen des Auftraggebers an den Auftragnehmer

\section{Variablen:}

$\mathrm{x}_{\mathrm{it}}=\left\{\begin{array}{l}1, \text { wenn Ereignis i zum Zeitpunkt } \mathrm{t} \text { stattfindet } \\ 0, \text { sonst }\end{array}\right.$

$y_{t}=\left\{\begin{array}{l}1, \text { wenn eine Fortschrittszahlung zum Zeitpunkt } t \text { stattfindet } \\ 0, \text { sonst }\end{array}\right.$

$z_{\mathrm{t}}$ : Höhe der Fortschrittszahlung zum Zeitpunkt $t$

Das Payment Scheduling Problem kann aus der Sicht des Auftraggebers damit wie folgt als gemischt-ganzzahliges lineares Programmierungsmodell formuliert werden. 
Die Zielfunktion (4.10) maximiert die Differenz aus dem Barwert der zu dem Zeitpunkt des Projektendes aus der Sicht des Auftraggebers erfolgenden Einzahlung aus den Projekterlösen und den Barwerten der zu den ermittelten Ereigniszeitpunkten geleisteten Zahlungen an den Auftragnehmer:

$\max E R L \cdot \sum_{t=0}^{T} \operatorname{DISKF}_{t} \cdot x_{1 t}-\sum_{t=0}^{T} \operatorname{DISKF}_{t} \cdot z_{t}$

Die folgenden Restriktionen als Bestandteil des hier vorgestellten Grundmodells zum Payment Scheduling werden im fünften Kapitel um die spezifischen Vertragscharakteristika ergänzt.

Die Nebenbedingung (4.11) legt fest, dass für jedes Ereignis i genau ein Zeitpunkt bestimmt wird, zu dem es stattfindet.

$\sum_{\mathrm{i}=0}^{\mathrm{T}} \mathrm{x}_{\mathrm{it}}=1 \quad \forall \mathrm{i}$

Durch die Bedingung (4.12) wird die Einhaltung der Vorgangsdauer und somit der Reihenfolgebeziehung unmittelbar aufeinander folgender Ereignisse gewährleistet. Die Menge $\mathrm{V}$ umfasst jeweils die einem Vorgang unmittelbar vorangehenden und unmittelbar nachfolgenden Ereignisse $i$ und $j$.

$\sum_{\mathrm{t}=0}^{\mathrm{T}} \mathrm{t} \cdot \mathrm{x}_{\mathrm{jt}}-\sum_{\mathrm{t}=0}^{\mathrm{T}} \mathrm{t} \cdot \mathrm{x}_{\mathrm{it}} \geq \mathrm{D}_{\mathrm{ij}} \quad \forall(\mathrm{i}, \mathrm{j}) \in \mathrm{V}$

Die Restriktion (4.13) stellt sicher, dass das vorgeschriebene spätesterlaubte Projektende DMax durch die Festlegung des Zeitpunktes des letzten Ereignisses I nicht überschritten wird.

$\sum_{\mathrm{t}=0}^{\mathrm{T}} \mathrm{t} \cdot \mathrm{x}_{\mathrm{It}} \leq \mathrm{DMax}$

Bei einer hier im Rahmen des Grundmodells modellierten Kostenerstattungsvereinbarung erhält der Auftragnehmer als Vergütung seiner geleisteten Arbeit Zahlungen durch den Auftraggeber, welche proportional zu den ihm entstandenen Kosten sind. Dabei setzt der Kostenerstattungsfaktor $\beta$ das Verhältnis zwischen Fortschrittszahlung und Kosten fest. Es muss daher durch eine Bedingung gewährleistet werden, dass die Summe der Fortschrittszahlungen zu jedem Zeitpunkt mindestens den mit dem Kostenerstattungsfaktor $\beta$ multiplizierten und bis zu diesem Zeitpunkt entstandenen Kosten der Leistungserstellung entspricht. In der Literatur zum Payment Scheduling wird 
die folgende Restriktion für die Bestimmung der zu einem Ereignis erfolgenden Kostenerstattungszahlung vorgeschlagen: ${ }^{111}$

$\sum_{\mathrm{t}=0}^{\mathrm{u}} \mathrm{z}_{\mathrm{t}} \geq \beta \cdot \sum_{\mathrm{t}=0}^{\mathrm{u}} \sum_{\mathrm{i}=1}^{\mathrm{I}} \mathrm{K}_{\mathrm{i}}^{\mathrm{AG}} \cdot \mathrm{x}_{\mathrm{it}} \quad \forall \mathrm{u}$

$\mathrm{Zu}$ jedem Zeitpunkt $\mathrm{u}$ muss mindestens eine Erstattung der mit dem vereinbarten Kostenerstattungsfaktor $\beta$ multiplizierten Summe der bis zu diesem Zeitpunkt entstandenen Kosten durch die Teilzahlung erfolgen.

Diese Bedingung führt in der dargestellten Form dazu, dass alle bis zu dem jeweiligen Zahlungszeitpunkt entstandenen Kosten anteilig erstattet werden, zusätzlich in dieser Fortschrittszahlung jedoch auch die Kosten der Vorgänge enthalten sind, die bis zu dem nächsten Zahlungszeitpunkt realisiert werden. Die Problematik wird anhand des in Abbildung 4.10 dargestellten Ausschnittes des Anwendungsbeispiels erläutert.

Die mit der Ausführung eines Vorgangs für den Auftragnehmer entstehenden Kosten sind an dem jeweiligen Vorgangspfeil kenntlich gemacht. Es wird für diesen Ausschnitt des Beispiels angenommen, dass zu dem Zeitpunkt $t=8$ sowie $z u$ dem Zeitpunkt $\mathrm{t}=18$ eine Fortschrittszahlung $\mathrm{z}_{\mathrm{t}}$ durch den Auftraggeber erfolgen soll. $\mathrm{Zu}$ dem Zeitpunkt der ersten Zahlung ist ausschließlich Vorgang A abgeschlossen, so dass bei einem Kostenerstattungsfaktor von $\beta=1,0$ eine Fortschrittszahlung in Höhe von mindestens $15.000 \mathrm{GE}$ erfolgen muss. Die Restriktion (4.14) ist für den Zeitpunkt $\mathrm{u}=8$ erfüllt, denn es gilt:

$$
\sum_{\mathrm{t}=0}^{8} \mathrm{z}_{\mathrm{t}}=15.000 \geq 1,0 \cdot \sum_{\mathrm{t}=0 \mathrm{i} \in\{1,2,3,6,9\}}^{8} \mathrm{~K}_{\mathrm{i}}^{\mathrm{AG}} \cdot \mathrm{x}_{\mathrm{it}}=15.000
$$

$\mathrm{Zu}$ dem Zeitpunkt $\mathrm{t}=16$ ist der Vorgang $\mathrm{B}$ abgeschlossen, der mit Kosten in Höhe von $20.000 \mathrm{GE}$ verbunden ist, das heißt, die Binärvariable $\mathrm{x}_{3,16}$ nimmt den Wert 1 an. $\mathrm{Zu}$ diesem Zeitpunkt weist die rechte Seite der Restriktion somit den Wert 35.000 auf. Die Erstattung dieser Kosten sollte jedoch erst zu dem Zeitpunkt $t=18$ der nächsten Zahlung erfolgen, zu dem auch die Vorgänge $\mathrm{F}$ und $\mathrm{G}$ beendet sind. Die Lösung des Optimierungsproblems unter Berücksichtigung der aufgezeigten Restriktion führt in diesem Fall zu einer Fortschrittszahlung zu dem Zeitpunkt $t=8$ in Höhe von 35.000 GE. Der Auftraggeber wird jedoch nur bereit sein, die Erstattung der Kosten der bis zu

11 Aus der Sicht des Auftraggebers vgl. Dayanand/Padman (2001b), S. 1657. Aus der Sicht des Auftragnehmers vgl. Dayanand/Padman (1997), S. 910; Dayanand/Padman (1998), S. 490. 
diesem Zeitpunkt $t=8$ erfolgreich durchgeführten und beendeten Vorgänge vorzunehmen.

Um eine realitätsnahe Modellierung dieser Restriktion zur Bestimmung der Höhe der Fortschrittszahlungen zu erzielen, muss gelten, dass diese Bedingung nur für diejenigen Ereigniszeitpunkte bindend ist, zu denen eine Zahlung erfolgt. Eine multiplikative Verknüpfung der rechten Seite der Restriktion (4.14) mit der Binärvariablen $y_{t}$, die den Wert 1 annimmt, sofern zu dem Zeitpunkt $t$ eine Zahlung erfolgt, führt zur Nichtlinearität. Stattdessen wird die folgende Formulierung der für die Bestimmung der Höhe der mindestens zu erstattenden Kosten als lineare Restriktion vorgeschlagen: ${ }^{112}$

$$
\sum_{\mathrm{t}=0}^{\mathrm{u}} \mathrm{z}_{\mathrm{t}} \geq \beta \cdot \sum_{\mathrm{t}=0}^{\mathrm{u}} \sum_{\mathrm{i}=1}^{\mathrm{I}} \mathrm{K}_{\mathrm{i}}^{\mathrm{AG}} \cdot \mathrm{x}_{\mathrm{it}}-\widehat{\mathrm{M}} \cdot\left(1-\mathrm{y}_{\mathrm{u}}\right) \quad \forall \mathrm{u}
$$

Der Parameter $\widehat{M}$ stellt eine hinreichend große Zahl dar und sollte mindestens der Höhe der Gesamtprojektkosten entsprechen, damit die Restriktion auch für den Fall nur einer Zahlung des Auftraggebers erfüllt ist. Nimmt die Entscheidungsvariable $y_{u}$ für alle Zeitpunkte einer Zahlung den Wert 1 an, besteht die Restriktion in ihrer ursprünglichen Form. Für alle Zeitpunkte, zu denen keine Zahlung erfolgt und $y_{u}$ den Wert 0 besitzt, ist die $\geq$-Restriktion durch die Subtraktion der großen Zahl $\widehat{M}$ auf der rechten Seite erfüllt. Die im Anschluss an diese Modellformulierung erfolgende Anwendung zeigt, dass die Bedingung (4.15) geeignet ist, die in der Literatur vorgeschlagene Restriktion (4.14) zu ersetzen.

Die folgende Nebenbedingung (4.16) fordert, dass die Summe der Teilzahlungen der gesamten durch den Auftraggeber an den Auftragnehmer zu leistenden Zahlung Zges entspricht.

$$
\sum_{\mathrm{t}=0}^{\mathrm{T}} \mathrm{z}_{\mathrm{t}}=\text { Zges }
$$

Restriktion (4.17) stellt sicher, dass die Anzahl der Teilzahlungen die zwischen Auftraggeber und Auftragnehmer vereinbarte Häufigkeit der Zahlungen mindestens erfüllt.

$\sum_{t=0}^{T} y_{t} \geq A N Z$

\footnotetext{
${ }^{112}$ Für die Modellierung der linearen Restriktion durch die Verknüpfung der Variablen in allgemeiner Form vgl. etwa Hillier/Liebermann (2001), S. 587.
} 
Die Bedingung (4.18) stellt eine Verknüpfung von Ereignissen und Zahlungen her, indem gefordert wird, dass zu dem Zeitpunkt, zu dem eine Zahlung erfolgt, auch mindestens ein Ereignis terminiert werden muss. Dies lässt sich damit begründen, dass der Auftraggeber Zahlungen an Projektereignisse bindet, die für den Projektfortschritt bedeutend sind.

$\sum_{i=1}^{1} x_{i t} \geq y_{t}$

$\forall \mathrm{t}$

Die Nebenbedingung (4.19) bildet die Verknüpfung der Variablen $y_{t}$ und $z_{t}$. Nimmt $y_{t}$ den Wert null an, das heißt zum Zeitpunkt $t$ erfolgt keine Zahlung, so folgt, dass auch $z_{t}$ den Wert null annimmt. Mit Zges als Summe aller Teilzahlungen ist die Ungleichung erfüllt, sofern zum Zeitpunkt t eine Zahlung erfolgt. Der Wert Zges kann in dieser Restriktion auch durch eine beliebig große Zahl $\widehat{M} \geq$ Zges ersetzt werden.

$\mathrm{z}_{\mathrm{t}}-$ Zges $\cdot \mathrm{y}_{\mathrm{t}} \leq 0$

Die Restriktionen (4.20), (4.21) und (4.22) legen den Definitionsbereich der Variablen des Modells fest.

$$
\begin{array}{ll}
\mathrm{x}_{\mathrm{it}} \in\{0,1\} & \forall \mathrm{i}, \mathrm{t} \\
\mathrm{y}_{\mathrm{t}} \in\{0,1\} & \forall \mathrm{t} \\
\mathrm{z}_{\mathrm{t}} \geq 0 & \forall \mathrm{t}
\end{array}
$$

Damit ist die formale Darstellung des Payment Scheduling Modells aus der Sicht des Auftraggebers abgeschlossen. Das folgende Kapitel wendet die Modelle sowohl des Project Schedulings als auch des Payment Schedulings anhand eines Beispiels an. Die Ergebnisse des Anwendungsbeispiels zeigen, dass die vorgeschlagenen quantitativen Modelle geeignet sind, diese Problemstellung abzubilden. Sie verdeutlichen insbesondere den Unterschied zwischen dem für die Planung des Auftragnehmers formulierten Project Scheduling Modell und dem die Planung des Auftraggebers unterstützenden Payment Scheduling Modell. 


\subsection{Anwendung der Projektplanung anhand eines Beispiels}

\subsubsection{Darstellung des Anwendungsbeispiels}

Die folgende Anwendung soll die Scheduling Problematik anschaulich verdeutlichen und zum Verständnis der in Kapitel 4.2.3 und 4.3.3 vorgestellten Modelle beitragen. Das Anwendungsbeispiel besteht aus 20 Vorgängen mit den in Tabelle 4.5 aufgefuihrten Reihenfolgebeziehungen, Vorgangsdauern in Wochen und Vorgangskosten. Aus diesen Daten kann der in Abbildung 4.11 dargestellte Vorgangspfeilnetzplan entwickelt werden. An jedem Ereignisknoten sind der frühestmögliche sowie der spätesterlaubte Startzeitpunkt eingetragen. ${ }^{113}$

Um eine realitätsnahe Modellierung der Problemstellung zu gewährleisten, wird weiterhin angenommen, dass die Auszahlungen des Auftragnehmers in Form der Vorgangskosten zu Beginn des jeweiligen Vorgangs anfallen und damit dem Ereignis zugeordnet werden, welches dem Vorgang vorangeht. Beauftragt der Auftragnehmer Subunternehmer, so kann abweichend hiervon angenommen werden, dass die mit einem Vorgang verbundenen Zahlungen nach Abschluss des Vorgangs an den Subunternehmer zu leisten sind und dem auf einen Vorgang folgenden Ereignis angerechnet werden. Die Zahlungen des Auftraggebers an den Auftragnehmer erfolgen nur zu einem Zeitpunkt, welcher einem Ereigniszeitpunkt entspricht. Hierdurch wird gewährleistet, dass Zahlungen ausschließlich zu den im zweiten Kapitel charakterisierten Meilensteinereignissen des aggregierten Projektplans stattfinden können.

Die aus der späteren Nutzung der Projektleistung resultierenden Erlöse des Auftraggebers werden mit 1.000.000 GE festgesetzt und entsprechen für dieses Beispiel somit der Summe der Vorgangskosten. Obwohl diese Annahme im Rahmen der folgenden Untersuchungen meist zu einem negativen Projektkapitalwert des Auftraggebers führt, zeigt das Ergebnis jedoch, wie groß der Barwert der über diese Erlöse hinausgehenden Zahlungen aus der Projektnutzung sein muss, um ein aus der Sicht des Auftraggebers gesetztes Zielkriterium, wie beispielsweise einen positiven Kapitalwert, zu erfüllen. Aufgrund des in dieser Arbeit nicht betrachteten Entscheidungsproblems der Projektauswahl sowie der hierfür notwendigen Projektbewertung werden durch den im Folgenden aus der Sicht des Auftraggebers ermittelten meist negativen Kapitalwert keine Aussagen über die Vorteilhaftigkeit des Projektes getroffen.

${ }^{113}$ Zur Bestimmung von Ereigniszeitpunkten in Vorgangspfeilnetzen vgl. etwa Schwarze (2001), S. $157 \mathrm{ff}$. 


\begin{tabular}{|cccc|}
\hline Vorgang & direkter Vorgänger & Dauer (Wochen) & Kosten \\
\hline A & - & 8 & 15.000 \\
\hline B & - & 16 & 20.000 \\
\hline C & - & 15 & 8.000 \\
\hline D & A & 8 & 4.000 \\
\hline E & A & 15 & 130.000 \\
\hline F & A & 2 & 70.000 \\
\hline G & B & 2 & 65.000 \\
\hline H & B & 13 & 80.000 \\
\hline I & C & 6 & 160.000 \\
\hline J & C & 10 & 13.000 \\
\hline K & D & 4 & 2.000 \\
\hline L & F, G & 5 & 60.000 \\
\hline M & F, G & 7 & 15.000 \\
\hline N & I & 8 & 50.000 \\
\hline O & J & 3 & 20.000 \\
\hline P & H, M & 16 & 150.000 \\
\hline Q & H, M & 2 & 10.000 \\
\hline R & E, K, L & 78.000 \\
\hline S & N, O, Q & 13 & 20.000 \\
\hline T & P, R, S & 7 & 30.000 \\
\hline & & & \\
\hline
\end{tabular}

Tabelle 4.5: Daten des Anwendungsbeispiels

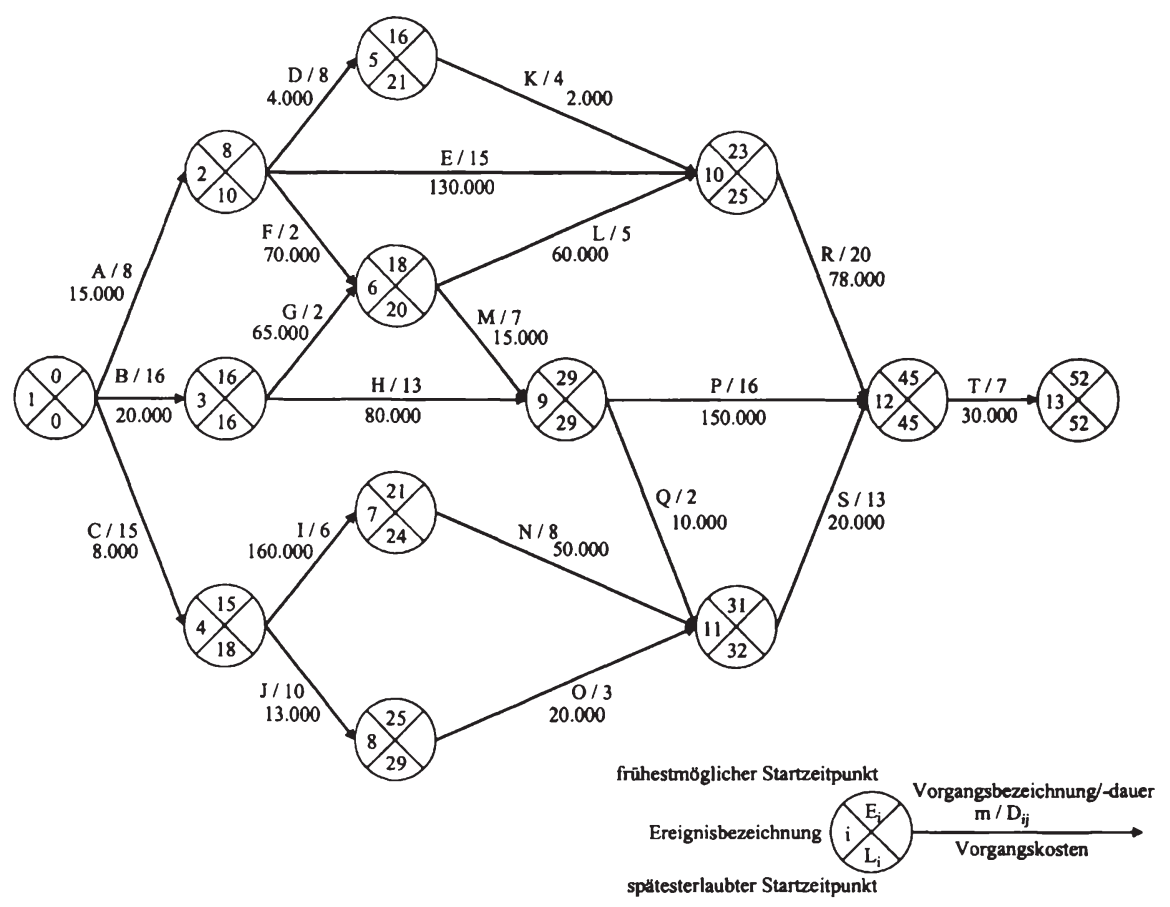

Abbildung 4.11: Projektplan des Anwendungsbeispiels 
Es ist bei einem Vergleich des Startzeitpunktes jedes Ereignisses des in Abbildung 4.11 dargestellten Projektplans erkennbar, dass neben den Start- und Endereignissen 1 und 13 die Ereignisse 3, 9 und 12 keine Pufferzeiten enthalten und somit auf dem kritischen Pfad liegen. Eine Über- oder Unterschreitung der Dauern auf dem kritischen Pfad liegender Vorgänge führt zu einer zeitlichen Verschiebung des Projektendes in Höhe der zeitlichen Abweichung von den geplanten Dauern. Diese Vorgänge bilden einen kritischen Pfad von dem Projektstart zu dem Projektende, sofern, wie es in diesem Beispiel der Fall ist, das frühestmögliche und das spätesterlaubte Projektende übereinstimmen. ${ }^{114}$

\subsubsection{Ergebnisse des Project Scheduling Problems}

Bei der Problemstellung des Project Schedulings werden für die Projektplanung des Auftragnehmers die Höhe der Vergütungen durch den Auftraggeber sowie die Ereignisse, zu denen die Zahlungen erfolgen, als bekannt vorausgesetzt. Für dieses Beispiel wird daher angenommen, dass zu den Zeitpunkten der Ereignisse 7 und 10 jeweils eine Zahlung des Auftraggebers an den Auftragnehmer in Höhe von 350.000 GE sowie zu dem Zeitpunkt des Ereignisses 12 eine Zahlung in Höhe von 300.000 GE erfolgt. Diese Einzahlungen sind durch die Verrechnung der mit den entsprechenden Ereignissen verbundenen Vorgangskosten als Nettozahlungen oberhalb der Ereignisknoten in Abbildung 4.12 angegeben. Bei Zugrundelegung eines jährlichen Diskontierungszinssatzes in Höhe von $10 \%{ }^{115}$ lassen sich die Ereigniszeitpunkte berechnen, die aus der Sicht des Auftragnehmers zu einem Projektplan mit einem maximalen Kapitalwert führen. ${ }^{116}$ Es ergibt sich für den Auftragnehmer ein optimaler Projektplan mit einem Kapitalwert von -19.714 GE. Aufgrund der zu Beginn dieses Kapitels getroffenen Annahme der durch den Auftraggeber zu leistenden Teilzahlungen in Höhe von insgesamt 1.000.000 GE wird hierbei kein zusätzliches Honorar berücksichtigt, welches zu einem positiven Kapitalwert führen kann. Daher erfolgt an dieser Stelle keine Beurteilung der Vorteilhaftigkeit des Projektes aus der Sicht des Auftragnehmers.

\footnotetext{
114 Vgl. Schwarze (2001), S. 164.

115 Das entspricht einem Diskontierungssatz pro Woche von annähernd 0,19\%. Welche Abhängigkeitsbeziehung zwischen dem optimalen Kapitalwert eines Investitionsprojektes und der maximalen Projektdauer sowie dem Kalkulationszinsfuß besteht, ist Gegenstand der Untersuchungen von SchwindtZimmermann (2002).

${ }^{116}$ Die Lösung aller in dieser Arbeit vorgestellten mathematischen Modelle für das Anwendungsbeispiel erfolgt durch den Einsatz der von Dash Optimization vertriebenen Software Xpress-MP 2003 in der Version XpressIve 1.14.57.
} 


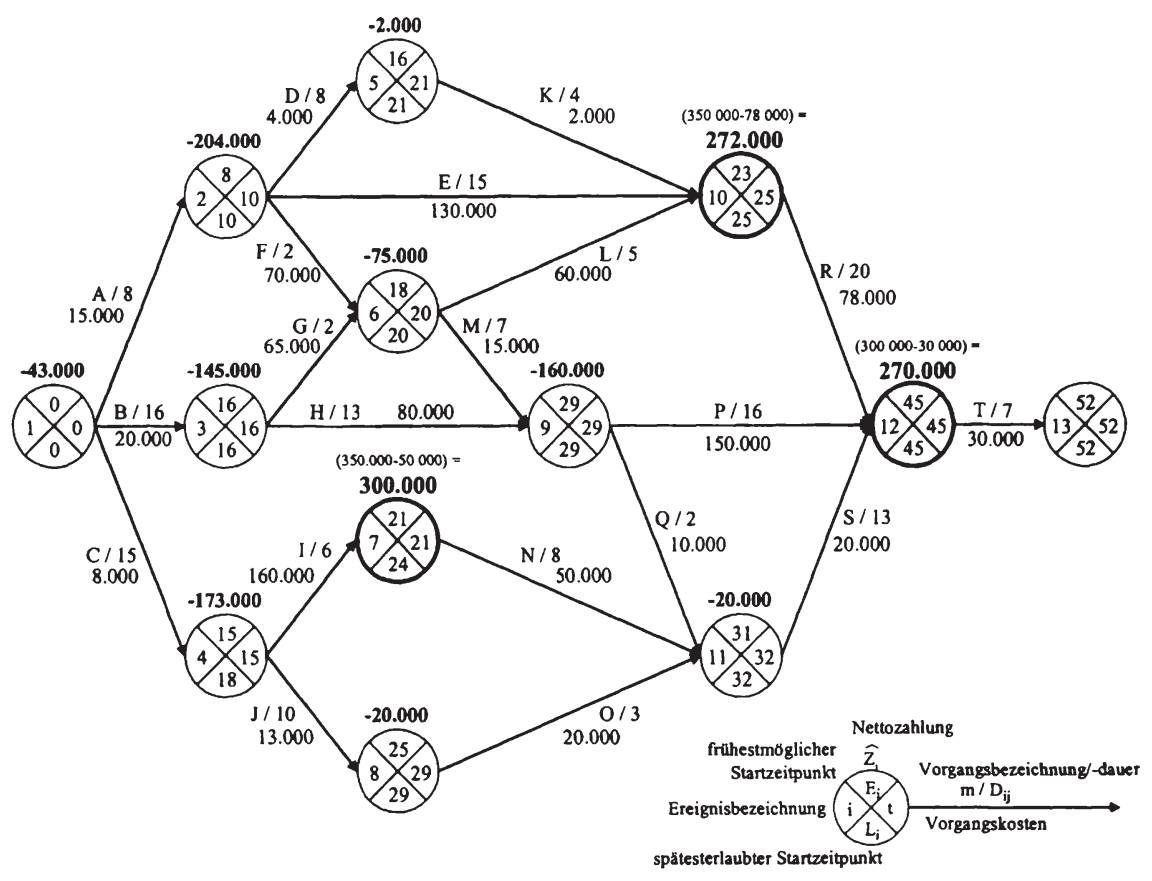

Abbildung 4.12: Optimale Lösung des Project Scheduling Problems

Die Vermutung, dass die mit der Kapitalwertmaximierung verbundene Zeitpräferenz für Zahlungen Ursache dafür ist, dass Ereignisse, zu denen eine Auszahlung erfolgt, zum spätesterlaubten Zeitpunkt und Ereignisse, mit denen eine Einzahlung verbunden ist, zum frühestmöglichen Zeitpunkt geplant werden, ist nicht zutreffend. Dies zeigt sich beispielsweise bei der Betrachtung des Ereignisses 4, zu dem eine Auszahlung erfolgt und zu dem frühestmöglichen Zeitpunkt geplant wird. Eine spätere Terminierung dieses Ereignisses hat zur Folge, dass das mit einer Einzahlung verbundene Ereignis 7 später stattfindet, was eine negative Wirkung auf den Kapitalwert hat.

Obwohl zu dem Ereignis 10 eine Einzahlung stattfindet, wird für den Ereigniszeitpunkt in der optimalen Lösung der spätesterlaubte Zeitpunkt gewählt. Dies ist damit zu erklären, dass die mit dem während der Projektdauer zeitlich frühen Ereignis 2 verbundene hohe Auszahlung möglichst spät erfolgt. Ein um zwei Zeiteinheiten früheres Stattfinden von Ereignis 2 vermindert den Projektkapitalwert in höherem Ausmaß als die um zwei Zeiteinheiten nach dem frühestmöglichen Starttermin von Ereignis 10 erfolgende Einzahlung. 
Unter Berücksichtigung der oben getroffenen Annahmen bezüglich der aus dem Projekt erzielbaren Erlöse für den Auftraggeber sowie eines mit dem Beispiel übereinstimmenden Diskontierungszinssatzes, lässt sich der Kapitalwert des Auftraggebers bei Zugrundelegung des ermittelten optimalen Projektplans ermitteln:

$\mathrm{KW}_{\mathrm{AG}}=\frac{1.000 .000 \mathrm{GE}}{(1+0,0019)^{52}}-\frac{350.000 \mathrm{GE}}{(1+0,0019)^{21}}-\frac{350.000 \mathrm{GE}}{(1+0,0019)^{25}}-\frac{300.000 \mathrm{GE}}{(1+0,0019)^{45}}=-39.531 \mathrm{GE}$

Obwohl das Project Scheduling Problem in seiner in der Literatur verwendeten Form ausschließlich die Projektplanung des Auftragnehmers unterstützt, wird der soeben ermittelte Kapitalwert des Auftraggebers in dem folgenden Kapitel zum Payment Scheduling als Referenzgröße herangezogen.

\subsubsection{Ergebnisse des Payment Scheduling Problems}

Das im vorherigen Kapitel aufgefuhrte Anwendungsbeispiel wird im Folgenden auf das die Projektplanung des Auftraggebers unterstützenden Payment Scheduling Problem übertragen. Es wird hierfür die Annahme aufgehoben, dass sowohl die Ereignisse, zu denen die Zahlungen des Auftraggebers an den Auftragnehmer erfolgen, als auch die Höhe dieser Zahlungen festgesetzt sind. Für die Berechnungen wird weiterhin unterstellt, dass die Summe der Zahlungen an den Auftragnehmer, welche aus der Sicht des Auftraggebers Auszahlungen darstellen, 1.000.000 GE beträgt. Dies entspricht damit der Summe der drei Zahlungen des Beispiels für das Project Scheduling Problem.

Die Anzahl der Zahlungen an den Auftragnehmer werden mit mindestens drei Teilzahlungen festgelegt. Die den Ereignissen zugeordneten Kosten der Leistungserstellung sind für beide Projektpartner bekannt und entsprechen denen des vorherigen Kapitels. Die Höhe der einzelnen Zahlungen des Auftraggebers an den Auftragnehmer muss mindestens die Summe der Kosten der bis zu dem Zahlungszeitpunkt abgeschlossenen Vorgänge decken (Kostenerstattungsfaktor $\beta=1,0$ ).

Die Lösung dieses Anwendungsbeispiels führt zu einer für den Auftraggeber optimalen Lösung mit den in Abbildung 4.13 dargestellten Ereignis- und Zahlungszeitpunkten sowie Zahlungsbeträgen: 


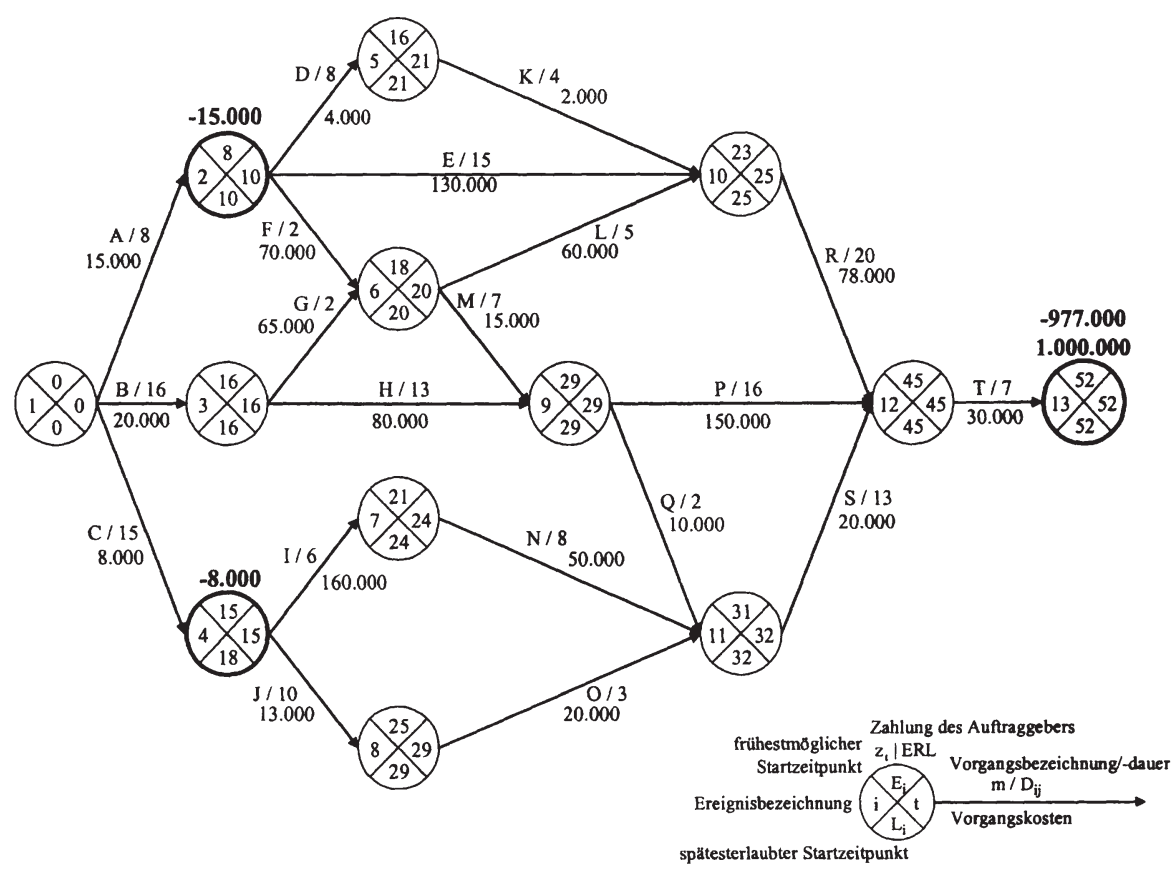

Abbildung 4.13: Optimale Lösung des Payment Scheduling Problems

Abbildung 4.13 zeigt, dass bei Ereignis 2 zu dem Zeitpunkt $t=10$ die erste Auszahlung in Höhe von $15.000 \mathrm{GE}$, bei Ereignis $4 \mathrm{zu}$ dem Zeitpunkt $\mathrm{t}=15$ die zweite Zahlung in Höhe von $8.000 \mathrm{GE}$ und bei Ereignis $13 \mathrm{zu}$ dem Zeitpunkt $\mathrm{t}=52$ die dritte und letzte Auszahlung in Höhe von $977.000 \mathrm{GE}$ erfolgt und somit die für die Modellierung der Kostenerstattungszahlungen modifizierte Restriktion (4.15) erfüllt wird. $\mathrm{Zu} \mathrm{dem}$ Zeitpunkt des Projektendes $t=52$ erhält der Auftraggeber eine Einzahlung der Erlöse in Höhe von 1.000.000 GE. Der Zielfunktionswert und somit der Kapitalwert des Auftraggebers beträgt -1.674 GE. Die Ermittlung des Kapitalwertes des Auftragnehmers ergibt für diesen Projektplan einen Wert in Höhe von -57.974 GE.

Entgegen der in Kapitel 4.3.1 getroffenen Vermutung, dass der Auftraggeber für die Erzielung des maximalen Kapitalwertes bemüht ist, mit möglichst späten während der Projektdauer stattfindenden Ereignissen Zahlungen zu leisten, zeigt sich für dieses Beispiel, dass es aufgrund der Kostenerstattungsvorschrift vorteilhaft ist, die ersten (ANZ-1) Zahlungen so früh wie möglich zu leisten und die letzte Zahlung mit dem 
Projektende zu verbinden. ${ }^{117}$ Die zu einem Ereignis zu leistende Zahlung an den Auftragnehmer sollte zu dem spätesterlaubten Ereigniszeitpunkt erfolgen, da dies den Kapitalwert des Auftraggebers positiv beeinflusst. Die zu Ereignis 4 erfolgende Zahlung in Höhe von $8.000 \mathrm{GE}$ wird jedoch zu dem Zeitpunkt $\mathrm{t}=15$ geplant, welcher nicht dem spätesterlaubten Ereigniszeitpunkt entspricht. Dies ist damit zu erklären, dass bei einer Zahlung zu dem Zeitpunkt $\mathrm{t}=18$ zusätzlich die mit Vorgang $\mathrm{B}$ verbundenen Kosten in Höhe von $20.000 \mathrm{GE}$ erstattet werden müssen.

Es zeigt sich im Vergleich zum Project Scheduling, dass der Auftraggeber durch die für ihn optimale Wahl der Zeitpunkte sowie Beträge der Fortschrittszahlungen einen höheren Kapitalwert erzielt. Der Auftragnehmer, dessen Kapitalwert sich im Vergleich vermindert hat, wird jedoch einer derartigen Zahlungsvereinbarung nicht zustimmen, da ein Großteil der entstandenen und durch ihn finanzierten Auszahlungen erst mit dem Projektende vergütet wird.

Auch die Annahme eines Kostenerstattungsfaktors $\beta=1,0$ ist aus der Sicht des Auftragnehmers nicht wünschenswert, vielmehr möchte dieser mit jeder Zahlung neben der Erstattung der bis zu den Zahlungszeitpunkten entstandenen Kosten auch einen Teil seines Honorars erhalten.

Sowohl die Höhe des Kostenerstattungsfaktors als auch die Häufigkeit und Höhe der Fortschrittszahlungen des Auftraggebers an den Auftragnehmer sind daher Bestandteil der vor der Projektdurchführung zwischen den Parteien stattfindenden Vertragsverhandlungen.

Das in den vorherigen Abschnitten vorgestellte Beispiel zeigt, dass die Zahlungsvereinbarungen maßgeblichen Einfluss auf den Projektkapitalwert beider Kooperationspartner haben. Dies bestätigt die Forderung, die unterschiedlichen im dritten Kapitel aufgezeigten Aspekte der Vertragsgestaltung sowie deren Konsequenzen in die quantitative Projektplanung einzubinden. Das Beispiel zeigt im Rahmen der Modellierung des Payment Scheduling Problems sowohl die Anwendbarkeit des vorgestellten Modells als auch Ansätze der Modellierung einer Kostenerstattungsvereinbarung.

${ }^{117}$ Vgl. Kapitel 4.3.1 sowie die Ergebnisse bei Dayanand/Padman (2001b). 


\subsection{Kritische Würdigung}

Eine Analyse der in den Kapiteln 4.2.3 sowie 4.3.3 vorgestellten mathematischen Modelle zum Project Scheduling sowie zum Payment Scheduling zeigt, dass insbesondere die in den letzten Jahren vorwiegend von Dayanand und Padman vorgestellten quantitativen Ansätze eine erste Verbindung von Contract Management und Projektplanung herstellen. Das für das Payment Scheduling Problem formulierte Modell beinhaltet die Möglichkeit für den Auftraggeber, Fortschrittszahlungen in Abhängigkeit von den entstandenen Kosten zu leisten. Dies entspricht der im dritten Kapitel vorgestellten Vereinbarung eines Kostenerstattungsvertrags. Obwohl Ansätze in der Literatur erkennbar sind, die auf eine Modellierung von typischen Charakteristika von Festpreisvereinbarungen etwa in Form gleicher Zahlungsintervalle hinweisen, ist eine systematische Verbindung dieser beiden betriebswirtschaftlichen Forschungsgebiete bisher unterblieben. Die Ergebnisse der Untersuchungen von Dayanand und Padman beruhen zudem auf einem für alle Veröffentlichungen identischen Anwendungsbeispiel. Es ist daher durch das in diesem Kapitel vorgestellte alternative Anwendungsbeispiel zu prüfen, ob die dort aufgrund der Lösung getroffenen Aussagen auf das in dieser Arbeit untersuchte Beispiel übertragbar sind.

Die für das Kooperationsverhältnis zwischen Auftraggeber und Auftragnehmer wichtigen und im dritten Kapitel aufgezeigten Teilnahme- und Anreizkompatibilitätsbedingungen werden im Rahmen der Prinzipal-Agenten-Theorie sowie der hierarchischen Planung mathematisch modelliert und theoretisch diskutiert, jedoch ist eine Einbindung dieser Bedingungen in die quantitativen Methoden der Projektplanung unter der Zielsetzung der Kapitalwertmaximierung bisher nicht erfolgt.

Ziel des folgenden fünften Kapitels ist es daher, die im dritten Kapitel vorgestellten Charakteristika möglicher Vertragsdesigns systematisch in ein erweitertes quantitatives Projektplanungsmodell $\mathrm{zu}$ integrieren sowie Aussagen $\mathrm{zu}$ der Vorteilhaftigkeit verschiedener Zahlungsvereinbarungen insbesondere aus dem Blickwinkel des Auftraggebers zu treffen. Zusätzlich sind für eine das Kooperationsverhältnis berücksichtigende und die Projektplanung des Auftraggebers unterstützende mathematische Modellierung Möglichkeiten aufzuzeigen, wie auch die Präferenzen des Auftragnehmers integriert werden können. Der Auftraggeber muss im Rahmen der Vertrags- und Projektplanung sowohl die Kooperationsteilnahme sicherstellen als auch das durch die Informationsasymmetrie geprägte Verhalten des Auftragnehmers berücksichtigen. 
Bernd Slaghuis - 978-3-631-75513-6 


\section{Einbindung des Vertragsdesigns in die Projektplanung des Auftraggebers}

Im Mittelpunkt dieses Kapitels steht die Einbindung der in Kapitel 3 aufgezeigten unterschiedlichen Vertragsdesigns in das im vierten Kapitel vorgestellte quantitative Payment Scheduling Modell. Die theoretischen Ansätze werden fortlaufend durch das zum Abschluss des letzten Kapitels eingeführte Anwendungsbeispiel ergänzt und zeigen so beispielhaft den Beitrag der Weiterentwicklungen des Modells für die Unterstützung der Vertrags- und Projektplanung des Auftraggebers auf.

Zunächst erfolgt eine Trennung der Vertragsdesigns in Kostenerstattungs- und Festpreisvereinbarungen. Bei einer Kostenerstattungsvereinbarung bestehen für den Auftraggeber unterschiedliche Handlungsalternativen hinsichtlich der Höhe des Kostenerstattungsfaktors und somit der Höhe der Zahlungen sowie der Anzahl der Teilzahlungen und damit der Zahlungszeitpunkte während der Projektlaufzeit. Das in Kapitel 4.3.3 vorgestellte Payment Scheduling Modell wird daher in Kapitel 5.1 erweitert und entsprechend modifiziert, so dass eine realitätsnahe Abbildung verschiedener Kostenerstattungsvereinbarungen möglich ist. Zu Beginn des Kapitels 5.2 werden unterschiedliche Zahlungsvereinbarungen für Festpreisverträge entwickelt und durch die Formulierung zusätzlicher Restriktionen in das Payment Scheduling Modell integriert. Anhand des Anwendungsbeispiels wird aufgezeigt, wie eine konkrete Umsetzung der Problemstellung möglich ist und zu einer Entscheidungsunterstützung des Auftraggebers genutzt werden kann. In Kapitel 5.3 erfolgt eine vergleichende Analyse und Beurteilung der Kostenerstattungs- und Festpreisvereinbarungen für das Anwendungsbeispiel.

Die Berücksichtigung der durch die Kooperationsbeziehung bestehenden hierarchischen Verbindung bei der Planung des Auftraggebers erfolgt in Kapitel 5.4 durch die Modellierung der Teilnahmebedingung des Auftragnehmers. Die Anwendung zeigt den Einfluss der Kooperationsbedingung auf den Projekterfolg des Auftraggebers für die hier betrachteten Vertragsformen konkret auf.

In Kapitel 5.5 wird für die Planung des Auftraggebers ein vierstufiges Vorgehen vorgeschlagen, welches das mögliche Auftragnehmerverhalten als Reaktion auf das Vertragsdesign antizipiert und den Auftraggeber zusätzlich bei der Einbindung von Anreizsystemen in das Vertragsdesign unterstützt. 


\subsection{Projektplanung bei Kostenerstattungsverträgen}

\subsubsection{Erweiterung des Payment Scheduling Modells um relevante Vertragscharakteristika}

Das in Kapitel 4.3.3 vorgestellte Payment Scheduling Modell beinhaltet bereits die Möglichkeit, Fortschrittszahlungen an den Auftragnehmer in Form einer Kostenerstattung durch den Auftraggeber zu leisten. Ziel dieses Kapitels ist eine das Grundmodell erweiternde und die relevanten Problemcharakteristika berücksichtigende Modellierung des Payment Scheduling Problems aus der Sicht des Auftraggebers, die die Grundlage der weiterführenden Untersuchungen einer Projektplanung bei Kostenerstattungsvereinbarungen darstellt.

Die im vierten Kapitel getroffenen Annahmen der Zuordnung der aus der Leistungserstellung des Auftragnehmers resultierenden Kosten zu Ereignissen werden im Folgenden für das fünfte Kapitel beibehalten. Insbesondere für eine Kostenerstattungsvereinbarung erscheint es sinnvoll, einerseits die für den Auftragnehmer entstehenden Kosten $\mathrm{K}_{\mathrm{i}}^{\mathrm{AN}}$ einem Ereigniszeitpunkt vor dem Beginn der Aktivität zuzurechnen und andererseits die Kosten $\mathrm{K}_{\mathrm{i}}^{\mathrm{AG}}$, welche die Grundlage für die Bestimmung der Höhe der durch den Auftraggeber zu den Zeitpunkten der Meilensteinereignisse zu leistenden Fortschrittszahlungen darstellen, Ereignissen zuzuordnen, welche zeitlich nach dem Abschluss der entsprechenden Aktivitäten geplant werden.

Restriktion (4.15) berücksichtigt einen Bestandteil der einem Kostenerstattungsvertrag zugrunde liegenden Zahlungsvereinbarung. Diese Bedingung enthält den Kostenerstattungsfaktor $\beta$, welcher den Anteil der während der Projektdauer zu leistenden Fortschrittszahlungen an den bis $\mathrm{zu}$ diesen Zeitpunkten entstandenen Kosten der Leistungserstellung angibt und somit die Mindesthöhe der Zahlungen des Auftraggebers an den Auftragnehmer festlegt.

Der Auftraggeber wird bemüht sein, einen Faktor kleiner als eins zu vereinbaren, um einerseits Anreize für ein frühes Erreichen des Projektendes und damit Zahlung des Restpreises zu schaffen und andererseits durch die Rückhaltung von Teilen der Vergütung Preisminderungen bei durch den Auftragnehmer verursachten Mängeln durchsetzen zu können. ${ }^{1}$

\footnotetext{
1 Vgl. Kapitel 3.3.2.
} 
Die Restriktion (4.16) des Payment Scheduling Modells gewährleistet, dass die Summe der Teilzahlungen der vertraglich vereinbarten Gesamtzahlung entspricht. Die Höhe der Summe der Fortschrittszahlungen wird bei einer Kostenerstattungsvereinbarung durch die dem Auftragnehmer für die Leistungserstellung entstandenen Kosten bestimmt, so dass eine Modifikation dieser Restriktion erforderlich ist. Je nach Wahl des Kostenerstattungsfaktors sind zwei Fälle zu unterscheiden:

\section{(1) Kostenerstattungsfaktor $\beta \leq 1,0$}

Ein Kostenerstattungsfaktor $\beta \leq 1,0$ bedeutet, dass die entstandenen Kosten maximal in voller Höhe mit jeder Teilzahlung erstattet werden, bei Faktoren kleiner eins erfolgt eine Einbehaltung von Beträgen während der Projektdauer und eine Abschlusszahlung in Höhe des zurückbehaltenen Betrags am Projektende mit dem letzten Ereignis. Zusätzlich kann der Auftraggeber mit dem Auftragnehmer eine Honorarzahlung für die Erbringung der Leistung vereinbaren. Um die mit diesen Kostenerstattungsfaktoren verbundenen Anreizwirkungen zu erhalten, empfiehlt sich die Verwendung eines festgesetzten Honorars HON, welches mit dem erfolgreichen Abschluss des Projektes mit der letzten Fortschrittszahlung gezahlt wird. Dies entspricht dem in Kapitel 3.2.3 vorgestellten Kostenerstattungsvertrag mit festem Honoraraufschlag (Cost-plus-fixedfee). Die Summe der Zahlungen $z_{t}$ muss somit mindestens der Summe der dem Auftragnehmer $\mathrm{zu}$ erstattenden Kosten $\mathrm{K}_{i}^{\mathrm{AN}}$ zuzüglich eines festen Honorars HON betragen. Für diesen Fall kann die Bedingung (4.16) des Modells wie folgt neu formuliert werden:

$\sum_{\mathrm{t}=0}^{\mathrm{T}} \mathrm{z}_{\mathrm{t}} \geq \sum_{\mathrm{i}=1}^{\mathrm{I}} \mathrm{K}_{\mathrm{i}}^{\mathrm{AN}}+\mathrm{HON}$

Die Kosten aus der Sicht des Auftragnehmers werden als Referenzgröße bei der Formulierung dieser Restriktion gewählt, um der Kostenerstattung der dem Auftragnehmer entstehenden Kosten Ausdruck zu verleihen, wenngleich gilt, dass die Summe der den einzelnen Ereignissen zugeordneten Kosten aus der Sicht von Auftragnehmer und Auftraggeber identisch ist.

$\sum_{\mathrm{i}=1}^{\mathrm{l}} \mathrm{K}_{\mathrm{i}}^{\mathrm{AN}}=\sum_{\mathrm{i}=1}^{\mathrm{l}} \mathrm{K}_{\mathrm{i}}^{\mathrm{AG}}$ 
(2) Kostenerstattungsfaktor $\beta>1,0$

Wird ein Kostenerstattungsfaktor $\beta>1,0$ vereinbart, so erhält der Auftragnehmer mit jeder Fortschrittszahlung $z_{t}$ neben der vollständigen Erstattung der Kosten zusätzlich einen Teil seines Honorars. Dieser Teil der Fortschrittszahlungen steht in proportionalem Verhältnis zu den entstehenden Kosten. Eine solche Vertragsvereinbarung wird im Rahmen des Contract Managements als Kostenerstattungsvertrag mit prozentualem Aufschlag bezeichnet (Cost-plus-percentage-fee). ${ }^{2}$ In diesem Fall beträgt die Summe der Fortschrittszahlungen das $\beta$-fache der Summe der Vorgangskosten, so dass eine die Restriktion (4.16) ersetzende Bedingung folgende Gestalt annimmt:

$$
\sum_{\mathrm{t}=0}^{\mathrm{T}} \mathrm{z}_{\mathrm{t}} \geq \beta \cdot \sum_{\mathrm{i}=1}^{\mathrm{I}} \mathrm{K}_{\mathrm{i}}^{\mathrm{AN}}
$$

Die Restriktionen (4.15), (5.1) und (5.3) sind als $\geq$-Restriktionen formuliert. Hierdurch wird einerseits gewährleistet, dass die Mindesthöhe der Fortschrittszahlungen eingehalten wird, jedoch andererseits die Möglichkeit für den Auftraggeber besteht, auch eine diese Mindesthöhe übersteigende Zahlung zu leisten. Obwohl dieses den Kapitalwert des Auftraggebers vermindert, werden in Kapitel 5.4 im Rahmen der Einbindung der Kooperationsbedingung Entscheidungssituationen aufgezeigt, die eine derartige Handlungsalternative als optimal ermitteln.

Unter Beibehaltung der übrigen Restriktionen (4.11) - (4.13), (4.15), (4.17) - (4.22) sowie der Zielfunktion (4.10) des Payment Scheduling Modells kann mit den in diesem Abschnitt formulierten Restriktionen der Fall eines Kostenerstattungsvertrags anwendungsnah modelliert werden. Dieses erweiterte Modell unterstützt den Auftraggeber als Entscheidungsträger bei der Projektplanung sowie der Vertragsgestaltung. Es ermöglicht neben der Bestimmung optimaler Ereignis- und Zahlungszeitpunkte auch Aussagen darüber, welchen Einfluss die unterschiedlichen Varianten einer Kostenerstattungsvereinbarung auf den Projekterfolg für den Auftraggeber in Form des Projektkapitalwertes haben.

Das folgende Kapitel zeigt anhand des am Ende des vierten Kapitels eingefuhrten Anwendungsbeispiels, wie sich die Höhe des Kostenerstattungsfaktors sowie die Anzahl der Zahlungen auf die Projektplanung und den Kapitalwert des Auftraggebers auswirken.

Vgl. Kapitel 3.3.2 sowie etwa Martin/Webster (1996), S. 167; Turner/Simister (2001), S. 459; Veld/Peeters (1989), S. 155. 


\subsubsection{Einfluss der Anzahl der Zahlungen und des Kostenerstattungsfaktors auf den Projekterfolg}

Für das Payment Scheduling Problem unter Berücksichtigung einer Kostenerstattungsvereinbarung müssen für die Berechnung eines optimalen Projektplans neben der Höhe des Auftragnehmerhonorars drei Parameter durch den Auftraggeber festgelegt werden: die Anzahl der Fortschrittszahlungen, der Kostenerstattungsfaktor und der Diskontierungszinssatz. Während die ersten beiden Parameter Gegenstand der vertraglichen Regelungen zwischen Auftraggeber und Auftragnehmer sind und somit bei der Projektplanung und -ausführung von Relevanz sind, hat die Wahl des Diskontierungszinssatzes ausschließlich Auswirkungen auf die kalkulatorische Größe des Kapitalwertes zur Beurteilung der Vorteilhaftigkeit des Vertragsdesigns sowie des Investitionsprojektes.

Die folgenden weiterführenden Untersuchungen des Anwendungsbeispiels verdeutlichen den Zusammenhang zwischen dem Projektkapitalwert und der Anzahl der Zahlungen sowie der Wahl des Kostenerstattungsfaktors. Die Berechnungen basieren auf den Daten aus Tabelle 4.5. Die Summe der Fortschrittszahlungen setzt sich bei Kostenerstattungsfaktoren $\beta \leq 1,0$ und keiner Honorarzahlung aus den zu erstattenden Kosten in Höhe von 1.000.000 GE zusammen.

Bei Kostenerstattungsfaktoren $\beta>1,0$ erhält der Auftragnehmer das $\beta$-fache der Kosten als Fortschrittszahlungen während der Projektdauer, so dass bei einem Faktor $\beta=1,2$ ein Honorar in Höhe von 200.000 GE durch den Auftraggeber gezahlt wird. Zusätzlich wird aus Gründen der Vergleichbarkeit aller Untersuchungsergebnisse zunächst unterstellt, dass, wie in Kapitel 4.4.1 erläutert, dem Auftraggeber am Ende des Projektes eine Einzahlung in Höhe von 1.000.000 GE als Projekterlös zukommt. Als Diskontierungssatz wird für die Berechnungen in diesem Kapitel ein jährlicher Zins von $10 \%$ festgesetzt.

Durch die Bindung der Zahlungen an Ereignisse, zu denen mindestens ein Vorgang abgeschlossen sein muss, beträgt die Anzahl der Fortschrittszahlungen für dieses Anwendungsbeispiel maximal zwölf. Mit Unterstützung der bereits in Kapitel 4 verwendeten Software Xpress-MP lässt sich das in Kapitel 4.3.3 aufgestellte und in Kapitel 5.1.1 erweiterte mathematische Modell lösen und ein aus der Sicht des Auftraggebers optimaler Projektplan ermitteln. Die folgenden Untersuchungen werden in den Fall eines Kostenerstattungsfaktors $\beta \leq 1,0$ sowie in den Fall eines Faktors $\beta>1,0$ unterteilt. 
(1) Kostenerstattungsfaktor $\beta \leq 1$

Die folgende Abbildung 5.1 zeigt das Ergebnis der Berechnungen für die Kostenerstattungsfaktoren $\beta \in\{0,7 ; 0,8 ; 0,9 ; 1,0\}$. Auf der Ordinate ist als Ergebnis der Optimierungen der maximale Projektkapitalwert des Auftraggebers abgetragen, auf der Abszisse die Anzahl der Fortschrittszahlungen während der Projektdurchführung. ${ }^{3}$

Es lässt sich für dieses Anwendungsbeispiel erkennen, dass eine Erhöhung der Anzahl der Fortschrittszahlungen den Projektkapitalwert nur in geringem Ausmaß beeinflusst, sofern nur wenige Zahlungen vereinbart werden. Bis zu einer Häufigkeit von fünf Zahlungen hat sowohl die Anzahl der Zahlungen als auch der gewählte Kostenerstattungsfaktor nur geringen Einfluss auf den Projektkapitalwert des Auftraggebers.

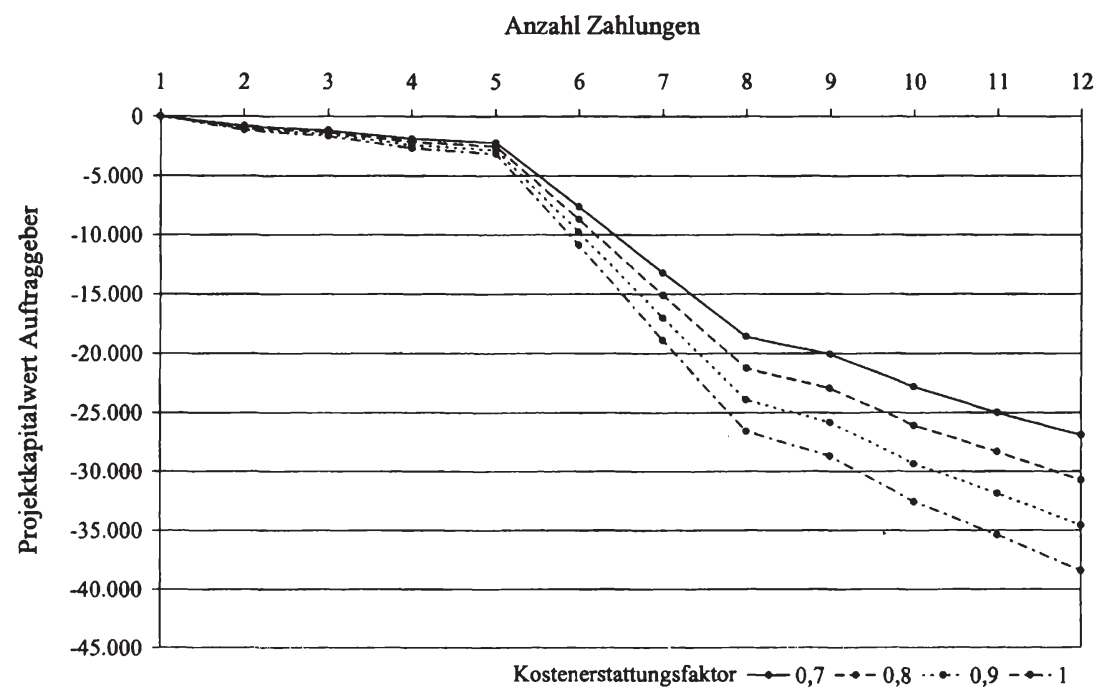

Abbildung 5.1: Kapitalwerte des Auftraggebers bei Kostenerstattungsfaktoren $\beta \leq 1,0$

Eine Erhöhung der Anzahl der Zahlungen von fünf auf sechs bedeutet bei einem Kostenerstattungsfaktor $\beta=1,0$ eine Verminderung des Kapitalwertes um 245\%. Dies ist mit der Struktur des Projektplans in Kombination mit den zu Ereignissen anfallenden Kosten wie folgt zu erklären:

3 Um die von der Anzahl der Zahlungen abhăngige Entwicklung des Kapitalwertes des Auftraggebers zu visualisieren, sind die zu einem Kostenerstattungsfaktor zugehorigen Punkte miteinander verbunden, obwohl es sich bei der Anzahl der Zahlungen um eine diskrete Größe handelt. 
In Tabelle 5.1 sind die aus der Sicht des Auftraggebers optimalen Zahlungszeitpunkte sowie die Beträge der Fortschrittszahlungen für einen Kostenerstattungsfaktor $\beta=1,0$ angegeben:

\begin{tabular}{|c|c|c|c|c|c|c|c|c|c|c|c|c|}
\hline & \multicolumn{12}{|c|}{ Anzahl der Zahlungen } \\
\hline $\begin{array}{l}\text { Zeipunkt } \\
\text { Hohe der Zzhlung }\end{array}$ & 1 & 2 & 3 & 4 & 5 & 6 & 7 & 8 & 9 & 10 & 11 & 12 \\
\hline 1. Zahlung & \begin{tabular}{c|}
52 \\
1.000 .000 \\
\end{tabular} & $\begin{array}{c}10 \\
15.000 \\
\end{array}$ & \begin{tabular}{|c|}
10 \\
15.000 \\
\end{tabular} & $\begin{array}{c}9 \\
15.000 \\
\end{array}$ & \begin{tabular}{|c|}
10 \\
15.000 \\
\end{tabular} & \begin{tabular}{|c|}
10 \\
15.000 \\
\end{tabular} & \begin{tabular}{|c|}
10 \\
15.000 \\
\end{tabular} & $\begin{array}{c}10 \\
15.000 \\
\end{array}$ & \begin{tabular}{|c|}
10 \\
15.000 \\
\end{tabular} & \begin{tabular}{|c|}
10 \\
15.000 \\
\end{tabular} & $\begin{array}{c}10 \\
15.000 \\
\end{array}$ & $\begin{array}{c}10 \\
15.000 \\
\end{array}$ \\
\hline 2. Zahlung & & $\begin{array}{c}52 \\
985.000 \\
\end{array}$ & $\begin{array}{c}15 \\
8.000 \\
\end{array}$ & \begin{tabular}{c|}
16 \\
20.000 \\
\end{tabular} & \begin{tabular}{|c|}
16 \\
20.000 \\
\end{tabular} & $\begin{array}{c}16 \\
20.000 \\
\end{array}$ & $\begin{array}{c}16 \\
20.000 \\
\end{array}$ & $\begin{array}{c}16 \\
20.000 \\
\end{array}$ & $\begin{array}{c}16 \\
20.000 \\
\end{array}$ & \begin{tabular}{|c|}
16 \\
20.000 \\
\end{tabular} & $\begin{array}{c}16 \\
20.000 \\
\end{array}$ & $\begin{array}{c}16 \\
20.000 \\
\end{array}$ \\
\hline 3. Zshlung & & & \begin{tabular}{c|}
52 \\
977.000 \\
\end{tabular} & $\begin{array}{c}17 \\
4.000 \\
\end{array}$ & $\begin{array}{c}18 \\
8.000 \\
\end{array}$ & $\begin{array}{c}18 \\
8.000\end{array}$ & $\begin{array}{c}18 \\
8.000 \\
\end{array}$ & $\begin{array}{c}18 \\
8.000 \\
\end{array}$ & $\begin{array}{c}18 \\
8.000 \\
\end{array}$ & \begin{tabular}{|c|}
18 \\
8.000 \\
\end{tabular} & \begin{tabular}{|c|}
18 \\
8.000 \\
\end{tabular} & $\begin{array}{c}18 \\
8.000 \\
\end{array}$ \\
\hline 4. Zahlung & & & & $\begin{array}{c}52 \\
961.000 \\
\end{array}$ & $\begin{array}{c}19 \\
4.000\end{array}$ & $\begin{array}{c}20 \\
135.000\end{array}$ & \begin{tabular}{c|}
20 \\
135.000 \\
\end{tabular} & $\begin{array}{c}20 \\
135.000\end{array}$ & $\begin{array}{c}20 \\
135.000 \\
\end{array}$ & \begin{tabular}{|c|}
20 \\
135.000 \\
\end{tabular} & $\begin{array}{c}20 \\
135.000 \\
\end{array}$ & $\begin{array}{c}20 \\
135.000 \\
\end{array}$ \\
\hline 5. Zahlung & & & & & \begin{tabular}{|c|}
52 \\
953.000 \\
\end{tabular} & $\begin{array}{c}21 \\
4.000 \\
\end{array}$ & $\begin{array}{c}21 \\
4.000 \\
\end{array}$ & $\begin{array}{c}21 \\
4.000 \\
\end{array}$ & $\begin{array}{c}21 \\
4.000 \\
\end{array}$ & $\begin{array}{c}21 \\
4.000 \\
\end{array}$ & $\begin{array}{c}21 \\
4.000 \\
\end{array}$ & $\begin{array}{c}21 \\
4.000 \\
\end{array}$ \\
\hline 6. Zahlung & & & & & & \begin{tabular}{c|}
52 \\
818.000 \\
\end{tabular} & \begin{tabular}{c|}
24 \\
160.000 \\
\end{tabular} & $\begin{array}{c}24 \\
160.000 \\
\end{array}$ & $\begin{array}{c}24 \\
160.000 \\
\end{array}$ & \begin{tabular}{|c|}
24 \\
160.000 \\
\end{tabular} & \begin{tabular}{|c|}
24 \\
160.000 \\
\end{tabular} & $\begin{array}{c}24 \\
160.000 \\
\end{array}$ \\
\hline 7. Zahlung & & & & & & & $\begin{array}{c}52 \\
658.000 \\
\end{array}$ & $\begin{array}{c}45 \\
628.000 \\
\end{array}$ & $\begin{array}{c}25 \\
192.000 \\
\end{array}$ & \begin{tabular}{|c|}
25 \\
192.000 \\
\end{tabular} & $\begin{array}{c}25 \\
192.000 \\
\end{array}$ & $\begin{array}{c}25 \\
192.000\end{array}$ \\
\hline 8. Zahlung & & & & & & & & $\begin{array}{c}52 \\
30.000 \\
\end{array}$ & $\begin{array}{c}28 \\
13.000 \\
\end{array}$ & \begin{tabular}{|c|}
28 \\
13.000 \\
\end{tabular} & $\begin{array}{c}28 \\
13.000 \\
\end{array}$ & $\begin{array}{c}28 \\
13.000 \\
\end{array}$ \\
\hline 9. Zahlung & & & & & & & & & $\begin{array}{c}52 \\
453.000 \\
\end{array}$ & \begin{tabular}{|c|}
29 \\
95.000 \\
\end{tabular} & $\begin{array}{c}29 \\
95.000 \\
\end{array}$ & \begin{tabular}{|c|}
29 \\
95.000 \\
\end{tabular} \\
\hline 10. Zshlung & & & & & & & & & & \begin{tabular}{|c|}
52 \\
358.000 \\
\end{tabular} & $\begin{array}{c}32 \\
80.000 \\
\end{array}$ & $\begin{array}{c}32 \\
80.000 \\
\end{array}$ \\
\hline 11. Zahlung & & & & & & & & & & & $\begin{array}{c}52 \\
278.000 \\
\end{array}$ & \begin{tabular}{|c|}
45 \\
248.000 \\
\end{tabular} \\
\hline 12. Zahlung & & & & & & & & & & & & $\begin{array}{c}52 \\
30.000 \\
\end{array}$ \\
\hline
\end{tabular}

Tabelle 5.1: Optimale Zahlungen und Zahlungszeitpunkte bei Kostenerstattung $(\beta=1,0)$

Das starke Sinken des Kapitalwertes bei einem Wechsel von fünf auf sechs Zahlungen ist mit den zu dem Zeitpunkt $\mathrm{t}=20$ abgeschlossenen und in das Ereignis 6 mündenden Vorgängen $\mathrm{F}$ und $\mathrm{G}$ zu erklären, so dass zu diesem Zeitpunkt eine Fortschrittszahlung in Höhe von $135.000 \mathrm{GE}$ fällig ist. Dieser im Vergleich zu den mit den Ereignissen 2, 3, 4 und 5 fälligen Zahlungen hohe Betrag ist bis zu einer Anzahl von einschließlich fünf Zahlungszeitpunkten in der Abschlusszahlung enthalten.

Aus der Sicht des ebenfalls den aus dem Projekt resultierenden Kapitalwert maximierenden Auftragnehmers besteht die Präferenz, eine Kompensationszahlung der entstandenen Kosten zu jedem Ereigniszeitpunkt und damit zwölf Zahlungen zu erhalten. ${ }^{4}$ Unter der Annahme, dass dieser die für die Projektdurchführung notwendigen Auszahlungen finanzieren muss, senkt eine Erhöhung der Anzahl der Zahlungen des Auftraggebers gleichzeitig die Finanzierungskosten des Auftragnehmers.

Der Auftraggeber erzielt den maximalen Kapitalwert bei einer Zahlung zum Abschluss des Projektes. Er hat ein Interesse daran, die Zahlungen an den Auftragnehmer so spät

\footnotetext{
4 Vgl. hierzu auch Gido/Clements (2003), S. 272.
} 
wie möglich zu leisten, um damit nicht nur monetäre Ziele zu verfolgen, sondern auch Anreize für die Arbeitsleistungen des Auftragnehmers zu setzen. Tabelle 5.2 zeigt für den Kostenerstattungsfaktor $\beta=1,0$ die Kapitalwerte für Auftraggeber und Auftragnehmer in Abhängigkeit von der Anzahl der Zahlungen. Während sich der maximale Kapitalwert des Auftraggebers mit jeder zusätzlichen Zahlung vermindert, erhöht sich mit Zunahme der Anzahl der Zahlungen der Kapitalwert des Auftragnehmers. ${ }^{5}$

\begin{tabular}{|l|c|c|c|c|c|c|c|c|c|c|c|c|}
\cline { 2 - 12 } \multicolumn{1}{c|}{} & \multicolumn{10}{c|}{ Anzahl der Zahlungen } \\
\cline { 2 - 12 } & $\mathbf{1}$ & $\mathbf{2}$ & $\mathbf{3}$ & $\mathbf{4}$ & $\mathbf{5}$ & $\mathbf{6}$ & $\mathbf{7}$ & $\mathbf{8}$ & $\mathbf{9}$ & $\mathbf{1 0}$ & $\mathbf{1 1}$ & $\mathbf{1 2}$ \\
\hline KW Auftraggeber & 0 & -1.141 & -1.674 & -2.717 & -3.162 & -10.894 & -18.896 & -26.590 & -28.702 & -32.586 & -35.421 & -38.460 \\
\hline KW Auftragnehmer & -58.675 & -57.534 & -57.967 & -56.358 & -55.520 & -47.781 & -39.779 & -32.084 & -30.010 & -26.126 & -23.290 & -20.251 \\
\hline
\end{tabular}

Tabelle 5.2: Kapitalwerte von Auftraggeber und Auftragnehmer bei Kostenerstattung $(\beta=1,0)$

Abbildung 5.2 zeigt die Entwicklung der Kapitalwerte von Auftraggeber und Auftragnehmer für die Kostenerstattungsfaktoren $\beta=0,8$ und $\beta=1,0$.

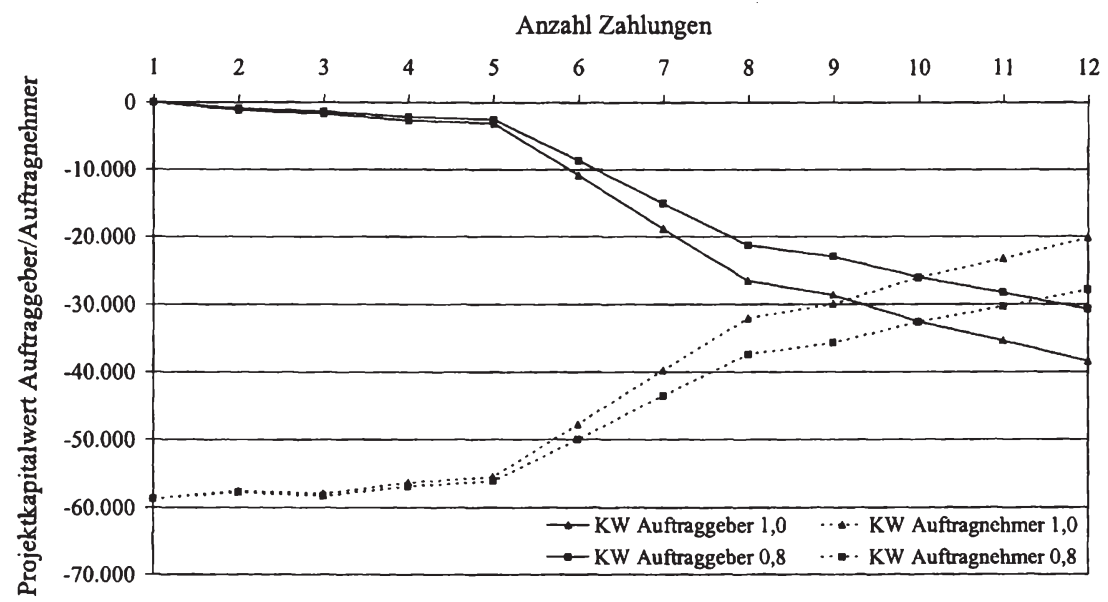

Abbildung 5.2: Kapitalwerte von Auftraggeber und Auftragnehmer bei Kostenerstattung

5 Eine Ausnahme bildet die Verănderung des Kapitalwertes bei einem Wechsel von zwei auf drei Zahlungen. Hier sinkt der Kapitalwert des Auftraggebers entgegen dem festgestellten Trend. Ereignis 4 muss im Zeitintervall $[15,18]$ liegen, Ereignis 5 im Intervall [16, 21]. Für den Auftraggeber ist es vorteilhaft, die drei Zahlungen an die zeitlich ersten beiden Ereignisse und an das letzte Ereignis zu koppeln. Um eine Fortschrittszahlung für Vorgang D zum Zeitpunkt des Ereignisses 5 in Verbindung mit Vorgang $C$ und Ereignis $4 \mathrm{zu}$ vermeiden, wird der Zeitpunkt des Ereignisses 4 auf $t=15$ terminiert. Aus der Sicht des Auftragnehmers ist mit Ereignis 4 eine Auszahlung in Höhe von 173.000 GE verbunden, welche zu diesem fruhen Ereigniszeitpunkt negative Auswirkungen auf den Kapitalwert des Auftragnehmers hat. 
Es bestätigt sich damit die Vermutung, dass der Auftraggeber niedrige Kostenerstattungsfaktoren und der Auftragnehmer hohe Kostenerstattungsfaktoren bevorzugt. Der Auftraggeber muss diesen Zielkonflikt zwischen den Kooperationspartnern im Rahmen der Vertragsgestaltung berücksichtigen. Ein hoher Kostenerstattungsfaktor kann den Auftragnehmer motivieren, dessen Arbeitsanstrengungen zu erhöhen und damit zu einem aus der Sicht des Auftraggebers besseren Ergebnis, etwa durch das Erreichen eines früheren Projektendes führen. Dieser Aspekt ist jedoch insbesondere unter der Annahme eines Nutzen maximierenden, opportunistischen Verhaltens des Auftragnehmers kritisch zu betrachten. Auch die Beurteilung der erbrachten Leistungen des Auftragnehmers ist für den Auftraggeber nicht immer möglich.

Die Ergebnisse der hier durchgeführten Berechnungen zeigen, welche quantitativen Auswirkungen die Festlegung der Anzahl der Zahlungen sowie der Höhe des Kostenerstattungsfaktors auf die Kapitalwerte der Kooperationspartner hat. Der Punkt, an dem sich die Verbindungslinien der Kapitalwerte von Auftraggeber und Auftragnehmer kreuzen, ist für die folgenden Untersuchungen nicht von Relevanz, da dieser maßgeblich durch die Höhe der Erlöse für den Auftraggeber aus der Projektnutzung bestimmt wird. Die für diese Berechnungen getroffene Annahme eines dem Auftraggeber zum Zeitpunkt des Projektendes zufließenden Projekterlöses in Höhe der für die Leistungserstellung notwendigen Kosten des Auftragnehmers von 1.000.000 GE führt zwar aufgrund der in der Realität später erfolgenden Einzahlungen hier zu einem höheren Kapitalwert, wird jedoch aus Gründen einer besseren Ergebnisinterpretation in dieser Höhe und zu diesem Zeitpunkt gewählt. Der Auftraggeber muss diese Daten bei realen Problemstellungen schätzen. Er kann jedoch mit Hilfe der durchgeführten Untersuchungen leicht ermitteln, in welcher Höhe je nach Wahl des Kostenerstattungsfaktors und der Anzahl der Zahlungen zusätzlich Einzahlungen aus dem Projekt erfolgen müssen, um einen positiven Kapitalwert zu erzielen.

Die Ermittlung eines aus der Sicht beider Vertragsparteien die individuellen Ziele in gleichem Ausmaß erfüllenden Projektplans findet im Rahmen der in dieser Arbeit unterstellten Beziehung zwischen dem den Vertrag gestaltenden Auftraggeber und dem das Projekt ausführenden Auftragnehmer keine weitere Berücksichtigung. ${ }^{6}$ Obwohl der Auftraggeber bei der Wahl des Vertragsdesigns die anschließende Entscheidung des Auftragnehmers über seine Arbeitsleistung in das Entscheidungsproblem einbezie-

6 Vgl. hierfür die Untersuchungen von Ulusoy/Cebelli (2000). 
hen muss und die Bestimmung eines Kostenerstattungsfaktors bzw. der Anzahl der Teilzahlungen Gegenstand von Vertragsverhandlungen sein können, wird davon ausgegangen, dass der Auftraggeber aufgrund seiner Machtposition innerhalb der Kooperationsbeziehung kein Interesse an einer Kompromisslösung hat, bei der beide Parteien in einem bestimmten Ausmaß von ihrer individuellen optimalen Lösung abweichen.

(2) Kostenerstattungsfaktor $\beta>1,0$

Wird im Rahmen einer Kostenerstattungsvereinbarung ein Faktor $\beta>1,0$ gewählt, erhält der Auftragnehmer neben einer Erstattung der Kosten abgeschlossener Vorgänge zusätzlich mit jeder Zahlung einen Teil seines Honorars. Für das Anwendungsbeispiel bedeutet dies, dass der Auftraggeber beispielsweise bei einem Faktor von $\beta=1,2$ Fortschrittszahlungen in Höhe von insgesamt 1.200.000 GE leistet.

Um einen Vergleich der Kapitalwerte des Auftraggebers für unterschiedliche Kostenerstattungsfaktoren zu gewährleisten, wird auch bei diesen Berechnungen eine Einzahlung am Ende der Projektlaufzeit in Höhe von 1.000.000 GE zugrunde gelegt. Diese Annahme führt wie zuvor zu negativen Kapitalwerten aus der Sicht des Auftraggebers. Die Tatsache, dass bei der betrachteten Problemstellung keine Auswahlentscheidung im Sinne einer Vorteilhaftigkeit des Projektes zu treffen ist und jeder alternative, höhere und somit eventuell zu einem positiven Kapitalwert führende Erlös im Rahmen dieses Anwendungsbeispiels auch eine Annahme darstellt, wird für eine Vergleichbarkeit der Ergebnisse weiterhin von Erlösen in Höhe von 1.000.000 GE ausgegangen.

Abbildung 5.3 zeigt die maximalen Projektkapitalwerte des Auftraggebers für die Kostenerstattungsfaktoren $\beta \in\{1,1 ; 1,2 ; 1,3\}$ in Abhängigkeit von der Anzahl der Zahlungen.

Erwartungsgemäß zeigt sich, dass eine Kostenerstattungsvereinbarung $\beta>1,0$ für den Fall einer einzigen, zu dem Zeitpunkt des Projektendes erfolgenden Zahlung zu einem sinkenden Kapitalwert gegenüber einem Faktor $\beta=1,0$ um den Betrag des auf den Zeitpunkt $\mathrm{t}=0$ diskontierten Honorars führt.

Mit jeder zusätzlichen Forschrittszahlung erhöht sich die Differenz der Kapitalwerte gegenüber der einfachen Kostenerstattung, da mit jeder Zahlung ein Teil des Honorars bereits während der Projektlaufzeit an den Auftragnehmer gezahlt wird. 


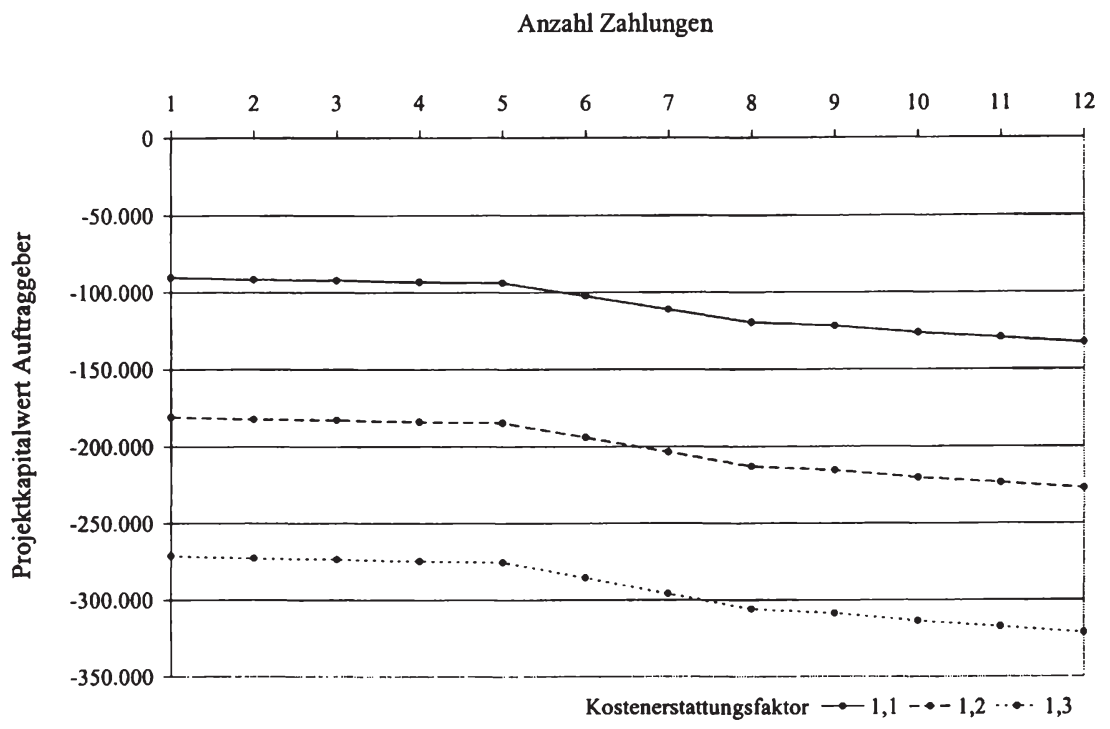

Abbildung 5.3: Kapitalwerte des Auftraggebers bei Kostenerstattungsfaktoren $\beta>1,0$

Diese Vereinbarung in Form eines prozentualen Honoraraufschlags kann mit einer Kostenerstattungsvereinbarung mit festem Honoraraufschlag verglichen werden. Unter Beibehaltung der Annahme der mit dem Projekt insgesamt entstehenden und zu erstattenden Kosten in Höhe von 1.000.000 GE ist die Summe der Fortschrittszahlungen bei den jeweiligen zu vergleichenden Kostenerstattungsvereinbarungen identisch. Für das Anwendungsbeispiel können daher beispielsweise die folgenden Kostenerstattungsvereinbarungen gegenübergestellt werden:

\begin{tabular}{|l|l|}
\hline $\begin{array}{l}\text { Kostenerstattung mit } \\
\text { prozentualem Honoraraufschlag }\end{array}$ & $\begin{array}{l}\text { Kostenerstattung mit } \\
\text { festem Honoraraufschlag }\end{array}$ \\
\hline$\beta=1,1$ & $\beta \leq 1,0+$ Honorar $100.000 \mathrm{GE}$ \\
\hline$\beta=1,2$ & $\beta \leq 1,0+$ Honorar $200.000 \mathrm{GE}$ \\
\hline$\beta=1,3$ & $\beta \leq 1,0+$ Honorar $300.000 \mathrm{GE}$ \\
\hline
\end{tabular}

Tabelle 5.3: Kostenerstattung mit prozentualem oder festem Honoraraufschlag 
Die folgende Abbildung 5.4 zeigt beispielhaft das Ergebnis der Untersuchungen für den Fall eines Kostenerstattungsfaktors $\beta=1,1$ im Vergleich mit einer Kostenerstattungsvereinbarung mit $\beta=1,0$ zuzüglich eines festen Honorars in Höhe von 100.000 GE. Dieser Vergleich entspricht der in der ersten Zeile der Tabelle 5.3 gegenübergestellten Kostenerstattungsvereinbarungen mit prozentualem bzw. festem Honoraraufschlag. Durch die Erstattung aller entstehenden Kosten führt auch eine Vereinbarung eines Faktors $\beta=0,9$ sowie des Honorars in Höhe von $100.000 \mathrm{GE}$ zu einer Gesamtzahlung von 1.100.000 GE, so dass auch dieser Fall in den Vergleich einbezogen werden kann.

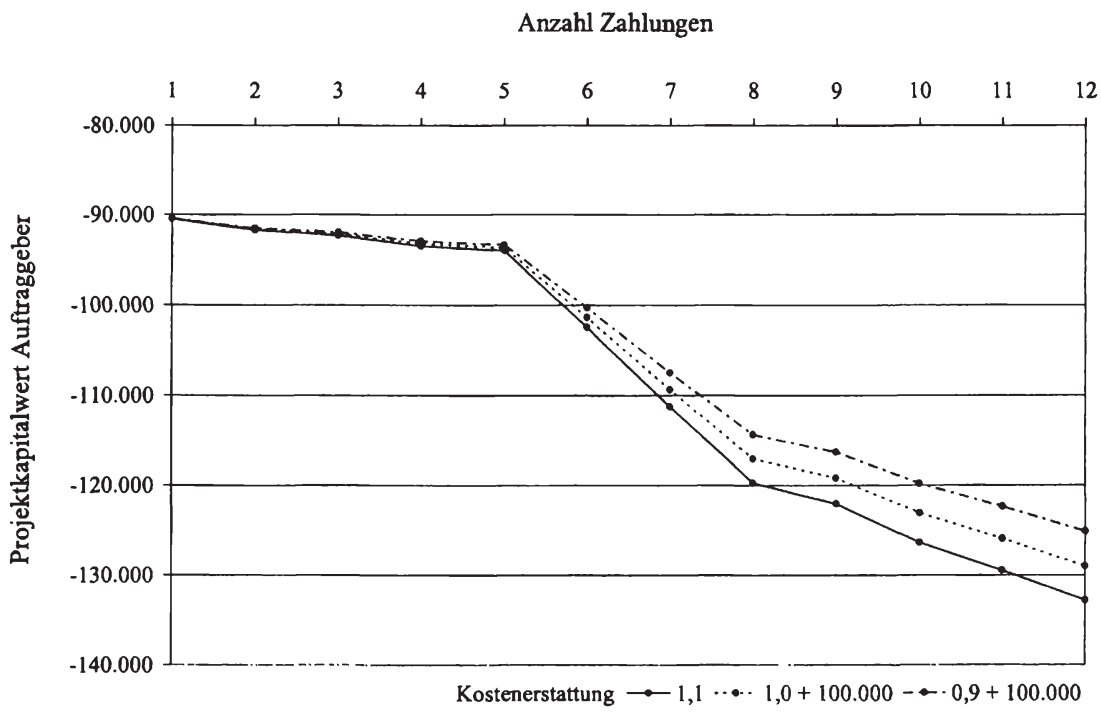

Abbildung 5.4: Kapitalwerte des Auftraggebers bei prozentualem oder festem Honoraraufschlag

Aus der Sicht des Auftraggebers ist es aufgrund der Zeitpräferenz seiner Auszahlungen vorteilhaft, das Honorar zum Abschluss des Projektes an den Auftragnehmer zu zahlen. Daher ist der Kapitalwert des Auftraggebers mit festem Honoraraufschlag und einer Kostenerstattung mit $\beta \leq 1,0$ immer gleich bzw. größer als der Kapitalwert bei prozentualer Kosten- und Honorarerstattung. Die Differenz der Kapitalwerte der beiden Kostenerstattungsvereinbarungen resultiert bei einem Faktor $\beta>1,0$ aus den während der Projektdauer zu den Zeitpunkten der Fortschrittszahlungen anteilig enthaltenen und diskontierten Honorarzahlungen an den Auftragnehmer. 
Die Berechnungen im Rahmen des Anwendungsbeispiels zeigen zusammenfassend, dass der Auftraggeber bei einer Kostenerstattungsvereinbarung die Anzahl der Zahlungen auf höchstens fünf Zahlungen begrenzen sollte. Weiterhin führt die Festlegung eines niedrigen Kostenerstattungsfaktors zu einem höheren Kapitalwert des Auftraggebers. Die Zahlung eines Honorars sollte mit dem Abschluss des Projektes erfolgen. Insgesamt kann das hier vorgestellte mathematische Modell den Auftraggeber bei der Festlegung der Kostenerstattungsvereinbarung unterstützen, indem die Konsequenzen unterschiedlicher Handlungsalternativen ermittelt und bewertet werden.

\subsection{Projektplanung bei Festpreisverträgen}

\subsubsection{Entwicklung von Zahlungsvereinbarungen für Festpreisverträge}

In Kapitel 3.3.3 sind die auf dem Gebiet des Contract Managements bekannten Formen vertraglicher Festpreisvereinbarungen vorgestellt sowie deren Chancen und Risiken für Auftraggeber und Auftragnehmer diskutiert worden. Im Folgenden wird entsprechend dem Vorgehen des vorherigen Kapitels das in Kapitel 4.3.3 aufgestellte Payment Scheduling Modell modifiziert und erweitert, um charakteristische Merkmale von Festpreisvereinbarungen abbilden zu können und deren Auswirkungen auf die Projektplanung des Auftraggebers zu analysieren.

Als Merkmal einer Festpreisvereinbarung ist die Zahlung eines bei Vertragsabschluss festgelegten Preises des Auftraggebers an den die vertragliche Leistung erbringenden Auftragnehmer während oder am Ende der Projektlaufzeit festgestellt worden. ${ }^{7}$ Die Höhe der Zahlung ist hierbei annahmegemäß unabhängig von den für die Leistungserbringung entstandenen Kosten. ${ }^{8}$ Insbesondere die Festlegung der Anzahl der Zahlungen bzw. Zahlungsintervalle sowie der Höhe der Zahlungen des Auftraggebers an den Auftragnehmer stellen wichtige Charakteristika der Festpreisvereinbarung dar.

Durch die Kombination dieser Charakteristika können unterschiedliche Zahlungsvereinbarungen für Festpreisverträge entwickelt werden. Bei der hier gewählten und in Abbildung 5.5 dargestellten Unterteilung in konstante und variierende Zahlungsintervalle sowie in konstante und variierende Zahlungsbeträge entstehen durch die Kombi-

\footnotetext{
Vgl. Kapitel 3.3.3.

8 Auf Festpreisverträge, die durch Anreizsysteme oder besondere zusätzliche Vereinbarungen die Hohe der tatsächlich entstandenen Kosten einbeziehen, wird in Kapitel 5.5.4 vertiefend eingegangen.
} 
nation der Merkmale vier Bereiche, für die in den Fällen variierender Zahlungsbeträge und/oder variierender Zahlungsintervalle beispielhaft verschiedene Möglichkeiten der Ausgestaltung von Festpreisvereinbarungen aufgeführt sind.

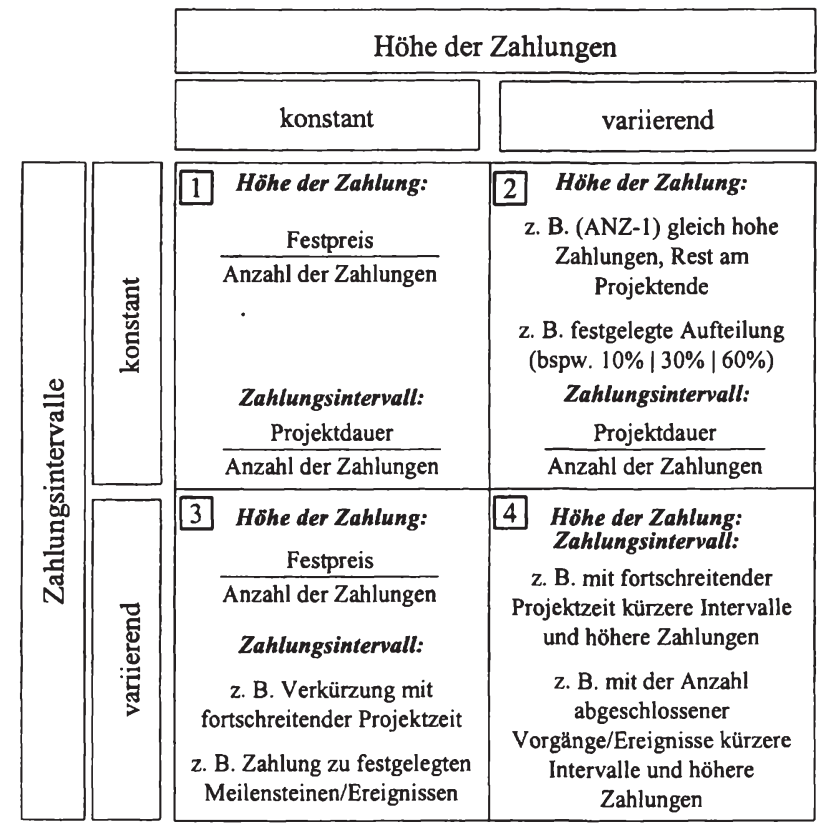

Abbildung 5.5: Zahlungsvereinbarungen für Festpreisverträge

Der erste Quadrant betrachtet den Fall, bei dem sowohl die Höhe der Zahlungen als auch die Zahlungsintervalle, die als zeitlicher Abstand zwischen zwei aufeinander folgenden Zahlungen definiert werden, konstant sind. Der Auftragnehmer erhält hierbei abhängig von der vereinbarten Anzahl der Zahlungen während der geplanten Projektzeit regelmäßig Zahlungen gleicher Höhe, die unabhängig von einem Projektfortschritt sowie den entstandenen Kosten sind. Diese Art der Zahlungsvereinbarung scheint für diejenigen Kooperationen sinnvoll zu sein, bei denen der Auftraggeber den Projektfortschritt bzw. die mit der Erstellung der vereinbarten Leistungen für den Auftragnehmer entstehenden Kosten nicht beobachten kann. In Kapitel 3.2.2 ist diese Art der Informationsasymmetrie mit dem Begriff Hidden Action bezeichnet worden. Eine derartige Form der Zahlungsvereinbarung enthält jedoch weder Anreize für den Auftragnehmer, die tatsächlich entstandenen Kosten offen zu legen, noch die Leistungen entsprechend der Präferenzen des Auftraggebers zu erbringen, da jede Möglichkeit der Einsparung von Kosten das Honorar des Auftragnehmers erhöht. 
Durch eine variierende Höhe der Zahlungen bei konstanten Zahlungsintervallen (2. Quadrant) hat der Auftraggeber die Möglichkeit, einen Anreiz zu setzen, das Projekt zu einem frühen Zeitpunkt abzuschließen. Vereinbaren die Parteien beispielsweise 5 Zahlungen in Höhe von $10 \%$ des Festpreises in konstanten Intervallen während der Projektdurchfuhrung und eine Abschlusszahlung in Höhe von 50\% des Festpreises zu dem Projektende, so kann der Auftraggeber den Auftragnehmer hierdurch zu einem Verhalten motivieren, so dass das geplante Projektende eingehalten bzw. sogar unterschritten wird. Es ist auch hier sicherzustellen, dass der für den Auftragnehmer bestehende Konflikt zwischen einem frühzeitigen Projektende und der Höhe der Abschlusszahlung keine Auswirkungen auf die Qualität der Leistungen hat.

Der dritte dargestellte Fall konstanter Zahlungen bei variierenden Zahlungsintervallen ist beispielsweise bei solchen Projekten anwendbar, bei denen ein Großteil der Kosten einer bestimmten Projektphase zugeordnet werden kann. Eine Verkürzung der Zahlungsintervalle mit fortschreitendem Projektverlauf kann dann vereinbart werden, wenn dem Auftragnehmer insbesondere in einer späten Projektphase hohe Kosten der Leistungserstellung entstehen. Möglich ist etwa eine Vereinbarung, bei der mit $\mathrm{Zu}$ nahme der Anzahl abgeschlossener Ereignisse die Zahlungsintervalle sinken und somit häufiger Zahlungen erfolgen, und die Höhe der Zahlungen konstant bleibt. Das Ausmaß der Veränderung der Zahlungsintervalle sollte bei Vertragsabschluss vereinbart werden und ist auch bei dieser Form eines Festpreisvertrags losgelöst von den tatsächlich entstandenen Kosten.

Die im vierten Quadranten aufgezeigten Beispiele einer Festpreisvereinbarung mit sowohl variierenden Zahlungen als auch variierenden Zahlungsintervallen sind aus betriebswirtschaftlicher Sicht nicht sinnvoll. Durch eine solche Zahlungsvereinbarung wird versucht, den während der Projektdauer in unterschiedlicher Höhe bestehenden Mittelbedarf des Auftragnehmers durch Teilzahlungen des Auftraggebers zeitnah zu befriedigen. Dies setzt jedoch voraus, dass der Auftraggeber bereits bei Vertragsabschluss Informationen über die zeitliche Struktur sowie die Höhe der kalkulierten Vorgangskosten durch den Auftragnehmer erhält, um die Teilzahlungen des Festpreises festsetzen zu können. Sind diese Informationen des Auftragnehmers bekannt, können die Projektpartner einen Kostenerstattungsvertrag abschließen und zusätzlich eine Preisobergrenze vereinbaren, so dass dieser Kostenerstattungsvertrag faktisch einem Festpreisvertrag entspricht. 
Aus der Sicht des Auftraggebers ist die Ursache für den Abschluss eines Festpreisvertrags zusätzlich zu der im dritten Kapitel diskutierten Übertragung des Kostenrisikos auf den Auftragnehmer darin zu sehen, dass sich die tatsächlichen Kosten während der Projektausführung nicht beobachten lassen oder der Auftragnehmer nicht bereit ist, diese offen zu legen und damit eine die Kosten erstattende Zahlungsvereinbarung nicht möglich ist. Auch die in Abbildung 5.5 im dritten und vierten Quadranten aufgefuihrten Festpreisvereinbarungen erfordern eine Kontrolle des Projektfortschrittes und werden daher im nächsten Kapitel bei der Modellierung des Payment Scheduling Problems bei Festpreisverträgen vernachlässigt. Es wird vielmehr eine geeignete Modellierung für die Fälle konstanter Zahlungsintervalle aufgezeigt sowie der Einfluss unterschiedlicher Festpreisvereinbarungen auf die Projektplanung des Auftraggebers analysiert.

\subsubsection{Projektplanung des Auftraggebers bei Festpreisvereinbarungen}

Vereinbaren Auftraggeber und Auftragnehmer die Zahlung des Festpreises in konstanten Teilzahlungen, sind die Restriktionen (4.15) und (4.16) des Payment Scheduling Modells wie folgt zu modifizieren:

Sowohl für den Fall konstanter als auch für den Fall variierender Zahlungen muss gelten, dass die Summe der Teilzahlungen $z_{t}$ dem vereinbarten Festpreis FP entspricht und somit die Restriktion (4.16) durch die folgende Bedingung ersetzt werden muss:

$$
\sum_{\mathrm{t}=0}^{\mathrm{T}} \mathrm{z}_{\mathrm{t}}=\text { FESTP }
$$

Der Fall konstanter Teilzahlungen kann derart modelliert werden, dass an solchen Ereigniszeitpunkten, zu denen eine Zahlung erfolgt, das heißt $y_{t}$ den Wert 1 annimmt, die Höhe der Zahlung durch den Quotienten aus Festpreis und Anzahl der Zahlungen bestimmt wird. Die folgende Restriktion ersetzt somit die Bedingung (4.15):

$\mathrm{z}_{\mathrm{t}}=\frac{\text { FESTP }}{\mathrm{ANZ}} \cdot \mathrm{y}_{\mathrm{t}} \quad \forall \mathrm{t}$

Vereinbaren Auftraggeber und Auftragnehmer die Zahlung gleich hoher Teilzahlungen in Höhe des prozentualen Anteils ANTFP des Festpreises sowie die Anzahl der Zahlungen, kann die Höhe der Teilzahlungen sowie der mit dem letzten Ereignis I ver- 
bundenen Abschlusszahlung durch die folgende die Bedingungen (4.15) bzw. (5.5) ersetzende Restriktion bestimmt werden:

$\mathrm{z}_{\mathrm{t}} \geq$ ANTFP $\cdot$ FESTP $+(1-($ ANTFP $\cdot$ ANZ $)) \cdot \operatorname{FESTP} \cdot \mathrm{x}_{\mathrm{lt}}-\widehat{\mathrm{M}} \cdot\left(1-\mathrm{y}_{\mathrm{t}}\right) \quad \forall \mathrm{t}$

Diese Restriktion (5.6) ist durch die Subtraktion der hinreichend großen Zahl $\widehat{M}$ von der rechten Seite der Ungleichung für die Zeitpunkte $t$ immer erfüllt, zu denen $y_{t}=0$ gilt, das heißt wenn zum Zeitpunkt $\mathrm{t}$ keine Zahlung erfolgt. Damit ist die Restriktion lediglich für solche Zeitpunkte bindend, zu denen eine Zahlung erfolgt. Die multiplikative Verknüpfung des zweiten Summanden mit der Binärvariablen $\mathrm{x}_{\mathrm{It}}$, die den Wert 1 annimmt, sofern das letzte Ereignis I zum Zeitpunkt $t$ stattfindet, gewährleistet, dass der noch nicht durch Teilzahlungen während der Projektdauer geleistete Anteil des Festpreises zum Zeitpunkt des Projektendes an den Auftragnehmer gezahlt wird.

Um zu gewährleisten, dass eine Aufteilung des Festpreises in ANZ identische Teilzahlungen möglich ist, sind nur solche prozentualen Anteile gleich hoher Teilzahlungen zulässig, die die folgende Bedingung erfüllen:

$\mathrm{ANTFP} \leq \frac{1}{\mathrm{ANZ}}$

Für die Modellierung konstanter Zahlungsintervalle sind die folgenden Aspekte zu berücksichtigen: Durch die vorausgesetzte Bindung der Zahlungen an die Ereigniszeitpunkte führt die Vorgabe der durch die Zahlungsintervalle als Quotient aus Projektdauer und Anzahl der Zahlungen ermittelten Zahlungszeitpunkte je nach frühestmöglichen und spätesterlaubten Zeitpunkten der Ereignisse nicht grundsätzlich zu einer zulässigen Lösung. Für die Einbindung von Zahlungszeitpunkten innerhalb konstanter Zeitintervalle in das Modell zum Payment Scheduling wird daher die folgende Restriktion zusätzlich eingefügt, die die Projektdauer DMax in identische Zeitabschnitte unterteilt, während derer genau eine Zahlung erfolgen muss:

$\mathrm{INT}=\frac{\mathrm{DMax}}{\mathrm{ANZ}}$

Um eine Überschneidung der Intervalle zu vermeiden, werden die folgenden Zeitpunkte als obere bzw. untere Grenzen für die $k=1, \ldots$, ANZ Intervalle bestimmt:

$$
\begin{array}{ll}
\mathrm{INT}_{\mathrm{k}}^{\mathrm{UG}}=\operatorname{runden}\langle(\mathrm{k}-1) \cdot \mathrm{INT}\rangle+1 & \forall \mathrm{k} \\
\mathrm{INT}_{\mathrm{k}}^{\mathrm{OG}}=\operatorname{runden}\langle\mathrm{k} \cdot \mathrm{INT}\rangle & \forall \mathrm{k}
\end{array}
$$


Der hier verwendete Ausdruck runden $\langle. .$.$\rangle rundet den Wert innerhalb der Klammer$ entsprechend der kaufmännischen Rundungsvorschriften. Damit wird der Zeitpunkt null, zu dem ausschließlich das erste Ereignis stattfinden kann, als untere Intervallgrenze ausgeschlossen, was jedoch durch die Vereinbarung, dass nur Zahlungen nach Abschluss von Vorgängen erfolgen können, möglich ist. Die in das Payment Scheduling Modell zu integrierende Restriktion, welche sicherstellt, dass genau eine Zahlung innerhalb der festgelegten Intervallgrenzen erfolgt, lautet somit:

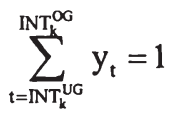

$\forall \mathrm{k}$

Die folgenden Untersuchungen verdeutlichen die Einsatzmöglichkeiten des auf die jeweiligen Festpreisvereinbarungen angepassten Payment Scheduling Modells zur Unterstützung des Auftraggebers bei der Vertragsgestaltung sowie bei der Projektplanung. Es wird zunächst der Fall konstanter Zahlungsintervalle sowie konstanter Zahlungen betrachtet. Unter Berücksichtigung der Restriktionen (5.8) - (5.11) wird die Projektdauer in ANZ identische Zeitabschnitte unterteilt. Für die Projektdauer DMax $=52$ des Anwendungsbeispiels zeigt die Abbildung 5.6 in Abhängigkeit von der Anzahl der Zahlungen die Zeitintervalle, innerhalb derer eine Zahlung erfolgen soll.

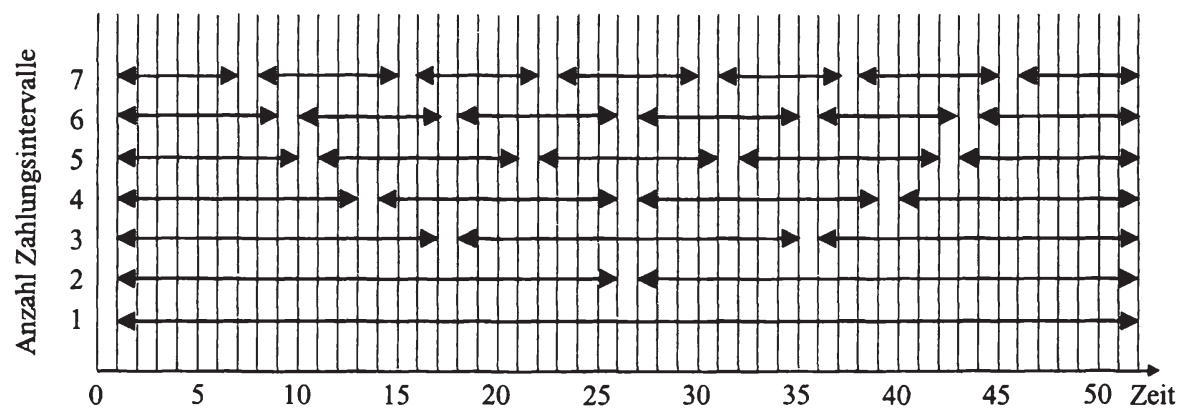

Abbildung 5.6: Konstante Zahlungsintervalle in Abhängigkeit von der Anzahl der Zahlungen

Die Zahlungszeitpunkte sind annahmegemäß an die Ereigniszeitpunkte gebunden, so dass durch eine Analyse der frühestmöglichen und spätesterlaubten Ereigniszeiten die Bestimmung aller möglichen Zahlungszeitpunkte erfolgen kann. Durch die Annahme, dass Zahlungen des Auftraggebers an den Auftragnehmer erst nach Abschluss mindestens eines Vorgangs erfolgen, stellt das erste Ereignis und somit der Zeitpunkt $t=0$ 
keinen Zahlungszeitpunkt dar. Es lassen sich für dieses Beispiel 22 mögliche Zahlungszeitpunkte ermitteln. Die Abbildung 5.7 zeigt für jedes Ereignis die entsprechenden Ereigniszeiten auf. Die mit einem Punkt dargestellten Ereigniszeiten sind Ereignissen auf dem kritischen Pfad zugeordnet, bei denen der frühestmögliche und spätesterlaubte Zeitpunkt übereinstimmen.

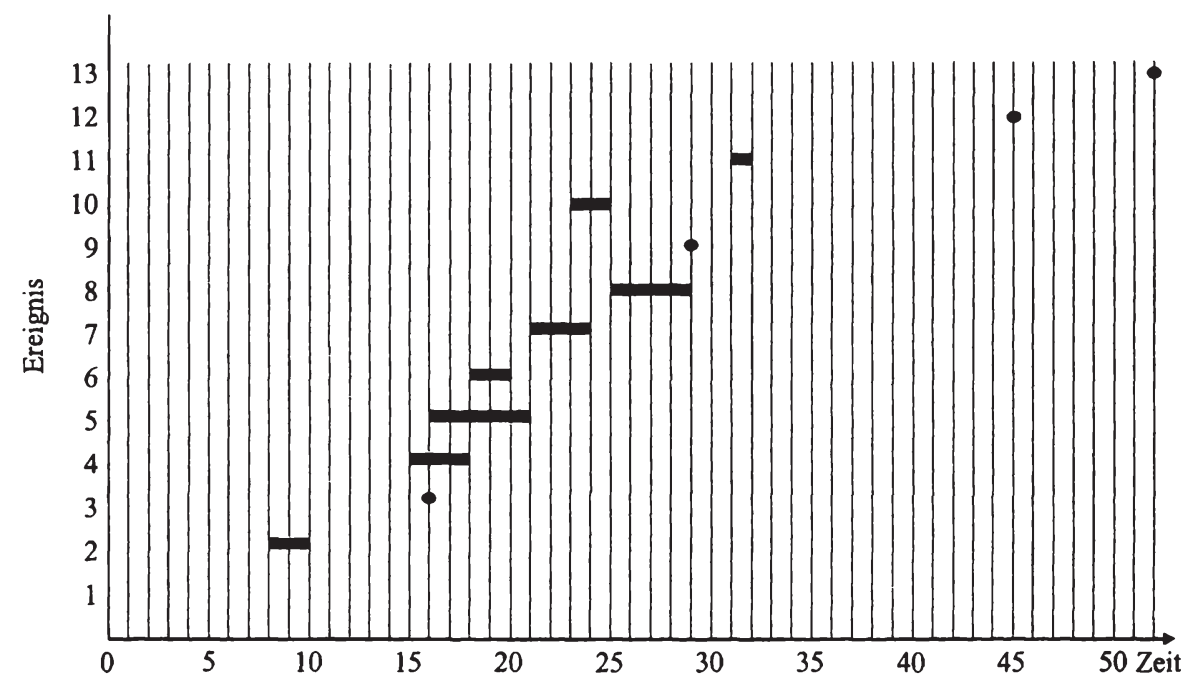

Abbildung 5.7: Mögliche Zahlungszeitpunkte des Anwendungsbeispiels

Ein Vergleich der Zahlungszeitpunkte in Abbildung 5.7 mit den durch die Anzahl der Zahlungen bestimmten Zahlungsintervalle in Abbildung 5.6 zeigt, dass für dieses Beispiel unter den getroffenen Annahmen bei einer Anzahl von Zahlungen ANZ $\geq 6$ keine zulässige Lösung des erweiterten Modells ermittelt werden kann, da die Zeitintervalle hierbei so kurz sind, dass in mindestens einem dieser Intervalle kein Ereignis stattfindet und damit keine Möglichkeit einer Zahlung existiert.

Angesichts der Problemgröße kann jedoch davon ausgegangen werden, dass die in der Realität zwischen den Vertragspartnern vereinbarte Anzahl der Teilzahlungen diesen Wert nicht übersteigt. Bei den folgenden Untersuchungen variiert die Anzahl der Zahlungen somit zwischen eins und fünf.

Das modifizierte Payment Scheduling Problem wird für die folgenden drei Fälle gelöst und die Kapitalwerte sowie die Zahlungen des Auftraggebers miteinander verglichen: 
(1) Festpreisvereinbarung mit konstanten Zahlungsbeträgen und konstanten Zeitintervallen.

(2) Festpreisvereinbarung mit variierenden Zahlungsbeträgen und konstanten Zeitintervallen, wobei jede Zahlung 10\% des Festpreises vergütet und eine Abschlusszahlung in Höhe der Differenz aus Festpreis und Teilzahlungen zu dem Projektende erfolgt.

(3) Festpreisvereinbarung mit variierenden Zahlungsbeträgen und konstanten Zeitintervallen, wobei jede Zahlung 20\% des Festpreises vergütet und eine Abschlusszahlung in Höhe der Differenz aus Festpreis und Teilzahlungen zu dem Projektende erfolgt.

Die Tabelle 5.4 zeigt das Ergebnis der Berechnungen beispielhaft für den Fall von vier Zahlungen auf. Für die Zahlungen werden die Zeitpunkte 10, 26, 32 und 52 ermittelt. Das bedeutet, dass zu den Ereignissen 2, 8, 11 und 13 eine Zahlung erfolgt. Die Zahlungszeitpunkte sowie die Zeiten aller Ereignisse sind für den Fall von vier Zahlungen bei den drei untersuchten Zahlungsvereinbarungen identisch. Die Höhe der Zahlungen resultiert aus den oben getroffenen Annahmen.

\begin{tabular}{|l|r|r|r|}
\cline { 2 - 4 } \multicolumn{1}{c|}{} & \multicolumn{1}{c|}{ (1) } & \multicolumn{2}{c|}{ (2) } \\
\hline Anzahl Zahlungen & \multicolumn{1}{c|}{4} & 4 & 4 \\
\hline Kapitalwert AG & -39.461 & -15.785 & -31.569 \\
\hline Kapitalwert AN & -19.966 & -43.643 & -27.858 \\
\hline Ereignis 1 & 0 & 0 & 0 \\
Ereignis 2 & 10 & 10 & 10 \\
Ereignis 3 & 16 & 16 & 16 \\
Ereignis 4 & 16 & 16 & 16 \\
Ereignis 5 & 21 & 21 & 21 \\
Ereignis 6 & 20 & 20 & 20 \\
Ereignis 7 & 24 & 24 & 24 \\
Ereignis 8 & 26 & 26 & 26 \\
Ereignis 9 & 29 & 29 & 29 \\
Ereignis 10 & 25 & 25 & 25 \\
Ereignis 11 & 32 & 32 & 32 \\
Ereignis 12 & 45 & 45 & 45 \\
Ereignis 13 & 52 & 52 & 52 \\
\hline \multirow{2}{*}{ Zahlung 1 } & 250.000 & 100.000 & 200.000 \\
& 10 & 10 & 10 \\
\hline Zahlung 2 & 250.000 & 100.000 & 200.000 \\
& 26 & 26 & 26 \\
\hline Zahlung 3 & 250.000 & 100.000 & 200.000 \\
& 32 & 32 & 32 \\
\hline Zahlung 4 & 250.000 & 700.000 & 400.000 \\
& 52 & 52 & 52 \\
\hline
\end{tabular}

Tabelle 5.4: Ergebnisse der Festpreisvereinbarungen bei vier Zahlungen 
In Abbildung 5.8 sind die Kapitalwerte des Auftraggebers in Abhängigkeit von der Anzahl der Zahlungen sowie den Zahlungsvereinbarungen dargestellt:

Anzahl Zahlungen

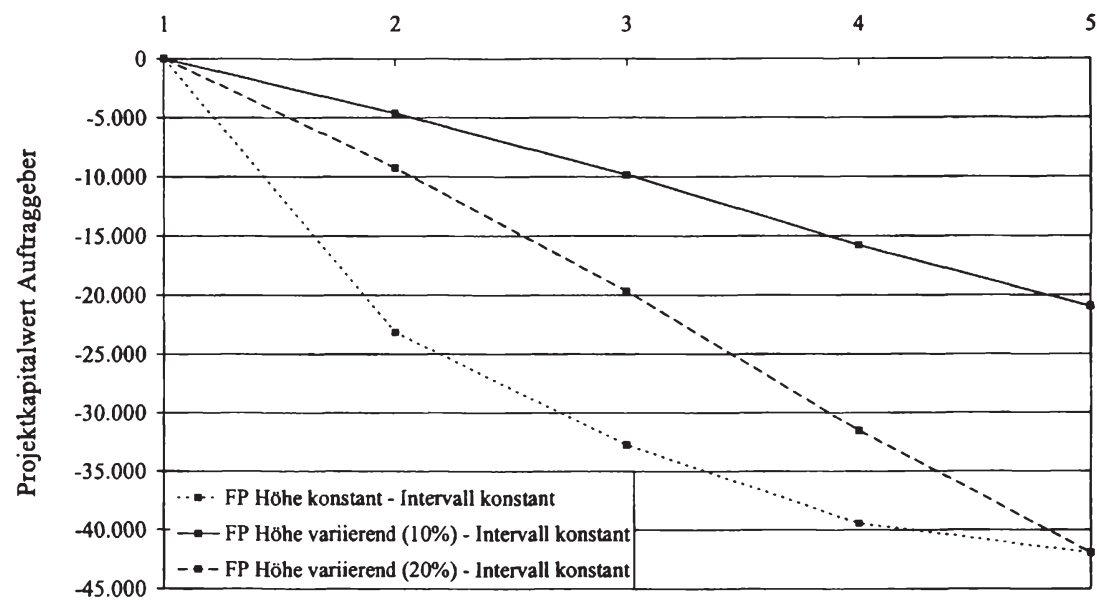

Abbildung 5.8: Kapitalwerte des Auftraggebers bei Festpreisvereinbarungen

Es zeigt sich, dass für dieses Anwendungsbeispiel eine Festpreisvereinbarung mit variierenden Teilzahlungen sowie einer Abschlusszahlung aus der Sicht des Auftraggebers gegenüber konstanten Zahlungen identischer Höhe vorzuziehen ist. Die bei variierender Zahlungshöhe insbesondere für den Fall weniger Zahlungen hohe Abschlusszahlung ist Ursache dafür, dass die Kapitalwerte des Auftraggebers bei dieser Art der Zahlungsvereinbarung größer sind als bei konstanten Festpreiszahlungen.

\subsection{Vergleichende Analyse und Beurteilung der Ergebnisse}

Die in den beiden vorherigen Kapiteln durchgeführten Berechnungen für das Anwendungsbeispiel lassen einen direkten Vergleich der Ergebnisse der unterschiedlichen Zahlungsvereinbarungen zu. Es werden jedoch nur diejenigen Alternativen verglichen, bei denen die Summe der Teilzahlungen an den Auftragnehmer 1.000.000 GE beträgt, so dass Kostenerstattungsfaktoren $\beta>1$ sowie prozentuale oder absolute Honoraraufschläge unberücksichtigt bleiben. Es werden daher die Ergebnisse aus der Sicht des Auftraggebers für folgende Zahlungsvereinbarungen miteinander verglichen: 


\begin{tabular}{|l|l|l|}
\hline Vertragsart & Zahlungsvereinbarung & Zahlungszeitpunkt \\
\hline FP 1 & (ANZ-1) Zahlungen 10\% des FP + Abschlusszahlung & Intervalle konstant \\
FP 2 & (ANZ-1) Zahlungen 20\% des FP + Abschlusszahlung & Intervalle konstant \\
FP 3 & Zahlungsbetrag konstant & Intervalle konstant \\
KE 1 & Kostenerstattung, Faktor 0,9 & freie Zahlungszeitpunkte \\
KE 2 & Kostenerstattung, Faktor 1,0 & freie Zahlungszeitpunkte \\
KE 3 & Kostenerstattung, Faktor 0,9 & Intervalle konstant \\
KE 4 & Kostenerstattung, Faktor 1,0 & Intervalle konstant \\
\hline
\end{tabular}

Tabelle 5.5: Charakteristika der betrachteten Zahlungsvereinbarungen

Die mit KE 1 und KE 2 bezeichneten Formen einer Kostenerstattungsvereinbarung entsprechen den in Kapitel 5.1.2 vorgestellten Zahlungsvereinbarungen mit den in Abbildung 5.1 visualisierten Ergebnissen. Bis zu einer Anzahl von fünf Zahlungen sinkt der Kapitalwert des Auftraggebers nur gering. Dies kann mit der Struktur des Projektplans sowie mit den während der Projektdauer früh stattfindenden Ereignissen und der mit diesen verbundenen Kosten erklärt werden: Bei einer Anzahl von fünf Zahlungen beträgt der Anteil dieser Zahlungen bei $\mathrm{KE} 1$ (KE 2) lediglich 4,23\% (4,7\%) der Gesamtzahlung, der Restbetrag wird erst zu dem Zeitpunkt des Projektendes gezahlt. Bei den Zahlungsvereinbarungen KE 3 bzw. KE 4 wird daher zusätzlich vorausgesetzt, dass die Zahlungen innerhalb konstanter Zeitintervalle erfolgen und die bis zu diesen Zeitpunkten entstandenen Kosten zu 90\% bzw. in voller Höhe abdecken, jedoch maximal 1.000.000 GE betragen. Diese Art der Vertragsvereinbarung vereinigt somit eine Kostenerstattungsvereinbarung für die Ermittlung der Höhe der Zahlungen mit der für Festpreisvereinbarungen vorgeschlagenen Bestimmung der Zahlungszeitpunkte. Während bei KE 1 und KE 2 keine konstanten Zahlungsintervalle vorgegeben sind und daher die Anzahl der Zahlungen zwischen eins und zwölf betragen kann, sind die übrigen betrachteten Formen wegen der zuvor dargestellten anwendungsspezifischen Problemstellung auf maximal fünf mögliche und in regelmäßigen Zeitintervallen stattfindende Zahlungen begrenzt.

Für den Auftraggeber als Entscheidungsträger erlauben diese Ergebnisse einen direkten Vergleich der verschiedenen Zahlungsvereinbarungen in Projektverträgen. Bezogen auf dieses Anwendungsbeispiel ist die Vorteilhaftigkeit eines Vertragsdesigns abhängig von der Anzahl der vereinbarten Zahlungen. Die folgende Abbildung 5.9 zeigt das Ergebnis der Berechnungen für die sieben untersuchten Zahlungsvereinbarungen: 


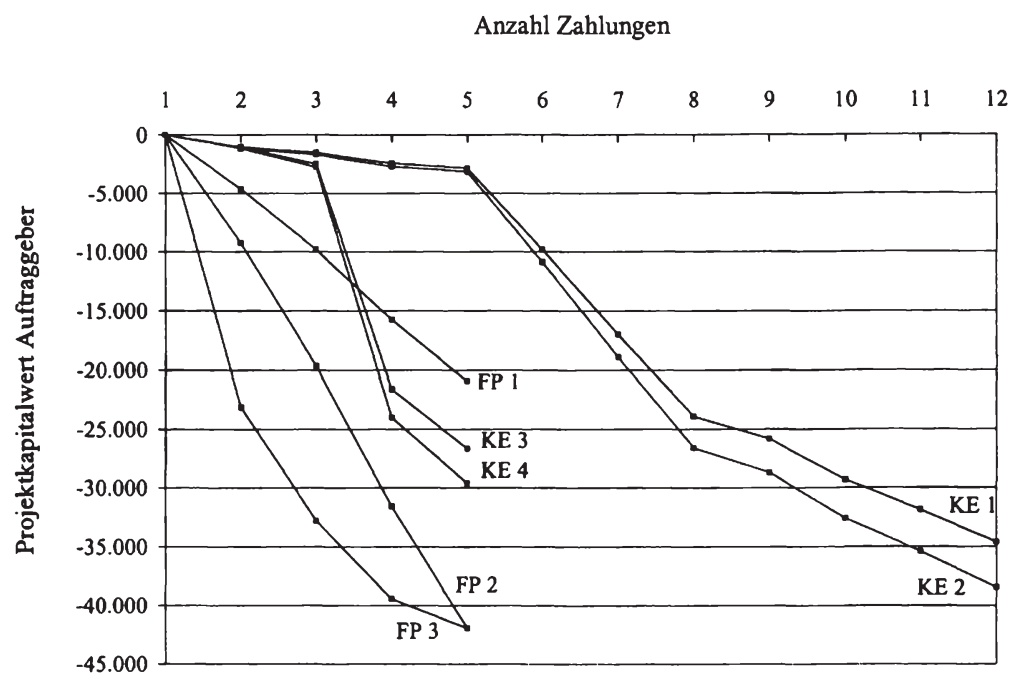

Abbildung 5.9: Kapitalwerte des Auftraggebers bei Kostenerstattungs- oder Festpreisvereinbarungen

Bei einer Anzahl bis zu drei Zahlungen hat der Auftraggeber unter der Zielsetzung der Kapitalwertmaximierung folgende Präferenz:

$\mathrm{KE} 1 \succ \mathrm{KE} 2 \succ \mathrm{KE} 3 \succ \mathrm{KE} 4 \succ \mathrm{FP} 1 \succ \mathrm{FP} 2 \succ \mathrm{FP} 3$

Entschließt er sich jedoch beispielsweise aufgrund der Verhandlungsstärke des Auftragnehmers, diesem vier oder fünf Teilzahlungen anzubieten, ändert sich diese Präferenz wie folgt:

$$
\mathrm{KE} 1 \succ \mathrm{KE} 2 \succ \mathrm{FP} 1 \succ \mathrm{KE} 3 \succ \mathrm{KE} 4 \succ \mathrm{FP} 2 \succ \mathrm{FP} 3
$$

Diese Ergebnisse lassen nicht die allgemeingültige Aussage zu, dass unter der Zielsetzung der Kapitalwertmaximierung Kostenerstattungsverträge Festpreisverträgen vorzuziehen sind. Liegt etwa eine Projektstruktur vor, bei der mit frühen Ereignissen hohe Kosten verbunden sind und somit bei einer Kostenerstattung zu Beginn des Projektes hohe Zahlungen anfallen, kann dies dazu führen, dass die Vereinbarung eines Festpreisvertrags mit konstanten Zahlungen während der Projektdauer mit einem höheren Kapitalwert für den Auftraggeber einhergeht. Der Auftraggeber kann diese Ergebnisse jedoch nutzen, um bei Kenntnis der Projektstruktur sowie der Kosten die aus seiner Sicht optimale Zahlungsvereinbarung zu identifizieren. Gleichzeitig ermöglicht die praxisnahe Modellierung der verschiedenen Zahlungsvereinbarungen durch die an die Problemstellung angepassten Payment Scheduling Modelle eine Abschätzung des mit der jeweils ausgewählten Vereinbarung verbundenen Kapitalwertes. 


\subsection{Integration der Kooperationsbedingung des Auftragnehmers in die Projektplanung}

\subsubsection{Einbindung der Kooperationsbedingung in das Payment Scheduling}

Bei den bisherigen Ausführungen stand das Ziel der Kapitalwertmaximierung des Auftraggebers im Mittelpunkt der Untersuchungen. Der Kapitalwert wird neben den Zahlungen an den Auftragnehmer auch von den nach Projektabschluss erfolgenden und aus der Nutzung des Projektes resultierenden Einzahlungen beeinflusst. Um eine Vergleichbarkeit der Untersuchungsergebnisse des Anwendungsbeispiels aus der Sicht des Auftraggebers zu gewährleisten, wurden Einzahlungen in Höhe der Kosten von 1.000.000 GE angenommen. Die Ergebnisse zeigen daher auf, welchen über die Kosten hinausgehenden Beitrag das Projekt während seiner Nutzungsphase leisten muss, um für den Auftraggeber einen positiven Kapitalwert und somit einen Wertbeitrag zu leisten.

Die Ausführungen und Berechnungen im Rahmen des Anwendungsbeispiels zeigen ebenfalls, dass bei nahezu allen vorgestellten Zahlungsvereinbarungen der Kapitalwert des Auftragnehmers negativ ist. Die in Kapitel 3.2.4 aus theoretischer Sicht begründete Teilnahmebedingung des Auftragnehmers wird daher in diesem sowie in dem folgenden Kapitel als Grundlage dienen, die Kooperationsbedingung für das Payment Scheduling Modell zu formulieren sowie die Auswirkungen auf die mit unterschiedlichen Zahlungsvereinbarungen verbundenen Vertragsdesigns beispielhaft anhand der Anwendung zu untersuchen.

Das in Kapitel 4.3.3 vorgestellte und später an verschiedene Vertragscharakteristika angepasste Payment Scheduling Modell verfolgt die Zielsetzung der Maximierung des Kapitalwertes des Auftraggebers unter Berücksichtigung der Projektplanungsrestriktionen und der Zahlungsvereinbarungen zwischen Auftraggeber und Auftragnehmer. Der Erfolg des Auftragnehmers aus der Projektkooperation wird maßgeblich durch seine Verhandlungsmacht gegenüber dem Auftraggeber beeinflusst. Die Ausführungen im Rahmen des Anwendungsbeispiels zeigen, dass eine Vereinbarung einer möglichst hohen Anzahl von Teilzahlungen sowie ein hoher Kostenerstattungsfaktor den Kapitalwert und somit den Erfolg der Projektkooperation des Auftragnehmers erhöhen. Der Auftragnehmer wird jedoch erst einen durch den Auftraggeber vorgegebenen Projekt- 
vertrag akzeptieren und damit an der Projektkooperation teilnehmen, wenn das Projekt nicht mit einem Wertverlust für ihn verbunden ist. ${ }^{9}$

Der Wertbeitrag, den der Auftragnehmer durch die Kooperation erzielt, lässt sich in Form der Differenz aus den für die Leistungserstellung notwendigen Zahlungen für beispielsweise Löhne, Roh-, Hilfs- und Betriebsstoffe sowie den Fortschrittszahlungen des Auftraggebers ermitteln. Um hierbei die Zeitpräferenz für Zahlungen zu berücksichtigen, wird im Folgenden auch für die Beurteilung des Projektes aus der Sicht des Auftragnehmers der Kapitalwert ermittelt. Dieser berechnet sich aus der Differenz der auf den Entscheidungszeitpunkt diskontierten Kosten sowie der diskontierten Erlöse in Form der Einzahlungen $\mathrm{z}_{\mathrm{t}}$ des Auftraggebers. Es wird weiterhin angenommen, dass die Kosten von Vorgängen den Zeitpunkten der Ereignisse zugerechnet werden, die unmittelbarer Vorgänger der jeweiligen Aktivitäten sind. Diese Kosten sind in Tabelle 4.4 mit $_{\mathrm{i}}^{\mathrm{AN}}$ bezeichnet worden. ${ }^{10}$

Die in das Payment Scheduling Modell des Auftraggebers einzubindende Kooperationsbedingung berücksichtigt den Kapitalwert des Auftragnehmers aus der Kooperation und stellt sicher, dass der für den Auftraggeber kapitalwertmaximale Projektplan mindestens einen Kapitalwert für den Auftragnehmer erbringt, welcher durch dessen Teilnahmebedingung TB $^{\text {AN }}$ vorgegeben ist. ${ }^{11}$ Die Kooperationsbedingung des Auftragnehmers stellt aus der Sicht des Auftraggebers ein Satisfizierungsziel dar, welches in Form einer zusätzlichen Restriktion in dem Modell berücksichtigt wird. ${ }^{12}$ Formal kann somit die folgende Formulierung als Kooperationsbedingung vorgeschlagen werden:

$$
\sum_{\mathrm{t}=0}^{\mathrm{T}} \operatorname{DISKF}_{\mathrm{t}}^{\mathrm{AN}} \cdot \mathrm{z}_{\mathrm{t}}-\sum_{\mathrm{t}=0}^{\mathrm{T}} \sum_{\mathrm{i}=1}^{\mathrm{I}} \operatorname{DISKF}_{\mathrm{t}}^{\mathrm{AN}} \cdot \mathrm{K}_{\mathrm{i}}^{\mathrm{AN}} \cdot \mathbf{x}_{\mathrm{it}} \geq \mathrm{TB}^{\mathrm{AN}}
$$

Obwohl bei den folgenden Untersuchungen angenommen wird, dass der Diskontierungszins DISKZ für Zahlungen sowohl aus der Sicht des Auftraggebers als auch aus der Sicht des Auftragnehmers identisch ist, soll die an dieser Stelle eingeführte Schreibweise $\mathrm{DISKF}_{t}^{\mathrm{AN}}=\left(1+\mathrm{DISKZ}^{\mathrm{AN}}\right)^{-t}$ signalisieren, dass die Zeitpräferenzen für

\footnotetext{
Vgl. Kapitel 3.2.4.

10 Vgl. Kapitel 5.1.1.

1 Es wird in diesem Abschnitt eine Situation unter Sicherheit unterstellt, bei der beide Kooperationspartner identische Vorstellungen über die Kosten und Dauern der Vorgănge besitzen. Die Einbeziehung von Risikonutzenfunktionen zur Ermittlung einer den Mindestnutzen des Auftragnehmers erfullenden Teilnahmebedingung ist daher an dieser Stelle nicht notwendig. Vgl. Kapitel 3.2.4.

12 Vgl. Dinkelbach/Kleine (1996), S. 183; Laux (2003), S. $23 \mathrm{ff}$.
} 
Zahlungen beider Vertragspartner unterschiedlich sein können und dies durch das vorgestellte Modell berücksichtigt werden kann. ${ }^{13}$

Kritisch anzumerken ist jedoch, dass der Auftraggeber als Entscheidungsträger kaum Informationen über den Diskontierungszins als Kalkulationsgröße des Auftragnehmers besitzt. ${ }^{14}$ Eine über dieses Problem hinausgehende Schwierigkeit besteht zudem darin, dass auch die Teilnahmebedingung des Auftragnehmers unbekannt ist, sofern diese nicht durch Signale an den Auftraggeber kommuniziert wird. ${ }^{15}$ Während ein Auftragnehmer als neuer Kooperationspartner ein großes Interesse an einem Zustandekommen der Kooperation und damit einer möglicherweise langfristigen Geschäftsbeziehung besitzen und daher als Teilnahmebedingung beispielsweise bereits einen Kapitalwert akzeptieren wird, welcher größer null ist, kann der Auftraggeber vermuten, dass ein Auftragnehmer einer wiederholten Kooperationsbeziehung einen Mindestkapitalwert fordert, der einem aus diesem Geschäft oder der einer alternativen Anlage mindestens zu erzielenden Wertbeitrag entspricht. ${ }^{16}$

Die Fragestellung, welche Bedeutung die Kooperationsbedingung für die quantitative Projektplanung sowie das Vertragsdesign des Auftraggebers hat, wird Bestandteil des folgenden Kapitels sein.

\subsubsection{Die Kooperationsbedingung bei Kostenerstattungsverträgen}

Als Fortführung der in Kapitel 5.1.2 durchgeführten Untersuchungen anhand des Anwendungsbeispiels wird zunächst der Einfluss der Kooperationsbedingung des Auftragnehmers auf den Projekterfolg des Auftraggebers bei Kostenerstattungsvereinbarungen analysiert. Wie in den vorherigen Kapiteln erfolgt auch hier eine Fallunterscheidung nach der Höhe des Kostenerstattungsfaktors.

\footnotetext{
13 Verschiedene Diskontierungsfaktoren für Auftraggeber und Auftragnehmer betrachtet Mohnen (2002), S. 42 und begründet dies durch unterschiedliche Risikopräferenzen. Aufgrund einer stärkeren Zeitprafferenz fur Zahlungen kann ebenfalls angenommen werden, dass der Diskontierungszins des Auftragnehmers hoher als der des Auftraggebers ist. Vgl. hierzu etwa Johannwille (2000), S. $23 \mathrm{ff}$.

14 Zur Problematik der Bestimmung sowie der Eignung des Diskontierungszinses zur Abbildung der Zeitpräferenz für Zahlungen vgl. die Ausführungen in Kapitel 4.1.

15 Zum Signalling vgl. Kapitel 3.2.2

16 Vgl. Wong/Cheung (2004), S. 440. Zum Verhältnis zwischen Auftraggeber und Auftragnehmer vgl. Vaaland (2004).
} 


\section{(1) Kostenerstattungsfaktor $\beta \leq 1,0$}

Wird ein Kostenerstattungsfaktor $\beta \leq 1,0$ vereinbart, so vergütet der Auftraggeber unter der Zielsetzung der Maximierung seines Kapitalwertes zu jedem Zahlungszeitpunkt höchstens das $\beta$-fache der bis dahin entstandenen Kosten abgeschlossener Vorgänge. Aus der Sicht des Auftragnehmers ergeben sich hieraus zwei negative Einflüsse auf dessen Kapitalwert: Zum einen besteht eine zeitliche Diskrepanz zwischen der Entstehung und somit Zahlung der Kosten durch den Auftragnehmer und Erstattung des $\beta$-fachen dieser Kosten durch den Auftraggeber, zum anderen erfolgt die Vergütung des $(1-\beta)$-fachen aller während der Projektdauer entstandenen Kosten erst mit dem Abschluss des Projektes. Wird kein zusätzliches Honorar vereinbart, führt diese Form der Kostenerstattung somit zu einem negativen Kapitalwert für den Auftragnehmer.

Durch die Einbindung der Kooperationsbedingung in die Projektplanung kann der Kapitalwert des Auftragnehmers durch die folgenden Modifikationen des Projekt- und Zahlungsplans erhöht werden:

- Die zu bestimmten Ereignissen stattfindenden Zahlungen betragen mehr als das $\beta$-fache der bis dahin entstandenen Kosten, die Gesamtzahlung entspricht jedoch den Gesamtkosten.

- Die Summe der Teilzahlungen übersteigt die Gesamtkosten. Dies entspricht der Zahlung eines zusätzlichen Honorars während bzw. zum Abschluss des Projektes.

- Ereignisse, zu denen keine Zahlungen erfolgen, werden zu ihren spätesterlaubten Zeitpunkten terminiert, sofern hierdurch keine folgenden mit Fortschrittszahlungen verbundenen Ereignisse später terminiert werden.

Während die erste und zweite Maßnahme die Kapitalwerte von Auftraggeber und Auftragnehmer in gegenläufigem Ausmaß beeinflusst, hat die dritte Maßnahme lediglich Einfluss auf den Kapitalwert des Auftragnehmers. Die folgenden Berechnungen verdeutlichen die aufgeführten Wirkungszusammenhänge anhand des Anwendungsbeispiels. Hierbei wird unterstellt, dass der Kapitalwert des Auftragnehmers als Kooperationsbedingung mindestens null betragen muss. Die mit den Vorgängen verbundenen Kosten sowie der Projektplan entsprechen den Ausführungen in Kapitel 4.4. Für die erste Untersuchung soll zusätzlich festgelegt werden, dass die Summe der Teilzahlungen die Gesamtkosten in Höhe von 1.000.000 GE nicht überschreiten soll. Nach mehrmaliger Durchführung der Optimierung unter Berücksichtigung der Koopera- 
tionsbedingung zeigt sich, dass mit Ausnahme des Falls einer einzigen Zahlung $(A N Z=1)$ eine Aufteilung der Teilzahlungen bestimmter Höhe zu bestimmten Ereigniszeitpunkten so möglich ist, dass der Kapitalwert des Auftraggebers unter Einhaltung der Kostenerstattungs- sowie der Kooperationsbedingung maximiert wird und unabhängig von der Anzahl der Zahlungen sowie des Kostenerstattungsfaktors identisch ist. Dieses Ergebnis resultiert aus den folgenden Überlegungen:

Der Auftragnehmer minimiert den Barwert aller entstehenden Kosten durch die zeitliche Festlegung der Ereignisse auf die spätesterlaubten Zeitpunkte. Für das Anwendungsbeispiel kann der Barwert der Auszahlungen des Auftragnehmers ermittelt werden und beträgt -963.599 GE. Für die Erfüllung der Kooperationsbedingung müssen diesem Barwert Einzahlungen des Auftraggebers mit einem Barwert in gleicher Höhe entgegenstehen, welche Auszahlungen aus der Sicht des Auftraggebers darstellen. Durch die für das Beispiel getroffene Annahme von Einzahlungen für den Auftraggeber aus der Nutzung des Projektes in Höhe von 1.000.000 GE am Ende des Projektes $(t=52)$ lässt sich deren Barwert in Höhe von 904.924 GE und somit der maximal mögliche Kapitalwert des Auftraggebers ermitteln. Dieser beträgt für das Beispiel $-58.675 \mathrm{GE}$.

Die folgenden Ergebnisse stellen eine Teilmenge der durch die wiederholte Optimierung ermittelten Lösungen dar, welche für zwei mögliche Teilzahlungen bei einem Kostenerstattungsfaktor $\beta=0,7 \mathrm{zu}$ dem oben berechneten maximalen Kapitalwert des Auftraggebers in Höhe von -58.675 GE sowie einem Kapitalwert des Auftragnehmers in Höhe von 0 GE führen. Sie zeigen, dass die Berücksichtigung der Kooperationsbedingung zu mehr als einer optimalen Lösung führen kann.

\begin{tabular}{|c|c|c|c|c|c|c|c|c|}
\hline \multirow{2}{*}{ Zahlung I } & Betrag & 153.161 & 332.714 & 376.604 & 572.107 & 727.690 & 905.425 & 960.549 \\
\hline & Zeitpunkt & 10 & 10 & 10 & 10 & 10 & 16 & 18 \\
\hline \multirow{2}{*}{ Zahlung 2} & Betrag & 846.839 & 667.286 & 623.396 & 427.893 & 272.310 & 94.575 & 39.451 \\
\hline & Zeitpunkt & 21 & 24 & 25 & 32 & 45 & 52 & 52 \\
\hline
\end{tabular}

Tabelle 5.6: Teilmenge optimaler Lösungen bei Kostenerstattung und Kooperationsbedingung

Während der Auftraggeber ohne Berücksichtigung der Kooperationsbedingung maximal das $\beta$-fache der entstandenen Kosten mit jeder Teilzahlung erstattet, da sich sein Kapitalwert mit jeder zusätzlichen Einheit vermindert und somit ausschließlich eine optimale Lösung existiert, ergeben sich für dieses Anwendungsbeispiel durch die Einbindung der Kooperationsbedingung des Auftragnehmers sowie der Zielsetzung der 
Kapitalwertmaximierung des Auftraggebers unterschiedliche Kombinationsmöglichkeiten aus Zeitpunkt und Höhe der Zahlungen, die bei Berücksichtigung der Kostenerstattungsbedingung zu einem Auftragnehmerkapitalwert von null führen. ${ }^{17}$

Es lässt sich darüber hinaus durch mehrfache Optimierung zeigen, dass sowohl für zwei oder mehr Teilzahlungen als auch für unterschiedliche Kostenerstattungsfaktoren verschiedene Lösungen mit identischem Zielfunktionswert, das heißt einem maximalen Kapitalwert des Auftraggebers in Höhe von -58.675 GE ermittelt werden können. Je höher die durch die Anzahl der Ereigniszeitpunkte bestimmte Anzahl der möglichen Zahlungszeitpunkte ist, desto mehr Kombinationsmöglichkeiten existieren, die Höhe der Teilzahlungen in Verbindung mit den Zahlungszeitpunkten unter Berücksichtigung der Teilnahmebedingung festzulegen.

Welche der optimalen Handlungsalternativen zur Zahlung des Preises bei einer Kostenerstattungsvereinbarung durch den Auftraggeber ausgewählt wird, ist abhängig von seinen zusätzlichen individuellen Präferenzen. Durch die Definition eines weiteren Ziels, wie beispielsweise die Minimierung der Summe der Differenz aus den Teilzahlungen und mindestens zu den Zahlungszeitpunkten zu erstattenden Kosten, können die Handlungsalternativen in eine eindeutige Präferenzreihenfolge überführt werden. ${ }^{18}$

Möchte der Auftraggeber etwa einen Anreiz schaffen, das Projekt frühzeitig abzuschließen, wird er sich entscheiden, während der Phase des Projektanfangs niedrige und mit fortschreitender Projektzeit höhere Zahlungen zu tätigen. Auch die Festlegung der Anzahl der Teilzahlungen muss durch Hinzuziehen individueller Kriterien erfolgen, da dies unter der Voraussetzung der Beachtung aller Restriktionen des Payment Scheduling Modells sowie der Kooperationsbedingung keinen Einfluss auf die quantitative Größe des Projekterfolgs in Form des Kapitalwertes hat.

17 Diese Aussage trifft für den Fall einer einzigen Zahlung nicht immer zu. Für dieses Beispiel existieren, wie in Kap. 5.2.2 ermittelt, 22 mögliche Zeitpunkte fur Zahlungen. Die Zahlung in Höhe von 1.000.000 GE erfolgt zu dem Zeitpunkt $\mathrm{t}=19$ und fuhrt somit zu einem Kapitalwert des Auftraggebers in Hohe von -59.231 GE und zu einem Kapitalwert des Auftragnehmers in Hohe von 548 GE. Eine Terminierung dieser Zahlung auf den nächsten späteren Zeitpunkt $(t=20)$ fuhrt zu einem negativen Kapitalwert für den Auftragnehmer und somit zu einer Verletzung der Kooperationsbedingung.

18 Durch die Berlucksichtigung des nächstwichtigen Zielkriteriums kann eine Ordnung der für das wichtigste Zielkriterium optimalen Handlungsaltemativen erfolgen. Fuhrt auch dies nicht zu einer eindeutigen Losung, wird ein drittes Ziel in die Entscheidungsfindung integriert. Für diese Entscheidungsregel der lexikographischen Ordnung vgl. etwa Bamberg/Coenenberg (2002), S. 56f.; Laux (2003), S. $96 f$. 
(2) Kostenerstattungsfaktor $\beta>1,0$

Vereinbaren die Vertragsparteien einen Kostenerstattungsfaktor $\beta>1,0$, so ist die Kooperationsbedingung unter der bisherigen Annahme eines aus der Sicht des Auftragnehmers mindestens zu realisierenden Kapitalwertes von null als Restriktion des Modells tendenziell nicht bindend, ${ }^{19}$ da mit jeder Teilzahlung zusätzlich eine Honorarzahlung erfolgt und sich diese positiv auf den Kapitalwert des Auftragnehmers auswirkt. Die Lösung des Payment Scheduling Modells unter Berücksichtigung der Kooperationsbedingung führt für das Anwendungsbeispiel zu den in Abbildung 5.3 dargestellten Kapitalwerten in Abhängigkeit von dem Kostenerstattungsfaktor sowie der Anzahl der Zahlungen. Es lässt sich erkennen, dass der Kapitalwert des Auftraggebers bei allen untersuchten Fällen niedriger als der maximal mögliche Kapitalwert in Höhe von $-58.675 \mathrm{GE}$ bei Wahl eines Kostenerstattungsfaktors $\beta \leq 1,0$ unter Berücksichtigung der Kooperationsbedingung ist. Auch die fur die Einbindung der Kooperationsbedingung ermittelten Ergebnisse des Anwendungsbeispiels zeigen, dass der Auftraggeber niedrige Kostenerstattungsfaktoren bevorzugen sollte.

Zusammenfassend kann an dieser Stelle festgestellt werden, dass der Auftraggeber im Rahmen einer Projektkooperation mit einer Kostenerstattungsvereinbarung das Payment Scheduling Modell für eine quantitative Planungsunterstützung unter Beachtung der Teilnahmebedingung einsetzen kann, um alternative Aufteilungen der Teilzahlungen sowie Zuordnungen zu Ereigniszeitpunkten zu ermitteln. Dabei ist es je nach Kosten- und Projektstruktur möglich, dass sowohl der Kostenerstattungsfaktor als auch die Anzahl der Zahlungen keinen Einfluss auf den Kapitalwert des Auftraggebers haben, sofern die Zahlung eines Honorars nicht vorgesehen ist. Der Auftraggeber kann mit Hilfe dieses Vorgehens solche Vertragsdesigns als Handlungsalternativen ausschlieBen, die die Kooperationsbedingung nicht erfüllen und daher durch den Auftragnehmer vermutlich nicht akzeptiert werden.

Durch die Festlegung zusätzlicher individueller Zeit- oder Höhenpräferenzen für Zahlungen hat der Auftraggeber die Möglichkeit, eine optimale Handlungsalternative zu

19 Es sind Fălle denkbar, bei denen diese allgemeine Aussage nicht zutreffend ist. Wird als Teilnahmebedingung ein von null unterschiedlicher positiver Kapitalwert vorausgesetzt, ist diese Restriktion auch bei Kostenerstattungsfaktoren größer eins je nach Höhe der Teilnahmebedingung bindend. Werden beispielsweise für das Anwendungsbeispiel eine einzige Zahlung sowie ein Kostenerstattungsfaktor von 1,05 vereinbart, so ist die Kooperationsbedingung mit einem geforderten Kapitalwert von null bindend und es erfolgt bereits zum Zeitpunkt $t=32$ eine Zahlung in Höhe von 1.050.000 GE, so dass der Kapitalwert des Auftragnehmers $23.653 \mathrm{GE}$ beträgt. Eine Zahlung zum nächsten später möglichen Zeitpunkt $t=45$ führt zu einem negativen Kapitalwert. 
identifizieren. Er kann darüber hinaus mit Hilfe des optimalen Zielfunktionswertes erkennen, wie hoch der Barwert der nach Projektende erfolgenden Einzahlungen sein muss, um die finanziellen Projektziele zu erreichen.

Ein Vergleich des unter Einbeziehung der Teilnahmebedingung maximal für den Auftraggeber erzielbaren Kapitalwertes mit den ohne Berücksichtigung der Kooperationsbedingung ermittelten Kapitalwerten für Kostenerstattungsverträge zeigt für das Anwendungsbeispiel, dass eine Projektplanung mit Kooperationsbedingung hinsichtlich des Kapitalwertkriteriums von allen Zahlungsvereinbarungen ohne Kooperationsbedingung dominiert wird. Damit enthält das unter Berücksichtigung der Kooperationsbedingung ermittelte Vertragsdesign einen Anreiz für den Auftragnehmer, an der Kooperation teilzunehmen. Diesen Anreiz ,bezahlt' der Auftraggeber mit einer Reduzierung seines maximal möglichen Kapitalwertes.

\subsubsection{Die Kooperationsbedingung bei Festpreisverträgen}

Die folgenden Überlegungen zeigen Aspekte auf, die bei der Einbindung der Kooperationsbedingung bei Festpreisverträgen von Bedeutung sind. Hierfür wird auf die in Kapitel 5.2.1 entwickelten Zahlungsvereinbarungen bei Festpreisverträgen zurückgegriffen und analysiert, welchen Einfluss die Teilnahmebedingung auf die Zahlungen und somit auf die Erfolgsgrößen von Auftraggeber und Auftragnehmer hat.

Während bei einer Kostenerstattungsvereinbarung die Höhe der Zahlungen an die Höhe der Kosten gebunden ist und somit ausschließlich der Kostenerstattungsfaktor sowie die Anzahl der Zahlungen Teil der Vertragsverhandlungen zwischen Auftraggeber und Auftragnehmer sind, werden bei Festpreisvereinbarungen die Höhe des Festpreises sowie die mit ihm verbundenen Teilzahlungen und Zahlungszeitpunkte festgelegt.

Wird das Payment Scheduling Modell unter der Zielsetzung der Kapitalwertmaximierung des Auftraggebers sowie der Berücksichtigung der Kooperationsbedingung für Festpreisvereinbarungen angewandt, so ergeben sich je nach Anzahl der Teilzahlungen unterschiedliche Festpreisbeträge als Summe der Teilzahlungen, wobei die Kombination aus Höhe sowie Zeitpunkt der Zahlungen durch die Diskontierung wie im vorherigen Kapitel zu unterschiedlichen Lösungen mit identischem Kapitalwert des Auftraggebers führt. 
Vereinbaren die Vertragsparteien konstante Teilzahlungen in konstanten Zeitintervallen (dies entspricht dem in dem ersten Quadranten der Abbildung 5.5 dargestellten Fall), kann der Auftraggeber im Rahmen seiner Planung analog zu dem Fall der Kostenerstattung zwischen unterschiedlichen Zahlungen wählen.

Für den Fall einer einzigen Zahlung ist die Zahlung des Festpreises theoretisch zu den in Abbildung 5.7 dargestellten 22 Ereigniszeitpunkten möglich. Je später die Zahlung erfolgt, desto größer muss der für die Kapitalwertberechnung zu diskontierende Festpreis sein, um die Teilnahmebedingung des Auftragnehmers zu erfüllen. Es wird auch bei diesen Untersuchungen als Kooperationsbedingung angenommen, dass der Kapitalwert des Auftragnehmers mindestens null betragen muss. Die Abbildung 5.10 zeigt für die 22 möglichen Zahlungszeitpunkte den für die Erfüllung der Teilnahmebedingung mindestens zu zahlenden Festpreisbetrag:

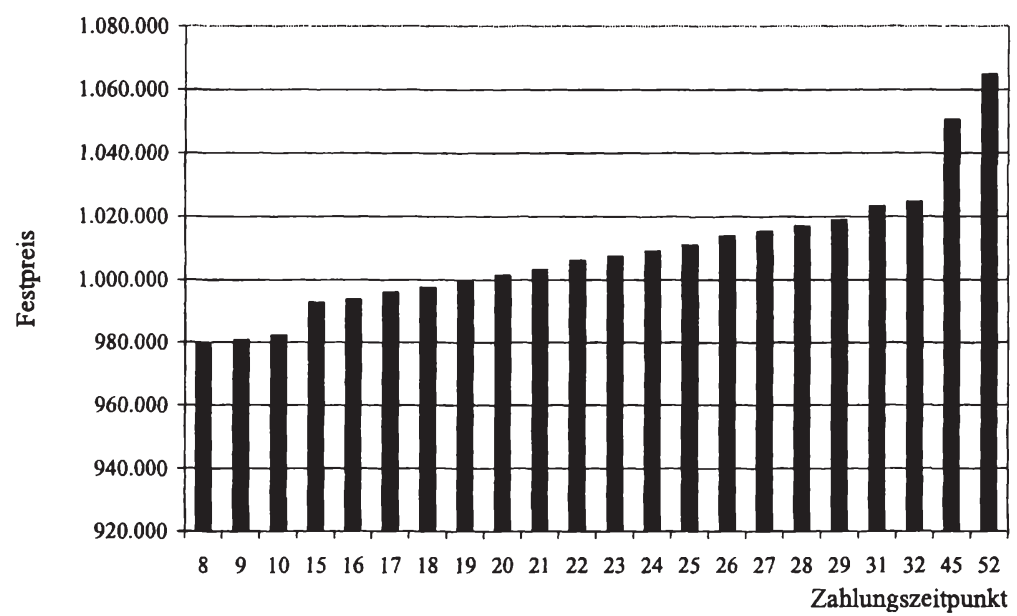

Abbildung 5.10: Höhe des Festpreises bei einer Zahlung in Abhängigkeit von dem Zahlungszeitpunkt

Erwartungsgemäß zeigt sich, dass die Höhe des notwendigen Festpreises steigt, je später der Zahlungszeitpunkt gewählt wird. Wie die Analysen der Untersuchungen für eine Kostenerstattungsvereinbarung bereits gezeigt haben, hängt auch bei einer Festpreisvereinbarung die Entscheidung des Auftraggebers für die Wahl einer Handlungsalternative und somit die Festlegung der Höhe des Festpreises von seinen zusätzlichen individuellen Präferenzen ab. Die während des Projektverlaufs erfolgende frühe Zahlung eines niedrigen Festpreises wird etwa aufgrund der bereits diskutierten Anreizproblematik und damit der Präferenz des Auftraggebers für späte Zahlungen durch die 
Zahlung eines höheren Festpreises zu einem späteren Ereigniszeitpunkt mit identischem Kapitalwert dominiert.

Durch die Vorgabe der Zahlungszeitpunkte findet auch zu Ereigniszeitpunkten, welche nicht den spätesterlaubten Zeitpunkten entsprechen, eine Zahlung statt, so dass für den Auftragnehmer in diesen Fällen durch die zu früheren Zeitpunkten diskontierten Auszahlungen der den Ereignissen zugerechneten Kosten der Barwert der Projektkosten steigt. Das bedeutet, dass der Auftraggeber für die Erfüllung der Teilnahmebedingung einen höheren Festpreis zahlen muss, so dass sich dessen maximaler Kapitalwert bei der Wahl dieser Zahlungszeitpunkte verringert. Bei allen Zahlungszeitpunkten, die einem spätesterlaubten Ereigniszeitpunkt entsprechen, beträgt der maximale Kapitalwert des Auftraggebers -58.675 GE und entspricht damit dem in dem vorherigen Kapitel ermittelten maximal möglichen Kapitalwert für dieses Anwendungsbeispiel unter Berücksichtigung der Kooperationsbedingung.

Abbildung 5.11 zeigt die Abweichungen der Kapitalwerte von dem maximal möglichen Kapitalwert bei einer Zahlung des Festpreises zu den vorgegebenen Zeitpunkten:

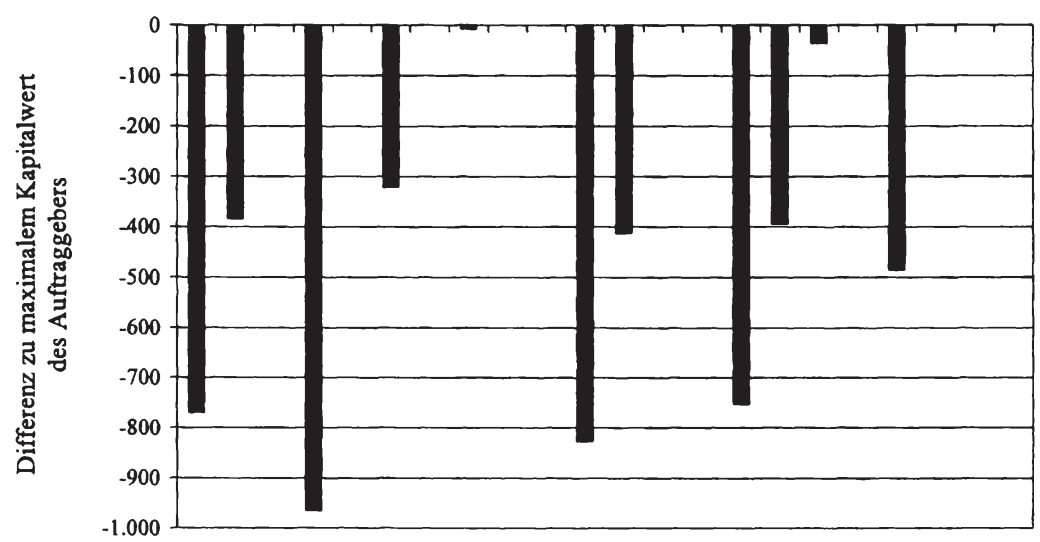

Abbildung 5.11: Kapitalwertdifferenzen zu maximalem Kapitalwert bei einer Zahlung

Für dieses Beispiel lässt sich die maximale Abweichung der Kapitalwerte aus dem Vergleich des $\mathrm{zu}$ dem Zahlungszeitpunkt $\mathrm{t}=15$ maximalen Kapitalwert sowie dem möglichen optimalen Kapitalwert in Höhe von 1,6\% ermitteln. Die Ergebnisse verdeutlichen den theoretischen Zusammenhang zwischen der notwendigen Höhe einer 
Festpreiszahlung und dem Kapitalwert des Auftraggebers unter Berücksichtigung der Kooperationsbedingung.

Im Gegensatz zu den Lösungen des Payment Scheduling Modells bei Kostenerstattung, die annahmegemäß zu einer Gesamtzahlung in Höhe von 1.000.000 GE führen, kann die Summe der Zahlungen bei einer Festpreisvereinbarung als Entscheidungsvariable des Modells betrachtet werden. Die Entscheidungssituation ist dadurch charakterisiert, dass der Auftraggeber die Höhe des Festpreises so festlegt, dass bei einer bestimmten Anzahl und zeitlichen Verteilung von Zahlungen sowie der Beachtung aller Restriktionen des Payment Scheduling Modells die Teilnahmebedingung des Auftragnehmers erfüllt ist und der Kapitalwert des Auftraggebers maximiert wird und somit für dieses Beispiel $-58.675 \mathrm{GE}$ beträgt.

Wird das Payment Scheduling Modell modifiziert, indem als Zielsetzung die Minimierung des Festpreises bei konstantem vorgegebenem Kapitalwert des Auftraggebers in Höhe von -58.675 GE gewählt wird, so kann für jede Anzahl von Teilzahlungen eine untere Grenze für den Festpreis ermittelt werden. In Tabelle 5.7 ist die optimale Lösung des Payment Scheduling Problems bei Festpreisminimierung, konstanten Zahlungsintervallen und konstanten Zahlungsbeträgen unter Berücksichtigung der Kooperationsbedingung sowie des maximalen Kapitalwertes des Auftraggebers dargestellt:

\begin{tabular}{|c|c|c|c|c|c|}
\hline Anzahl Zahlungen & 1 & 2 & 3 & 4 & 5 \\
\hline \multirow{2}{*}{ Zahlung 1} & 982.291 & 500.109 & 336.434 & 252.669 & 202.067 \\
\hline & 10 & 10 & 10 & 10 & 10 \\
\hline \multirow{2}{*}{ Zahlung 2} & & 500.109 & 336.434 & 252.669 & 202.067 \\
\hline & & 29 & 18 & 16 & 16 \\
\hline \multirow{2}{*}{ Zahlung 3} & & & 336.434 & 252.669 & 202.067 \\
\hline & & & 45 & 29 & 21 \\
\hline \multirow{2}{*}{ Zahlung 4} & & & & 252.669 & 202.067 \\
\hline & & & & 45 & 32 \\
\hline Zahlung 5 & & & & & 202.067 \\
\hline Festpreis & 982.291 & 1.000 .218 & 1.009 .302 & 1.010 .676 & 1.010 .335 \\
\hline Kapitalwert AG & -58.675 & -58.675 & -58.675 & -58.675 & -58.675 \\
\hline Kapitalwert AN & 0 & 0 & 0 & 0 & 0 \\
\hline
\end{tabular}

Tabelle 5.7: Ergebnisse bei Minimierung des Festpreises und Kooperationsbedingung

Für die Zielsetzung der Festpreisminimierung kann allgemein festgestellt werden, dass der Festpreis mit der Anzahl der Zahlungen tendenziell steigt. Des Weiteren erfolgen die Zahlungen zu möglichst frühen Zeitpunkten während der jeweiligen Zahlungsin- 
tervalle, so dass die diskontierten Zahlungen positiv auf die Einhaltung der Kooperationsbedingung wirken. Die hier bestimmten Festpreise können als untere Festpreisgrenze interpretiert werden. Die Zahlung dieses Festpreises ist mindestens notwendig, um unter Berücksichtung der Kapitalwertmaximierung des Auftraggebers die Teilnahmebedingung des Auftragnehmers zu erfüllen. Die untere Festpreisgrenze ist in Abbildung 5.12 dargestellt. $^{20}$

In dieser Abbildung sind zusätzlich beispielhaft drei weitere in Abhängigkeit von der Anzahl der Zahlungen alternative Festpreise dargestellt, welche die Teilnahmebedingung unter der Zielsetzung der Kapitalwertmaximierung des Auftraggebers erfüllen. Die variierende Höhe des jeweiligen Festpreises resultiert aus der zuvor diskutierten Möglichkeit der Zahlung des Festpreises zu unterschiedlichen Zeitpunkten.

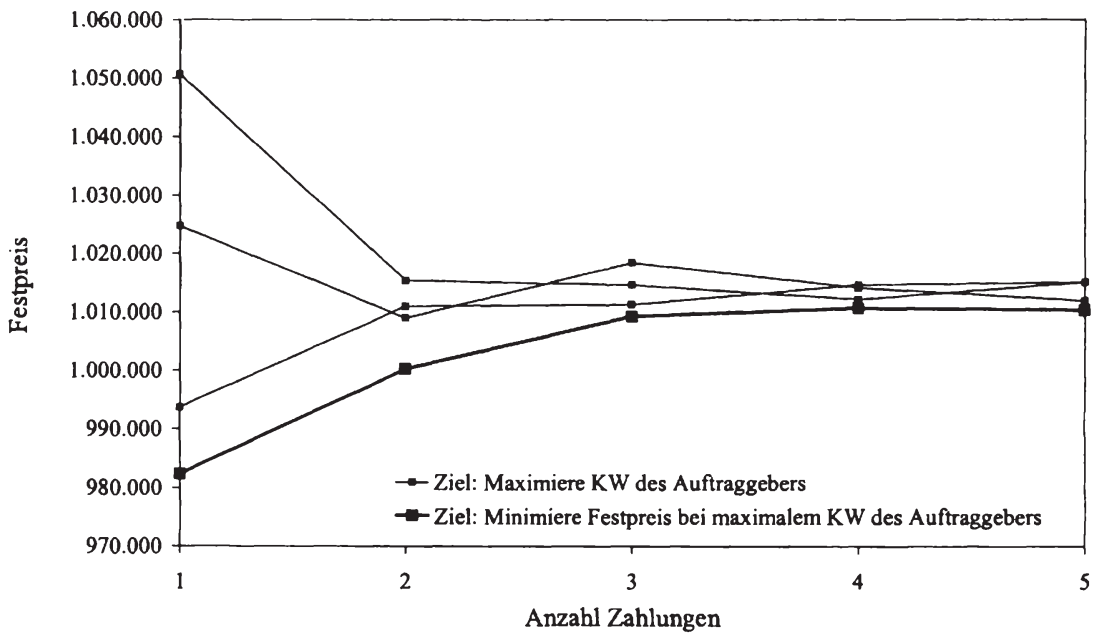

Abbildung 5.12: Höhe der Festpreise und untere Festpreisgrenze

Die durchgeführten Berechnungen zeigen, welcher mindestens zu zahlende Festpreis die Kooperationsbedingung erfüllt und zu dem maximal möglichen Kapitalwert des Auftraggebers führt. Der Auftraggeber muss durch die Einbeziehung zusätzlicher Prä-

20 Die Bestimmung einer oberen Festpreisgrenze ist unter der Annahme eines zu realisierenden Auftragnehmerkapitalwertes von null (das heißt, dass die Kooperationsbedingung genau erfullt ist) möglich, jedoch aus betriebswirtschaftlicher Sicht nicht sinnvoll, da zu erwarten ist, dass der Auftraggeber eine Präferenz für niedrige Festpreise besitzt. Diese obere Festpreisgrenze würde den Bereich der Festpreise eingrenzen, die je nach Anzahl und Zeitpunkten der Zahlungen sowie Berücksichtigung der Teilnahmebedingung zu dem maximal mőglichen Kapitalwert des Auftraggebers führen. 
ferenzen entscheiden, ob er die zeitlich frühe Zahlung eines niedrigen Festpreises der späteren Zahlung eines höheren Festpreises vorzieht.

Existiert für den Auftraggeber neben dem Ziel der Kapitalwertmaximierung beispielsweise das Ziel der Erreichung eines maximalen Liquiditätsstatus, ${ }^{21}$ so wird er eine möglichst hohe Anzahl Teilzahlungen vereinbaren und damit eine gleichmäßige Verteilung der Zahlungen während der Projektdauer erreichen. Dies führt zusätzlich dazu, dass die Höhe jeder Teilzahlung sinkt und somit insbesondere bei frühen Zahlungen keine in hohem Ausmaß über die entstandenen Kosten des Auftragnehmers hinausgehenden Zahlungen durch den Auftraggeber erfolgen.

\subsection{Payment Scheduling unter Berücksichtigung des Auftragneh- merverhaltens}

\subsubsection{Erweiterung der Problemstellung um alternative Arbeitsniveaus}

Um die zusätzlich zu der Teilnahmebedingung des Auftragnehmers in Kapitel 3.2.4 eingeführte Antizipation des Verhaltens des Auftragnehmers bei der Vertragsentscheidung sowie der Projektplanung des Auftraggebers zu berücksichtigen, wird im Folgenden zunächst erläutert, welche zusätzlichen Annahmen für eine realitätsnahe $\mathrm{Ab}$ bildung eines um alternative Arbeitsniveaus erweiterten Anwendungsbeispiels getroffen werden. Insbesondere sind hierbei Aspekte einzubeziehen, die die Informationsasymmetrie zwischen den Kooperationspartnern beachten. Das Entscheidungsproblem des Auftraggebers wird daher wie folgt formuliert:

Der Auftragnehmer übermittelt an den Auftraggeber ein Angebot für die Erstellung der in der Projektausschreibung spezifizierten Leistungen. Dieses Angebot enthält sowohl die für die Ausführung kalkulierten Vorgangsdauern sowie die mit den Vorgängen verbundenen und den Meilensteinereignissen zurechenbaren kalkulierten Kosten. Auf der Grundlage dieses Angebotes ermittelt der Auftraggeber ein Vertragsdesign. Hierbei muss er berücksichtigen, dass der Auftragnehmer ausschließlich solche Verträge akzeptiert, die die Kooperationsbedingung erfüllen. Das heißt, dass im Rahmen

${ }^{21} \mathrm{Zu}$ dem Begriff des Liquiditătsstatus vgl. etwa Schmidt/Terberger (1997), S. 28. Zu einer Finanzplanung unter Berücksichtigung der Liquiditătssituation vgl. Corsten/Corsten (2000), S. $232 \mathrm{ff}$. 
des Vertragsdesigns nur solche Handlungsalternativen berücksichtigt werden, die die Einhaltung der Teilnahmebedingung gewährleisten.

Aufgrund der zu dem Zeitpunkt des Vertragsabschlusses vorherrschenden Informationsasymmetrie über die als Reaktion auf den Vertrag durch den Auftragnehmer gewählte Arbeitsanstrengung muss der Auftraggeber zusätzlich berücksichtigen, dass der Auftragnehmer in Abhängigkeit von dem Vertragsdesign die Ausführung der für die Leistungserstellung notwendigen Aktivitäten in der Form realisieren wird, dass diese seinen Kapitalwert maximiert. ${ }^{22}$ Entsprechen diese Arbeitsanstrengung sowie die hieraus resultierenden Projektkonsequenzen nicht denen, welche der Auftraggeber für die Maximierung seines Kapitalwertes präferiert, sind Anreize so in das Vertragsdesign zu integrieren, dass das nach Vertragsabschluss vermutlich durch den Auftragnehmer realisierte Arbeitsniveau gleichzeitig den Auftraggeberpräferenzen entspricht.

Um das Anwendungsbeispiel fortführen zu können, wird im Folgenden angenommen, dass der Auftragnehmer für die Erstellung der Projektleistungen neben dem geplanten und durch das Angebot quantifizierten Arbeitsniveau nach Vertragsabschluss zusätzlich ein niedriges und ein hohes Arbeitsniveau realisieren kann. Die Entscheidung für eines dieser drei Arbeitsniveaus trifft der Auftragnehmer unmittelbar nach Vertragsabschluss mit dem Beginn der Leistungserstellung. Er führt sämtliche Vorgänge des Projektes entsprechend dem gewählten Arbeitsniveau aus, so dass ein Wechsel der Arbeitsanstrengung während der Projektdauer bei den folgenden Betrachtungen ausgeschlossen wird. ${ }^{23}$

Analog zu dem aus dem Bereich von Projektsteuerungsproblemen bekannten ZeitKosten-Konflikt wird unterstellt, dass bei einem niedrigeren Arbeitsniveau niedrigere Kosten sowie längere Dauern erwartet werden. Eine über das geplante Arbeitsniveau hinausgehende Anstrengung ist mit kürzeren erwarteten Vorgangsdauern und höheren erwarteten Kosten verbunden. ${ }^{24}$ Die aus den unterschiedlichen Arbeitsniveaus resultierenden Kosten und Dauern der Vorgänge sowie die für den Auftraggeber in Abhän-

\footnotetext{
22 Fur die formale Darstellung vgl. Formel (3.8) in Kapitel 3.2.4.

23 Obwohl diese Annahme die Problemstellung vereinfacht, spiegelt sie dennoch die im Rahmen der PrinzipalAgenten-Theorie vorgestellte Entscheidungssituation des Auftragnehmers wider. Wird die hier getroffene Annahme aufgehoben, resultiert daraus eine Entscheidungssituation, die als Problem einer Projektsteuerung aufgefasst werden kann. Die mit einem hohen Arbeitsniveau verbundene Zahlung von höheren Vorgangskosten können hierbei als Beschleunigungskosten zur Realisierung eines früheren Projektendes interpretiert werden. Vgl. zu diesem insbesondere wăhrend des Projektverlaufs vorliegenden Entscheidungsproblem Wolf (2004).

24 Vgl. Gido/Clements (2003), S. 218ff.; Meredith/Mantel (2003), S. 445ff.; Werners/Wolf (2004), S. 97.
} 
gigkeit von dem Zeitpunkt des Projektendes erzielbaren Erlöse aus der Projektnutzung werden zunächst näher betrachtet.

Der Auftraggeber besitzt durch das Angebot des Auftragnehmers hinsichtlich der im Folgenden unter der Bezeichnung des geplanten Arbeitsniveaus zusammengefassten Leistungen des Auftragnehmers sowohl Informationen über die erwarteten Dauern der Vorgänge als auch über deren erwartete Kosten. Mit Hilfe dieser Informationen ist es für den Auftraggeber möglich, unter der Annahme frühestmöglicher Ausführungszeiten der Vorgänge mit den Methoden der Netzplantechnik die kürzeste Projektdauer und somit das frühestmögliche Projektende zu ermitteln.

Für das Anwendungsbeispiel wird im Folgenden angenommen, dass die am Ende des vierten Kapitels dargestellten und auch für die weiterführenden Analysen des fünften Kapitels verwendeten Vorgangsdauern und -kosten aus der Leistungserstellung dem geplanten Arbeitsniveau entsprechen. Somit lässt sich für das geplante Arbeitsniveau ein frühestmögliches Projektende zu dem Zeitpunkt $t=52$ berechnen. Um eine Vergleichbarkeit der Ergebnisse zu gewährleisten, wird unterstellt, dass die erwarteten Erlöse für den Auftraggeber 1.000.000 GE betragen, sofern das Projekt zu dem Zeitpunkt $\mathrm{t}=52$ abgeschlossen wird und ab diesem Zeitpunkt eine Nutzung des Projektgegenstandes möglich ist.

Über die alternativen Arbeitsniveaus des Auftragnehmers besitzt der Auftraggeber zunächst keine Informationen. Da der Auftragnehmer im Rahmen der Vertragsverhandlungen diese Informationen nicht preisgeben wird, muss der Auftraggeber den durch die unterschiedlichen Arbeitsanstrengungen begründeten Zeit-Kosten-Konflikt auf der Grundlage von Erfahrungswerten schätzen. Die folgende Abbildung 5.8 zeigt für das Anwendungsbeispiel zusätzlich zu den geplanten Dauern und Kosten auch die aus dem niedrigen sowie dem hohen Arbeitsniveau des Auftragnehmers resultierenden vermuteten Vorgangsdauern und -kosten: 


\begin{tabular}{|c|rr|rr|rr|}
\cline { 2 - 7 } \multicolumn{1}{c|}{} & \multicolumn{2}{c|}{ niedriges Arbeitsniveau } & \multicolumn{2}{c|}{ geplantes Arbeitsniveau } & \multicolumn{2}{c|}{ hohes Arbeitsniveau } \\
\hline Vorgang & Vorgangsdauer & Kosten & Vorgangsdauer & Kosten & Vorgangsdauer & Kosten \\
\hline A & 10 & 12.000 & 8 & 15.000 & 6 & 16.500 \\
B & 19 & 16.000 & 16 & 20.000 & 13 & 22.000 \\
C & 18 & 6.400 & 15 & 8.000 & 12 & 8.800 \\
D & 10 & 3.200 & 8 & 4.000 & 6 & 4.400 \\
E & 18 & 104.000 & 15 & 130.000 & 12 & 143.000 \\
F & 2 & 56.000 & 2 & 70.000 & 2 & 77.000 \\
G & 2 & 52.000 & 2 & 65.000 & 2 & 71.500 \\
H & 16 & 64.000 & 13 & 80.000 & 10 & 88.000 \\
I & 7 & 128.000 & 6 & 160.000 & 5 & 176.000 \\
J & 12 & 10.400 & 10 & 13.000 & 8 & 14.300 \\
K & 5 & 1.600 & 4 & 2.000 & 3 & 2.200 \\
L & 6 & 48.000 & 5 & 60.000 & 4 & 66.000 \\
M & 8 & 12.000 & 7 & 15.000 & 6 & 16.500 \\
N & 10 & 40.000 & 8 & 50.000 & 6 & 55.000 \\
O & 4 & 16.000 & 3 & 20.000 & 2 & 22.000 \\
P & 19 & 62.400 & 16 & 78.000 & 13 & 85.800 \\
Q & 2 & 120.000 & 2 & 150.000 & 2 & 165.000 \\
R & 24 & 16.000 & 20 & 20.000 & 16 & 22.000 \\
S & 16 & 8.000 & 13 & 10.000 & 10 & 11.000 \\
T & 8 & 24.000 & 7 & 30.000 & 6 & 33.000 \\
\hline
\end{tabular}

Tabelle 5.8: Vorgangsdauern und -kosten als Konsequenz des Arbeitsniveaus

Aus der Sicht des Auftraggebers werden als Konsequenzen eines höheren Arbeitsniveaus durch die damit verbundenen kürzeren Vorgangsdauern ein früheres Projektende und somit höhere Erlöse aus der Nutzung des Projektgegenstandes erwartet. Das gegenüber dem geplanten Projektende durch die höhere Leistungsintensität mögliche frühere Projektende ist für den Auftragnehmer mit zusätzlichen Kosten verbunden. Diese können als Kosten einer beschleunigten Projektausführung interpretiert werden. Übersteigen die aus einem frühen Projektende resultierenden zusätzlichen diskontierten Erlöse des Auftraggebers die diskontierten Beschleunigungskosten, so besitzt der Auftraggeber die Präferenz, dass der Auftragnehmer die Projektleistung durch einen erhöhten Arbeitseinsatz erbringt. Werden alle Vorgänge eines Arbeitsniveaus zu ihren frühestmöglichen Zeitpunkten geplant, so können die folgenden Zeitpunkte als frühestmögliche Zeitpunkte des Projektendes in Abhängigkeit von dem Arbeitsniveau berechnet werden:

\begin{tabular}{|c|c|c|c|}
\cline { 2 - 4 } \multicolumn{1}{c|}{} & niedriges Arbeitsniveau & geplantes Arbeitsniveau & hohes Arbeitsniveau \\
\hline $\mathrm{E}_{\mathrm{la}}$ & 62 & 52 & 42 \\
\hline
\end{tabular}

Tabelle 5.9: Frühestmögliches Projektende in Abhängigkeit von dem Arbeitsniveau

Die von dem Arbeitseinsatz abhängigen erwarteten Erlöse sind in Abbildung 5.13 dargestellt. Es wird unterstellt, dass ein gegenüber dem geplanten Projektende zum Zeit- 
punkt $\mathrm{t}=52$ um eine Woche früheres/späteres Projektende zu einem um 40.000 GE höheren/niedrigeren Erlös führt. Kann das Projekt nicht bis zum Zeitpunkt $t=70$ fertig gestellt werden, muss das Projekt abgebrochen werden und führt damit zu keinem Erlös für den Auftraggeber.

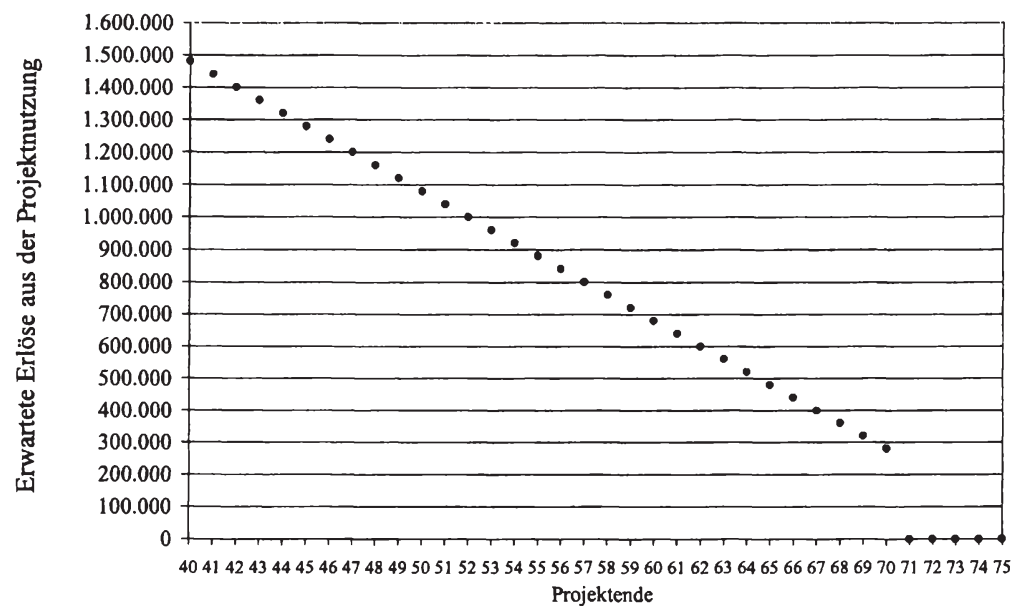

Abbildung 5.13: Erlöse in Abhängigkeit von dem Projektende

Ein alternativer Verlauf der Erlösfunktion kann aus der Sicht des Marketings etwa damit begründet werden, dass zusätzlich zu der durch ein früheres Projektende möglichen früheren Nutzung des Projektgegenstandes auch ein gegenüber Konkurrenten früherer Markteintritt zu überproportional steigenden Erlösen führt. Es wird jedoch für die folgenden Analysen der in Abbildung 5.13 aufgezeigte lineare Verlauf der Erlösfunktion unterstellt.

\subsubsection{Einbindung der Verhaltensantizipation in das Payment Scheduling}

Das in diesem Kapitel vorgestellte vierstufige Vorgehen verfolgt das Ziel, die im Rahmen des Contract Managements erfolgende Festlegung des Vertragsdesigns durch die quantitative Projektplanung zu unterstützten und insbesondere die zu dem Zeitpunkt des Vertragsabschlusses bestehende Informationsasymmetrie zwischen Auftraggeber und Auftragnehmer zu berücksichtigen. Hierfür beachtet der Auftraggeber bei der Vertragsplanung zusätzlich zu der Teilnahmebedingung unterschiedliche mögliche Arbeitsniveaus des Auftragnehmers und antizipiert somit das als Reaktion auf das Ver- 
tragsdesign mögliche Verhalten des Auftragnehmers. Abbildung 5.14 gibt einen Überblick über das entwickelte vierstufige Vorgehen:
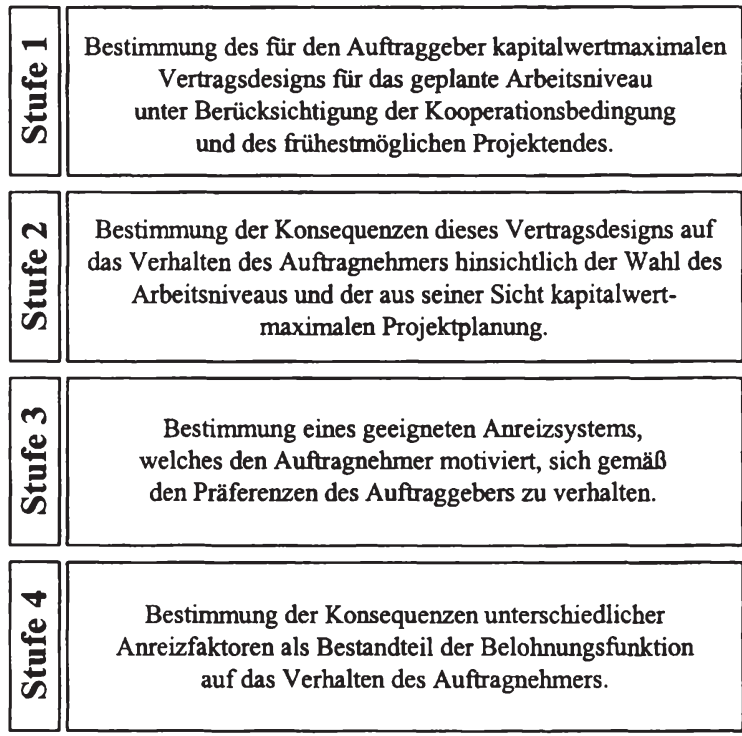

Abbildung 5.14: Vierstufiges Vorgehen für die Festlegung des Vertragsdesigns

\section{Stufe I}

Auf der ersten Stufe legt der Auftraggeber auf der Grundlage der Vorgangsdauern und -kosten des geplanten Arbeitsniveaus ein seinen Kapitalwert maximierendes Vertragsdesign fest, welches die Teilnahmebedingung des Auftragnehmers für dieses Arbeitsniveau erfüllt. Der Auftraggeber ist durch die mit der Projektnutzung erwarteten Erlöse tendenziell an einem möglichst frühen Projektende interessiert. Daher wird für die Bestimmung des Vertragsdesigns auf dieser Stufe als spätestes Projektende der frühestmögliche Zeitpunkt des letzten Ereignisses des geplanten Arbeitsniveaus festgelegt.

So wird zunächst die Zahlungsvereinbarung ermittelt, welche die Teilnahme des Auftragnehmers gewährleistet und zu einem optimalen Kapitalwert des Auftraggebers führt. Die Lösung dieses die Kooperationsbedingung berücksichtigenden und den Kapitalwert des Auftraggebers maximierenden Planungsproblems ist in den Kapiteln 5.4.2 und 5.4.3 für Kostenerstattungs- sowie Festpreisvereinbarungen vorgestellt worden. 


\section{Stufe 2}

$\mathrm{Da}$ der Auftraggeber zu dem Zeitpunkt des Vertragsdesigns keine Informationen besitzt, mit welcher Arbeitsanstrengung der Auftragnehmer auf den Vertrag reagieren wird, erfolgt auf der zweiten Stufe eine Antizipation dieser Reaktion. Hierfür versetzt sich der Auftraggeber in die Entscheidungssituation des Auftragnehmers und ermittelt unter Berücksichtigung der in der ersten Stufe festgelegten und für alle Arbeitsniveaus identischen Zahlungsvereinbarung, welches Verhalten und somit welches Arbeitsniveau aus der Sicht des Auftragnehmers zu einem kapitalwertmaximalen Projektplan führt. Das Ergebnis der zweiten Stufe bildet somit die Konsequenzen des aus der Sicht des Auftraggebers vermuteten Verhaltens des Auftragnehmers ab. Der Auftraggeber kann in Abhängigkeit von dem Arbeitsniveau erkennen, zu welchen Zeitpunkten das Projektende erreicht wird und welche Auswirkungen sich für seinen Kapitalwert ergeben. Aufgrund der Tatsache, dass ein möglichst frühes Projektende zu hohen Erlösen für den Auftraggeber führt, besteht aus seiner Sicht tendenziell die Präferenz, durch die Möglichkeit der beschleunigten Ausführung der Leistungen zusätzliche Kosten zu tragen, sofern dies zu einem früheren Projektende führt. Übersteigen die diskontierten Kosten der Beschleunigung jedoch die zusätzlich realisierbaren diskontierten Erlöse und bleiben weitere Zielkriterien unberücksichtigt, ist diese Präferenz nicht mehr vorhanden und der Auftraggeber bevorzugt tendenziell eine Leistungserstellung entsprechend des geplanten Arbeitsniveaus.

\section{Stufe 3}

Zeigen die Ergebnisse der zweiten Stufe, dass der Auftragnehmer aus seiner Sicht vermutlich nicht das durch den Auftraggeber bevorzugte frühe Projektende realisieren wird, kann die Motivation des Auftragnehmers durch die Einbindung von Anreizen in das Vertragsdesign beeinflusst werden, so dass sich dieser tendenziell entsprechend den Auftraggeberpräferenzen verhält. ${ }^{25}$ Bevorzugt der Auftraggeber beispielsweise durch die Präferenz für ein früheres Projektende das höhere Arbeitsniveau $a_{3}$ gegenüber dem geplanten sowie dem niedrigen Niveau, so muss er dem Auftragnehmer die Möglichkeit geben, durch die Wahl des hohen Arbeitsniveaus $a_{3}$ einen höheren Kapitalwert zu erzielen als bei Realisation von $a_{1}$ oder $a_{2}$. Ist zum Beispiel mit einem vor dem geplanten Projektende erreichten Abschluss des Projektes eine Bonuszahlung verbunden, so kann diese den Auftragnehmer für die Wahl des hohen Arbeitsniveaus,

25 Einen Überblick über Motivationstheorien geben beispielsweise Comelli/Rosenstiel (2003); Jost (2000); Kressler (2001). 
welches mit einem möglichen frühzeitigen Projektabschluss einhergeht, motivieren. Auf der dritten Stufe muss daher eine Anreizfunktion als Teil des Anreizsystems bestimmt werden, welche durch die Einbindung in das Vertragsdesign dazu führt, dass der Auftragnehmer die Projektleistungen entsprechend den Präferenzen des Auftraggebers ausführt. Das Ergebnis der dritten Stufe zeigt die Konsequenzen des Anreizsystems sowohl für die Projektplanung des Auftragnehmers und dessen Kapitalwert als auch für die mit dem vermutlich realisierten Projektende verbundenen Erlöse für den Auftraggeber und dessen Kapitalwert auf.

\section{Stufe 4}

Ziel der vierten Stufe ist die Bestimmung einer Anreizfunktion, welche aus der Sicht des Auftraggebers zu einem maximalen Kapitalwert führt. Diese Situation ist in Kapitel 3.2.4 in der Abbildung 3.6 durch die Bezeichnungen der First-Best-Lösung sowie der Second-Best-Lösung dargestellt worden. Bedingt durch die Informationsasymmetrie bezüglich des Auftragnehmerverhaltens als Reaktion auf das Vertragsdesign können somit für den Auftraggeber zusätzliche Zahlungen entstehen, um dieses PrinzipalAgenten-Problem zu lösen. Es muss somit derjenige Teilungsfaktor ermittelt werden, welcher mit möglichst niedrigen Anreizzahlungen die gewünschte Änderung des Auftragnehmerverhaltens herbeiführt. Das hier in allgemeiner Form vorgeschlagene vierstufige Vorgehen für das das Auftragnehmerverhalten antizipierende Payment Scheduling aus der Sicht des Auftraggebers wird in den folgenden zwei Kapiteln sowohl für Kostenerstattungs- als auch für Festpreisvereinbarungen umgesetzt und auf das Beispiel angewandt.

\subsubsection{Antizipierendes Payment Scheduling bei Kostenerstattungsverträgen}

Im Rahmen der bisherigen Ausführungen sind die charakteristischen Merkmale von Kostenerstattungsvereinbarungen zwischen Auftraggeber und Auftragnehmer vorgestellt und in das Payment Scheduling Modell integriert sowie die Auswirkungen einer Berücksichtigung der Kooperationsbedingung aufgezeigt worden. Der Schwerpunkt des folgenden Abschnittes ist es daher, das allgemein im vorherigen Kapitel definierte vierstufige Vorgehen für die Antizipation des Auftragnehmerverhaltens bei der Vertragsentscheidung des Auftraggebers anhand des Beispiels zu veranschaulichen. Das Ziel ist hierbei die Umsetzung des entwickelten Konzeptes, welches auch auf alternative, hier nicht berücksichtigte Zahlungsvereinbarungen übertragen werden kann. 
Folgende die Zahlungen und Zahlungszeitpunkte betreffenden Vertragsvereinbarungen werden im Rahmen der hier untersuchten Modellierung einer Kostenerstattungsvereinbarung angenommen:

- Der Auftraggeber vergütet mit ANZ Teilzahlungen jeweils genau das $\beta$-fache der Kosten, die seit der vorangegangenen Zahlung bzw. dem Projektbeginn planmäßig entstanden sind. Die Zahlungen müssen zu Zeitpunkten innerhalb ANZ konstanter Zeitintervalle erfolgen, so dass die Fortschrittszahlungen zu möglichst regelmäßigen Zeitpunkten während der Projektdauer stattfinden.

- Der Auftraggeber bevorzugt durch die aus der Projektnutzung nach Abschluss des Projektes resultierenden Erlöse ein Projektende, welches dem frühestmöglichen Projektende des jeweiligen Arbeitsniveaus entspricht. Ein spätesterlaubtes Projektende wird zusätzlich festgelegt.

- Der Auftraggeber berücksichtigt bei seiner Vertragsentscheidung auf der ersten Stufe die Kooperationsbedingung des Auftragnehmers, so dass der Kapitalwert des Auftragnehmers bei Realisation des geplanten Arbeitsniveaus und einem geplanten Projektende $\mathrm{zu}$ dem frühestmöglichen Zeitpunkt mindestens der Teilnahmebedingung $\mathrm{TB}^{\mathrm{AN}}$ entspricht. Durch die mit den Teilzahlungen erfolgende Erstattung des genau $\beta$-fachen der Kosten ist es bei Kostenerstattungsfaktoren $\beta \leq 1,0$ notwendig, im Rahmen der Optimierung ein Honorar so zu bestimmen, dass die Kooperationsbedingung erfüllt ist. Die Zahlung des Honorars erfolgt mit dem Abschluss des Projektes zu dem Zeitpunkt des letzten Ereignisses.

\section{Stufe 1}

Das im Anschluss aufgeführte Modell für die Bestimmung der aus der Sicht des Auftraggebers für das geplante Arbeitsniveau optimalen Zahlungsvereinbarung berücksichtigt die hier beschriebenen Vereinbarungen einer Kostenerstattung. Zunächst erfolgt eine Darstellung aller in dem Modell verwendeten Indizes, Daten und Variablen:

\section{Indizes und Indexmengen:}

$\mathrm{a}=1, \ldots, \mathrm{A} \quad$ : Arbeitsniveaus des Auftragnehmers

$a_{\text {plan }} \quad:$ Geplantes Arbeitsniveau

$\mathrm{i}, \mathrm{j}=1, \ldots, \mathrm{I} \quad$ : Ereignisse

$\mathbf{k}=1, \ldots$, ANZ : Anzahl der Teilzahlungen

$\mathrm{t}, \mathrm{u}=0, \ldots, \mathrm{T} \quad:$ Ereigniszeitpunkte 


\section{Daten:}

ANZ : Anzahl der durch den Auftraggeber zu leistenden Zahlungen

$\beta \quad$ : Kostenerstattungsfaktor

$\mathrm{D}_{\mathrm{ija}} \quad$ : Dauer des Vorgangs zwischen Ereignissen $\mathrm{i}$ und $\mathrm{j}$ bei Arbeitsniveau a

DISKZ $^{\text {AG/AN }}$ : Diskontierungszinssatz des Auftraggebers/Auftragnehmers ${ }^{26}$

DISKF $_{t}^{\mathrm{AG} / \mathrm{AN}}$ : Diskontierungsfaktor des Auftraggebers/Auftragnehmers ${ }^{26}$

$\mathrm{E}_{\mathrm{ia}} \quad:$ Frühestmöglicher Zeitpunkt des Ereignisses $\mathrm{i}$ bei Arbeitsniveau a

ERL $_{\mathrm{t}} \quad$ : Erlöse des Auftraggebers bei einem Projektende zum Zeitpunkt $t$

INT : Dauer der Zahlungsintervalle

$\mathrm{NNT}_{\mathrm{k}}^{\mathrm{UG}} \quad$ : Untere Grenze der Zahlungsintervalle

$\mathrm{INT}_{\mathrm{k}}^{\mathrm{OG}} \quad$ : Obere Grenze der Zahlungsintervalle

$\mathrm{K}_{\mathrm{ia}}^{\mathrm{AG} / \mathrm{AN}} \quad$ : Kosten bei Ereignis i und Arbeitsniveau a aus der Sicht des AG/AN ${ }^{26}$

$\widehat{\mathrm{M}} \quad$ : Große Zahl

$\mathrm{TB}^{\mathrm{AN}} \quad:$ Teilnahmebedingung des Auftragnehmers

$\mathrm{V} \quad$ : Menge der einem Vorgang unmittelbar vorangehenden und unmittelbar nachfolgenden Ereignisse (i,j)

\section{Variablen:}

$\mathbf{x}_{\mathrm{ita}}=\left\{\begin{array}{l}1, \text { wenn Ereignis i zum Zeitpunkt } \mathrm{t} \text { bei Arbeitsniveau a stattfindet } \\ 0, \text { sonst }\end{array}\right.$

$y_{t a}=\left\{\begin{array}{l}1, \text { wenn eine Zahlung zum Zeitpunkt } t \text { bei Arbeitsniveau a stattfindet } \\ 0, \text { sonst }\end{array}\right.$

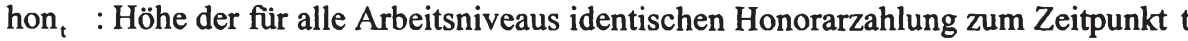

$\mathrm{z}_{\mathrm{ta}} \quad$ : Höhe der Zahlung zum Zeitpunkt t bei Arbeitsniveau a

Das für Kostenerstattungsvereinbarungen entwickelte Payment Scheduling Modell der ersten Stufe kann als gemischt-ganzzahliges lineares Programmierungsmodell formuliert werden. Das Modell bildet die Entscheidungssituation für das geplante Arbeitsniveau $a_{\text {plan }}$ unter Beachtung der Kooperationsbedingung $a b$.

26 Für eine übersichtlichere Darstellung werden die Daten zusammengefasst, welche sich ausschließlich durch den Index für den Auftraggeber bzw. den Auftragnehmer (AG/AN) unterscheiden. 


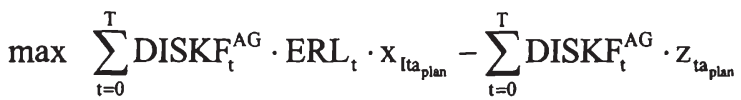

so dass

$\sum_{\mathrm{t}=0}^{\mathrm{T}} \mathrm{x}_{\mathrm{ita}_{\text {plun }}}=1$

$\forall \mathrm{i}$

$\sum_{\mathrm{t}=0}^{\mathrm{T}} \mathrm{t} \cdot \mathrm{x}_{\mathrm{jta} \text { pan }}-\sum_{\mathrm{t}=0}^{\mathrm{T}} \mathrm{t} \cdot \mathrm{x}_{\mathrm{ita}_{\mathrm{plan}}} \geq \mathrm{D}_{\mathrm{ija} \mathrm{alan}_{\mathrm{pan}}} \quad \forall(\mathrm{i}, \mathrm{j}) \in \mathrm{V}$

$\sum_{\mathrm{t}=0}^{\mathrm{T}} \mathrm{t} \cdot \mathrm{x}_{\mathrm{Itaplan}}=\mathrm{E}_{\mathrm{Ia}_{\text {plan }}}$

$\sum_{\mathrm{t}=0}^{\mathrm{T}} \mathrm{z}_{\mathrm{ta}_{\mathrm{plan}}}=\sum_{\mathrm{i}=1}^{\mathrm{I}} \mathrm{K}_{\mathrm{it}_{\mathrm{pan}}}^{\mathrm{AG}}+\sum_{\mathrm{t}=0}^{\mathrm{T}}$ hon $_{\mathrm{t}} \quad$ für $\beta \leq 1,0$

$\sum_{\mathrm{t}=0}^{\mathrm{T}} \mathrm{z}_{\mathrm{ta}_{p \operatorname{lan}}}=\beta \cdot \sum_{\mathrm{i}=1}^{\mathrm{I}} \mathrm{K}_{\mathrm{i}_{\mathrm{p} p \operatorname{las}}^{\mathrm{AG}}}^{\mathrm{AG}} \quad$ für $\beta>1,0$

$\sum_{t=0}^{T} y_{t_{\text {plax }}}=\mathrm{ANZ}$

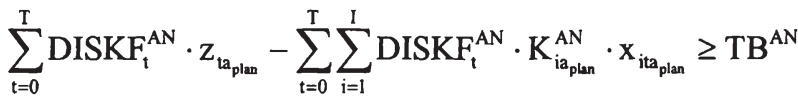

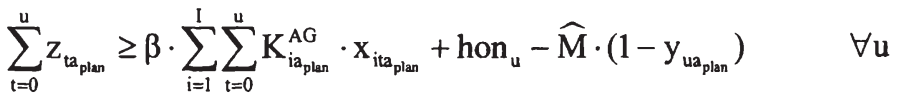

$\sum_{\mathrm{t}=0}^{\mathrm{u}} \mathrm{z}_{\mathrm{ta}_{\mathrm{alus}}}-\widehat{\mathrm{M}} \cdot\left(1-\mathrm{y}_{\mathrm{ua}_{\mathrm{pun}}}\right) \leq \beta \cdot \sum_{\mathrm{i}=1}^{1} \sum_{\mathrm{t}=0}^{\mathrm{u}} \mathrm{K}_{\mathrm{i}_{\mathrm{p} \text { pun }}}^{\mathrm{AG}} \cdot \mathrm{x}_{\mathrm{ita}_{\mathrm{plan}}}+$ hon $_{\mathrm{u}} \quad \forall \mathrm{u}$

$\sum_{t=\mathbb{N T}_{k}^{\mathrm{UG}}}^{\mathrm{NN}_{\mathrm{K}}^{\mathrm{OG}}} \mathrm{y}_{\mathrm{ta}_{\mathrm{plan}}}=1$

$\forall \mathrm{k}$

$\sum_{i=1}^{1} x_{\text {ita }_{\text {plan }}} \geq y_{t_{\text {plan }}}$

$\forall \mathrm{t}$

hon $_{\mathrm{t}} \leq \widehat{\mathrm{M}} \cdot \mathrm{x}_{\text {Ita plen }}$

$\forall \mathrm{t}$

hon $_{\mathrm{t}} \geq 0$

$\forall \mathrm{t}$

$\mathbf{x}_{\text {ita }_{\text {plan }}} \in\{0,1\}$

$\forall \mathrm{i}, \mathrm{t}$

$\mathrm{y}_{\mathrm{ta}_{\mathrm{plax}}} \in\{0,1\}$

$\forall \mathrm{t}$

$\mathrm{z}_{\mathrm{ta}_{\mathrm{plan}}} \geq 0$

$\forall \mathrm{t}$ 
Die Zielfunktion (5.13) maximiert den Kapitalwert des Auftraggebers für das geplante Arbeitsniveau. Dieser Kapitalwert setzt sich aus der Differenz des Barwertes der von dem Zeitpunkt des Projektendes abhängigen Erlöse aus der Projektnutzung sowie der Barwerte der Zahlungen an den Auftragnehmer zusammen.

Die Restriktionen (5.14) und (5.15) sind aus dem Grundmodell des vierten Kapitels bekannt. Sie gewährleisten, dass jedes Ereignis zu genau einem Zeitpunkt stattfindet und hierbei die Reihenfolgebeziehung unmittelbar aufeinander folgender Ereignisse sowie die Vorgangsdauern eingehalten werden. Bedingung (5.16) legt den Zeitpunkt des letzten Ereignisses I auf dessen frühestmöglichen Zeitpunkt fest, so dass das Projektende zu seinem frühestmöglichen Zeitpunkt geplant wird.

Die Restriktionen (5.17) bzw. (5.18) gewährleisten, dass bei einem Kostenerstattungsfaktor $\beta \leq 1,0$ die Summe der Zahlungen den Gesamtkosten zuzüglich des notwendigen Honorars bzw. bei einem Kostenerstattungsfaktor $\beta>1,0$ der Summe der mit diesem Faktor multiplizierten Gesamtkosten entspricht. Es wird hierbei ersichtlich, dass die Honorarzahlung als Bestandteil der Kostenerstattungszahlungen modelliert wird. Durch die Bedingung (5.19) wird die Anzahl der Teilzahlungen auf die vereinbarte Anzahl begrenzt.

Die für das geplante Arbeitsniveau durchgeführte Modellierung berücksichtigt durch die Restriktion (5.20) die Kooperationsbedingung des Auftragnehmers. Die Differenz aus den Barwerten der Summe der Kostenerstattungszahlungen und der Barwerte der Vorgangskosten muss mindestens der Teilnahmebedingung des Auftragnehmers entsprechen.

Die folgenden zwei Restriktionen stellen sicher, dass mit jeder Zahlung genau die bis zu diesem Zeitpunkt entstandenen und noch nicht durch vorherige Zahlungen vergüteten Kosten erstattet werden. Hierfür legt die Bedingung (5.21) fest, dass zu den Zeitpunkten, zu denen eine Zahlung erfolgt, die Summe der bis zu diesem Zeitpunkt geleisteten Zahlungen mindestens den bis dahin entstandenen und mit dem Kostenerstattungsfaktor $\beta$ multiplizierten Kosten entspricht. Die Vereinbarung, mit jeder Teilzahlung genau das $\beta$-fache der Kosten zu erstatten, erfordert die Modellierung der maximal erlaubten Höhe der Zahlungen durch die Restriktion (5.22). Die Formulierung dieser zwei Bedingungen in Form einer einzigen Gleichheitsrestriktion ist aufgrund der mit $\widehat{M}$ additiv verknüpften Ausdrücke nicht möglich. Das zu dem Zeitpunkt des Pro- 
jektendes zu zahlende Honorar ist Bestandteil der letzten Zahlung und wird daher in den Restriktionen (5.21) und (5.22) berücksichtigt.

Die Zahlungen sollen zu ANZ innerhalb konstanter Zeitintervalle liegenden Zeitpunkten gezahlt werden, so dass eine möglichst gleichmäßige Aufteilung der Zahlungen während der Projektdauer erreicht wird. Daher ist die aus der Modellierung der Festpreisvereinbarung bekannte Restriktion (5.23) Teil dieses Modells. Die Bedingung gewährleistet, dass genau eine Zahlung innerhalb der von der Anzahl der Zahlungen abhängigen Intervalle erfolgt. Die Restriktion (5.24) stellt die Verbindung zwischen den Binärvariablen des Modells her, indem gewährleistet wird, dass zum Zeitpunkt einer Zahlung mindestens ein Ereignis stattfindet. Obwohl der Zeitpunkt des Projektendes für das geplante Arbeitsniveau mit dem frühestmöglichen Zeitpunkt des letzten Ereignisses festgelegt und damit bekannt ist, enthält die Variable der Honorarzahlung den Index t. Dies ist für die weiterführenden Modelle notwendig, da der Zeitpunkt des letzten Ereignisses im Rahmen dieser Modelle eine Entscheidungsvariable darstellt und somit auch der Zeitpunkt der Honorarzahlung als Entscheidungsvariable angesehen werden muss. Durch den Index $t$ wird die Nichtlinearität des Modells durch die für die Bindung der Zahlung an den Zeitpunkt notwendige multiplikative Verknüpfung der Variablen des Honorars mit der Binärvariablen $\mathrm{y}_{\mathrm{ta}}$ vermieden. Zusätzlich gewährleistet die Bedingung (5.25), dass die Honorarzahlung mit dem Abschluss des Projektes erfolgt. Schließlich legen die Bedingungen (5.26) bis (5.29) den Definitionsbereich der Variablen des Modells fest.

Damit ist die formale Darstellung des quantitativen Modells für die ausschließlich das geplante Arbeitsniveau des Auftragnehmers berücksichtigende erste Stufe abgeschlossen. Für eine Vorstellung des entwickelten vierstufigen Vorgehens wird im Folgenden auf einen Vorteilhaftigkeitsvergleich unterschiedlicher Kostenerstattungsvereinbarungen durch den Auftraggeber, etwa durch alternative Kostenerstattungsfaktoren oder durch eine alternative Anzahl an Teilzahlungen verzichtet und auf die Ausführungen des Kapitels 5.4.2 verwiesen.

Es wird zusätzlich zu den zuvor aufgezeigten allgemeinen Vertragsvereinbarungen für die anschließenden Untersuchungen beispielhaft angenommen, dass eine Anzahl $\mathrm{ANZ}=3$ Teilzahlungen sowie ein Kostenerstattungsfaktor $\beta=1,0$ vereinbart werden. Das spätesterlaubte Projektende wird zu dem Zeitpunkt $t=70$ festgelegt. 
Die Lösung dieses Optimierungsproblems führt zu den optimalen Zahlungszeitpunkten $\mathrm{t}=10, \mathrm{t}=32$ sowie $\mathrm{t}=52$. Damit werden die mit Zahlungen verbundenen Ereignisse 2, 11 und 13 festgelegt. Zusätzlich wird eine für die Erfüllung der Kooperationsbedingung notwendige Honorarzahlung für das hier betrachtete Beispiel in Höhe von 35.884 GE bestimmt. Durch den Abschluss des Projektes zu seinem frühestmöglichen Zeitpunkt $t=52$ erwartet der Auftraggeber Erlöse in Höhe von 1.000.000 GE aus der Projektnutzung. Die Berücksichtigung des niedrigen sowie des hohen Arbeitsniveaus ist im Rahmen der ersten Stufe nicht erforderlich, da ausschließlich das geplante Arbeitsniveau Bestandteil der Vertragsverhandlungen ist und dieses somit für die Bestimmung des für den Auftraggeber optimalen Vertragsdesigns maßgeblich ist. Tabelle 5.10 zeigt die Ergebnisse der Optimierung für das geplante Arbeitsniveau:

\begin{tabular}{|c|c|c|c|}
\hline \multirow{2}{*}{ Stufe 1} & \multicolumn{3}{|c|}{ Arbeitsniveau } \\
\hline & niedrig & geplant & hoch \\
\hline $\begin{array}{lc}\text { 1. Zahlung } & \begin{array}{c}\text { Betrag } \\
\text { Zeitpunkt }\end{array} \\
\end{array}$ & & $\begin{array}{c}15.000 \\
10 \\
\end{array}$ & \\
\hline $\begin{array}{lc}\text { 2. Zahlung } & \begin{array}{c}\text { Betrag } \\
\text { Zeitpunkt }\end{array} \\
\end{array}$ & & $\begin{array}{c}707.000 \\
32 \\
\end{array}$ & \\
\hline $\begin{array}{lc}\text { 3. Zahlung } & \text { Betrag } \\
\text { (inkl. Honorat) } & \text { Zeitpunkt } \\
\end{array}$ & & $\begin{array}{c}313.884 \\
52 \\
\end{array}$ & \\
\hline Honorar & & 35.884 & \\
\hline Erlöse Auftraggeber & & 1.000 .000 & \\
\hline Kapitalwert Auftraggeber & & -58.675 & \\
\hline Kapitalwert Auftragnehmer & & 0 & \\
\hline
\end{tabular}

Tabelle 5.10: Ergebnisse für das geplante Arbeitsniveau bei Kostenerstattung

Die Höhe der ersten und zweiten Zahlung entspricht den bis zu diesen Zeitpunkten entstandenen Kosten. Die dritte Zahlung zu dem Zeitpunkt des frühestmöglichen Projektendes enthält zusätzlich zu der mit Ereignis 13 erforderlichen Kostenerstattungszahlung das Honorar in Höhe von 35.884 GE. Es zeigt sich, dass auch hier der maximale Kapitalwert des Auftraggebers dem in den vorigen Kapiteln unter Berücksichtigung der Kooperationsbedingung ermittelten Kapitalwert entspricht.

Der Auftraggeber entwirft auf der Grundlage der hier ermittelten Lösung ein Vertragsdesign, welches für dieses Anwendungsbeispiel die folgenden Zahlungsvereinbarungen enthält:

- Zahlungen erfolgen zu den Zeitpunkten der Ereignisse 2, 11 und 13. Die Höhe der Zahlungen richtet sich nach den bis zu diesen Ereignissen entstandenen Kosten. Es wird genau das 1-fache der Kosten erstattet. 
- Das Projekt muss spätestens zu dem Zeitpunkt $t=70$ fertig gestellt sein. Mit Abschluss des Projektes wird ein Honorar in Höhe von 35.884 GE gezahlt. ${ }^{27}$

Die erste Stufe ist mit der Festlegung der aus der Sicht des Auftraggebers optimalen Zahlungsvereinbarungen, welche die Grundlage der Analysen der zweiten Stufe bilden, abgeschlossen.

\section{Stufe 2}

Für die Antizipation des Auftragnehmerverhaltens wird das vorgestellte Modell so modifiziert, dass es das vermutete Verhalten des Auftragnehmers bezüglich der Realisation eines Arbeitsniveaus abbildet. Der Auftraggeber versetzt sich in die Entscheidungssituation des Auftragnehmers, um das mögliche Verhalten als Reaktion auf die Zahlungsvereinbarung beurteilen zu können. Der Auftraggeber wendet dieses Modell für alle vermuteten möglichen Arbeitsniveaus an, so dass die Ausdrücke, welche in dem zuvor vorgestellten Modell den Index $a_{\text {plan }}$ beinhalten, so modifiziert werden, dass sie den Index des jeweils betrachteten Arbeitsniveaus enthalten.

Die Zielfunktion muss die Zielsetzung des Auftragnehmers berücksichtigen und wird daher für das Modell der zweiten Stufe durch die Formulierung (5.30) ersetzt:

$\max \sum_{\mathrm{t}=0}^{\mathrm{T}} \operatorname{DISKF}_{t}^{\mathrm{AN}} \cdot \mathrm{z}_{\mathrm{ta}}-\sum_{\mathrm{t}=0}^{\mathrm{T}} \sum_{\mathrm{i}=1}^{1} \operatorname{DISKF}_{\mathrm{t}}^{\mathrm{AN}} \cdot \mathrm{K}_{\mathrm{ia}}^{\mathrm{AN}} \cdot \mathbf{x}_{\mathrm{ita}}$

Die Restriktionen (5.14) und (5.15) bleiben Bestandteil des Modells. Die Forderung, dass das Projekt zu dem frühestmöglichen Ende abgeschlossen wird, entfällt, da dies nicht Bestandteil der Vertragsvereinbarung ist. Stattdessen wird mit der Bedingung (5.31) der Zeitpunkt DMax = 70 als spätesterlaubtes Projektende festgelegt:

$\mathrm{t} \cdot \mathrm{x}_{\mathrm{Ita}} \leq \mathrm{DMax}$

Der Auftragnehmer wird daher entscheiden, welche Ereigniszeitpunkte zu einem aus seiner Sicht besten Ergebnis führen. Die Bedingung (5.17) bzw. (5.18) muss gelten, so dass insbesondere unter dieser Zielsetzung die Gesamtsumme der Kostenerstattungs-

27 Dies ist das für die Erfüllung der Kooperationsbedingung durch die Optimierung bestimmte mindestens notwendige Honorar. Bei realen Problemstellungen kann davon ausgegangen werden, dass die Vereinbarung des Honorars Gegenstand der Vertragsverhandlungen zwischen Auftraggeber und Auftragnehmer ist und beispielsweise auf $36.000 \mathrm{GE}$ gerundet wird. 
zahlungen durch die Höhe der gesamten Kosten sowie des Honorars bzw. der mit dem Kostenerstattungsfaktor multiplizierten gesamten Kosten begrenzt ist. Dabei wird die Variable hon ${ }_{t}$ durch den Parameter $\mathrm{HON}_{\mathrm{t}}$ ersetzt, welcher das auf der ersten Stufe festgesetzte und zu dem Zeitpunkt des Projektendes durch den Auftraggeber an den Auftragnehmer zu zahlende Honorar festsetzt. Hierfür werden die Restriktionen (5.25) und (5.26) durch den folgenden Ausdruck ersetzt, welcher sowohl die Höhe des Honorars als auch den Zeitpunkt der Zahlung des Honorars mit dem Zeitpunkt des letzten Ereignisses I bei Arbeitsniveau a festsetzt:

$\mathrm{HON}_{\mathrm{t}}=$ hon $_{\mathrm{t}} \cdot \mathrm{x}_{\text {Ita }} \quad \forall \mathrm{t}$

Auch die Anzahl der Teilzahlungen ist weiterhin durch die vertragliche Vereimbarung vorgegeben, so dass auch Restriktion (5.19) Gültigkeit behält. Aus der Sicht des Auftragnehmers wird das durch die Kooperationsbedingung für die Planung des Auftraggebers berücksichtigte Satisfizierungsziel zu einem Maximierungsziel, so dass die Kooperationsbedingung (5.20) für das Modell der zweiten Stufe nicht berücksichtigt wird. Die Restriktionen (5.21) und (5.22) sind notwendig, um die Höhe der Kostenerstattungszahlungen festzulegen. Auf der ersten Stufe sind die Ereignisse festgelegt worden, zu denen eine Kostenerstattungszahlung erfolgen soll. Die Restriktion (5.33) verknüpft in Verbindung mit Restriktion (5.24) die Zeitpunkte der Ereignisse 2, 11 und 13 mit den Zahlungszeitpunkten und ersetzt somit die Bedingung (5.23). Diese Zahlungszeitpunkte müssen innerhalb des Intervalls der möglichen Ereigniszeitpunkte liegen, welche durch den frühestmöglichen Beginn und das spätesterlaubte Ende jedes Ereignisses bestimmt werden. Durch die Formulierung dieser Restriktion als $\leq-\mathrm{Be}$ dingung wird die Möglichkeit berücksichtig, dass sich die Zeitintervalle der für Zahlungen vorgesehenen Ereignisse überschneiden.

$$
\mathrm{t} \cdot \mathrm{x}_{\mathrm{ita}} \leq \mathrm{t} \cdot \mathrm{y}_{\mathrm{ta}} \quad \forall \mathrm{t}, \mathrm{i} \in\{2,11,13\}
$$

Die Restriktionen (5.27) bis (5.29) legen den Definitionsbereich der Variablen für das Modell der zweiten Stufe fest. Die Lösung dieses für die Sichtweise des Auftragnehmers modifizierten Modells zeigt dem Auftraggeber die Konsequenzen des Vertragsdesigns hinsichtlich des durch den Auftragnehmer als Reaktion auf das Vertragsdesign gewählten Arbeitsniveaus auf und führt zu den durch die Optimierung ermittelten und in Tabelle 5.11 dargestellten Ergebnissen: 


\begin{tabular}{|cc|c|c|c|}
\hline \multirow{2}{*}{ Stufe 2 } & \multicolumn{3}{c|}{ Arbeitsniveau } \\
\cline { 3 - 5 } & Betrag & niedrig & geplant & hoch \\
\hline \multirow{2}{*}{ 1. Zahlung } & 28.000 & 203.000 & 223.300 \\
& Zeitpunkt & 19 & 21 & 17 \\
\hline \multirow{2}{*}{ 2. Zahlung } & Betrag & 549.600 & 519.000 & 570.900 \\
& Zeitpunkt & 37 & 36 & 29 \\
\hline \multirow{2}{*}{$\begin{array}{c}\text { 3. Zahlung } \\
\text { (inkl. Honorar) }\end{array}$} & Betrag & 258.284 & 313.884 & 341.684 \\
\hline Honorar & & $\mathbf{6 9}$ & $\mathbf{6 3}$ & $\mathbf{5 1}$ \\
\hline \multicolumn{2}{|c|}{ Erlöse Auftraggeber } & 35.884 & 35.884 & 35.884 \\
\hline \multicolumn{2}{|c|}{ Kapitalwert Auftraggeber } & 320.000 & 560.000 & 1.040 .000 \\
\hline \multicolumn{2}{|c|}{ Kapitalwert Auftragnehmer } & -484.830 & -461.231 & -122.948 \\
\hline
\end{tabular}

Tabelle 5.11: Ergebnisse antizipierendes Payment Scheduling bei Kostenerstattung für Stufe 2

Es lässt sich erkennen, dass die Kapitalwerte für den Auftragnehmer bei allen Arbeitsniveaus positiv sind und der höchste Kapitalwert bei Realisation des hohen Arbeitsniveaus erreicht werden kann. Dieses Ergebnis bestätigt die im dritten Kapitel aufgestellten Vermutungen, dass bei einer Kostenerstattungsvereinbarung kein Verlustrisiko für den Auftragnehmer besteht und damit kein Anreiz existiert, die Leistungen zu niedrigen Kosten zu erbringen.

Diese Untersuchungen zeigen außerdem, dass der Auftragnehmer bei Realisation des geplanten Arbeitsniveaus von dem aus der Sicht des Auftraggebers kapitalwertmaximalen Projektplan abweicht und eine spätere Terminierung der Ereignisse und damit auch ein späteres Projektende wählt. Auch die zu einem maximalen Auftragnehmerkapitalwert führenden Zeitpunkte des Projektendes bei Realisation des hohen sowie des niedrigen Arbeitsniveaus werden später als die frühestmöglichen Zeitpunkte festgelegt.

Für den Auftraggeber ist das hohe Arbeitsniveau ebenfalls mit dem höchsten Kapitalwert verbunden. Die Wahl der aus der Sicht des Auftragnehmers optimalen Zeitpunkte für die Zahlungen und somit für die Ereignisse 2, 11 und 13 zeigt jedoch, dass die Ereignisse nicht zu ihren frühestmöglichen Zeitpunkten geplant werden. Obwohl das Projektende bei Wahl des hohen Arbeitsniveaus zum Zeitpunkt $t=51$ festgelegt wird und damit das geplante Projektende aus der Sicht des Auftraggebers um eine Woche unterschreitet, wird der Auftraggeber nicht bereit sein, die mit dem hohen Arbeitsniveau entstehenden hohen Kosten zu erstatten, wenn ein Projektende zum Zeitpunkt $t=52$ bei Realisation des geplanten und mit niedrigeren Kosten verbundenen Arbeitsniveaus möglich ist. Es ist für den Auftraggeber jedoch möglich, seinen Kapitalwert zu erhöhen, sofern die Vorgänge und Ereignisse des hohen Arbeitsniveaus zu früheren 
Zeitpunkten durchgeführt werden und somit ein früheres Projektende bzw. das frühestmögliche Projektende zum Zeitpunkt $t=42$ erreicht wird. Ziel der folgenden dritten Stufe ist es daher, durch zusätzliche Zahlungen die zu einer kürzeren Projektdauer führenden Unterschreitungen der geplanten Projektdauer zu belohnen, gleichzeitig jedoch auch Überschreitungen der geplanten Projektdauer durch eine Reduzierung des Honorars zu bestrafen.

\section{Stufe 3}

Der Auftraggeber kann auf der dritten Stufe prüfen, ob dieses Ziel durch die Einbindung von Anreizen erreicht werden kann. Durch die Modellierung unterschiedlicher Anreizfunktionen, welche sowohl ein gewünschtes Verhalten belohnen als auch ein unerwünschtes Verhalten bestrafen, kann das erwartete Verhalten des Auftragnehmers beeinflusst werden. Bei der zuvor aufgezeigten Problemstellung ist der Auftraggeber tendenziell an einem frühen Projektende interessiert. Dies ist insbesondere durch eine Projektdurchführung in Form des hohen Arbeitsniveaus möglich. Es wird daher eine Anreizfunktion entwickelt, welche bei einer Unterschreitung des frühestmöglichen Zeitpunktes des letzten Ereignisses des geplanten Arbeitsniveaus $(t=52)$ zu einer zusätzlichen Zahlung an den Auftragnehmer zum Zeitpunkt des tatsächlichen Projektendes führt und eine Überschreitung bestraft. ${ }^{28}$ Die Anreizfunktion wird in das auf der zweiten Stufe vorgestellte Modell für jedes Arbeitsniveau eingefügt und hat folgende Gestalt:

$$
\operatorname{ANRD}_{\mathrm{ta}}=\operatorname{ANTD} \cdot\left(\mathrm{E}_{\mathrm{Ia}_{\mathrm{plan}}}-\mathrm{t}\right) \cdot \mathrm{x}_{\mathrm{Ita}} \quad \forall \mathrm{t}
$$

Die Höhe der zusätzlich zu der Honorar- sowie der letzten Kostenerstattungszahlung mit dem Zeitpunkt des letzten Ereignisses I bei Arbeitsniveau a verbundenen Zahlung $\mathrm{ANRD}_{\mathrm{ta}}$ berechnet sich durch die Multiplikation des Faktors ANTD mit der Differenz aus dem frühestmöglichen Projektende des geplanten Arbeitsniveaus und dem tatsächlichen Projektende. ANTD gibt an, in welcher Höhe das Honorar bei einer Un-

28 Die Einbindung einer Abweichung von den geplanten Kosten in die Anreizfunktion und somit die Bestrafung bzw. Belohnung von Kostenüber- bzw. -unterschreitungen ist besonders bei Kostenerstattungsvereinbarungen von großer Bedeutung, um die Leistungserstellung sowie die hiermit verbundenen Kosten zu beeinflussen. Auch das Setzen einer Höchstgrenze maximal erstattungsfähiger Kosten ist im Rahmen des dritten Kapitels diskutiert worden. Für dieses Anwendungsbeispiel besitzt der Auftraggeber jedoch eine Präferenz für das mit høheren Kosten verbundene Arbeitsniveau, so dass im Folgenden das durch Hidden Information verursachte Problem einer fehlenden Kontrollmöglichkeit der Kosten durch den Auftraggeber ausgeklammert wird und Kostenanreize nicht Bestandteil der Anreizfunktion sind. Für eine Kombination aus Zeit- und Kostenanreizen vgl. beispielsweise Arditi/Yasamis (1998), S. 362. 
ter- bzw. Überschreitung der Projektdauer um eine Woche vermehrt oder vermindert wird. Tabelle 5.4 zeigt die durch die Lösung der Modelle ermittelten Ergebnisse für einen bei diesen Berechnungen unterstellten Faktor ANTD $=1.000 \mathrm{GE} /$ Woche:

\begin{tabular}{|lc|c|c|c|}
\hline \multirow{2}{*}{ Stufe 3 } & \multicolumn{3}{c|}{ Arbeitsniveau } \\
\cline { 2 - 5 } & niedrig & geplant & hoch \\
\hline \multirow{2}{*}{ 1. Zahlung } & Betrag & 12.000 & 15.000 & 16.500 \\
& Zeitpunkt & 12 & 10 & 7 \\
\hline \multirow{2}{*}{ 2. Zahlung } & Betrag & 565.600 & 707.000 & 777.700 \\
& Zeitpunkt & 37 & 31 & 25 \\
\hline 3. Zahlung & Betrag & 248.284 & 313.884 & 351.684 \\
(inkl. Honorar) & Zeitpunkt & 62 & 52 & $\mathbf{4 2}$ \\
\hline Honorar & & 25.884 & 35.884 & 45.884 \\
\hline Erlöse Auftraggeber & 600.000 & 1.000 .000 & 1.400 .000 \\
\hline Kapitalwert Auftraggeber & -226.296 & -59.953 & $\mathbf{2 0 9 . 5 4 0}$ \\
\hline Kapitalwert Auftragnehmer & -6.921 & 793 & $\mathbf{1 3 . 4 8 2}$ \\
\hline
\end{tabular}

Tabelle 5.12: Ergebnisse antizipierendes Payment Scheduling bei Kostenerstattung für Stufe 3

Es lässt sich erkennen, dass der Auftragnehmer durch den Einsatz des Anreizsystems motiviert wird, das Projekt bei jedem Arbeitsniveau zu dem in Tabelle 5.9 dargestellten frühestmöglichen Zeitpunkt zu beenden. Im Hinblick auf das von beiden Partnern bevorzugte hohe Arbeitsniveau lassen sich zwei Auswirkungen beobachten:

- Der Kapitalwert des Auftraggebers erhöht sich im Vergleich zu dem auf Stufe 2 ohne Anreize ermittelten Kapitalwert. Dies ist mit dem frühzeitigen Projektende und der hierdurch frühen Möglichkeit der Projektnutzung verbunden. Im Gegenzug wird der Kapitalwert durch die zu den früheren Zeitpunkten der Ereignisse 2, 11 und 13 stattfindenden Zahlungen gemindert. Die Wirkung der zeitlichen Verschiebung der Zahlungen auf frühere Termine wird jedoch durch das frühere Projektende kompensiert und trägt zu einer Erhöhung des Kapitalwertes bei.

- Auch der Kapitalwert des Auftragnehmers erhöht sich gegenüber dem auf Stufe 2 ermittelten maximalen Kapitalwert. Durch den bei Realisierung des hohen Arbeitsniveaus um 10 Wochen vor dem geplanten Ende möglichen Projektabschluss erhält der Auftragnehmer zum Zeitpunkt des Projektendes zusätzlich zu seinem Honorar eine Zahlung in Höhe von 10.000 GE. Gleichzeitig erfolgen die Kostenerstattungszahlungen des Auftraggebers zu früheren Zeitpunkten, was einen zusätzlichen positiven Effekt auf den Kapitalwert des Auftragnehmers hat. Diese positiven Wirkungen auf den Kapitalwert übersteigen die negativen Effekte durch die zu früheren Zeitpunkten entstehenden Vorgangskosten. 
Die Einbindung dieser vorgestellten Anreizfunktion führt somit für beide Projektpartner zu einer Erhöhung des Projektkapitalwertes. Die Höhe des Faktors ANTD ist unabhängig von Optimalitätsüberlegungen auf der dritten Stufe festgelegt worden. Daher ist es die Zielsetzung der folgenden vierten Stufe, den Faktor zu ermitteln, der für den Auftraggeber zu möglichst niedrigen Anreizzahlungen und gleichzeitig zu dem gewünschten Projektende führt.

\section{Stufe 4}

Die Entscheidung, in welcher Höhe eine Abweichung von dem geplanten Projektende bestraft oder belohnt wird, trifft der Auftraggeber als Teil des Vertragsdesigns. Bei der Festlegung des für die hier vorgestellte Anreizfunktion und zu einer gewünschten Verkürzung der Projektdauer führenden notwendigen Faktors ANTD kann das Modell der vierten Stufe unterstützen. Aufgrund des auf der dritten Stufe verwendeten und zu einer maximalen Verkürzung der Projektdauer führenden Faktors ANTD $=1.000$ GE/Woche muss für den auf dieser Stufe zu ermittelnden Faktor gelten, dass dessen Höhe unterhalb von $1.000 \mathrm{GE} /$ Woche liegt. Die Lösung des vorgestellten Modells der dritten Stufe für unterschiedliche Faktoren ANTD führt zu den in der folgenden Abbildung 5.15 dargestellten Kapitalwerten des Auftraggebers:

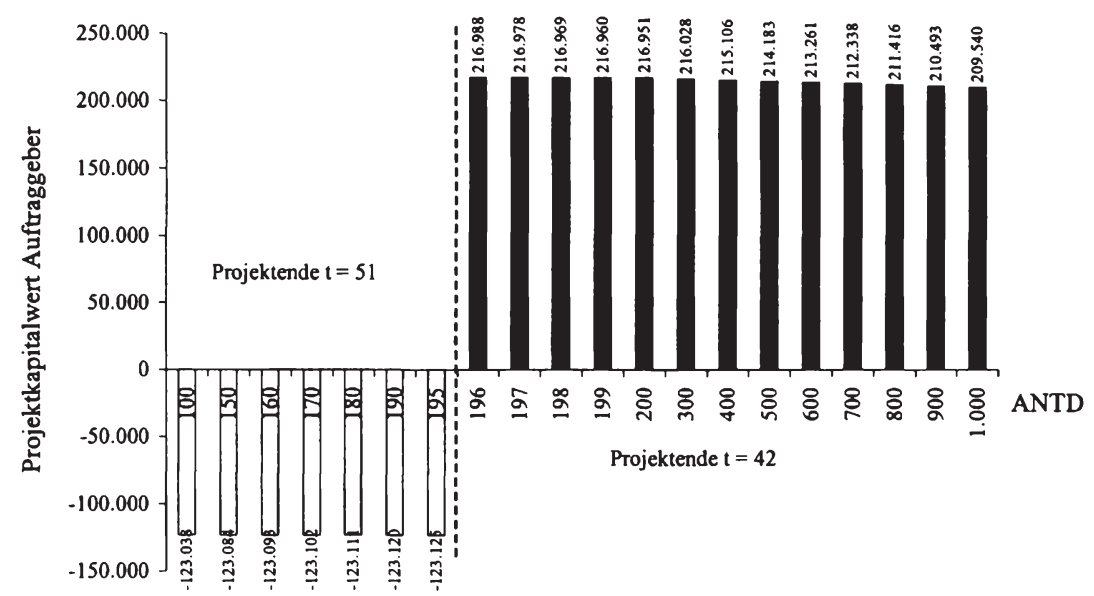

Abbildung 5.15: Bestimmung des optimalen Faktors ANTD bei Kostenerstattung auf Stufe 4

Es ist zu erkennen, dass der Faktor ANTD $=196$ GE/Woche zu einem sprunghaften Anstieg des Kapitalwertes des Auftraggebers führt. Eine Analyse der Projektpläne bei 
unterschiedlichen Faktoren zeigt, dass der Auftragnehmer im Rahmen der Optimierung bei Faktoren ANTD $\leq 195$ GE/Woche ein optimales Projektende zum Zeitpunkt $t=51$ ermittelt, was zu einem aus seiner Sicht maximalen Kapitalwert führt, aufgrund des späten Projektendes jedoch für den Auftraggeber nur Erlöse in Höhe von 1.040.000 GE resultieren. Bei Anreizfaktoren ANTD>195 GE/Woche ist die Anreizwirkung so stark, dass die Vorgänge so früh terminiert werden, dass ein Projektende zum Zeitpunkt $t=42$ angestrebt wird und somit Erlöse in Höhe von 1.400.000 GE für den Auftraggeber möglich sind. Die Abbildung 5.15 zeigt ebenfalls, dass für Faktoren ANTD > 195 GE/Woche mit zunehmender Höhe des Anreizfaktors der Kapitalwert des Auftraggebers in geringem Ausmaß sinkt. Dies resultiert aus der mit zunehmendem Faktor steigenden Höhe der durch das frühe Projektende notwendigen Bonuszahlungen an den Auftragnehmer. Eine zusätzliche Verkürzung der Projektdauer ist mit steigenden Bonuszahlungen nicht möglich, da das frühestmögliche Projektende bereits realisiert wird. Das Ergebnis der Untersuchungen dieser vierten Stufe zeigt somit, dass der Faktor ANTD $=196$ GE/Woche aus der Sicht des Auftraggebers zu einem maximalen Kapitalwert sowie einem frühestmöglichen Projektende führt.

Abbildung 5.16 stellt die Entwicklung des Kapitalwertes des Auftragnehmers für die untersuchten Faktoren dar:

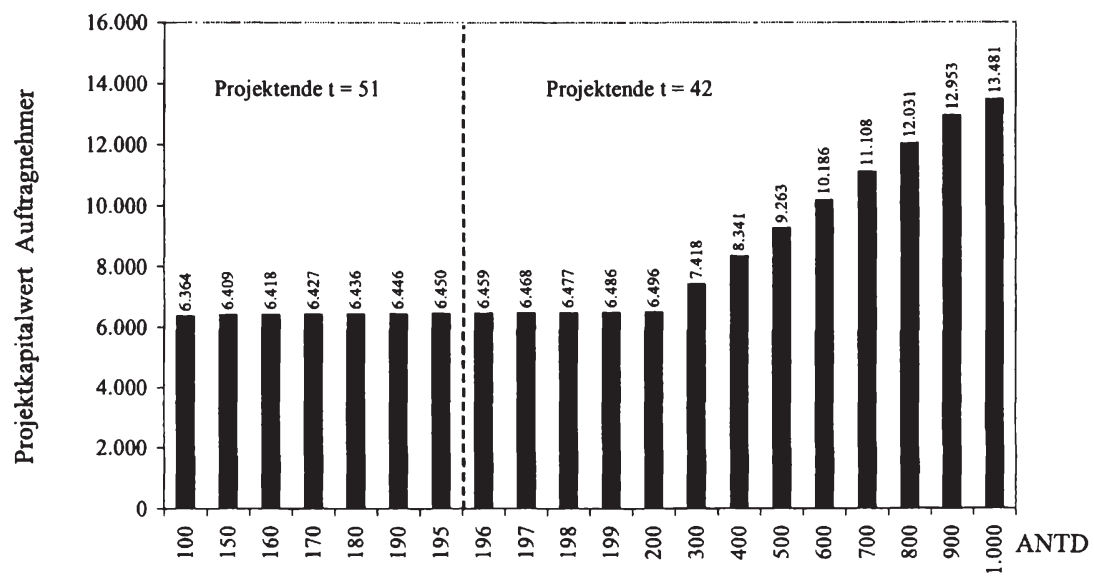

Abbildung 5.16: Kapitalwerte des Auftragnehmers bei unterschiedlichen Faktoren ANTD bei einer Kostenerstattungsvereinbarung 
Der Kapitalwert des Auftragnehmers steigt mit zunehmendem Faktor ANTD . Ein höherer Faktor ist somit mit einer höheren Anreizwirkung verbunden. Der Auftraggeber, der durch den Einsatz dieser Modelle das Verhalten des Auftragnehmers bei seiner Planung antizipiert, kann hier lediglich Aussagen über das vermutete Verhalten treffen, da mit den untersuchten Vertragsvereinbarungen keine zwanghaften Regeln verbunden sind. Aus diesem Grund muss der Auftraggeber entscheiden, welche Anreizintensität er durch die Wahl der Höhe des über ANTD=196 GE/Woche hinausgehenden Anreizfaktors wählt. Aufgrund der hohen Kapitalwertdifferenz zwischen einem Projektende zum Zeitpunkt $\mathrm{t}=51$ und dem präferierten Projektende zum Zeitpunkt $t=42$ kann dem Auftraggeber empfohlen werden, durch die Wahl eines höheren Anreizfaktors ein Signal für den Auftragnehmer zu setzen, die Leistungen entsprechend dem hohen Arbeitsniveau auszuführen und somit eine Bonuszahlung durch die Realisierung eines frühen Projektendes zu erhalten, welche seinen Kapitalwert erhöht.

\subsubsection{Antizipierendes Payment Scheduling bei Festpreisverträgen}

Analog zu der im vorherigen Kapitel im Rahmen der Kostenerstattungsvereinbarungen gewählten Struktur werden zunächst die Modifikationen vorgestellt, welche für die die unterschiedlichen Arbeitsniveaus antizipierenden Payment Scheduling Modelle für Festpreisvereinbarungen notwendig sind. Anschließend erfolgt auch hier die konkrete Umsetzung und Lösung dieser Modelle anhand der Anwendung.

Wie in Kapitel 5.2.1 gezeigt, sind unterschiedliche Zahlungsvereinbarungen bei Festpreisverträgen möglich. ${ }^{29}$ Gemeinsames Merkmal aller Vereinbarungen ist die Bestimmung des Festpreises und damit die von den Vorgangskosten unabhängige Höhe der Teilzahlungen sowie deren Zahlungszeitpunkte. Bei der folgenden Analyse des hier vorgestellten Konzeptes wird der Fall konstanter Zahlungsintervalle und konstanter Zahlungen betrachtet. ${ }^{30}$ Eine Übertragung der Modellmodifikationen auf andere Zahlungsvereinbarungen ist möglich und aus der Sicht des Auftraggebers erforderlich, um die Konsequenzen unterschiedlicher Vertragsdesigns sowohl auf den Projekterfolg als auch auf das vermutete Verhalten des Auftragnehmers beurteilen zu können.

29 Vgl. Kapitel 5.2.1, Abbildung 5.5: Zahlungsvereinbarungen für Festpreisverträge.

30 Dies ist der im ersten Quadranten der Abbildung 5.5 dargestellte Fall einer Zahlungsvereinbarung. 


\section{Stufe 1}

Das folgende mathematische Modell bildet zunächst das Entscheidungsproblem des Auftraggebers der ersten Stufe hinsichtlich der Wahl einer Festpreisvereinbarung für das geplante Arbeitsniveau ab. Aufgrund des hohen Anteils der durchgeführten Modifikationen wird auch das für Festpreisverträge entwickelte Modell an dieser Stelle vollständig aufgeführt.

\section{Indizes und Indexmengen:}

$\mathrm{a}=1, \ldots, \mathrm{A} \quad$ : Arbeitsniveaus des Auftragnehmers

$a_{\mathrm{plan}} \quad:$ Geplantes Arbeitsniveau

$\mathrm{i}, \mathrm{j}=1, \ldots, \mathrm{I} \quad$ : Ereignisse

$\mathrm{k}=1, \ldots, \mathrm{ANZ}$ : Anzahl der Teilzahlungen

$\mathrm{t}, \mathrm{u}=0, \ldots, \mathrm{T} \quad$ : Ereigniszeitpunkte

\section{Daten:}

ANZ : Anzahl der durch den Auftraggeber zu leistenden Zahlungen

$\mathrm{D}_{\mathrm{ij} a} \quad$ : Dauer des Vorgangs zwischen Ereignissen $\mathrm{i}$ und $\mathrm{j}$ bei Arbeitsniveau a

DISKZ $^{\text {AG/AN }}$ : Diskontierungszinssatz des Auftraggebers/Auftragnehmers ${ }^{31}$

DISKF $_{\mathrm{t}}^{\mathrm{AG} / \mathrm{AN}}$ : Diskontierungsfaktor des Auftraggebers/Auftragnehmers ${ }^{31}$

$\mathrm{E}_{\mathrm{ia}} \quad$ : Frühestmöglicher Zeitpunkt des Ereignisses i bei Arbeitsniveau a

ERL $_{\mathrm{t}} \quad$ : Erlöse des Auftraggebers bei einem Projektende zum Zeitpunkt $t$

INT : Dauer der Zahlungsintervalle

$\mathrm{INT}_{\mathrm{k}}^{\mathrm{UG}} \quad$ : Untere Grenze der Zahlungsintervalle

$\mathrm{INT}_{\mathrm{k}}^{\mathrm{OG}} \quad$ : Obere Grenze der Zahlungsintervalle

$\mathrm{K}_{\mathrm{i} a}^{\mathrm{AN}} \quad$ : Kosten bei Ereignis i und Arbeitsniveau a aus der Sicht des AN

$\widehat{\mathrm{M}} \quad$ : Große Zahl

$\mathrm{TB}^{\mathrm{AN}} \quad$ : Teilnahmebedingung des Auftragnehmers

$\mathrm{V} \quad$ : Menge der einem Vorgang unmittelbar vorangehenden und unmittelbar nachfolgenden Ereignisse (i,j)

31 Für eine ubersichtlichere Darstellung werden die Daten zusammengefasst, welche sich ausschließlich durch den Index fur den Auftraggeber bzw. den Auftragnehmer (AG/AN) unterscheiden. 


\section{Variablen:}

$\mathrm{x}_{\mathrm{ita}}=\left\{\begin{array}{l}1, \text { wenn Ereignis i zum Zeitpunkt } \mathrm{t} \text { bei Arbeitsniveau a stattfindet } \\ 0, \text { sonst }\end{array}\right.$

$y_{t a}=\left\{\begin{array}{l}1, \text { wenn eine Zahlung zum Zeitpunkt } t \text { bei Arbeitsniveau a stattfindet } \\ 0, \text { sonst }\end{array}\right.$

festp : Höhe des Festpreises

$\mathrm{z}_{\mathrm{ta}} \quad$ : Höhe der Zahlung zum Zeitpunkt $\mathrm{t}$ bei Arbeitsniveau a

Das Payment Scheduling Problem der ersten Stufe kann als gemischt-ganzzahliges lineares Programmierungsmodell für das geplante Arbeitsniveau formuliert werden:

$\max \sum_{t=0}^{T} \operatorname{DISKF}_{t}^{A G} \cdot \mathrm{ERL}_{t} \cdot \mathbf{x}_{\mathrm{Ita}_{\mathrm{plen}}}-\sum_{\mathrm{t}=0}^{\mathrm{T}} \operatorname{DISKF}_{\mathrm{t}}^{\mathrm{AG}} \cdot \mathrm{z}_{\mathrm{ta}_{\mathrm{plan}}}$

so dass

$\sum_{i=0}^{\mathrm{T}} \mathbf{x}_{\mathrm{ita} \text { plen }}=$

$\forall \mathrm{i}$

$\sum_{\mathrm{t}=0}^{\mathrm{T}} \mathrm{t} \cdot \mathrm{x}_{\mathrm{jta} \mathrm{plan}_{\mathrm{p}}}-\sum_{\mathrm{t}=0}^{\mathrm{T}} \mathrm{t} \cdot \mathrm{x}_{\mathrm{ita}_{\mathrm{plan}}} \geq \mathrm{D}_{\mathrm{ija}_{\mathrm{plan}}} \quad \forall(\mathrm{i}, \mathrm{j}) \in \mathrm{V}$

$\mathrm{y}_{\mathrm{ta}_{\mathrm{p} \operatorname{lon}}}=1$

$\mathrm{t}=\mathrm{E}_{\mathrm{Laplem}_{\mathrm{pla}}}$

$\sum_{\mathrm{i}=0}^{\mathrm{T}} \mathrm{z}_{\mathrm{ta}_{\mathrm{plam}}}=$ festp

$\sum_{t=0}^{\mathrm{T}} \operatorname{DISKF}_{t}^{\mathrm{AN}} \cdot \mathrm{z}_{\mathrm{ta}_{\mathrm{plan}}}-\sum_{\mathrm{t}=0}^{\mathrm{T}} \sum_{\mathrm{i}=1}^{\mathrm{I}} \mathrm{DISKF}_{t}^{\mathrm{AN}} \cdot \mathrm{K}_{\mathrm{ia}_{\mathrm{plan}}}^{\mathrm{AN}} \cdot \mathbf{x}_{\mathrm{ita}_{\mathrm{plam}}} \geq \mathrm{TB}^{\mathrm{AN}}$

$\mathrm{z}_{\mathrm{ta}_{\mathrm{plen}}} \geq \frac{\text { festp }}{\mathrm{ANZ}}-\widehat{\mathrm{M}} \cdot\left(1-\mathrm{y}_{\mathrm{ta}_{\mathrm{plan}}}\right)$

$\forall \mathrm{t}$

$\sum_{t=\mathbb{N} T_{k}^{U G}}^{\mathbb{N N T}} y_{t_{\text {plan }}}^{\text {OG }}=1$

$\forall \mathbf{k}$

$\sum_{i=1}^{1} x_{\text {ita plen }_{\text {plas }}} \geq y_{\text {ta }_{\text {ples }}}$

$\forall \mathrm{t}$

festp $\geq 0$

$\mathbf{x}_{\text {ita }_{\text {plen }}} \in\{0,1\}$

$\mathrm{y}_{\mathrm{ta}_{\mathrm{plam}}} \in\{0,1\}$

$\mathrm{z}_{\mathrm{ta}_{\mathrm{ples}}} \geq 0$

$\forall \mathrm{t}$ 
Die Zielfunktion (5.35) maximiert den Kapitalwert des Auftraggebers unter der Annahme der Daten des geplanten Arbeitsniveaus. Sie wird daher gegenüber der in Kapitel 4.3.3 formulierten Zielfunktion des Payment Scheduling Modells um den Index für das geplante Arbeitsniveau erweitert. Obwohl dieses Modell ausschließlich das geplante Arbeitsniveau berücksichtigt, wird diese allgemeine Formulierung gewählt, um das Modell für auf die durchzuführenden Analysen auf den folgenden Stufen auch auf alternative Arbeitsniveaus übertragen zu können. Die Restriktionen (5.36) und (5.37) entsprechen den aus Kapitel 4 bekannten Restriktionen und legen fest, dass jedes Ereignis zu genau einem Zeitpunkt stattfinden muss, wobei die durch die Vorgänge und Vorgangsdauern bestimmten Anordnungsbeziehungen unmittelbar aufeinander folgender Ereignisse eingehalten werden. Der Auftraggeber präferiert durch die Annahme der mit dem Projektende verbundenen Erlöse einen Projektabschluss zu dem frühestmöglichen Zeitpunkt des letzten Ereignisses. Die Gleichung (5.38) legt daher den Zeitpunkt der Abschlusszahlung und somit das Projektende für das geplante Arbeitsniveau mit dem frühestmöglichen Zeitpunkt des letzten Ereignisses fest. Bedingung (5.39) gewährleistet, dass die Summe der Teilzahlungen dem Festpreis entspricht. Die Teilnahmebedingung des Auftragnehmers in Form der Restriktion (5.40) stellt sicher, dass die auf dieser Stufe ermittelte Zahlungsvereinbarung das Anspruchsniveau des Auftragnehmers hinsichtlich dessen Zielvorstellungen erfüllt. Die Höhe der Teilzahlungen als Quotient aus Festpreis und Anzahl der Zahlungen wird durch die Bedingung (5.41) bestimmt, wobei diese Bedingung nur bindend ist, sofern die Binärvariable $y_{t a}$ den Wert 1 annimmt und $\mathrm{zu}$ dem Zeitpunkt $\mathrm{t}$ eine Zahlung erfolgt. Durch die Vorgabe, dass die Zahlungen bei dieser Festpreisvereinbarung innerhalb konstanter Zeitintervalle erfolgen müssen, wird durch die Restriktion (5.42) die kürzeste Projektdauer für das geplante Arbeitsniveau in ANZ Intervalle unterteilt und gefordert, dass genau eine Teilzahlung innerhalb dieser Zeitintervalle erfolgt. Die Restriktion (5.43) legt fest, dass Zahlungen ausschließlich zu Ereigniszeitpunkten erfolgen können. Die Restriktionen (5.44) bis (5.47) legen den Definitionsbereich der Variablen des Modells fest.

Durch die Lösung dieses Optimierungsproblems lassen sich die für das geplante Arbeitsniveau und unter Beachtung der Teilnahmebedingung zu einem maximalen Kapitalwert des Auftraggebers führenden Zahlungen und Zahlungszeitpunkte sowie der Festpreis ermitteln. Die Ergebnisse sind in Tabelle 5.13 für den Fall von $\mathrm{ANZ}=3$ Teilzahlungen dargestellt: 


\begin{tabular}{|c|c|c|c|}
\hline \multirow{2}{*}{ Stufe 1} & \multicolumn{3}{|c|}{ Arbeitsniveau } \\
\hline & niedrig & geplant & hoch \\
\hline $\begin{array}{lc}\text { 1. Zahlung } & \text { Betrag } \\
\text { Zeitpunkt }\end{array}$ & & $\begin{array}{c}339.415 \\
10\end{array}$ & \\
\hline $\begin{array}{c}\text { Betrag } \\
\text { Zeitpunkt }\end{array}$ & & $\begin{array}{c}339.415 \\
25\end{array}$ & \\
\hline $\begin{array}{cc}\text { 3. Zahlung } & \text { Betrag } \\
\text { Zeitpunkt }\end{array}$ & & $\begin{array}{c}339.415 \\
52 \\
\end{array}$ & \\
\hline Festpreis & & 1.018 .245 & \\
\hline Erlöse Auftraggeber & & 1.000 .000 & \\
\hline Kapitalwert Auftraggeber & & -58.675 & \\
\hline Kapitalwert Auftragnehmer & & $\mathbf{0}$ & \\
\hline
\end{tabular}

Tabelle 5.13: Ergebnisse für das geplante Arbeitsniveau bei Festpreisvereinbarung

Das in der Tabelle dargestellte Ergebnis der ersten Stufe zeigt, dass für die Erfüllung der Kooperationsbedingung ein Festpreis in Höhe von 1.018.245 GE notwendig ist. Die Höhe der Differenz aus Festpreis und Gesamtkosten kann als Honorar definiert werden und beträgt für dieses Beispiel 18.245 GE. Somit ist die Gesamtzahlung des Auftraggebers an den Auftragnehmer bei der Festpreisvereinbarung gegenüber der Kostenerstattungsvereinbarung für das geplante Arbeitsniveau um 17.639 GE niedriger. Auch hier beträgt der maximale Kapitalwert des Auftraggebers durch die Teilnahmebedingung $-58.675 \mathrm{GE}$.

Für das Vertragsdesign einer Kostenerstattungsvereinbarung sind durch die auf der ersten Stufe ermittelten Zahlungszeitpunkte die Ereignisse festgelegt worden, zu denen eine Zahlung erfolgen soll. Obwohl zunächst im Rahmen der Modellierung weiterhin angenommen wird, dass zu einem Zahlungszeitpunkt auch mindestens ein Ereignis stattfinden muss, legt die Zahlungsvereinbarung bei einem Festpreisvertrag die Zahlungszeitpunkte fest. Die Aufteilung des Festpreises auf die innerhalb der Zahlungsintervalle erfolgenden drei gleich hohen Zahlungen führt zu den Zahlungszeitpunkten $\mathrm{t}=10, \mathrm{t}=25$ sowie durch die Abschlusszahlung zu $\mathrm{t}=52$. Der Auftragnehmer erhält bei dem hier vorgestellten Ergebnis die erste Teilzahlung in Höhe eines Drittels der Gesamtzahlung zum Zeitpunkt $\mathrm{t}=10$, zu dem die Ereignisse 1 und 2 abgeschlossen sind. Aus der Sicht des Auftragnehmers sind bis zu diesem Zeitpunkt Kosten in Höhe von 247.000 GE entstanden, so dass mit der ersten Festpreiszahlung eine um 92.415 GE höhere, über die Kosten hinausgehende Zahlung erfolgt. Dies ist abhängig von der Kostenstruktur des Projektes und kann durch die besondere Charakteristik eines von den tatsächlich entstandenen Kosten unabhängigen Festpreisvertrags erklärt werden. 


\section{Stufe 2}

Unter der Annahme der für das geplante Arbeitsniveau für den Auftraggeber optimalen Zahlungsvereinbarung erfolgt auf der zweiten Stufe die Berechnung der Kapitalwerte für die alternativen Arbeitsniveaus aus der Sicht des Auftragnehmers. Hierfür wird das vorgestellte Modell wie folgt modifiziert:

Die das geplante Arbeitsniveau des Auftragnehmers bezeichnenden Indizes in der Zielfunktion sowie in allen der im Folgenden modifizierten Modell enthaltenen Restriktionen werden durch die Bezeichnung für das jeweils betrachtete Arbeitsniveau ersetzt. Der Auftraggeber versetzt sich in die Entscheidungssituation des Auftragnehmers und muss somit die Zielfunktion den Zielvorstellungen des Auftragnehmers anpassen:

$\max \sum_{t=0}^{T} \operatorname{DISKF}_{t}^{A N} \cdot z_{t a}-\sum_{t=0}^{T} \sum_{i=1}^{I} \operatorname{DISKF}_{t}^{A N} \cdot K_{i a}^{A N} \cdot x_{i t a}$

Der Auftragnehmer maximiert den Projektkapitalwert als Differenz der diskontierten identischen Festpreiszahlungen $z_{t a}$ und der mit den Vorgängen verbundenen diskontierten Kosten.

Die bei der Planung des Auftraggebers berücksichtigte Teilnahmebedingung (5.40) wird für dieses modifizierte Modell durch die den Kapitalwert des Auftragnehmers maximierende Zielfunktion ersetzt. Die Festpreisvereinbarung legt die Zahlungszeitpunkte für alle Arbeitsniveaus fest. Daher wird die Restriktion (5.42), welche gewährleistet, dass die Zahlungen innerhalb der identischen Intervalle stattfinden, durch die folgende Gleichung ersetzt, die die Zahlungszeitpunkte, zu denen die Teilzahlungen erfolgen, als Parameter in das Modell einbindet:

$\mathrm{y}_{\mathrm{ta}}=1 \quad \forall \mathrm{t} \in\{10,25\}$

Der Zeitpunkt der zu dem Projektende erfolgenden Abschlusszahlung wird nicht vorgegeben, sondern im Rahmen der Optimierung bestimmt, so dass für diese Anwendung die Zahlungen ausschließlich an die Zeitpunkte $t=10$ und $t=25$ gebunden werden. Auch die Annahme des Projektendes zu dem frühestmöglichen Zeitpunkt des letzten Ereignisses, welche durch die Bedingung (5.38) formuliert worden ist, entfällt für das modifizierte Modell, da diese Bedingung nicht Gegenstand der Vertragsvereinbarung ist. Diese Bedingung wird durch die Restriktion (5.50) ersetzt, welche das spä- 
testerlaubte Projektende aufgrund der in Kapitel 4.2.1 diskutierten Problematik auf den Zeitpunkt DMax $=70$ festlegt.

$\mathrm{t} \cdot \mathrm{x}_{\mathrm{Ita}} \leq \mathrm{DMax}$

Der Festpreis ist mit der Lösung der ersten Stufe bestimmt worden und beträgt 1.018.245 GE. Dieser Wert geht als Parameter FESTP in das Modell ein und ersetzt die Restriktion (5.44). Sämtliche hier nicht modifizierten bzw. ausgegliederten Restriktionen sind auch Bestandteil des Modells der zweiten Stufe. Die Lösung dieses das vermutete Verhalten des Auftragnehmers antizipierenden Modells führt für die drei betrachteten Arbeitsniveaus zu den folgenden Ergebnissen:

\begin{tabular}{|lc|c|c|c|}
\hline \multirow{2}{*}{ Stufe 2 } & \multicolumn{3}{c|}{ Arbeitsniveau } \\
\cline { 3 - 5 } & & niedrig & geplant & hoch \\
\hline \multirow{2}{*}{ 1. Zahlung } & Betrag & 339.415 & 339.415 & 339.415 \\
& Zeitpunkt & 10 & 10 & 10 \\
\hline \multirow{2}{*}{ 2. Zahlung } & Betrag & 339.415 & 339.415 & 339.415 \\
& Zeitpunkt & 25 & 25 & 25 \\
\hline \multirow{2}{*}{ 3. Zahlung } & Betrag & 339.415 & 339.415 & 339.415 \\
& Zeitpunkt & $\mathbf{7 0}$ & $\mathbf{7 0}$ & $\mathbf{7 0}$ \\
\hline Festpreis & & 1.018 .245 & 1.018 .245 & 1.018 .245 \\
\hline Erlöse Auftraggeber & 280.000 & 280.000 & 280.000 \\
\hline Kapitalwert Auftraggeber & -708.393 & -708.393 & -708.393 \\
\hline Kapitalwert Auftragnehmer & 195.786 & 20.553 & -64.468 \\
\hline
\end{tabular}

Tabelle 5.14: Ergebnisse antizipierendes Payment Scheduling bei Festpreisvereinbarung für Stufe 2

Die Ergebnisse der zweiten Stufe zeigen, dass die fehlende Vereinbarung der Übereinstimmung des Projektendes mit dem frühestmöglichen Zeitpunkt des letzten Ereignisses zur Folge hat, dass der Auftragnehmer bei einer Festpreisvereinbarung für jedes Arbeitsniveau als Projektende den Zeitpunkt $t=70$ wählt, welcher dem spätesterlaubten Zeitpunkt des Projektendes entspricht. Für das geplante Arbeitsniveau ist es somit für den Auftragnehmer möglich, einen Kapitalwert zu erzielen, der die auf der ersten Stufe berücksichtigte Kooperationsbedingung um 20.553 GE übersteigt. Durch die Festlegung des Festpreises auf der Grundlage der geplanten Kosten ist der Kapitalwert des Auftragnehmers für den Fall des mit höheren Kosten verbundenen höheren Arbeitseinsatzes negativ und für den Fall des mit niedrigeren Kosten verbundenen niedrigen Arbeitseinsatzes am größten, so dass der Auftraggeber vermuten kann, dass der Auftragnehmer auf das Vertragsangebot mit der Realisierung des niedrigen Arbeitsniveaus reagiert und somit die Differenz der Barwerte aus Festpreis und Kosten der Leistungserstellung maximiert. 
Durch den für alle Arbeitsniveaus durch den Auftragnehmer geplanten identischen späten Zeitpunkt des Projektabschlusses erwartet der Auftraggeber Erlöse in Höhe von $280.000 \mathrm{GE}$. Der Auftraggeber wird daher nicht bereit sein, diesen für das geplante Arbeitsniveau ermittelten Festpreis für eine mit einem niedrigeren Arbeitseinsatz erbrachte Leistung zu zahlen, welche zusätzlich zu einem späten Projektabschluss und damit zu niedrigen Erlösen führt. Vielmehr präferiert der Auftraggeber auch hier die Projektdurchführung auf der Grundlage des hohen Arbeitsniveaus, um das Projekt möglichst früh abzuschließen und von den hieraus resultierenden hohen Erlösen zu profitieren.

Es zeigt sich somit, dass für den Fall dieser Festpreisvereinbarung die Präferenzen für die Wahl des Arbeitsniveaus aus der Sicht des Auftraggebers, welcher tendenziell das hohe Arbeitsniveau bevorzugt, sowie aus der Sicht des Auftragnehmers, welcher bei einer Festpreisvereinbarung generell ein niedriges Arbeitsniveau realisieren wird, nicht übereinstimmen. Ziel der dritten Stufe ist es daher, den Auftragnehmer durch die Integration eines Anreizsystems in die Zahlungsvereinbarung zu motivieren, ein hohes Arbeitsniveau zu realisieren und das Projekt zu einem möglichst frühen Zeitpunkt abzuschließen.

\section{Stufe 3}

Der Auftraggeber kann das auf der zweiten Stufe modifizierte Payment Scheduling Modell nutzen, um die Konsequenzen unterschiedlicher Anreizsysteme auf das mögliche Verhalten des Auftragnehmers bei der Vertragsgestaltung zu berücksichtigen. Der auf der ersten Stufe ermittelte Festpreis und somit die Höhe der Teilzahlungen sowie deren Zahlungszeitpunkte gehen auch hier als Parameter in das Modell der dritten Stufe ein.

Der Auftraggeber, welcher den Auftragnehmer durch die Einbindung einer Anreizfunktion motivieren möchte, durch die Wahl des hohen Arbeitsniveaus ein frühes Projektende zu realisieren, kann mit Hilfe des erweiterten Modells den aus der Sicht des Auftragnehmers maximalen Kapitalwert für jedes Arbeitsniveau bestimmen und durch die Wahl des Parameters ANTD den Auftragnehmer zur Realisation des gewünschten Arbeitsniveaus motivieren. Während es aus der Sicht des Auftraggebers durch das Problem der Informationsasymmetrie nicht grundsätzlich möglich ist, die tatsächlich entstandenen Kosten oder die Qualität der erbrachten Leistungen zu kontrollieren und 
als Bemessungsgrundlage eines Anreizsystems zu nutzen, ist die Bestimmung des Projektendes mit dem Abschluss der Leistungen des Auftragnehmers leicht möglich.

Der Parameter ANTD gibt daher auch im Rahmen der Festpreisvereinbarung an, um welchen Betrag die Abschlusszahlung reduziert oder erhöht wird, sofern das tatsächliche Projektende von dem geplanten Projektende abweicht. Diese Definition zeigt, dass durch die Anreizfunktion nicht nur Bonuszahlungen bei einer Verkürzung der Projektdauer gezahlt, sondern auch Strafzahlungen bei einer Überschreitung des geplanten Projektendes festgesetzt werden.

Die Gesamtzahlung setzt sich bei dieser Anwendung somit aus ANZ-1=2 identischen und durch die in der ersten Stufe ermittelten Teilzahlungen und Zahlungszeitpunkte sowie einer mit dem letzten Ereignis verbundenen Abschlusszahlung zusammen. Die Abschlusszahlung ist durch die Anreizfunktion abhängig von dem durch die Wahl des jeweiligen Arbeitsniveaus realisierten Projektende. Als Beispiel für die Modellierung einer Anreizfunktion kann die bereits in Kapitel 5.5.3 vorgeschlagene Restriktion zur Bestimmung der Höhe der mit dem Zeitpunkt der letzten Zahlung verbundenen Anreizzahlung für jedes Arbeitsniveau in das Modell integriert werden:

$$
\mathrm{ANRD}_{\mathrm{ta}}=\mathrm{ANTD} \cdot\left(\mathrm{E}_{\mathrm{la}_{\mathrm{plan}}}-\mathrm{t}\right) \cdot \mathrm{x}_{\mathrm{Ita}} \quad \forall \mathrm{t}
$$

Die durch die Festpreisvereinbarung bestimmte letzte Teilzahlung wird somit um eine Bonuszahlung bei Unterschreitung des geplanten Projektendes anteilig erhöht bzw. bei einer Dauerüberschreitung anteilig gemindert. Dies führt dazu, dass die Restriktionen (5.39) und (5.41) für alle auf dieser dritten Stufe untersuchten Arbeitsniveaus entsprechend um die mit Gleichung (5.51) berechnete Anreizzahlung erweitert werden müssen:

$$
\begin{aligned}
& \sum_{\mathrm{t}=0}^{\mathrm{T}} \mathrm{z}_{\mathrm{ta}}=\text { FESTP }+\sum_{\mathrm{t}=0}^{\mathrm{T}} \text { ANRD }_{\mathrm{t} a} \\
& \mathrm{z}_{\mathrm{t} \mathrm{a}} \geq \frac{\text { FESTP }}{\mathrm{ANZ}}+\text { ANRD }_{\mathrm{ta}}-\widehat{\mathrm{M}} \cdot\left(1-\mathrm{y}_{\mathrm{ta}}\right) \quad \forall \mathrm{t}
\end{aligned}
$$

Im Rahmen der Untersuchung dieser Stufe wird der Faktor für den Anreiz der Einhaltung der geplanten Projektdauer von 52 Wochen mit ANTD $=25.000 \mathrm{GE} /$ Woche festgelegt. Endet das Projekt beispielsweise zwei Wochen nach dem geplanten Termin, vermindert sich die Abschlusszahlung um 50.000 GE. Die Ergebnisse der Lösung des Payment Scheduling Problems der dritten Stufe sind in Tabelle 5.15 dargestellt: 


\begin{tabular}{|lc|c|c|c|}
\hline \multirow{2}{*}{ Stufe 3 } & \multicolumn{3}{c|}{ Arbeitsniveau } \\
\cline { 2 - 5 } & Betrag & 339.415 & 339.415 & 339.415 \\
& niedrig & geplant & hoch \\
\hline \multirow{2}{*}{ 1. Zahlung } & 10 & 10 & 10 \\
\hline \multirow{2}{*}{ 2. Zahlung } & Betrag & 339.415 & 339.415 & 339.415 \\
& Zeitpunkt & 25 & 25 & 25 \\
\hline \multirow{2}{*}{ 3. Zahlung } & Betrag & 89.415 & 339.415 & 539.415 \\
& Zeitpunkt & 62 & 52 & 44 \\
\hline Gesamtzahlung Auftraggeber & 768.245 & 1.018 .245 & 1.218 .245 \\
\hline Erlöse Auftraggeber & 600.000 & 1.000 .000 & 1.320 .000 \\
\hline Kapitalwert Auftraggeber & -203.206 & -58.675 & $\mathbf{6 0 . 8 5 7}$ \\
\hline \multicolumn{2}{|c|}{ Kapitalwert Auftragnehmer } & -30.239 & 0 & $\mathbf{8 8 . 5 7 2}$ \\
\hline
\end{tabular}

Tabelle 5.15: Ergebnisse antizipierendes Payment Scheduling bei Festpreisvereinbarung für Stufe 3

Der Vergleich der Kapitalwerte des Auftragnehmers zeigt, dass dieser durch die Berücksichtigung der Anreizfunktion motiviert wird, das hohe Arbeitsniveau zu realisieren, um das Projekt zu einem frühen Termin abzuschließen. Beide Kooperationspartner können durch die Ausführung dieses mit dem hohen Arbeitsniveau bezeichneten Projektplans den höchsten Kapitalwert erzielen. Das Anreizsystem erfüllt somit die Funktion, den Auftragnehmer im Rahmen der Festpreisvereinbarung nicht für den mit den niedrigsten Kosten verbundenen, sondern für einen höheren Arbeitseinsatz zu motivieren.

Aus der Sicht des Auftragnehmers wird für das hohe Arbeitsniveau ein Projektende zu dem Zeitpunkt $t=44$ geplant, welcher nicht dem frühestmöglichen Zeitpunkt $t=42$ dieses Arbeitsniveaus entspricht. Die Vermutung, dass eine zu geringe Anreizwirkung durch die Vorgabe des Anreizfaktors in Höhe von ANTD = 25.000 GE/Woche besteht, das frühestmögliche Projektende zu realisieren, ist nicht zutreffend. Vielmehr kann das Projekt durch die Vorgabe der zwei Zahlungs- und somit Ereigniszeitpunkte $t=10$ und $\mathrm{t}=25$ unter Berücksichtigung der mit dem hohen Arbeitsniveau verbundenen Vorgangsdauern sowie der Projektstruktur frühestens zum Zeitpunkt $t=44$ beendet werden. Konkret führt die Vorgabe des Zahlungszeitpunktes $t=25$ durch die Bindung der Zahlung an mindestens einen Ereigniszeitpunkt dazu, dass das auf dem kritischen Pfad liegende Ereignis 9 nicht zu seinem mit dem hohen Arbeitsniveau möglichen frühesten Zeitpunkt $t=23$, sondern zwei Wochen später geplant wird, was zu der Verzögerung des Projektendes um zwei Wochen führt.

Wird die Bindung der Zahlungszeitpunkte an Ereigniszeitpunkte aufgehoben, bedeutet dies, dass die Planung der Ereigniszeiten durch den Auftragnehmer ausschließlich durch die Bindung der Abschlusszahlung an den Zeitpunkt des letzten Ereignisses, 
welcher dem Projektende entspricht, beeinflusst wird. Die Bedingung der Übereinstimmung der während der Projektdurchführung zu planenden Zahlungszeitpunkte mit mindestens einem Ereigniszeitpunkt kann durch die Nichtberücksichtigung der Restriktion (5.43) aufgehoben werden. Unter Beibehaltung der auf der ersten Stufe ermittelten Zahlungszeitpunkte sowie der zuvor dargestellten Anreizfunktion ergeben sich hieraus die folgenden in Tabelle 5.15 dargestellten Lösungen:

\begin{tabular}{|lc|c|c|c|}
\hline \multirow{2}{*}{ Stufe 3 } & \multicolumn{3}{c|}{ Arbeitsniveau } \\
\cline { 2 - 5 } & niedrig & geplant & hoch \\
\hline \multirow{2}{*}{ 1. Zahlung } & Betrag & 339.415 & 339.415 & 339.415 \\
& Zcitpunkt & 10 & 10 & 10 \\
\hline \multirow{2}{*}{ 2. Zahlung } & Betrag & 339.415 & 339.415 & 339.415 \\
& Zeitpunkt & 25 & 25 & 25 \\
\hline \multirow{2}{*}{ 3. Zahlung } & Betrag & 89.415 & 339.415 & 589.415 \\
& Zeitpunkt & 62 & 52 & $\mathbf{4 2}$ \\
\hline Gesamtzahlung Auftraggeber & 768.245 & 1.018 .245 & 1.268 .245 \\
\hline Erlöse Auftraggeber & 600.000 & 1.000 .000 & 1.400 .000 \\
\hline Kapitalwert Auftraggeber & -203.206 & -58.675 & $\mathbf{9 1 . 2 9 3}$ \\
\hline Kapitalwert Auftragnehmer & -29.625 & 0 & $\mathbf{1 3 2 . 6 9 2}$ \\
\hline
\end{tabular}

Tabelle 5.16: Ergebnisse antizipierendes Payment Scheduling bei Festpreisvereinbarung für Stufe 3 ohne Bindung von Zahlungs- und Ereigniszeitpunkten

Insbesondere das für das hohe Arbeitsniveau ermittelte Ergebnis zeigt, dass durch die durchgeführte Modifikation das frühestmögliche Projektende erreicht werden kann. Dies führt durch die für den Auftraggeber zusätzlichen und zu einem früheren Zeitpunkt möglichen Erlöse zu einer Erhöhung des Kapitalwertes um 44.120 GE. Der Kapitalwert des Auftragnehmers erhöht sich insbesondere durch die Anreizzahlung um $30.436 \mathrm{GE}$. Es ist somit für beide Kooperationspartner vorteilhaft, durch die Einbindung der Anreizfunktion in das Vertragsdesign das frühestmögliche Projektende als Ergebnis eines erhöhten Arbeitseinsatzes zu realisieren.

\section{Stufe 4}

Welche Anreizwirkungen der Auftraggeber durch die Einbindung des Anreizsystems in das Vertragsdesign hervorruft, hängt maßgeblich von der Gestaltung der Anreizfunktion ab. ${ }^{32}$ Die hier gewählte Anreizfunktion enthält den Parameter ANTD, welcher die Über- oder Unterschreitung der geplanten Projektdauer bestraft bzw. belohnt. Das Payment Scheduling Modell der dritten Stufe kann den Auftraggeber auf der vierten Stufe bei der Wahl dieses Faktors unterstützen. Während im Rahmen der Untersu-

32 Vgl. hierzu die Ausführungen in Kapitel 3.4.1. 
chungen einer Kostenerstattungsvereinbarung der für die gewünschte Anreizwirkung optimale Anreizfaktor dadurch charakterisiert worden ist, dass dieser zu einem mit dem hohen Arbeitsniveau verbundenen frühen Projektende führt, muss der Anreizfaktor den Auftragnehmer im Rahmen einer Festpreisvereinbarung erst für die Wahl des hohen Arbeitsniveaus motivieren. Darüber hinaus ist sicherzustellen, dass die Leistungserstellung bei der Wahl des hohen Arbeitsniveaus zu dem frühestmöglichen Projektende führt.

Der Auftragnehmer wird das hohe Arbeitsniveau nur dann realisieren, sofern hiermit für ihn ein Kapitalwert erzielbar ist, welcher höher als der mit alternativen Arbeitsniveaus mögliche Kapitalwert ist. Wählt der Auftragnehmer das geplante Arbeitsniveau, wird er durch die Anreizfunktion das Projektende zum Zeitpunkt $t=52$ planen und somit durch die auf der ersten Stufe berücksichtigte Kooperationsbedingung einen Kapitalwert in Höhe von $0 \mathrm{GE}$ erreichen. Daher zeigt die Abbildung 5.17 ausschließlich den Vergleich der Kapitalwerte des Auftragnehmers für unterschiedliche Anreizfaktoren ANTD sowohl bei Realisation des niedrigen als auch des hohen Arbeitsniveaus:

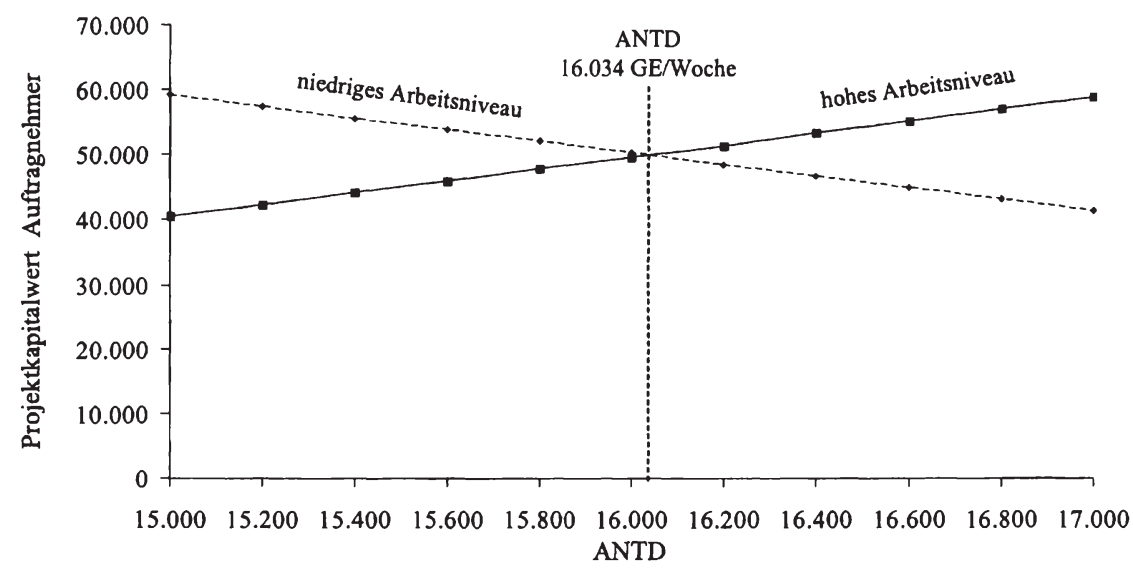

Abbildung 5.17: Bestimmung des optimalen Faktors ANTD bei Festpreisvereinbarung auf Stufe 4

Es lässt sich erkennen, dass der Auftragnehmer vermutlich das niedrige Arbeitsniveau wählen wird, wenn als Anreizfaktor ein Wert ausgewählt wird, welcher kleiner als 16.034 GE/Woche ist. Dieser Wert resultiert aus dem Vergleich der Kapitalwerte des Auftragnehmers als Ergebnis der Lösung des Modells der dritten Stufe für unterschiedliche Faktoren ANTD sowohl für das niedrige als auch für das hohe Arbeitsni- 
veau. Durch die aus dem niedrigen Arbeitseinsatz resultierenden niedrigen Kosten sowie dem für das geplante Niveau festgesetzten Festpreis ist es für den Auftragnehmer bei niedrigen Anreizfaktoren vorteilhaft, die mit der langsameren Leistungserstellung fälligen Strafzahlungen für das verspätete Projektende zum Zeitpunkt $t=62$ zu leisten.

Werden Anreizfaktoren ANTD $\geq 16.034 \mathrm{GE} /$ Woche gewählt, wird der Auftragnehmer vermutlich durch die Wahl des hohen Arbeitseinsatzes ein Projektende zu dem Zeitpunkt $\mathrm{t}=42$ realisieren und durch die hiermit verbundene Bonuszahlung die aus dem hohen Arbeitsniveau entstehenden höheren Kosten kompensieren.

Aus der Sicht des Auftraggebers ist daher zu empfehlen, bei der für das um die alternativen Arbeitsniveaus erweiterte Anwendungsbeispiel untersuchten Festpreisvereinbarung in Form von drei identischen und zu den festgelegten Zeitpunkten erfolgenden Zahlungen Anreizfaktoren festzulegen, welche größer als 16.034 GE/Woche sind und den Auftragnehmer motivieren, die Leistungen entsprechend einem höheren Arbeitsniveau auszuführen. Die folgende Abbildung zeigt die Kapitalwerte des Auftraggebers für unterschiedliche Anreizfaktoren auf. Hierbei wird unterstellt, dass sich der Auftragnehmer für das aus seiner Sicht kapitalwertmaximale, von der Höhe des Anreizfaktors abhängige Arbeitsniveau entscheidet.

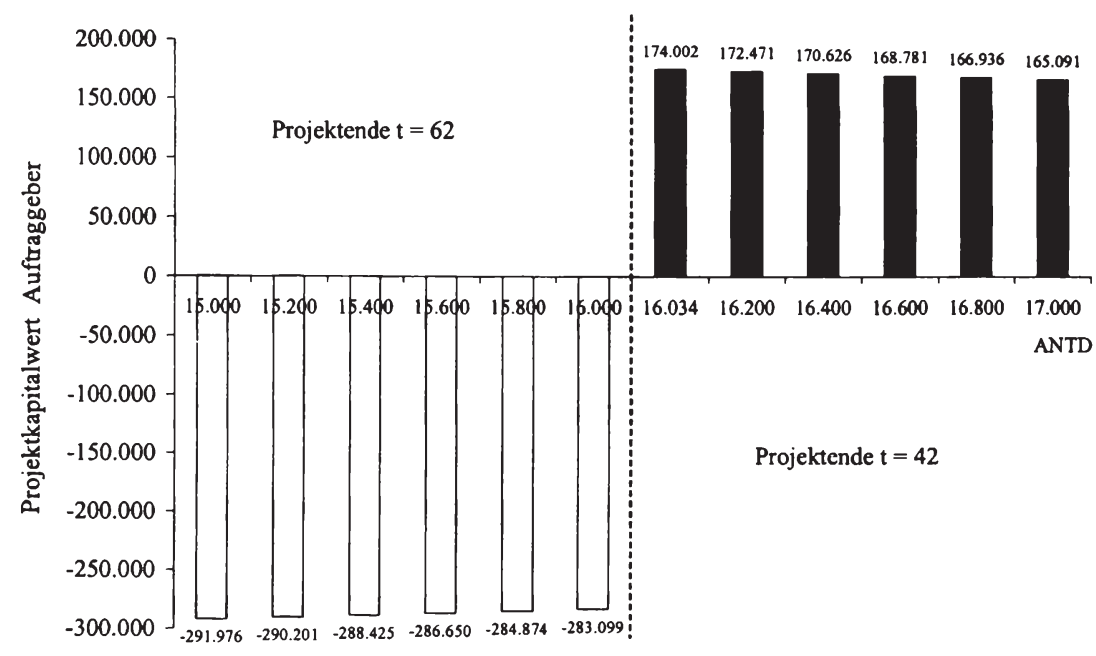

Abbildung 5.18: Kapitalwerte des Auftraggebers in Abhängigkeit von der Entscheidung des Auftragnehmers bei Festpreisvereinbarungen für Stufe 4 
Die in Abbildung 5.18 dargestellten Ergebnisse zeigen, dass die aus der Wahl des niedrigen Arbeitsniveaus resultierenden für den Auftraggeber möglichen Kapitalwerte negativ sind. Aufgrund der hohen Differenz der Kapitalwerte zwischen dem frühen und dem späten Projektende kann dem Auftraggeber empfohlen werden, einen Anreizfaktor zu wählen, welcher eine so hohe Signalwirkung auf den Auftragnehmer ausübt, dass dieser bereit ist, die Leistungen mit dem gewünschten hohen Arbeitseinsatz zu erstellen. Der Auftraggeber sollte daher bei seiner Entscheidung über die Ausgestaltung des Anreizsystems auch bei Festpreisverträgen insbesondere das durch die Kooperationsbeziehung geprägte Verhältnis sowie die eventuell durch wiederholte Kooperationen gewonnenen Erfahrungen hinsichtlich des Auftragnehmerverhaltens berücksichtigen.

\subsection{Ergebnisse der Planungsunterstützung für das Vertragsdesign}

Die zu Beginn dieses Kapitels vorgestellten Erweiterungen und Modifikationen des am Ende des vierten Kapitels aufgezeigten Payment Scheduling Grundmodells ermöglichen neben der Abbildung der in der Arbeit betrachteten Problemstellung der Projektplanung des Auftraggebers in Form eines quantitativen Modells zusätzlich die Möglichkeit der Einbindung der im dritten Kapitel dargestellten verschiedenen Arten von Zahlungsvereinbarungen zwischen einem Auftraggeber und einem Auftragnehmer. Es ist durch die Lösung der mathematischen Modelle für das Anwendungsbeispiel aufgezeigt worden, dass die Berücksichtigung der unterschiedlichen Vertragscharakteristika das Ergebnis der Projektplanung und somit den Kapitalwert von Auftraggeber und Auftragnehmer maßgeblich beeinflusst. Es zeigt sich, dass der Auftraggeber für das Ziel der Kapitalwertmaximierung niedrige Kostenerstattungsfaktoren, eine niedrige Anzahl Teilzahlungen sowie die Zahlung eines Honorars zu dem Zeitpunkt des Projektendes bevorzugt. Somit ist die Zahlung eines festen Honorars einem Kostenerstattungsvertrag mit prozentualem Honoraraufschlag, das heißt Kostenerstattungsfaktoren $\beta>1$ vorzuziehen. Für einen Festpreisvertrag sind vier unterschiedliche Zahlungsvereinbarungen vorgestellt und diskutiert worden. Hiervon sind die Fälle konstanter Zahlungsintervalle sowie konstanter bzw. variierender Zahlungen als anwendungsnah ausgewählt und jeweils in das Payment Scheduling Modell eingebunden worden. Erwartungsgemäß ist aus der Sicht des Auftraggebers eine Festpreisvereinbarung mit niedrigen Teilzahlungen und einer hohen Abschlusszahlung gegenüber einer Vereinbarung mit über die Projektdauer verteilten identischen Zahlungen vorzuziehen. 
Durch die Integration der Kooperationsbedingung des Auftragnehmers in die Planung des Auftraggebers erfolgt eine über die bisher aus der Literatur zum Payment Scheduling bekannten Ansätze hinausgehende Berücksichtigung der Präferenzen des Auftragnehmers durch den Auftraggeber. Für das Anwendungsbeispiel zeigt sich, dass der Auftraggeber für die Erfüllung der Teilnahmebedingung einen höheren Festpreis vereinbart bzw. höhere Teilzahlungen und/oder diese zu früheren Zeitpunkten während der Projektdurchführung leistet und daraus eine Reduzierung seines Kapitalwertes gegenüber der Nichtberücksichtigung der Kooperationsbedingung resultiert.

Das sowohl aufgrund der hierarchischen Struktur der Kooperationsbeziehung als auch durch das Verhalten der Kooperationspartner bestehende Problem der Informationsasymmetrie sowie das eigennutzorientierte Verhalten des Auftragnehmers führen zu der Notwendigkeit, das vermutete Verhalten des Auftragnehmers als Reaktion auf das Vertragsangebot des Auftraggebers bei der Planung zu berücksichtigen. Hierfür ist ein vierstufiges Vorgehen vorgeschlagen worden, das geeignet ist, dem Auftraggeber die Konsequenzen unterschiedlicher Zahlungsvereinbarungen unter Berücksichtigung mehrerer möglicher Arbeitsniveaus des Auftragnehmers aufzuzeigen.

Bei einem Kostenerstattungsvertrag wird der Auftragnehmer tendenziell ein mit höheren Kosten verbundenes hohes Arbeitsniveau wählen. Im Gegensatz hierzu entscheidet sich der Auftragnehmer bei einer Festpreisvereinbarung für eine mit niedrigen Kosten verbundene Arbeitsanstrengung. Stimmen die Präferenzen der Projektpartner hinsichtlich der Wahl der Arbeitsanstrengung nicht überein, kann gezeigt werden, dass durch die Berücksichtigung von Anreizsystemen bei der Vertrags- und Projektplanung des Auftraggebers Anreize gesetzt werden können, welche die Motivation des Auftragnehmers für ein bestimmtes Verhalten beeinflussen.

Das vierstufige Vorgehen schließt sowohl die Teilnahme- als auch die Leistungsentscheidung des Auftragnehmers in die Planung des Auftraggebers ein. Für eine Bestimmung des aus der Sicht des Auftraggebers am besten geeigneten Vertragsdesigns müssen die Berechnungen für verschiedene Zahlungsvereinbarungen durchgeführt und deren Ergebnisse analysiert werden.

Für das Anwendungsbeispiel und einer Kostenerstattungsvereinbarung mit drei Teilzahlungen ergibt sich beispielsweise eine Modellgröße von etwa 395 Restriktionen und etwa 994 Binär- sowie 142 stetigen Variablen. Die Rechenzeit beträgt auf einem Computer mit Pentium-Prozessor mit $1,8 \mathrm{GHz}$. und $512 \mathrm{MB}$ RAM weniger als eine 
Minute. Auch die für andere Stufen sowie für alternative Vertragsdesigns im Rahmen des Anwendungsbeispiels erfolgten Berechnungen führten maximal innerhalb weniger Minuten zu der optimalen Lösung des Modells. Dies ermöglicht eine Umsetzung des vorgestellten Vorgehens zur Lösung praktischer Problemstellungen.

Die Entscheidung für eine Kostenerstattungs- oder eine Festpreisvereinbarung sollte nicht ausschließlich auf der Grundlage einer Kapitalwertmaximierung getroffen werden. Der Auftraggeber muss hierfür sowohl die mit dem Projekt verbundenen Chancen und Risiken als auch das sozio-ökonomische Verhältnis zu dem Auftragnehmer in das Vertragsmanagement einbeziehen. 


\section{Zusammenfassung und Ausblick}

Die Verbindung der zwei aktuell diskutierten Forschungsbereiche des Contract Managements sowie der quantitativen Projektplanung bildet den Schwerpunkt der vorliegenden Arbeit. Das Contract Management, welches insbesondere durch die in der Volkswirtschaftstheorie entwickelten Ansätze der Neuen Institutionenökonomik geprägt und durch die betriebswirtschaftliche Organisationstheorie ergänzt wird, betrachtet die Fragestellung, wie Verträge dazu beitragen können, ökonomische Transaktionen zwischen verschiedenen Akteuren zu organisieren. Ein konkreter betriebswirtschaftlicher Anwendungsbezug lässt sich beispielsweise zwischen einem Projektauftraggeber und den das Projekt ausführenden Auftragnehmern herstellen. Als Teilaspekt wurde für diese Arbeit die Aufgabe der Planung von vertraglich geregelten Zahlungsbestimmungen ausgewählt und als Organisationsform eine interorganisationale Projektkooperation zwischen dem ein Investitionsprojekt in Auftrag gebenden Projekteigentümer als Kooperationsträger sowie einem ausführenden Auftragnehmer betrachtet, so dass die Methoden und Verfahren des Projektmanagements als Grundlage für dieses kombinierte Vertrags- und Projektplanungsproblem dienen.

Zunächst sind die betriebswirtschaftlichen Anforderungen an das Vertragsmanagement aufgezeigt sowie die mit einer derartigen Kooperationsform einhergehenden Probleme analysiert worden. Es zeigt sich, dass die mit der Ausführung des Investitionsprojektes verbundene Unsicherheit sowie die insbesondere bei mittel- und langfristigen Kooperationen vorliegende Spezifität der Projektleistungen Ursache ist, unvollständige Verträge abzufassen. Damit muss das Vertragsmanagement als ein das Projekt begleitender Prozess verstanden werden, welcher durch das Vertragscontrolling ergänzt wird. Auch die aus der Informationsasymmetrie zwischen den Kooperationspartnern resultierenden Probleme, wie beispielsweise das durch den Auftraggeber nach Vertragsabschluss nicht beobachtbare Verhalten des Auftragnehmers bieten ein Potenzial für opportunistisches Verhalten und sollten daher im Rahmen des Vertragsmanagements Beachtung finden.

Die in dieser Arbeit betrachtete Organisationsform einer interorganisationalen Projektkooperation erlaubt es, eine Verbindung zu dem Konzept der hierarchischen Planung aufzuzeigen und somit die Auftraggeber-Auftragnehmer-Beziehung als hierarchische Prinzipal-Agenten-Situation darzustellen. Diese Betrachtungen führen zu dem Ergebnis, dass der Auftraggeber sowohl die Präferenzen als auch das mögliche Verhalten 
des Auftragnehmers als Reaktion auf seine Entscheidungen bei der Auswahl der optimalen Handlungsalternative berücksichtigen muss. Hierfür wurden die Kooperationsund Anreizkompatibilitätsbedingungen für das Grundmodell einer Prinzipal-AgentenBeziehung diskutiert und formal abgebildet. Die Kooperationsbedingung gewährleistet, dass der Auftraggeber für die Vertragsgestaltung nur solche Alternativen einbezieht, welche ein vorgegebenes Anspruchsniveau aus der Sicht des Auftragnehmers erfüllen und dieser somit bereit ist, an der Kooperation teilzunehmen. Unter der Annahme eines den individuellen Nutzen maximierenden Verhaltens beider Kooperationspartner kann der Auftraggeber zusätzlich durch die Einbeziehung der Anreizkompatibilitätsbedingung ein Vertragsdesign vorschlagen, welches den Auftragnehmer motiviert, den durch den Auftraggeber gewünschten Arbeitseinsatz zu erbringen. Darüber hinaus besteht die Möglichkeit, die durch die Informationsasymmetrie bedingten Probleme durch ein Informationsmanagement zu reduzieren. Hierfür wurden die Anforderungen an den Einsatz sowie die Anwendungsbereiche von Informations- und Kommunikationssystemen für die unterschiedlichen Kooperationsphasen vorgestellt.

Die durchgeführte Analyse aktueller, auf dem Gebiet des Contract Managements veröffentlichter Fachbeiträge zeigt, dass das Vertragsdesign in die zwei Gruppen der Kostenerstattungs- sowie der Festpreisverträge unterschieden wird. Durch die Einbindung von Anreizsystemen in die Vertragsgestaltung können die Kooperationsrisiken auf die Vertragspartner aufgeteilt und diese somit zu einem bestimmten Verhalten motiviert werden. Hierbei stehen die Bestimmung einer geeigneten Anreizfunktion sowie die Auswahl der hierfür benötigten Bemessungsgrundlagen im Mittelpunkt. Aufgrund der Vielzahl bereits entwickelter, meist formaler Ansätze auf diesem Gebiet, wurde beispielhaft eine lineare, die Projektkosten als Bemessungsgrundlage verwendende Anreizfunktion formuliert sowie deren ökonomische Konsequenzen auf die Projektkooperation diskutiert. Das Ziel dieser Anreizfunktion besteht häufig darin, den Auftragnehmer zu motivieren, die vertraglich vereinbarten Leistungen zu niedrigen Kosten zu erstellen. Das bei realen Problemstellungen bestehende Problem der Kontrolle des Auftragnehmers durch den Auftraggeber führt dazu, dass eine Überprüfung der tatsächlichen Kosten durch den Auftraggeber oft nicht möglich ist. Daher ist im fünften Kapitel für das Anwendungsbeispiel eine Anreizfunktion verwendet worden, bei der der Zeitpunkt des Projektabschlusses als Bemessungsgrundlage dient. Zusammenfassend ließen sich sechs Faktoren identifizieren, welche die Projektkooperation sowie die hieraus resultierenden Probleme interdependent beeinflussen. Eine Berücksichtigung aller Einflussfaktoren bei der Vertrags- und Projektplanung des Auftraggebers ist 
durch die Komplexität der Problemstellung sowie das Zusammenwirken der verschiedenen Einflüsse kaum realisierbar. Die unterschiedlichen hier analysierten Zusammenhänge zeigen jedoch die Relevanz des Contract Managements für das Management von Projektkooperationen auf.

Für eine Verbindung des Contract Managements mit den Methoden der quantitativen Projektplanung dient das als Project Scheduling Problem with Discounted Cash Flows bekannte und in der Literatur aktuell diskutierte Planungsproblem als Grundlage der weiterführenden Untersuchungen. Ein Literaturüberblick über die Charakteristika von Project Scheduling Problemen hat unter anderem gezeigt, dass hierbei eine Lösung des Planungsproblems des Auftragnehmers angestrebt wird und die Höhe und Zeitpunkte der Zahlungen des Auftraggebers an den Auftragnehmer als bekannt vorausgesetzt werden. Das Project Scheduling Problem kann somit das in dieser Arbeit untersuchte Planungsproblem des Auftraggebers nicht ausreichend abbilden. Die Analyse der in den letzten Jahren erfolgten Weiterentwicklungen zur Lösung dieser Problemstellungen unter der Bezeichnung des Payment Scheduling Problems zeigt, dass eine Berücksichtigung von Vertragscharakteristika möglich und in den die Planung des Auftraggebers unterstützenden quantitativen Modellen bereits ansatzweise erfolgt ist.

Ausgehend von einem Grundmodell des Payment Schedulings sind in dieser Arbeit Modellerweiterungen und -modifikationen durchgeführt worden, um das anhand einer Anwendung konkret dargestellte Planungsproblem sowohl für Kostenerstattungs- als auch für Festpreisvereinbarungen realitätsnah abzubilden. Für das Vertragsdesign sind die Vertragsart und insbesondere die Anzahl und die Höhe der Teilzahlungen festzulegen. Die Ergebnisse der Umsetzung und Lösung der als lineare Programmierungsmodelle allgemein formulierten Probleme zeigen für das Anwendungsbeispiel konkret die Konsequenzen unterschiedlicher Zahlungsvereinbarungen auf den Kapitalwert als Erfolgsgröße von Auftraggeber und Auftragnehmer auf.

Durch die Integration der Kooperationsbedingung des Auftragnehmers in die Projektplanung des Auftraggebers kann dieser ermitteln, welcher aus seiner Sicht kapitalwertmaximale Projekt- und Zahlungsplan die Teilnahmebedingung des Auftragnehmers erfüllt. Für beide Vertragsarten lassen sich durch die von der Projektstruktur abhängigen Kombinationsmöglichkeiten von Zahlungen und mit den Ereignissen verbundenen Zahlungszeitpunkten mehrere optimale Zahlungspläne ermitteln, die unter Einhaltung dieser Bedingung zu einem maximalen Kapitalwert des Auftraggebers füh- 
ren. Durch die Berücksichtigung zusätzlicher Präferenzen des Auftraggebers etwa in Form von Liquiditäts- oder Zeitpräferenzen ist die Bestimmung einer optimalen Handlungsalternative möglich.

Um darüber hinaus das vermutete Verhalten des Auftragnehmers als Reaktion auf die Vertragsentscheidung des Auftraggebers zu berücksichtigen, ist ein vierstufiges Vorgehen vorgeschlagen worden. Der Auftraggeber vermutet, dass der Auftragnehmer neben dem geplanten Arbeitsniveau auch eine niedrigere bzw. eine höhere Arbeitsanstrengung realisieren kann, wobei die höhere Arbeitsanstrengung mit höheren Kosten verbunden ist, jedoch durch die Verkürzung der Vorgangsdauern zu einem früheren Projektende und somit höheren Erlösen für den Auftraggeber führen kann. Die Untersuchungen zeigen, dass der Auftragnehmer tendenziell bei einer Festpreisvereinbarung das niedrige und bei einer Kostenerstattungsvereinbarung das hohe Arbeitsniveau realisieren wird. Dies bestätigt die Vermutungen der im Rahmen des Contract Managements diskutierten Verhaltensweisen des Auftragnehmers. Zusätzlich konnte gezeigt werden, dass der Auftraggeber den Auftragnehmer durch die Einbindung eines Anreizsystems sowohl hinsichtlich der Wahl des gewünschten Arbeitsniveaus als auch der Realisierung eines frühen Projektendes motivierend beeinflussen kann. Die vorgeschlagenen Planungsmodelle unterstützen den Auftraggeber bei der Bestimmung einer für die Motivation des Auftragnehmers notwendigen Anreizfunktion, welche ein Abweichen des tatsächlich realisierten Projektendes von dem geplanten Zeitpunkt des Projektabschlusses bestraft oder belohnt. Obwohl bei der vorgestellten Problemstellung die Belohnung einer mit dem hohen Arbeitseinsatz verbundenen Leistungserstellung im Vordergrund stand, kann dieses Vorgehen auch verwendet werden, um eine Anreizfunktion zu bestimmen, welche die Motivation des Auftragnehmers durch die Möglichkeit der Festsetzung einer Vertragsstrafe beeinflusst, ein durch den Auftraggeber nicht gewünschtes Verhalten zu vermeiden.

Zusammengefasst zeigt die Arbeit ein anwendungsorientiertes Vorgehen auf, welches die speziell bei Auftraggeber-Auftragnehmer-Beziehungen in Investitionsprojekten vorliegenden Kooperationsmerkmale in die Vertrags- und Projektplanung des Auftraggebers integriert. Der Auftraggeber kann bei Kenntnis der Problemstruktur durch die Unterstützung der hier weiterentwickelten quantitativen Planungsmodelle unter Berücksichtigung der Präferenzen sowie des Verhaltens des Auftragnehmers die Konsequenzen verschiedener Zahlungsvereinbarungen ermitteln und somit ein für die Projektkooperation geeignetes Vertragsdesign bestimmen. 
Die Notwendigkeit von Vertragsvereinbarungen ist nicht auf Investitionsprojekte beschränkt, sondern erstreckt sich auf vielfältige zwischen Kooperationspartnern durchzuführende Transaktionen. Beispielsweise erfordern die teilweise langfristigen Kooperationsbeziehungen zwischen einem Produzenten und dessen Zulieferern die Integration eines an den Unternehmungszielen ausgerichteten Vertragsmanagements als Teil des Supply Chain Managements. Zusätzliche Anforderungen, welche insbesondere aus weiteren anwendungsspezifischen Fragestellungen resultieren können, sind in das vorgestellte Vorgehen zu integrieren.

Die vertraglichen, insbesondere mit ökonomischen Konsequenzen verbundenen Regelungen stellen die Grundlage der Kooperationsbeziehung dar und bestimmen maßgeblich deren Erfolg. Es kann daher erwartet werden, dass das Contract Management als angewandte ökonomische Vertragstheorie durch die Tendenz einer zunehmend globalen und durch unterschiedliche Formen der Arbeitsteilung geprägten Leistungserstellung an Bedeutung gewinnen wird. 
Bernd Slaghuis - 978-3-631-75513-6 


\section{Literaturverzeichnis}

Abbasi, Ghaleb Y.; Arabiat, Yasser A. (2001): A heuristic to maximize the net present value for resource-constrained project-scheduling problems, in: Project Management Journal, Vol. 32, No. 2, 2001, S. 17-24.

Adam, Dietrich (1996): Planung und Entscheidung - Modelle, Ziele, Methoden, 4., vollst. überarb. u. wesentl. erw. Aufl., Wiesbaden 1996.

Adam, Dietrich (2000): Investitionscontrolling, 3., völlig neu bearb. u. wesentl. erw. Aufl., München, Wien 2000.

Adler, Terry R.; Scherer, Robert F.; Barton, Sidney L.; Katerberg, Ralph (1998): An empirical test of transaction cost theory - validating contract typology, in: Journal of Applied Management Studies, Vol. 7, No. 2, 1998, S. 185-200.

Ahuja, Ravindra K.; Magnanti, Thomas L.; Orlin, James B. (1993): Network Flows Theory, Algorithms, and Applications, Englewood Cliffs u. a. 1993.

Allen, Douglas W.; Lueck, Dean (1999): The role of risk in contract choice, in: The Journal of Law, Economics, and Organization, Vol. 15, No. 3, 1999, S. 704-725.

Al-Subhi Al-Harbi, Kamal M. (1998): Sharing fractions in cost-plus-incentive-fee contracts, in: International Journal of Project Management, Vol. 16, No. 2, 1998, S. 73-80.

Altrogge, Günter (1996): Netzplantechnik, 3. Aufl., München, Wien 1996.

Archibald, Russell D. (2001): Role management: the integrative roles in project management, in: Knutson, Joan (Hrsg.): Project Management for Business Professionals, New York u. a. 2001, S. 440-457.

Arditi, David; Yasamis, Firuzan (1998): Incentive/disincentive contracts: perceptions of owners and contractors, in: Journal of Construction Engineering and Management, Vol. 124, No. 5, 1998, S. 361-373.

Arrow, Kenneth J. (1986): Agency and the market, in: Handbook of Mathematical Economics, Vol. 3, 1986, S. 1183-1195. 
Artz, Kendall W.; Norman, Patricia M. (2002): Buyer-supplier contracting: contract choice and ex post negotiation costs, in: Journal of Managerial Issues, Vol. 14, No. 4, 2002, S. 399-417.

Backhaus, Klaus (1980): Auftragsplanung im industriellen Anlagengeschäft, Stuttgart 1980.

Backhaus, Klaus (2003): Industriegütermarketing, 7., erw. u. überarb. Aufl., München 2003.

Backhaus, Klaus; Köhl, Thomas (1999): Claim-Management im internationalen Anlagengeschäft, in: Hübner, Ulrich (Hrsg.): Festschrift für Bernhard Großfeld zum 65. Geburtstag, Heidelberg 1999, S. 17-34.

Backhaus, Klaus; Piltz, Klaus (1990): Strategische Allianzen - eine neue Form kooperativen Wettbewerbs?, in: Backhaus, Klaus; Piltz, Klaus (Hrsg.): Strategische Allianzen, Düsseldorf 1990, S. 1-10.

Baker, George (2000): The use of performance measures in incentive contracting, in: American Economic Review, Vol. 90, No. 2, 2000, S. 415-420.

Baker, George; Gibbons, Robert; Murphy, Kevin J. (1994): Subjective performance measures in optimal incentive contracts, in: Quarterly Journal of Economics, Vol. 109, No. 4, 1994, S. 1125-1156.

Bamberg, Günter; Coenenberg, Adolf G. (2002): Betriebswirtschaftliche Entscheidungslehre, 11., überarb. Aufl., München 2002.

Bamberg, Günter (1991): Extended contractual incentives to reduce project cost, in: OR Spektrum, Heft 13, 1991, S. 95-98.

Baroum, Sami M.; Patterson, James H. (1996): The development of cash flow weight procedures for maximizing the net present value of a project, in: Journal of Operations Management, Vol. 14, 1996, S. 209-227.

Baroum, Sami M.; Patterson, James H. (1998): An exact solution procedure for maximizing the net present value of cash flows in a network, in: Weglarz, Jan (Hrsg.): Project Scheduling: Recent Models, Algorithms and Applications, Boston u. a. 1998, S. 107-134. 
Bauer, Siegfried (2003): Perspektiven in der Organisationsgestaltung, in: Bullinger, Hans-Jörg; Warnecke, Hans Jürgen; Westkämper, Engelbert (Hrsg.): Neue Organisationsformen im Unternehmen, 2., neu bearb. u. erw. Aufl., Berlin u. a. 2003, S. 93-128.

Beck, Christoph (1994): Interorganisationales Projekt-Management, eine alternative Kooperationsform - Ein Beitrag unter Berücksichtigung des integrativen Informations-Managements als Basis für die projektbezogene Kooperation, Diss. Universität Hamburg 1994.

Beck, Thomas (1996): Die Projektorganisation und ihre Gestaltung, Berlin 1996, zugl. Diss. Eberhard-Karls-Universität Tübingen 1995.

Beier, Dirk (2002): Informationsmanagement aus Sicht der Betriebswirtschaftslehre: Theoretische Ansätze und das Beispiel Mobile Business, Frankfurt am Main u. a. 2002, zugl. Diss. Ruhr-Universität Bochum 2001.

Berends, T. C. (2000): Cost plus incentive fee contracting - experiences and structuring, in: International Journal of Project Management, Vol. 18, 2000, S. 165-171.

Blohm, Hans; Lüder, Klaus (1995): Investition - Schwachstellenanalyse des Investitionsbereichs und Investitionsrechnung, 8., aktual. und erg. Aufl., München 1995.

Bloodgood, James M.; Salisbury, W. David (2001): Understanding the influence of organizational change strategies on information technology and knowledge management strategies, in: Decision Support Systems, Vol. 31, 2001, S. 55-69.

Bode, Jürgen (1997): Der Informationsbegriff in der Betriebswirtschaftslehre, in: Zeitschrift für betriebswirtschaftliche Forschung, Jg. 49, Heft 5, 1997, S. 449-468.

Borgwardt, Karl Heinz (2001): Optimierung, Operations Research, Spieltheorie Mathematische Grundlagen, Basel u. a. 2001.

Bosse, Christian (2000): Investitionsmanagement in divisionalen Unternehmen Strategiebestimmung, Koordination von Investitionsentscheidungen und Anreizsysteme, Chemnitz 2000, zugl. Diss. Technische Universität Chemnitz 2000 . 
Boute, R.; Demeulemeester, Erik L.; Herroelen, Willy S. (2004): A real options approach to project management, in: International Journal of Production Research, Vol. 42, No. 9., 2004, S. 1715-1725.

Bowman, Edward H.; Moskowitz, Gary T. (2001): Real options analysis and strategic decision making, in: Organization Science, Vol. 12, No. 6, 2001, S. 772-777.

Branconi, Christof; Loch, Christoph H. (2004): Contracting for major projects - eight business levers for top management, in: International Journal of Project Management, Vol. 22, No. 2, 2004, S. 119-130.

Breid, Volker (1995): Aussagefähigkeit agencytheoretischer Ansätze im Hinblick auf die Verhaltenssteuerung von Entscheidungsträgern, in: Zeitschrift für betriebswirtschaftliche Forschung, Jg. 47, Heft 9, 1995, S. 821-854.

Breuer, Wolfgang (2002): Investition I, Entscheidungen bei Sicherheit, 2., korr. Aufl., Wiesbaden 2002.

Breuer, Wolfgang; Gürtler, Marc; Schuhmacher, Joachim (1999): Die Bewertung betrieblicher Realoptionen, in: Betriebswirtschaftliche Forschung und Praxis, Heft 2, 1999, S. 213-232.

Brockhoff, Klaus (2000): Problems of evaluating R\&D projects as real options, in: Frenkel, Michael; Hommel, Ulrich; Rudolf, Markus (Hrsg.): Risk Management, Berlin u. a. 2000, S. 203-212.

Bröker, Erich W. (1993): Erfolgsrechnung im industriellen Anlagengeschäft - Ein dynamischer Ansatz auf Zahlungsbasis, Wiesbaden 1993, zugl. Diss. RuhrUniversität Bochum 1993.

Brucker, Peter (2001): Scheduling Algorithms, 3rd ed., Berlin u. a. 2001.

Brucker, Peter; Drexl, Andreas; Möhring, Rolf; Neumann, Klaus; Pesch, Erwin (1999): Resource-constrained project scheduling - notation, classification, models, and methods, in: European Journal of Operational Research, Vol. 112, 1999, S. 3-41.

Bubshait, Abdulaziz A. (2003): Incentive/disincentive contracts and its effects on industrial projects, in: International Journal of Project Management, Vol. 21, 2003, S. 63-70. 
Burghardt, Manfred (2002a): Projektmanagement - Leitfaden für die Planung, Überwachung und Steuerung von Entwicklungsprojekten, 6., wesentl. überarb. u. erw. Aufl., Berlin, München 2002.

Burghardt, Manfred (2002b): Einführung in Projektmanagement - Definition, Planung, Kontrolle, Abschluss, 4., überarb. u. erw. Aufl., Berlin, München 2002.

Buss, Arnold H.; Rosenblatt, Meir J. (1997): Activity delay in stochastic project networks, in: Operations Research, Vol. 45, No. 1, S. 126-139.

Busse von Colbe, Walther; Laßmann, Gert (1990): Betriebswirtschaftstheorie, Band 3: Investitionstheorie, 3., durchges. Aufl., Berlin u. a. 1990.

Cachon, Gérard P. (2003): Supply chain coordination with contracts, in: Handbooks in Operations Research and Management Science, Vol. 11, 2003, S. 229-339.

Canes, Michael E. (1975): The simple economics of incentive contracting - Note, in: The American Economic Review, Vol. 65, No. 3, S. 478-483.

Cappels, Thomas M. (2003): Financially focused project management, Boca Raton 2003.

Carbon, Martin (1999): Folgen der Dezentralisierung - Anforderungen an das Informationsmanagement, in: Bullinger, Hans-Jörg (Hrsg.): Effizientes Informationsmanagement in dezentralen Organisationsstrukturen, Berlin u. a. 1999, S. 43-71.

Carr, Robert I. (1977): Paying the price for construction risk, in: Journal of the Construction Division, Vol. 103, 1977, S. 153-161.

Chapman, Chris; Ward, Stephen (1994): The efficient allocation of risk in contracts, in: Omega International Journal of Management Science, Vol. 22, No. 6, 1994, S. 537-552.

Chapman, Chris; Ward, Stephen (2003): Project Risk Management - Processes, Techniques and Insights, 2nd ed., Chichester u. a. 2003.

Chiappori, Pierre A.; Salanié, Bernard (2002): Testing contract theory - a survey of some recent work, CESIFO Working Paper No. 738, München 2002.

Chiu, Huan Neng; Tsai, Deng Maw (2002): An efficient search procedure for the resource-constrained multi-project scheduling problem with discounted cash flows, in: Construction Management and Economics, Vol. 20, 2002, S. 55-66. 
Chopra, Sunil; Meindl, Peter (2004): Supply Chain Management, 2nd ed., New Jersey 2004.

Cleland, David I. (2001): Overview: the discipline of project management, in: Knutson, Joan (Hrsg.): Project Management for Business Professionals, New York u. a. 2001, S. 3-22.

Coase, R. H. (1937): The nature of the firm, in: Econometrica, Vol. 9, 1937, S. 386-405.

Comelli, Gerhard; von Rosenstiel, Lutz (2003): Führung durch Motivation: Mitarbeiter für Organisationsziele gewinnen, 3., erw. u. überarb. Aufl., München 2003.

Cooke-Davies, Terry (2002): The "real" success factors on projects, in: International Journal of Project Management, Vol. 20, 2002, S. 185-190.

Corsten, Hans; Corsten, Hilde (2000): Projektmanagement - Einführung, München, Wien, Oldenbourg 2000.

Crasselt, Nils (2003): Wertorientierte Managemententlohnung, Unternehmensrechnung und Investitionssteuerung - Analyse unter Berücksichtigung von Realoptionen, Frankfurt am Main u. a. 2003, zugl. Diss. Ruhr-Universität Bochum 2002.

Crocker, Keith J.; Masten, Scott E. (1991): Pretia ex machina? Price and process in long-term contracts, in: Journal of Law and Economics, Vol. 34, 1991, S. 69-99.

Crocker, Keith J.; Reynolds, Kenneth J. (1993): The efficiency of incomplete contracts - an empirical analysis of air force engine procurement, in: RAND Journal of Economics, Vol. 24, No. 1, 1993, S. 126-146.

Cummins, Michael J. (1977): Incentive contracting for national defense - a problem of optimal risk sharing, in: Bell Journal of Economics, Vol. 8, No. 1, 1977, S. $169-185$.

Dahlstrom, Robert; Nygaard, Arne (1999): An empirical investigation of ex post transaction costs in franchised distribution channels, in: Journal of Marketing Research, Vol. 36, 1999, S. 160-170. 
Dayanand, Nalini; Padman, Rema (1997): On modelling payments in projects, in: Journal of the Operational Research Society, Vol. 48, 1997, S. 906-918.

Dayanand, Nalini; Padman, Rema (1998): On payment schedules in contractor client negotiations in projects - an overview of the problem and research issues, in: Weglarz, Jan (Hrsg.): Payment scheduling: Recent Models, Algorithms and Applications, Boston u. a. 1998, S. 476-508.

Dayanand, Nalini; Padman, Rema (2001a): A two stage search heuristic for scheduling payments in projects, in: Annals of Operations Research, Vol. 102, 2001, S. 197-220.

Dayanand, Nalini; Padman, Rema (2001b): Project contracts and payment schedules - the client's problem, in: Management Science, Vol. 27, No. 2, 2001, S. 1654-1667.

De Maio, Adriano; Varganti, Roberto; Corso, Mariano (1994): A multi-project management framework for new product development, in: European Journal of Operational Research, Vol. 78, 1994, S. 178-191.

De Reyck, Bert; Herroelen, Willy S. (1998): An optimal procedure for the resourceconstrained project scheduling problem with discounted cash flows and generalized precedence relations, in: Computers and Operations Research, Vol. 25, No. 1, 1998, S. 1-17.

Demeulemeester, Erik L.; Herroelen, Willy S. (2002): Project Scheduling A Research Handbook, Boston u. a. 2002.

Deutsches Institut für Normung (1987): DIN 69 901, Projektwirtschaft, Projektmanagement, Begriffe, Deutsches Institut für Normung e.V., Berlin 1987.

Dinkelbach, Werner; Kleine, Andreas (1996): Elemente einer betriebswirtschaftlichen Entscheidungslehre, New York u. a. 1996.

Disterer, Georg (2001): Wissensmanagement bei Unternehmensberatungen, in: Wirtschaftswissenschaftliches Studium, Jg. 30, Heft 12, 2001, S. 677-680.

Dixit, Avinash K.; Pindyck, Robert S. (1994): Investment under Uncertainty, Princeton 1994.

Dixit, Avinash; Skeath, Susan (2004): Games of Strategy, 2nd ed., New York, London, 2004. 
Doersch, Robert H.; Patterson, James H. (1977): Scheduling a project to maximize its present value - a zero-one programming approach, in: Management Science, Vol. 23, No. 8, 1977, S. 882-889.

Domschke, Wolfgang; Drexl, Andreas (2002): Einführung in Operations Research, 5., überarb. und erw. Aufl., Berlin u. a. 2002.

Domschke, Wolfgang; Scholl, Armin (2003): Grundlagen der Betriebswirtschaftslehre - Eine Einführung aus entscheidungsorientierter Sicht, 2. verb. Aufl., Berlin u. a. 2003.

Domschke, Wolfgang; Scholl, Armin; Voß, Stefan (1997): Produktionsplanung - Ablauforganisatorische Aspekte, 2., überarb. u. erw. Aufl., Berlin u. a. 1997.

Drews, Hanno (2001): Instrumente des Kooperationscontrollings - Anpassung bedeutender Controllinginstrumente an die Anforderungen des Managements von Unternehmenskooperationen, Wiesbaden 2001, zugl. Diss. Universität Trier 2001 .

Drukarczyk, Jochen (2003): Unternehmensbewertung, 4., überarb. und erw. Aufl., München 2003.

Dudek, Gregor (2004): Collaborative Planning in Supply Chains - A NegotiationBased Approach, Berlin u. a. 2004, zugl. Diss Universität Darmstadt 2003.

Dvir, Dov; Raz, Tzvi; Shenhar, Aaron J. (2003): An empirical analysis of the relationship between project planning and project success, in: International Journal of Project Management, Vol. 21, 2003, S. 89-95.

Eisenführ, Franz; Weber, Martin (2003): Rationales Entscheiden, 4., neu bearb. Aufl., Berlin u. a. 2003.

Eisenhard, Kathleen M. (1989): Agency theory - an assessment and review, in: Academy of Management Review, Vol. 14, No. 1, 1989, S. 57-74.

Ellinger, Theodor; Beuermann, Günter; Leisten, Rainer (2003): Operations Research - Eine Einführung, 6., durchges. Aufl., Berlin u. a. 2003.

Elmaghraby, Salah E.; Ferreira, J. Antunes; Tavares, L. Valadares (2000): Optimal start times under stochastic activity durations, in: International Journal of Production Economics, Vol. 64, 2000, S. 153-164. 
Elmaghraby, Salah E. (1990): Project bidding under deterministic and probabilistic activity durations, in: European Journal of Operational Research, Vol. 49, 1990, S. 14-34.

Elmaghraby, Salah E.; Herroelen, Willy S. (1990): The scheduling of activities to maximize the net present value of projects, in: European Journal of Operational Research, Vol. 49, 1990, S. 35-49.

Elmaghraby, Wedad J. (2000): Supply contract competition and sourcing policies, in: Manufacturing and Service Operations Management, Vol. 2, No. 4, 2000, S. 350-371.

Engelhardt, Werner H.; Gersch, Martin (1995): Informationsmanagement als Instrument zur erfolgreichen Gestaltung von Geschäftsbeziehungen - am Beispiel des deutschen Pharagroßhandels, in: Handelsforschung, Heft 10, 1995, S. 201-222.

Erenguc, S. Selcuk; Tufekci, Suleyman; Zappe, Christopher J. (1993): Solving time/cost trade-off problems with discounted cash flows using generalized benders decomposition, in: Naval Research Logistics, Vol. 40, 1993, S. 25-50.

Erlei, Mathias (1998): Institutionen, Märkte und Marktphasen - Allgemeine Transaktionskostentheorie unter spezieller Berücksichtigung der Entwicklungsphasen von Märkten, Tübingen 1998.

Erlei, Mathias; Leschke, Martin; Sauerland, Dirk (1999): Neue Institutionenökonomik, Stuttgart 1999.

Erner, Carsten; Olinger, Dennis; Werthschulte, Holger (2003): Einsatz des Realoptionsansatzes zur Bewertung von Projektfinanzierungsvorhaben, in: Backhaus, Klaus; Werthschulte, Holger (Hrsg.): Projektfinanzierung, 2., völlig neu bearb. u. erw. Aufl., Stuttgart 2003, S. 223-249.

Etgar, Ran; Shtub, Avraham (1999): Scheduling project activities to maximize the net present value - the case of time-dependent cash flows, in: International Journal of Production Research, Vol. 37, No. 2, 1999, S. 329-339.

Etgar, Ran; Shtub, Avraham; LeBlanc, Larry J. (1996): Scheduling projects to maximize net present value - the case of time-dependent, contingent cash flows, in: European Journal of Operational Research, Vol. 96, 1996, S. 90-96. 
Fandel, Günter; Heuft, Birgit; Paff, Andrea; Pitz, Thomas (2004): Kostenrechnung, 2., neu bearb. u. erw. Aufl., Berlin u. a. 2004.

Farrell, L. M. (2003): Principal-agency risk in project finance, in: International Journal of Project Management, Vol. 21, 2003, S. 547-561.

Federhen, Jens; Adlbrecht, Gerald (2003): Earned-Value-Managementsysteme für Großprojekte, in: Bullinger, Hans-Jörg; Warnecke, Hans Jürgen; Westkämper, Engelbert (Hrsg.): Neue Organisationsformen im Unternehmen, 2., neu bearb. u. erw. Aufl., Berlin u. a. 2003, S. 804-812.

Fiedler, Rudolf (2003): Controlling von Projekten - Projektplanung, Projektsteuerung und Risikomanagement, 2., verb. u. erw. Aufl., Braunschweig 2003.

Fleischer, Sonja (1997): Strategische Kooperationen - Planung, Steuerung, Kontrolle, Köln 1997, zugl. Diss. Universität Köln 1996.

Frey, Bruno S.; Osterloh, Margit (2002): Motivation - a dual-edged factor of production, in: Frey, Bruno S.; Osterloh, Margit (Hrsg.): Successful Management by Motivation - Balancing Intrinsic and Extrinsic Incentives, Berlin u. a. 2002, S. 3-26.

Friedli, Thomas (2000): Die Architektur von Kooperationen, Diss. Universität St. Gallen 2000.

Fuchs, Marius (1999): Projektmanagement für Kooperationen - eine integrative Methodik, Stuttgart, Wien 1999, zugl. Diss. Universität St. Gallen 1999.

Fuest, Ute (1998): Die Organisation von Kooperationen - Rahmen, Parameter und Modelle der Gestaltung von Interorganizational Relations, Diss. Universität St. Gallen 1998.

Funk, Joachim (1986): Volkswirtschaftliche Bedeutung und betriebswirtschaftliche Besonderheiten des industriellen Anlagengeschäfts, in: Funk, Joachim; Laßmann, Gert (Hrsg.): Langfristiges Anlagengeschäft - Risiko-Management und Controlling, in: Zeitschrift für betriebswirtschaftliche Forschung, Sonderheft 20, 1986, S. 9-19.

Gabriel, Roland; Beier, Dirk (2003): Informationsmanagement in Organisationen, Stuttgart 2003. 
Gabriel, Roland; Knittel, Friedrich; Taday, Holger; Reif-Mosel, Ane-Kristin (2002): Computergestützte Informations- und Kommunikationssysteme in der Unternehmung - Technologien, Anwendungen, Gestaltungskonzepte, 2. Aufl., Berlin u. a. 2002.

Gandhi, Devinder K. (1979): A strategy for risk reduction in incentive contracting, in: Decision Sciences, Vol. 10, No. 3, 1979, S. 371-386.

Gardiner, Paul D.; Stewart, Kenneth (2000): Revisiting the golden triangle of cost, time and quality - the role of NPV in project control, success and failure, in: International Journal of Project Management, Vol. 18, 2000, S. 251-256.

George, Gunnar (1999): Kennzahlen für das Projektmanagement - Projektbezogene Kennzahlen und Kennzahlensysteme; Ein Ansatz zur Unterstützung des Projektmanagements, Frankfurt am Main u. a. 1999, zugl. Diss. Brandenburgische Technische Universität Cottbus 1998.

Gersch, Martin (1998): Vernetzte Geschäftsbeziehungen: Die Nutzung von EDI als Instrument des Geschäftsbeziehungsmanagement, Wiesbaden 1998, zugl. Diss. Ruhr-Universität Bochum 1998.

Gido, Jack; Clements, James P. (2003): Successful Project Management, 2nd ed., Mason 2003.

Gierl, Heribert (2000): Opportunismus in langfristigen Geschäftsbeziehungen, in: Zeitschrift für betriebswirtschaftliche Forschung, Heft 52, 2000, S. 107-140.

Gilbreath, Robert D. (1992): Managing Construction Contracts - Operational Controls for Commercial Risk, 2nd ed., Chichester u. a. 1992.

Girmscheid, Gerhard (2004): Projektabwicklung in der Bauwirtschaft - Wege zur Win-Win-Situation für Auftraggeber und Auftragnehmer, Berlin u. a. 2004.

Göbel, Elisabeth (2002): Neue Institutionenökonomik - Konzeption und betriebswirtschaftliche Anwendungen, Stuttgart 2002.

Goldberg, Victor P.; Erickson, John R. (1987): Quantity and price adjustment in longterm contracts - a case study of petroleum coke, in: Journal of Law and Economics, Vol. 30, 1987, S. 369-398. 
Göx, Robert F.; Budde, Jörg; Schöndube, Jens Robert (2002): Das lineare Agency Modell bei asymmetrischer Information über den Agentennutzen, in: Zeitschrift für Betriebswirtschaft, Jg. 72, Heft 1, 2002, S. 65-79.

Grinold, Richard C. (1972): The payment scheduling problem, in: Naval Research Logistics Quarterly, Vol. 19, 1972, S. 123-136.

Grossman, Sandford J.; Hart, Oliver D. (1983a): An analysis of the principal-agent problem, in: Econometrica, Vol. 51, No. 1, 1983, S. 7-45.

Grossman, Sandford J.; Hart, Oliver D. (1983b): Implicit contracts under asymmetric information, in: The Quarterly Journal of Economics, Vol. 98, 1983, S. 123-156.

Günther, Thomas; Grüning, Michael (2001): Performance Measurement-Systeme ein Konzeptvergleich, in: Zeitschrift für Planung, Jg. 12, Heft 3, 2001, S. 283-306.

Guthof, Philipp (1995): Strategische Anreizsysteme - Gestaltungsoptionen im Rahmen der Unternehmungsentwicklung, Wiesbaden 1995, zugl. Diss. Universität St. Gallen 1994.

Hammann, Peter; Palupski, Rainer; von der Gathen, Andreas; Welling, Michael (2001): Markt und Unternehmung - Handlungsfelder des Marketing, 4. korr. u. erg. Aufl., Aachen 2001.

Hansel, Jürgen; Lomnitz, Gero (2003): Projektleiter-Praxis: Optimale Kommunikation und Kooperation in der Projektarbeit, 4., überarb. und erw. Aufl., Berlin u. a. 2003.

Hardtmann, Guido (1996): Die Wertsteigerungsanalyse im Managementprozess, Wiesbaden 1996, zugl. Diss. Universität Köln 1995.

Harris, Milton; Raviv, Artur (1979): Optimal incentive contracts with imperfect information, in: Journal of Economic Theory, Vol. 20, 1979, S. 231-259.

Hart, Oliver D. (1987): Incomplete contracts, in: Eatwell, J. et al. (Hrsg.): The New Palgrave, London, New York 1987, S. 752-758.

Hart, Oliver D. (1995): Firms, Contracts and Financial Structure, Oxford 1995. 
Hartmann-Wendels, Thomas (1989): Principal-Agent-Theorie und asymmetrische Informationsverteilung, in: Zeitschrift für betriebswirtschaftliche Forschung, Jg. 59, Heft 7, 1989, S. 714-734.

Hartmann-Wendels, Thomas (1992): Agency Theorie, in: Frese, Erich (Hrsg.): Handwörterbuch der Organisation, 3., völlig neu gestaltete Aufl., Stuttgart 1992.

Hasan, Helen; Gould, Edward (2001): Support for the sense-making activity of managers, in: Decision Support Systems, Vol. 31, 2001, S. 71-86.

Hax, Herbert (1991): Theorie der Unternehmung - Information, Anreize und Vertragsgestaltung, in: Ordelheide, Dieter; Rudolph, Bernd; Büsselmann, Elke (Hrsg.): Betriebswirtschaftslehre und ökonomische Theorie, Stuttgart 1991, S. 51-72.

Hax, Herbert (1993): Investitionstheorie, korr. Nachdr. der 5., bearb. Aufl., Heidelberg 1993.

Heinrich, Andreas (2002): Management von Softwareprojekten, München, Wien 2002.

Henderson, Linda S. (2004): Encoding and decoding communication competencies in project management - an exploratory study, in: International Journal of Project Management, Vol. 22, 2004, S. 469-476.

Herbsman, Zohar J.; Chen, Wei T.; Epstein, William C. (1995): Time is money - innovative contracting methods in highway construction, in: Journal of Construction Engineering and Management, Vol. 123, No. 1, 1995, S. 273-281.

Herroelen, Willy S.; Gallens, Els (1993): Computational experience with an optimal procedure for the scheduling of activities to maximize the net present value of projects, in: European Journal of Operational Research, Vol. 65, 1993, S. 274-277.

Herroelen, Willy S.; van Dommelen, Patrick; Demeulemeester, Erik L. (1997): Project network models with discounted cash flows - a guided tour through recent developments, in: European Journal of Operational Research, Vol. 100, 1997, S. 97-121. 
Herroelen, Willy S.; De Reyck, Bert; Demeulemeester, Erik L. (1998): Resourceconstrained project scheduling - a survey of recent developments, in: Computers and Operations Research, Vol. 25, No. 4, 1998, S. 279-302.

Heussen, Benno (Hrsg.) (2002): Handbuch Vertragsverhandlung und Vertragsmanagement, 2. Aufl., Köln 2002.

Hillier, Frederick S.; Liebermann, Gerald J. (2001): Introduction to Operations Research, 7th ed., New York 2001.

Höffken, Ernst; Schweitzer, Marcell (Hrsg.) (1991): Beiträge zur Betriebswirtschaft des Anlagenbaus, Arbeitskreis Internes Rechnungswesen der SchmalenbachGesellschaft, in: Zeitschrift für betriebswirtschaftliche Forschung, Sonderheft 28, 1991.

Holler, Manfred; Illing, Gerhard (2003): Einführung in die Spieltheorie, 5., überarb. Aufl., Berlin u. a. 2003.

Holmström, Bengt (1979): Moral hazard and observability, in: Bell Journal of Economics, Vol. 10, No. 1, 1979, S. 74-91.

Homburg, Carsten (1996): Hierarchische Aushandlungen in Organisationen, Heidelberg 1996.

Homburg, Carsten (2001): Hierarchische Controllingkonzeption - Theoretische Fundierung eines koordinationsorientierten Controlling, Heidelberg 2001.

Homburg, Christian (2000): Quantitative Betriebwirtschaftslehre - Entscheidungsunterstützung durch Modelle, 3., überarb. Aufl., Wiesbaden 2000.

Horsch, Jürgen (2003): Innovations- und Projektmanagement - Von der strategischen Konzeption bis zur operativen Umsetzung, Wiesbaden 2003.

Horváth, Péter (Hrsg.) (2002): Performance Controlling - Strategie, Leistung und Anreizsystem effektiv verbinden, Stuttgart 2002.

Horváth, Péter (2003): Controlling, 9., vollst. überarb. Aufl., München 2003.

Icmeli, Oya; Erenguc, S. Selcuk (1996): A branch and bound procedure for the resource constrained project scheduling problem with discounted cash flows, in: Management Science, Vol. 42, No. 10, 1996, S. 1395-1408. 
Icmeli, Oya; Erenguc, S. Selcuk; Zappe, Christopher J. (1993): Project scheduling problems - a survey, in: International Journal of Operations and Production Management, Vol. 13, No. 11, 1993, S. 80-91.

Ip, W. H.; Yung, K. L.; Wang, Dingwei (2004): A branch and bound algorithm for sub-contractor selection in agile manufacturing environments, in: International Journal of Production Economics, Vol. 87, 2004, S. 195-205.

Johannwille, Ulrich (2000): Arbitragefreie Bewertung unternehmerischer Investitionsprojekte, Köln 2000, zugl. Diss. Westfälische Wilhelms-Universität Münster 1999.

Jórasz, William (2003): Kosten- und Leistungsrechnung, 3., überarb. und erw. Aufl., Stuttgart 2003.

Jost, Peter-J. (2000): Organisation und Motivation - Eine ökonomisch-psychologische Einführung, Wiesbaden 2000.

Jost, Peter-J. (Hrsg.) (2001): Die Prinzipal-Agenten-Theorie in der Betriebswirtschaftslehre, Stuttgart 2001.

Kaluza, Bernd; Dullnig, Herwig; Malle, Franz (2003): Principal-Agent-Probleme in der Supply Chain - Problemanalyse und Diskussion von Lösungsvorschlägen, Klagenfurt 2003.

Kazaz, Burak; Sepil, Canan (1996): Project scheduling with discounted cash flows and progress payments, in: Journal of the Operational Research Society, Vol. 47, 1996, S. 1262-1272.

Kerzner, Harold (2003): Project Management - A Systems Approach to Planning, Scheduling, and Controlling, 8th ed., New Jersey 2003.

Keßler, Heinrich; Winkelhofer, Georg (2004): Projektmanagement - Leitfaden zur Steuerung und Führung von Projekten, 4., überarb. Aufl., Berlin u. a. 2004.

Kiener, Stefan (1990): Die Principal-Agent-Theorie aus informationsökonomischer Sicht, Heidelberg 1990, zugl. Diss. Universität Regensburg 1989.

Kim, Son Ku; Wang, Susheng (2004): Robustness of a fixed-rent contract in a standard agency model, in: Economic Theory, Vol. 24, 2004, S. 111-128.

Kimms, Alf (2001): Mathematical Programming and Financial Objectives for Scheduling Projects, Boston u. a. 2001. 
Kirchler, Erich; Rodler, Christa (2001): Motivation in Organisationen - Organisationspsychologie 1, Wien 2001.

Kirkpatrick, S.; Gelatt, C.; Vecchi, M. (1983): Optimization by simulated annealing, in: Science, Vol. 220, S. 671-680.

Klein, Robert (2000): Scheduling of Resource-Constrained Projects, Boston u. a. 2000.

Kleine, Andreas (1995): Entscheidungstheoretische Aspekte der Principal-AgentTheorie, Heidelberg 1995, zugl. Diss. Universität Saarbrücken 1995.

Klingebiel, Norbert (1998): Performance management - performance measurement, in: Zeitschrift für Planung, Jg. 9, Heft 1, S. 1-15.

Kolisch, Rainer (2001): Entwicklungen und Anwendungen in der Projektplanung Ein Überblick, in: Zeitschrift für betriebswirtschaftliche Forschung und Praxis, Heft 3, 2001, S. 212-226.

Kolisch, Rainer; Padman, Rema (2001): An integrated survey of deterministic project scheduling, in: Omega International Journal of Management Science, Vol. 29, 2001, S. 249-272.

Krcmar, Helmut (2003): Informationsmanagement, 3. Aufl., Berlin u. a. 2003.

Kressler, Herwig W. (2001): Leistungsbeurteilung und Anreizsysteme - Motivation, Vergütung, Incentives, Frankfurt, Wien 2001.

Kriwet, Carla Katharina (1997): Inter- and Intraorganizational Knowledge Transfer, Diss. Universität St. Gallen 1997.

Krüger, Wilfried (1994): Organisation der Unternehmung, 3., verb. Aufl., Stuttgart, Berlin, Köln 1994.

Kuhlmann, Axel (2003): Entwicklung eines praxisnahen Project Scheduling Ansatzes auf der Basis von Genetischen Algorithmen, Diss. FernUniversitätGesamthochschule Hagen 2003.

Küpper, Hans-Ulrich (2001): Controlling - Konzeption, Aufgaben und Instrumente, 3., überarb. und erw. Aufl., Stuttgart 2001.

Laffont, Jean-Jacques; Martimort, David (2002): The Theory of Incentives - The Principal-agent Model, Princeton, Oxford 2002. 
Lambert, Richard A. (1983): Long-term contracts and moral hazard, in: Bell Journal of Economics, Vol. 14, No. 2, 1983, S. 441-452.

Laux, Helmut (1990): Risiko, Anreiz und Kontrolle - Principal-Agent-Theorie, Berlin u. a. 1990.

Laux, Helmut (1992): Anreizsysteme - ökonomische Dimension, in: Frese, Erich (Hrsg.): Handwörterbuch der Organisation, 3., völlig neu gestaltete Aufl., Stuttgart 1992, Sp. 112-122.

Laux, Helmut (1999): Unternehmensrechnung, Anreiz und Kontrolle, Berlin u. a. 1999.

Laux, Helmut (2003): Entscheidungstheorie, 5., verb. Aufl., Berlin u. a. 2003.

Laux, Helmut; Liermann, Felix (1997): Grundlagen der Organisation - Die Steuerung von Entscheidungen als Grundproblem der Betriebswirtschaftslehre, 4. vollst. überarb. Aufl., Berlin u. a. 1997.

Laux, Helmut; Schenk-Mathes, Heike Y. (1992): Lineare und nichtlineare Anreizsysteme, Heidelberg 1992.

Levitt, Raymond E.; Ashley, David B.; Logcher, Robert D. (1980): Allocating risk and incentive in construction, in: Journal of the Construction Division, Vol. 106, 1980, S. 297-305.

Ling, Forence Yean Yng (2004): How project managers can better control the performance of design-build projects, in: International Journal of Project Management, Vol. 22, 2004, S. 477-488.

Link, Patrick; Marxt, Christian (2004): Integration of risk- and chance management in the co-operation process, in: International Journal of Production Economics, Vol. 90, 2004, S. 71-78.

Litke, Hans-Dieter (2004): Projektmanagement - Methoden, Techniken, Verhaltensweisen, 4., überarb. und erw. Aufl., München, Wien 2004.

Lockyer, Keith; Gordon, James (1991): Critical Path Analysis and other Project Network Techniques, 5th ed., London 1991.

Lova, Antonio; Tormos, Pilar (2001): Analysis of scheduling schemes and heuristic rules performance in resource-constrained multiproject scheduling, in: Annals of Operations Research, Vol. 102, 2001, S. 263-286. 
Lucke, Claus (2001): Investitionsprojekte mit mehreren Realoptionen - Bewertung und Analyse, Sternenfels, 2001, zugl. Diss. Universität Karlsruhe 2000.

Lücke, Wolfgang (1991): Investitionslexikon, 2., völlig neu bearb. Aufl., München 1991.

Macha, Roman (2003): Grundlagen der Kosten- und Leistungsrechnung, 3., völlig überarb. Aufl., München 2003

Macho-Stadler, Inés; Pérez-Castrillo, J. David (2001): An Introduction to the Economics of Information, Incentives and Contracts, 2nd ed., New York u. a. 2001 .

Madauss, Bernd (2000): Handbuch Projektmanagement, 6., überarb. u. erw. Aufl., Stuttgart 2000.

Mag, Wolfgang (1990): Grundzüge der Entscheidungstheorie, München 1990.

Magni, Carlo Alberto (2002): Investment decisions in the theory of finance - some antinomies and inconsistencies, in: European Journal of Operational Research, Vol. 137, 2002, S. 206-217.

Martin, M. Dean; Webster, Francis M. (1986): Contract type and the measurement of project success, in: Project Management Institute Seminar/Symposium Montreal, Canada, 1986, S. 165-172.

Martiny, Lutz; Klotz, Michael (1989): Strategisches Informationsmanagement Bedeutung und organisatorische Umsetzung, München u. a. 1989.

Marzouk, M.; Moselhi, O. (2003): A decision support tool for construction bidding, in: Construction Innovation, Vol. 3., 2003, S. 111-124.

Mc Call, J. J. (1970): The simple economics of incentive contracting, in: The American Economic Review, Vol. 60, 1970, S. 837-846.

Meier, Markus (2003): Projektmanagement, Stuttgart 2003.

Meinhövel, Harald (1999): Defizite der Principal-Agent-Theorie, Köln 1999, zugl. Diss. Ruhr-Universität Bochum 1998.

Mensch, Gerhard (2002): Investition - Investitionsrechnung in der Planung und Beurteilung von Investitionen, München, Wien 2002. 
Meredith, Jack R.; Mantel, Samuel J. (2003): Project Management - A Managerial Approach, 5th ed., New York 2003.

Meyer, Axel (1992): Finanzinnovationen - Optimale Verträge im Rahmen von Einund Mehr-Agenten-Ansätzen der Prinzipal-Agent-Theorie, Frankfurt am Main 1992.

Meyer, Matthias (2002): Die Heuristik des normativen Prinzipal-Agenten-Modells Wechselseitige Abstimmung vs. einseitige Verhaltenssteuerung, Diss. Ludwig-Maximilians-Universität München 2002.

Mikus, Barbara (1998): ZP-Stichwort: Prinzipal-Agent-Theorie, in: Zeitschrift für Planung, Jg. 9, Heft 4, 1998, S. 451-458.

Miller, Robert W. (1970): Zeit-Planung und Kosten-Kontrolle durch PERT - Ein Leitfaden für die Anwendung in Entwicklung und Fertigung, 2. Aufl., Hamburg u. a. 1970.

Mohnen, Alwine (2002): Performancemessung und die Steuerung von Investitionsentscheidungen, Wiesbaden 2002, zugl. Diss. Universität Köln 2002.

Mohr, Niko (1995): Kommunikation als Interaktionsvariable im Kooperationsmanagement, in: Schertler, Walter (Hrsg.): Management von Unternehmenskooperationen - Branchenspezifische Analysen, Wien 1995, S. 319-385.

Monczka, Robert; Trent, Robert; Handfield, Robert (2002): Purchasing and Supply Chain Management, 2nd ed., Cincinnati 2002.

Mulcahy, Rita (2001): Contracts and procurement management - why should you care?, in: Knutson, Joan (Hrsg.): Project Management for Business Professionals, New York 2001, S. 151-165.

Müller, Christian (2003): Projektmanagement in FuE-Kooperationen - Eine empirische Analyse in der Biotechnologie, Norderstedt 2003, zugl. Diss. Technische Universität Hamburg-Harburg 2003.

Nissen, Volker (1997): Einführung in evolutionäre Algorithmen - Optimierung nach dem Vorbild der Evolution, Braunschweig, Wiesbaden 1997.

Nordqvist, Stefan; Hovmark, Svante; Zika-Viktorsson, Annika (2004): Perceived time pressure and social processes in project teams, in: International Journal of Project Management, Vol. 22, 2004, S. 463-468. 
Olfert, Klaus (2003): Investition, 9., durchges. u. aktual. Aufl., Ludwigshafen 2003.

Olfert, Klaus; Steinbuch, Pitter A. (2003): Organisation, 13., völlig überarb. Aufl., Ludwigshafen 2003.

Özdamar, Linet; Dündar, Hakan (1997): A flexible heuristic for a multi-mode capital constrained project scheduling problem with probabilistic cash inflows, in: Computers and Operations Research, Vol. 24, No. 12, 1997, S. 1187-1200.

Padman, Rema; Roehrig, Stephen R. (1997): A genetic programming approach for heuristic selection in contrained project scheduling, in: Barr, Richard S.; Helgason, Richard V.; Kennington, Jeffery L. (Hrsg.): Interfaces in Computer Science and Operations Research - Advances in Metaheuristics, Optimization, and Stochastic Modeling Technologies, Boston u. a. 1997, S. 405-421.

Padman, Rema; Smith-Daniels, Dwight E. (1993): Early-tardy cost trade-offs in resource constrained projects with cash flows - an optimization-guided heuristic approach, in: European Journal of Operational Research, Vol. 64, 1993, S. 295-311.

Pedersen, Mogens Kühn; Larsen, Michael Holm (2001): Distributed knowledge management based on product state models - the case of decision support in health care administration, in: Decision Support Systems, Vol. 31, 2001, S. $139-158$.

Perridon, Louis; Steiner, Manfred (2003): Finanzwirtschaft der Unternehmung, 12., verb. Aufl., München 2003.

Petersen, Thomas (1989): Optimale Anreizsysteme - Betriebswirtschaftliche Implikationen der Prinzipal-Agenten-Theorie, Wiesbaden 1989, zugl. Diss. Universität Bonn 1988.

Pfaff, Dieter; Zweifel, Peter (1998): Die Principal-Agent Theorie - Ein fruchtbarer Beitrag der Wirtschaftstheorie zur Praxis, in: Wirtschaftswissenschaftliches Studium, Jg. 27, Heft 4, 1998, S. 184-190.

Pfaffmann, Eric (1996): Die vertragstheoretische Perspektive in der Neuen Institutionenökonomik, in: Wirtschaftswissenschaftliches Studium, Jg. 25, Heft 12, 1996, S. 646-648. 
Picot, Arnold (1991): Ökonomische Theorie der Organisation - Ein Überblick über neuere Ansätze und deren betriebswirtschaftliches Anwendungspotential, in: Ordelheide, D.; Rudolph, B.; Büsselmann, E. (Hrsg.): Betriebswirtschaftslehre und ökonomische Theorie, Stuttgart 1991, S. 143-170.

Picot, Arnold; Dietl, Helmut; Franck, Egon (2002): Organisation - Eine ökonomische Perspektive, 3., überarb. und erw. Aufl., Stuttgart 2002.

Picot, Arnold; Reichwald, Ralf; Wigand, Rolf T. (2003): Die grenzenlose Unternehmung - Information, Organisation und Management, 5., aktual. Aufl., Wiesbaden 2003.

Pinder, Jonathan P.; Marucheck, Ann S. (1996): Using discounted cash flow heuristics to improve project net present value, in: Journal of Operations Management, Vol. 14, 1996, S. 229-240.

Project Management Institute (2000): A Guide to the Project Management Body of Knowledge (PMBOK-guide), Newton Square 2000.

Raghu, T. S.; Sen, P. K.; Rao, H. R. (2003): Relative performance of incentive mechanisms - computational modelling and simulation of delegated investment decisions, in: Management Science, Vol. 49, No. 2, 2003, S. 160-178.

Raiffa, Howard; Richardson, John; Metcalfe, David (2003): Negotiation Analysis The Science and Art of Collaborative Decision Making, Cambridge u. a. 2003.

Ramesh, Balasubramaniam; Tiwana, Amrit (1999): Supporting collaborative process knowledge management in new product development teams, in: Decision Support Systems, Vol. 27, 1999, S. 213-235.

Rey, Michael (1999): Informations- und Kommunikationssysteme in Kooperationen, Lohmar 1999, zugl. Diss. Technische Hochschule Aachen 1999.

Richter, Rudolf (1991): Institutionenökonomische Aspekte der Theorie der Unternehmung, in: Ordelheide, Dieter; Rudolph, Bernd; Büsselmann, Elke (Hrsg.): Betriebswirtschaftslehre und ökonomische Theorie, Stuttgart 1991, S. 395-429.

Richter, Rudolf; Furubotn, Eirik G. (2003): Neue Institutionenökonomik - Eine Einführung und kritische Würdigung, 3., überarb. u. erw. Aufl., Tübingen 2003. 
Riegler, Christian (2000): Hierarchische Anreizsysteme im wertorientierten Management - Eine Agency-theoretische Untersuchung, Stuttgart 2000.

Rolfes, Bernd (2003): Moderne Investitionsrechnung - Einführung in die klassische Investitionstheorie und Grundlagen marktorientierter Investitionsentscheidungen, 3., unwes. veränd. Aufl., München, Wien 2003.

Rose, Thomas; Fünffinger, Martin; Knublauch, Holger; Ru, Christian (2002): Prozessorientiertes Wissensmanagement, in: Künstliche Intelligenz, Heft 1, 2002, S. 19-24.

Ross, Stephen A. (1973): The economic theory of agency - the principal's problem, in: American Economic Review, Vol. 63, No. 1, 1973, S. 134-139.

Russell, A. H. (1970): Cash flows in networks, in: Management Science, Vol. 16, No. 5,1972 , S. 357-373.

Sadeh, Arye; Dvir, Dov; Shenhar, Aaron (2000): The role of contract type in the success of $R \& D$ defense projects under increasing uncertainty, in: Project Management Journal, Vol. 31, No. 3, 2000, S. 14-22.

Saliger, Edgar (2003): Betriebswirtschaftliche Entscheidungstheorie - Einführung in die Logik individueller und kollektiver Entscheidungen, 5., durchges. Aufl., München 2003.

Samuelson, William (1986): Bidding for contracts, in: Management Science, Vol. 32, No. 12,1986 , S. $1533-1550$.

Schäffer, Utz (2002): Strategien zur Vermeidung opportunistischen Verhaltens, in: Betriebswirtschaftliche Forschung und Praxis, Jg. 54, Heft 1, 2002, S. 86-99.

Schanz, Günther (1991): Handbuch Anreizsysteme in Wirtschaft und Verwaltung, Stuttgart 1991.

Scherer, F. M. (1964): The theory of contractual incentives for cost reduction, in: Quarterly Journal of Economics, Vol. 78, No. 2, 1964, S. 257-280.

Schertler, Walter (1995): Management von Unternehmenskooperationen - Entwurf eines Bezugsrahmens, in: Schertler, Walter (Hrsg.): Management von Unternehmenskooperationen - Branchenspezifische Analysen, Wien 1995, S. 19-54. 
Scheubrein, Ralph (2003): Informationssysteme zur Unterstützung betrieblicher Entscheidungen, in: Habenicht, Walter; Scheubrein, Beate; Scheubrein, Ralph (Hrsg.): Multi-Cirteria- und Fuzzy-Systeme in Theorie und Praxis, Wiesbaden 2003, S. 221-243.

Schiller, Ulf (1994): Vertikale Unternehmensbeziehungen - Vertrags- und oligopoltheoretische Analysen, Heidelberg 1994, zugl. Diss. Universität Köln 1993.

Schlosser, Andreas (2001): Unternehmenswertsteigerung durch Strategische Allianzen - Ein Ansatz zum wertorientierten Kooperationsmanagement, Diss. Universität St. Gallen 2001.

Schmid, Karl-Heinz (2001): Vertragsmanagement zur Risikominimierung bei externen Entwicklungsprojekten, in: Gassmann, Oliver; Kobe, Carmen; Voit, Eugen (Hrsg.): High-Risk-Projekte - Quantensprünge in der Entwicklung erfolgreich managen, Berlin u. a. 2001, S. 391-419.

Schmidt, Jörg (2004): Der Fachplanervertrag unter Berücksichtigung der Vertragsbeziehungen zwischen dem Auftraggeber des Fachplaners und dessen Auftraggeber, Renningen 2004, zugl. Diss. Universität Rostock 2002.

Schmidt, Reinhard H.; Terberger, Eva (1997): Grundzüge der Investitions- und Finanzierungstheorie, 4., aktual. Aufl., Wiesbaden 1997.

Schmitt, Markus (2002): Optionsorientierte Projektbewertung - Kapitalmarktgerechte Entscheidungen mit der Black-Scholes-Formel, in: Kostenrechnungspraxis, Jg. 46, Heft 3, 2002, S. 147-150.

Schnedler, Wendelin (2004): The Value of Signals in Hidden Action Models - Concepts, Application and Empirical Evidence, Heidelberg, New York 2004, zugl. Diss. Universität Bonn 2003.

Schneeweiß, Christoph (1995): Hierarchical structures in organisations - a conceptual framework, in: European Journal of Operational Research, Vol. 86, S. 4-41.

Schneeweiß, Christoph (2003a): Distributed Decision Making, 2. Aufl., Berlin u. a. 2003.

Schneeweiß, Christoph (2003b): Distributed decision making - a unified approach, in: European Journal of Operational Research, Vol. 150, 2003, S. 237-252. 
Schneeweiß, Christoph; Zimmer, Kirstin (2004): Hierarchical coordination mechanisms within the supply chain, in: European Journal of Operational Research, Vol. 153, 2004, S. 687-704.

Schneider, Dieter (1992): Investition, Finanzierung und Besteuerung, 7., vollst. überarb. u. erw. Aufl., Studentenausg., Wiesbaden 1992.

Schneider, Dieter (1995): Betriebswirtschaftslehre, Band 1 - Grundlagen, 2., verb. und erg. Aufl., München u. a. 1995.

Schneider, Dieter (1997): Betriebswirtschaftslehre, Band 2 - Rechnungswesen, 2., vollst. überarb. und erw. Aufl., München, Wien 1997.

Scholtis, Thomas (1998): Vertragsgestaltung bei Informationsasymmetrie - Probleme und Lösungen bei der Zertifizierung von QM-Systemen nach ISO 9000ff., Wiesbaden 1998, zugl. Diss. Universität Frankfurt am Main 1997.

Schrader, Stephan (1990): Zwischenbetrieblicher Informationstransfer - Eine empirische Analyse kooperativen Verhaltens, Berlin 1990, zugl. Diss. Universität München 1989.

Schwartz, Alan (1992): Legal contract theories and incomplete contracts, in: Werin, Lars; Wijkander, Hans (Hrsg.): Contract Economics, Cambridge 1992, S. 76-108.

Schwarze, Jochen (2001): Projektmanagement mit Netzplantechnik, 8., vollst. überarb. u. wesentl. erw. Aufl., Herne, Berlin 2001.

Schwindt, Christoph; Zimmermann, Jürgen (2002): Parametrische Optimierung als Instrument zur Bewertung von Investitionsprojekten, in: Zeitschrift für Betriebswirtschaft, Jg. 72, Heft 6, 2002, S. 593-617.

Sepil, C.; Ortac, N. (1997): Performance of the heuristic procedures for constrained projects with progress payments, in: Journal of the Operational Research Society, Vol. 48, 1997, S. 1123-1130.

Shelanski, Howard A.; Klein, Peter G. (1995): Empirical research in transaction cost economics - a review and assessment, in: The Journal of Law, Economics, and Organization, Vol. 11, No. 12, 1995, S. 335-361. 
Shenhar, Aaron J.; Tishler, Asher; Dvir, Dov; Lipovetsky, Stanislav; Lechler, Thomas (2002): Refining the search for project success factors - a multivariate, typological approach, in: R\&D Management, Vol. 32, No. 2, 2002, S. 111-126.

Shr, Jin F.; Chen, Wei T. (2003): A method to determine minimum contract bids for incentive highway projects, in: International Journal of Project Management, Vol. 21, 2003, S. 601-615.

Shtub, Avraham (1986): The trade-off between the net present cost of a project and the probability to complete it on schedule, in: Journal of Operations Management, Vol. 6, No. 4, S. 461-470.

Shtub, Avraham; Etgar, R. (1997): A branch and bound algorithm for scheduling projects to maximize net present value - the case of time dependent, contingent cash flows, in: International Journal of Production Research, Vol. 35, No. 12, 1997, S. 3367-3378.

Sieg, Gernot (2000): Spieltheorie, München, Wien 2000.

Simister, Stephen (2003): Bidding, in: Turner, Rodney (Hrsg.): Contracting for Project Management, Burlington 2003, S. 117-124.

Smith-Daniels, Dwight E.; Aquilano, Nicholas J. (1987): Using a late-start resourceconstrained project schedule to improve project net present value, in: Decision Sciences, Vol. 18, No. 4, 1987, S. 617-630.

Smith-Daniels, Dwight E.; Padman, Rema; Smith-Daniels, Vicki L. (1996): Heuristic scheduling of capital constrained projects, in: Journal of Operations Management, Vol. 14, 1996, S. 241-254.

Smith-Daniels, Dwight E.; Smith-Daniels, Vicki L. (1987): Maximizing the net present value of a project subject to materials and capital constraints, in: Journal of Operations Management, Vol. 7, No. 1, S. 33-45.

Specht, Dieter; Kahmann, Joachim (2000): Regelung kooperativer Tätigkeit im virtuellen Unternehmen, in: Zeitschrift für Betriebswirtschaft, Ergänzungsheft 2, 2000, S. 55-73.

Spremann, Klaus (1990): Asymmetrische Information, in: Zeitschrift für Betriebswirtschaft, Jg. 60, Heft 5/6, 1990, S. 561-586. 
Staehle, Wolfgang H. (1999): Management - Eine verhaltenswissenschaftliche Perspektive, 8., überarb. Aufl., München 1999.

Steinmann, Horst; Schreyögg, Georg (2000): Management - Grundlagen der Unternehmensführung, 5., überarb. Aufl., Wiesbaden 2000.

Stelzer, Dirk (2003): Informations- versus Wissensmanagement - Versuch einer Abgrenzung, in: Kemper, Hans-Georg; Mülder, Wilhelm (Hrsg.): Informationsmanagement, Lohmar 2003, S. 25-41.

Steuer, Ralph E.; Na, Paul (2003): Multiple criteria decision making combined with finance - a categorized bibliographic study, in: European Journal of Operational Research, Vol. 150, 2003, S. 496-515.

Steven, Marion; Otterpohl, Lars (2000): Virtuelle Unternehmen aus spieltheoretischer Sicht, in: Zeitschrift für Betriebswirtschaft, Ergänzungsheft 2, 2000, S. 177-200.

Stiasni, Christian (1994): Entscheidungsgestützte Projektplanung - Darstellung eines rechnerbasierten Modells, Wiesbaden 1994, zugl. Diss. Johann-Wolfgang Goethe-Universität Frankfurt am Main 1993.

Streubel, Frauke (2000): Organisatorische Gestaltung und Informationsmanagement in der lernenden Unternehmung - Bausteine eines Managementkonzeptes organisationalen Lernens, Frankfurt am Main u. a. 2000, zugl. Diss. RuhrUniversität Bochum 1999.

Talbot, F. Brian (1982): Resource-constrained project scheduling with time-resource tradeoffs - the nonpreempitve case, in: Management Science, Vol. 28, No. 10,1982 , S. $1197-1210$.

Tavares, L. Valadares (1994): A stochastic model to control project duration and expenditure, in: European Journal of Operational Research, Vol. 78, 1994, S. 262-266.

Tavares, L. Valadares (2002): A review of the contribution of operational research to project management, in: European Journal of Operational Research, Vol. 136, 2002, S. 1-18.

Tavares, L. Valadares; Ferreira, J. Antunes; Coelho, J. Silva (1998): On the optimal management of project risk, in: European Journal of Operational Research, Vol. 197, 1998, S. 451-469. 
Theilen, Bernd (1996): Optimal Contract and Organizational Design under Moral Hazard and Adverse Selection, Köln, 1996, zugl. Diss. Universität Köln 1995.

Trebilcock, Michael J. (1993): The Limits of Freedom of Contract, Cambridge, London 1993.

Tröndle, Dirk (1986): Kooperationsmanagement - Steuerung interaktioneller Prozesse bei Unternehmungskooperationen, Diss. Albert-Ludwigs-Universität Freiburg im Breisgau 1986.

Trumpp, Andreas (1995): Kooperation unter asymmetrischer Information - Eine Verbindung von Prinzipal-Agenten-Theorie und Transaktionskostenansatz, Neuried 1995, zugl. Diss. Universität Marburg 1994.

Turner, J. Rodney (2003): Contracting for Project Management, Hampshire 2003.

Turner, J. Rodney (2004): Farsighted project contract management - incomplete in its entirety, in: Construction Management and Economics, Vol. 22, 2004, S. 75-83.

Turner, J. Rodney; Müller, Ralf (2003): On the nature of the project as a temporary organization, in: International Journal of Project Management, Vol. 21, 2003, S. $1-8$.

Turner, J. Rodney; Simister, Stephen J. (2001): Project contract management and a theory of organization, in: International Journal of Project Management, Vol. 19, 2001, S. 457-464.

Ulusoy, Gündüz; Cebelli, Serkan (2000): An equitable approach to the payment scheduling problem in project management, in: European Journal of Operational Research, Vol. 127, 2000, S. 262-278.

Ulusoy, Gündüz; Özdamar, Linet (1995): A heuristic scheduling algorithm for improving the duration and net present value of a project, in: International Journal of Operations and Production Management, Vol. 15, No. 1, 1995, S. 89-98.

Ulusoy, Gündüz; Sivrikaya-Serifoglu, Funda; Sahin, Sule (2001): Four payment models for the multi-mode resource constrained project scheduling problem with discounted cash flows, in: Annals of Operations Research, Vol. 102, 2001, S. 237-261. 
Vaaland, Terje I. (2004): Improving project collaboration - start with the conflicts, in: International Journal of Project Management, Vol. 22, 2004, S. 447-454.

Vanhoucke, Mario; Demeulemeester, Erik L. (2003): The application of project scheduling techniques in a real-life environment, in: Project Management Journal, Vol. 34, No. 1, 2003, S. 30-42.

Vanhoucke, Mario; Demeulemeester, Erik L.; Herroelen, Willy S. (2001a): On maximizing the net present value of a project under renewable resource constraints, in: Management Science, Vol. 47, No. 8, 2001, S. 1113-1121.

Vanhoucke, Mario; Demeulemeester, Erik L.; Herroelen, Willy S. (2001b): Maximizing the net present value of a project with linear time-dependent cash flows, in: International Journal of Production Research, Vol. 39, No. 14, 2001, S. $3159-3181$.

Vanhoucke, Mario; Demeulemeester, Erik L.; Herroelen, Willy S. (2003): Progress payments in project scheduling problems, in: European Journal of Operational Research, Vol. 148, 2003, S. 604-620.

Veld, J.; Peeters, W. A. (1989): Keeping large projects under control - the importance of contract type selection, in: Project Management, Vol. 7, No. 3, 1989, S. $155-162$.

Vincenti, Aurelio J. (2002): Wirkungen asymmetrischer Informationsverteilung auf die Unternehmensbewertung, in: Betriebswirtschaftliche Forschung und Praxis, Jg. 54, Heft 1, 2002, S. 55-68.

Völkner, Peer (1998): Modellbasierte Planung von Geschäftsprozessabläufen - Entwicklung eines Entscheidungsunterstützungssystems auf Grundlage objektorientierter Simulation, Wiesbaden 1998, zugl. Diss. Ruhr-Universität Bochum 1998.

Völkner, Peer; Werners, Brigitte (2000): A decision support system for business process planning, in: European Journal of Operational Research, Vol. 125, 2000, S. 633-647.

von Spreckelsen, Burkhard (2004): Performance Measurement von F\&E-Kooperationsprojekten, Diss. Universität St. Gallen 2004.

Voß, Stefan; Gutenschwager, Kai (2001): Informationsmanagement, Berlin u. a. 2001. 
Voß, Stefan; Schneidereit, Gabriele (2002): Interdependencies between supply contracts and transaction costs, in: Seuring, Stefan; Goldbach, Maria (Hrsg.): Cost Management in Supply Chains, Heidelberg, New York 2002.

Vroom, Victor H. (1964): Work and Motivation, New York u. a. 1964.

Walker, Anthony (2002): Project Management in Construction, 4th ed., Oxford 2002.

Wall, Friederike (2003): Die normative Prinzipal-Agenten-Theorie als Untersuchungsansatz für Management-Support-Systeme, in: Wirtschaftsinformatik, Jg. 45, Heft 5, 2003, S. 521-526.

Ward, Stephen; Chapman, Chris (1994): Choosing contractor payment terms, in: International Journal of Project Management, Vol. 12, No. 4, 1994, S. 216-221.

Ward, Stephen; Chapman, Chris (1995): Evaluating fixed price incentive contracts, in: Omega International Journal of Management Science, Vol. 23, No. 1, 1995, S. 49-62.

Ward, Stephen; Chapman, Chris (2003): Transforming project risk management into project uncertainty management, in: International Journal of Project Management, Vol. 21, 2003, S. 97-105.

Weber, Kurt E. (2003): Vertragsinhalte und -management, in: Projektmanagement Fachmann, Band 2, Eschborn 2003, S. 963-1001.

Weihrich, Heinz; Koontz, Harold (1993): Management - A Global Perspective, 10th ed., New York u. a. 1993.

Weinert, Ansfried B. (1992): Anreizsysteme - Verhaltenswissenschaftliche Dimension, in: Frese, Erich (Hrsg.): Handwörterbuch der Organisation, 3., völlig neu gestaltete Aufl., Stuttgart 1992, Sp. 122-133.

Welge, Martin K.; Al-Laham, Andreas (2003): Strategisches Management - Grundlagen, Prozess, Implementierung, 4., aktual. Aufl., Wiesbaden 2003.

Wenger, Ekkehard; Terberger, Eva (1988): Die Beziehung zwischen Agent und Prinzipal als Baustein einer ökonomischen Theorie, in: Wirtschaftswissenschaftliches Studium, Jg. 17, Heft 10, 1988, S. 506-514.

Werin, Lars; Wijkander, Hans (1992): Contract Economics, Cambridge 1992. 
Werners, Brigitte (1993): Unterstützung der strategischen Technologieplanung durch wissensbasierte Systeme, Aachen 1993.

Werners, Brigitte (2000): Projektsteuerung durch die Zuweisung von Vorgangspuffern, in: Wirtschaftswissenschaftliches Studium, Jg. 29, Heft 8, 2000, S. $422-427$.

Werners, Brigitte; Slaghuis, Bernd (2004): Contract Management - Vertragsdesign und Anreizsysteme am Beispiel von Investitionsprojekten, in: Wirtschaftswissenschaftliches Studium, Jg. 33, Heft 6, 2004, S. 352-358.

Werners, Brigitte; Thorn, Jens (2003): Collaborative Supply Chain Planning, in: Wirtschaftswissenschaftliches Studium, Jg. 32, Heft 10, 2003, S. 590-595.

Werners, Brigitte; Thorn, Jens; Freiwald, Stephanie (2003): Performance-Kriterien für das Supply Chain Design, in: Supply Chain Management, Jg. 3, Heft 3, 2003, S. 7-16.

Werners, Brigitte; Wolf, Andreas (2004): Simulationsbasierte Steuerung risikobehafteter Projekte, in: Biethahn, Jörg (Hrsg.): Proceedings zum 9. Symposium: Simulation als betriebliche Entscheidungshilfe - Neuere Werkzeuge und Anwendungen aus der Praxis, Göttingen 2004, S. 95-114.

Wicher, Hans (2003): Projektorganisation, in: Bernecker, Michael; Eckrich, Klaus (Hrsg.): Handbuch Projektmanagement, München 2003, S. 69-84.

Widemann, Max R. (1992): Project and Program Risk Management - A Guide to Managing Project Risks and Opportunities, PMBOK Handbook Series No. 6, Newton Square 1992.

Wienhold, Klaus (2003): Prozess- und controllingorientiertes Projektmanagement für komplexe Projektfertigung, Diss. Universität Dortmund 2003.

Wildförster, Ricarda; Wingen, Sascha (2001): Projektmanagement und Probleme Systemische Perspektiven auf Organisationsberatung und Begleitforschung, Heidelberg 2001.

Willenbrock, Jack H. (1973): Utility function determination for bidding models, in: Journal of the Construction Division, Vol. 99, 1973, S. 133-153.

Williamson, Oliver E. (1975): Markets and Hierarchies - Analysis and Antitrust Implications, New York 1975. 
Wittmann, Waldemar (1959): Unternehmung und unvollkommene Information Unternehmerische Voraussicht, Ungewissheit und Planung, Köln 1959.

Wolf, Andreas (2004): Dynamische Projektsteuerung unter Risiko - Entscheidungsunterstützung mit Anpassung an die vorliegende Risikoeinstellung, Diss. Ruhr-Universität Bochum 2004.

Wolf, Joachim (2003): Organisation, Management, Unternehmensführung - Theorien und Kritik, Wiesbaden 2003.

Wolff, Birgitta (1995): Organisation durch Verträge - Koordination und Motivation in Unternehmen, Wiesbaden 1995.

Wolff, Birgitta (2000): Ronald Coase und die ökonomische Theorie der Organisation, in: Pies, Ingo; Leschke, Martin (Hrsg.): Ronald Coase' TransaktionskostenAnsatz, Tübingen 2000, S. 31-57.

Wollnik, Michael (1988): Ein Referenzmodell des Informations-Managements, in: Informations Management, Jg. 3, 1988, S. 34-43.

Wong, Peter Shek-Pui; Cheung, Sai-On (2004): Trust in construction partnering views from parties of the partnering dance, in: International Journal of Project Management, Vol. 22, 2004, S. 437-446.

Yang, Kum Khiong; Talbot, F. Brian; Patterson, James H. (1992): Scheduling a project to maximize its net present value - an integer programming approach, in: European Journal of Operational Research, Vol. 64, 1992, S. 188-198.

Yang, Kum Khiong; Tay, Lee Choo; Sum, Chee Chuong (1995): A comparison of stochastic scheduling rules for maximizing project net present value, in: European Journal of Operational Research, Vol. 85, 1995, S. 327-339.

Zell, Helmut (2003): Grundbegriffe und Grundstrukturen von Projekten, in: Bernecker, Michael; Eckrich, Klaus (Hrsg.): Handbuch Projektmanagement, München 2003, S. 53-68.

Zimmermann, Hans-Jürgen (1971): Netzplantechnik, Berlin u. a. 1971. 


\title{
Bochumer Belträge zur Unternehmensführung
}

\author{
Herausgegeben vom Direktorium des Instituts \\ für Unternehmensführung der Ruhr-Universität Bochum
}

Band 1 Busse von Colbe, Walther/Mattessich, Richard (Hrsg.): Der Computer im Dienste der Unternehmungsführung (1968)

Band 2 Busse von Colbe, Walther/Meyer-Dohm, Peter (Hrsg.): Unternehmerische Planung und Entscheidung (1969)

Band 3 Anthony, Robert N.: Harvard-Fälle aus der Praxis des betrieblichen Rechnungswesens. Herausgegeben von Richard V. Mattessich unter Mitarbeit von Klaus Herrnberger und Wolf Lange (1969)

Band 4 Mattessich, Richard: Die wissenschaftlichen Grundlagen des Rechnungswesens (1970)

Band 5 Schweim, Joachim: Integrierte Unternehmungsplanung (1969)

Band 6 Busse von Colbe, Walther (Hrsg.): Das Rechnungswesen als Instrument der Unternehmungsführung (1969)

Band 7 Domsch, Michel: Simultane Personal- und Investitionsplanung im Produktionsbereich (1970)

Band 8 Leunig, Manfred: Die Bilanzierung von Beteiligungen. Eine bilanztheoretische Untersuchung (1970)

Band 9 Franke, Reimund: Betriebsmodelle. Rechensystem für Zwecke der kurzfristigen Planung, Kontrolle und Kalkulation (1972)

Band 10 Wittenbrink, Hartwig: Kurztristige Erfolgsplanung und Erfolgskontrolle mit Betriebsmodellen (1975)

Band 11 Lutter, Marcus (Hrsg.): Recht und Steuer der internationalen Unternehmensverbindungen (1972)

Band 12 Niebling, Helmut: Kurzfristige Finanzrechnung auf der Grundlage von Kosten- und Erlösmodellen (1973)

Band 13 Perlitz, Manfred: Die Prognose des Unternehmenswachstums aus Jahresabschlüssen deutscher Aktiengesellschaften (1973)

Band 14 Niggemann, Walter: Optimale Informationsprozesse in betriebswirtschaftlichen Entscheidungssituationen (1973)

Band 15 Richardt, Harald: Der aktienrechtliche Abhängigkeitsbericht unter ökonomischen Aspekten (1974)

Band 16 Backhaus, Klaus: Direktvertrieb in der Investitionsgüterindustrie - Eine Marketing-Entscheiung (1974)

Band 17 Plinke, Wulff: Kapitalsteuerung in Filialbanken (1975)

Band 18 Steffen, Rainer: Produktionsplanung bei Fließbandfertigung (1977)

Band 19 Kolb, Jürgen: Industrielle Erlösrechnung - Grundlagen und Anwendungen (1978)

Band 20 Busse von Colbe, Walther/Lutter, Marcus (Hrsg.): Wirtschaftsprüfung heute: Entwicklung oder Reform? (1977)

Band 21 Uphues, Peter: Unternehmerische Anpassung in der Rezession (1979)

Band 22 Gebhardt, Günther: Insolvenzprognosen aus aktienrechtlichen Jahresabschlüssen (1980)

Band 23 Domsch, Michel: Systemgestützte Personalarbeit (1980)

Band 24 Schmied, Volker: Alternativen der Arbeitsgestaltung und ihre Bewertung (1982) 
Band 25 Wäscher, Gerhard: Innerbetriebliche Standortplanung bei einfacher und mehrfacher Zielsetzung (1982)

Band 26 Weber, Martin: Entscheidungen bei Mehrfachzielen - Verfahren zur Unterstützung von Individual- und Gruppenentscheidungen (1983)

Band 27 Kroesen, Alfred: Instandhaltungsplanung und Betriebsplankostenrechnung (1983)

Band 28 Plinke, Wulf: Erlösplanung im industriellen Anlagengeschäft (1985)

Band 29 Chamoni, Peter: Simulation störanfälliger Systeme (1986)

Band 30 Arning, Andreas: Die wirtschaftliche Bewertung der Zentrenfertigung - Dargestellt am Beispiel einer Fertigungsinsel (1987)

Band 31 Gebhardt, Günther: Finanzielle Planung und Kontrolle bei internationaler Unternehmenstãtigkeit

Band 32 Markiewicz, Michael: Ersatzteildisposition im Maschinenbau - Betriebswirtschaftliche Methoden der Planung und Überwachung (1988)

Band 33 Pellens, Bernd: Der Informationswert von Konzernabschlüssen - Eine empirische Untersuchung deutscher Börsengesellschaften (1989)

Band 34 Mrotzek, Rüdiger: Bewertung direkter Auslandsinvestitionen mit Hilfe betrieblicher Investitionskalkūle (1989)

Band 35 Deppe, Joachim: Quality Circle und Lernstatt - Ein integrativer Ansatz (1989, 3. Auflage 1993)

Band 36 Rademacher, Michael: Arbeitszeitverkürzung und -flexibilisierung - Formen und betriebliche Auswirkungen (1990)

Band 37 Kaiser, Klaus: Kosten- und Leistungsrechung bei automatisierter Produktion (1991, 2. Auflage 1993)

Band 38 Müller, Hermann: Industrielle Abfallbewältigung - Entscheidungsprobleme aus betriebswirtschaftlicher Sicht (1991)

Band 39 Schörner, Peter: Gesetzliches Insiderhandelsverbot - Eine ordnungspolitische Analyse (1991)

Band 40 Bentler, Martin: Grundsätze ordnungsmäßiger Bilanzierung für die Equitymethode (1991)

Band 41 Brüggerhoff, Jürgen: Management von Desinvestitionen (1992)

Band 42 Bröker, Erich W.: Erfolgsrechnung im industriellen Anlagengeschäft - Ein dynamischer Ansatz auf Zahlungsbasis - (1993)

Band 43 Frankenberg, Peter: Transnationale Analyse US-amerikanischer und deutscher Jahresabschlüsse - Eine theoretische und empirische Untersuchung (1993)

Band 44 Kleinaltenkamp, Michael: Standardisierung und Marktprozeß - Entwicklungen und Auswirkungen im CIM-Bereich (1993)

Band 45 Pellens, Bernhard: Aktionärsschutz im Konzern - Empirische und theoretische Analyse der Reformvorschläge der Konzernverfassung (1994)

Band 46 Reckenfelderbäumer, Martin: Marketing-Accounting im Dienstleistungsbereich - Konzeption eines prozeßkostengestūtzten Instrumentariums (1995)

Band 47 Knittel, Friedrich: Technikgestützte Kommunikation und Kooperation im Büro. Entwicklungshindernisse - Einsatzstrategien - Gestaltungskonzepte (1995)

Band 48 Riezler, Stephan: Lebenszyklusrechnung - Instrument des Controlling strategischer Projekte (1996)

Band 49 Schulte, Jörn: Rechnungslegung und Aktienkursentwicklung - Erklärung und Prognose von Aktienrenditen durch Einzel- und Konzernabschlußdaten (1996) 
Band 50 Muhr, Martin: Zeitsparmodelle in der Industrie - Grundlagen und betriebswirtschaftliche Bedeutung mehrjähriger Arbeitszeitkonten (1996)

Band 51 Brotte, Jörg: US-amerikanische und deutsche Geschäftsberichte. Notwendigkeit, Regulierung und Praxis jahresabschlußergänzender Informationen (1997)

Band 52 Gersch, Martin: Vernetzte Geschäftsbeziehungen. Die Nutzung von EDI als Instrument des Geschäftsbeziehungsmanagement (1998)

Band 53 Währisch, Michael: Kostenrechnungspraxis in der deutschen Industrie. Eine empirische Studie (1998)

Band 54 Völkner, Peer: Modellbasierte Planung von Geschäftsprozeßabläufen (1998)

Band 55 Fülbier, Rolf Uwe: Regulierung der Ad-hoc-Publizität. Ein Beitrag zur ökonomischen Analyse des Rechts (1998)

\section{Band 1 - 55 erschienen beim Gabler Verlag Wiesbaden}

Band 56 Ane-Kristin Reif-Mosel: Computergestützte Kooperation im Büro. Gestaltung unter Berücksichtigung der Elemente Aufgabe, Struktur, Technik und Personal (2000)

Band 57 Claude Tomaszewski: Bewertung strategischer Flexibilität beim Unternehmenserwerb. Der Wertbeitrag von Realoptionen (2000)

Band 58 Thomas Erler: Business Objects als Gestaltungskonzept strategischer Informationssystemplanung (2000)

Band 59 Joachim Gassen: Datenbankgestützte Rechnungslegungspublizität. Ein Beitrag zur Evolution der Rechnungslegung (2000)

Band 60 Frauke Streubel: Organisatorische Gestaltung und Informationsmanagement in der lernenden Unternehmung. Bausteine eines Managementkonzeptes organisationalen Lernens (2000)

Band 61 Andreas von der Gathen: Marken in Jahresabschluß und Lagebericht (2001)

Band 62 Lars Otterpohl: Koordination in nichtlinearen dynamischen Systemen (2002)

Band 63 Ralf Schremper: Aktienrückkauf und Kapitalmarkt. Eine theoretische und empirische Analyse deutscher Aktienrückkaufprogramme (2002)

Band 64 Peter Ruhwedel: Aufsichtsratsplanungssysteme. Theoretische Grundlagen und praktische Ausgestaltung in Publikumsaktiengesellschaften (2002)

Band 65 Jens Thorn: Taktisches Supply Chain Planning. Planungsunterstützung durch deterministische und stochastische Optimierungsmodelle (2002)

Band 66 Dirk Beier: Informationsmanagement aus Sicht der Betriebswirtschaftslehre. Theoretische Ansătze und das Beispiel Mobile Business. (2002)

Band 67 Nils Crasselt: Wertorientierte Managemententlohnung, Unternehmensrechnung und Investitionssteuerung. Analyse unter Berücksichtigung von Realoptionen. (2003)

Band 68 Franca Ruhwedel: Eigentümerstruktur und Unternehmenserfolg. Eine theoretische und empirische Analyse deutscher börsennotierter Unternehmen. (2003)

Band 69 Andreas Bonse: Informationsgehalt von Konzernabschlüssen nach HGB, IAS und USGAAP. Eine empirische Analyse aus Sicht der Eigenkapitalgeber. (2004)

Band 70 Thorsten Sellhorn: Goodwill Impairment. An Empirical Investigation of Write-Offs under SFAS 142. (2004) 
Band 71 Bernd Slaghuis: Vertragsmanagement für Investitionsprojekte. Quantitative Projektplanung zur Unterstützung des Contract Managements unter Berücksichtigung von Informationsasymmetrie. (2005)

wuw.peterlang.de 
Bernd Slaghuis - 978-3-631-75513-6 


\section{Prozess- und controllingorientiertes Projektmanagement für komplexe Projektfertigung}

Frankfurt am Main, Berlin, Bern, Bruxelles, New York, Oxford, Wien, 2004.

XXII, 508 S., 214 Abb.

(1) Controlling und Management.

Herausgegeben von Thomas Reichmann und Martin K. Welge. Bd. 27

ISBN 3-631-52230-4 · br. € 79.50*

Die Zielsetzung der Arbeit liegt im Aufbau eines modularen prozess- und controllingorientierten Projektmanagementsystems für komplexe Projektfertiger, das sämtliche projektrelevanten Teilaufgaben einschließt. Das entworfene System ist multiprojektfähig und somit mehrjahresfähig. Aufbauend auf den (1) grundlegenden Methoden der Prozessgestaltung (Workflow-Modellierung mit eEPK) und -optimierung (Business Process Reengineering) wird für die Projekthauptphasen (Projektvorbereitungsphase, Projektabwicklungsphase und Projektnachbereitungsphase) eine prozessorientierte Controlling-Konzeption für das Projektmanagementsystem entwickelt. Es wird gezeigt, wie durch das umgesetzte integrierte, intranetgestützte Informations- und Kommunikationssystem die Prozessunterstützung erreicht wird.

Aus dem Inhalt: Grundlagen des Projektmanagements · Konzeption eines prozess- und controllingorientierten Projektmanagementsystems .

둥 DV-Implementierung des Projektmanagementsystems

Frankfurt am Main · Berlin · Bern · Bruxelles · New York · Oxford · Wien

Auslieferung: Verlag Peter Lang AG

Moosstr. 1, CH-2542 Pieterlen

Telefax 0041 (0) $32 / 3761727$

*inklusive der in Deutschland gültigen Mehrwertsteuer

Preisänderungen vorbehalten

Homepage http://umw.peterlang.de 
Bernd Slaghuis - 978-3-631-75513-6 